\title{
Characterization of Past and Present Solid Waste Streams from the Plutonium Finishing Plant
}

D. R. Duncan

B. A. Mayancsik

Westinghouse Hanford Company

J. A. Pottmeyer

E. J. Vejvoda

J. A. Reddick

K. M. Sheldon

M. I. Weyns

Los Alamos Technical Associates

Date Published

February 1993

Prepared for the U.S. Department of Energy Office of Environmental Restoration and Waste Management

\section{(2) Westinghouse P.O. Box 1970 \\ (W) Hanford Company Richland. Washington 99352}

Hanford Operations and Engineering Contractor for the

U.S. Department of Energy under Contract DE-AC06-87RL 10930

\section{MASTER}

DISTRIBUTION OF THIS DOCUMENT IS UNLIMITED 


\section{LEGAL DISCLAIMER}

This report was prepared as an account of work sponsored by an agency of the United States Government. Neither the United States Government nor any agency thereof, nor any of their employees, nor any of their contractors, subcontractors or their employees, makes any warranly, express or implied. or assumes any legal liability or responsibility for the accuracy, completeness, or any third party's use or the results of such use of any information, apparatus, product, or process disclosed, or represents that its use would not infringe privately owned rights. Reference herein to any specific commercial product, process, or service by trade name, trademark, manufacturer, or otherwise, does not necessarily constitute or imply its endorsement, recommendation, or favoring by the United States Government or any agency thereof or its contractors or subcontractors. The views and opinions of authors expressed herein do not necessarily state or reflect those of the United States Government or any agency thereof.

This report has been reproduced from the best available copy. Available in paper copy and microfiche.

Available to the U.S. Department of Energy and its contractors from

Office of Scientific and Technical Information

P.O. Box 62

Oak Ridge, TN 3783

(615) $576-8401$

Available to the public from the U.S. Department of Commerce National Technical information Service

5285 Port Royal Foad

Springfield, VA 22161

(703) 487.4650

Printed in the United States of America 
Prepared by:

Approved by:
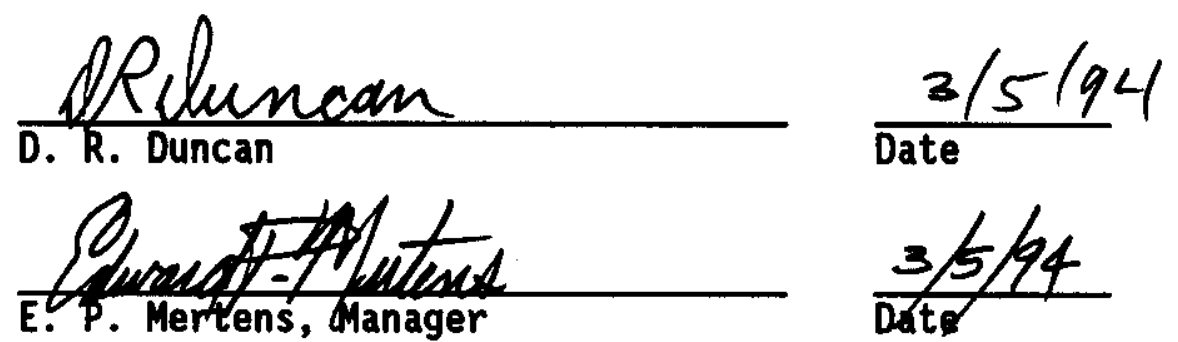

Waste Characterization
Date

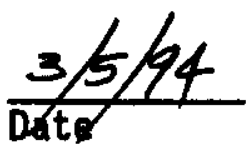

\section{DISCLAIMER}

This report was prepared as an account of work sponsored by an aseacy of the Unitod States Government. Neither the United Stutes Government nor any agency thereof, nor any of their employecs, makes any warranty, express or implied, or ascumes any legal liability or responsibility for the accuracy, completeneas, or usefulneas of any information, apparatus, product, or process discloeed, or represents that its use would not infringe privately owned rights. Reference berein to any upecific commercial product, proceas, or service by trede name, trademark, manufacturer, or otherwive does not necesarily conatitute or imply its endorsement, recommendation, of favoring by the United States Government or any agency thereof. The views and opinions of suthors expreased herein do not necesestily state or reflect those of the United States Government or any agency thereof. 


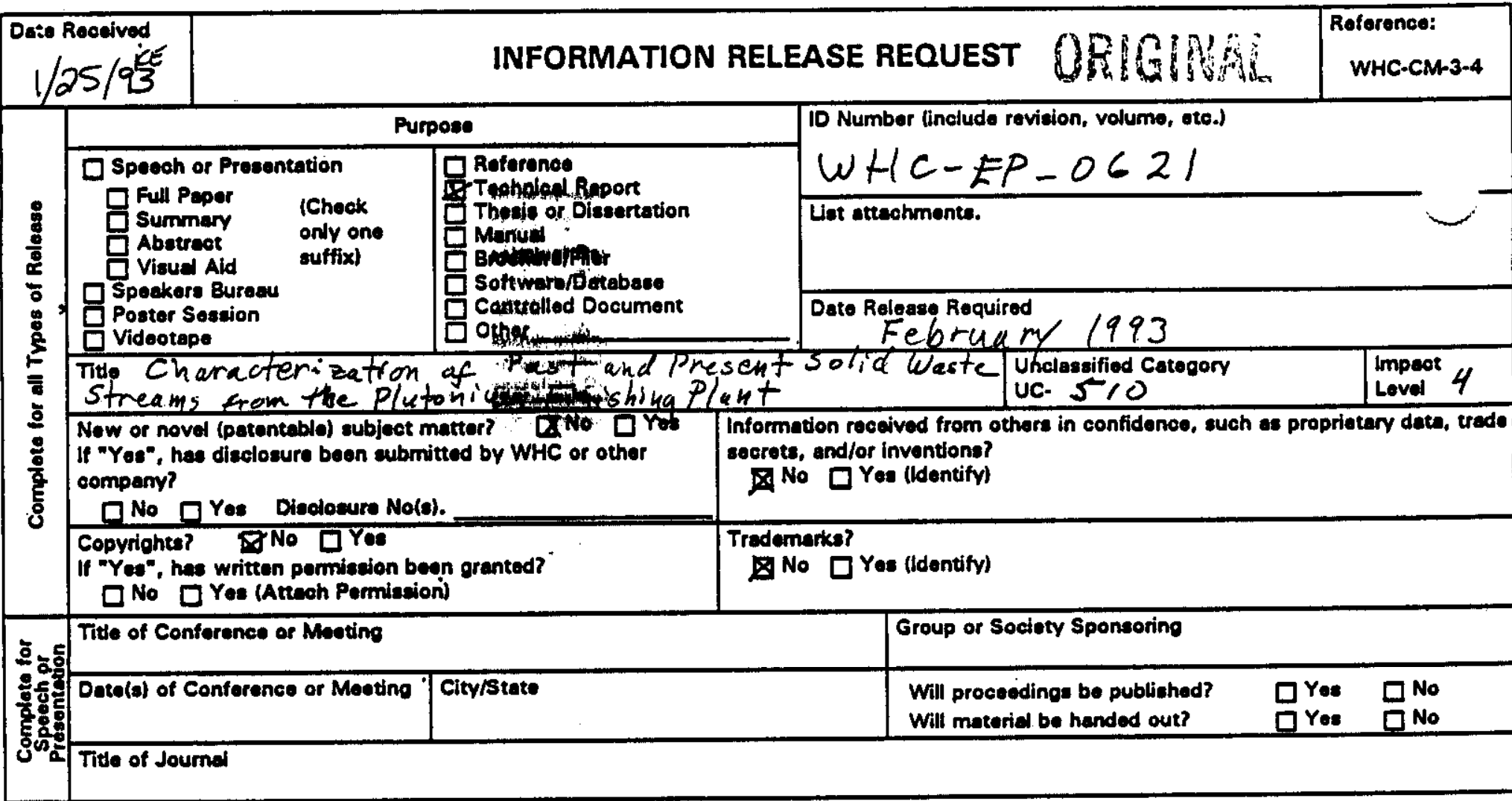

\section{CHECKLIST FOR SIGNATORIES}

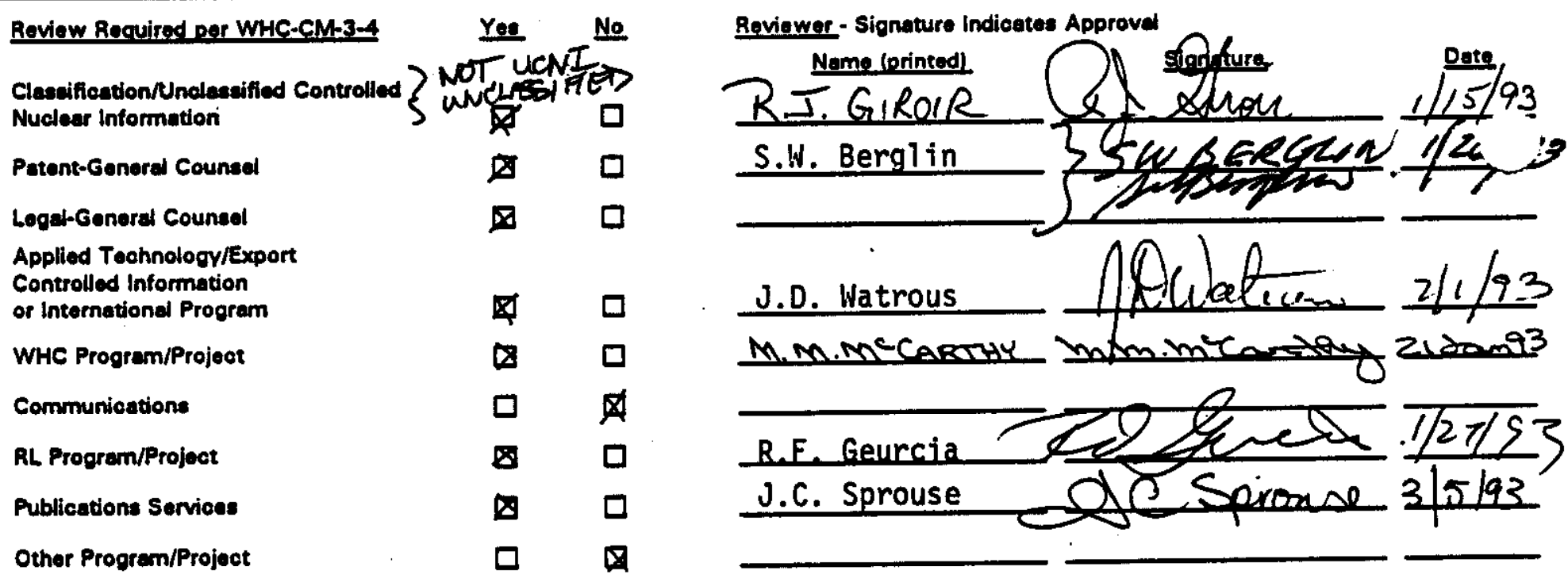

Information conforms to all applicable requirements. The above information is certified to be correct.

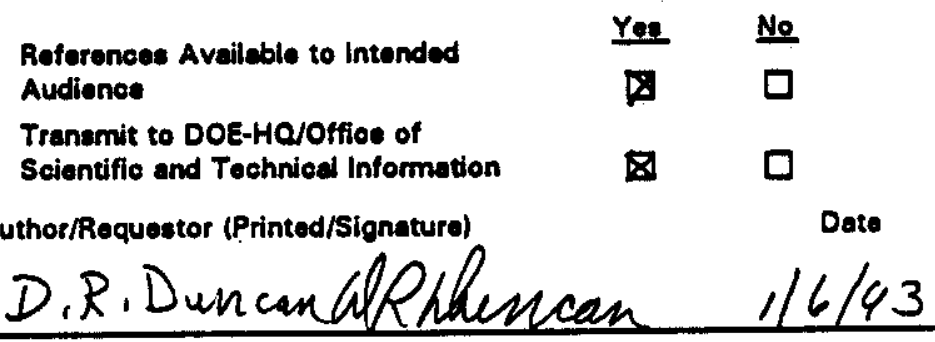

Intended Audience

$\square$ Internal $\square$ Sponsor Externel

Responsible Manager (Printed/ganaturet) Mentexs
INFORMATION RELEASE ADMINISTRATION APPROVAL STAMP

Stamp is required before release. Releese is contingent upon resolution of mandatory commants.

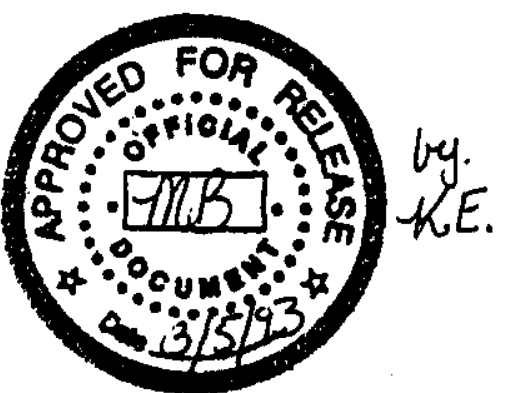

Date Cancelled

Date Disapproved 


\section{WHC-EP-0621}

\section{ACKNONLEDGEMENTS}

The following people generously shared their time and knowledge of the Plutonium Finishing Plant Operations and Solid Waste generation:
W. R. Buchanan
S. L. Claybrook
H. V. Colton
L. J. Estey
B. R. Fitzpatrick
S. L. Galstad
S. T. Hurlbut
D. J. Hart
E. J. Manthos
P. A. Meyer
E. C. Mincey
M. L. Sheriff
J. A. Teal
F. F. Walters 
WHC-EP-0621

\section{EXECUTIVE SURMARY}

During the next two decades the transuranic (TRU) wastes now stored in the burial trenches and storage facilities at the Hanford Site are to be retrieved, processed at the Waste Receiving and Processing (WRAP) Facility, and shipped to the Waste Isolation Pilot Plant (WIPP) near Carlsbad, New Mexico for final disposal. Over 50\% of the TRU waste to be retrieved for shipment to the WIPP has been generated at the Plutonium Finishing Plant (PFP), also known as the Plutonium Processing and Storage Facility and Z Plant.

The purpose of this report is to characterize the radioactive solid wastes generated by the PFP since its construction in 1947 using process knowledge, existing records, and history obtainned from interviews. The PFP is currently operated by Westinghouse Hanford Company (WHC) for the U.S. Department of Energy (DOE). This report specifically excludes radioactive wastes generated at the Plutonium Metallurgy Laboratory (231-Z facility), which is operated for the DOE by the Pacific Northwest Laboratory (PNL). The wastes generated at the $231-Z$ facility will be covered in a later report.

The PFP is a collection of facilities that have been used primarily to produce plutonium metal and plutonfum oxide to support national defense activities. The special nuclear materials (SNM) that form the feed for the PFP processing include, but are not limited to, plutonium bearing scrap, rework plutonium, incinerator ash, unirradiated fuel rods, and unique plutonium bearing solutions (e.g., plutonium nitrate solution from the Plutonium-Uranium Reduction and Extraction [PUREX] Plant). The purpose of the 
initial feed processing is to prepare a purified plutonium nitrate solution that can be processed through a solvent extraction step and subsequently reduced to product. Processing at the PFP has included the fabrication of plutonium metal into useful parts, al though that activity has been discontinued.

Four significant waste generating activities at the PFP have been identified. They are as follows:

- Plutonium processing

- Maintenance

- Housekeeping

- Waste processing.

The types and estimated quantities of waste resulting from these activities are discussed in detail in Section 3.0.

All solid wastes originating at the PFP are packaged for onsite or offsite storage or disposal. The waste packages are designed to safely contain the waste during transportation and storage, and to meet the criteria of the storage or disposal unit. Waste packaging and reporting requirements have undergone significant changes throughout the history of the PFP. Current and historical packaging and handling procedures for radioactive wastes at the PFP are provided in Section 4.0 .

Information on the radioactive wastes generated at the PFP can be found in a number of existing documents and databases. The most important of these 
are the Solid Waste Information and Tracking System (SWITS) database and Solid Waste Burial Records (SWBRs). Facility personnel also provide excellent information about past waste generation and the procedures used to handle that waste. Section 5.0 was compiled using these sources to characterize the radioactive wastes, especially TRU wastes, generated at the PFP.

Between 1970 and 1991 33,546 containers of radioactive solid waste were generated at the PFP. These containers represent over 4.6 million kilograms (10 million pounds) of waste occupying $13,053 \mathrm{~m}^{3}\left(460,959.84 \mathrm{ft}^{3}\right)$ of space. Section 5.0 provides an in-depth look at this waste including the following:

- Weight and volume of the waste

- Container types and numbers

- Physical description of the waste

- Radiological components

- Hazardous constituents

- Classified waste

- Current storage and/or disposal locations. 
WHC-EP-0621

\section{CONTENTS}

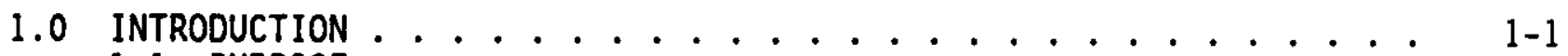

1.1 PURPOSE .......................... . . . . . $1-1$

1.2 BACKGROUND . . . . . . . . . . . . . . . . . . . . . $1-1$

1.3 SOURCES ......................... . . . . . . . .

1.4 SCOPE . . . . . . . . . . . . . . . . . . $1-3$

2.0 DESCRIPTION OF THE PFP .................... 2-1

2.1 LOCATION . . . . . . . . . . . . . . . . . . 2-1

2.2 PROCESS SUMMARY ...................... 2-1

2.3 PFP PHYSICAL PLANT . . . . . . . . . . . . . . . . 2-2

2.3.1 The 234-5Z Building . . . . . . . . . . . . . . . $2-2$

2.3.2 The 236-z Building................... . . . . . . $2-3$

2.3.3 The 232-z Building ................ $2-3$

2.3.4 The 241-z Building................. . $2-4$

2.3.5 The 242-Z Building................. . $2-4$

2.3.6 The 270-Z Building ................ . 2-4

2.3.7 The 291-Z Building ................ . 2-5

2.3.8 The 2736-Z Building . . . . . . . . . . . . . . $2-5$

2.3.9 The 2736-ZB Building. . . . . . . . . . . . . . . 2-5

2.4 PROCESS AND FACILITY HISTORY ................ . . . . $2-5$

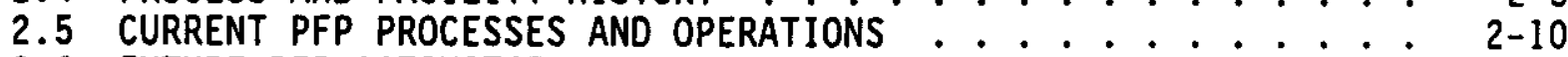

2.6 FUTURE PFP ACTIVITIES ................... $2-11$

3.0 PFP SOLID WASTE STREAMS ................ 3-1

3.1 INTRODUCTION . . . . . . . . . . . . . . . 3-1

3.2 RADIOACTIVE WASTE SEGREGATION .............. . . 3-1

3.3 WASTE-GENERATING ACTIVITIES . . . . . . . . . . . . 3-1

3.3.1 Plutonium Production Processes . . . . . . . . . 3-2

3.3.2 Maintenance Requirements ............ $3-4$

3.3.3 Housekeeping Activities ............ . 3-5

3.3.4 Waste Processing Activities . . . . . . . . . 3-5

3.4 HIGH PERIODS OF SOLID WASTE GENERATION ........ $3-6$

3.5 CURRENT PFP SOLID WASTE INVENTORY AND

NOMINAL GENERATION RATES . . . . . . . . 3-7

3.6 FUTURE FACILITY CHANGES AND RESULTING

4.0 SOLID WASTE HANDLING PRACTICES AND PROCEDURES ........ . 4-1

4.1 HISTORICAL WASTE PACKAGING AND HANDLING PROCEDURES ..... $4-1$

4.2 CURRENT WASTE HANDLING AND PACKAGING . . . . . . . . . 4-2

4.2.1 Equipment and System Description........... 4-3

4.2.2 Operating Procedures .............. . 4-4

5.0 CHARACTERIZATION OF RETRIEVABLY STORED SOLID WASTE
GENERATED BY PFP ....... 5-1

5.1 SUMMARY OF PFP WASTE GENERATION $: \cdots \cdots$

5.1.1 Waste Stored in 55-Gallon Steel Drums . . . . . . 5-1

5.1.2 Waste Stored in Containers Other Than $5-3$

5.1.3 Summary of Waste Generation Rates at PFP $\ldots$ 
CONTENTS (cont.)

5.2 WASTE CONTAINERS .............. . . . 5-7

5.2.1 Non-TRU Waste Containers . . . . . . . . . . . 5-7

5.2.2 TRU Waste Containers............. . 5-7

5.3 TRU WASTE STORAGE LOCATIONS ............. 5-7

5.4 PHYSICAL CONTENTS OF TRU WASTE CONTAINERS . . . . . . . . 5-8

5.5 RADIOLOGICAL DESCRIPTION OF PFP SOLID WASTE . . . . . . . 5-9

5.6 HAZARDOUS CONSTITUENTS OF SOLID WASTE GENERATED BY PFP . . . 5-10

5.6.1 Chemical Inventories ........... 5-10

5.6.2 Solid Waste Information and Tracking System ..... 5-10

5.6.3 Burial Records .............. 5-11

5.6.4 Interviews With PFP Analytical Laboratory

5.7 CLASSIFIED WASTE Faciltty Personnel ......... $. .5-115$

6.0 REFERENCES .......................... 6- 6 . .

APPENDIXES

A PFP FLOWSHEETS ................... A A-1

B DATA ON 234-5Z WASTE GENERATION FROM THE SOLID WASTE

INFORMATION TRACKING SYSTEM ............... B-1

B.1 CONTAINER NUMBER AND WEIGHT OF TRU WASTE IN 55-GALLON

DRUMS - 4.0 SORTED BY WASTE TYPE ........... B-7

B.2 TRU WASTE IN CONTAINERS OTHER THAN 55-GALLON DRUMS - SORTED BY WASTE TYPE ............. B-15

B.3 ANNUAL VOLUME OF TRU AND NON-TRU WASTE IN 55-GALLON DRUMS - SORTED BY WASTE TYPE ........... B-25

B.4 ANNUAL VOLUME OF TRU WASTE IN CONTAINERS OTHER THAN 55-GALLON DRUMS - SORTED BY WASTE TYPE ......... B-33

B.5 ANNUAL VOLUMES OF NON-TRU WASTE IN CONTAINERS OTHER THAN 55-GALLON DRUMS ............... B-41

B.6 ANNUAL VOLUMES OF WASTE WITH SECONDARY WASTE CODES GREATER THAN 2A STORED IN WASTE CONTAINERS OTHER THAN 55-GALLON DRUMS .......................... B-49

B.7 TRU WASTE STORAGE FACILITIES - SORTED BY PRIMARY WASTE TYPE, CONTAINER TYPE, AND YEAR ......... B-55

B.8 RADIOLOGICAL DATA ON TRU WASTE CONTAINERS - SORTED BY DATE, PRIMARY WASTE TYPE, AND STORAGE FACILITY $\ldots$ B-65

B.9 PHYSICAL CONTENTS DESCRIPTION FOR 55-GALLON DRUMS CONTAINING TRU WASTE - SORTED BY DATE, PRIMARY WASTE TYPE, AND STORAGE FACILITY 


$$
\text { WHC-EP-0621 }
$$

CONTENTS (cont.)

APPENDIXES (cont.)

B.10 PHYSICAL CONTENTS DESCRIPTION FOR TRU WASTE CONTAINERS

OTHER THAN 55-GALLON DRUMS - SORTED BY DATE,

PRIMARY WASTE TYPE, AND STORAGE FACILITY . . . . . . .

B.11 HAZARDOUS CONSTITUENTS LISTED FOR 55-GALLON DRUMS

CONTAINING TRU WASTE . . . . . . . . . . . . B-99

B.12 HAZARDOUS CONSTITUENTS LISTED FOR TRU WASTE CONTAINERS OTHER THAN 55-GALLON DRUMS ......... B-105

B.13 ISOTOPES IN 55-GALLON DRUMS THAT CONTAIN TRU WASTES ... . B-111

B.14 ISOTOPES IN TRU WASTE CONTAINERS OTHER THAN 55-GALLON ORUMS ....................... B-121

C ORIGINAL SOLID WASTE INFORMATION AND TRACKING SYSTEM

RECORDS FOR ALL 55-GALLON WASTE DRUMS WITH

WEIGHTS $\geq 150$ KILOGRAMS .................... c-1

D ORIGINAL SOLID WASTE INFORMATION TRACKING SYSTEM

RECORDS FOR ALL 55-GALLON WASTE DRUMS WITH

GREATER THAN 300 GRAMS OF PLUTONIUM ............. . . D-1

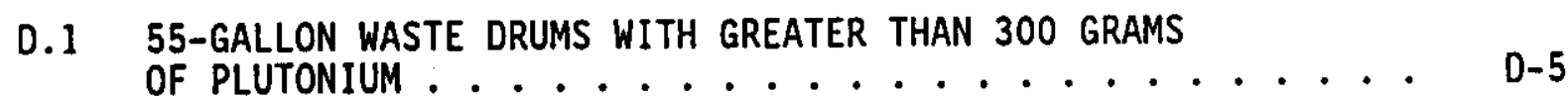

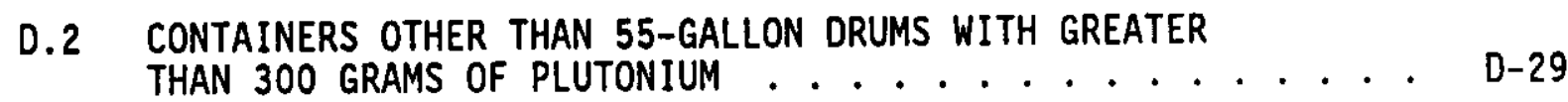

E SOLID WASTE INFORMATION AND TRACKING SYSTEM RECORD FOR TRU CLASSIFIED DRUM RS-83-6-1 
WHC-EP-0621

\section{LIST OF FIGURES}

2-1 Location of the Hanford Site .............. 2-12

2-2 Hanford Site Map ................. 2-13

2-3 200 West Area ................. 2-14

2-4 Aerial Photograph of the PFP ............ 2-15

2-5 Main PFP structures.............. 2-16

3-1 Destination of Nonradioactive, Nondangerous Solid Waste for Storage/Disposal ............... 3-11

3-2 Destination of Nonradioactive, Dangerous Solid Waste for Disposal ...................... 3-12

3-3 Destination of Low-Level Radioactive Solid Waste for Storage . . 3-13

3-4 Destination of WIPP-Certifiable TRU Waste for Storage . . . . . 3-14

3-5 Destination of Non-WIPP-Certifiable TRU Waste for Storage . . . 3-15

3-6 Recoverable Material Dissolution Flowsheet . . . . . . . 3-16

3-7 Slag and Crucible Material Dissolution Flowsheet . . . . . 3-17

3-8 Typical Solvent Extraction Flowsheet ........... 3-19

3-9 Remote Mechanical C Line Flow Diagram, Nitrate Solution Through Plutonium Fluoride Production ........... 3-21

3-10 Remote Mechanical C Line Flow Diagram, Plutonium Fluoride Through Plutonium Button Production ............ 3-22

5-1 PFP 55-Gallon Drums Generated Annually . . . . . . . . . 5-16

5-2 Percentage of TRU and Non-TRU 55-Gallon Drums Generated Annually at the PFP................ 5-17

5-3 Total Weight of Radioactive Waste Stored in 55-Gallon Drums Generated Annually at the PFP. . . . . . . . . . . 5-18

5-4 Percent Weight of TRU and Non-TRU 55-Galion Drums Generated Annually at the PFP............. 5-19

5-5 Volume of Radioactive Waste Stored in 55-Gallon Drums Generated Annually at the PFP ........... . . . 5-20

5-6 Percent Volume of TRU and Non-TRU 55-Gallon Drums Generated Annualiy at the PFP . . . . . . . . . . . . 


\section{WHC-EP-0621}

\section{LIST OF FIGURES (cont.)}

5-7 Volume of Radioactive Waste Stored in Containers Other Than 55-Galion Drums Generated Annually at the PFP........ . 5-22

5-8 Percent Volume of TRU and Non-TRU COntainers Other Than 55-Gallon Drums Generated Annually at the PFP . . . . . . . . . . . 5-23

5-9 Containers Other Than 55-Gallon Drums Generated Annually at the PFP...........................

5-10 Percentage of TRU and Non-TRU Containers Other Than 55-Galion Drums Generated Annually at the PFP. 5-25

5-11 Total Weight of Radioactive Waste Stored in Containers Other Than 55-Gallon Drums Generated Annually at the PFP . . . . . . . . 5-26

5-12 Percent Weight of TRU and Non-TRU Containers Other Than 55-Galion Drums Generated Annually at the PFP . . . . . . . . . 5-27

5-13 Total Number of Waste Containers Generated by PFP Annually . . . 5-28

5-14 Total Weight of Waste Generated by PFP Annually . . . . . . . . 5-29

5-15 Total Volume of Waste Generated by PFP Annually . . . . . . . . . 5-30

5-16 Grams of TRU Stored in Waste Generated by PFP . . . . . . . . . . 5-31 
WHC-EP-0621

\section{LIST OF TABLES}

4-1 TRU Storage Requirements for the Hanford Site . . . . . . . . 4-9

5-1 Waste Summary Data for 55-Gallon Drums Generated at the PFP from 1970 to $1991 \ldots \ldots$ 5-32

5-2 PFP 55-Gallon Drums Over 150 Kilograms .......... 5-33

5-3 Waste Summary Data for Containers Other Than 55-Gallon Drums Generated at the PFP from 1970 to $1991 \ldots . . . . . .5$ 5-35

5-4 Summary of Low-Level Waste Generated at 234-5Z from 1970 through $1991 \ldots \ldots$ 5-36

5-5 TRU Waste Generated at 234-5Z by Container Type and Year . . . 5-37

5-6 TRU Waste in 55-Gallon Drums: Drum Count by Storage Factlity . . 5-42

5-7 TRU Waste in Containers Other Than 55-Gallon Drums:

Container Count by Storage Facility ........... 5-43

5-8 Distribution and Percentage of TRU 55-Gallon Drum Physical

Contents by Storage Location - 218W3A ........... 5-44

5-9 Distribution and Percentage of TRU 55-Galion Drum Physical Contents by Storage Location $-218 \mathrm{~W} 4 \mathrm{~B}$. . . . . . . . . . 5-45

5-10 Distribution and Percentage of TRU 55-Gallon Drum Physical Contents by Storage Location - 218W4C . . . . . . . . . 5-46

5-11 Distribution and Percentage of TRU 55-Gallon Drum Physical Contents by Storage Location - 218W5 ........... 5-48

5-12 Distribution and Percentage of TRU 55-Gallon Drum Physical Contents by Storage Location - 224T ............ 5-49

5-13 Distribution and Percentage of TRU 55-Gallon Drum Physical Contents by Storage Location - $2401 \mathrm{~W} . . . . . . . .5-51$

5-14 Distribution and Percentage of TRU 55-Gallon Drum Physical Contents by Storage Location - 2402WB ............ . 5-52

5-15 Distribution and Percentage of TRU Container Physical Contents by Storage Location -218 W3A .......... 5-53

5-16 Distribution and Percentage of TRU Container Physical Contents by Storage Location -218 W4B ........... 5-54

5-17 Distribution and Percentage of TRU Container Physical Contents by Storage Location - 218 W4C 
WHC-EP-0621

LIST OF TABLES (cont.)

5-18 Distribution and Percentage of TRU Container Physical

Contents by Storage Location - 2401W ........... . 5-56

5-19 Distribution and Percentage of TRU Container Physical

Contents by Storage Location $-2402 W \ldots$. . . . . . . . . 5-57

5-20 Distribution and Percentage of TRU Container Physical

Contents by Storage Location - 2402 WB .......... 5-58

5-21 Distribution and Percentage of TRU Container Physical

Contents by Storage Location - FS8 ........ . . . . 5-59

5-22 TRU Waste in 55-Gallon Drums: Total Grams of TRU

by Storage Facility .................. 5-60

5-23 TRU Waste in Containers Other Than 55-Gallon Drums:

Total Grams of TRU by Storage Facility ............ . 5-61

5-24 TRU Waste from 234-5Z Containing High quantities of ${ }^{238} \mathrm{Pu}$. . . . 5-62

5-25 PFP Waste in Other Containers Containing More Than

300 Grams of TRU ................. 5-63

5-26 PFP ( $Z$ Plant) Plutonium Conversion to Oxide

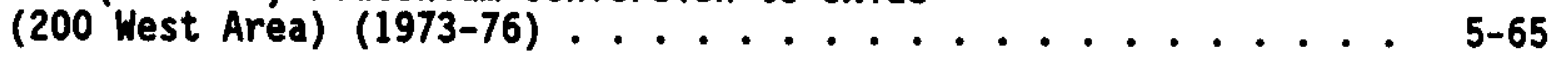

5-27 PFP (Z Plant): Plutonium Reclamation and Waste Treatment

(200 West Area) $(1973-76) \ldots . . . . . . . . .55-66$

5-28 PFP (Z Plant): Analytical Laboratory (200 West Area)

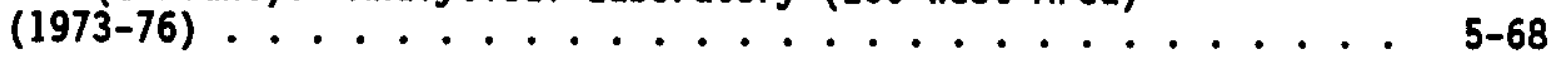

5-29 TRU Mixed Waste Stored in 55-Gallon Drums by Storage Facility . . 5-71

5-30 TRU Mixed Waste Containers Other Than 55-Gallon Drums by Storage Facility ................ 5-72

5-31 Number and Percent of TRU Mixed Waste Drums Stored at 218W4C Containing Hazardous Constituents ............. 5-73

5-32 Number and Percent of TRU Mixed Waste Drums Stored at 224-T

Containing Hazardous Constituents ............ 5-74

5-33 Number and Percent of TRU Mixed Waste Drums Stored at 2401W

Containing Hazardous Constituents ............ 5-75

5-34 Number and Percent of TRU Mixed Waste Drums Stored at 2402 WB

Containing Hazardous Constituents ........... 5-76 


\section{LIST OF TABLES (cont.)}

5-35 Number and Percentage of TRU-Mixed Waste Containers Other Than 55-Gallon Drums Stored at 218W3A Containing Hazardous Constituents ............... 5-77

5-36 Number and Percentage of TRU-Mixed Waste Containers Other Than 55-Gallon Drums Stored at 218W4C Containing Hazardous Constituents ............. 5-78

5-37 Number and Percentage of TRU-Mixed Waste Containers Other Than 55-Gallon Drums Stored at 2401W Containing Hazardous Constituents .............. 5-79

5-38 Number and Percentage of TRU-Mixed Waste Containers Other Than 55-Gallon Drums Stored at 2402W Containing Hazardous Constituents .............. 5-80

5-39 Number and Percentage of TRU-Mixed Waste Containers Other Than 55-Gallon Drums Stored at 2402WB Containing Hazardous Constituents ............... 5-81

5-40 Number and Percentage of TRU-Mixed Waste Containers Other Than 55-Galion Drums Stored at FS8 Containing Hazardous Constituents .............. 5-82

5-41 Summary of Information from Sol id Waste Storage Disposal Records for Containers of TRU Mixed Waste .......... 
WHC-EP-0621

\section{LIST OF TERMS}

\section{ABBREVIATIONS, ACRONYMS, NDO INITIALISHS}

AEC

ALARA

CERCLA

$C P$

CSMO

D\&D

$\mathrm{DL}$

DMA

DOE

DOT

$\mathrm{ECL}$

Ecology

EDE

EDL

EHW

EIS

EL

EPA

ERDA-RL

\section{FFTF}

FG

FRP

FSAR

GPP

HEDL

HEHF

HEPA

HVAC

$H / X$

IDLH

LANL

LI/LO

LLW

LLWTF

LWDF

MSDS

MW

NDA

NRC

O\&M

PCB

PFD

PFP

PFPEL

PNL

PPO
U.S. Atomic Energy Commission (now DOE)

as low as reasonably achievable

Comprehensive Environmental Response Compensation and Liability Act of 1980

Chemical Processing

Central Scrap Management Organization

decontamination and decommissioning

Development Laboratory

Division of Military Applications

U.S. Department of Energy

U.S. Department of Transportation

environmental control 11st

Washington State Department of Ecology

effective dose equivalent

economic discard 1 imit

extremely hazardous waste

environmental impact statement

Engineering Laboratory

U.S. Environmental Protection Agency

Energy Research and Development Administration, Richland Operations (now RL)

Fast Flux Test Facility

fuels grade

fiberglass reinforced polyester

final safety analysis report

general plant projects

Hanford Environmental Development Laboratory

Hanford Environmental Health Foundation

high-efficiency particulate air (filter)

heating, ventflating, and air conditioning

hydrogen-to-fissionable material atom ratio

immediately dangerous to Iffe and health

Los Al amos National Laboratory

load-in/load-out

low-level waste

Low-Leve1 Waste Treatment Facility

Liquid Waste Disposal Facility (241-Z Building)

Material Safety Datasheet

mixed waste

nondestructive assay

U.S. Nuclear Regulatory Commission

Operations and Maintenance

polychlorinated biphenyl

process flowsheet documents

Plutonium Finishing Plant

Plutonium Fintshing Plant Engineering Laboratory

Pacific Northwest Laboratory

Plutonium Process Operations (234-5Z Building) 


\section{ABBREVIATIONS, ACRONYMS, AND INITIALISMS}

PRF
PRFP
PSAR
PSE
PSF
PUREX
QC
R-SWIMS
RCRA
RHO
RIDS
RL
RMA
RMC
RMW
S\&S
SAR
SARP
SDAR
SGSAS
SNM
SRP
SS\&C
SWBR
SWE
SWITS
SWSOR
TBP
TCO
TRU
TRUSAF
WAC
WG
WHC
WIPP
WRAP
WT

\section{BUILDINGS}

Plutonium Reclamation Facility (236-Z Building) Plutonium Reclamation Facility Plant prel iminary safety analys is report prel iminary safety evaluation Plutonium Storage Facility Plutonium-Uranium Reduction Extraction Plant quality control Richland Solid Waste Information Management System Resource Conservation and Recovery Act of 1976 Rockwell Hanford Operations Records Inventory Disposition Sheet U.S. Department of Energy, Richland Field Office remote mechanical $A$ remote mechanical $C$ radioactive mixed waste Safeguards and Security safety analysis report safety analys is report for packaging Storage/Disposal Approval Record Segmented Gamma Scan Assay System special nuclear material Savannah River Plant sand, slag, and crucible Solid Waste Burial Record Solid Waste Engineering Solid Waste Information and Tracking System Solid Waste Storage and Disposal Record tributylphosphate terminal cleanout operations transuranic Transuranic Storage and Assay Facility waste acceptance criteria weapons grade Westinghouse Hanford Company Waste Isolation Pilot Plant Waste Receiving and Processing Facility waste treatment

Waste Incinerator Layaway Building Plutonium Process Operations (PPO) Plutonium Reclamation Facility Liquid Waste Disposal Facility (LWDF) Waste Treatment Layaway Building Support Storage Complex Building Ventilation Exhaust Fans and Mechanical Service Equipment Building 
WHC-EP-0621

\subsection{INTRODUCTION}

\subsection{PURPOSE}

The purpose of this report is to characterize, as far as possible, the solid waste generated by the Plutonium Finishing Plant (PFP) since its construction in 1947. This characterization is of particular interest in the planning of transuranic (TRU) waste retrieval operations (including the Waste Receiving and Processing [WRAP] Facility) because the PFP has generated approximately $55 \%$ of the TRU 55-gal drums currently stored at the Hanford site. This report specifically excludes radioactive wastes generated at the Plutonium Metallurgy Laboratory (231-Z facility), which is operated for the U.S. Department of Energy (DOE) by the Pacific Northwest Laboratory (PNL). The wastes generated by this facility will be covered in a separate report.

\subsection{BACKGROUND}

Since 1944, the production of defense related materials at the Hanford Site has generated radioactive wastes. The bulk of these wastes have been disposed of or stored in the 200 East Area and 200 West Area burial grounds and waste storage facilities.

In the period between 1944 and 1970 both TRU and low-level wastes (LLW) were disposed of in shallow land trenches with no.attempt to segregate these materials by their chemical or radfoactive natures. In 1970 the U.S. Atomic Energy Commission (AEC) directed that AEC sites segregate "waste with known or detectable contamination of transuranium nuclides" from other waste types (Immediate Action Directive 0511-21 [AEC 1970]). The TRU radionuclides are those with an atomic number greater than 92 . The AEC further directed that these wastes be packaged and stored as contamination-free packages for at least 20 years. The 20-year interim storage period was to allow time to study permanent disposal options for TRU contaminated wastes.

The Immediate Action Directive 0511-21 did not provide a detailed definition for TRU waste in 1970. The AEC contractors implemented the Immediate Action Directive 0511-21 to the best of their ability with the instrumentation then available. In 1973, the Atomic Energy Commission Manual (AEC 1973) further defined TRU waste as material contaminated with certain alpha-emitting radionuclides with half-lives greater than 20 years and activity greater than $10 \mathrm{nCi} / \mathrm{g}$. The radionuclides included were $233 \mathrm{U}$ and its daughter products, as well as plutonium, and transplutonium nuclides with the exception of ${ }^{238} \mathrm{Pu}$ and ${ }^{241} \mathrm{Pu}$. In 1982, the TRU waste segregation limit was raised to $100 \mathrm{nCi} / \mathrm{g}$ by DOE Order 5820.1, Management of Transuranic Material (DOE 1982).

In addition to radioactive materials, Hanford site production plants and support operations used a wide variety of chemicals. Many of these chemicals are currently classified as dangerous or hazardous by the U.S. Environmental Protection Agency (EPA) and the Washington State Department of Ecology (Ecology). When dangerous or hazardous wastes are found in radioactive waste they are termed "mixed" wastes. 
At the time much of the mixed waste was generated there were no definitions or regulations governing the storage, disposal, or documentation of mixed wastes. In 1987, the DOE issued a mixed by-product ruling stating that the hazardous components of mixed waste are regulated by the Resource Conservation and Recovery Act of 1976 (RCRA) (DOE 1987). In November 1987, the EPA authorized Ecology to regulate the hazardous constituents of mixed waste at the Hanford Site (EPA 1987).

During the next two decades the TRU waste now stored in the burial trenches and storage facilities is to be retrieved, processed at the WRAP, and shipped to the Waste Isolation Pilot Plant (WIPP) near Carlsbad, New Mexico for final disposal. Over $50 \%$ of the TRU waste to be retrieved for shipment to the WIPP has been generated by the PFP.

\subsection{SOURCES}

Data for this study were compiled from a variety of sources. Each of the major sources used is listed with a few explanatory notes. Greater detail on each of the data sources can be found in the body of this document as the information from each is discussed.

Documents that describe the PFP processes - These documents include the Plutonium Finishing Plant Safety Analysis Report (WHC 1991), the 200 Areas Fact Book (RHO 1985), and Geometrically Favorable Plutonium Scrap Recovery Plant (Bruns 1965), which were used to determine the solid wastes that may have been generated at the PFP during its 40-year history.

Documents that describe the PFP waste packaging and handling procedures These documents include laboratory solid waste procedures (Louk 1991) and Hanford Site Solid Waste Acceptance Criteria (Willis and Triner 1991), which were used to describe packaging and handing practices.

Interviews - Interviews with current and former PFP personnel were used to gather information regarding unusual waste contents and historical waste handling and packaging procedures. The individuals interviewed represented both process and laboratory personnel, some of whom have been at the PFP for over 30 years.

Characterization data - These data were primarily derived from the Solid Waste Information and Tracking System (SWITS) database. The SWITS database was created in 1991 primarily with the solid waste data from the Richland Solid Waste Information Management System (R-SWIMS). The SWITS database contains information about radioactive wastes, both TRU and LLW, buried or stored in the 200 Areas since 1970. Data in SWITS were originally taken from Solid Waste Burial Records (SWBRs) and their replacement the Sol id Waste Storage/ Disposal Records (SWSDRs). The SWBRs and SWSDRs, which will be jointly referred to as "burial records" in this document, often contain supplementary forms such as shipment manifests, U.S. Nuclear Regulatory Commission (NRC) 741 Forms, etc. 
WHC-EP-0621

Hazardous waste components - These components were determined using information from the SWITS database, SWBRs, SWSDRs, and personnel interviews. Additional information was obtained from Inventory of Chemicals Used at Hanford Site Production Plants and Support Operations (1944-80) (Klem 1990) and Unstable and Reactive Chemicals in TRU Retrievable Waste at the Hanford Site - A Review of Available Data (Reddinger 1992).

\subsection{SCOPE}

The major sections of this document and the topics they cover are outlined briefly below. Because of the number of tables and figures included in this report, they appear at the end of each section.

Section 2.0 provides a brief description of the PFP physical plant and the operations that occur there. A short history of the significant occurrences and changes at the PFP follows this description.

Section 3.0 identifies the solid waste streams that arise from the PFP processes, maintenance operations, housekeeping operations, and waste processing. The types and amounts of solid waste that may have been generated from each of the waste streams are estimated.

Section 4.0 discusses the waste handling and packaging procedures used in the PFP operational facilities and in the PFP Analytical Laboratories. Historical changes in waste handling, packaging, and recordkeeping are also reviewed in this section.

Section 5.0 contains the results of a search for actual waste container data. This section describes what is known about the physical, radiological and hazardous characteristics of the radioactive solid waste from the PFP.

Section 6.0 lists the references used in the compilation of this report.

Appendix A contains the PFP process flowsheets taken from the Plutonium Finishing Plant Safety Analysis Report (SAR) (WHC 1991).

Appendix $B$ contains the original SWITS data run that forms the basis for most of the tables and figures found in Section 5.0.

Appendix $C$ is composed of individual SWITS records for the heaviest containers generated by the PFP.

Appendix 0 is composed of individual SWITS records for the TRU waste containers with the heaviest gram loadings.

Appendix E contains the SWITS record for the one classified waste container listed as generated at the PFP. 
WHC-EP-0621

This page intentionally left blank. 
WHC-EP-0621

\subsection{DESCRIPTION OF THE PFP}

The PFP, al so referred to as 234-5Z and Z Plant among others, is a collection of facilities that has primarily been used to produce plutonium metal and plutonium oxide to support national defense activities. The special nuclear materials (SNM) that form the feed for the PFP processing include, but are not limited to, plutonium-bearing caps, incinerator ash, silicon nitride crucibles, polystyrene cubes, plutonium-uranium mixtures, siag and crucible fragments, unirradiated fuel rods, plutonium-beryllium sources, and unique plutonium-bearing solutions (e.g., plutonium nitrate solution from the Plutonium-Uranium Reduction Extraction Plant [PUREX]). The PFP uses separate process lines to handle different feeds.

Numerous changes to equipment and processes have been made since the PFP was first started. The purpose of this section is to describe the PFP and to summarize the process history, so that solid waste generation activities can be compared to status of the PFP.

\subsection{LOCATION}

The PFP is located within DOE's Hanford Site in south-central Washington State (Figure 2-1). The PFP is situated in the west-central region of the Hanford Site within the 200 West Area (Figure 2-2). The location of the PFP relative to other plants in the 200 West Area is shown in Figure 2-3. A photograph of the PFP can be found in Figure 2-4.

\subsection{PROCESS SUMMARY}

The recently renamed PFP has been known as the Plutonium Process and Storage Facility, and more familiarly known as Z Plant. The plant was first operated in 1949. Both manual and remote mechanical processing have been employed at the plant.

Three types of feed materials are processed at the PFP to produce plutonium metal. Feed material types are handled differently in different process lines. The purpose of initial feed processing is to prepare a purified plutonfum nitrate solution that can be processed through a solvent extraction step and subsequently reduced to product. Processing at the PFP has included fabrication of plutonium metal into useful parts, al though that activity has been discontinued.

Historically, the main feed for the PFP was purified plutonium nitrate solution that was produced el sewhere in a fuel reprocessing plant. This feed was charged directly to one of the main process lines, which was initially a glovebox 1 ine. The glovebox 1 ine was replaced by remote mechanical 1 ines, which were upgraded over the years. In time, processes were added to handle rework and scrap plutonium. These processes were used to convert the rework and scrap materials into a purified plutonium nitrate solution that could be handled by the main process. The PFP facilities have included laboratory 
WHC-EP-0621

facilities; shops for maintenance, chemical development, and instrumentation; and an incinerator that was used for processing scrap. Significant process changes in the PFP are described in the following section.

\subsection{PFP PHYSICAL PLANT}

The buildings that compose the PFP are shown in Figure 2-5. The primary PFP process and support buildings include 232-Z, 234-5Z,236-Z, 241-Z, 242-Z, 291-Z, 2736-Z, and 2736-ZB. Some processes within the PFP buildings are no longer operated. For clarity, therefore, each of the process support butldings and their functions are described as follows.

- Active - An active process is currently operating or is scheduled for future operation.

- Standby - A standby process is not currently operating but is operable with appropriate repairs/upgrades. Future operations are not currently scheduled.

- Layaway - A layaway process is not operable without major repairs/upgrades. No operations are planned. This category is scheduled for terminal cleanout operations (TCO)/decontamination and decommissionting (D\&D).

- Future - A future bullding, process, and/or enhancement that is planned for construction and operation.

\subsubsection{The 234-5Z Building (Active)}

The 234-5Z Building, also referred to as the 234-5 8uilding, has approximate dimensions of $54.9 \mathrm{~m}$ (180 ft) wide by $152.4 \mathrm{~m}$ (500 ft) long and extends from $2.9 \mathrm{~m}(9.5 \mathrm{ft})$ below grade to $14.3 \mathrm{~m}(46.8 \mathrm{ft})$ above grade. Floor levels are designated as basement, first floor, duct level, second floor, and roof level.

The 234-5Z basement mostly consists of pipe tunnels carrying drain piping to sumps. The first floor houses the two plutonium processing lines (remote mechanical $A$ [RMA] and remote mechanical $C$ [RMC] lines) and their control rooms; scrap stabilization gloveboxes; plutonium storage vaults; the plutonium nitrate feed load-in/load-out (LI/LO), blending, and storage facilities; the Engineering Laboratory and the Development Laboratory; the instrument maintenance shops; the building maintenance shops; the locker rooms with change facilities and restrooms; and office spaces. The duct level contains most of the service piping, ventilation ducts, and some filterboxes. The lunchroom, conference room, materials storage room, chemical feed preparation and aqueous make-up rooms, locker rooms with change facilities and restrooms, and office spaces are on the second floor. Also located on the second floor are exhaust air duct works, including filterboxes and filter rooms. The fan room, located on the northwestern corner of the second floor, houses the ventilation supply fans, the steam inlet and distribution system, air dryers, the distilled water still, air chilling units, and the vent and balance control room. 


\subsubsection{The 236-Z Building (Active)}

The 236-Z Building is located south of the southeastern corner of the 234-5Z Building and is connected to it by the 242-Z Building. The 236-Z Building, built as the CAC-880 Project, houses the Plutonium Reclamation Facility (PRF). It is also referred to as 880 , Plutonium Reclamation Facility Plant (PRFP), Plutonium Nitrate Production Facility, and 236.

The building is essentialiy a four-story structure, surmounted by a twostory penthouse. Its dimensions are about $24.1 \mathrm{~m}(79 \mathrm{ft})$ wide by $21.6 \mathrm{~m}$ (71 ft) long. Its outstanding internal structural feature is a single process equipment cell that is $9.8 \mathrm{~m}(32 \mathrm{ft})$ wide by $15.8 \mathrm{~m}(52 \mathrm{ft})$ long.

Maintenance shop facilities are located on the service (east) side of the bullding on the ground floor. The second floor of the service side is used for a maintenance glovebox and ventilation exhaust filters. Building service equipment and electrical switch gear are on the third floor of the service area. The fourth floor is used for chemical preparation, miscellaneous treatment, the operating control room, slag and crucible dissolver equipment, and a column room in which vertical sections of two liquid-liquid extraction columns penetrating the room from above and below are housed in a glovebox. The first through the fourth floors are serviced by a service elevator located within the east side of the building.

The process cell has a $0.6-\mathrm{m}-(2-\mathrm{ft}-)$ thick concrete wall between the cell and access hoods. These access hoods are stainless steel paneled hoods containing glass viewing windows and Hypalon hood gloves. These hoods are located on both sides of the cell on the first two floors. The hoods contain process piping, pumps, valves, flowmeters, and other equipment that frequently requires maintenance.

The cell floor is covered with a stainless steel liner extending $45.7 \mathrm{~cm}$ (18 in.) up the side wall. The remaining cell wall and ceiling surfaces are covered with chemical-resistant coatings. Water-filled viewing windows on the third floor have adjacent remote control stations for the cell crane.

\subsubsection{The 232-Z Buitding (Layaway)}

The 232-Z Building houses the layaway contaminated waste recovery process. It was commonly called the incinerator. It was constructed by Project CGC-013, plutonfum recovery process, and was partially decontaminated and decommissioned in 1984.

The 232-Z Building is located about $61 \mathrm{~m}(200 \mathrm{ft})$ south of the main portion of the 234-5Z Building and is about $30.5 \mathrm{~m}(100 \mathrm{ft})$ west of the 291-Z stack. Its approximate dimensions are $11.3 \mathrm{~m}(37 \mathrm{ft})$ wide by $17.4 \mathrm{~m}$ (57 ft) long. It is divided into areas for process, storage, changeroom, chemical preparation, ventilation and electrical equipment. Except for

\footnotetext{
"Hypalon is a trademark of E.I. duPont de Nemours and Company.
} 
WHC-EP-0621

ventilation supply and exhaust filtration, the 232-Z Building uses services from the 234-5Z Building. The 232-Z Building has been isolated from the 291-Z Building, although they were connected in the past.

\subsubsection{The 241-Z Building (Active)}

The 241-Z Building is designated as the Waste Treatment Facility. It is commonly called the 241-Z Sumps and in the past was called the 216-Z Large Waste Sump Tanks. The 241-Z Building is a buried structure, with a sheet metal enclosure over the top which houses a hoist for removing cell covers. It consists of five separate enclosures or ventilated cells, each containing a 20,000-L (5,283.5-gal) tank used to accumulate the liquid wastes generated in the PFP before transfer to the tank farms. Built of reinforced concrete, its approximate dimensions are $6.1 \mathrm{~m}(20 \mathrm{ft})$ wide by $28 \mathrm{~m}(92 \mathrm{ft})$ long.

At the southwest corner of the 241-Z vault deck is the equipment for the $241-Z$ vessel vent and vault ventilation system. The $7.3-\mathrm{m}-(24-\mathrm{ft}-)$ high stack (296-Z-3) and its associated fans, filters, and controls are located on a $4.3-$ by $5.5-m$ (14- by $18-\mathrm{ft}$ ) concrete pad.

\subsubsection{The 242-Z Buflding (Layaway)}

The 242-Z Building houses portions of the Waste Treatment and Americium Facility, which are in layaway and planned for future D\&D. Built primarily by Project CGC-912, this building is usually referred to as "912" or "WT."

The 242-Z structure is between the southeastern corner of the 234-5Z and 236-Z Buildings. Along its east side runs a corridor connecting the 234-5Z, $242-Z$, and 236-Z Buildings. At its west end is an entrance enclosure for outside entry into both $242-Z$ and the Engineering Laboratory area in the 234-5Z Building.

The 242-Z Building's approximate dimensions are $12.2 \mathrm{~m}(40 \mathrm{ft})$ wide, $7.9 \mathrm{~m}(26 \mathrm{ft})$ long, and $7 \mathrm{~m}(23 \mathrm{ft})$ high. The south portion, approximately $12.2 \mathrm{~m}$ (40 ft) wide by $3 \mathrm{~m}$ (10 ft) long, is the tank room (tank cell). This room extends the full inside building height. The north portion, designated the control room, has a mezzanine over its west half for chemical addition tanks.

The tank room in the 242-Z Building houses large process vessels, which are piped to the process gloveboxes of the control room. The 242- $Z$ Building shares the main ventilation system in common with the 234-5Z and 236-Z Buildings and is equipped with the other PFP utilities and services.

\subsubsection{The 270-Z Building (Active)}

The 270-Z Building, also known as the PFP Operations Support Building, is a wood-frame structure with plaster board inner walls. This building houses Plant Management, Engineering, and Nuclear Facility Safety Personnel. 


\subsubsection{The 291-Z Butlding (Active)}

The 291-Z Building, also known as the Exhaust Fan House, Exhaust Air Stack Building, and Compressor and Fan House, is a reinforced concrete structure located approximately $16.2 \mathrm{~m}(53 \mathrm{ft})$ south of the central part of the 234-5Z Buflding. Of irregular shape, its approximate dimensions are $22.6 \mathrm{~m}(74 \mathrm{ft})$ wide by $43.6 \mathrm{~m}(143 \mathrm{ft})$ long. Its overall height is approximately $7 \mathrm{~m}(23 \mathrm{ft})$, with only $1.2 \mathrm{~m}(4 \mathrm{ft})$ above grade. This building houses the exhaust fans, the mechanical service equipment, and the substation. Auxiliary to $291-\mathrm{Z}$ is the 61-m- (200-ft-) high 291-z-1 stack.

\subsubsection{The 2736-Z Butlding (Active)}

Building 2736- $Z$ is the primary PFP Plutonium Storage Facility (PSF). Building 2736-Z is approximately $19.8 \mathrm{~m}(65 \mathrm{ft})$ long by $17 \mathrm{~m}(56 \mathrm{ft})$ wide. The building consists of four rooms for the storage of SNM, divided by a corridor running the width of the building. The building is constructed of reinforced concrete walls, $35.6 \mathrm{~cm}$ (14 in.) thick, supported by a cast-inplace concrete slab, $16.5 \mathrm{~cm}(6.5 \mathrm{in}$.) thick. Each storage room is approximately 8.5 by $8.5 \mathrm{~m}$ (28 by $28 \mathrm{ft}$ ). Rooms 1,3 , and 4 contain storage cubicles while room 2 has steel shelves and open floor storage.

\subsubsection{The 2736-ZB Building (Active)}

Building 2736-ZB, located immediately to the south of Building $2736-Z$, is approximately 40.2 by $27.4 \mathrm{~m}$ (132 by $90 \mathrm{ft}$ ) with reinforced concrete walls (except for administrative areas) and roof.

The 2736-ZB shipping and receiving areas each provide approximately $92.9 \mathrm{~m}^{2}\left(1,000 \mathrm{ft}^{2}\right)$ of floor space to accommodate a maximum of 100 shipping containers the size of 55-gal drums. Containers are spaced to meet Westinghouse Hanford Company's criticality prevention and personnel exposure specifications as well as allowing corridor access to staging areas. The two areas are physically separated by a wall. The majority of shipping containers handled contain plutonium oxide powder, plutonium metal, or miscellaneous solid scrap materials from various onsite and offsite sources.

\subsection{PROCESS AND FACILITY HISTORY} the PFP.

The following is a chronological list of historical events relating to

- July 1949 - Project C-198. Factlity starts up with rubber glove/remote button line.

- June 1951 - Project C-198 is mostly complete, including installation (but not startup) of the remote mechanical line (now known as the RMA line) that would replace the rubber glove/remote button line. 
- August 1951 - Development work is initiated on the Recuplex solvent extraction process for recovering piutonium from waste materials and from process solutions originating at separations plants.

- May 1953 - Project C-413 is completed. This project provided improvements to the RMA line, including increased efficiency, capacity, and instrumentation, and added facilities. The project also added a second partial remote mechanical line called RMB. The RMA line started up and the rubber glove line shut down shortly before this project was completed.

- April 1955 - Project C-496 is completed. This project installed the Recuplex Facility at the PFP.

- May 1955 - Project CG-551 is completed. This project installed modifications to the fluorination and metal reduction steps of the RMA line to increase capacity.

- September 1955 - Project CG-617 is completed. This project installed new process air drying facilities.

- May 1958 - Project CG-691 is completed. This project installed equipment for continuous processing steps for oxalate formation, filtration, calcining to oxide, and conversion to plutonium fluoride. The purpose of this project was to decrease costs and increase output.

- August 1958 - Project CG-756 provides fire protection modifications, including replacement of combustible exhaust filters with noncombustible filters.

- June 1959 - Project CG-745 adds plutonium fabrication equipment to what was known as the RMC fabrication line.

- September 1959 - Project CGC-800 installs radiation shielding and other equipment to reduce exposure to personnel working on the RMA line.

- October 1959 - Project CG-723 converts the Recuplex Facility from a semi-works to a manufacturing facility. Safety, contamination control, and process control are improved. Conversion supports increased plutonium production activity.

- January 1960 - Project CGC-826 replaces the plant 66-cm (26-in.) mercury vacuum system, including supplementary piping, modified filters, and cleanout facilities.

- July 1960 - Project CG-734 installs a second remote mechanical line, with improved radiation protection, for producing plutonium metal. This line, known as RMC, is to operate in addition to the RMA line.

- March 1961 - Project CG-789 installs additional fire protection systems. 
- September 1961 - Project CGC-813 installs the 232-Z Incinerator Building. The building includes equipment for leaching plutonium from wastes, for burning combustible plutonium-bearing materials, and for transferring the resulting product to cans.

- April 1962 - An accidental nuclear excursion occurs in the Recuplex facility, hastening its $\mathrm{pl}$ anned abandonment. No mechanical damage or spread of contamination occurred.

- July 1962 - Project CG-811 provides modernization of plutonium fabrication facilities and provides inspection facilities.

- February 1963 - Project CGC-978 provides interim facilities to process the filtrate from the RMC 1 ine (pending installation of a replacement system for Recuplex). Ion exchange columns are installed in glovebox HA-46 and tanks are provided in cell 46 to process filtrate.

- June 1964 - Project CGC-912 installs equipment to treat liquid process wastes that are routed to cribs. The purpose of the Project is to reduce the amount of plutonium discharged, since processing was increasing. The 242-Z Building is equipped with an ion exchange facility, solvent extraction column, process tanks, instrumentation, and a chemical makeup area.

- June 1964 - Project CAC-880 installs the new PRF in the 236-Z Building. The PRF replaces the functions performed by the Recuplex facility. It is designed to concentrate liquid feeds, dissolve or otherwise process solid material, and to perform solvent extraction recovery of plutonium from aqueous streams.

- May 1965 - Project CAC-121 installs a batch process americium recovery facility in the $242-Z$ Building. Feed to the facility is the solvent extraction waste stream from the 236-Z Building.

- May 1965 - Project CGC-180 installs the Plutonium Buy-Back Facility in room 227 of the 234-5Z Building. The new facility handles shipping activities.

- December 1965 - Fabrication of plutonium metal nuclear weapon components (Division of Military Applications line) ceases at the PFP.

- June 1970 - Project HCP-640 upgrades fire protection systems.

- October 1970 - Project HCE-637 upgrades the liquid waste treatment processes at $242-Z$ by providing a continuous system, and by increasing recovery of plutonium and americium.

- September 1971 - Project HCE-658 modifies the PFP to produce plutonium oxide for use in the Fast Flux Test Facility (FFTF) program. The RMA line fluorinator is modified to be used as a second stage calciner. Production scale capability is provided for sieving, blending, sampling, canning, and weighing plutonium oxide. 
- November 1971 - Project HCE-651 modifies the PRF to recover plutonium from mixed plutonium/uranium scraps. The PRF was originally designed to process plutonium-bearing scrap in support of metal preparation and fabrication. It previously had only a limited capability for separating plutonium from uranium.

- April 1973 - 232-Z Incinerator is shut down. The RMC line is placed in standby.

- October 1973 - Project HCP-653 upgrades fire protection systems.

- November 1973 - Project HCP-669 upgrades the PFP effluent control. The RMC line fluorinator is equipped with a new recirculating offgas scrubber, reducing the volume of some aqueous plutonium-bearing waste streams, comprised mainly of jet water. A new, underground encased piping system is installed to the 242-T Evaporator, for the transfer of all aqueous process waste to the tank farms. (This waste was previously sent to a crib.)

- May 1974 to May 1976 - Decommissioning and disposal of DMA line equipment.

- August 1976 - An explosion occurs in a 242-Z cation exchange column. The 242-Z Waste Treatment and Americium Recovery Facility is shut down.

- February 1979 - The PRF is placed in standby.

- December 1979 - The RMA line is shut down. Plutonium oxide blending and production end.

- 1979 - Central Scrap Management Organization (CSMO) inventory reduction begins and lasts through 1982 .

- September 1980 - The $242-Z$ Building is restabilized.

- October 1980 - A fire in a plutonium-scrap can occurs in the 234-5Z Building, room 230-C, contaminating most of the Zone 3 operating area.

- January 1981 - Decontamination of room $230-C$ is completed.

- February 1981 - A new central station alarm facility is completed.

- March 1981 - A new 43-cm (17-in.) mercury vacuum system is installed, replacing the $25-\mathrm{cm}(10-i n$.$) vacuum system.$

- January 1982 - Room 308 in the 234-5Z Building is contaminated during a filter change.

- February 1982 - Decontamination of room 308 in the 234-5Z Building is completed. 
- May 1982 - The PFP TCO programs are initiated. During the next three years, the TCO program successfully completes $232-Z$ (Incinerator), the RMA line, and removes the $66-\mathrm{cm}(26-i n$.$) vacuum$ system. Approximately $1,4000.61-$ by $0.61-$ by $0.30-m$ (2- by $2-$ by $1-f t$ ) high-efficiency particulate air (HEPA) filters were removed with the vacuum system and sent to TRU waste.

- August 1982 - Downloading and stabilization of the PRF is initiated.

- December 1982 - The PRF downloading is completed.

- June 1983 - The D\&D is initiated in 232-Z (Incinerator). Highlights included removal of about $50 \%$ of the $232-2$ gloveboxes, removal of about $66 \%$ of the Radioactive Acid Digestion Test Unit gloveboxes, and gloveboxes $\mathrm{HC}-30, \mathrm{HC}-58$, and $\mathrm{HC}-227$.

- 1984 - The RMC cleanout takes place from 1984 to 1985.

- January 1984 - The PRF is restarted. Initial feed is low weight percent plutonium scrap feed.

- January 1984 - The PRF processing campaign began. The dates for the four processing campaigns follow:

\begin{tabular}{|c|c|c|}
\hline PRF & Start date. & End date \\
\hline 1984 & January 1, 1984 & November 18,1984 \\
1985 & December 17, 1984 & May 31, 1985 \\
1986 & December 23,1985 & March 31, 1986 \\
1987 & July 1, 1987 & December 18, 1987 \\
\hline
\end{tabular}

- June 1985 - The RMC line is restarted, marking the beginning of seven RMC line campaigns.

\begin{tabular}{|c|c|c|}
\hline RMC & Start date & End date \\
\hline 1985 & July 1, 1985 & October 31, 1985 \\
$1986-1$ & April 5, 1986 & May 21, 1986 \\
$1986-2$ & June 6, 1986 & July 22, 1986 \\
$1986-3$ & September 1, 1986 & October 30,1986 \\
$1988-1$ & July 11, 1988 & August 18, 1988 \\
$1988-2$ & September 12, 1988 & November 10, 1988 \\
$1988-3$ & March 15, 1989 & June 2, 1989 \\
\hline
\end{tabular}


WHC-EP-0621

- January 1986 - The RMC Halon dry fire suppressant system is installed.

- August 1986 - Fire line safety upgrades completed.

- September 1986 - The PFP operations are halted for 9 months to assess administrative controls. Procedures and training are enhanced.

- March 1987 - A new 9AB filter box is installed.

- May 1987 - The PFP roof security upgrades are completed.

- June 1987 - Polychlorinated biphenyl (PCB) removal initiated.

- March 1989 - The 270-Z Operational Office Support Building is constructed.

- January 1991 - The PRF and RMC lines are operated sequentially.

In the forty-odd years that PFP has been in operation various processing missions have been accomplished. These include the following:

- Plutonium purification

- Oxide production

- Metal production

- Parts fabrication

- Plutonium recovery

- Recovery of $241 \mathrm{Am}$.

The PFP has been used for receipt and large-scale storage of onsite and offsite plutonium scrap and product materials.

\subsection{CURRENT PFP PROCESSES AND OPERATIONS}

The PFP complex is used to perform diversified plutonium processing, handling, storage, and support operations. Included in these operations are the following:

- SNM handling and storage

- Plutonium recovery

- Conversion of plutonium solutions into oxide and metal

- Laboratory support

- Radioactive waste handling

- Shutdown facility surveillance.

For a detailed narrative description of the PFP process systems refer to the PFP SAR (WHC 1991). The flow diagrams and the text are found in Appendix $A$ of this report. These diagrams show the flow of materials and

"Halon is a trademark of Allied-Signal, Incorporated. 
material balances for the plutonium handling and storage, plutonium recovery, and plutonium conversion operations. Many of the processes are "batch"

operations; consequently, material balances are based on the normal "batch" size.

\subsection{FUTURE PFP ACTIVITIES}

An operational readiness review is currently being done for the resumption of operations at the PFP. After DOE acceptance of a Declaration of Readiness, a sequential startup of the PFP will begin. Startup is planned for 1993 and will begin in the PRF. Once the PRF is online other processes will be prepared for readiness.

The next startup is planned for glovebox HC-60. The RMC will then undergo a series startup to resume production of stable plutonium oxides. HA-21-I will then begin burning scrap. After process and material treatment requirements are met, characterization and process evaluation will occur in preparation of the transition to standby status. 
WHC-EP-0621

Figure 2-1. Location of the Hanford Site.

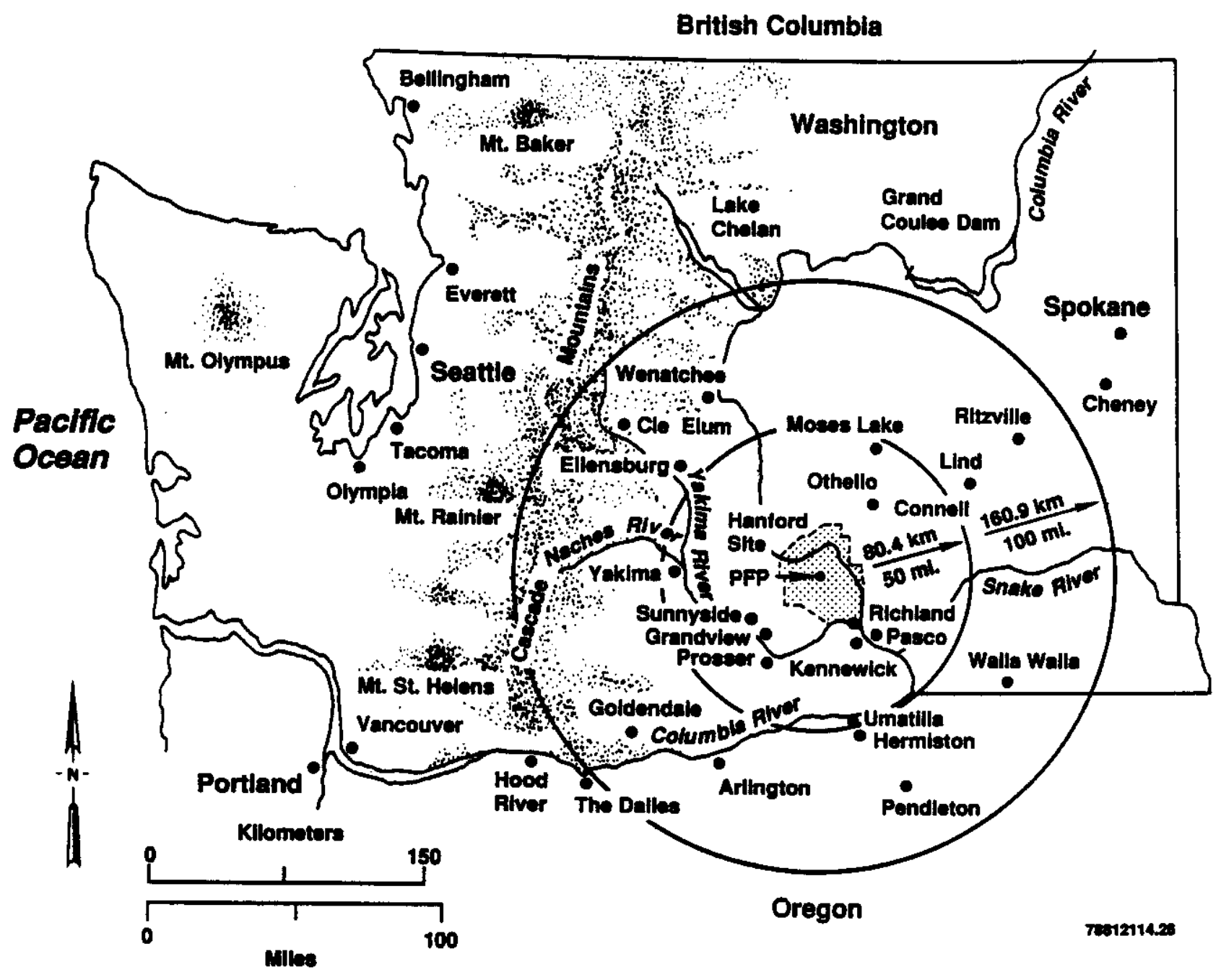


Figure 2-2. Hanford Site Map.

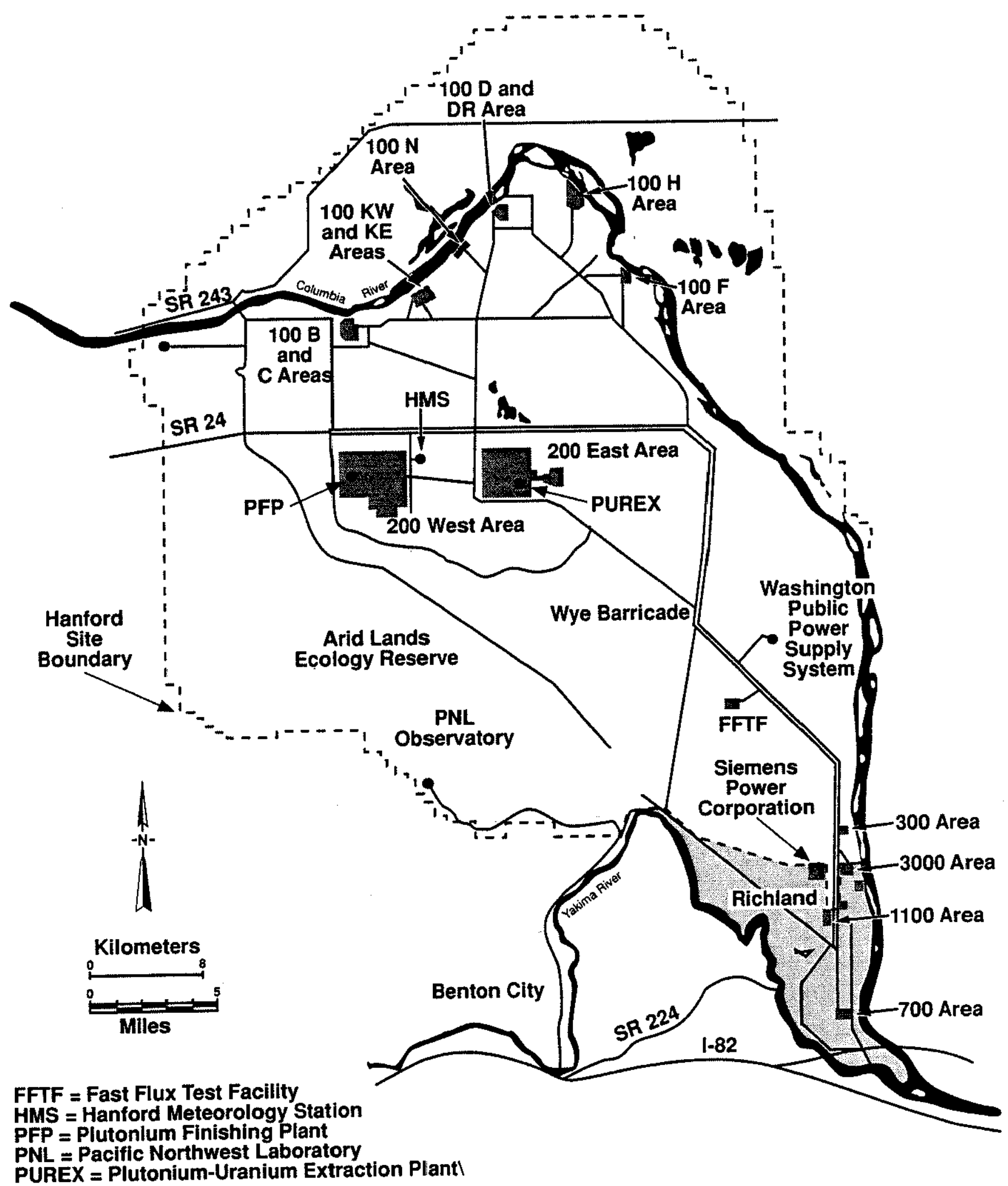


WHC-EP-0621

Figure 2-3. 200 West Area.

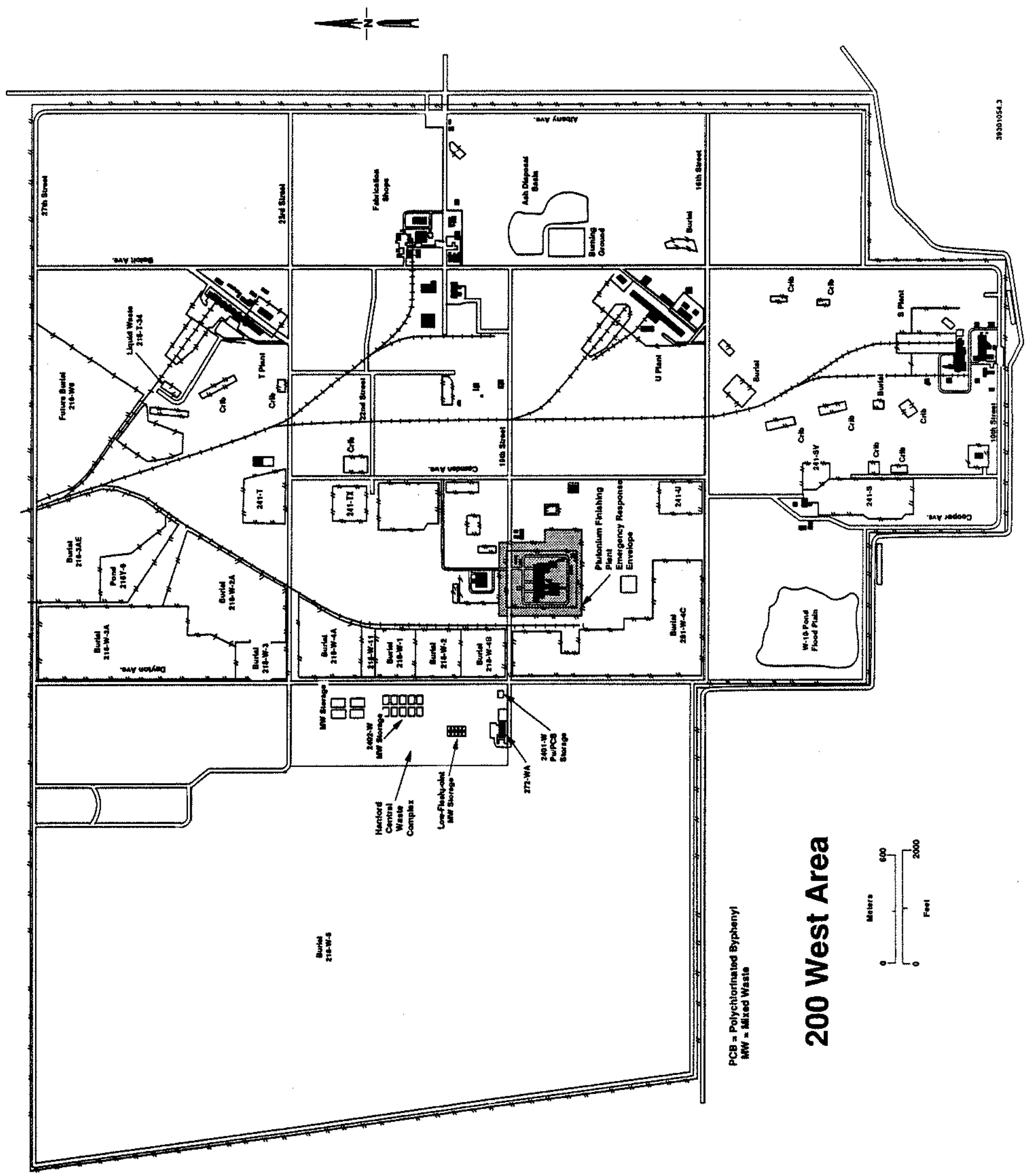


Figure 2-4. Aerial Photograph of the PFP.

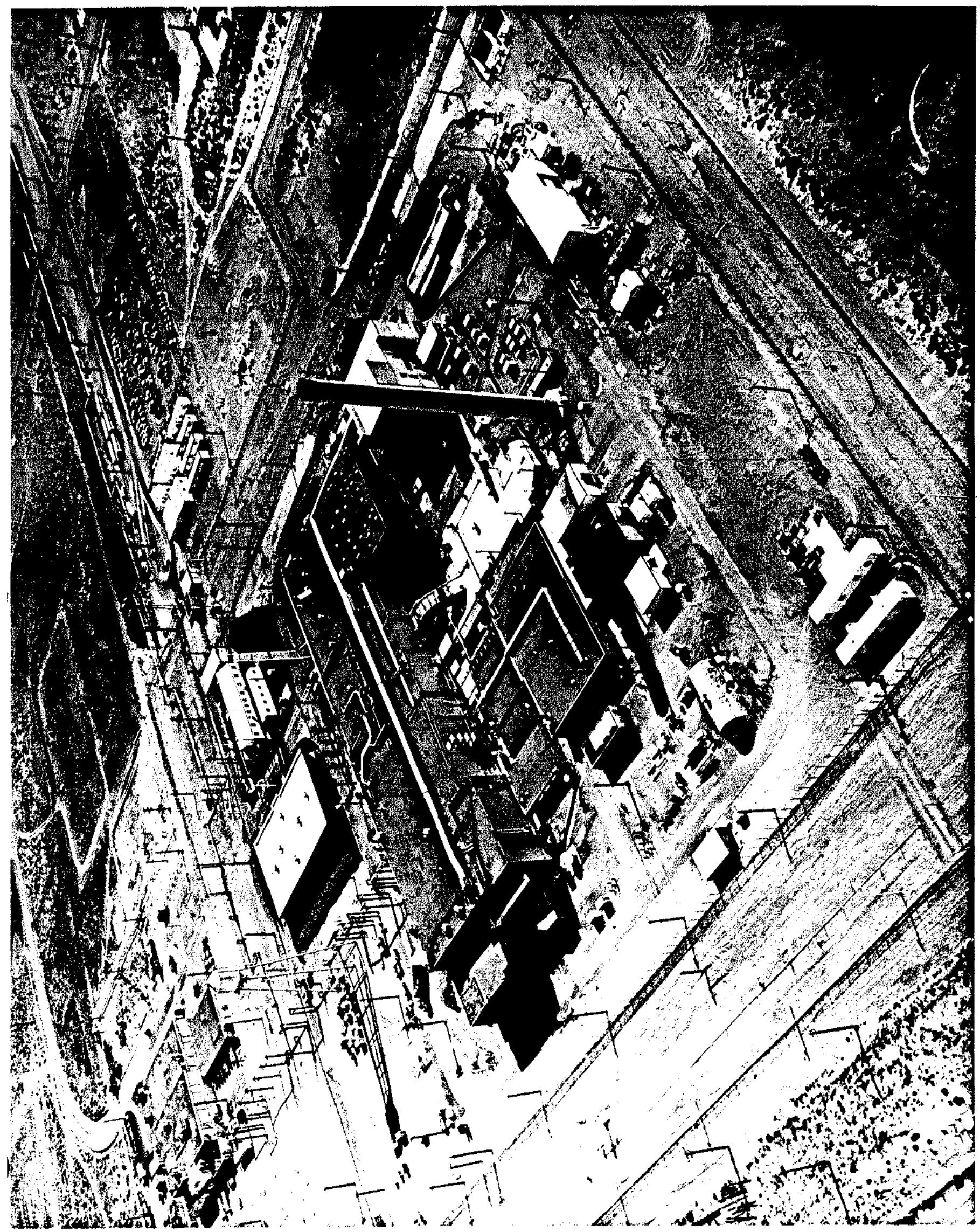


WHC-EP-0621

Figure 2-5. Main PFP Structures.

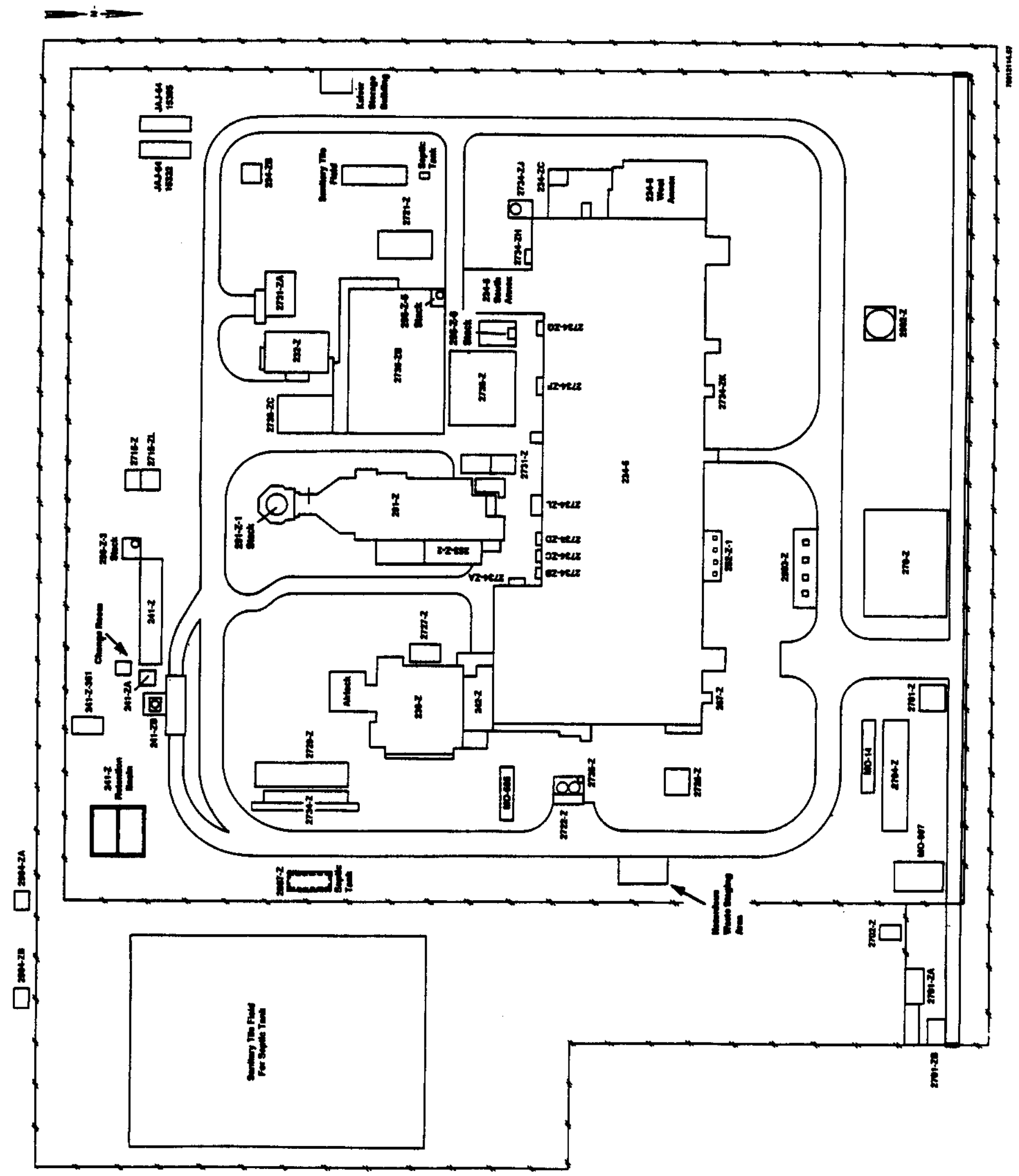


WHC-EP-0621

\subsection{PFP SOLID WASTE STREAMS}

\subsection{INTRODUCTION}

All solid wastes originating from the PFP are packaged for onsite or offsite storage or disposal. Radioactive solid wastes are packaged in metal drums or burial boxes, depending on the size of the wastes. Burial boxes are used to dispose of failed equipment, HEPA filters, and other radioactive solids too large to fit into 55-gal drums. Dangerous solid wastes are collected in metal drums. Nonradioactive, nondangerous solid wastes are generally collected in metal dumpsters. Figures 3-1 through 3-5 show the destination points for the PFP solid wastes.

Waste packages generated at the PFP must meet the criteria of the storage/disposal unit. For TRU wastes, these criteria are the WIPP Waste Acceptance Certification Criteria (WEC 1989). For LLWs these criteria are those established by the LLW burial grounds (Willis and Triner 1991). Hazardous and mixed waste must meet regulatory standards established by Ecology (1989) and the U.S. Department of Transportation (DOT) (1989).

\subsection{RADIOACTIVE WASTE SEGREGATION}

All radioactive solid wastes generated at the PFP are segregated at the point of generation depending on waste category. Solid wastes removed from gloveboxes and other wastes potentially contaminated with TRU elements are segregated as TRU wastes. These wastes are often referred to as "hood wastes." All other solid wastes generated within radiation control zones are segregated as LLW and are referred to as "room wastes." The TRU or LLWs that al so contain regulated hazardous wastes are segregated as radioactive mixed waste (RMW), and noncertified TRU wastes are segregated from other TRU wastes.

Before 1988, TRU and LLWs were segregated mainly as combustibles, noncombustibles, and absorbed organics. Noncombustibles could include glass, metal equipment, piping, etc.; combustibles could include paper cartons, plastic bottles, rags, wood, plastic bags, drybox gloves, etc.

\subsection{WASTE-GENERATING ACTIVITIES}

Four significant waste-generating activities at the PFP have been identified:

1. Plutonium processing

2. Required maintenance

3. Housekeeping

4. Waste processing.

These activities and the waste streams associated with each are discussed in the following sections. 
WHC-EP-0621

\subsubsection{Plutonium Production Processes} the PFP:

The following major plutonium production processes have occurred at

1. Plutonium recovery as plutonium nitrate solution from plutoniumbearing solid scrap and residues and mixed plutonium-uranium solid scrap and residues

2. Conversion of purified plutonium nitrate solutions to a plutonium oxide powder

3. Conversion of plutonium oxide to plutonium metal

4. Fabrication of nuclear weapon components from plutonium metal $(1960-65)$.

5. Incineration of combustible plutonium-bearing materials (1964-73).

The waste streams associated with each of these processes are discussed below. For a more complete discussion of these processes and the location of each facility involved refer to Section 2.0 and Appendix $A$.

\subsubsection{Plutonium Nitrate Feed Preparation. Plutonium-bearing solid} materials are dissolved in a variable mixture of nitric acid and fluoride ions using hydrofluoric acid or fluoride salts as a fluoride ion source. The plutonfum nitrate solution is transferred to the solvent extraction process while the dissolver heels are assayed for their plutonium content.

A simplified flowsheet is shown in Figure 3-6, "Recoverable Material Dissolution Flowsheet. " If a dissolver heel is more than the economic discard limit (EDL), the heel is saved for reprocessing. If the heel is less than the EOL, it is transferred to solid waste treatment as TRU waste.

The plutonium-bearing solid materials processed through the dissolving systems in the PFP have encompassed more than 50 different types of scrap. consequently, the composition of the discarded heels has varied accordingly. The most significant (by quantity) plutonium-bearing scrap processed has been sand, slag, and crucible (SS\&C) waste generated by the metal reduction process. The SS\&C consists of magnesium oxide sand, magnesium oxide crucible chips, calcium fluoride slag, calcium iodide booster, plutonium oxide, plutonium fluoride, free calcium metal, and traces of plutonium metal shot that did not coalesce during the bomb reduction. The composition of the discarded heel is shown in Figure 3-7, "Slag and Crucible Material Dissolution Flowsheet." As indicated in Figure 3-7, the major constituent of the dissolver heel is calcium fluoride $(92.3 \%$ ) with minor constituents of magnesium oxide $(3.0 \%$ ) and aluminum fluoride $(4.4 \%)$ and trace quantities of silicon oxide, plutonium oxide, plutonium, and plutonium fluoride. Another major contributor to dissolver heels was the processing of incinerator ash. From 1964 to 1973, an incinerator operated in 232-Z and burned plutoniumcontaminated gloves, rags, plastic bottles, paper cartons, and other

"The dissolution heels generated in dissolvers MT-5 and HC-46-F are assayed for plutonium content. 
miscellaneous combustible materials. The resulting ash was processed to recover the plutonium, which correspondingly generated an insoluble ash heel. The major constituents were silicon oxide (35-45\%) and carbon (10-40\%) with metal oxides making up the remainder. This insoluble heel was discarded to solid waste treatment as TRU waste once the plutonium was less than the EDL.

From 1965 to 1977, plutonium nitrate solutions were shipped to industrial contractors engaged in nuclear fuel preparation. The solutions employed criticality-safe plastic cylinders (about 10-L volume) as the primary containers. A considerable number of these plastic cylinders were often discarded in TRU waste, because of exterior contamination.

A tantalum crucible 1 id was employed in the metal reduction process. This lid was leached to remove any spattered plutonium metal and was subsequently discarded as TRU waste.

Other plutonium-bearing solid scrap was received from onsite- and offsite-generated dissolver heels. These heels were generally refractory metal oxides and sflicates and leached insoluble metal scrap such as stainless steels and tantalum items. These heels and substrates were discarded as TRU waste.

3.3.1.2 Solvent Extraction Process. The solvent extraction process generates a small amount of solid waste since essentially all processing reagents are liquids. Consequently, spent liquid reagents are either recycled or discarded to the tank farm as 1 iquid waste. A small quantity of solid waste is collected from the CAW centrifuge as shown in Figure 3-8, "Typical Solvent Extraction Flowsheet." The collected solids are insoluble particulates representing refractory metal oxides, ceramics, carbon, and high-fired plutonium oxide. If the plutonium is less than the EDL, these collected particulates are discharged to solid waste treatment as TRU waste.

3.3.1.3 Plutonium Nitrate to Plutonium Tetrafluoride Conversion. The plutonium nitrate to plutonium tetrafluoride conversion process is shown in Figure 3-9, "Remote Mechanical C Line Flow Diagram, Nitrate Solution Through plutonium fluoride Production." As indicated in Figure 3-9, no solid process waste is generated except for glovebox sweepings and other housekeeping activities. Usually, the glovebox sweepings are high enough in plutonium to warrant processing. The liquid wastes are discharged to the tank farm or to the 241-Z Building. The scrubber step indicated by the dashed 1 ines is a relatively recent addition to the process.

3.3.1.4 Plutonium Fluoride to Metal Conversion. The conversion of plutonium fluoride to a plutonium metal button is shown in Figure 3-10, "Remote Mechanical C Line Flow Diagram, plutonium fluoride Through Plutonium Metal Button Production." As shown in Figure 3-9, no solid process waste is generated. However, the slag and crucible produced is a high source of feed to the dissolvers.

3.3.1.5 Summary. The solid process wastes generated by the PRF and RMC 1 ine processing is minimal except for dissolver heels. The main source of TRU waste is from dissolver heels. The quantities of heels generated over the years of processing at the PFP are directly proportional to the plutionium scrap processed and the metal produced. A review of the metal production 
quantities per year and the kilograms of scrap processed can provide an estimate of the dissolver heels generated and discarded as TRU waste. The dissolver heels and other process solids were placed in plastic bottles and bagged out of the glovebox line.

3.3.1.6 Plutonium Component Fabrication. The fabrication of plutonium metal nuclear weapon components from 1960 to 1965 generated both TRU waste and classified waste. The TRU waste generated from foundry castings would be graphite molds, tantalum crucibles and funnels, ceramics, and furnace insulation. The TRU waste generated by machining and inspection operations would be spent machine tools, fixturing and gauging. Combustible waste would have been sent to the incinerator.

\subsubsection{Maintenance Requirements}

Solid waste generated by maintenance requirements was the result of five PFP mission assignments:

1. Plutonium metal schedules and requirements

2. Refurbishments/restarts to accomplish (1)

3. New processing assignments like the CSMO

4. Dismantling processes and D\&D work

5. Safety upgrades to the facility and supporting systems.

The extent of maintenance activities with respect to the five assignments above requires searching the facility records and archives. Time does not permit an extensive search. It is assumed that the following events would have produced significant maintenance activities:

- Replaced combustible exhaust filters with noncombustible filters and installed other fire protection modifications (1958)

- RMC line weapon fabrication equipment removed (1970-72)

- Provided the 236-Z Building with the partitioning capability to recover plutonium from mixed plutonium-uranium scrap (1972)

- Restarted the PRF (1984)

- Restarted RMC plutonium metal line.

Solid wastes generated by maintenance activities are packaged by the PFP operators, not maintenance personnel. Consequently, there is a consistency associated with solid waste packaging and with glovebox-generated waste. Both drums and boxes are used.

Maintenance solid wastes include the following:

- Spent and falled processing equipment constructed from a combination of glass, rubber, metal, ceramics, and plastics

- Piping, tubing, flanges, fittings, valves, traps, vessels, furnaces, and hot plates 
WHC-EP-0621

- Leaded glovebox windows (when unable to be salvaged)

- Miscellaneous items including bolts/nuts, straps, screws/nails, wire, connectors, and broken tools

- Sheet metal items including brackets, shelves, covers, and hoods.

\subsubsection{Housekeeping Activities}

The majority of the solid radioactive waste generated at the PFP consists of rags, paper cartons, HEPA filters, gloves, plastic bags, etc., which ąre generated mainly by housekeeping activities. The majority of the gloveboxgenerated housekeeping wastes are TRU, primarily from plutonium content. Glovebox sweepings and spill cleanups which contain recoverable quantities of plutonfum are designated to the PRF for processing.

Cleanup of SNM physical inventories will generate a considerable quantity of housekeeping wastes.

\subsubsection{Waste Processing Activities}

The majority of waste generated by the PFP processes is liquid waste which is handled by the tank farm system or the crib system. Wastes generated by waste processing at the PFP may include those from the following sources.

- Process-generated scrap and residues are recycled through the dissolution processes which generate dissolver heels. These heels are eventually discarded as TRU waste when (reduced to) less than the EDL. The largest heels generated are from the recycling of slag and crucible from the bomb reduction process.

- Discarding the spent carbon tetrachloride-tributylphosphate (TBP) extraction solvent generates TRU-mixed waste with a very hazardous carbon tetrachloride component. This change out of extraction solvent occurs very infrequently and is not a routine change out.

- The SNM physical inventories generate wastes, as all plutoniumbearing materials must be measured for their plutonium content. In addition, process equipment is dismantled to measure plutonium holdups. At the same time, the dismantled equipment usually undergoes maintenance, which generates spent equipment waste. Large quantities of rags are usually generated via cleanup requirements.

- A caustic scrubber is used for the fluorination of plutonium oxide to plutonium fluoride to capture and neutralize the HF gas released. The spent scrubber (potassium hydroxide $[\mathrm{KOH}]$ ) solution is sent to high salt waste.

- The operational history and TRU waste solids generated by the $242-Z$ waste solution treatment $\mathrm{plant}$ should be reviewed. It operated from 1964 to 1976, and was then shut down because of the accident involving americium. 
WHC-EP-0621

\subsection{HIGH PERIODS OF SOLID WASTE GENERATION}

High amounts of solid waste may have been generated by the PFP as a result of one or more of the following events:

- Restarting any process facility such as the PRF and RMC line requires refurbishment, upgrades, and modifications. These maintenance efforts generate solid wastes via strip-out of equipment, equipment overhauls, etc.

- Periods of high plutonium metal production, like the period from 1985 to 1988, will generate solid wastes as described herein.

- Campaigns to reduce the plutonium inventory at the Hanford Site, such as those in the late 1960's and early 1970's, and in the early 1980 's generate solid wastes related to producing plutonium oxide rather than metal. Classified production data must be reviewed to identify processing periods and production quantities.

- Strip-outs of facilities such as the Hanford Environmental Development Laboratory (HEDL) Radioactive Acid Digestion Test (RAD 2) facility, the 232-Z incinerator, the RMA and RMC weapon lines, and the Recuplex facility generate large quantities of solid waste.

- High periods of intense production at the PFP and other programmatic activities should be correlated with higher solid waste generation. These may include the following events.

- Button lines (metal production) operated from 1950 to 1973 with varying metal outputs. See metal production schedules.

- The RMC button line was restarted in 1985. From 1985 until 1988 the PFP ran seven processing campaigns.

- Waste Treatment Building, 242-Z, was placed onstream to remove plutonium and ${ }^{241} \mathrm{Am}$ from the PFP waste effluent streams, and operated from July 1964 to August 1976. Americium-241 recovered and sent to PNL for further purification and used for alpha/gamma sources.

- The PRF was cleaned out and refurbished (1984-85).

- The PRF is restarted and runs from 1987 to 1988.

- The RMA line is operated from 1971 to 1973 , and then is cleaned out from 1974 to 1979. This was the plutonium oxide blending program for Babcock and Wilcox. 
WHC-EP-0621

\subsection{CURRENT PFP SOLID HASTE INVENTORY AND NONINAL GENERATION RATES}

The estimated radiological wastes listed below are taken from the PFP SAR (WHC 1991).

Radioactive waste generation rates are likely to have been estimated on the low side. This is done to avoid expenses that would result from a high estimate. The quantities of nonradioactive hazardous waste generated are likely to decrease because of new practices whereby non-Westinghouse Hanford Company contractors dispose of the nonradioactive wastes generated by their activities.

- WIPP-certifiable waste includes plastics (bags), cloth (rags), rubber, metal, and glass. The nominal generation rate for this type of waste is $96.28 \mathrm{~m}^{3} / \mathrm{yr}\left(3,400 \mathrm{ft}^{3} / \mathrm{yr}\right)$ or $46055-\mathrm{gal} \mathrm{drums} / \mathrm{yr}$.

Burial boxes of mixed wastes (lead panels and leaded glass windows) are alşo WIPP-certifiable. Their nominal generation rate is $2.12 \mathrm{~m}^{3} / \mathrm{yr}\left(75 \mathrm{ft}^{3} / \mathrm{yr}\right)$.

- Non-WIPP-certifiable waste includes waste-containing HEPA filters, aerosol cans, and leaded glovebox gloves. Nominal generation rates are as follows:

- HEPA fj.jters 20.32 by $20.32 \mathrm{~cm}(8$ by $8 \mathrm{in.})-0.42 \mathrm{~m}^{3} / \mathrm{yr}$ $\left(15 \mathrm{ft}^{3} / \mathrm{yr}\right)$ or two 55 -gal drums/yr

- Leaded glovebox gloves mixed waste - $2.12 \mathrm{~m}^{3} / \mathrm{yr}\left(75 \mathrm{ft}^{3} / \mathrm{yr}\right)$ or ten 55-gal drums/yr

- Burial boxes for items too large to fit into 55-gal drums $198.21 \mathrm{~m}^{3} / \mathrm{yr}\left(7,000 \mathrm{ft}^{3} / \mathrm{yr}\right)$.

These are currently stored at the PFP, then shipped to retrievable storage.

- Low-level radioactive nonhazardous waste (mainly room waste) is transferred to burial grounds for disposal. In 1991, the total low-1evvel radioactive nonhazardous waste was 1,035 55-gal drums or $221 \mathrm{~m}^{3}\left(7,804.5 \mathrm{ft}^{3}\right)$.

- Nonhazardous, zone 3 office waste includes paper, plastic, rubber, metal, and glass items; and clothing. The nominal generation rate is $45.87 \mathrm{~m}^{3} / \mathrm{yr}\left(1,620 \mathrm{ft}^{3} / \mathrm{yr}\right)$ compacted or $22055-\mathrm{gal} \mathrm{drums} / \mathrm{yr}$.

- Nonhazardous, zone 3 room waste includes paper, plastic, cloth, rubber, metal, and $g$ lazss items. The nominal generation rate is $150.08 \mathrm{~m}^{3} / \mathrm{yr}\left(5,300 \mathrm{ft}^{3} / \mathrm{yr}\right)$ compacted or $1,44655-\mathrm{gal}$ drums $/ \mathrm{yr}$.

- Nonhazardous aerosol cans are generated at a nominal rate of $0.42 \mathrm{~m}^{3} / \mathrm{yr}$ (15 $\mathrm{ft}^{3} / \mathrm{yr}$ ) or two $55-\mathrm{gal} \mathrm{drums} / \mathrm{yr}$. 
- The nonhazardous 0.61 - by $0.61-\mathrm{m}$ (2- by 2-ft) HEPA filters, including plywood- and metal-covered filters, are generated at a nominal rate of $16.99 \mathrm{~m}^{3} / \mathrm{yr}\left(600 \mathrm{ft}^{3} / \mathrm{yr}\right)$.

- The nonhazardous, low-level burial box nominal waste generation rate is $2.83 \mathrm{~m}^{3} / \mathrm{yr}\left(100 \mathrm{ft}^{3} / \mathrm{yr}\right)$.

- Hazardous fluorescent lamps nominal waste generation rate is $0.11 \mathrm{~m}^{3} / \mathrm{yr}\left(4 \mathrm{ft}^{3} / \mathrm{yr}\right)$ or $0.555-\mathrm{gal} \mathrm{drum} / \mathrm{yr}$.

- Hazardous lead alkaline dry cell batterieş, and wet cell batteries are generated at a nominal rate of $0.23 \mathrm{~m}^{3} / \mathrm{yr}\left(8 \mathrm{ft}^{3} / \mathrm{yr}\right)$ or one 55-gal drum/yr.

- PCB-contaminated and absorbed organic wastes include TRU-absorbed organics (carbon tetrachloride, TBP, TBP in normal paraffin hydrocarbons, xylene, trimethyi benzene, nonionic detergent, and trioctal phosphine oxide) that are absorbed onto diatomaceous earth materials and placed into 55-gal drums. The nominal waste generation rate is $1.47 \mathrm{~m}^{3} / \mathrm{yr}\left(52 \mathrm{ft}^{3} / \mathrm{yr}\right)$ or $7 \mathrm{drums} / \mathrm{yr}$.

- TRU PCB-contaminated waste may consist of rags, protective clothing, tools, hydraulic hoses, and general equipment. The nominal waste generation rate is $0.84 \mathrm{~m}^{3} / \mathrm{yr}\left(30 \mathrm{ft}^{3} / \mathrm{yr}\right)$ or four $55-\mathrm{gal}$ drums $/ \mathrm{yr}$. The TRU PCB-contaminated burial box waste nominal generation rate is $1.41 \mathrm{~m}^{3} / \mathrm{yr}\left(50 \mathrm{ft}^{3} / \mathrm{yr}\right)$. For TRU-PCB-hydraulic fluids (greater than $55 \mathrm{ppm}$ and less than 70,000 ppm PCB in normal paraffin hydrocarbon or polyphenyl phosphate-based hydraulic fluids) the nominal waste generation rate is $1.41 \mathrm{~m}^{3} / \mathrm{yr}\left(50 \mathrm{ft}^{3} / \mathrm{yr}\right)$ or seven 55 -gal drums/yr.

- Low-level absorbed organics (12 L [3.17 gal] per drum) such as carbon tetrachloride and TBP are generated at a nominal rate of $1.41 \mathrm{~m}^{3} / \mathrm{yr}\left(50 \mathrm{ft}^{3} / \mathrm{yr}\right)$.

- For low-level PCB hydraul ic fluids (greater than 50 ppm and less than $70,000 \mathrm{ppm}$ PCB in diesel or PYDRAUL or FYRQUEL" hydraulic fluids ( $30 \mathrm{~L}$ per drum) the nominal waste generation rate is $1.87 \mathrm{~m}^{3} / \mathrm{yr}$ (66 $\mathrm{ft}^{3} / \mathrm{yr}$ ) or ninety $55-\mathrm{gal}$ drums $/ \mathrm{yr}$.

- Other typical PFP wastes estimated on an annual basis are as follows:

- Waste nitrates and other oxidizers - 2,481 kg $(5,469.58 \mathrm{lb})$

- Benzenes and other halogenated benzenes - $65 \mathrm{~kg}(143.30 \mathrm{lb})$

- Toxic process chemicals - $840 \mathrm{~kg}(1,851.85 \mathrm{lb})$

- Carbon tetrachloride and other extremely hazardous hydrocarbons - $14 \mathrm{~kg}(30.86 \mathrm{lb})$

"FYRQUEL is a trademark of Akzo Chemicals Incorporated. 
WHC-EP-0621

- Acids - $437 \mathrm{~kg}(963.40 \mathrm{lb})$

- Sodium hydroxide and alkaline liquids - 1,720 kg $(3,791.89 \mathrm{lb})$

- Poisonous laboratory chemicals - $4 \mathrm{~kg}(8.82 \mathrm{lb})$

- Miscellaneous laboratory chemicals - $7.5 \mathrm{~kg}$ (16.53 lb)

- Flammable liquids - $17 \mathrm{~kg}(37.48 \mathrm{lb})$

- Paint, thinners, resins, and asphalt - $127 \mathrm{~kg}$ (279.98 lb)

- Nonflammable refrigerated gas - $280 \mathrm{~kg}(617.28 \mathrm{lb})$.

\subsection{FUTURE FACILITY CHANGES AND RESULTING WASTE OPERATION}

The future facility changes described in the PFP SAR (WHC 1991) will produce radioactive, hazardous, and mixed wastes. Although accurate forecasts of solid waste quantities have not been made, personnel interviews indicate the possible scope of waste generation. Due to their conjectural nature, the actual wastes encountered, and the quantities produced, are likely to be greater than the following estimates. Since the PFP SAR was published, many of the projects listed have been either completed or canceled; only those projects likely to be undertaken will be described.

- PFP Supply Air Pressure Control System upgrade - This project will provide an upgrade of the 234-5Z Building supply air system. The damper units have already been replaced, and the controller will be replaced in the future. The controller, PRC-1, contains approximately $450 \mathrm{~g}$ (16 oz) of mercury.

- Carbon tetrachloride pumping station and storage areas - This project will provide a 3,000-gal stainless steel tank for storage of carbon tetrachloride. The waste generated in this project will be in the form of piping and tanks.

- 2736-ZB tile field - This project will add an additional 1,000-gal septic tank and approximately 91.44 linear m (300 linear $\mathrm{ft}$ ) of drain pipe to supplement the existing drain field. Quantities of radioactive soil will be excavated and require disposal.

- 232-Z Isolation Project - This project will isolate the 232-Z ducting and building. Part of this project will involve the replacement of zone controllers in 234-52 and the PRF. These controllers contain approximately $566 \mathrm{~g}(20 \mathrm{oz})$ of Gargoyle, an oil which is classified as a hazardous waste.

"Gargoyle is a trademark of the Mobile 0il Corporation. 
- PFP liquid effluent treatment facilities - This project will provide a TRU extraction system to remove TRU elements from the liquid effluents presently routed to the $241-Z$ Waste Retention Facility, and provide upgrades to 241- $Z$. Hazardous and mixed wastes will be generated by this project, primarily in the form of large quantities of contaminated soil.

- PFP Liquid LLW System modification - This project will provide a new LLW treatment facility, and the installation of a closed-loop cooling system. Radioactive and/or mixed waste may be generated in modest quantities, principaliy in the form of contaminated soil.

- PFP fire safety upgrade - This project will provide upgrades to the 234-5Z Building to meet a current DOE requirement. Radioactive waste will be generated in the changeout of some alarms and horns. 
Figure 3-1. Destination of Nonradioactive, Nondangerous Solid Waste for Storage/Disposal.

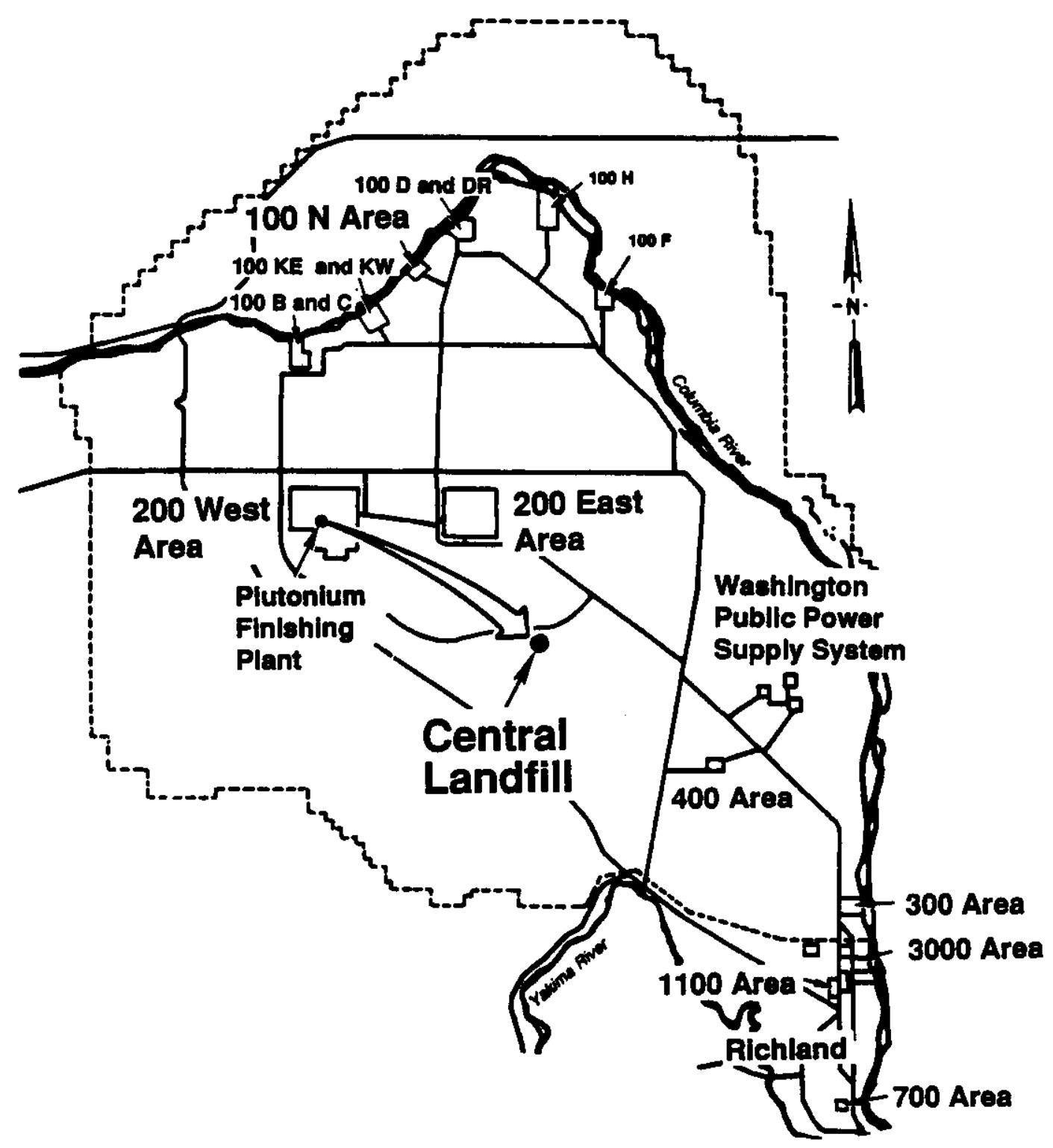

70001048-122 
Figure 3-2. Destination of Nonradioactive, Dangerous Solid Waste for Disposal.

Offsite

Treatment

Disposal

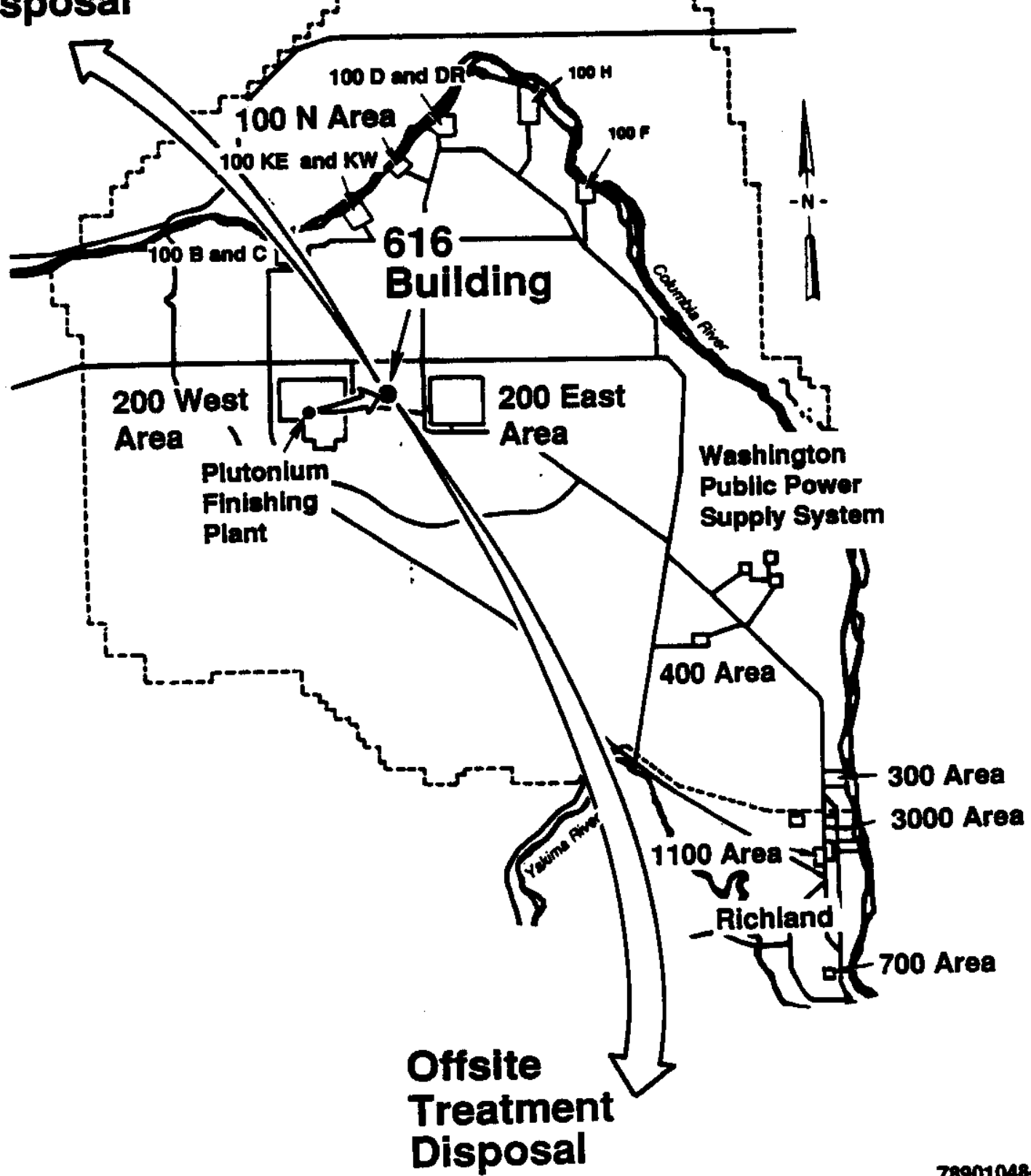

78901048-121 
Figure 3-3. Destination of Low-Level Radioactive Solid Waste for Storage.

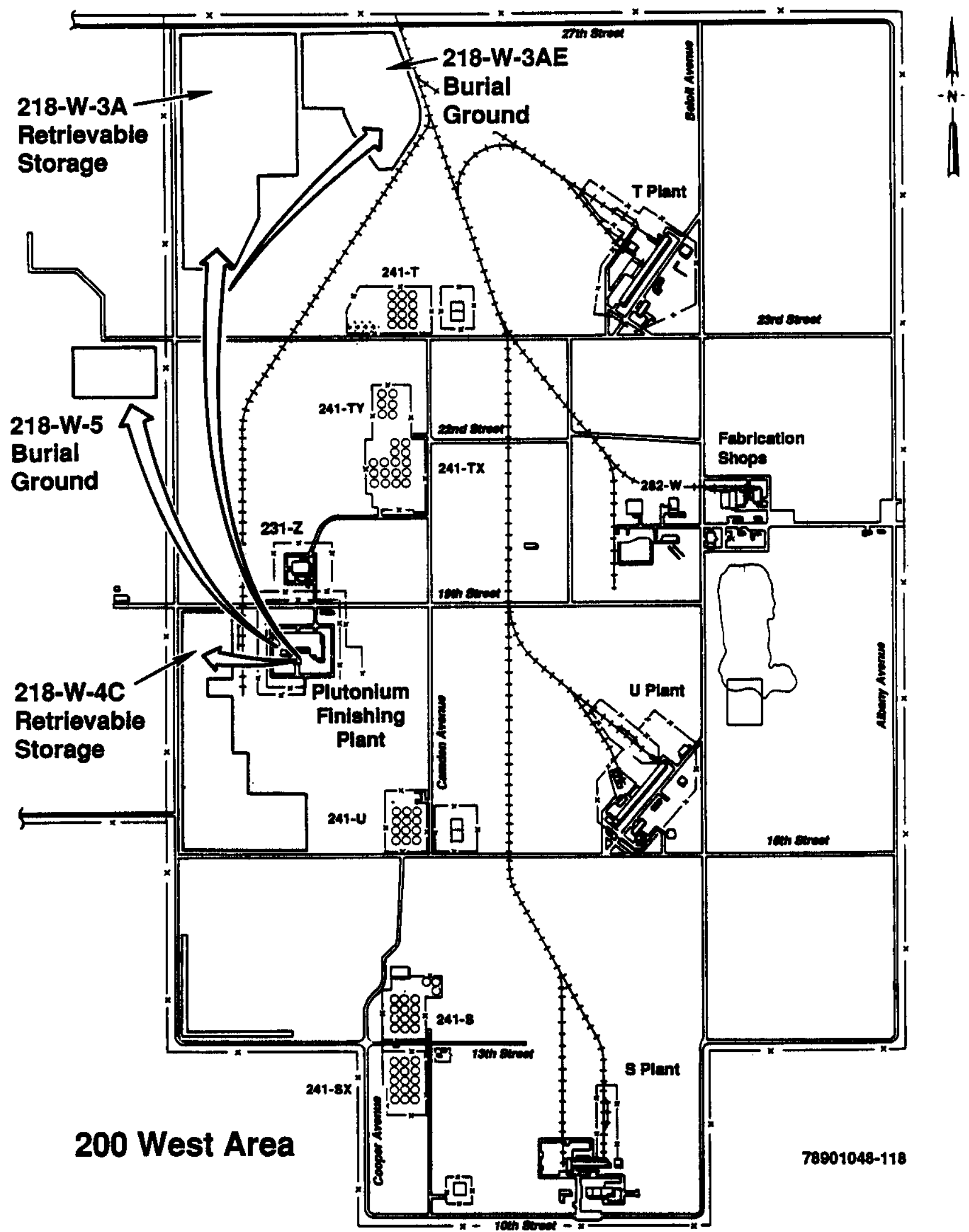


Figure 3-4. Destination of WIPP-Certifiable TRU Waste for Storage.

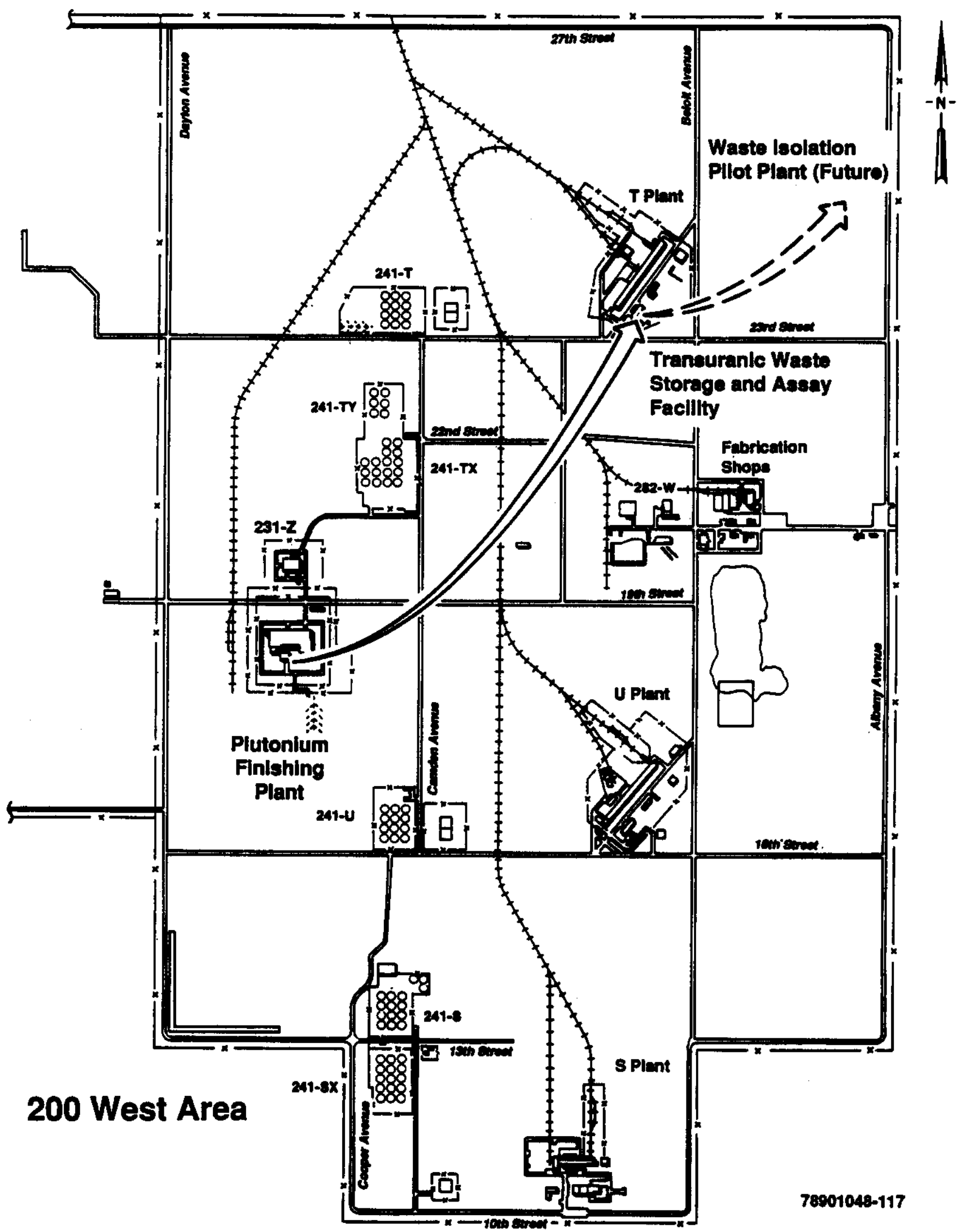


Figure 3-5. Destination of Non-WIPP-Certifiable TRU Waste for Storage.

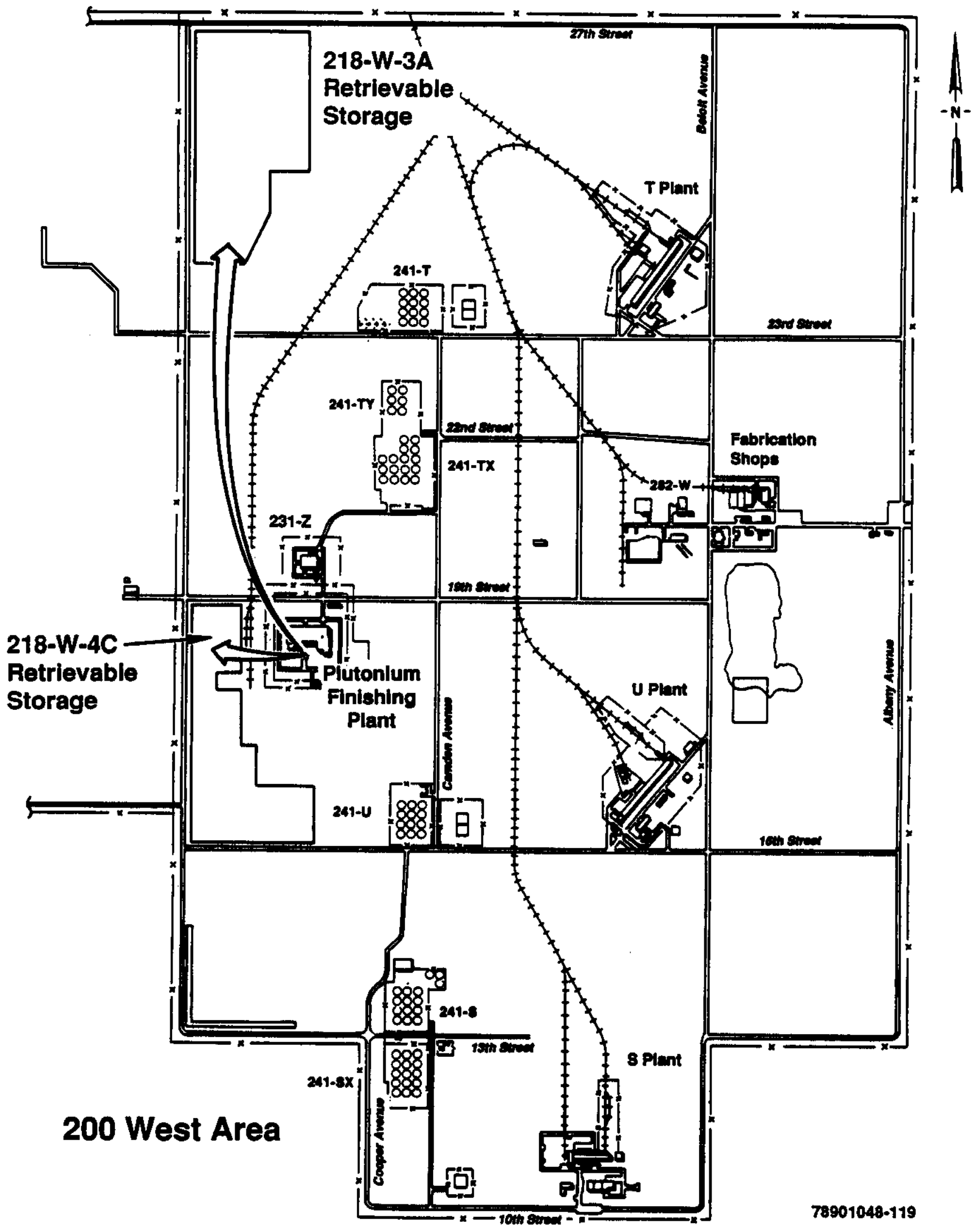


WHC-EP-0621

Figure 3-6. Recoverable Material Dissolution Flowsheet.

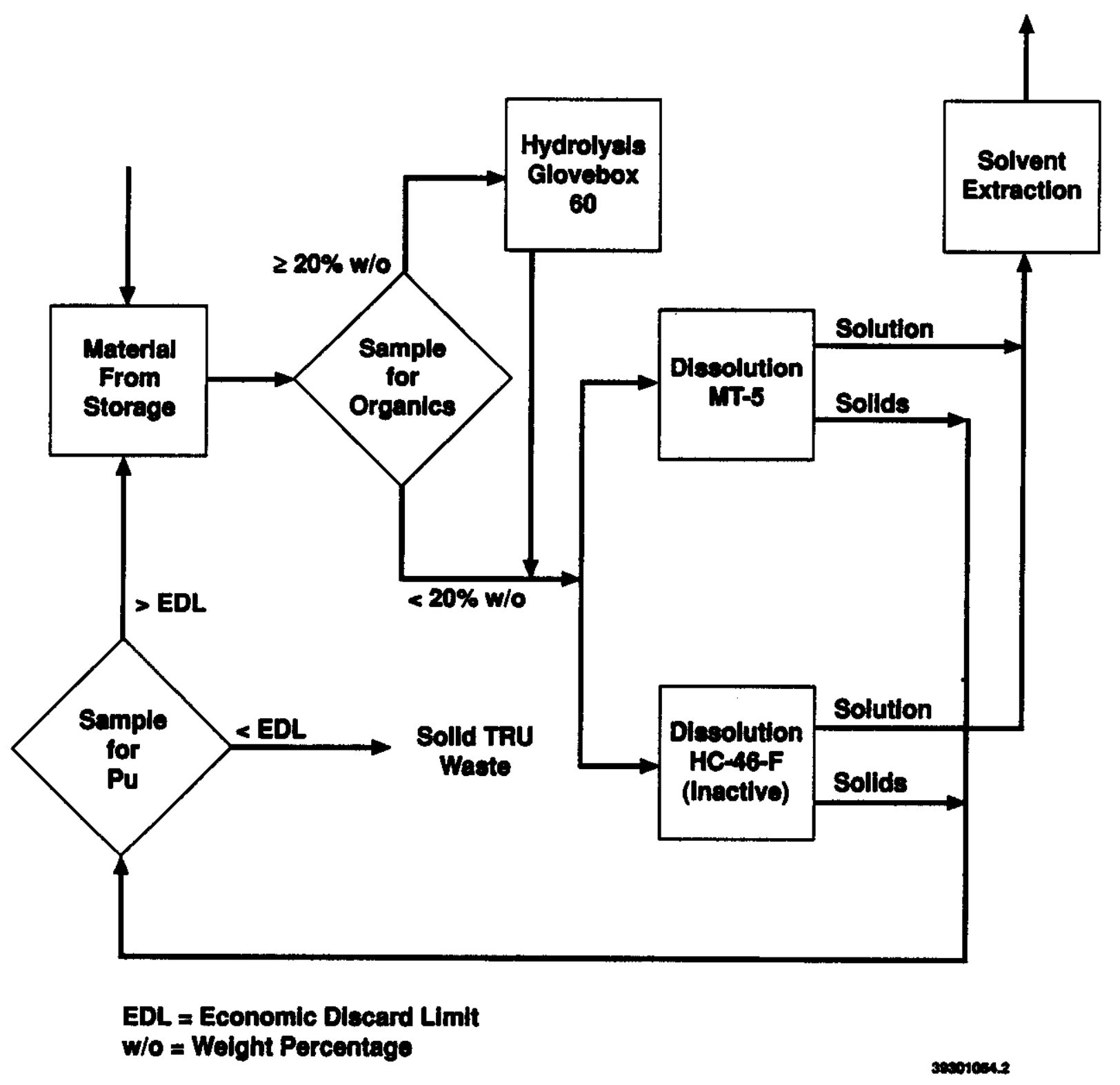




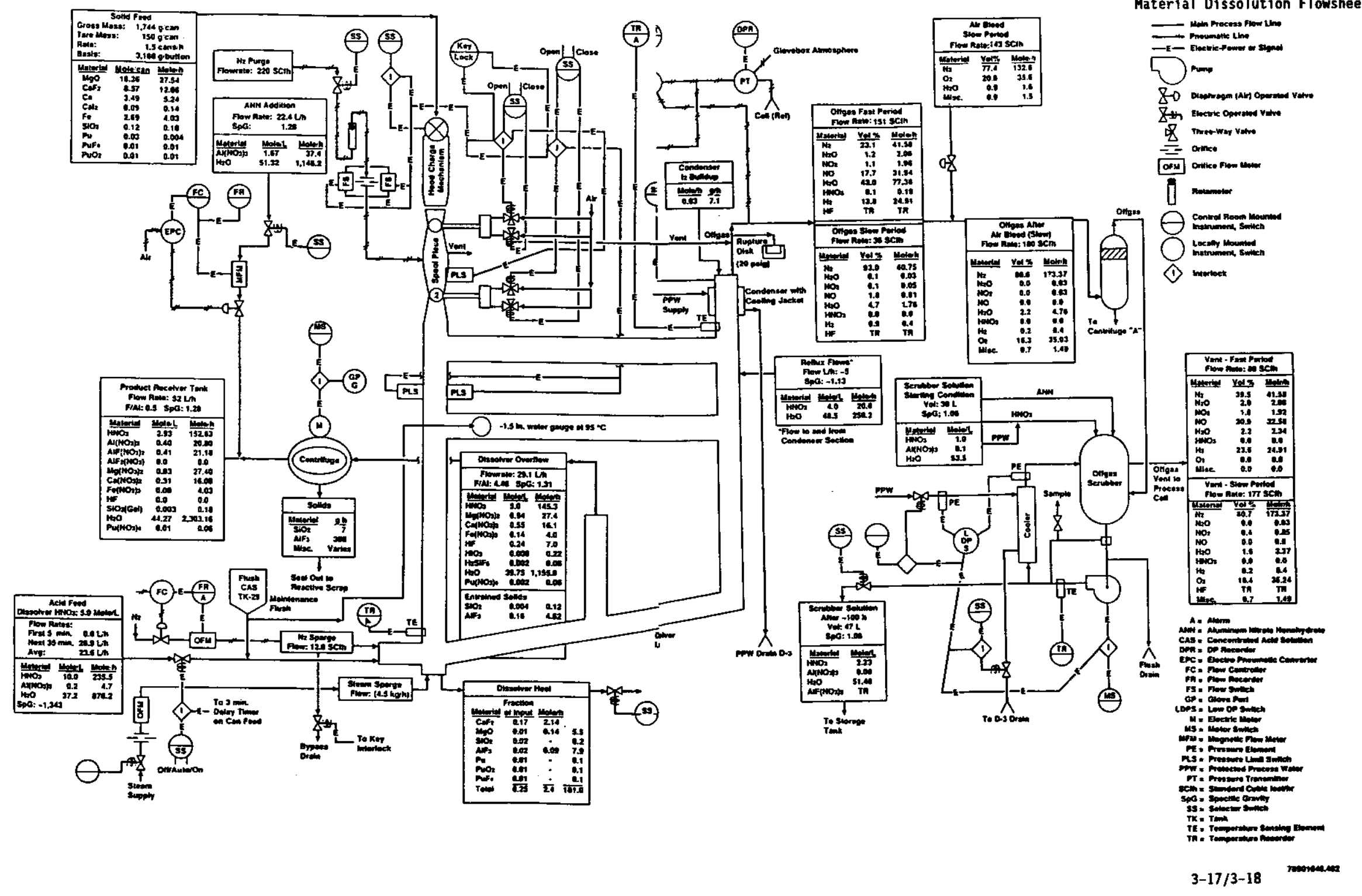


Figure 3-8. Typical Solvent

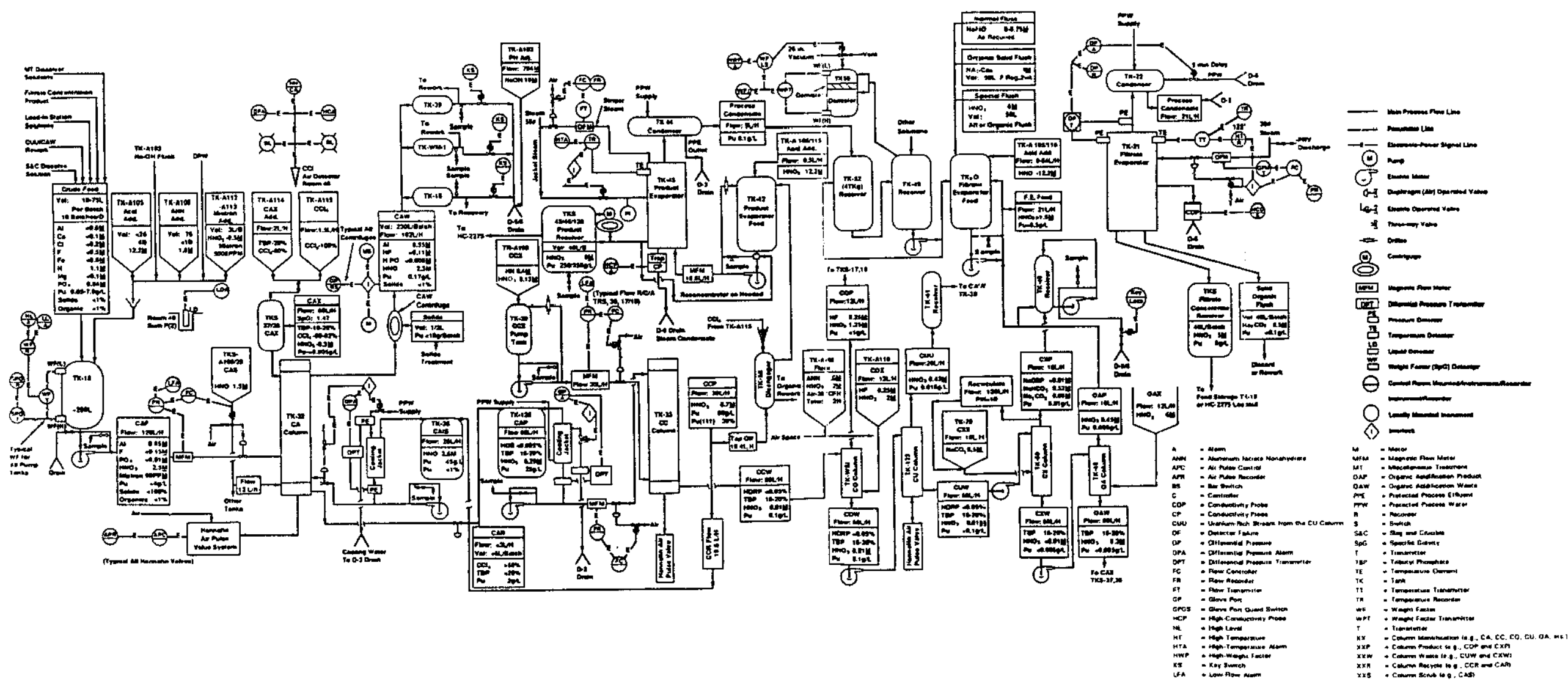

$3-19 / 3-20$ 
WHC-EP-0621

Figure 3-9. Remote Mechanical C Line Flow Diagram, Nitrate Solution Through Plutonium Fluoride Production.

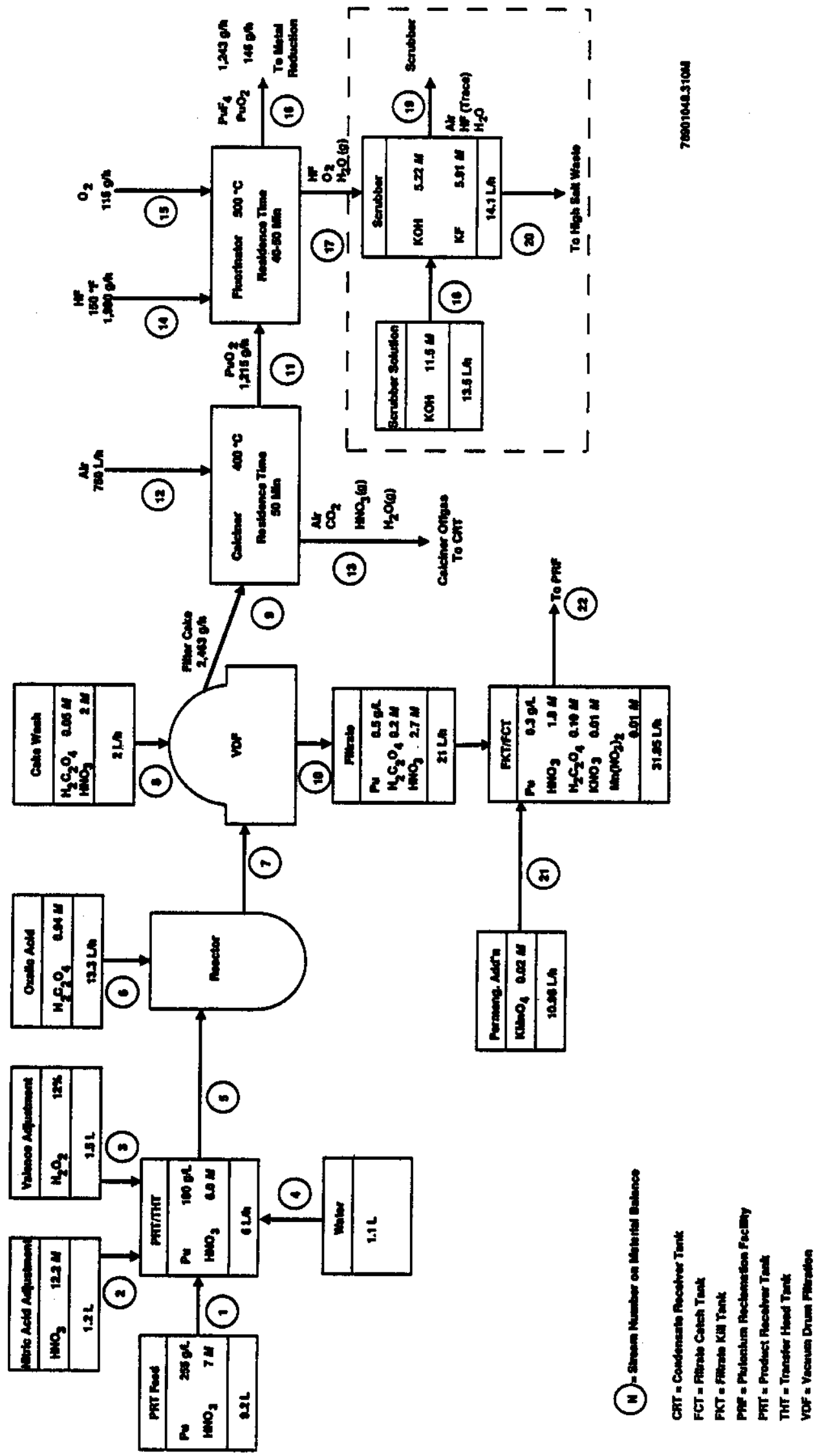


Figure 3-10. Remote Mechanical C Line Flow Diagram, Plutonium Fluoride Through Plutonium Button Production.

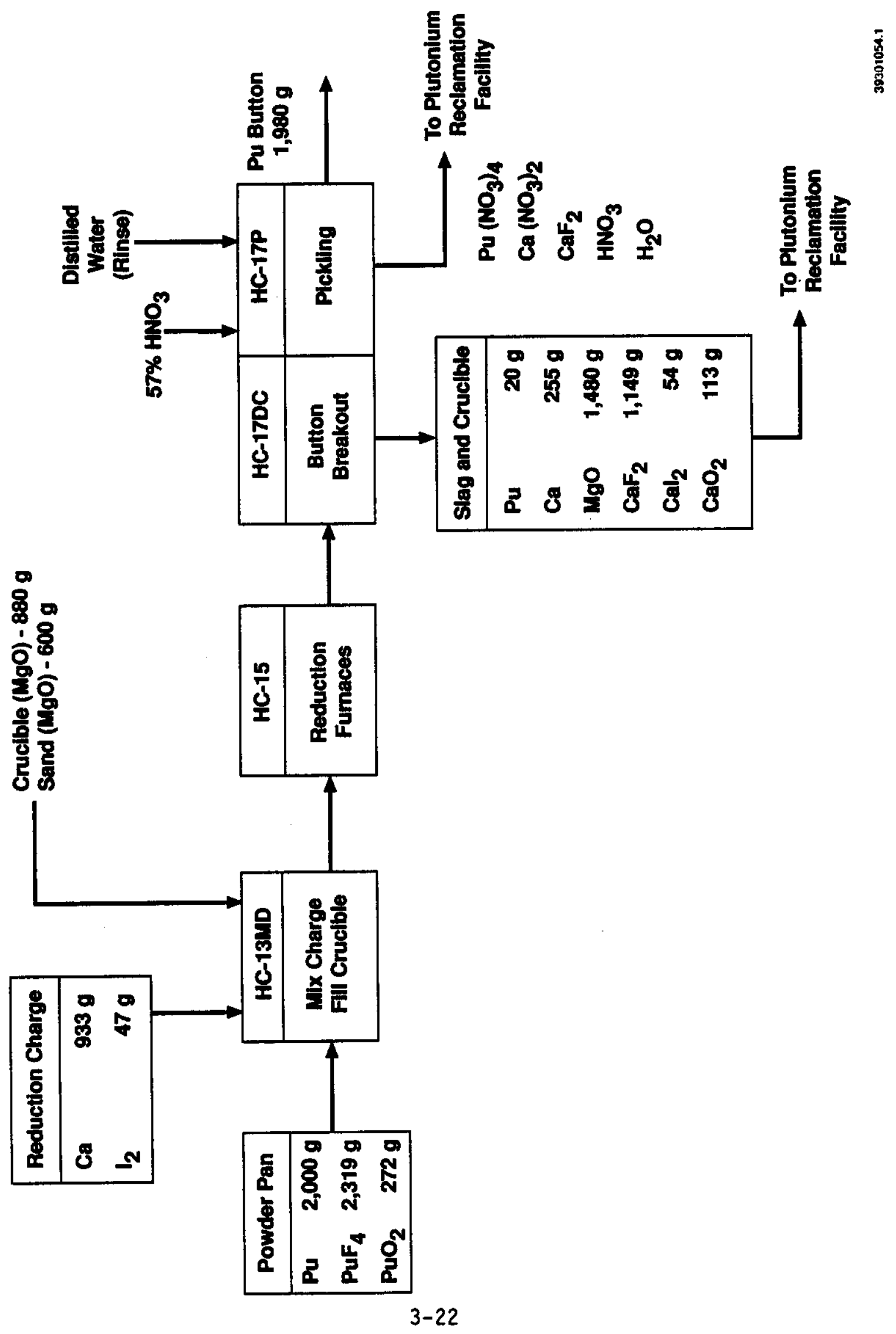


WHC-EP-0621

\subsection{SOLID WASTE HANDLING PRACTICES AND PROCEDURES}

\subsection{HISTORICAL WASTE PACKAGING AND HANDLING PROCEDURES}

Eleven historical waste packaging manuals were reviewed for information regarding packaging and labeling requirements for TRU waste in retrievable storage at the Hanford Site. These manuals covered requirements from 1974 to 1988 .

Packaging requirements have changed over time and have become more stringent. Table 4-1 summarizes TRU waste storage requirements for the Hanford Site from 1970 through 1988. The columns in Table 4-1 are not symmetrical; where a definition or requirement is consistent between manuals, text is shared under several document number columns. A blank spot indicates that no requirements or definition are found in that manual. Definitions (i.e., hazardous and toxic materials, etc.) change from manual to manual and require reference to the particular manual.

Some of the more significant changes that have occurred in the past 20 years with regard to the packaging, handling, and recordkeeping for radioactive wastes from the PFP follow.

- The definition of TRU waste has changed since 1970 , and the designation of waste packages as TRU has also changed. During the period from 1970 to 1973 TRU segregation was based on generator practice. From 1974 unti1 1982, TRU waste was segregated if the concentration of TRU was greater than $10 \mathrm{nCi} / \mathrm{g}$. In 1982, the current $100 \mathrm{nCi} / \mathrm{g}$ definition for TRU waste was implemented by DOE.

- Because of the varied contents of the waste containers, chemical reactions can occur. Accumulation of gases, including hydrogen, may contribute to fire and/or explosion. Since 1978, all waste drums from PFP have been fitted with vent clips to allow continual release of gases and catalyst packs to recombine any hydrogen that may be produced.

- Criticality specifications limited the amount of TRU to less than $250 \mathrm{~g}(8.82 \mathrm{oz}) / 55$-gal drum from 1975 to 1978 and to $200 \mathrm{~g}$ ( $7.05 \mathrm{oz}) /$ drum after 1978 . Before 1975 there were no criticality limits.

- Individual container weights were not required before 1977; however, est imated weights for pre-1977 containers were added to the records in the R-SWIMS database during the data re-entry program in the mid1980 's. Standard weights were given for each container type. Al1 55-gal drums, for example, were given a value of $68 \mathrm{~kg}$ (150 lb).

- Originally, waste burial records for TRU waste were not done for individual containers, but for entire shipments. In 1982, TRU waste burial and storage records began to be based on an individual container basis. The entry of unique data for each waste container into the R-SWIMS database was not fully implemented until 1984. 
WHC-EP-0621

During the R-SWIMS re-entry program, historical TRU records were converted to an individual basis so that today there is one SWITS record for each TRU container stored at the Hanford Site.

The conversion from group data to individual container data required that some assumptions about the containers in the group be made. Chief among these assumptions was that an even distribution of radiological and hazardous constituents existed among the members of the group.

LLW records are still kept for multiple containers rather than for individual containers.

- Information on the hazardous constituents of waste containers was not required before 1986. During the R-SWIMS re-entry program any avallable information from the SWBRs was added. However, this information is limited.

- Physical descriptions of the waste contents were not required on the SWBR before 1978. The SWITS records for waste buried before 1978 list the contents as "miscellaneous" in most cases. In more recent years the physical contents description has become more detailed. Many of the records now include the weight and volume percentage of a given component in the waste container.

- Between 1972 and 1978 combustible and noncombustible waste components were segregated. Although the SWITS database did not retain a data field for this information, it was added to the physical contents description field.

- The most common TRU waste container used at the Hanford Site is the 55-gal steel drum. The DOT $17 \mathrm{C}$ or $17 \mathrm{H}$ drums with minimum wall thicknesses of 0.13 and $0.11 \mathrm{~cm}(0.053$ and 0.043 in.), respectively, were both used. Before 1982 most of the drums were painted, so there is approximately $0.01 \mathrm{~cm}(0.005 \mathrm{in}$.$) of paint on both the$ exterior and interior of the drums. In 1982, galvanized drums replaced the painted drums. Recycled 55-gal drums were also permitted for the storage of TRU waste from 1973 to 1978.

- Data for wastes generated before 1970 are not included in the SWITS database.

\subsection{CURRENT WASTE HANDLING AND PACKAGING}

All solid wastes originating at the PFP are packaged for onsite or offsite storage or disposal. The design objective for waste packaging is to safely contain the waste during temporary storage at the PFP and during transportation to storage or disposal units. The waste packages must also meet the criteria of the storage/disposal unit. For TRU wastes, these criteria are the WIPP waste acceptance/certification criteria (WEC 1989) and Hanford Site Solid Waste Acceptance Criteria (Willis and Triner 1991). For LLWs these criteria are those established by the LLW burial grounds

(Willis and Triner 1991). Hazardous and mixed waste must meet regulatory 
WHC-EP-0621

standards established by Ecology and DOT. The objectives of solid waste management activities also include proper segregation of all types of solid wastes.

This section describes the equipment and procedures for packaging radioactive solid wastes currently in use at the PFP. Additional information can be found in the PFP SAR (WHC 1991); Hanford Site Solid Waste Acceptance Criteria (Willis and Triner 1991); and Solid Waste Repackaging and Disposal - PFP Engineering Laboratories (Louk 1991).

\subsubsection{Equipment and System Description}

Radioactive solid wastes are packaged in metal drums or burial boxes, depending on the size of the wastes. Burial boxes are used to dispose of failed equipment, HEPA filters, and other radioactive solids too large to fit into 55-gal drums. Dangerous solid wastes are collected in metal drums. Nonradioactive, nondangerous solid wastes are generally collected in metal dumpsters.

All radioactive solid wastes generated at the PFP are segregated at the point of generation depending on waste category. Sol id wastes removed from gloveboxes and other wastes potentially contaminated with TRU elements are segregated as TRU wastes (hood wastes). All other solid wastes generated within radiation control zones are segregated as LLW (room wastes). In addition, TRU or LLWs that is also RMW are segregated, and noncertifiable TRU wastes are segregated from other TRU wastes.

Radioactive waste drums are not compacted at the PFP because of the potential for rupturing radioactively contaminated packages contained within the drum, which could result in a spread of radioactive contamination.

Radioactive waste drums consist of DOT 17C 55-gal galvanized or aluminized drums. Each drum is quality control (QC) inspected and approved before use and is provided with a trace number. Each drum contains $3 \mathrm{~L}$ (0.79 gal) of diatomaceous earth absorbent in the bottom of the drum, a minimum of 4-mil polyethylene liner, and $3 \mathrm{~L}(0.79 \mathrm{gal})$ of diatomaceous earth in the bottom of the liner. Drums are sealed with lock ring seals with lock bolts tightened to 54 joules (40 ft-lb) torque. Starting in 1979, the PFP drums were equipped with a carbon-filter vented lid. Those drums not equipped with the vented lid require that a vent $c l i p$ and a hydrogen-oxygen recombinant catalyst pack be added to the drum to prevent pressurization resulting from the decomposition and/or interaction of organics that may be present.

Burial boxes consist of steel boxes that are painted inside and outside so that no bare metal is exposed. A box may be lined with a 6-mil polyethylene liner. Boxes are designed to be watertight and are kept closed when not filled. Once filled, boxes are bolted shut with bolts tightened to specified torques, and security seals are applied and inspected by QC staff.

The plutonium content of each drum or waste package is determined through nondestructive assay (NDA) techniques. The NDA is performed using a sodium iodide counter or small table Segmented Gamma Scan Assay System (SGSAS) counters. Drums are temporarily staged inside the PFP before NDA. Following 
WHC-EP-0621

NDA, room waste drums are stored before they are transferred to the burial grounds. Hood waste drums are transferred to the 2736-ZB Building before they are transferred to the Transuranic Storage and Assay Facility (TRUSAF). Burial boxes are stored outside of the PFP, to the north, south, and west of the 234-5Z Building.

Al1 drums are assayed when they reach TRUSAF. If a LLW drum assays at less that $100 \mathrm{nCi} / \mathrm{g}$, it is sent to the burial grounds; if the LLW drum assays at greater than $100 \mathrm{nCi} / \mathrm{g}$, it is sent back to the plant to be repackaged as TRU waste. If a TRU drum assays at less then $100 \mathrm{nCi} / \mathrm{g}$, it is sent back to the plant to be repackaged as LLW.

A drum repackage facility is located in the 234-5Z Building. The repackaging facility consists of a glovebox with an attached greenhouse. Drums are opened and the contents removed and sealed into the glovebox for examination.

\subsubsection{Operating Procedures}

4.2.2.1 Monitoring of Solid Waste. Solid waste monitoring procedures include the use of administrative controls to control the quantities of radioactive materials placed in each waste container, and the use of NDA to determine the amount of plutonium present. As each waste package is placed into a waste container, a logsheet is filled out specifying the total amount of plutonium present. Room waste packages (i.e., packages obviously containing less than $1 \mathrm{~g}[0.03 \mathrm{oz}]$ plutonium) may be placed directly into the waste container after being surveyed with a portable alpha radiation detector to verify that there is no surface contamination. Other waste packages must be placed in transfer containers and taken for NDA to determine the plutonium inventory before placement in the waste container. Management must be notified if the waste package contains greater than $200 \mathrm{~g}(7.05 \mathrm{oz})$ of plutonium. After NDA, the waste package is placed into the container. Waste packages are added until the waste container is determined to be ful1. Drums are considered full when any of the following conditions are met:

- PFP drums - When plutonium content reaches $200 \mathrm{~g}(7.05 \mathrm{oz})$ (WIPP 1 imit)

- PRF drums - When plutonium content reached $150 \mathrm{~g}(5.29 \mathrm{oz}$ ) (based on the PRF operating experience; in some cases, total drum assay is higher than sum of individual package assays)

- HEPA filter drums - When the plutonium content reaches $200 \mathrm{~g}$ $(7.05 \mathrm{oz})$, or $100 \mathrm{~g}(3.53 \mathrm{oz})$ if a filter contains more than $40 \mathrm{~g}$ $(1.41 \mathrm{oz})$, which is the criticality limit.

- When the drum surface dose rate reaches $100 \mathrm{mrem} / \mathrm{h}$ at any point (TRUSAF limit). Drums with surface dose rates of up to $200 \mathrm{mrem} / \mathrm{h}$ may be accepted at the TRUSAF with a signed waiver. Two hundred mrem/h is the WIPP limit 
- When the drum estimated gross weight reaches $136 \mathrm{~kg}(300 \mathrm{lb})$ (the PFP limit based on personnel drum handling safety) or up to $227 \mathrm{~kg}$ (500 lb) with management approval

- When waste reaches level of the top rolling hoop on the drum (Safety Analysis Report for Packaging [SARP] 1 imit, to ensure compliance with DOE authorized shipping criteria).

After each drum is filled, the entire drum undergoes NDA to determine the plutonium inventory. Initially, drums are weighed and counted on the sodium iodide counter. Further actions depend upon the results of the assay, as follows.

- If less than $1 \mathrm{~g}(0.03 \mathrm{Oz})$ - Attach standard DOT radioactive materials label, and remove fissile labels.

- If between 1 and $10 \mathrm{~g}(0.03$ and $0.35 \mathrm{oz})$ - Attach standard package label recording identification number, type of material, gross weight, grams of fissile material, net weight; hydrogen-tofissionable material atom ratio $(H / X)$, and seal number. The $H / X$ value is a gauge of the extent of moderation of fizzile materials. The $H / X$ value reflects the moisture content of the package. The higher the $H / X$ value, the greater the affinity of the waste package for water. Packages with high $H / X$ values tend to attract and absorb water. The water acts as a moderator, making the material less stable. Low $H / X$ values are desirable.

- If greater than $10 \mathrm{~g}(0.35 \mathrm{Oz})$ - Transfer the drum to SGSAS in the 2736-ZB Butlding. Have NDA personnel count the drum. If greater than $20.0 \mathrm{~g}(7.05 \mathrm{oz})$ plutonium, notify management.

Waste packages and containers are also surveyed to control radiation exposure. The external surface dose from a waste drum may not exceed $200 \mathrm{mrem} / \mathrm{h}$. Each waste package is limited to $20 \mathrm{dpm} / 100 \mathrm{~cm}^{2}$ alpha smearable contamination.

4.2.2.2 Solid Waste Packaging and Handling Safety and Criticality Prevention. Criticality safety limits for solid waste management are based on determination of the fissionable material content of each waste package placed in a waste container and on observation of fissionable material limits and handling procedures. For 55-gal waste drums and transfer cans, the following procedures and limits have been established.

- Maximum plutonium or ${ }^{235} \mathrm{U}$ in $55-g a l$ drums is $200 \mathrm{~g}(7.05 \mathrm{oz})$.

- Maximum plutonium permitted in a 55-gal drum containing HEPA filters is $200 \mathrm{~g}(7.05 \mathrm{Oz})$ for filters with less than $40 \mathrm{~g}(1.41 \mathrm{oz})$ per filter or $100 \mathrm{~g}(3.53 \mathrm{oz})$ for filters with more than $40 \mathrm{~g}(1.41 \mathrm{oz})$ per filter.

- Maximum plutonium and ${ }^{235} \mathrm{U}$ in a yellow $23-\mathrm{kg}(50-1 \mathrm{~b}) \mathrm{lard}$ can containing solid gloveboxes waste is $250 \mathrm{~g}(8.82 \mathrm{oz})$. Yellow cans are to be used for transportation only and not for storage. 
WHC-EP-0621

The following criticality safety-related limits and procedures have been established for burial boxes.

- Plutonium content for each HEPA filter is not to exceed $40 \mathrm{~g}$ ( $1.41 \mathrm{oz})$ for a $60.96-$ by $60.96-$ by $30.48-\mathrm{cm}$ (24- by $24-$ by $12-\mathrm{in}$.) filter, $20 \mathrm{~g}(0.71 \mathrm{oz})$ for a $60.96-$ by $60.96-$ by $30.48-\mathrm{cm}$ (24- by 24- by 6-in.) filter, and $2 \mathrm{~g}(0.07 \mathrm{oz})$ for an 20.32 - by $20.32-$ by $15.24-\mathrm{cm}$ (8- by 8- by 6 -in.) filter.

- Maximum plutonium content is $350 \mathrm{~g}(12.34 \mathrm{oz})$ per piece of equipment and $1,000 \mathrm{~g}$ (35.27 $\mathrm{oz})$ per burial box.

- Burial boxes will contain a total of no more than $15 \mathrm{~g}(0.53 \mathrm{oz})$ of plutonium in all waste material other than equipment pieces or HEPA filters.

Additional safety procedures established for waste containers include the following.

- All water-soaked rags are wrung out and dried before loading. Acidsoaked rags are treated with dilute nitric acid, wrung out in a glovebox, and then neutralized with sodium hydroxide, wrung out in a glovebox again, and allowed to dry using drying bars in the glovebox. Diatomaceous earth is added to all packages that might contain inorganic liquid to help ensure that all free liquids are absorbed.

- Sharp corners and edges are padded and taped before packages are loaded into drums and boxes.

- Aerosol cans are to be disposed of in drums marked "Aerosol Cans Only." The cans are periodically removed and processed before disposal. Aerosol cans must be permanently vented before disposal.

- The HEPA filters are to be disposed of in drums marked "HEPA Filters Only" and are not placed in room or hood waste drums.

The following items are prohibited from waste drums and fiberboard boxes:

- Chemically incompatible materials in any waste container (40 CFR 265.313, [EPA 1989a])

- Explosives (10 CFR 61.56, [EPA 1990])

- Pyrophonics (10 CFR 61.56, [EPA 1990])

- Gas cylinders (including aerosol cans) that are not permanently vented

- Chelating compounds (prohibited from disposal only; they may be included in future treatment on a case-by-case bas is (DOE Order 5820.2A [DOE 1988]) 


\section{WHC-EP-0621}

- Liquids, except as packaged in accordance with WHC-EP-0063-3

(Willis and Triner 1991) (40 CFR 265.314 [EPA 1989a] and 10 CFR 61.56 [EPA 1990]).

Administrative controls are relied upon for solid waste segregation. Separate waste containers are provided for each type of waste (i.e., room waste, certifiable hood waste, noncertifiable hood waste, mixed room waste, mixed hood waste, and dangerous waste). Logsheets are used to track the inventory of each waste container to ensure that unauthorized wastes are not present. In addition, written procedures exist for each waste and container type. 
WHC-EP-0621

This page intentionally left blank. 
Table 4-1. Comparison of Past and Present TRU

Storage Requirements for the Hanford S1 te.

$(3$ sheets)

\begin{tabular}{|c|c|c|c|c|c|c|c|c|}
\hline \multicolumn{9}{|c|}{ Document numbers and dates } \\
\hline $4 / 74:-4032$ & 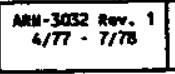 & 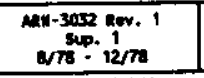 & 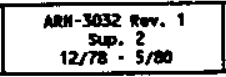 & $\frac{500-m-222}{3 / 00}=6 / 20$ & 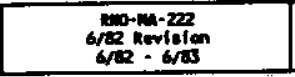 & 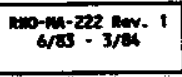 & 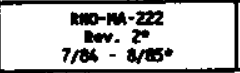 & 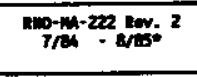 \\
\hline \multicolumn{9}{|c|}{ TRu definition } \\
\hline 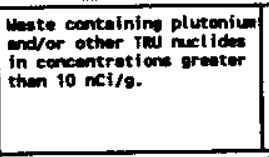 & 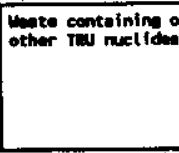 & 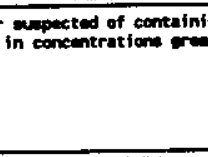 & 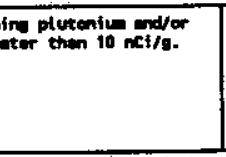 & somentitite. & 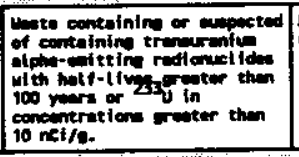 & 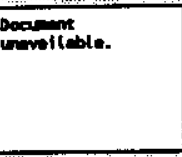 & \multicolumn{2}{|c|}{ 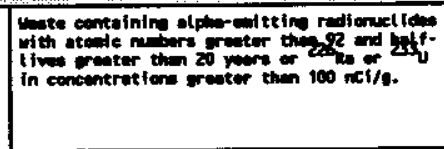 } \\
\hline \multirow{2}{*}{\multicolumn{3}{|c|}{ 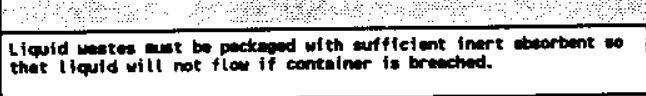 }} & Prohibited waste & types and & ackeging requirements & es & . & \\
\hline & & & to fros liquitch. & & 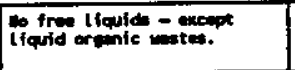 & & 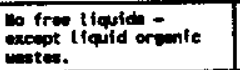 & Mo froe lifaldis. \\
\hline & \multicolumn{2}{|c|}{ 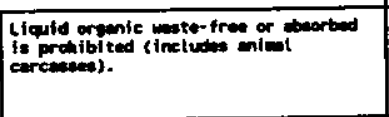 } & 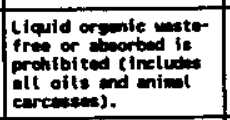 & & 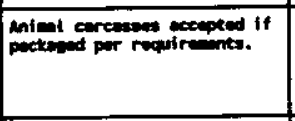 & & \multicolumn{2}{|c|}{ 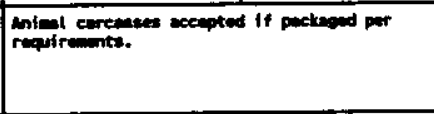 } \\
\hline & \multicolumn{2}{|c|}{ 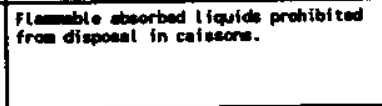 } & 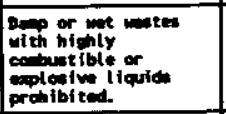 & & & & \multicolumn{2}{|c|}{ 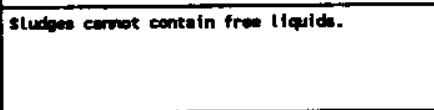 } \\
\hline & \multicolumn{3}{|c|}{ 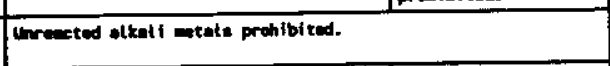 } & & 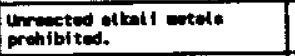 & & \multicolumn{2}{|c|}{ 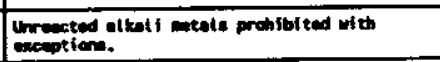 } \\
\hline & & & $\begin{array}{l}\text { Epplosive } \\
\text { prohibitites. }\end{array}$ & & Explosives protibitited. & & \multirow{2}{*}{ 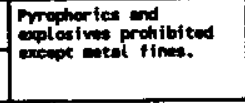 } & $\begin{array}{l}\text { Eplouived. } \\
\text { protibited. }\end{array}$ \\
\hline & & & 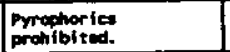 & & mraphoricas protibit ted. & & & 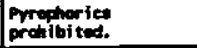 \\
\hline & & & & & & & 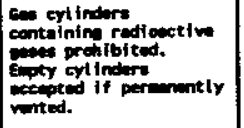 & 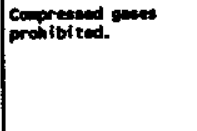 \\
\hline & & \multicolumn{2}{|c|}{ 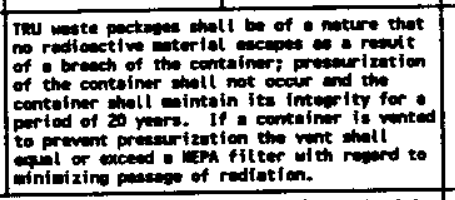 } & & 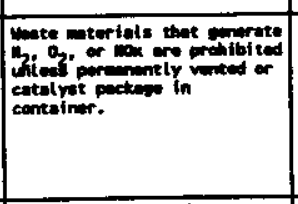 & & 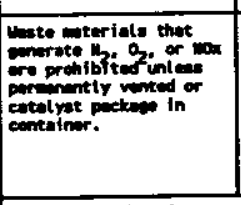 & 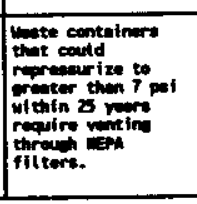 \\
\hline & nexererdote end & 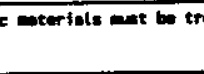 & asted pritor to buriel. & & 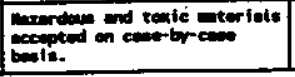 & & \multicolumn{2}{|c|}{ 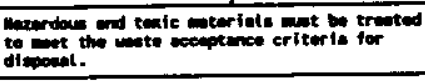 } \\
\hline
\end{tabular}


WHC-EP-0621

Table 4-1. Comparison of Past and Present TRU Storage Requirements for the Hanford Site. (3 sheets)

\begin{tabular}{|c|c|c|c|c|c|c|c|c|}
\hline 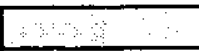 & & & & ent number & and dates & & & \\
\hline 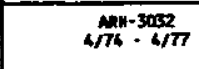 & 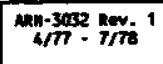 & 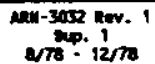 & 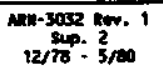 & 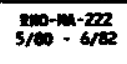 & 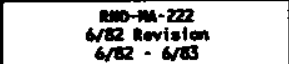 & 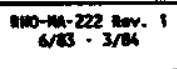 & 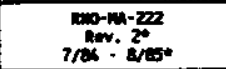 & 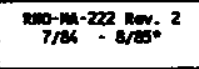 \\
\hline 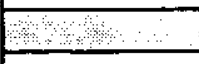 & Ye. & (1) & ited mastes: & es and pact & ging requifrements (con & t.1 & 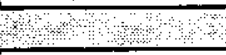 & \\
\hline & & & & & & & & 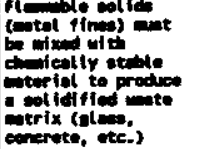 \\
\hline & 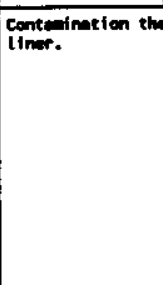 & 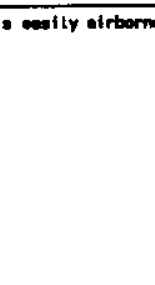 & to in an Imerer & & 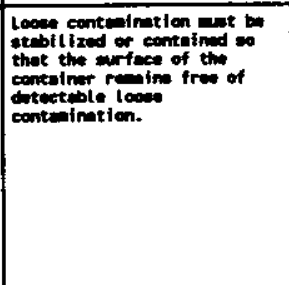 & & 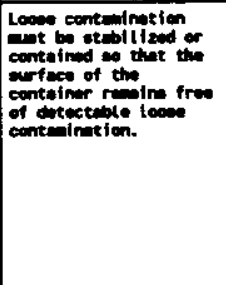 & 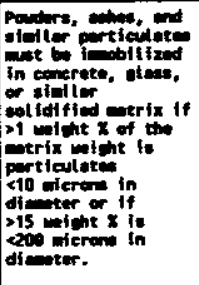 \\
\hline 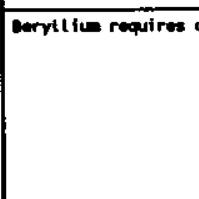 & anterirmm. & & & & 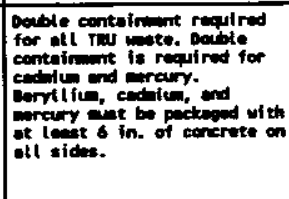 & & 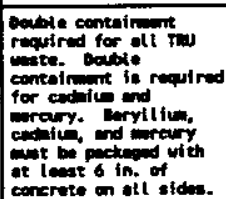 & 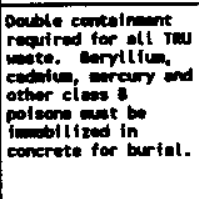 \\
\hline & & & & & 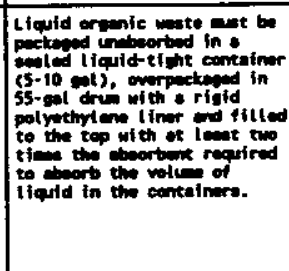 & & 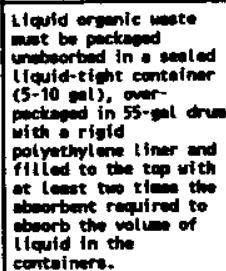 & 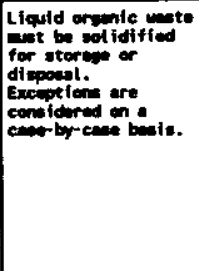 \\
\hline
\end{tabular}

4-11/4-12 
Table 4-1. Comparison of Past and Present TRU

Storage Requirements for the Hanford Site.

Storage Requirements for the
(3 sheets)

\begin{tabular}{|c|c|c|c|c|c|c|c|}
\hline $4 / 74 \cdot-3032$ & 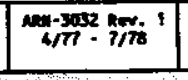 & 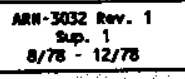 & 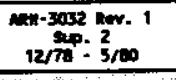 & 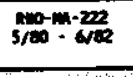 & 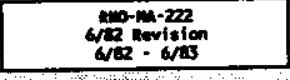 & $\frac{2100-m-222 n+1}{6 / 3}-3 / 2.1$ & 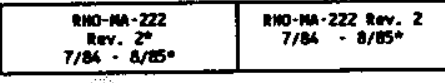 \\
\hline \multicolumn{4}{|c|}{ 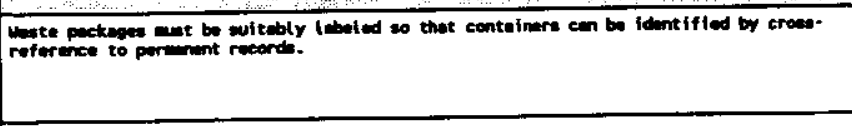 } & elling requ & 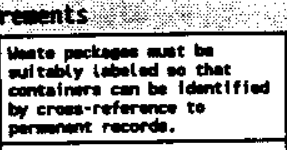 & & 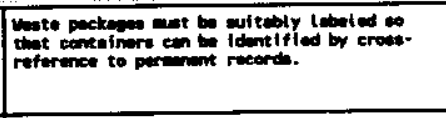 \\
\hline \multicolumn{4}{|c|}{ Coryltilu mat be labeled. } & & 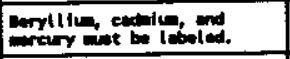 & & \multirow{2}{*}{ 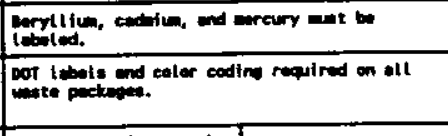 } \\
\hline & \multicolumn{3}{|c|}{ 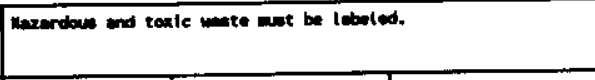 } & & 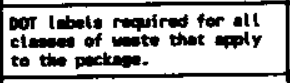 & & \\
\hline & & & & & 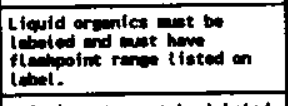 & & 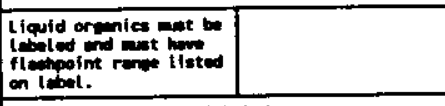 \\
\hline & & & & & Animit mente mart be tobiled. & & 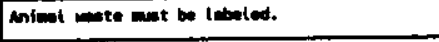 \\
\hline & & & & & 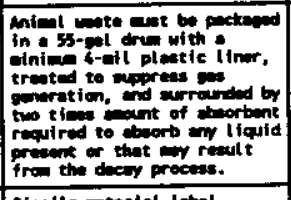 & & 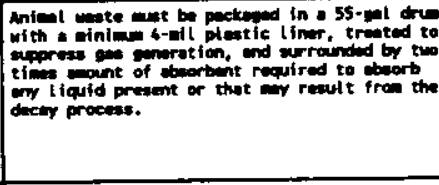 \\
\hline & & & & & 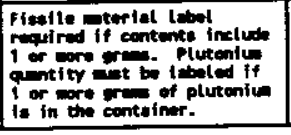 & & 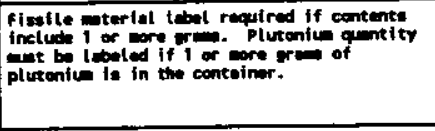 \\
\hline
\end{tabular}

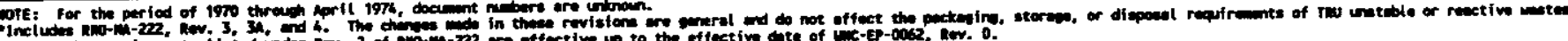

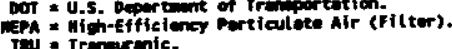


WHC-EP-0621

\subsection{CHARACTERIZATION OF RETRIEVABLY STORED SOLID WASTE GENERATED BY PFP}

The information found in this section is based primarily on data from the SWITS database. This database, which incorporated the older R-SWIMS database, is used to track information on radioactive and other wastes stored or disposed of at the Hanford Site. Radioactive solid waste packages have been tracked since 1970. In the intervening years, changes in the requirements and regulations governing radioactive wastes have affected quantity and quality of the data tracked in this database. Caveats are included in the text to alert the reader to changes that may affect the interpretation of the data provided.

The bulk of the data provided is limited to information about the TRU waste that was generated at the PFP; however, some general information on the non-TRU waste is included for completeness. The term non-TRU waste is used instead of LLW because a small percentage of the unsegregated waste is designated only as not TRU. The data on the TRU wastes are further segregated in this report by container type. Since the initial retrieval efforts and WRAP 1 will focus on 55-gal drums, these containers are considered separately from all other container types. Therefore, the term "other containers" in this report will refer to all container types, except 55-gal drums, combined. The term "drum" refers to 55-gal drums only.

The original SWITS data that form the basis for most of the tables and figures in this section can be found in Appendix B. Each computer run is preceded by the query used to generate the data.

\subsection{SUMMARY OF PFP WASTE GENERATION}

\subsubsection{Waste Stored in 55-Gallon Steel Drums}

The most common waste container for TRU waste stored at the Hanford Site is the 55-gal steel drum. The drums used are either DOT $17 \mathrm{C}$ or $17 \mathrm{H}$ drums with minimum wall thicknesses of 0.135 and $0.109 \mathrm{~cm}(0.053$ and $0.043 \mathrm{in.})$, respectively. Before 1982 most of the drums were painted, so these drums have approximately $0.0127 \mathrm{~cm}(0.005 \mathrm{in.})$ of paint on both the exterior and interior of the drums. In 1982, galvanized drums replaced painted drums. Recycled 55-gal drums were al so permitted for the storage of TRU waste between 1973 and 1978 .

The 1970 Immediate Action Directive (AEC 1970) stipulated that TRU wastes be packaged and stored as contamination-free packages for at least 20 years. The 20-year interim period was to allow time to study permanent disposal options for TRU wastes.

As more of the 55-gal drums reach and exceed the 20-year storage mark, more attention has been given to ascertaining the condition of these drums. A discussion of the previous studies of steel drum corrosion and degradation can be found in WHC-EP-0225, Rev. I (Anderson et a1. 1991). 
Between 1970 and 1991, 28,269 55-gal drums of radioactive waste were generated at the PFP. This total includes 7,073 drums of LLW and 21,196 drums of TRU waste. The TRU waste drums, generated by the PFP, account for approximately 55\% of the 55-gal TRU waste drums in retrievable storage at the Hanford Site.

Table 5-1 contains waste summary data for 55-gal drums of radioactive waste generated at PFP between 1970 and 1991 . The upper portion of this table indicates the number, total weight, and total volume of TRU waste drums. TRU mixed waste drums are also indicated. The same information for non-TRU waste follows. At the bottom of the table are the relative percentages of TRU and non-TRU wastes by container number, weight, and volume. Figures 5-1 through 5-6 present this information graphicaliy.

\subsubsection{Number of Drums. Figure 5-1 provides a graph of the number of} 55-gal drums of TRU and LLW generated at the PFP by year. The general trend shown in this figure is a gradual decrease in the ratio of TRU waste to LLW drums. This trend is shown more clearly in Figure 5-2 which shows the relative percentages of TRU and non-TRU waste drums by year. There are several reasons for this trend.

1. During the period from 1970 to 1973 segregation of TRU wastes was based on generator practice, rather than on concentration limits.

2. From 1974 to 1982 , TRU waste was defined with a concentration of greater than $10 \mathrm{nC} 1 / \mathrm{g}$.

3. In 1982, the current definition of greater than $100 \mathrm{nCi} / \mathrm{g}$ was implemented; by DOE.

4. In 1985 TRUSAF was completed. TRUSAF has the ability to assay packaged waste accurately, and, as a result, the amount of waste designated as TRU declined dramatically during 1986 and all subsequent years.

There are two notable peaks in the number of TRU waste drums generated:

- 1979-80 - This period corresponds with the reduction in the Hanford Site plutonium-bearing scrap and residue inventory which was mandated by the Energy Research and Development AdministrationRichland Operations office (ERDA-RL) in 1978. This mandate led to material stabilization and subsequent onsite storage of a portion of this inventory during the 1979-82 time frame. The peak in onsite storage for these materials was in 1980 .

- 1984-85 - With the PRF restart in January 1984 and the RMC line restart in June 1985, the peak in waste production corresponds to a peak in processing.

Non-TRU radioactive wastes were not disposed of in 55-gal drums in seven of the years since 1970: 1971-75, 1978, and 1984. Instead, most non-TRU waste was disposed of in a variety of different container types. These wastes and waste containers will be discussed in more detail in Section 5.1.2. 
Since 1986, however, the number of 55-gal drums containing non-TRU radioactive waste has been steadily increasing as a proportion of the total number of drums. This is shown in Figure 5-2.

5.1.1.2 Weight. In the period between 1970 and 1991 approximately $1,426,060 \mathrm{~kg}(3,143,871.2 \mathrm{lb})$ of TRU waste and $414,744 \mathrm{~kg}(914,338.62 \mathrm{lb})$ of non-TRU waste generated at the PFP were stored in 55-gal drums. These numbers are estimates since container weights were not required for individual containers before 1977. During the R-SWIMS data re-entry program in the mid-1980's, all pre-1977 containers were assigned standard weights. Al1 55-gal drums, for instance, were assigned a value of $68 \mathrm{~kg} \cdot(150 \mathrm{lb})$, which most probably underestimates the true container weight. Since 1978 the mean weight for 55-gal drum is $79 \mathrm{~kg}(174 \mathrm{lb})$.

The results of a search for the heaviest drums generated at the PFP can be found in Table 5-2. For the reasons discussed in the paragraph above, the record for twenty six 55-gal drums weighing greater than $150 \mathrm{~kg}$ (330.69 ib) were all from the period between 1984 and 1992 . The contents of these "heavy drums" are also included in Table 5-2. It should be noted that five of these drums contain more than $100 \mathrm{~g}(0.92 \mathrm{oz})$ of TRU waste. Appendix $C$ contains the complete SWITS records for all 26 drums.

Figure 5-3 presents an annual look at the total weight of both TRU and non-TRU radioactive wastes stored in 55-gal drums. The relative percentages of weight for TRU and non-TRU drums generated annually at the PFP is shown in Figure 5-4. These figures show, once again, the dip in the amount of waste designated as TRU after TRUSAF began operations.

5.1.1.3 Volume. The total volume of TRU waste generated at the PFP and stored in 55-gal drums is $4,443 \mathrm{~m}^{3}\left(156,902.21 \mathrm{ft}^{3}\right)$. The total volume of nonTRU radioactlve waste generated at the PFP and stored in 55-gal drums is 1,503 $\mathrm{m}^{3}\left(53,077.66 \mathrm{ft}^{3}\right)$. The volume of TRU and non-TRU waste generated between 1970 and 1991 at the PFP and stored in 55-gal drums is shown in Figure 5-5. The relative percentages of the total amount of radioactive waste stored in 55-gal drums is provided in Figure 5-6. Since all 55-gal drums have the same volume, the patterns shown in Figures 5-5 and 5-6 are the same as those described for. Figures 5-1 and 5-2 (drum number), respectively. They are included here so that comparisons with Figures 5-7 and 5-8 can be made.

\subsubsection{Waste Stored in Containers Other Than 55-Gallon Steel Drums}

Radioactive solid wastes have been stored or disposed of in a wide variety of containers other than 55-gal steel drums. In this discussion these containers will be referred to as "other containers." Container types used for solid waste generated at the PFP include the following:

- Burlap, cloth, paper, or plastic bags

- Concrete boxes

- Fiberboard/plastic boxes, cartons, or cases

- Metal drums, barrels, and kegs (other than 55-gal drums) 
- Metal boxes, cartons, or cases

- Fiberglass reinforced polyester (FRP) boxes

- Wooden boxes, cartons, or cases.

In addition some items such as gloveboxes; trucks, flatbeds, compactors or loadluggers; and self-contained equipment were disposed of or stored without additional packaging. These items are also included in the "other containers" category.

Between 1970 and 1991, 5,277 other containers of radioactive waste were generated at the PFP. Of these containers, 1,070 hold TRU waste, while 4,207 hold LLW. During this time a total of 28,269 55-gal drums of radioactive solid waste.were generated at the PFP.

Table 5-3 summarizes the waste data for containers of radioactive waste other than 55-gal drums that were generated at the PFP between 1970 and 1991. The upper portion of this table indicates the number, total weight, and total volume of TRU waste stored in other containers. The number of other containers of TRU mixed waste is also provided. The same information for non-TRU waste follows. At the bottom of this table are the relative percentages of TRU and non-TRU wastes by container number, weight, and volume. Figures 5-7 through 5-12 present these data graphically.

5.1.2.1 Number of Other Containers. Figure 5-9 is a graph of the number of other containers of TRU and LLW generated at the PFP by year. Note that no TRU waste was packaged in containers other than 55-gal drums during the following six years: 1979, 1986, 1987, 1988, 1990, and 1991 .

The largest number of other containers containing TRU waste (625) was generated in 1970. A second minor peak in the packaging of TRU waste in other containers is found during the period from 1981 and 1983. One reason for the peak during this time period is the reduction of Hanford Site's inventory of scrap and residue items mandated by ERDA-RL in 1978. This mandate led to the material stabilization and subsequent onsite storage of a portion of this inventory during 1979-82. Other contributors may be the decontamination of room 230-C in 1981 and the TCO of room 308 in 1982 .

The greatest number of other containers containing non-TRU waste was recorded for 1970 and 1979-81. The latter peak is probably a result of the scrap inventory reduction.

Figure 5-10 presents the relative percentages of TRU and non-TRU containers other than 55-gal drums that were generated at the PFP on an annual basis. After 1976, other containers of TRU waste represent less than $40 \%$ of the yearly total.

5.1.2.2 Weight. In the period between 1970 and 1991 approximately $2,764,655 \mathrm{~kg}(6,094,918.4 \mathrm{lb})$ of radioactive solid waste was generated at the PFP and Placed in containers other than 55-gal drums. This total weight includes approximately $1,464,454 \mathrm{~kg}(3,228,514.1$ lb) of TRU and 1,300,201 kg $(2,866,404.3 \mathrm{lb})$ of non-TRU contaminated material. These weights are approximations because individual container weights were not required to be recorded for individual containers before 1977. During an update of the R-SWIMS database in the mid-1980's all waste containers without a recorded 
weight were assigned standard values. For example, all 2- $\mathrm{ft}^{3}$ boxes were given a standard weight of $5.5 \mathrm{~kg}(12 \mathrm{lb})$ and 5 -gal 1 ard cans were assigned a standard weight of $22.7 \mathrm{~kg}(50 \mathrm{lb})$.

Figure 5-11 depicts the weights of TRU and non-TRU waste stored in containers other than 55-gal drums on an annual basis. The following peaks in waste production on a weight bas is are shown in Figure 5-11:

- 1975 - The greatest total weight of TRU waste was generated in 1975, when 51 containers accounted for $643,173 \mathrm{~kg}(1,417,929.8 \mathrm{1b})$ of weight. Unfortunately the records for these containers list the contents as "miscellaneous." The reason for this peak is unknown.

- 1976-78 - In August 1976 there was an explosion in the 242-Z cation exchange column, and the 242-Z Waste Treatment and Americium Recovery facility was shut down. This may have resulted in the high weight of TRU contaminated waste disposed of between 1976 and 1978 .

- 1980-81 - The greatest annual weight of radioactive waste generated by the PFP was found in 1980. The peak during this period is a result of the scrap inventory reduction, which was discussed previously. The plutonium scrap can fire in room 230-C of the 234-5Z Building that occurred in October 1980 involved scrap from the inventory mentioned above. The bulk of the weight disposed of during this period was composed of non-TRU waste.

- 1985 - In 1985, both PRF and the RMC line were operating. The weight peak during this period may be a result of restart and processing activities.

- 1989 - The peaks in weight and volume of other container TRU waste in 1989 are a result of the disposal of approximately 12 burial boxes of mixed waste. This was primarily large equipment that would not fit into 55-gal drums, including leaded glass, fluorescent lamps, and PCB ballast.

Figure 5-12 shows the percent contribution of TRU and non-TRU waste to the total annual weight of waste packaged in other containers. The relatively high proportion of TRU to non-TRU waste during the period from 1970 and 1978 reflects the TRU segregation limit of greater than or equal to $10 \mathrm{nCi} / \mathrm{g}$ used during those years.

The peak in the relative weight of TRU waste in the early 1980's is probably a result of some or all of the following:

- Scrap and residue inventory reduction

- Decontamination of rooms $230-C$ and 308 in the 234-5Z Building

- TCO programs for the 232-Z (incinerator), the RMA line, and the $66.04-\mathrm{cm}(26-i n$.$) vacuum system.$ 
WHC-EP-0621

5.1.2.3 Volume. The volume of waste generated at the PFP and packaged in containers other than 55-gal drums during 1970-91 is $7,089 \mathrm{~m}^{3}$ $\left(250,344.31 \mathrm{ft}^{3}\right)$. TRU wastes account for $3,987 \mathrm{~m}^{3}\left(140,798.81 \mathrm{ft}^{3}\right)$ of the total volume; non-TRU wastes account for $3,102 \mathrm{~m}^{3}\left(109,545.5 \mathrm{ft}^{3}\right)$.

Figure 5-7 shows annual volumes of TRU and non-TRU wastes generated by the PFP and packaged in containers other than 55-gal drums. Figure 5-8 shows the relative contribution of TRU and non-TRU wastes to the total volume of waste in other containers for each year.

Over $400 \mathrm{~m}^{3}\left(14,125.79 \mathrm{ft}^{3}\right)$ of radioactive waste was packaged in other containers during each of the following years: 1975, 1976, 1978, 1980, 1981, and 1985.

By far the largest annual volume of waste packaged in other containers was $1,675 \mathrm{~m}^{3}\left(59,151.75 \mathrm{ft}^{3}\right)$ in 1975 , with TRU waste making up $98.5 \%$ of this volume.

\subsubsection{Summary of Waste Generation Rates at PFP Between 1970 and 1991}

5.1.3.1 Number of Waste Containers. Between 1970 and 1991 there were 33,546 containers of radioactive solid waste generated at the PFP. 55-gal drums account for $84 \%$ of this total; other containers account for the remaining $16 \%$. Section 5.2 describes the container types used.

Overall, waste designated as TRU is stored in about two-thirds $(66 \%)$ of the containers generated between 1970 and 1991. Non-TRU waste, mostly LLW, can be found in $34 \%$ of the containers. Figure 5-13 shows the total numbers of both TRU and non-TRU waste containers generated annually at the PFP during this period.

It should be remembered that several changes (discussed in Section 4.2) between 1970 and 1991 resulted in more waste being designated as LLW and less waste being designated as TRU. This trend is clearly seen in Figure 5-13. A significant portion of the waste stored as TRU may be able to be redesignated as LLW upon characterization at WRAP.

5.1.3.2 Total Weight. Over 4.6 million kilograms ( 10.14 million pounds) of radioactive solid waste was generated at the PFP between 1970 and 1991 . Waste packaged in 55-gal drums accounts for about $40 \%$ of the total weight of waste from the PFP, with $60 \%$ of the total weight composed of waste packaged in some other type of waste container.

Waste designated as TRU comprises $63 \%$ of the total weight, while waste designated as non-TRU makes up the remainder. Figure 5-14 provides a graph of the total weight of TRU and non-TRU waste generated at the PFP on a yearly basis between 1970 and 1991 .

5.1.3.3 Total Volume. In the 21-year period between 1970 and $1991,13,035 \mathrm{~m}^{3}$ $\left(460,324.18 \mathrm{ft}^{3}\right)$ of radioactive solid wastes were generated at the PFP. A little less than one-half (46\%) of this volume is composed of 55-gal drums; 
WHC-EP-0621

the remaining 54\% of the volume is made up of other container types, ranging from cardboard boxes to self-contained equipment. Section 5.2 discusses the other container types and their respective volumetric capacities.

As was seen when looking at the total number and weight of containers, waste designated as TRU accounts for approximately two-thirds (65\%) of the total waste volume and non-TRU waste accounts for the remainder. Figure 5-15 shows the total volume of TRU and non-TRU waste generated annually at the PFP between 1970 and 1991 .

\subsection{WASTE CONTAINERS}

\subsubsection{Non-TRU Waste Containers}

Table 5-4 provides a summary of the non-TRU waste generated at the PFP from 1970 to 1991 by container type. The most common containers were 55-gal drums, but significant numbers of fiberboard/plastic boxes; burlap, cloth, paper or plastic bags; metal drums other than 55-gal; and selfcontained equipment were al so found. The greatest weight of non-TRU from the PFP is stored in 55-gal drums, followed by trucks, flatbeds, compactors, and loadluggers; wooden boxes; self-contained equipment; and metal drums (other than 55-gal) in decreasing order.

\subsubsection{TRU Waste Containers}

Table 5-5 provides a summary of the TRU waste generated annually at the PFP from 1970 to 1991 and is sorted by container type. A significant majority of TRU waste was stored in 55-gal drums. The percentages for all container types and the total weight of these containers are shown for each year in this table.

\subsection{TRU WASTE STORAGE LOCATIONS}

Table 5-6 provides the storage locations for TRU waste packaged in 55-gal drums by year. In general, the bulk of the drums generated in 1 year are stored in one or two facilities. Storage locations for TRU waste packaged in other containers are shown in Table 5-7. A summary of the PFP waste storage by storage location follows:

- 218-W-3A - Burial Ground 218-W-3A consists of 14 earthen-bottom, gravel filled trenches, with waste emplaced from May 1970 to April 1988. The PFP 55-gal drum waste generated predominantly from 1970 to 1971 is stored here, with one additional drum from 1984. other containers of waste generated from 1970 to 1978 are located in this area, including one known to contain lead, a hazardous constituent. 
- 218-W-4B - Burial Ground 218-W-4B, consisting of 14 trenches, accepted waste from August 1970 through September 1978. Drum and other container waste from the PFP was buried here during this time period. No record of hazardous material was made during the burial time.

- 218-W-4C - Burial Ground 218-W-4C consists of six trenches, with the first waste emplaced in March 1978. PFP waste in drums has been sent to this area from 1978 to the present, with other containers emplaced from 1980 to 1985. Waste containers recorded as having hazardous constituents were buried from 1981 to 1987 . These waste containers included a total of 63 drums and 10 other containers. The predominantly listed contaminants are asbestos and beryllium.

A record search was done for waste containers with more than $300 \mathrm{~g}$ (10.58 0z) of TRU. Trench T01 contains heavily loaded TRU waste from the 1980 to 1982 period, corresponding to the CSMO inventory reduction. There are a total of ten 55-gal drums and 22 other containers with more than $300 \mathrm{~g}(10.58 \mathrm{oz})$ of TRU elements. The contents of these containers are listed as metal/iron/galvanized/sheet, with no hazardous constituents noted.

- 218-W-5 - Burial Ground 218-W-5 contains one 55-gal drum from the PFP, which does not contain mixed waste.

- 224-T - The TRUSAF is used to store TRU waste certified for transfer to the WIPP. 55-gal drums from the PFP have been stored at this site from 1986 to the present, with mixed waste drums beginning in 1989. The primary hazardous contaminants are lead products, mercury, and heavy metals.

- 2401-W, 2402-W, and 2402-WB - These buildings are located at the Hanford Central Waste Complex, in the 200 West Area. All PFP waste containers stored at these locations contain mixed waste. A total of forty four 55-gal drums and 14 other containers were stored here from 1982 to 1990. The principal hazardous constituents are lead and lead compounds, PCB, hydraulic fluid, and heavy metals.

- FS8 - This is a Low Flashpoint Storage Module, and contains one non-drum container of mixed waste.

\subsection{PHYSICAL CONTENTS OF TRU WASTE CONTAINERS}

The physical contents for 55-gal drums are shown in Tables 5-8 through 5-14. There is a separate table for each storage location. The top portion of each table indicates the number of drums for which a given component is listed; the bottom portion of the table indicates the percentage of the total drums that number represents. It should be noted that before 1978 physical contents were not required to be listed on the burial records, so a great many of the contents on early records are listed only as "Miscell aneous." 
WHC-EP-0621

The physical contents for TRU waste containers other than 55-gal drums can be found in Tables 5-15 through 5-21.

\subsection{RADIOLOGICAL DESCRIPTION OF PFP SOLID WASTE}

Table 5-22 shows the number of grams of TRU stored in 55-gal drums each year by storage location; Table 5-23 provides the same information for TRU waste containers other than 55-gal drums. Figure 5-16 combines the information on these tables in a graph that shows the total number of grams of TRU present in waste packages from the PFP by year. It should also be noted that not all SWITS records for TRU waste containers list the number of grams of TRU, particularly in the early 1970's.

The most striking observation about the TRU gram loading in containers from the PFP is the peak in 1980 when over $60,000 \mathrm{~g}(2,116.40 \mathrm{oz})$ of TRU were stored in just under 1,700 containers. The reason for this peak was the effort to reduce plutonium-bearing scrap and residue being stored at the Hanford Site.

In the late 1960's or early 1970's the Hanford Site was designated by the AEC as the CSMO for plutonium-bearing materials. The function of the CSMO was to accept plutonium scrap and residues from AEC sites and industrial contractors when recovery capability/capacity was not available.

The CSMO was to locate a recovery plant that could do the recovery and fund that plant for the recovery work. If not, CSMO was to carry out the recovery mission in the PRF/PFP. As the result of CSMO, the Hanford Site received a wide variety of plutonium-bearing material for recovery which generated a very large scrap and residue inventory.

The packaging for many of the materials in the Hanford Site inventory was marginal. The chemical stability for many of the scrap and residue items was questionable. Consequently, in 1978, ERDA-RL decided to reduce the Hanford Site inventory by carrying out the following three options:

1. Ship appropriate material to other sites (Los Alamos National Laboratory [LANL], Rocky Flats Plant [RFP], and Savannah River Plant [SRP]) for processing.

2. Process material with a low concentration of plutonium (e.g., ash and sand, slag, and crucible) so that it may be discarded.

3. Stabilize remaining material for long-term storage and repackage in a safe, storable configuration.

The three options were carried out during 1979-82 with activities peaking in 1980. The high TRU values shown in Figure 5-16 for 1979-82 are attributed to material stabilization, processing, and repackaging for offsite shipment and onsite storage, and the discard of low-level residues. The EDLs were changed to allow these low-level materials to be discarded. This discard was the largest contributor to the TRU values shown in Figure 5-16. 
WHC-EP-0621

Tables 5-24 and 5-25 show the results of a R-SWIMS search for waste containers with more than $300 \mathrm{~g}(10.58 \mathrm{oz})$ of TRU. Notice that the 10 drums and 22 other containers all contain metal scrap, and were disposed of from 1980 to 1982. Appendix C contains the complete SWITS records for these waste containers.

Additional information about the radiological components of the TRU waste from the PFP can be found in Appendix B, Sections B.13 and B.14. Additional information found in these sections includes maximum dose rates and TRU isotope lists. None of the maximum dose rates found exceeded the limit for contact handled containers.

\subsection{HAZARDOUS CONSTITUENTS OF SOLID WASTE GENERATED BY THE PFP}

This section provides a review of the data on the hazardous components found in the SWITS database and on the original burial records. In addition, an inventory of the chemicals known to be used at the PFP and information from personnel interviews are included in this section. Because information on the nonradioactive, hazardous chemicals in waste containers was not required on burial records before 1987, information before that date is meager.

\subsubsection{Chemical Inventories}

Tables 5-26 through 5-28 provide several lists of chemicals used at the PFP and the PFP Analytical Laboratory between 1949 and 1980 . These 1 ists were developed by Klem (1990) to support waste characterization efforts for the single-shell tanks. Although most liquid wastes from the PFP did end up in the tanks, it may be possible to find small amounts of these chemicals in solid waste containers. These lists are based on chemical process flowsheets, essential material consumption records, letters, reports, and other historical data.

The final two columns in each of the tables cited above contain information on the toxicity and hazards associated with each of the chemicals listed. The first of these columns lists the EPA toxicity category, which was determined using the EPA "Spill Table" (EPA 1989b). Category X marks the most toxic chemicals, followed by Categories $A, B, C$, and $D$, listed in order of decreasing toxicity. The EPA "Spill Table" is incorporated by reference into the Washington State Administrative Code, Chapter 173-303, "Dangerous Waste Regulations." The final column lists the DOT designation found in the DOT "Hazardous Materials Table" (49 CFR 172.101).

\subsubsection{Solid Waste Information and Tracking System}

The information about the hazardous constituents of the solid waste from PFP is limited in the years before 1987. According to the SWITS database only 51 containers from the PFP are designated as mixed waste before 1986. Since 1986, reporting of hazardous constituents has improved considerably;

107 containers have been designated as mixed waste in the past 5 years. The 
WHC-EP-0621

greatest percentage of mixed waste was found in 1989 when $39 \%$ of all 55-gal drums and $100 \%$ of all other container types generated by the PFP contained mixed waste. A summary of the number of mixed waste containers and the percentages they represent can be found in Tables 5-29 and 5-30.

The hazardous constituent field in the SWITS database was searched for all containers from the PFP that contained radioactive mixed waste. Tables 5-31 through 5-34 show the hazardous constituents of TRU 55-gal drums sorted by storage location and year. In addition, these figures indicate the percentage of the total number of containers generated in that year and stored at that location that contain each constituent. Tables 5-35 through 5-40 show the same information for TRU containers other than 55-gal drums.

\subsubsection{Burial Records}

The SWBR or SWSDR for a given container is the source of waste container information abstracted for the SWITS database. Often these records will have more detailed information on the hazardous components of a waste container than is found in SWITS. Additional data can also be found on the supplementary forms often attached to the SWBR or SWSDR. These supplementary documents include Uniform Hazardous Waste Manifests, Contents Inventory Sheets, NRC 741 Forms, and Storage/Disposal Approval Records (SDARs).

In the current study, a review of the burials records for all TRU mixed waste containers was made. Table 5-41 contains a summary of the information obtained.

\subsubsection{Interviews With PFP Analytical Laboratory and Process Facility Personnel}

A list of known or suspected components in the solid waste stream was produced from the information gained in interviews with the Analytical Laboratory and process facility personnel.

The list of known or suspected hazardous constituents was compiled using the information gained from interviewing process and Analytical Laboratory personnel. The SWITS records for waste shipments received from the PFP do not specify the part of the facility from which the waste originated. It is impossible to discern the PFP analytical waste from PFP process waste by solely analyzing the computer records.

The following constituents are either suspected or known components of the solid waste stream from the PFP. Interviewees were asked to provide information concerning the quantity, form, packaging, and relevant use of the constituents. It is important to note that the liquids below were probably disposed of in a nonliquid form, often absorbed by kitty litter, rags, cotton swabs, laboratory coats, etc.

Acetone - Acetone was part of the laboratory process before being replaced by a) cohol. 
WHC-EP-0621

Asbestos - The PFP personnel estimated the amount of asbestos that was part of the waste stream was small. The asbestos was primarily in the form of insulation removed during remodeling or maintenance work. old steamline installations and some old gaskets (high heat exposure) within certain process vessels located in glovebox lines and outside glovebox lines contained some asbestos. The asbestos was present in the insulation used in furnaces, steamlines and gaskets. The asbestos was packaged in lined burial boxes and galvanized 55-gal drums.

In the Analytical Laboratory, asbestos was part of the emission spectroscopy process and was possibly in floor tiles. Cleanup and packaging is believed to have been in accordance with regulations at the time.

Benzene - During the 1960's a lot of benzene was used in the Analytical Laboratory. In the 1970's there was a big push to get rid of it, thus a lot less was used.

Beryllium - Former PFP process personnel recalled that very little beryllium is present in solid wastes. However, beryllium was part of the PFP Analytical Laboratory in several capacities. It served as a standard for metals. Beryllium was also used in neutron multipliers and NDA.

Calcium - Calcium metal is employed in the PFP to reduce plutonium fluoride powder to a plutonium metal button. The reduction charge contains about $10-20 \%$ over the stoichiometric amount of calcium required. Consequently, the unreacted free calcium metal becomes part of the reduction slag, which is mainly calcium fluoride. The reduction slag was recycled aqueously to recover residual amounts of plutonium remaining in the slag. Therefore, unreacted calcium was destroyed and its corresponding solution species was sent to the tank farms.

Any calcium metal spilled outside the glovebox line was treated to produce calcium oxide or calcium hydroxide and properly disposed of according to the disposal practices in effect at that time.

There is a remote chance, on an infrequent basis, that spilled calcium metal in a glovebox line could have been swept up with glovebox sweepings and trash. The quantity would have been very small (less than $50 \mathrm{~g}[1.76 \mathrm{oz}]$ ) and would probably have reacted with the carbon dioxide and water in the glovebox to form less reactive calcium salts. These calcium salts would probably be in the form of pellets or shot.

One interview respondent recalled a sample of calcium being part of a carbon analysis.

Carbon Tetrachloride - A considerable amount of carbon tetrachloride was used in the Analytical Laboratory for the analys is of gallium. There were dilute samples, process samples, and a percentage of organic solutions involved.

Ethers - Before 1970, ethers were a part of the laboratory process.

Hydrazine - Small amounts of hydrazine were used in the laboratory as a standard solution test. 
Hydrogen Peroxide - 20\% hydrogen peroxide was used in mass spectroscopy in the Analytical Laboratory.

Lithium Fluoride - Lithium fluoride was used in emission spectroscopy in the Analytical Laboratory.

Mercuric Salts - Before 1983, mercuric salts were a part of the processes at the PFP Analytical Laboratory.

Mercuric Nitrate - One respondent thought that mercuric nitrate may have been used for an emission spectroscopy chemical assay in the PFP Analytical Laboratory. Another described its use in assisting the dissolution of plutonium-aluminum fuel pins that were not irradiated.

Mercuric Sulfate - Mercuric sulfate was used for a chemical assay.

Mercuric Thiocyanate - Small amounts of mercuric thiocyanate were used in the PFP Analytical Laboratory.

Mercury - As a result of broken instrumentation, manometers, etc., minute amounts of mercury are suspected in solid waste burial. According to the 1975 environmental impact statement (EIS), ERDA-1538, mercury is not a part of the 200 Area process chemical inventory. However, mercuric nitrate was purchased and stored in polylined ice cream cartons for use at the PFP. About $45.36 \mathrm{~kg} / \mathrm{yr}$ (100 $\mathrm{ib} / \mathrm{yr})$ were used.

During the 1950's and 1960's the Analytical Laboratory carried out triple distillations to purify mercury for analytical equipment and vacuum diffusion pumps. The method used for the disposal of distillation bottoms was not known by; the personnel interviewed. A large amount of mercury was also used in the galometry process at the PFP. Analytical Laboratory.

Nitric Acid - Two mechanisms could have introduced nitric acid into the solid waste stream. The first is through rags used to clean up glovebox spills, whereby all of the nitric acid was not removed by subsequent water rinses and drying. Nitric acid has a tendency to nitrate the cellulose in the rags, significantly lowering the ignition point and thereby increasing the fire risk.

The second mechanism involved residual nitric acid solution left in discarded equipment that was removed by maintenance, such as valves, pumps, filters, etc. These items have a variety of hold-up cavities that are not amenable to convenient, easy drainage. Consequently, small quantities of nitric acid (less than $50 \mathrm{~mL}[1.69 \mathrm{fl} 0 \mathrm{oz}]$ ) could have been sent out with the sol id waste stream. However, with time, these solutions would have probably dried up and the nitric acid reacted with the surrounding material. Nevertheless, it is still a concern and warrants appropriate precautions.

Both concentrated nitric acid and fuming concentrated nitric acid were used in the PFP Analytical Laboratory. None of the Analytical Laboratory respondents noted amounts or packaging.

Oxalic Acid - Oxalic acid, in both liquid and powder form, was part of the PFP Analytical Laboratory. Oxalic acid was used for non-rad cold procedures. 


$$
\text { WHC-EP-0621 }
$$

PCBs - PCBs were used in several capacities in the Analytical Laboratory. They were used in lathes, in equipment such as elevator pumps, and in laboratory pumps. It is possible that the PCBs also were used in bomb parts. Any of the oils used in the machine shop also could contain PCBs.

Potassium Permanganate - Potassium permanganate was premade at the 222-S Analytical Laboratory and shipped to the PFP.

Propane - From 1965 to $1973,0.45-\mathrm{kg}$ (1-1b) propane cylinders were used in processing plutonium. About one to three cylinders were used per week. Exhausted cylinders were discarded to TRU waste and packaged in cardboard boxes. The cylinders were not punctured to release any residual propane. Initially, the cylinder control valve was removed before discarding the spent cylinder. Consequently, the valve was discarded along with the cylinder to TRU waste. Occasionally, the valve would malfunction, causing the discard of cylinders having higher levels of propane. The amount of time this practice continued after January of 1973 is not known.

Rhodium/Platinum - Rhodium and platinum are tightly controlled precious metals that were, as a whole, accounted for. There is a possibility that small amounts ended up in solid waste.

Sodium Fluoride - During the 1960's, sodium fluoride powder was used in a uranium procedure. It was also used for chemical assays and as a soak for electrodes in the PFP Analytical Laboratory.

Sodium Hydroxide - During the 1960's, sodium hydroxide was used in a highly concentrated form, approximately $50 \%$. Since then the concentration has dropped to $6 \%$ or less. Rags coated with sodium hydroxide were disposed of in numerous ways. Some were incinerated and others went into boxes and drums. It is possible that some sodium-hydroxide may be double bagged and stored in 20-year retrievable drums.

Sodium Nitrate - Sodium nitrate was used in the PFP Analytical Laboratory as a standard and a reagent.

Sodium 0xalate - One of the interview respondents recalled sodium oxalate being used as a standard in the PFP Analytical Laboratory.

Sulfuric Acid - Approximately $1 M$ sulfuric acid was used as a chemical assay in the PFP Analytical Laboratory. Another use was for nonradioactive cold procedures.

TBP-Carbon Tetrachloride - On several occasions the solvent extractant tributyl phosphate - carbon tetrachloride (TBP/carbon tetrachloride) employed in PRF would emulsify. Faflure to break the emulsion would result in a change out. The emulsified TBP/Carbon tetrachloride would be placed in polybottles (probably 4-L size) along with conwed absorbent pads to absorb the liquid phase. The polybottles were then placed in 55-gal drums for disposal. The polybottles were secured within the drums with absorbent and padding.

A complete changeout would generate a significant (greater than 10) number of drums. 
The following constituents were also used in Analytical Laboratory processes at the PFP and may show up in solid waste packages:

- Acetic acid

- Iron and tungsten (in form of rings/chips)

- Isopropyl ethyl alcohol

- Metal oxides (emission spectroscopy standards)

- Potassium hydroxide

- Silver chloride

- Silver nitrate

- Silver sulfate.

\subsection{CLASSIFIED WASTE}

Only one container from the PFP is designated as classified TRU waste in the SWITS database. This container is a 55-gal drum stored at $218 \mathrm{~W} 3 \mathrm{~A}$. It contains $26 \mathrm{~g}(0.92 \mathrm{oz})$ of plutonium and $2.455 \mathrm{~kg}(5.41 \mathrm{lb})$ of enriched uranium. The full SWITS record for this container can be found in Appendix E. 
WHC-EP-0621

Figure 5-1. PFP 55-Gallon Drums Generated Annually.

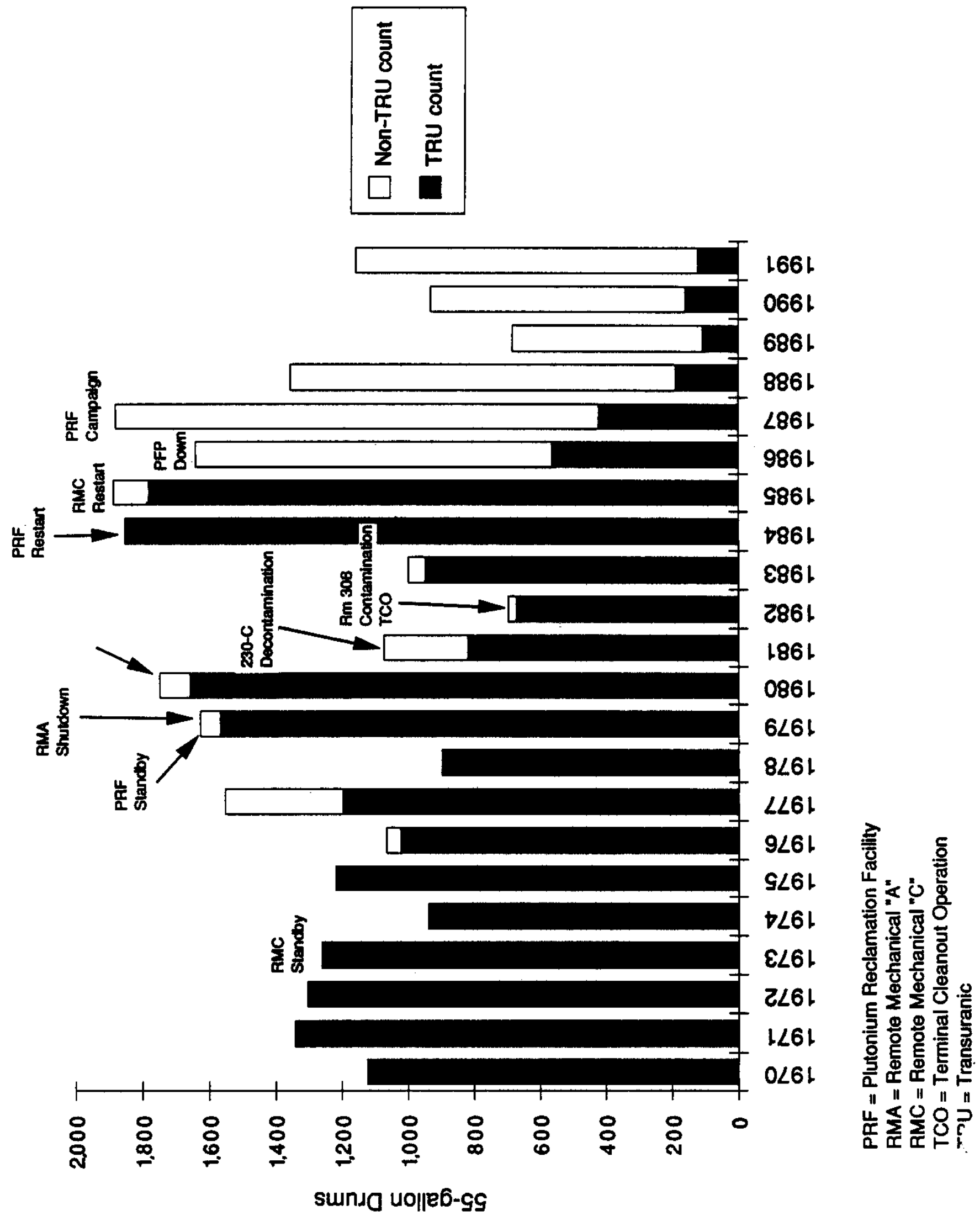




\section{WHC-EP-0621}

Figure 5-2. Percentage of TRU and Non-TRU 55-Gallon Drums Generated Annually at the PFP.
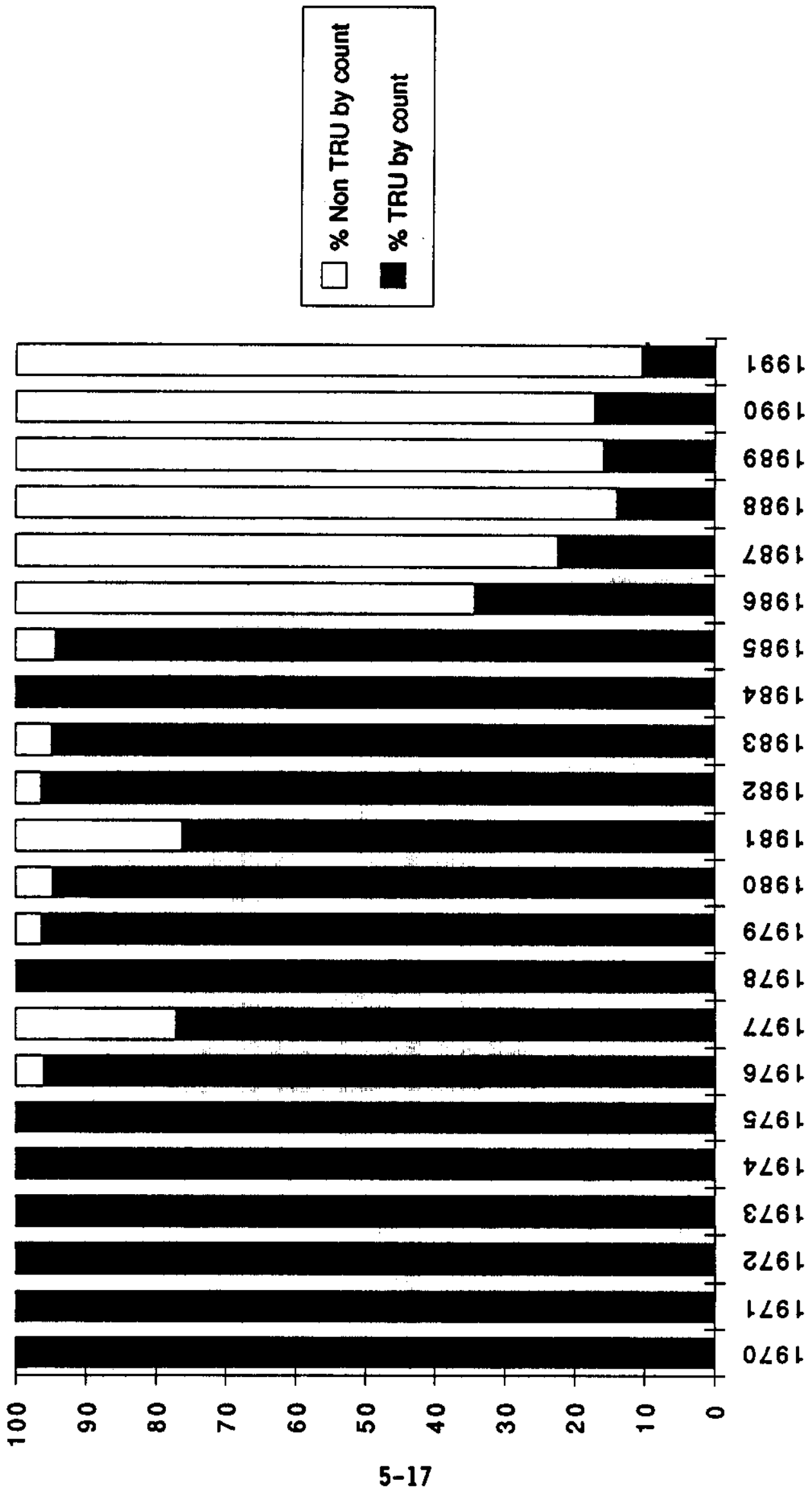
Figure 5-3. Total Weight of Radioactive Waste Stored in 55-Gallon Drums Generated Annually at the PFP.

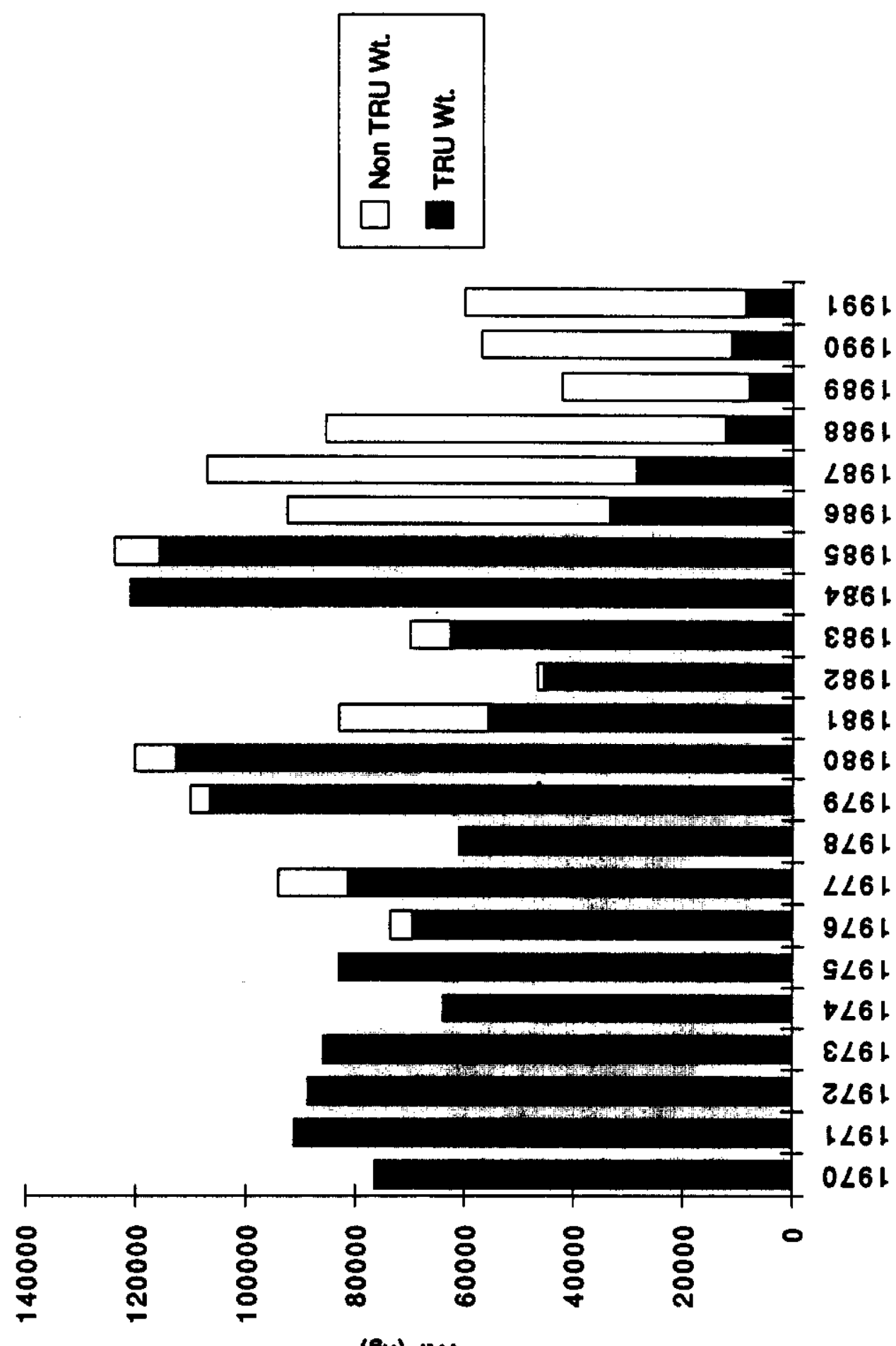

(By) $7 M$ 
WHC-EP-0621

Figure 5-4. Percent Weight of TRU and Non-TRU 55-Gallon Drums Generated Annually at the PFP.
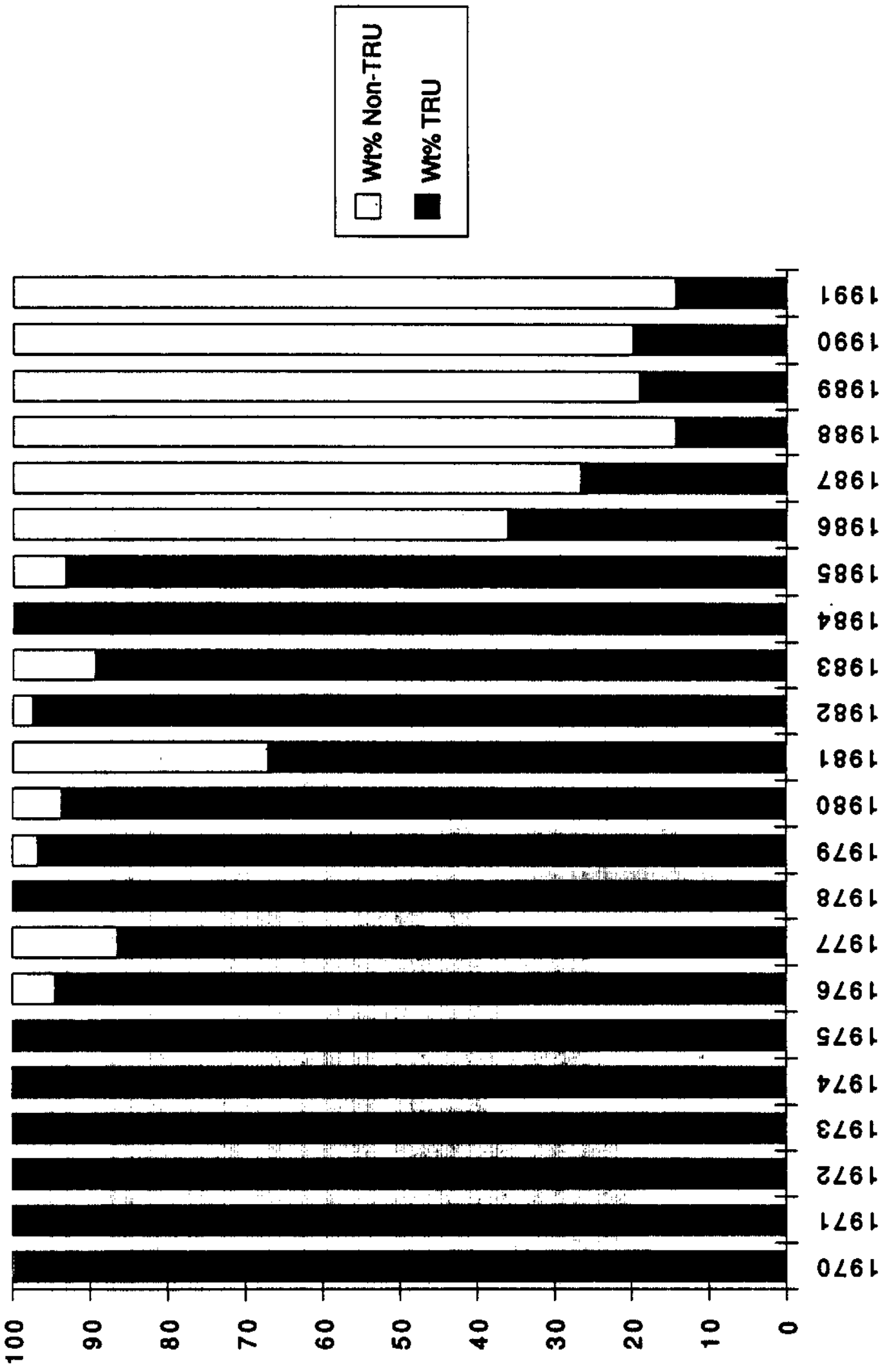
WHC-EP-0621

Figure 5-5. Volume of Radioactive Waste Stored in 55-Gallon Drums Generated Annually at the PFP.

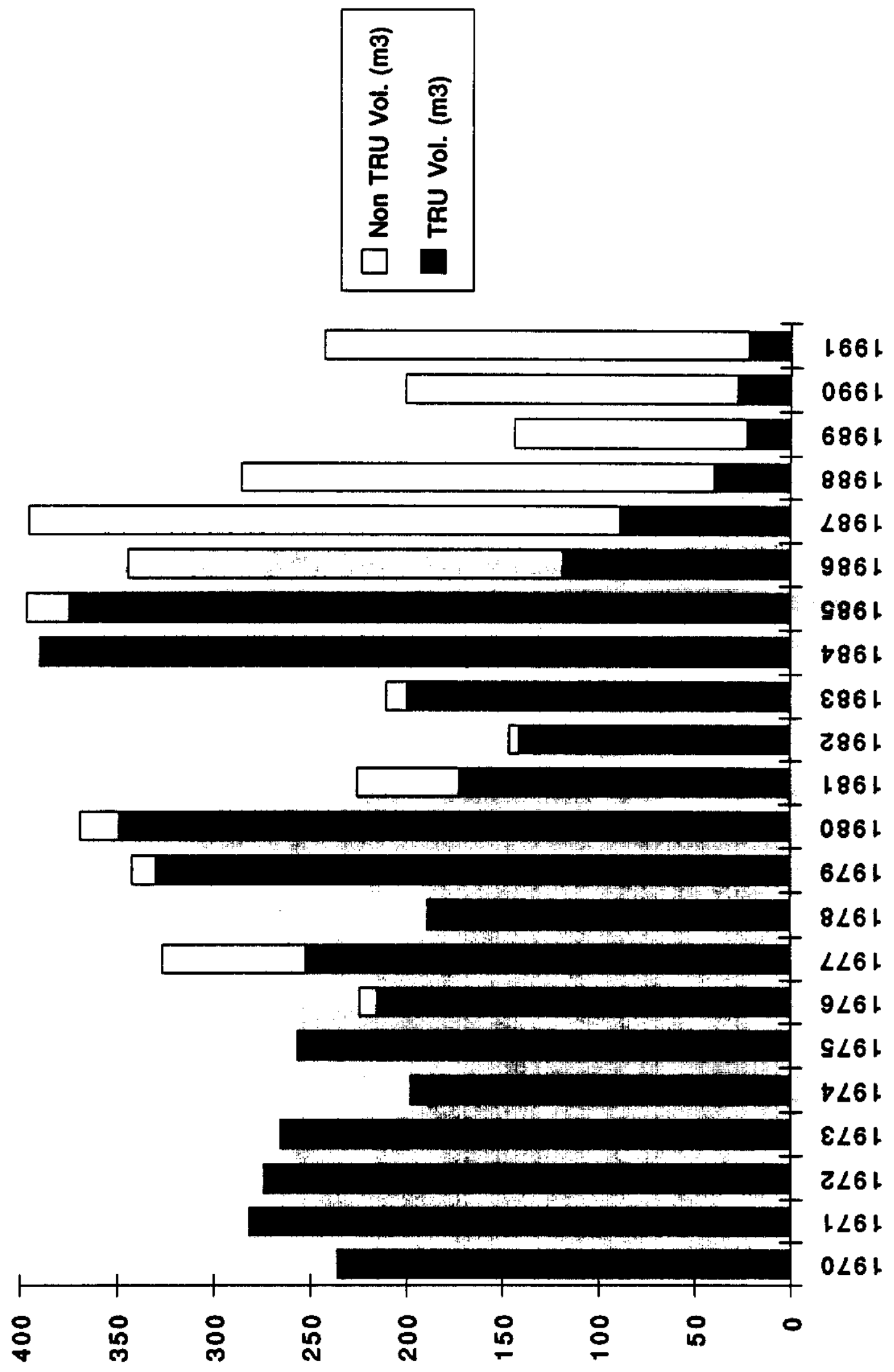


Figure 5-6. Percent Volume of TRU and Non-TRU 55-Gallon Drums Generated Annually at the PFP.
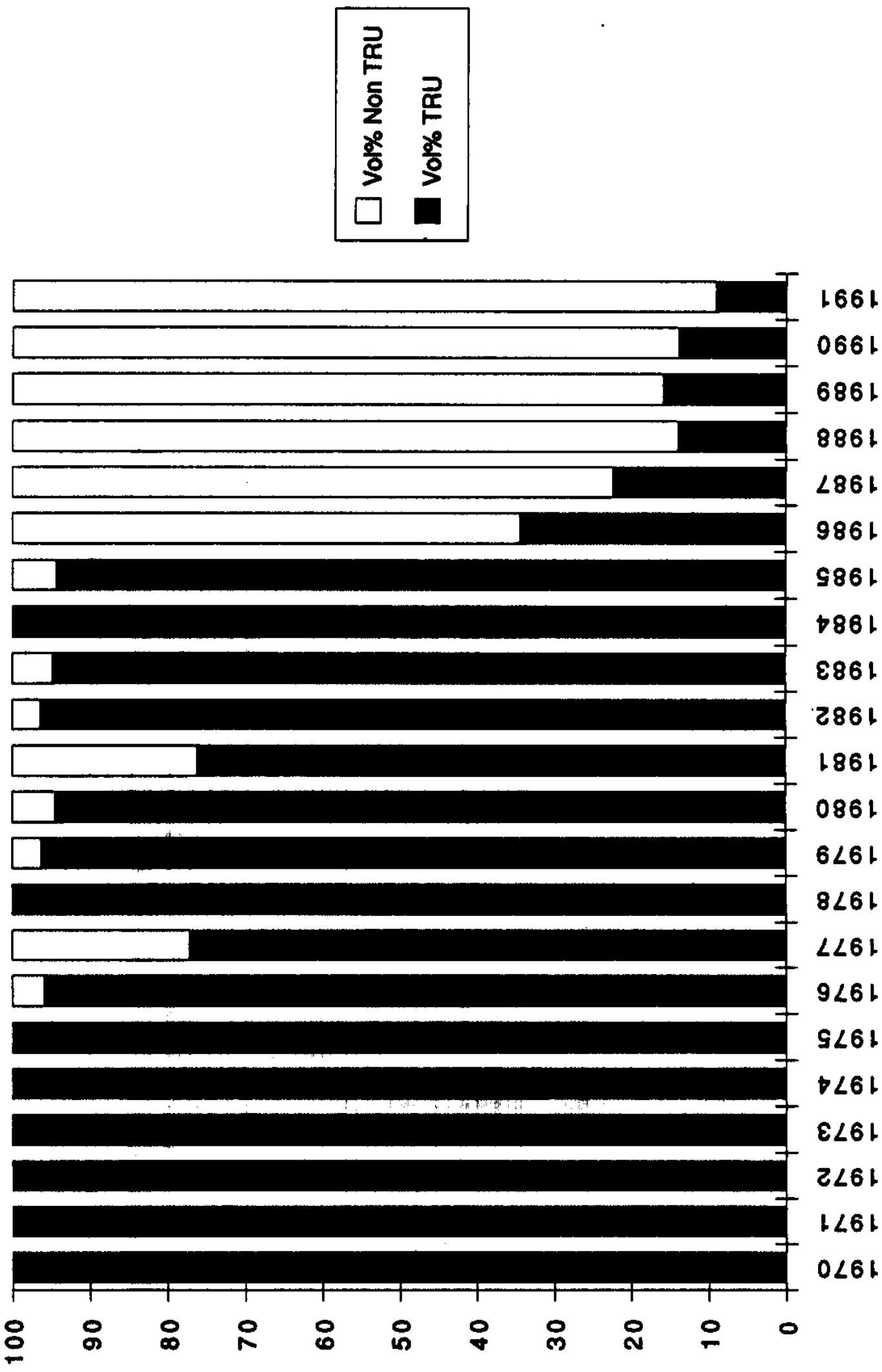


\section{WHC-EP-0621}

Figure 5-7. Volume of Radioactive Waste Stored in Containers Other Than 55-Gallon Drums Generated Annually at the PFP.

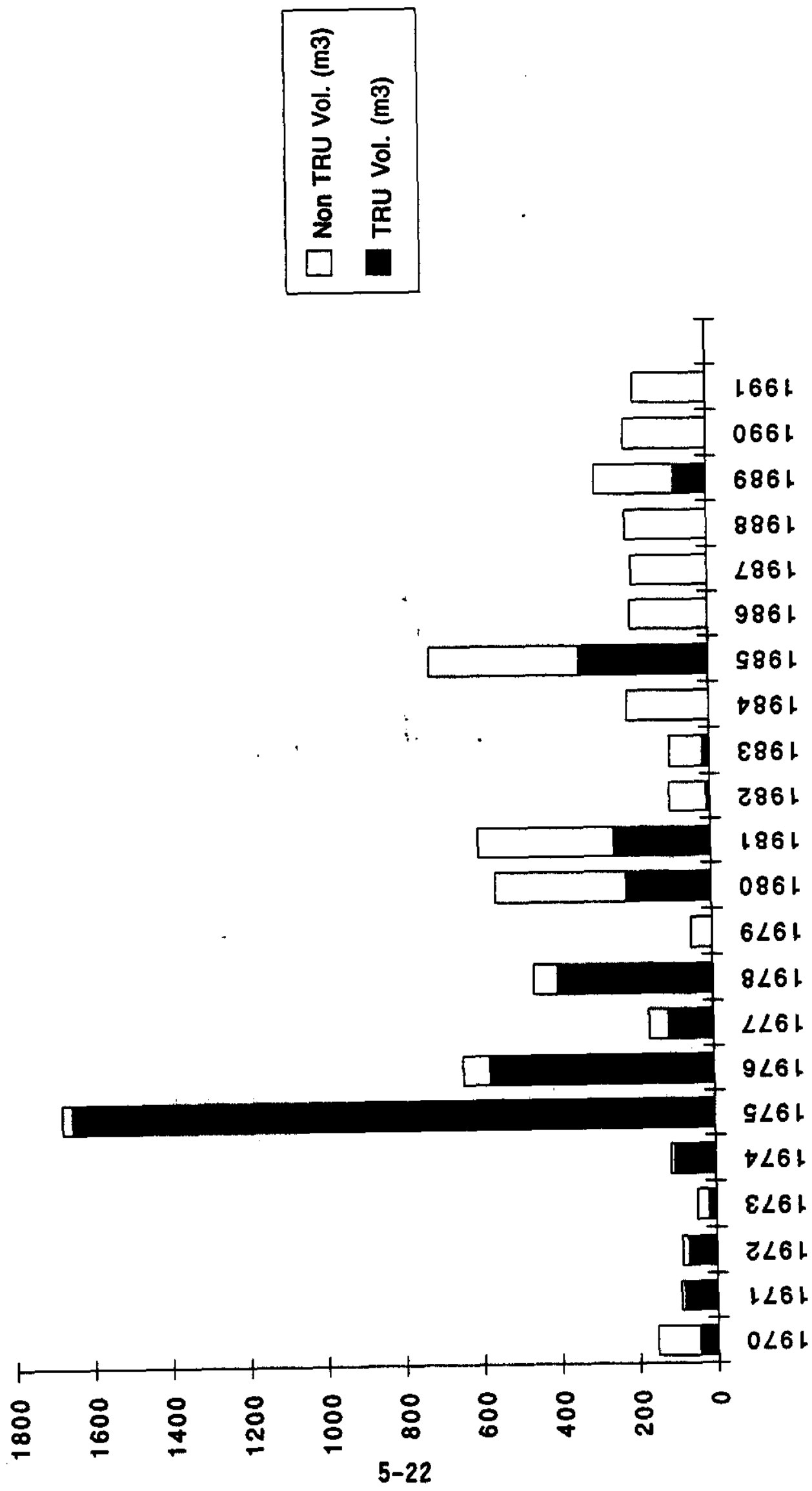


Figure 5-8. Percent Volume of TRU and Non-TRU Containers Other Than 55-Gallon Drums Generated Annually at the PFP.

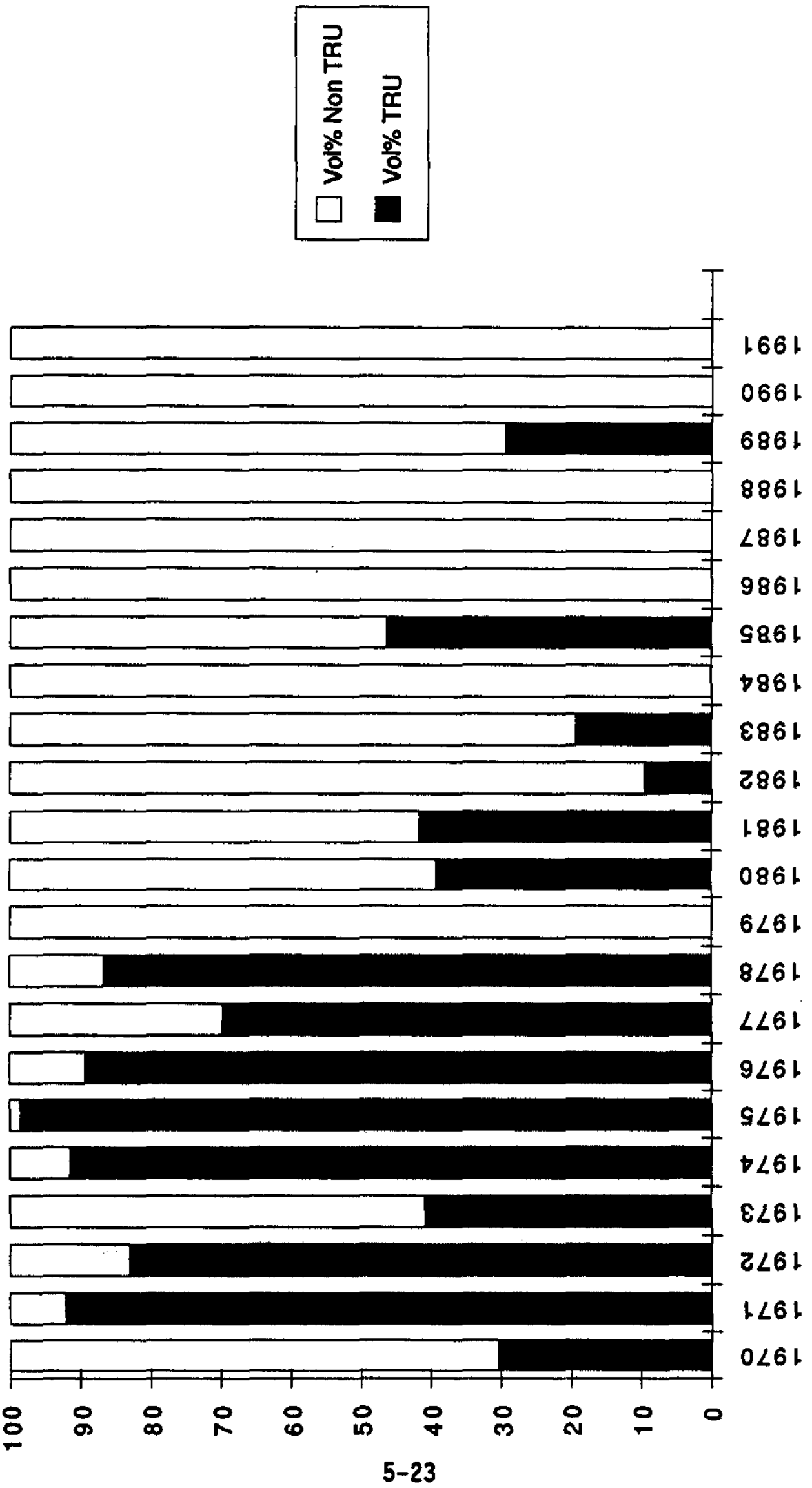


Figure 5-9. Containers Other Than 55-Gallons Drums Generated Annually at the PFP.

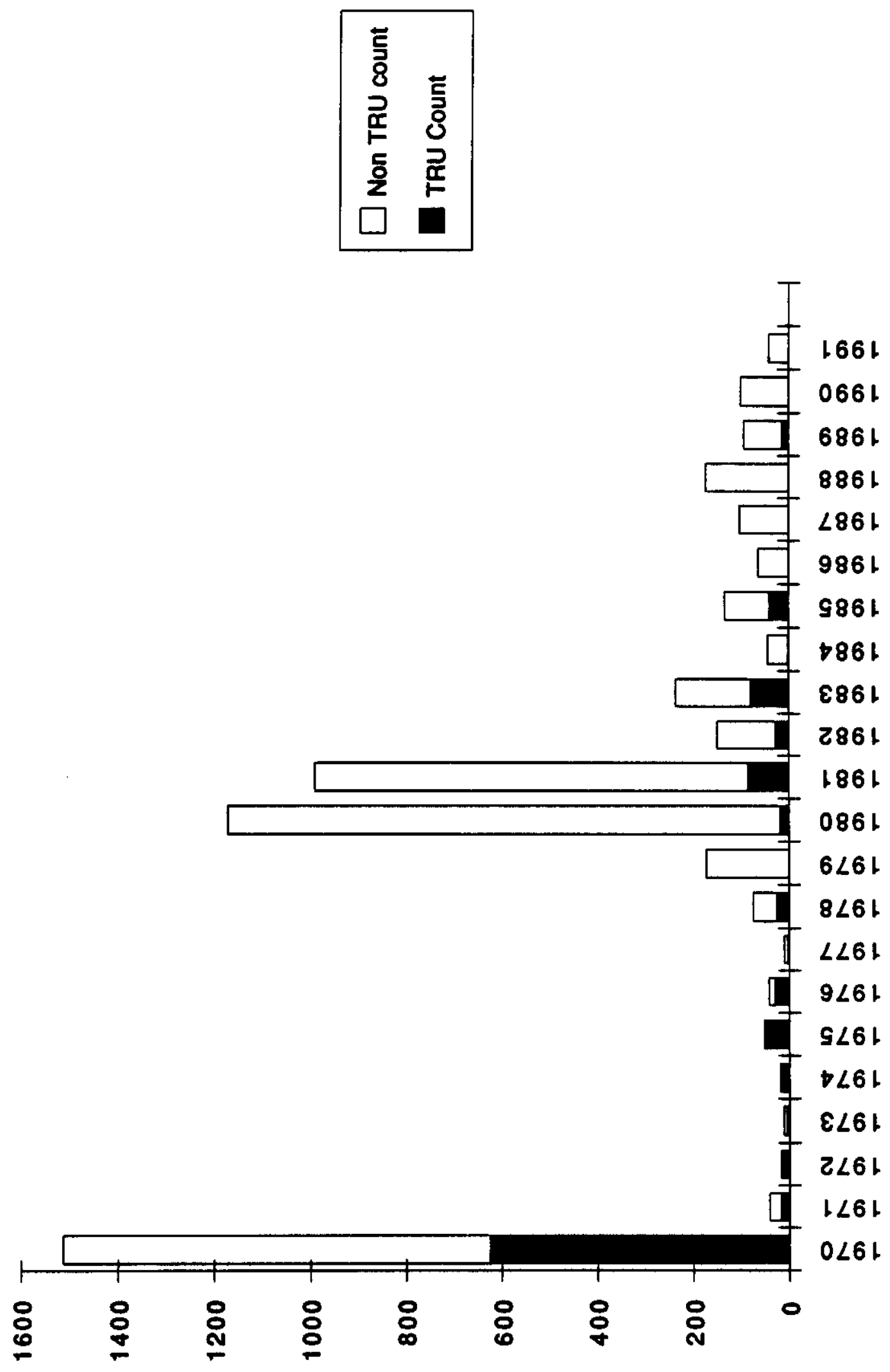


Figure 5-10. Percentage of TRU and Non-TRU Containers Other Than 55-Gallon Drums Generated Annually at the PFP.

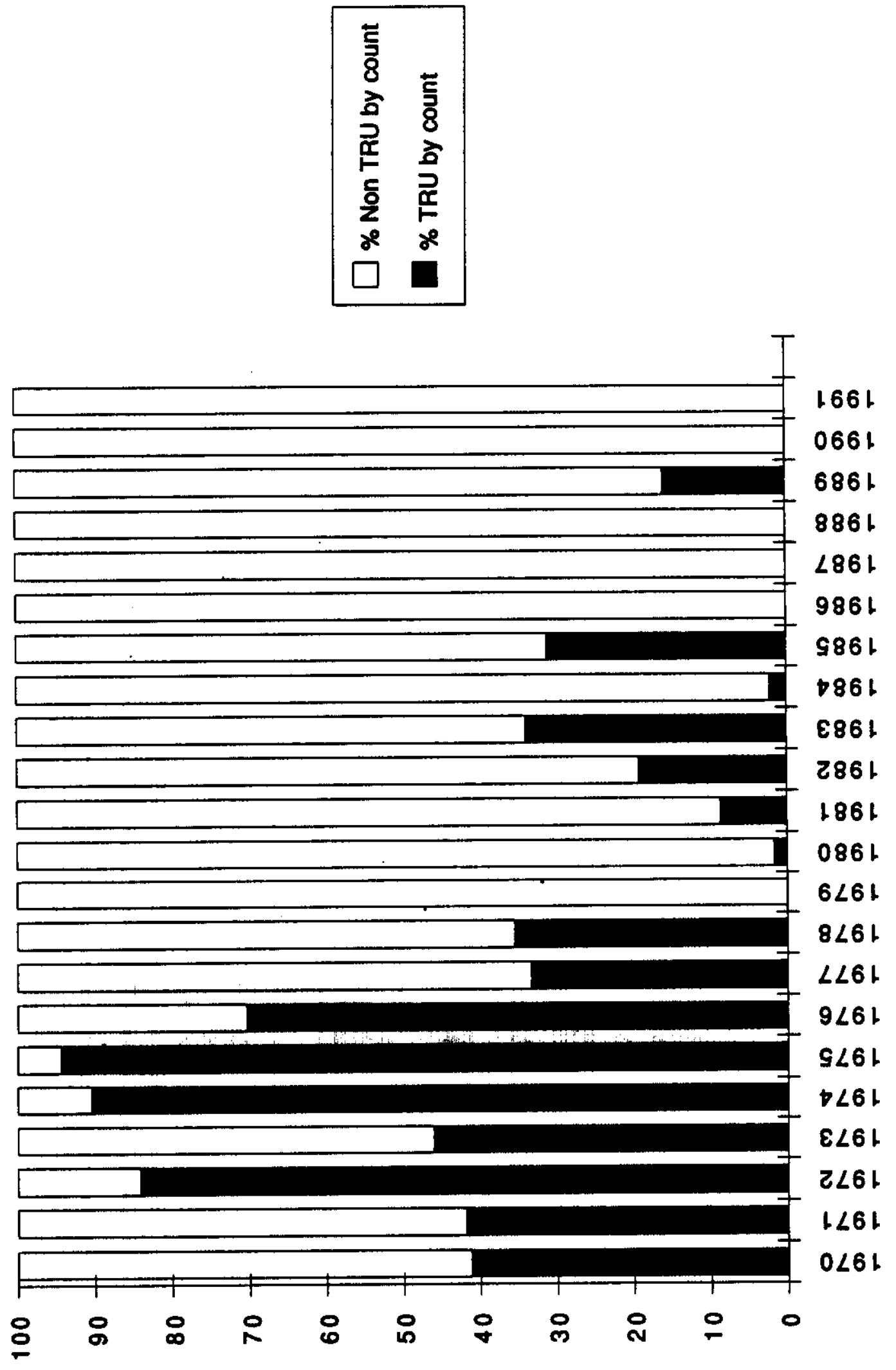


Figure 5-11. Total Weight of Radioactive Waste Stored in Containers Other Than 55-Galion Drums Generated Annually at the PFP.

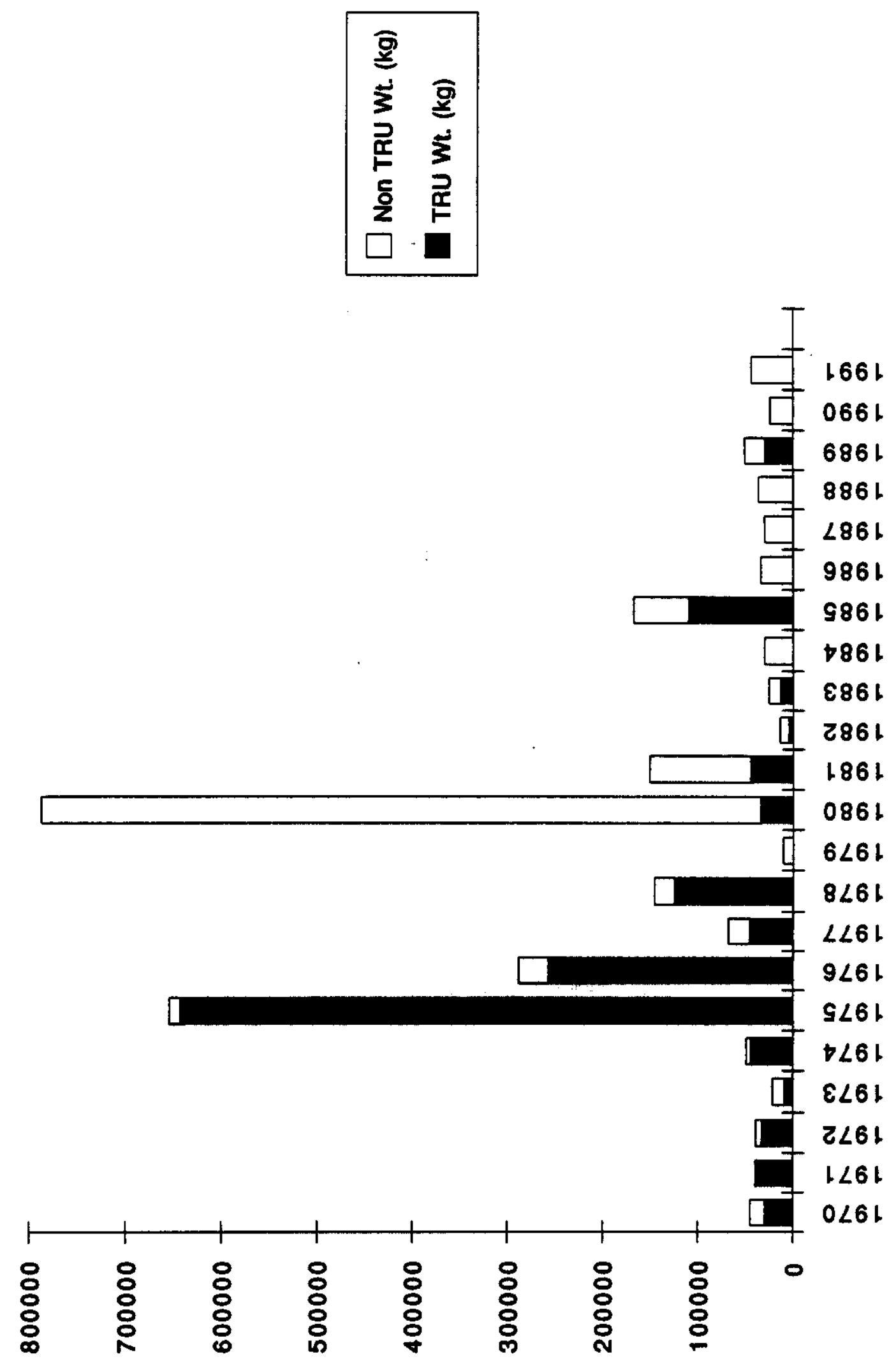


Figure 5-12. Percent Weight of TRU and Non-TRU Containers Other Than 55-Galilon Drums Generated Annually at the PFP.

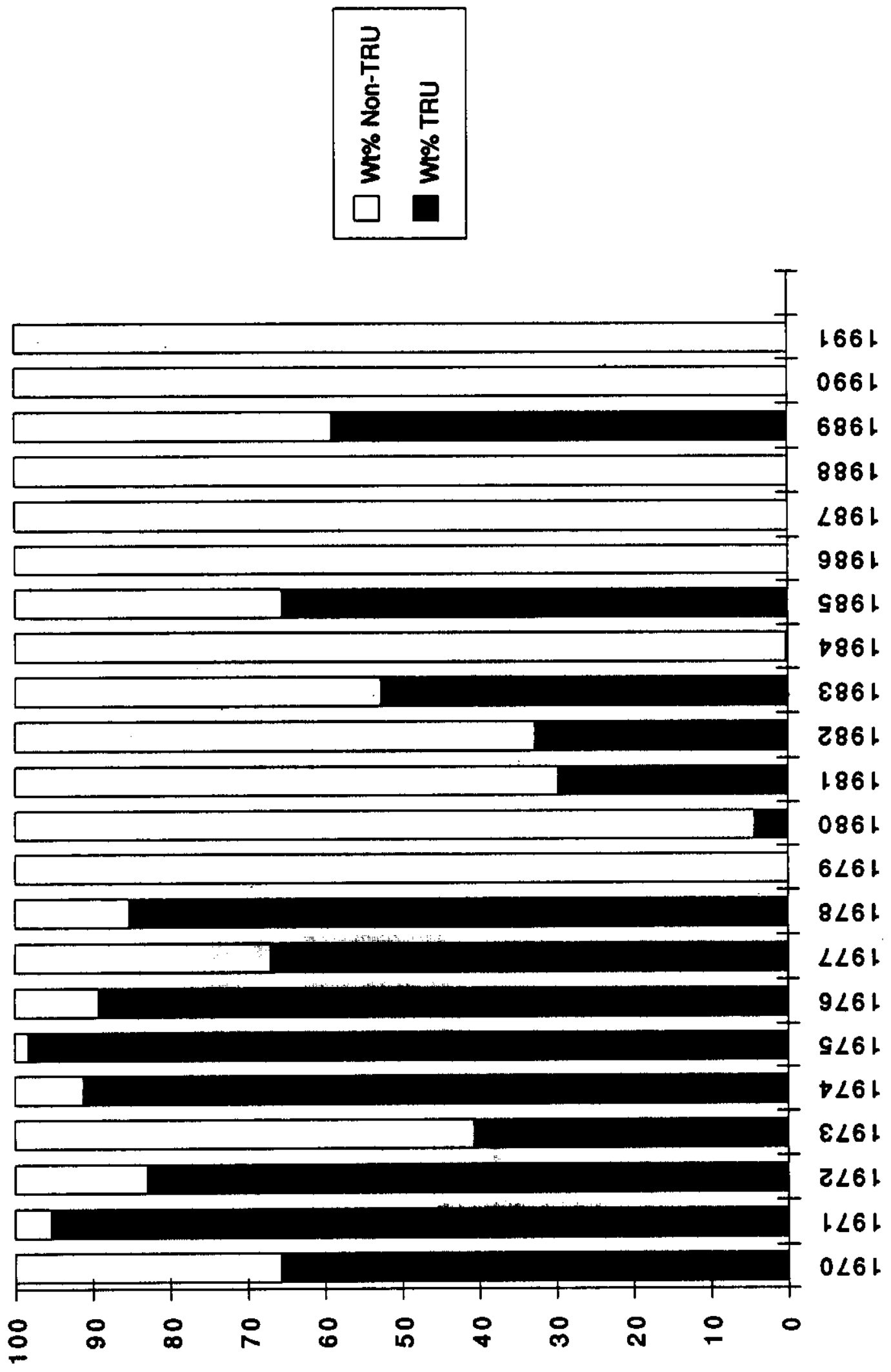


WHC-EP-0621

Figure 5-13. Total Number of Waste Containers Generated by PFP Annually.

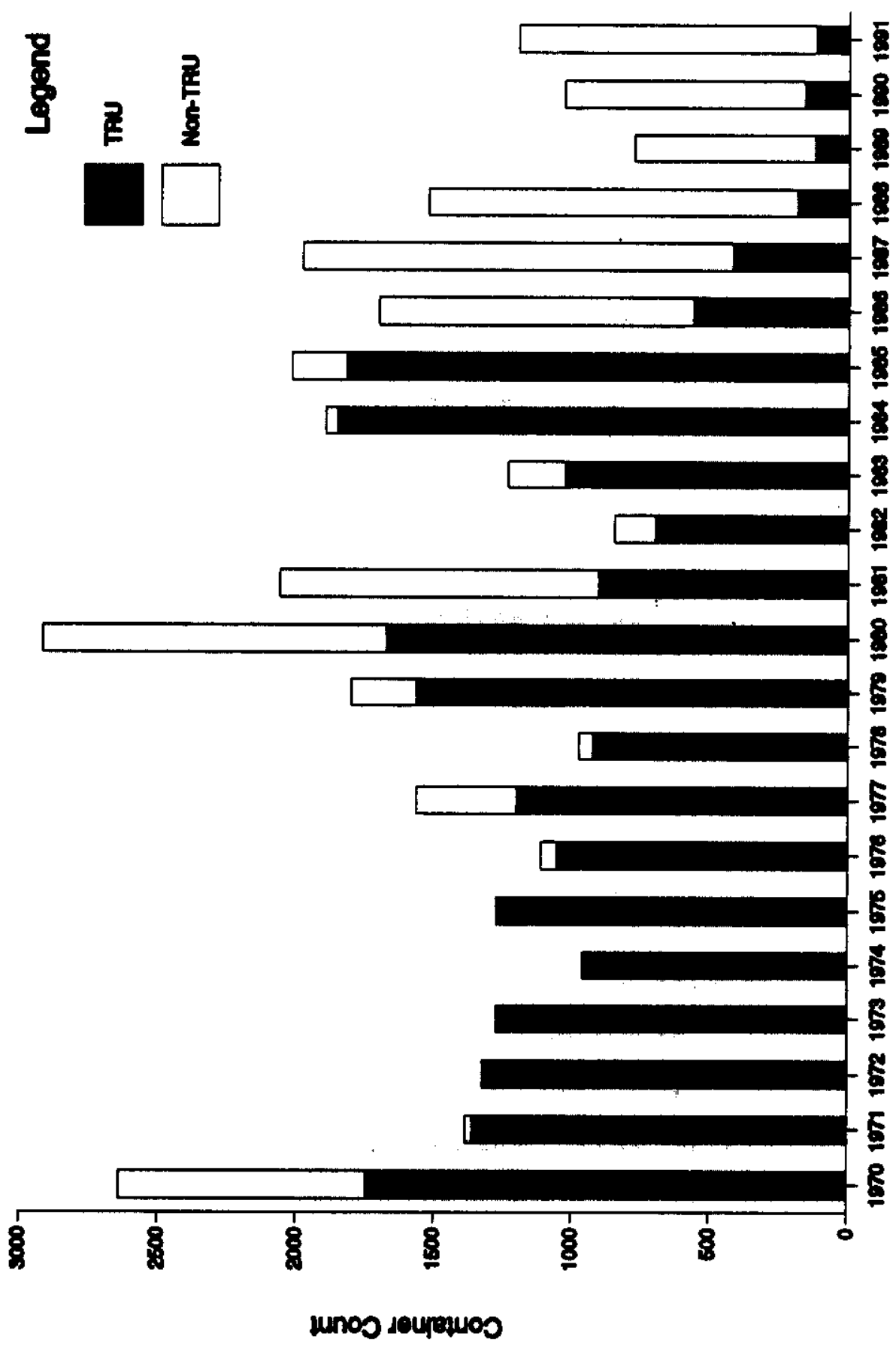


WHC-EP-0621

Figure 5-14. Total Weight of Waste Generated by PFP Annually.

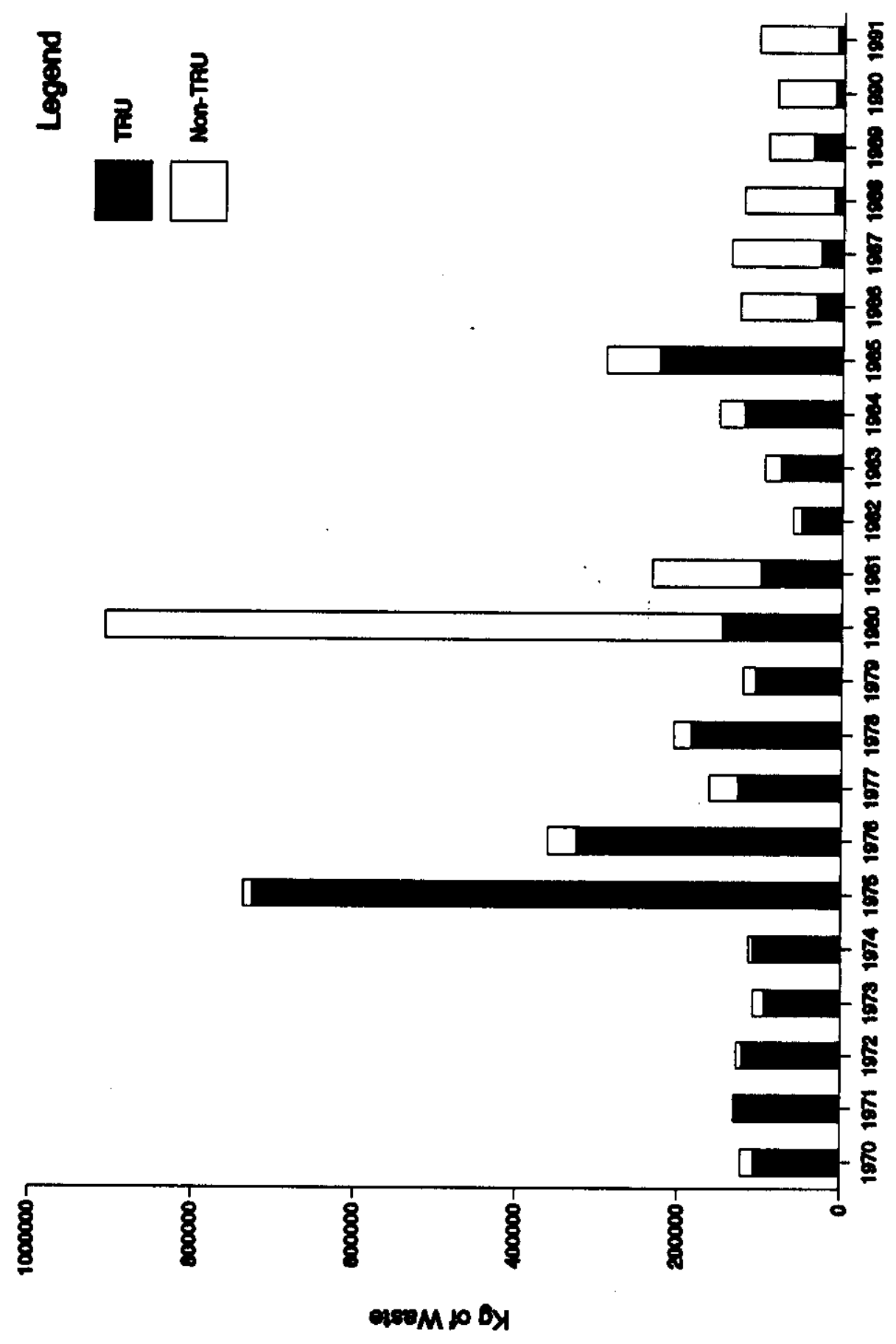


WHC-EP-0621

Figure 5-15. Total Volume of Waste Generated by PFP Annually.
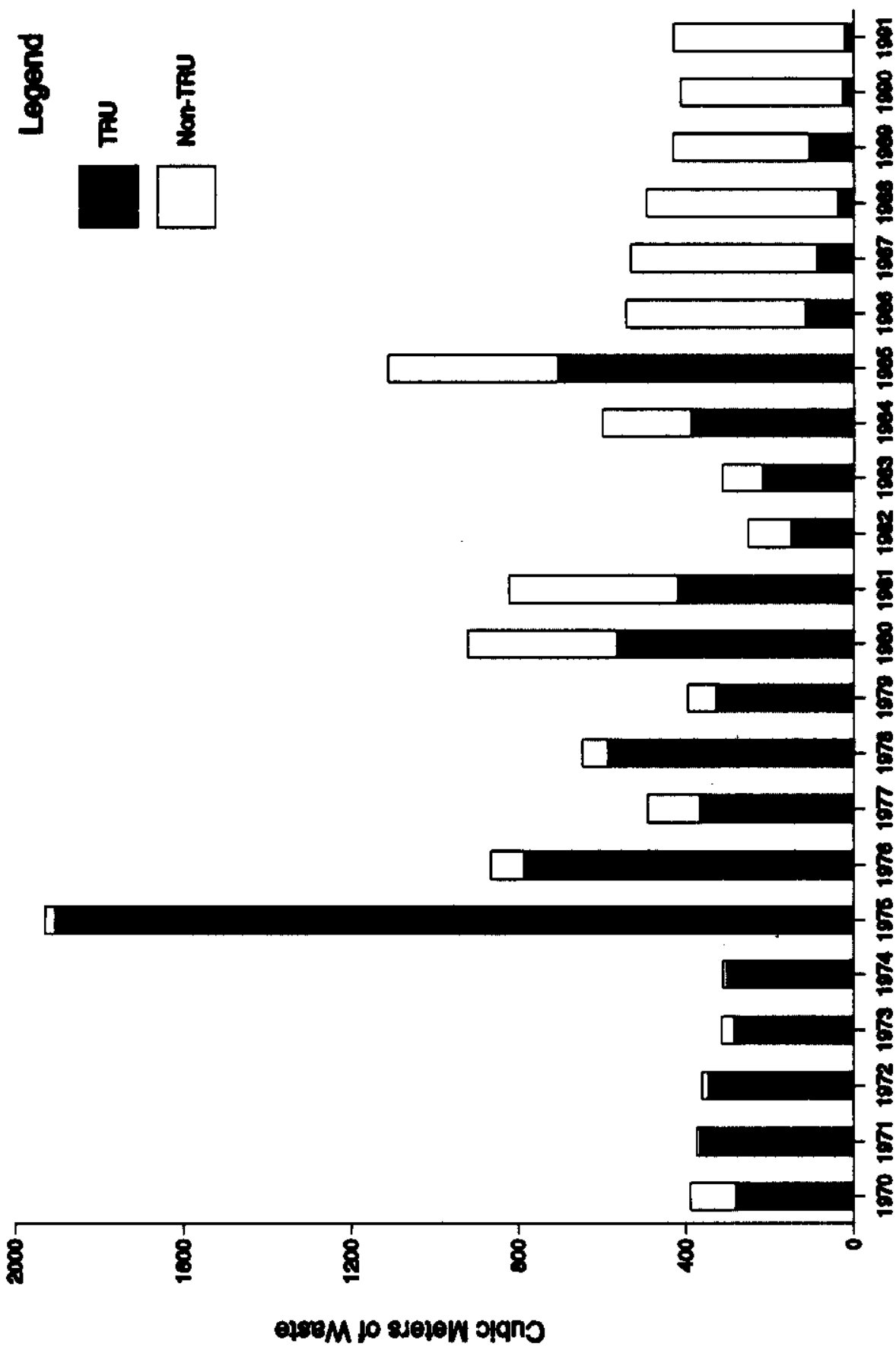


\section{WHC-EP-0621}

Figure 5-16. Grams of TRU Stored in Waste Generated by PFP.

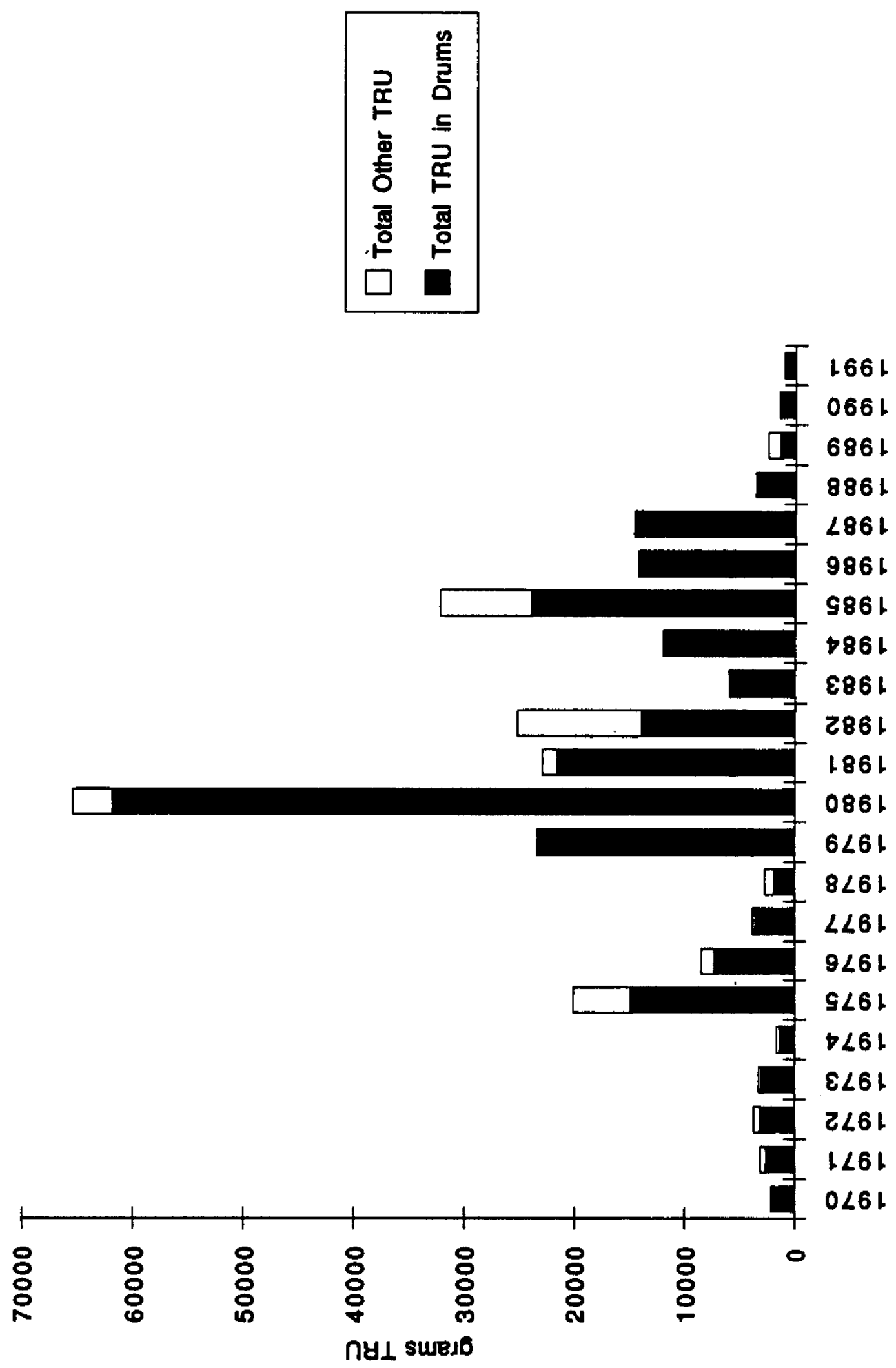


Table 5-1. Waste Summary Data for 55-Gallon Drums Generated at the PFP from 1970 to 1991.

\begin{tabular}{|c|c|c|c|c|c|c|c|c|c|c|c|c|c|c|c|c|c|c|c|c|c|c|}
\hline \multicolumn{23}{|c|}{ 234-5Z Waste Summary Data for 55 Galion Drums } \\
\hline 55 Ga. Drums & 1970 & 1971 & 1972 & 1973 & 1974 & 1975 & 1978 & $19 \pi$ & 1978 & 1979 & 1980 & 1981 & 1982 & 1983 & 1984 & 1985 & 1986 & 1987 & 1988 & 1989 & 1990 & 1991 \\
\hline TRU count & 1123 & 1343 & 1306 & 1264 & 942 & 1222 & 1025 & 1199 & 800 & 1569 & 1658 & 819 & 672 & 949 & 1855 & 1782 & 564 & 422 & 190 & 109 & 161 & 122 \\
\hline TRU Wh (kg) & 76409 & 91357 & 88860 & 86071 & 640094 & 83145 & 69657 & 81444 & 61236 & 106746 & 112897 & 55819 & 45650 & 62774 & 121391 & 115894 & 33501 & 28650 & 12349 & 8085 & 11351 & 8680 \\
\hline TRU Vol. (m3) & 236 & 282 & 274 & 266 & 198 & 257 & 215 & 252 & 189 & 329 & 348 & 172 & 141 & 200 & 390 & 374 & 118 & 8 & 40 & 23 & 28 & 22 \\
\hline TRU MW count & & & & & & & & & & & & & 6 & 4 & 3 & 27 & 0 & 0 & 10 & 43 & 21 & 18 \\
\hline Non TRU count & 3 & 0 & 0 & 0 & 0 & 0 & 43 & 355 & 0 & 60 & $\infty 2$ & 255 & 25 & 51 & 0 & 106 & 1078 & 1459 & 1165 & 575 & 771 & 1035 \\
\hline Non TRU Wh (kg) & 204 & 0 & 0 & 0 & 0 & 0 & 4037 & 12741 & 0 & 3512 & 7574 & 27337 & 1173 & 7438 & 0 & 8285 & 59198 & 78666 & 73283 & 34203 & 45648 & 51445 \\
\hline Non TAU Vol. (m3) & 1 & 0 & 0 & 0 & 0 & 0 & 9 & 74 & 0 & 13 & 20 & 54 & 5 & 11 & 0 & 22 & 226 & 307 & 246 & 121 & 173 & 221 \\
\hline \% TRU by count & 100 & 100 & 100 & 100 & 100 & 100 & 96 & 77 & 100 & 86 & 85 & 76 & 96 & 95 & 100 & 94 & 34 & 22 & 14 & 16 & 17 & 11 \\
\hline \% Non TRU by count & 0.27 & 0 & 0 & 0 & 0 & 0 & 4 & 23 & 0 & 4 & $\mathbf{5}$ & 24 & 4 & 5 & 0 & 6 & 66 & 78 & 86 & 84 & 83 & $\infty$ \\
\hline We\% TRU & 100 & 100 & 100 & 100 & 100 & 100 & 95 & 86 & 100 & 97 & 94 & 67 & 97 & 89 & 100 & 93 & 36 & 27 & 14 & 19 & 20 & 14 \\
\hline W\% Non-TRU & 0.27 & 0 & 0 & 0 & 0 & 0 & 5 & 14 & 0 & 3 & 6 & 33 & 3 & 11 & 0 & 7 & 64 & 73 & 86 & 81 & 80 & 86 \\
\hline Vol\% TRU & 100 & 100 & 100 & 100 & 100 & 100 & 96 & 77 & 100 & 86 & 86 & 76 & 96 & 95 & 100 & 94 & 34 & 22 & 14 & 16 & 14 & 9 \\
\hline Vol\% Non TRU & 0.27 & 0 & 0 & 0 & 0 & 0 & 4 & 23 & $\mathbf{0}$ & 4 & 5 & 24 & 4 & 5 & 0 & 6 & 66 & 78 & 86 & 84 & 86 & 91 \\
\hline \multicolumn{13}{|c|}{$x_{0}$ of TRU Drums that are TRU NW by coumt } & 1 & 0.42 & 0.16 & 2 & 0 & 0 & 5 & 39 & 13 & 15 \\
\hline
\end{tabular}


Table 5-2. PFP 55-Gallon Drums Over 150 Kilograms. (2 sheets)

\begin{tabular}{|c|c|c|c|c|c|}
\hline Year & $\begin{array}{c}\text { Container } \\
\text { identification }\end{array}$ & $\begin{array}{c}\text { Drum } \\
\text { weight } \\
\text { (kg) }\end{array}$ & $\begin{array}{c}\text { TRU } \\
\text { waste } \\
\text { (g) }\end{array}$ & Physical contents & Hazardous components \\
\hline \multirow{4}{*}{1984} & A11392 & 162 & 1.00 & Metal/iron/galvanized/sheet & \\
\hline & A11478 & 189 & 6.00 & Metal/iron/galvanized/sheet & \\
\hline & Al1778 & 159 & 1.00 & Metal/iron/galvanized/sheet & \\
\hline & A11787 & 180 & 1.00 & Metal/iron/galvanized/sheet & \\
\hline \multirow{12}{*}{1985} & A11608 & 178 & 179.00 & $\begin{array}{l}\text { Metal/iron/galvanized/sheet/ } \\
\text { plastic/polyurethane }\end{array}$ & \\
\hline & A12236 & 150 & 125.00 & Plastic/polyurethane & \\
\hline & A12615 & 192 & 1.00 & Paper/cardboard & \\
\hline & A12764 & 150 & 1.00 & Metal/iron/galvanized/sheet & \\
\hline & A13135 & 165 & 82.00 & Plastic/polyurethane & . \\
\hline & A13200 & 177 & 1.00 & Dirt/soil/diatomaceous earth & \\
\hline & A13201 & 154 & 2.00 & Dirt/soil/diatomaceous earth & \\
\hline & A13203 & 154 & 1.00 & Dirt/soil/diatomaceous earth & \\
\hline & A13205 & 182 & 1.00 & Dirt/soil/diatomaceous earth & \\
\hline & A13209 & 159 & 3.00 & Dirt/soil/diatomaceous earth & . \\
\hline & A13220 & 177 & 1.00 & Dirt/soil/diatomaceous earth & \\
\hline & A13237 & 216 & 1.00 & Metal/iron/galvanized/sheet & \\
\hline \multirow{3}{*}{1986} & A14053 & 153 & 123.00 & Metal/iron/galvanized/sheet & \\
\hline & Al5015 & 155 & 145.00 & Metal/iron/galvanized/sheet & \\
\hline & A10107 & 154 & 103.00 & Metal/iron/galvanized/sheet & \\
\hline 1987 & A17826 & 193 & 24.00 & Metal/iron/galvanized/sheet & \\
\hline
\end{tabular}


Table 5-2. PFP 55-Gallon Drums Over 150 Kilograms. (2 sheets)

\begin{tabular}{|c|c|c|c|l|l|}
\hline \multirow{4}{*}{1990} & $\begin{array}{c}\text { Container } \\
\text { identification }\end{array}$ & $\begin{array}{c}\text { Drum } \\
\text { weight } \\
(\mathrm{kg})\end{array}$ & $\begin{array}{c}\text { TRU } \\
\text { waste } \\
(\mathrm{g})\end{array}$ & \multicolumn{1}{|c|}{ Physical contents } & \multicolumn{1}{|c|}{ Hazardous components } \\
\cline { 2 - 6 } & $220-A 20220$ & 208 & 26.00 & Lead & Lead, acid \\
\cline { 2 - 7 } & $212-A 21207$ & 152 & 39.00 & Lead shielding & Lead, lead chromate \\
\hline \multirow{3}{*}{1992} & $220-A 21295$ & 150 & 44.00 & Rubber/lead & $\begin{array}{l}\text { Lead, lead chromate, lead acid, } \\
\text { barium, cadmium, mercury }\end{array}$ \\
\cline { 2 - 7 } & RHZ-213-A22341 & 173 & 13.00 & Rubber/hazardous constituents & Lead, lead chromate \\
\cline { 2 - 7 } & RHZ-213-A22515 & 154 & 74.90 & Hazardous constituents & Barium, cadmium, mercury \\
\hline
\end{tabular}

TRU = Transuranic.

$\underset{\substack{\omega \\ \leftrightarrow}}{\omega}$ 
Table 5-3. Waste Summary Data for Containers 0ther Than 55-Gallon Drums Generated at the PFP from 1970 to 1991.

\begin{tabular}{|c|c|c|c|c|c|c|c|c|c|c|c|c|c|c|c|c|c|c|c|c|c|c|}
\hline \multicolumn{23}{|c|}{ 234-5Z TRU Waste Summany Data for other containers (not 55 gal drums) } \\
\hline & 1970 & 1971 & 1972 & 1973 & 1974 & 1975 & 1976 & 1977 & $\cdot 1978$ & 1070 & 1980 & 1981 & 1982 & 1983 & 1984 & 1985 & 1886 & 1987 & 1988 & 1989 & 1990 . & 1991 \\
\hline TRU Count & 625 & 18 & 16 & 6 & 19 & 51 & 31 & 4 & 27 & 0 & 20 & 86 & 29 & 80 & 1 & 42 & 0 & 0 & 0 & 15 & 0 & 0 \\
\hline TRU W (kg) & 29906 & 39446 & 33117 & 8006 & 45000 & 643173 & 257352 & 45985 & 124427 & 0 & 34638 & 44912 & 4760 & 13528 & 86 & 109829 & 0 & 0 & 0 & 30100 & 0 & 0 \\
\hline IRU Vol. (m3) & 47 & 86 & 74 & 20 & 105 & 1650 & 575 & 116 & 390 & 0 & 218 & 249 & 10 & 20 & 0.21 & 333 & 0 & 0 & 0 & 85 & 0 & 0 \\
\hline TRU MW count & & & & & & 1 & & & & & & 7 & 3 & & & & & & & 15 & & \\
\hline $\begin{array}{l}\text { Non TRU count } \\
\text { Non TRU Wh (kg) } \\
\text { Non TRU Vol }(\mathrm{m} 3)\end{array}$ & $\begin{array}{c}890 \\
15507 \\
107 \\
\end{array}$ & $\begin{array}{c}26 \\
1898 \\
7 \\
\end{array}$ & $\begin{array}{c}3 \\
6782 \\
15 \\
\end{array}$ & $\begin{array}{c}7 \\
13106 \\
30 \\
\end{array}$ & $\begin{array}{c}2 \\
434 \\
10 \\
\end{array}$ & $\begin{array}{r}3 \\
11226 \\
25 \\
\end{array}$ & $\begin{array}{c}13 \\
31056 \\
69 \\
\end{array}$ & $\begin{array}{c}8 \\
22587 \\
50 \\
\end{array}$ & $\begin{array}{c}49 \\
21423 \\
61 \\
\end{array}$ & $\begin{array}{c}174 \\
10663 \\
55 \\
\end{array}$ & $\begin{array}{c}1151 \\
753601 \\
338 \\
\end{array}$ & $\begin{array}{c}904 \\
106053 \\
349 \\
\end{array}$ & $\begin{array}{c}122 \\
9753 \\
96 \\
\end{array}$ & $\begin{array}{c}156 \\
12099 \\
84 \\
\end{array}$ & $\begin{array}{c}44 \\
30309 \\
211 \\
\end{array}$ & $\begin{array}{r}93 \\
57588 \\
385 \\
\end{array}$ & $\begin{array}{r}64 \\
34473 \\
200 \\
\end{array}$ & $\begin{array}{c}103 \\
30543 \\
197 \\
\end{array}$ & $\begin{array}{c}173 \\
36879 \\
210 \\
\end{array}$ & $\begin{array}{c}79 \\
20820 \\
203 \\
\end{array}$ & $\begin{array}{c}101 \\
24866 \\
213 \\
\end{array}$ & $\begin{array}{c}43 \\
44365 \\
187 \\
\end{array}$ \\
\hline $\begin{array}{l}\text { \% TRU by count } \\
\text { \% Non TRU by count } \\
\text { W\% TRU }\end{array}$ & $\begin{array}{l}41 \\
59 \\
66\end{array}$ & $\begin{array}{l}42 \\
58 \\
95\end{array}$ & $\begin{array}{l}84 \\
16 \\
83\end{array}$ & $\begin{array}{l}46 \\
54 \\
41\end{array}$ & $\begin{array}{l}90 \\
10 \\
91\end{array}$ & $\begin{array}{c}94 \\
6 \\
98\end{array}$ & $\begin{array}{l}70 \\
30 \\
89\end{array}$ & $\begin{array}{l}33 \\
67 \\
67\end{array}$ & $\begin{array}{l}36 \\
64 \\
85\end{array}$ & $\begin{array}{c}0 \\
100 \\
0\end{array}$ & $\begin{array}{c}2 \\
88 \\
4\end{array}$ & $\begin{array}{l}9 \\
91 \\
30\end{array}$ & $\begin{array}{l}19 \\
81 \\
33\end{array}$ & $\begin{array}{l}34 \\
66 \\
53\end{array}$ & $\begin{array}{c}2 \\
98 \\
0\end{array}$ & $\begin{array}{l}31 \\
69 \\
66\end{array}$ & $\begin{array}{c}0 \\
100 \\
0\end{array}$ & $\begin{array}{c}0 \\
100 \\
0\end{array}$ & $\begin{array}{c}0 \\
100 \\
0\end{array}$ & $\begin{array}{l}16 \\
84 \\
59\end{array}$ & $\begin{array}{c}0 \\
100 \\
0\end{array}$ & $\begin{array}{c}0 \\
100 \\
0\end{array}$ \\
\hline W\% Non-TRU & 34 & 5 & 17 & 59 & 9 & 2 & 11 & 33 & 15 & 100 & 96 & 70 & 67 & 47 & 100 & 34 & 100 & 100 & 100 & 41 & 100 & 100 \\
\hline Volx TRU & 30 & 92 & 83 & 41 & 92 & 9 & 89 & 70 & 87 & 0 & 39 & 42 & 10 & 19 & 0 & 46 & 0 & 0 & 0 & 29 & 0 & 0 \\
\hline Vol\% Non TRU & 70 & 8 & 17 & 59 & 8 & 1 & 11 & 30 & 13 & 100 & 61 & 58 & 90 & 81 & 100 & 54. & 100 & 100 & 100 & 71 & 100 & 100 \\
\hline \multicolumn{6}{|c|}{ \% TRU Containers that are TRU MW by count } & 2 & & & & & & 8 & 10 & & & & & & & 100 & & \\
\hline
\end{tabular}


Table 5-4. Summary of Low-Level Waste Generated at 234-5Z from 1970 through 1991.

\begin{tabular}{|l|r|r|}
\hline \multicolumn{1}{|c|}{ Container type } & Number & Weight (kg) \\
\hline 55-gal steel drums & 7395 & $426,968.37$ \\
\hline Burlap, cloth, paper, or plastic bags & 772 & $34,465.02$ \\
\hline Concrete boxes (1970 only) & 10 & 331.12 \\
\hline Dump trucks & 24 & $3,149.02$ \\
\hline Fiberboard/plastic boxes, cartons, and cases & 1235 & $15,857.06$ \\
\hline Gloveboxes (1976 and 1981) & 3 & $2,626.28$ \\
\hline HEPA filters & 365 & $11,206.44$ \\
\hline Metal boxes, cartons, and cases & 87 & $23,036.94$ \\
\hline Metal drums, barrels, and kegs (other than 55-gal drums) & 514 & $43,898.93$ \\
\hline Miscellaneous scrap & 122 & $24,600.01$ \\
\hline Self-contained equipment & 817 & $59,019.76$ \\
\hline Portable tanks & 4 & 734.82 \\
\hline Trucks, flatbeds, compactors, and loadluggers & 201 & $326,525.07$ \\
\hline Wooden boxes, cartons, and cases & 11,620 & $69,156.63$ \\
\hline Total & $1,069,921.47$ \\
\hline
\end{tabular}

HEPA = High-efficiency particulate air. 
Table 5-5. TRU Waste Generated at 234-5Z by Container Type and Year. (5 sheets)

\begin{tabular}{|c|c|c|c|c|c|c|c|}
\hline Container type & $\begin{array}{l}\text { Containar } \\
\text { size }\end{array}$ & $\begin{array}{l}\text { Container } \\
\text { numibor }\end{array}$ & $\begin{array}{c}\text { Percent } \\
\text { of } \\
\text { total }\end{array}$ & $\begin{array}{l}\text { Totel } \\
\text { conteiner } \\
\text { wight } \\
\text { (kg) }\end{array}$ & $\begin{array}{l}\text { Percent } \\
\text { weight }\end{array}$ & Volune & $\begin{array}{l}\text { Percent } \\
\text { volume }\end{array}$ \\
\hline \multicolumn{8}{|c|}{1010} \\
\hline $\begin{array}{l}\text { Burlap, cloth, paper, or plastic } \\
\text { bage }\end{array}$ & $\boldsymbol{N} / \mathbf{A}$ & 6 & 0.3 & 130 & 0.1 & & \\
\hline Concrete boxes & $N / A$ & 1 & 0.1 & 20,638 & 19.4 & 46.02 & 16.3 \\
\hline $\begin{array}{l}\text { Fibarboard/plastic boxes, } \\
\text { cartons, cases }\end{array}$ & $\begin{array}{l}2 \mathrm{ft}^{3} \\
\text { Unienoim }\end{array}$ & 523 & $\begin{array}{r}0.2 \\
29.9\end{array}$ & 6.641 & $\begin{array}{r}80.1 \\
6.2\end{array}$ & & \\
\hline Gloveboxes & $\mathbf{n} / \mathbf{A}$ & & & & & & \\
\hline Motal drume, barrels, kege & $\begin{array}{l}55 \text { ot } \\
30 \text { ol } \\
5 \cdot 91\end{array}$ & $\begin{array}{r}1,123 \\
8 \\
55\end{array}$ & $\begin{array}{r}64.2 \\
0.5 \\
3.2 \\
\end{array}$ & $\begin{array}{r}76,409 \\
327 \\
1,267\end{array}$ & $\begin{array}{r}71.8 \\
0.3 \\
1.2\end{array}$ & $\begin{array}{r}235.83 \\
0.93\end{array}$ & $\begin{array}{c}83.4 \\
0.3\end{array}$ \\
\hline Miscellaneous serap & $N / \boldsymbol{A}$ & 1 & 0.1 & 25 & $>0.1$ & & \\
\hline self-conteined equipenent & $N / A$ & 26 & 1.5 & 920 & 0.9 & & \\
\hline $\begin{array}{l}\text { Trucks, flatbeds, compectors, } \\
\text { toedlugger }\end{array}$ & $N / \boldsymbol{A}$ & 1 & 0.1 & 45 & $<0.1$ & & \\
\hline Total & & 1,748 & & 106,404 & & 282.78 & \\
\hline \multicolumn{8}{|c|}{181} \\
\hline Gloveboxes & $N / A$ & 2 & 0.2 & 4,877 & 3.8 & 10.87 & 3.0 \\
\hline Matal boxes, cartons, cases & $\begin{array}{l}3 * 3 * 6 \\
3 * 5 * 8 \\
4 * 4 * 5 \\
4 * 6 * 16.5 \\
5 * 7 * 17 \\
\text { Unknown }\end{array}$ & $\begin{array}{l}3 \\
1 \\
1 \\
1 \\
1 \\
1\end{array}$ & $\begin{array}{l}0.2 \\
0.1 \\
0.1 \\
0.1 \\
0.1 \\
0.1\end{array}$ & $\begin{array}{l}1,372 \\
1,524 \\
1,016 \\
5,030 \\
7,557 \\
3,048\end{array}$ & $\begin{array}{l}1.1 \\
1.2 \\
0.8 \\
3.9 \\
5.8 \\
2.4\end{array}$ & $\begin{array}{r}3.06 \\
3.40 \\
2.27 \\
11.21 \\
16.85 \\
6.80 \\
\end{array}$ & $\begin{array}{l}0.8 \\
0.9 \\
0.6 \\
3.0 \\
4.6 \\
1.9\end{array}$ \\
\hline Metal drume, barrels, kege & $\begin{array}{l}30 \mathrm{gal} \\
55 \mathrm{gal}\end{array}$ & 1,343 & $\begin{array}{r}0.6 \\
98.7\end{array}$ & 91,357 & $\begin{array}{r}0.2 \\
70.4\end{array}$ & $\begin{array}{r}8,203 \\
0.58 \\
\end{array}$ & $\begin{array}{r}76.7 \\
0.2\end{array}$ \\
\hline Self-contained equipment & $n / A$ & 2 & 0.2 & 3,658 & 2.8 & 8.16 & 2.2 \\
\hline Wooden boxes, cartons, cases & $4 * 5 * 6$ & 1 & 0.8 & 10,160 & 7.8 & 22.65 & 6.2 \\
\hline Totel & & 1,361 & & 129,803 & & $8,288.85$ & \\
\hline \multicolumn{8}{|c|}{108} \\
\hline Gloveboxes & $N / \mathbf{A}$ & 4 & 0.3 & 6,096 & 5.0 & 3.40 & 1.0 \\
\hline Metal boxes, cartons, cuses & $\begin{array}{l}80.8 \mathrm{ft}^{3} \\
115 \mathrm{ft}^{3} \\
149 \mathrm{ft}^{3} \\
136 \mathrm{ft}^{3} \\
197 \mathrm{ft}^{3} \\
232 \mathrm{ft}^{3} \\
269 \mathrm{ft}^{3} \\
3 * 4 * 10 \\
4 * 5.5 * 16 \\
4 * 5 * 6 * 10\end{array}$ & $\begin{array}{l}1 \\
1 \\
1 \\
2 \\
1 \\
1 \\
1 \\
1 \\
1 \\
1\end{array}$ & $\begin{array}{l}0.1 \\
0.1 \\
0.1 \\
0.2 \\
0.1 \\
0.1 \\
0.1 \\
0.1 \\
0.1 \\
0.1\end{array}$ & $\begin{array}{l}1,026 \\
1,461 \\
1,892 \\
3,963 \\
2,502 \\
3,201 \\
3,416 \\
1,524 \\
4,471 \\
3,429\end{array}$ & $\begin{array}{l}0.8 \\
1.2 \\
1.6 \\
3.3 \\
2.9 \\
2.6 \\
2.8 \\
1.2 \\
3.7 \\
2.8\end{array}$ & $\begin{array}{l}2.29 \\
3.26 \\
4.22 \\
8.83 \\
5.58 \\
7.14 \\
7.62 \\
3.40 \\
9.97 \\
7.65\end{array}$ & $\begin{array}{l}0.7 \\
1.0 \\
1.3 \\
2.6 \\
1.7 \\
2.1 \\
2.3 \\
1.0 \\
3.0 \\
2.3\end{array}$ \\
\hline Metal drums, barrels, kegs & $\begin{array}{l}55 \mathrm{gal} \\
110 \mathrm{gal}\end{array}$ & $\begin{array}{r}1,306 \\
1\end{array}$ & $\begin{array}{r}98.8 \\
0.1\end{array}$ & $\begin{array}{r}88,860 \\
136\end{array}$ & $\begin{array}{r}72.9 \\
0.1\end{array}$ & $\begin{array}{r}274.26 \\
0.42\end{array}$ & $\begin{array}{r}81.1 \\
0.1\end{array}$ \\
\hline Total & & 1,322 & & 121,977 & & 338.04 & \\
\hline
\end{tabular}


Table 5-5. TRU Waste Generated at 234-5Z by Container Type and Year. (5 sheets)

\begin{tabular}{|c|c|c|c|c|c|c|c|}
\hline Conteinar type & $\begin{array}{l}\text { Container } \\
\text { size }\end{array}$ & $\begin{array}{c}\text { Contoiner } \\
\text { mutbor }\end{array}$ & $\begin{array}{c}\text { Parcent } \\
\text { of } \\
\text { total }\end{array}$ & $\begin{array}{c}\text { Totol } \\
\text { containar } \\
\text { wight } \\
\text { (kg) }\end{array}$ & $\begin{array}{l}\text { Percent } \\
\text { weight }\end{array}$ & Volume & $\begin{array}{l}\text { Percent } \\
\text { volume }\end{array}$ \\
\hline \multicolumn{8}{|c|}{$1 \% 3$} \\
\hline Metal boxes, cartons, caeses & $\begin{array}{l}197+t^{3} \\
4+6 * 10\end{array}$ & $\begin{array}{l}1 \\
2\end{array}$ & $\begin{array}{l}0.1 \\
0.2\end{array}$ & $\begin{array}{l}2,502 \\
6,096\end{array}$ & $\begin{array}{l}2.6 \\
6.4\end{array}$ & $\begin{array}{r}5.58 \\
13.59\end{array}$ & $\begin{array}{l}2.0 \\
4.8\end{array}$ \\
\hline Motal drum, barrels, kege & $\begin{array}{r}55 \\
110 \mathrm{gal}\end{array}$ & 1,264 & $\begin{array}{r}9.5 \\
0.2\end{array}$ & $\begin{array}{r}86,071 \\
408\end{array}$ & $\begin{array}{r}90.5 \\
0.6\end{array}$ & $\begin{array}{r}265.65 \\
1.26\end{array}$ & $\begin{array}{r}92.9 \\
0.4\end{array}$ \\
\hline Total & & 1,270 & & $\%, 077$ & & 286.08 & \\
\hline \multicolumn{8}{|c|}{$1 \%$} \\
\hline Metal boxes, cartons, cases & $\begin{array}{l}2 * 3 * 15 \\
3 * 6 * 10 \\
4 * 6 * 10 \\
4 * 8 * 10 \\
4 * 6 * 16 \\
5 * 10 * 13 \\
5.2 * 7.1 * 10.5 \\
5.2 * 7.1 * 16.5\end{array}$ & $\begin{array}{l}1 \\
1 \\
2 \\
1 \\
1 \\
1 \\
2 \\
1\end{array}$ & $\begin{array}{l}0.1 \\
0.1 \\
0.2 \\
0.1 \\
0.1 \\
0.1 \\
0.2 \\
0.1\end{array}$ & $\begin{array}{l}1,143 \\
152 \\
6,096 \\
4,064 \\
6,504 \\
8,255 \\
9,879 \\
7,779\end{array}$ & $\begin{array}{l}1.1 \\
0.1 \\
5.6 \\
3.7 \\
6.0 \\
7.6 \\
9.1 \\
7.1\end{array}$ & $\begin{array}{r}2.55 \\
3.40 \\
13.59 \\
9.06 \\
14.50 \\
18.41 \\
22.03 \\
17.34\end{array}$ & $\begin{array}{l}0.8 \\
1.1 \\
4.5 \\
3 . \\
4.8 \\
6.1 \\
7.3 \\
5.7\end{array}$ \\
\hline Metal drume, barrela, keges & $\begin{array}{l}55 \text { el } \\
110 \text { की }\end{array}$ & 942 & $\begin{array}{r}98.0 \\
0.9\end{array}$ & $\begin{array}{r}6,004 \\
1,226\end{array}$ & $\begin{array}{r}58.7 \\
1.1\end{array}$ & $\begin{array}{r}197.82 \\
3.77\end{array}$ & $\begin{array}{r}65.4 \\
1.3\end{array}$ \\
\hline Total & & 961 & & 109,191 & & 302.47 & \\
\hline \multicolumn{8}{|c|}{$1 \%$} \\
\hline $\begin{array}{l}\text { Fiberglass reinforeed polyester } \\
\text { boxes }\end{array}$ & $\begin{array}{l}9 * 10.67 * 16 \\
10.5 * 10.7+12 \\
4 * 6 * 7 \\
4.83 * 3 * 8 \\
6.3 * 9 * 16.7 \\
9 * 10.69+20 \\
9 * 10.7+12 \\
9 * 11.6 * 20\end{array}$ & $\begin{array}{r}6 \\
1 \\
6 \\
2 \\
1 \\
10 \\
11 \\
6\end{array}$ & $\begin{array}{l}0.3 \\
0.1 \\
0.5 \\
0.2 \\
0.1 \\
0.8 \\
0.9 \\
0.5\end{array}$ & $\begin{array}{r}56,050 \\
17,069 \\
8,560 \\
4,923 \\
9,652 \\
20,070 \\
154,946 \\
134,408\end{array}$ & $\begin{array}{r}10.3 \\
3.3 \\
1.6 \\
0.9 \\
1.8 \\
3.7 \\
28.4 \\
24.6\end{array}$ & $\begin{array}{r}38.06 \\
19.03 \\
10.93 \\
21.52 \\
173.98 \\
543.91 \\
358.83 \\
59.49\end{array}$ & $\begin{array}{r}2.4 \\
1.2 \\
0.7 \\
1.4 \\
11.1 \\
34.5 \\
22.8 \\
3.8\end{array}$ \\
\hline Metal boxes, cartons, ceses & $\begin{array}{l}3.7 * 6.5 * 13.2 \\
5.2 * 7.1 * 10.5 \\
5.2 * 7.1 * 10.5 \\
5.2 * 7.1 * 16.5\end{array}$ & $\begin{array}{r}1 \\
5 \\
3 \\
3\end{array}$ & $\begin{array}{l}0.1 \\
0.4 \\
0.1 \\
0.2\end{array}$ & $\begin{array}{r}3,937 \\
26,698 \\
4,940 \\
23,305\end{array}$ & $\begin{array}{l}0.7 \\
4.5 \\
0.9 \\
4.3\end{array}$ & $\begin{array}{r}8.78 \\
11.02 \\
55.08 \\
17.32\end{array}$ & $\begin{array}{l}0.6 \\
0.7 \\
3.5 \\
1.1\end{array}$ \\
\hline Metal drume, berrels, kege & 55 gal & 1,222 & 96.0 & 83,145 & 15.2 & 256.62 & 16.3 \\
\hline Total & & 1.273 & & 545,683 & & $1,574.57$ & \\
\hline \multicolumn{8}{|c|}{106} \\
\hline $\begin{array}{l}\text { Fiberglase reinforced polyester } \\
\text { boxes }\end{array}$ & 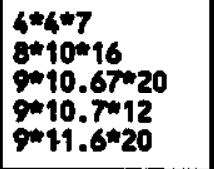 & $\begin{array}{l}5 \\
1 \\
7 \\
3 \\
1\end{array}$ & $\begin{array}{l}0.5 \\
0.1 \\
0.7 \\
0.3 \\
0.1\end{array}$ & $\begin{array}{r}7,265 \\
17,490 \\
170,790 \\
43,898 \\
16,005\end{array}$ & $\begin{array}{r}2.2 \\
5.4 \\
52.2 \\
13.4 \\
4.9\end{array}$ & $\begin{array}{r}16.20 \\
38.99 \\
380.78 \\
97.86 \\
35.68\end{array}$ & $\begin{array}{r}2.1 \\
4.9 \\
48.2 \\
12.4 \\
4.5\end{array}$ \\
\hline Motal drum, barrels, kege & $\begin{array}{l}55 \text { gal } \\
110 \text { gal }\end{array}$ & 1,025 & $\begin{array}{r}97.1 \\
1.3\end{array}$ & $\begin{array}{r}69,657 \\
1,905\end{array}$ & $\begin{array}{r}21.3 \\
0.6\end{array}$ & $\begin{array}{r}215.25 \\
5.87\end{array}$ & $\begin{array}{r}27.2 \\
0.7\end{array}$ \\
\hline Total & & 1,056 & & 327,008 & & 790.63 & \\
\hline
\end{tabular}


Table 5-5. TRU Waste Generated at 234-5Z by Container Type and Year. (5 sheets)

\begin{tabular}{|c|c|c|c|c|c|c|c|}
\hline Conteiner type & $\begin{array}{c}\text { Containor } \\
\text { size }\end{array}$ & $\begin{array}{l}\text { Containar } \\
\text { nubar }\end{array}$ & $\begin{array}{c}\text { Percent } \\
\text { of } \\
\text { totel }\end{array}$ & $\begin{array}{l}\text { Total } \\
\text { conteinor } \\
\text { wight } \\
\text { (kg) }\end{array}$ & $\begin{array}{l}\text { Percent } \\
\text { weight }\end{array}$ & Volum & $\begin{array}{l}\text { Percent } \\
\text { volume }\end{array}$ \\
\hline \multicolumn{8}{|c|}{$1 \% 8$} \\
\hline $\begin{array}{l}\text { Flbergleas reinforced polyeater } \\
\text { boxee }\end{array}$ & $\begin{array}{l}8 * 10 * 16 \\
9 * 10.7 * 12\end{array}$ & 2 & $\begin{array}{l}0.2 \\
0.1\end{array}$ & $\begin{array}{l}27.215 \\
12.619\end{array}$ & $\begin{array}{r}21.4 \\
9.8\end{array}$ & $\begin{array}{l}72.49 \\
32.62\end{array}$ & $\begin{array}{r}19.7 \\
8.9\end{array}$ \\
\hline Metal boxes, cartons, cases & $5.2 * 7.1 * 10.5$ & 1 & 0.1 & 6,350 & 5.0 & 11.02 & 3. \\
\hline Motal drum, barrole, kees & $55 \mathrm{gal}$ & 1,199 & 9.7 & 81,446 & 63.9 & 252.08 & 68.5 \\
\hline Total & & 1,203 & & 127,628 & & 368.21 & \\
\hline \multicolumn{8}{|c|}{18.3} \\
\hline $\begin{array}{l}\text { Fibergless reinforced polyeater } \\
\text { boxes }\end{array}$ & $\begin{array}{l}4 * 4 * 7 \\
6.5 * 8 * 14.6 \\
6.5 * 8 * 18.5 \\
8 * 8 * 10.7 \\
9.5 * 9.9 * 12\end{array}$ & $\begin{array}{l}9 \\
9 \\
3 \\
2 \\
1\end{array}$ & $\begin{array}{l}1.0 \\
1.0 \\
0.3 \\
0.2 \\
0.1\end{array}$ & $\begin{array}{r}3.629 \\
73.110 \\
23.713 \\
6.917 \\
4.423\end{array}$ & $\begin{array}{r}2.0 \\
39.4 \\
13.9 \\
3.7 \\
2.4\end{array}$ & $\begin{array}{r}28.55 \\
193.69 \\
81.38 \\
39.36 \\
32.08\end{array}$ & \\
\hline Metal boxes, cartons, cases & $\begin{array}{l}4 * 4 * 7 \\
4 * 6 * 15\end{array}$ & $\begin{array}{l}1 \\
2\end{array}$ & $\begin{array}{l}0.1 \\
0.2\end{array}$ & $\begin{array}{l}1.432 \\
9,144\end{array}$ & $\begin{array}{l}0.8 \\
4.9\end{array}$ & $\begin{array}{r}3.17 \\
20.39\end{array}$ & \\
\hline Motal drum, barrels, kegs & $55 \mathrm{gal}$ & 900 & 97.1 & 61,236 & 33.0 & 189.00 & \\
\hline Total & & 927 & & 165,664 & & 587.62 & \\
\hline \multicolumn{8}{|c|}{$10 \%$} \\
\hline Matal druma, barrels, kegs & $35 \mathrm{gal}$ & 1,569 & 100 & 106,746 & 100 & 329.49 & 100 \\
\hline Total & & 1,569 & & 106,766 & & 329.49 & \\
\hline \multicolumn{8}{|c|}{ x/s } \\
\hline $\begin{array}{l}\text { Fiberglaes reinforeed polyester } \\
\text { boxes }\end{array}$ & $\begin{array}{l}4 * 6 * 7 \\
9 * 10.67 * 16 \\
9 * 10.7 * 12\end{array}$ & $\begin{array}{l}2 \\
4 \\
1\end{array}$ & $\begin{array}{l}0.1 \\
0.2 \\
0.1\end{array}$ & $\begin{array}{r}2.343 \\
25.009 \\
4.423\end{array}$ & $\begin{array}{r}1.6 \\
17.5 \\
3.0\end{array}$ & $\begin{array}{r}6.34 \\
173.87 \\
32.59\end{array}$ & $\begin{array}{r}1.1 \\
30.7 \\
5.8\end{array}$ \\
\hline Motal drume, berrels, kegs & $\begin{array}{l}55 \text { gal } \\
\text { Unknown }\end{array}$ & 1,658 & $\begin{array}{r}98.8 \\
0.8\end{array}$ & $\begin{array}{r}112,896 \\
2,064\end{array}$ & $\begin{array}{r}76.5 \\
1.4\end{array}$ & $\begin{array}{r}348.39 \\
5.45\end{array}$ & $\begin{array}{r}61.5 \\
1.0\end{array}$ \\
\hline Total & & 1,670 & & 147,535 & & 566.64 & \\
\hline \multicolumn{8}{|c|}{ 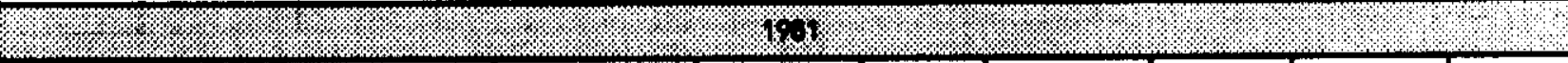 } \\
\hline $\begin{array}{l}\text { Fibarglase relnforced polyeater } \\
\text { boxes }\end{array}$ & $9+10.67 * 16$ & $\mathbf{5}$ & 0.6 & 30,234 & 30.0 & 217.34 & 51.6 \\
\hline Metal drum, berrels, kege & $\begin{array}{l}55 \text { eal } \\
\text { Uniknoin } \\
\text { Uniknown }\end{array}$ & $\begin{array}{r}819 \\
76 \\
74\end{array}$ & $\begin{array}{r}90.5 \\
8.2 \\
0.8\end{array}$ & $\begin{array}{r}55,819 \\
13.042 \\
1,576\end{array}$ & $\begin{array}{r}55.4 \\
13.0 \\
1.6 \\
\end{array}$ & $\begin{array}{r}172.05 \\
0.89 \\
31.01\end{array}$ & $\begin{array}{r}40.8 \\
0.2 \\
7.4\end{array}$ \\
\hline Total & & 905 & & 100,751 & & 421.29 & \\
\hline
\end{tabular}


Table 5-5. TRU Waste Generated at 234-5Z by Container Type and Year. (5 sheets)

\begin{tabular}{|c|c|c|c|c|c|c|c|}
\hline Container type & $\begin{array}{c}\text { Container } \\
\text { size }\end{array}$ & $\begin{array}{c}\text { Container } \\
\text { muber }\end{array}$ & $\begin{array}{c}\text { Percent } \\
\text { of } \\
\text { totet }\end{array}$ & $\begin{array}{l}\text { Total } \\
\text { conteinar } \\
\text { welght } \\
\text { (kg) }\end{array}$ & $\begin{array}{l}\text { Percent } \\
\text { wight }\end{array}$ & Volune & $\begin{array}{l}\text { Percent } \\
\text { volume }\end{array}$ \\
\hline \multicolumn{8}{|c|}{$1 \%$} \\
\hline Metal drums, barrels, kegs & $\begin{array}{l}30 \text { gal } \\
55 \text { gal } \\
55 \text { gal } \\
55 \text { gat } \\
110 \text { gal } \\
\text { Unienown } \\
\text { Unimenn }\end{array}$ & $\begin{array}{r}4 \\
665 \\
1 * \\
6 \\
15 \\
3 \\
7\end{array}$ & $\begin{array}{r}0.6 \\
94.9 \\
0.1 \\
0.9 \\
2.1 \\
0.4 \\
1.0\end{array}$ & $\begin{array}{r}794 \\
45,099 \\
68 \\
4.3 \\
2,041 \\
1,344\end{array}$ & $\begin{array}{r}1.6 \\
89.5 \\
0.1 \\
1.0 \\
4.1 \\
1.1 \\
2.7\end{array}$ & $\begin{array}{r}0.53 \\
0.21 \\
1.26 \\
139.71 \\
6.29 \\
0.38 \\
2.93\end{array}$ & $\begin{array}{r}0.4 \\
0.1 \\
0.8 \\
92.3 \\
4.2 \\
1.9\end{array}$ \\
\hline Total & & 701 & & 50,610 & & 151.31 & \\
\hline \multicolumn{8}{|c|}{401} \\
\hline Motal boxes, cartons, cases & $4 * 6 * 10$ & 1 & 0.1 & 3,706 & 5.5 & 6.99 & 3.2 \\
\hline Metal drunis, barrels, kegs & $\begin{array}{l}55 \text { gal } \\
55 \text { gol } \\
55 \text { gol } \\
\text { Unknown }\end{array}$ & $\begin{array}{l}929 \\
16 \\
4 * 1 \\
79\end{array}$ & $\begin{array}{l}90.3 \\
1.6 \\
0.4 \\
7.7\end{array}$ & $\begin{array}{r}61,249 \\
1,207 \\
319 \\
1,381\end{array}$ & $\begin{array}{r}90.3 \\
1.8 \\
0.5 \\
2.0\end{array}$ & $\begin{array}{r}3.36 \\
0.84 \\
195.30 \\
13.09\end{array}$ & $\begin{array}{r}1.5 \\
0.4 \\
88.9 \\
6.0\end{array}$ \\
\hline Total & & 1,029 & & 67,860 & & 219.58 & \\
\hline \multicolumn{8}{|c|}{10,4} \\
\hline Metal drum, barrels, kege & $55 \mathrm{sml}$ & 1,855 & 9.9 & 121,391 & 99.9 & 389.54 & 100 \\
\hline Miscellaneous serep & $\mathbf{N} / \mathbf{A}$ & 1 & 0.1 & 86 & 0.1 & 0.21 & $<0.1$ \\
\hline Total & & 1,856 & & 121,677 & & 389.75 & \\
\hline \multicolumn{8}{|c|}{$1 \%$} \\
\hline Matal boxes, cartons, cases & 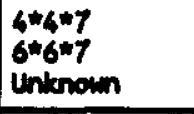 & $\begin{array}{r}2 \\
2 \\
38 \\
\end{array}$ & $\begin{array}{l}0.1 \\
0.1 \\
2.1\end{array}$ & $\begin{array}{r}1,471 \\
2,907 \\
105,451\end{array}$ & $\begin{array}{r}0.7 \\
1.3 \\
46.7 \\
\end{array}$ & $\begin{array}{r}5.60^{\circ} \\
15.00 \\
312.31\end{array}$ & $\begin{array}{r}0.85 \\
2.1 \\
44.2\end{array}$ \\
\hline Metal drune, berrels, kegs & $\begin{array}{ll}55 & \text { gal } \\
55 & \text { gal } \\
55 & \text { gel }\end{array}$ & $\begin{array}{l}1,755 \\
13^{*} \\
14^{*}\end{array}$ & $\begin{array}{l}9.2 \\
0.7 \\
0.8\end{array}$ & $\begin{array}{c}113,551 \\
1.2 \\
3 \\
1,320\end{array}$ & $\begin{array}{r}50.3 \\
0.5 \\
0.6\end{array}$ & $\begin{array}{r}2.73 \\
2.94 \\
368.55\end{array}$ & $\begin{array}{r}0.4 \\
0.4 \\
52.1\end{array}$ \\
\hline Total & . & 1,826 & & $224,704.2$ & & 707.13 & \\
\hline \multicolumn{8}{|c|}{198} \\
\hline Metal drum, berrels, kegs & 55 gal & 564 & 100 & 33,502 & 100 & 118.44 & 100 \\
\hline Total & & 564 & & 33,502 & & & \\
\hline \multicolumn{8}{|c|}{$1 \% 1 \%$} \\
\hline Metal druss, barrels, keges & $55 \mathrm{eat}$ & 442 & 100 & 28,650 & 100 & 88.62 & 100 \\
\hline Total & & 442 & & 28,650 & & 88.62 & \\
\hline \multicolumn{8}{|c|}{$1 \%$} \\
\hline Metal drume, barrels, kegs & $\begin{array}{l}55 \text { gel } \\
55 \text { gall }\end{array}$ & $\begin{array}{r}180 \\
10^{4}\end{array}$ & $\begin{array}{r}94.7 \\
5.3\end{array}$ & $\begin{array}{r}11,649 \\
700\end{array}$ & $\begin{array}{r}94.3 \\
5.7\end{array}$ & $\begin{array}{r}2.10 \\
37.80\end{array}$ & $\begin{array}{r}5.3 \\
94.7\end{array}$ \\
\hline Total & & 190 & & 12,349 & & 39.90 & \\
\hline
\end{tabular}


Table 5-5. TRU Waste Generated at 234-5Z by Container Type and Year. (5 sheets)

\begin{tabular}{|c|c|c|c|c|c|c|c|}
\hline Container type & $\begin{array}{c}\text { Container } \\
\text { size }\end{array}$ & $\begin{array}{c}\text { Container } \\
\text { number }\end{array}$ & $\begin{array}{c}\text { Percent } \\
\text { of } \\
\text { total }\end{array}$ & $\begin{array}{l}\text { Total } \\
\text { container } \\
\text { weight } \\
(k g)\end{array}$ & $\begin{array}{l}\text { Percent } \\
\text { wight }\end{array}$ & Volune & $\begin{array}{l}\text { Percent } \\
\text { volume }\end{array}$ \\
\hline \multicolumn{8}{|c|}{$100 \%$} \\
\hline Motal boxes, cartons, cases & $\begin{array}{l}149+t^{3} \\
6 * 6 * 7 \\
4 * 6 * 7 \\
4.5 * 4.5 * 7.3 \\
5.6 * 6.5 * 9.3 \\
6 * 6 * 7 \\
6 * 6 * 7\end{array}$ & $\underset{1+\infty}{j \neq i \neq}$ & $\begin{array}{l}0.8 \\
0.8 \\
0.8 \\
4.0 \\
3.2 \\
0.8 \\
0.8\end{array}$ & $\begin{array}{r}1,361 \\
1,043 \\
1,724 \\
9,344 \\
11,804 \\
2,177 \\
2,449\end{array}$ & $\begin{array}{r}3.6 \\
2.7 \\
4.5 \\
24.5 \\
31.1 \\
5.7 \\
6.4\end{array}$ & $\begin{array}{r}3.17 \\
3.17 \\
21.03 \\
38.44 \\
7.14 \\
7.14 \\
4.21\end{array}$ & $\begin{array}{r}3.0 \\
3.0 \\
19.6 \\
35.8 \\
6.6 \\
6.6 \\
3.9\end{array}$ \\
\hline Motal druns, barrels, kegs & $\begin{array}{ll}55 & \mathrm{al} \\
55 & \mathrm{all} \\
55 & \mathrm{al} \\
85 & \mathrm{al}\end{array}$ & $\begin{array}{c}66 \\
37 * \\
6 *\end{array}$ & $\begin{array}{r}53.2 \\
29.8 \\
4.8 \\
0.8\end{array}$ & $\begin{array}{r}4,393 \\
3,254 \\
439 \\
118\end{array}$ & $\begin{array}{r}11.5 \\
8.5 \\
1.2 \\
0.3\end{array}$ & $\begin{array}{r}7.77 \\
1.26 \\
13.86 \\
0.28\end{array}$ & $\begin{array}{r}7.2 \\
1.2 \\
12.9 \\
0.9\end{array}$ \\
\hline Total & & 124 & & 38,186 & & 107.47 & \\
\hline \multicolumn{8}{|c|}{$19 \%$} \\
\hline Metal drums, berrels, kegs & أl $55 \mathrm{gal}$ & $\begin{array}{l}140 \\
20 * \\
9 \neq\end{array}$ & $\begin{array}{r}87.0 \\
12.4 \\
0.6\end{array}$ & $\begin{array}{r}8,836 \\
123 \\
46\end{array}$ & $\begin{array}{r}98.1 \\
1.4 \\
0.5\end{array}$ & $\begin{array}{r}4.20 \\
0.21 \\
23.521\end{array}$ & \\
\hline Total & & 161 & 100 & 9,005 & 100 & & \\
\hline \multicolumn{8}{|c|}{1948} \\
\hline Motal drum, berrels, kegs & $\begin{array}{l}55 \text { gal } \\
55 \text { gal }\end{array}$ & $\begin{array}{l}104 \\
18\end{array}$ & $\begin{array}{l}85.3 \\
14.8\end{array}$ & $\begin{array}{l}6,968 \\
1,712\end{array}$ & $\begin{array}{l}80.3 \\
19.7\end{array}$ & $\begin{array}{r}3.772 \\
18.45^{2}\end{array}$ & $\begin{array}{l}17.0 \\
83.0\end{array}$ \\
\hline Total & & 122 & & 8,680 & & 22.22 & \\
\hline
\end{tabular}

WOTE: To convert from gollons to liters multiply by 3.785. To convert from cubic feet to cubic neter mult iply by 2,831,685 E-02.

This volum is besed on 112 55-gal drums.

This volume is besed on eighty eight 55-gal drum.

- Mixed waste.

wh a Mixed waste with PCEs.

MA - Not eppl iceble.

PCB = Polychlorinated biphemyls. 
Table 5-6. TRU Waste in 55-Gallon Drums: Drum Count by Storage Facility.

\begin{tabular}{|c|c|c|c|c|c|c|c|c|c|c|c|c|c|c|c|c|c|c|c|c|c|c|}
\hline $234-52$ & $\begin{array}{l}5 \mathrm{Gall} \\
1970 \\
\end{array}$ & $\begin{array}{l}\text { on Dru } \\
1971\end{array}$ & $\begin{array}{l}\text { mss: } \\
1972\end{array}$ & $\begin{array}{c}\text { Drum c } \\
1973\end{array}$ & $\begin{array}{l}\text { ount S } \\
1974\end{array}$ & $\begin{array}{l}\text { tored } \\
1975\end{array}$ & $\begin{array}{l}\text { by Lo } \\
1976\end{array}$ & $\begin{array}{c}\text { cation } \\
1977\end{array}$ & 1978 & 1979 & 1980 & 1981 & 1982 & 1983 & 1984 & 1985 & 1986 & 1987 & 1988 & 1989 & 1990 & 1991 \\
\hline $218 \sqrt{3 A}$ & 1112 & 537 & & & & & & & & & & & & & 1 & & & & & & & \\
\hline $\begin{array}{l}218 \mathrm{~m} 4 \mathrm{~B} \\
218 \mathrm{4} 4 \mathrm{C}\end{array}$ & & 806 & 1306 & 1264 & 942 & 1222 & 1025 & 1199 & 900 & 1569 & 1658 & 819 & 666 & 945 & 1854 & 1768 & 445 & 10 & 11 & & 53 & 18 \\
\hline 21815 & & & & & & & & & & & & & & & & & & & & & 1 & \\
\hline 224-T & & & & & & & & & & & & & & & & & 119 & 412 & 169 & 100 & 106 & 104 \\
\hline $2401-4$ & & & & & & & & & & & & & 6 & 4 & & 14 & & & 10 & 8 & 1 & \\
\hline $\begin{array}{l}24024 \\
240218 \\
\text { FSO }\end{array}$ & & & & & & & & & & & & & & & & & & & & 1 & & \\
\hline Total & 1112 & 1343 & 1306 & 1264 & 942 & 1222 & 1025 & 1199 & 900 & 1569 & 1658 & 819 & 672 & 949 & 1855 & 1782 & 564 & 422 & 190 & 109 & 161 & 122 \\
\hline
\end{tabular}

$\stackrel{1}{1}$ 
Table 5-7. TRU Waste in Containers Other Than 55-Gallon Drums: Container Count by Storage Facility.

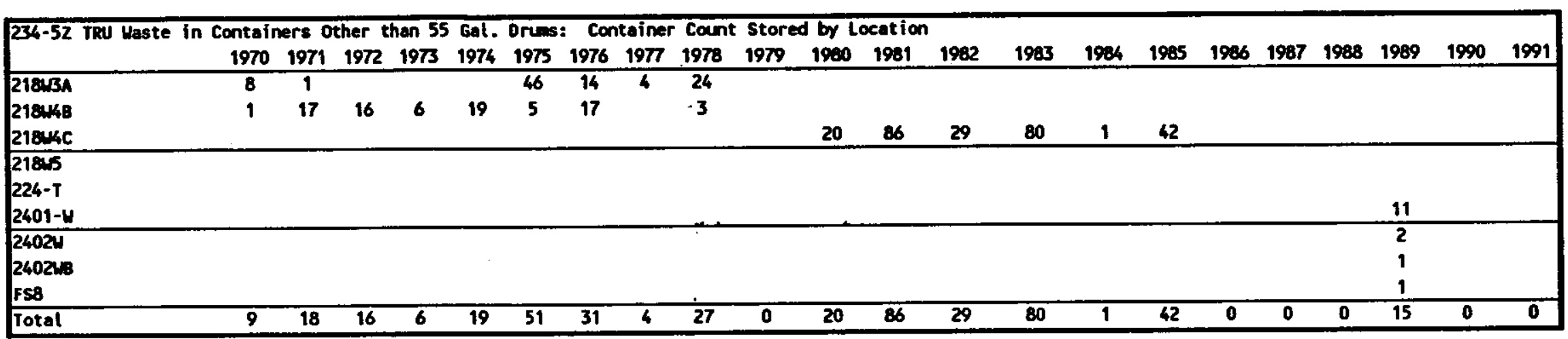


Table 5-8. Distribution and Percentage of TRU 55-Gallon Drum Physical Contents by Storage Location - 218W3A.

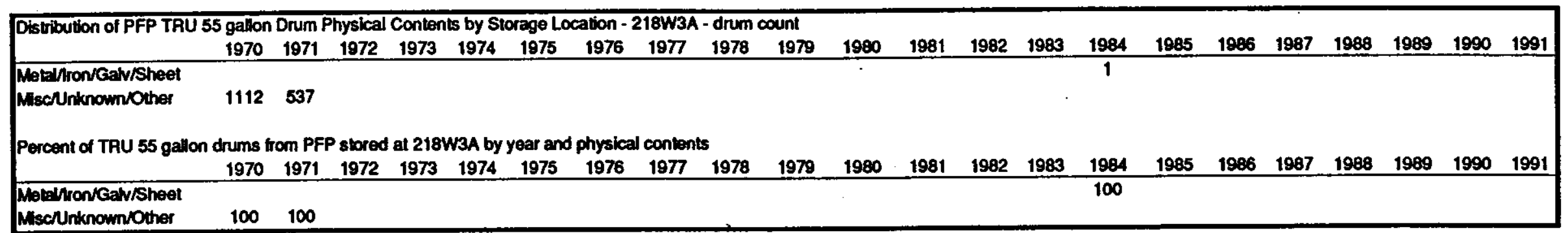


Table 5-9. Distribution and Percentage of TRU 55-Gallon Drum Physical Contents by Storage Location - 218W4B.

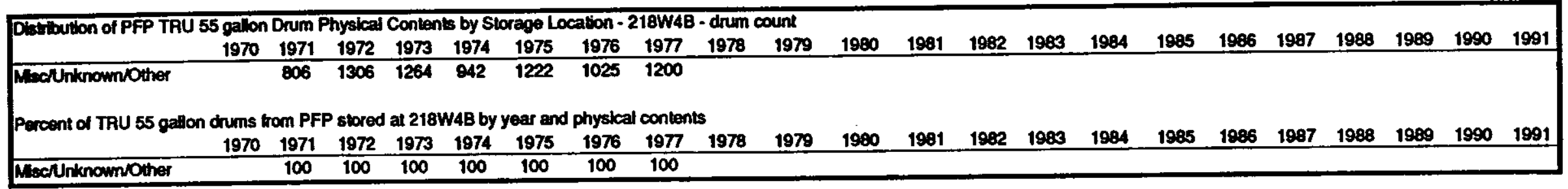

! 
Table 5-10. Distribution and Percentage of TRU 55-Gallon Drum Physical Contents by Storage Location - 218144. (2 sheets)

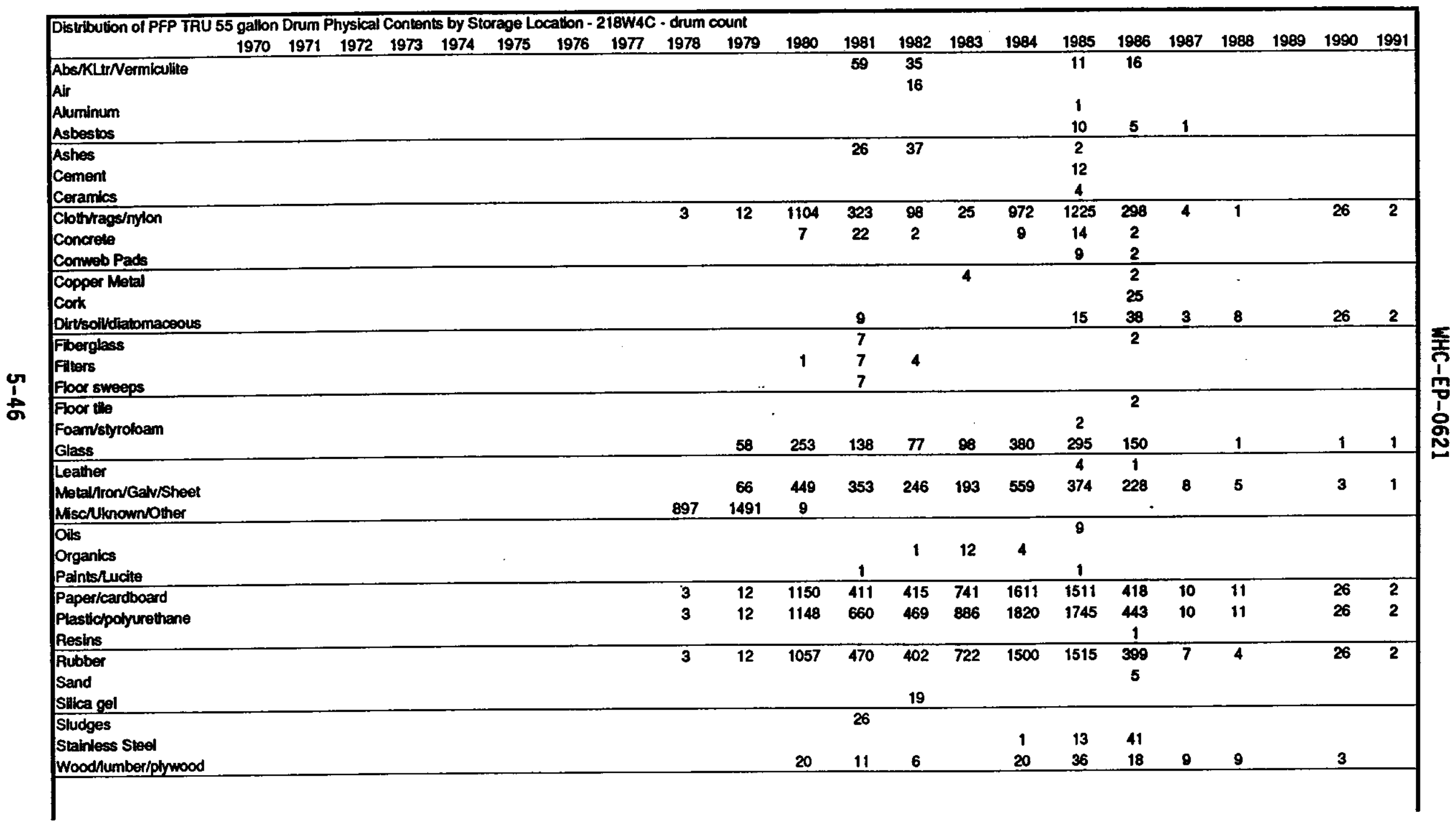


Table 5-10. Distribution and Percentage of TRU 55-Gallon Drum Physical Contents by Storage Location $-218 W 4 C$. (2 sheets)

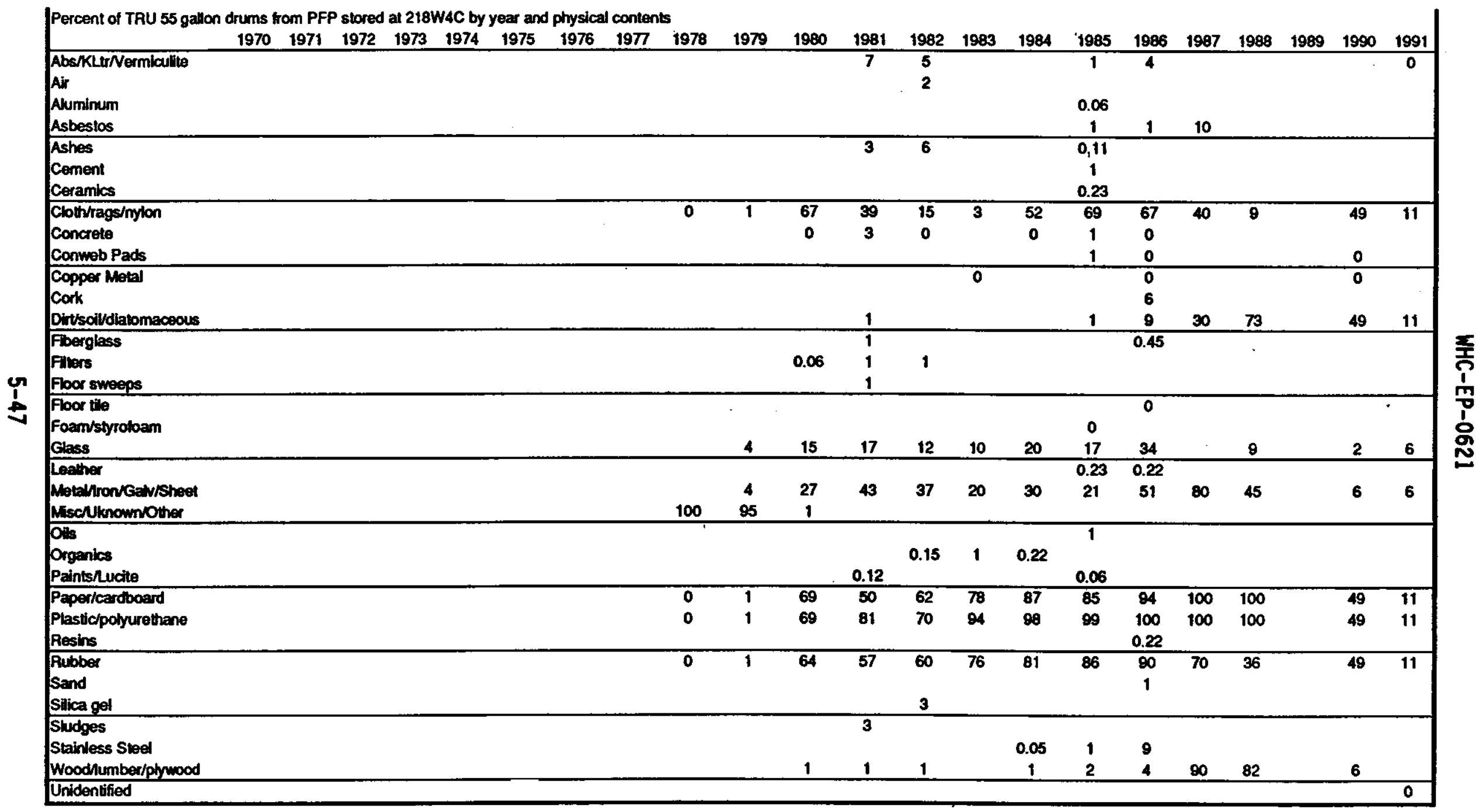


Table 5-11. Distribution and Percentage of TRU 55-Gallon Drum Physical Contents by Storage Location $-218 \mathrm{~W} 5$.

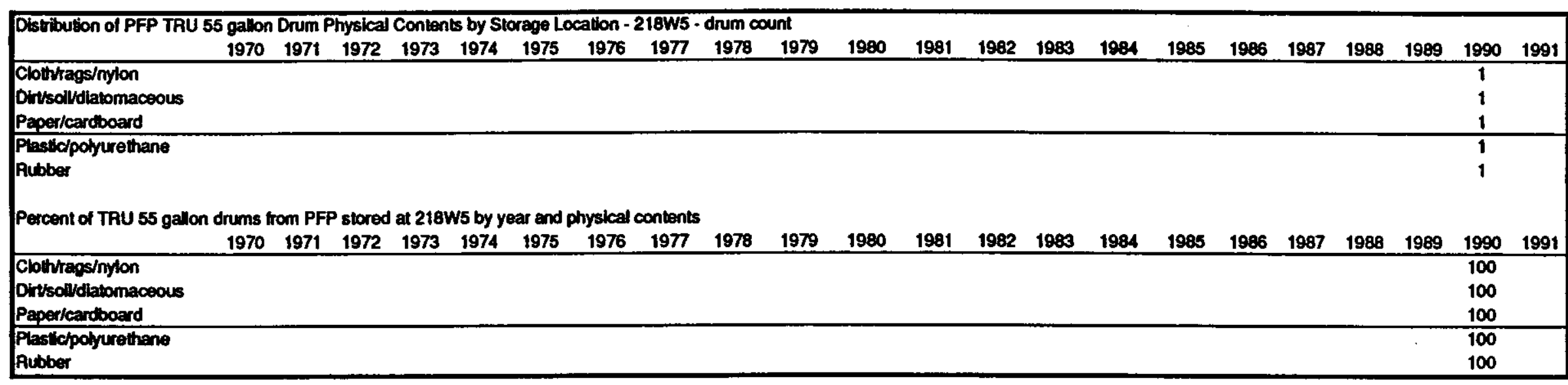

$\stackrel{1}{\infty}$ 
Table 5-12. Distribution and Percentage of TRU 55-Gallon Drum Physical Contents by Storage Location - 224T. (2 sheets)

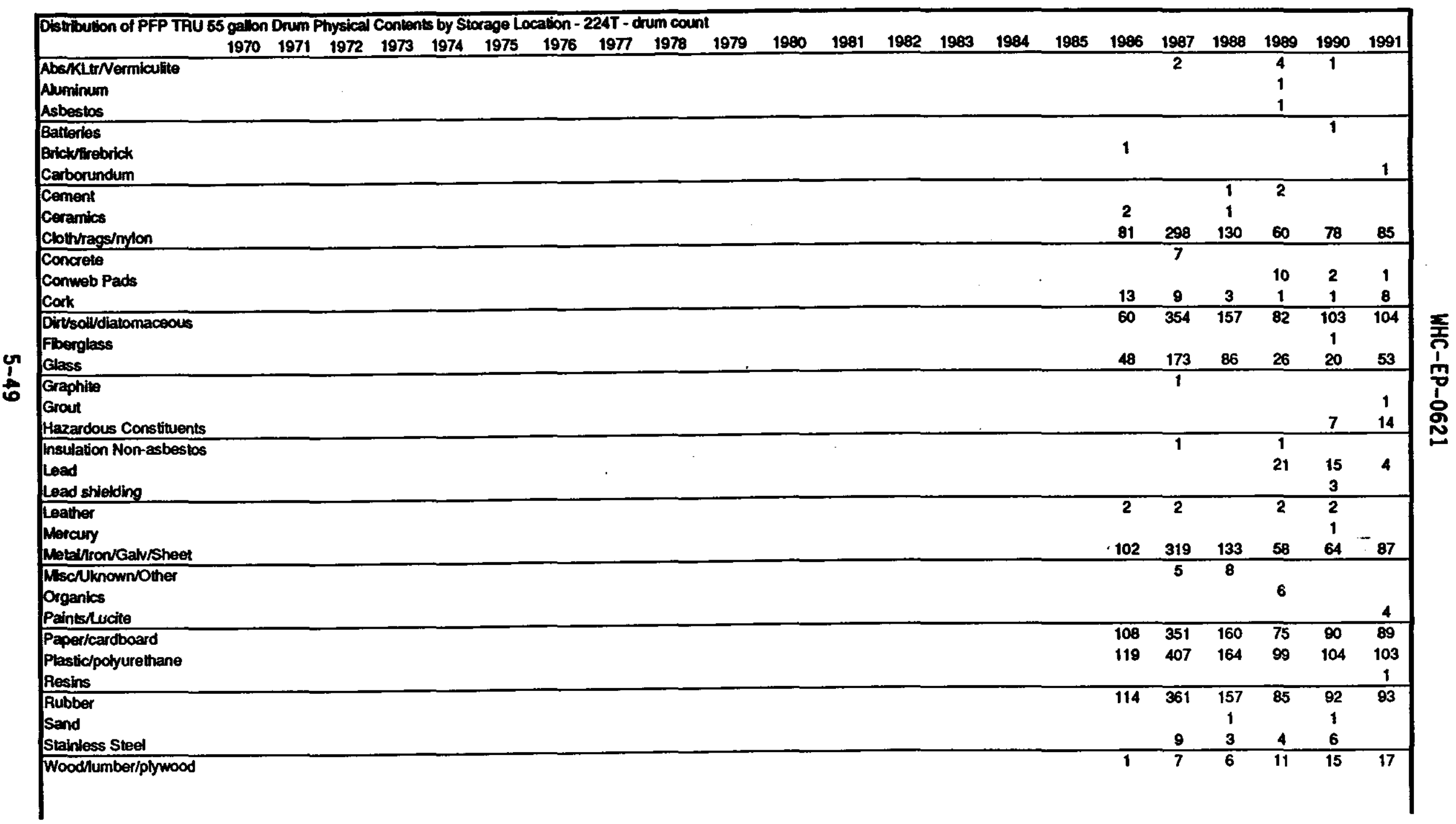


Table 5-12. Distribution and Percentage of TRU 55-Gallon Drum Physical Contents by Storage Location - 224T. (2 sheets)

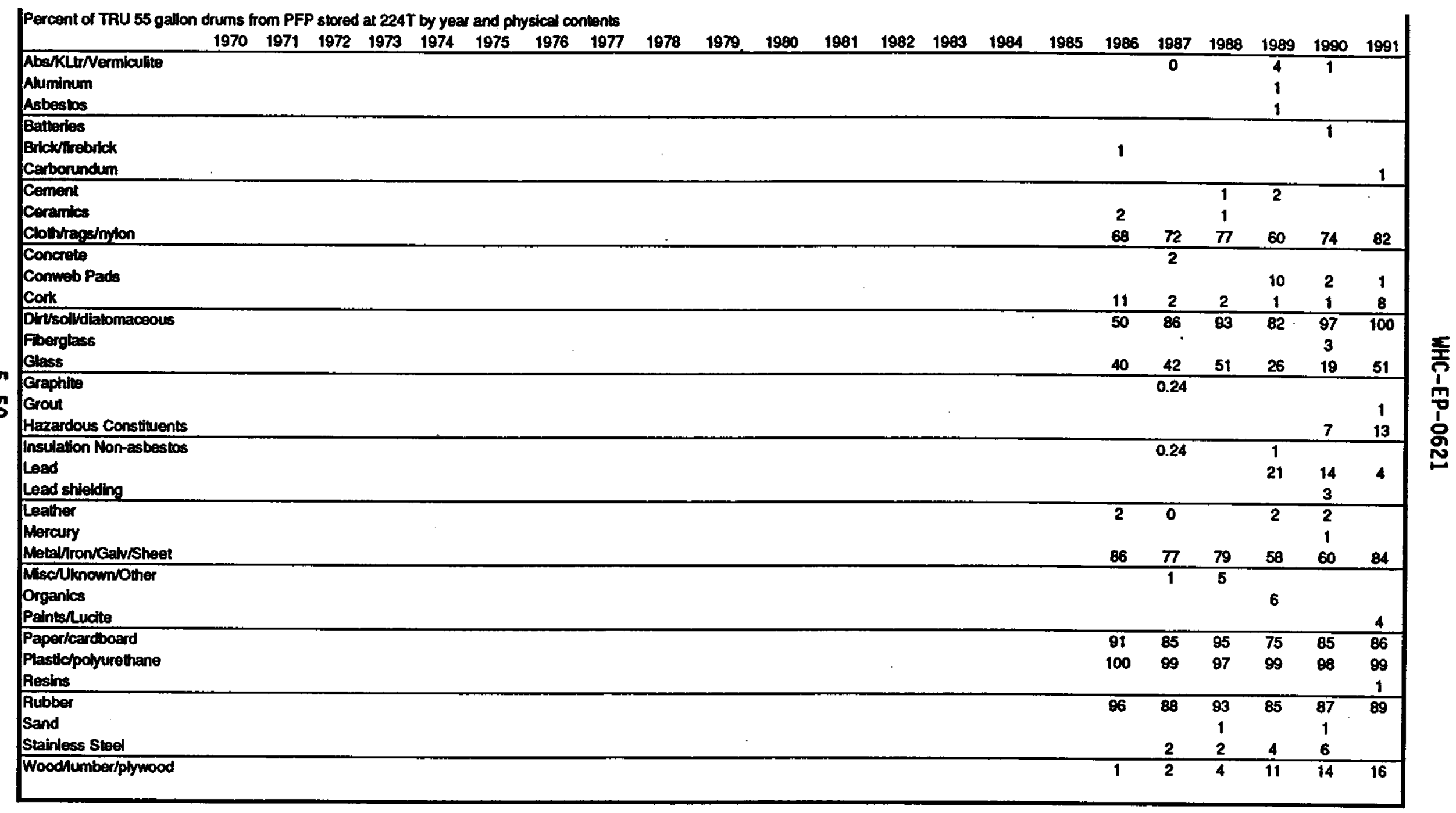


Table 5-13. Distribution and Percentage of TRU 55-Gallon Drum Physical Contents by Storage Location - 2401W.

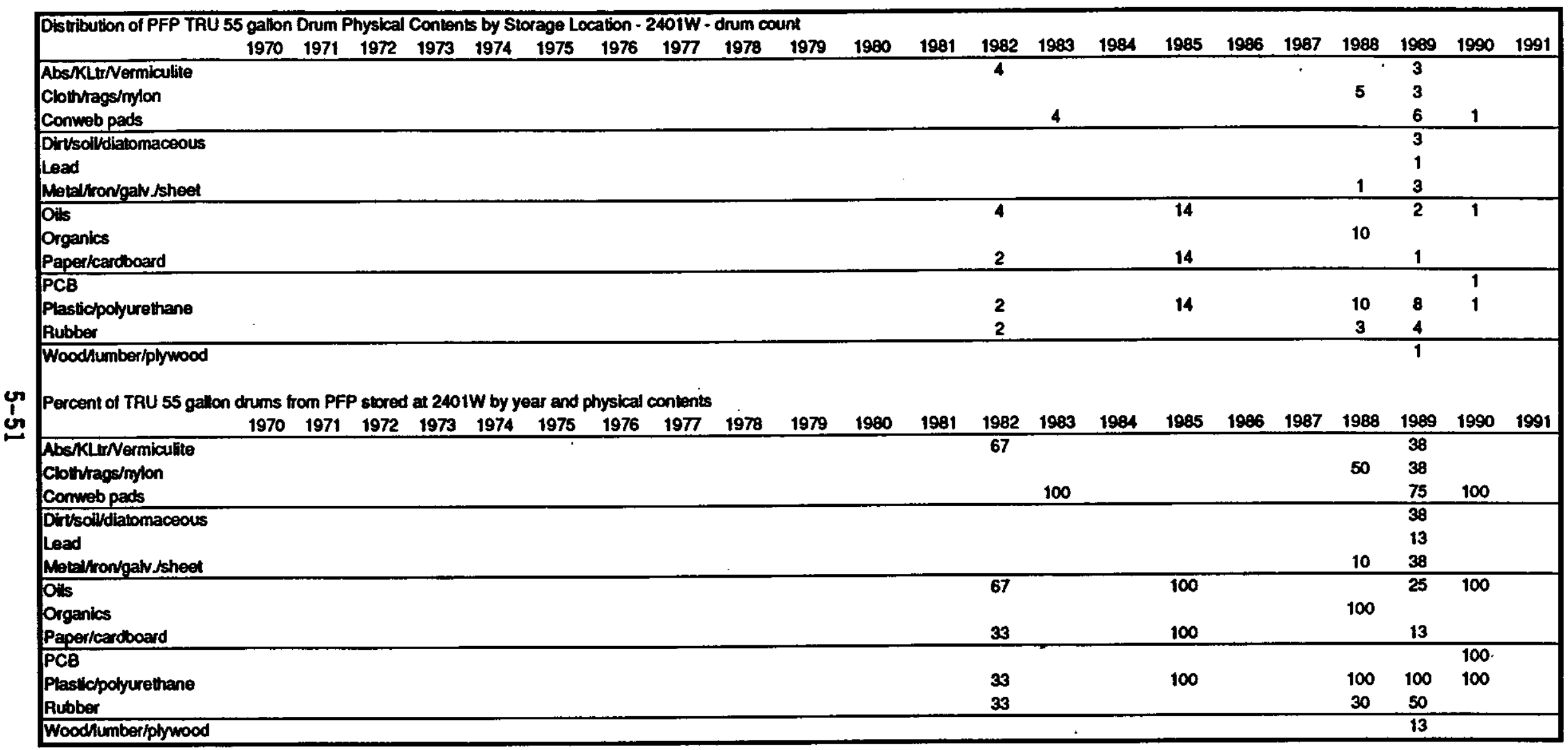


Table 5-14. Distribution and Percentage of TRU 55-Gallon Drum Physical Contents by Storage Location - 2402WB.

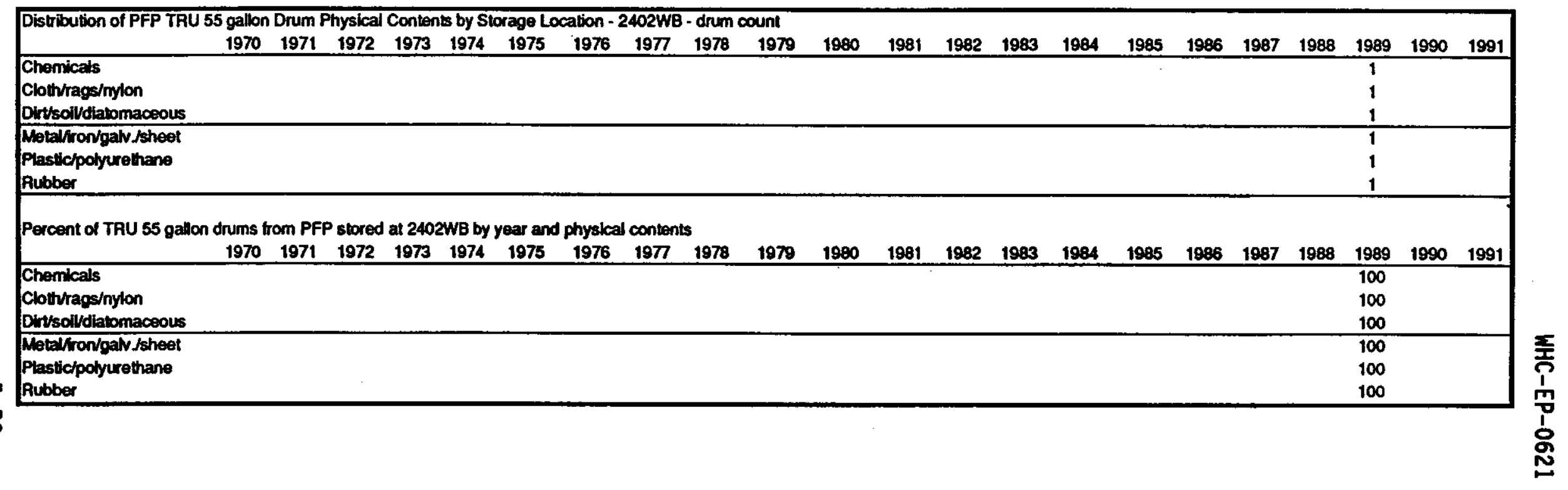


Table 5-15. Distribution and Percentage of TRU Container Physical Contents by Storage Location - 218 W3A.

\begin{tabular}{|c|c|c|c|c|c|c|c|c|c|c|c|c|c|c|c|c|c|c|c|c|c|c|}
\hline \multicolumn{23}{|c|}{ Distribution of PFP TRU Other Container Physical Contents by Storage Location - 218W3A - container count } \\
\hline & 1970 & 1971 & 1972 & 1973 & 1974 & 1975 & 1976 & 1977 & 1978 & 1979 & 1980 & 1981 & 1982 & 1983 & 1984 . & 1985 & 1986 & 1987 & 1988 & 1989 & 1990 & 1991 \\
\hline Misc.Nnknownother & 8 & 1 & & & 1 & 46 & 14 & 4 & 24 & & & & & & & & & & & & & \\
\hline \multicolumn{23}{|c|}{ Percent of TRU Other Containers from PFP stored at 218W3A by year and physical contents } \\
\hline & 1970 & 1971 & 1972 & 1973 & 1974 & 1975 & 1976 & 1977 & 1978 & 1979 & 1980 & 1981 & 1982 & 1983 & 1984 & 1985 & 1986 & 1987 & 1988 & 1989 & 1990 & 1991 \\
\hline Misc/Unknown/Other & 100 & 100 & & & 13 & 100 & 100 & 100 & 100 & & & & & & & & & & & & & \\
\hline
\end{tabular}


Table 5-16. Distribution and Percentage of TRU Container Physical Contents by Storage Location - 218 W4B.

\begin{tabular}{|c|c|c|c|c|c|c|c|c|c|c|c|c|c|c|c|c|c|c|c|c|c|c|}
\hline \multicolumn{23}{|c|}{ Distribution of PFP TRU Other Container Physical Contents by Storage Location - 218W4B - container counf } \\
\hline & 1970 & 1971 & 1972 & 1973 & 1974 & 1975 & 1976 & 1977 & 1978 & 1979 & 1980 & 1981 & 1982 & 1983 & 1984 & 1985 & 1986 & 1987 & 1988 & 1989 & 1990 & 1991 \\
\hline Misc/Unknown/Other & 1 & 17 & 16 & 6 & 18 & 5 & 17 & & 3 & & & & & & & & & & & & & \\
\hline \multicolumn{23}{|c|}{ Percent of TRU Other Containers from PFP stored at 218W3A by year and physical contentș. } \\
\hline & 1970 & 1971 & 1972 & 1973 & 1974 & 1975 & 1976 & 1977 & 1978 & 1979 & 1980 & 1981 & 1982 & 1983 & 1984 & 1985 & 1986 & 1987 & 1988 & 1989 & 1990 & 1991 \\
\hline Misc,Nnknownother & & 100 & 100 & & & 100 & & & 100 & & & & & & & & & & & & & 1 \\
\hline
\end{tabular}


Table 5-17. Distribution and Percentage of TRU Container Physical Contents by Storage Location - 218 W4C.

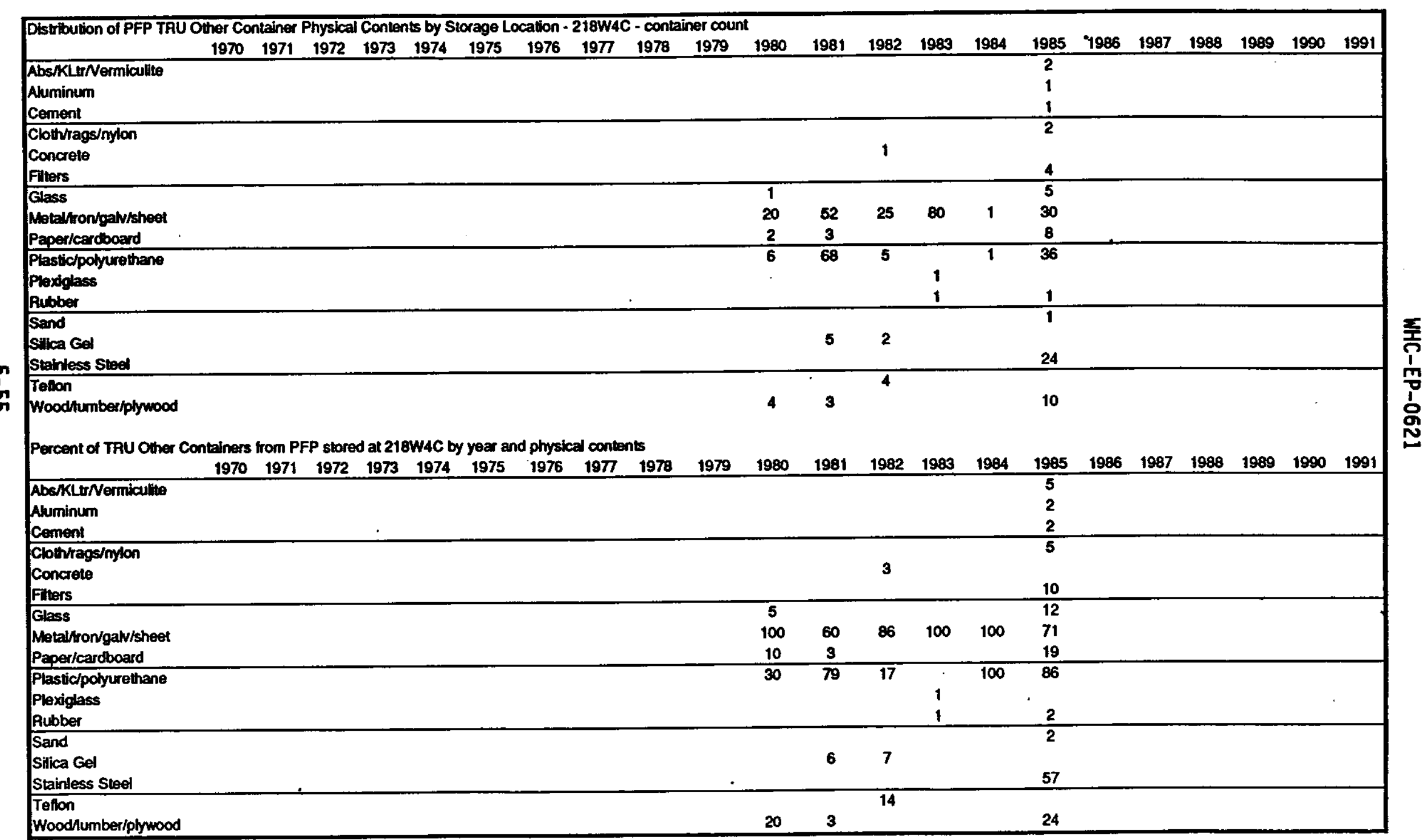


Table 5-18. Distribution and Percentage of TRU Container Physical Contents by Storage Location - $2401 \mathrm{~W}$.

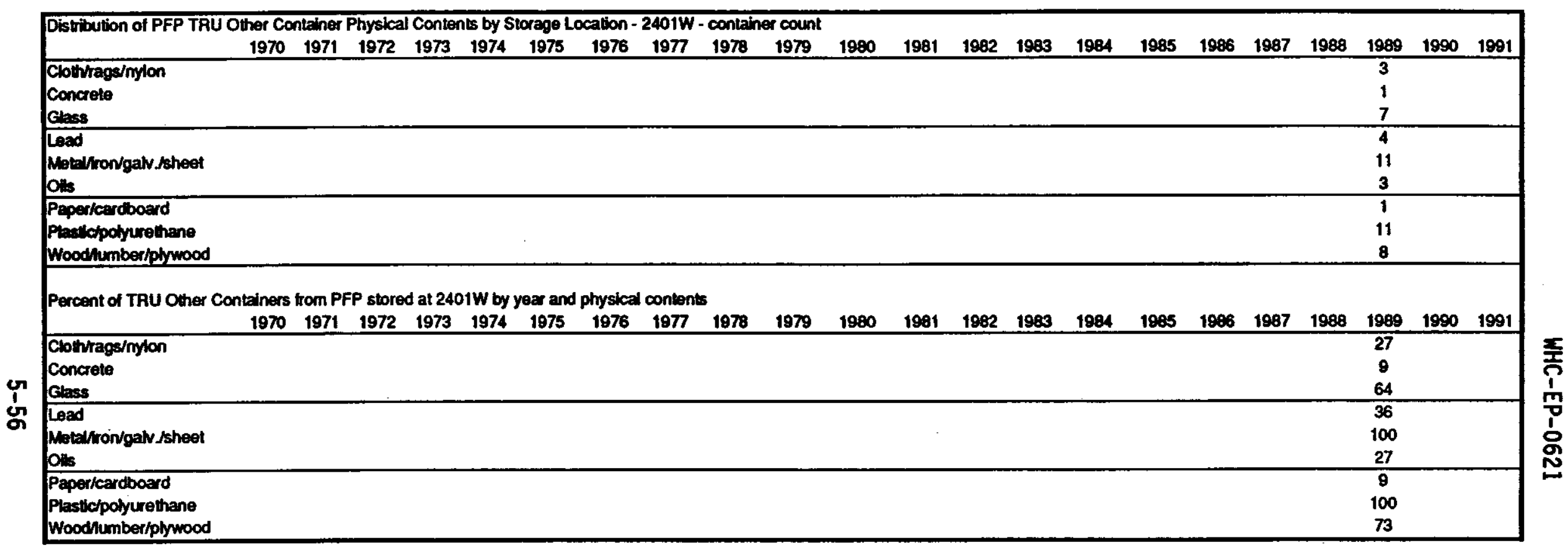


Table 5-19. Distribution and Percentage of TRU Container Physical Contents by Storage Location - 2402W.

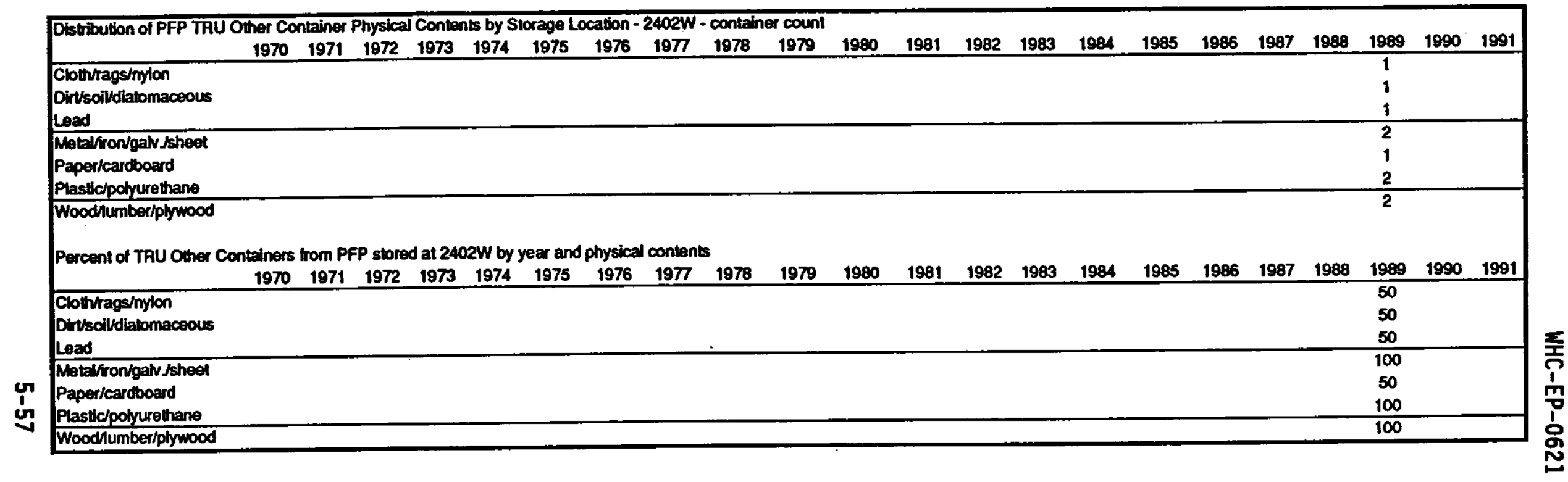


Table 5-20. Distribution and Percentage of TRU Container Physical Contents by Storage Location - 2402 WB.

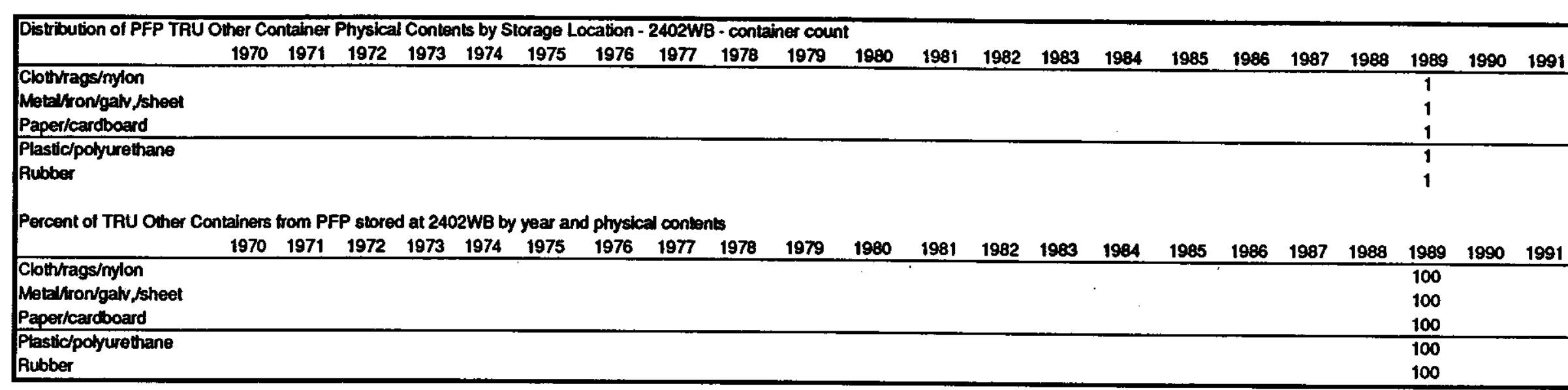

离 
Table 5-21. Distribution and Percentage of TRU Container Physical Contents by Storage Location - FS8.

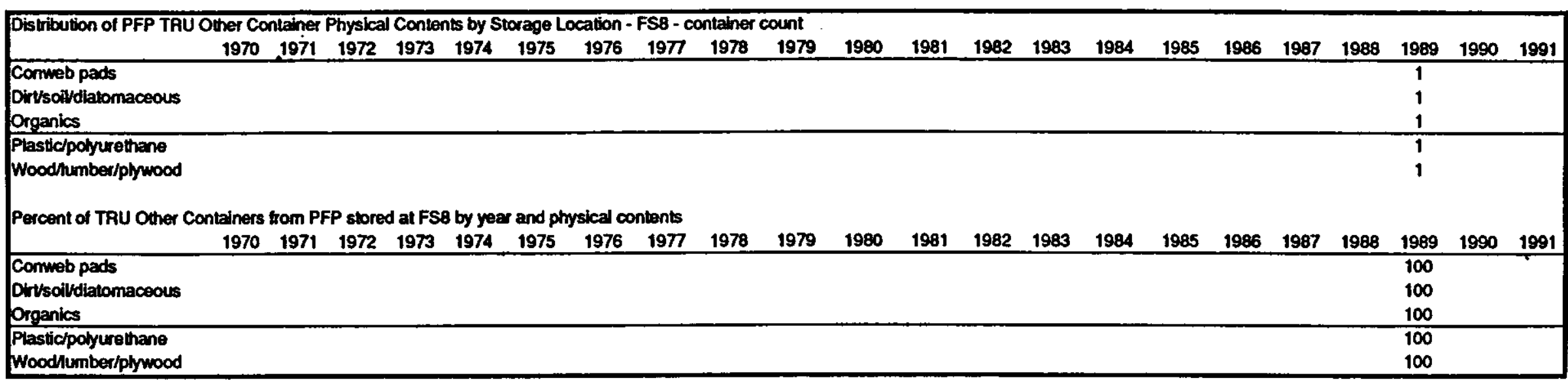

乡. 
Table 5-22. TRU Waste in 55-Gallon Drums: Total Grams of TRU by Storage Facility.

\begin{tabular}{|c|c|c|c|c|c|c|c|c|c|c|c|c|c|c|c|c|c|c|c|c|c|c|}
\hline $234-52$ rRU Waste in & $\begin{array}{l}5 \mathrm{Gall} \\
1970\end{array}$ & $\begin{array}{l}\text { on Drt } \\
1971\end{array}$ & Ims: & $\begin{array}{r}\text { Total } \\
1973\end{array}$ & $\begin{array}{r}\text { Grams } \\
1974\end{array}$ & $\begin{array}{l}\text { TRU St } \\
1975\end{array}$ & $\begin{array}{r}\text { tored } \\
1976 \\
\end{array}$ & $\begin{array}{l}\text { by } 100 \\
1977\end{array}$ & $\begin{array}{l}\text { tion } \\
1978\end{array}$ & 1979 & 1980 & 1981 & 1982 & 1983 & 1984 & 1985 & 1986 & 1987 & 1988 & 1989 & 1990 & 1991 \\
\hline $218 \sqrt{3 A}$ & 2189 & 1067 & & & & & & & & & & & & & 26 & & & & & & & \\
\hline $\begin{array}{l}218448 \\
218 \text {. }\end{array}$ & & 1581 & 3209 & 3070 & 1396 & 14941 & 7333 & 3620 & 1897 & 23472 & 61909 & 21582 & 13901 & 5983 & 11960 & 23928 & 8770 & 395 & 215 & & & 16 \\
\hline $\begin{array}{l}21845 \\
224-\tau \\
2401-4\end{array}$ & & & & & & & & & & & & & & & & 2 & 5487 & 14290 & $\begin{array}{c}3480 \\
3 \\
\end{array}$ & $\begin{array}{c}1381 \\
11 \\
\end{array}$ & $\begin{array}{c}1561 \\
0\end{array}$ & 1090 \\
\hline $\begin{array}{l}2402 \mathrm{~W} \\
2402 \mathrm{~B} \\
\mathrm{FS8}\end{array}$ & & & & & & & & & & & & & & & & & & & & 1 & & \\
\hline Total TRU in Drums & 2189 & 2649 & 3209 & 3070 & 1396 & 14941 & 7333 & 3620 & 1897 & 23472 & 61909 & 21582 & 13901 & 5983 & 11986 & 23930 & 14256 & 14685 & 3698 & 1393 & 1561 & 1106 \\
\hline
\end{tabular}

on 
Table 5-23. TRU Waste in Containers Other Than 55-Gallon Drums: Total Grams of TRU by Storage Facility.

\begin{tabular}{|c|c|c|c|c|c|c|c|c|c|c|c|c|c|c|c|c|c|c|c|c|c|c|}
\hline 234-52 TRU Waste & $\begin{array}{c}\text { ontain } \\
1970\end{array}$ & $\begin{array}{l}\text { ers } 0 \\
1971 \\
\end{array}$ & $\begin{array}{c}\text { her } t \\
1972\end{array}$ & $\begin{array}{l}\operatorname{han} 55 \\
1973\end{array}$ & $\begin{array}{l}\text { Gal. } \\
1974 \\
\end{array}$ & $\begin{array}{l}\text { Druns : } \\
1975\end{array}$ & $\begin{array}{c}\text { Tot } \\
1976\end{array}$ & $\begin{array}{l}\text { af Gr: } \\
1977\end{array}$ & $\begin{array}{c}\text { Is TR } \\
1978\end{array}$ & $\begin{array}{l}\text { Store } \\
1979\end{array}$ & $\begin{array}{c}d \text { by L } \\
1980\end{array}$ & $\begin{array}{l}\text { xation } \\
1981\end{array}$ & 1982 & 1983 & 1984 & 1985 & 1986 & 1987 & 1988 & 1989 & 1990 & 1991 \\
\hline $21843 A$ & 16 & 0 & & & 240 & 4619 & 1171 & 274 & 829 & & & & & & & & & & & & & \\
\hline $218 \times 46$ & 20 & 543 & 586 & 274 & 84 & 551 & 3 & & 21 & & & & & & & & & & & & & \\
\hline $218 \mathrm{MC}$ & & & & & & & & & & & 3551 & 1361 & 11332 & 36 & 5 & 8353 & & & & & & \\
\hline \multicolumn{23}{|l|}{$\begin{array}{l}21845 \\
224-T\end{array}$} \\
\hline $2401-4$ & & & & & & & & & & & & & & & & & & & & 964 & & \\
\hline $2402 W$ & & & & & & & & & & & & & & & & & & & & 153 & & \\
\hline 240218 & & & & & & & & & & & & & & & & & & & & 11 & & \\
\hline FS8 & & & & & & & & & & & & & & & & & & & & 0 & & \\
\hline Total Other TRU & 36 & 543 & 586 & 274 & 324 & 5170 & 1174 & 274 & 850 & 0 & 3551 & 1361 & 11332 & 36 & 5 & 8353 & $\mathbf{0}$ & $\overline{0}$ & 0 & 1129 & 0 & 0 \\
\hline
\end{tabular}


Table 5-24. TRU Waste from 234-5Z Containing High Quantities of ${ }^{238} \mathrm{Pu}$.

\begin{tabular}{|c|c|c|c|c|c|c|c|}
\hline $\begin{array}{c}\text { Disposal } \\
\text { date }\end{array}$ & $\begin{array}{c}\text { SuSDR } \\
\text { number }\end{array}$ & $\begin{array}{c}\text { Volume } \\
\left(\mathrm{ft}^{3}\right)^{*}\end{array}$ & $\begin{array}{c}\text { Weight } \\
(\mathrm{kg})\end{array}$ & $\begin{array}{c}\text { Total dose } \\
\text { rate } \\
\text { (mrem) }\end{array}$ & $\begin{array}{c}\text { Pu } \\
(\mathrm{g})\end{array}$ & $\begin{array}{c}\text { Mixed } \\
\text { fission } \\
\text { products } \\
\text { (g) }\end{array}$ & $\begin{array}{c}238 \mathrm{Pu} \\
\text { (g) }\end{array}$ \\
\hline $10 / 28 / 80$ & 801541 & $7.400 \mathrm{E}+00$ & 68.04 & $1.000 \mathrm{E}+00$ & $1.000 \mathrm{E}-04$ & $1.000 \mathrm{E}-03$ & $5.125 \mathrm{E}+02$ \\
\hline $10 / 28 / 80$ & 801542 & $7.400 \mathrm{E}+00$ & 68.04 & $1.000 \mathrm{E}+00$ & $1.000 \mathrm{E}-04$ & $1.000 \mathrm{E}-03$ & $5.150 \mathrm{E}+02$ \\
\hline $10 / 28 / 80$ & 801543 & $7.400 \mathrm{E}+00$ & 68.04 & $1.000 \mathrm{E}+00$ & $1.000 \mathrm{E}-04$ & $1.000 \mathrm{E}-03$ & $2.221 \mathrm{E}+02$ \\
\hline $10 / 28 / 80$ & 801544 & $7.400 \mathrm{E}+00$ & 68.04 & $1.000 \mathrm{E}+00$ & $1.000 \mathrm{E}-04$ & $1.000 \mathrm{E}-03$ & $3.134 \mathrm{E}+02$ \\
\hline $10 / 28 / 80$ & 801545 & $7.400 \mathrm{E}+00$ & 68.04 & $1.000 \mathrm{E}+00$ & $1.000 \mathrm{E}-04$ & $1.000 \mathrm{E}-03$ & $5.013 \mathrm{E}+02$ \\
\hline $10 / 28 / 80$ & 801546 & $7.400 \mathrm{E}+00$ & 68.04 & $1.000 \mathrm{E}+00$ & $1.000 \mathrm{E}-04$ & $1.000 \mathrm{E}-03$ & $3.972 \mathrm{E}+02$ \\
\hline $10 / 28 / 80$ & 801547 & $7.400 \mathrm{E}+00$ & 68.04 & $1.000 \mathrm{E}+00$ & $1.000 \mathrm{E}-04$ & $1.000 \mathrm{E}-03$ & $5.126 \mathrm{E}+02$ \\
\hline $10 / 28 / 80$ & 801548 & $7.400 \mathrm{E}+00$ & 68.04 & $1.000 \mathrm{E}+00$ & $1.000 \mathrm{E}-04$ & $1.000 \mathrm{E}-03$ & $5.143 \mathrm{E}+02$ \\
\hline $10 / 28 / 80$ & 801549 & $7.400 \mathrm{E}+00$ & 68.04 & $1.000 \mathrm{E}+00$ & $1.000 \mathrm{E}-04$ & $1.000 \mathrm{E}-03$ & $5.117 \mathrm{E}+02$ \\
\hline $10 / 28 / 80$ & 801550 & $7.400 \mathrm{E}+00$ & 68.04 & $1.000 \mathrm{E}+00$ & $1.000 \mathrm{E}-04$ & $1.000 \mathrm{E}-03$ & $4.948 \mathrm{E}+02$ \\
\hline $10 / 28 / 80$ & 801551 & $7.400 \mathrm{E}+00$ & 68.04 & $1.000 \mathrm{E}+00$ & $1.000 \mathrm{E}-04$ & $1.000 \mathrm{E}-03$ & $4.839 \mathrm{E}+02$ \\
\hline $10 / 28 / 80$ & 801552 & $7.400 \mathrm{E}+00$ & 68.04 & $1.000 \mathrm{E}+00$ & $1.000 \mathrm{E}-04$ & $1.000 \mathrm{E}-03$ & $2.517 \mathrm{E}+02$ \\
\hline Total & & $8.880 \mathrm{E}+01$ & 816.48 & & $1.200 \mathrm{E}-03$ & $1.200 \mathrm{E}-02$ & $5.230 \mathrm{E}+03$ \\
\hline
\end{tabular}

NOTE: Stored in 55-gal drums in burial ground 4C, trench T01, no known hazardous constituents present. All 12 drums contain metal/iron/galvanized/steel. The information in this table should be considered suspect because of the uniformity of the 12 drums reactivities, weights, plutonium contents, and mixed fission product contents. Also, no fission products are expected to be in PFP waste.

*To convert from cubic feet to cubic meters multiply by $2.831,685$ E-02. SWSDR = Solid Waste Storage and Disposal Record. 
Table 5-25. PFP Waste in Other Containers Containing More Than 300 Grams of TRU. (2 sheets)

\begin{tabular}{|c|c|c|c|c|c|c|c|}
\hline Container ID & $\begin{array}{l}\text { Disposal } \\
\text { date }\end{array}$ & $\begin{array}{l}\text { Volyme } \\
\left(n^{3}\right)\end{array}$ & $\begin{array}{l}\text { Weight } \\
\text { (kg) }\end{array}$ & $\begin{array}{c}\text { Total dose } \\
\text { rate } \\
\text { (mrena) }\end{array}$ & $\begin{array}{l}\text { Plutonium } \\
\text { (g) }\end{array}$ & $\begin{array}{l}\text { Uranium } \\
\text { (natural) }\end{array}$ & $\begin{array}{l}\text { Uranium } \\
\text { (depleted) }\end{array}$ \\
\hline R409 & $10 / 16 / 80$ & 0.419 & 159 & $1.00 E+99$ & $3.2 E+02$ & $9.29 E+03$ & \\
\hline R488 & $10 / 16 / 80$ & 0.419 & 159 & $1.00 E+00$ & $3.1 \mathrm{E}+02$ & $9.29 \mathrm{E}+03$ & \\
\hline CCS74-137 & $3 / 04 / 82$ & 0.419 & 197 & $2.00 E+00$ & $8.49 E+02$ & $4.30 E+04$ & \\
\hline $\operatorname{ccS75-138}$ & $3 / 04 / 82$ & 0.419 & 197 & $2.00 E+00$ & $6.87 \mathrm{E}+02$ & $4.30 E+04$ & \\
\hline $\operatorname{ccs} 74-141$ & $3 / 04 / 82$ & 0.419 & 197 & $2.00 E+00$ & $6.87 E+02$ & $4.30 E+04$ & \\
\hline CCS74-142 & $3 / 04 / 82$ & 0.419 & 197 & $2.00 E+00$ & $7.68 E+02$ & $4.30 E+04$ & \\
\hline CCS74-143 & $3 / 04 / 82$ & 0.419 & 197 & $2.00 E+00$ & $3.94 \mathrm{E}+02$ & $4.30 E+04$ & \\
\hline CCS74-149 & $3 / 04 / 82$ & 0.419 & 136 & $2.00 E+00$ & $9.10 \mathrm{E}+02$ & $4.30 E+04$ & \\
\hline $7774-412$ & $3 / 25 / 82$ & 0.419 & 136 & $1.00 E+00$ & $4.76 E+02$ & $1.43 \mathrm{E}+04$ & $4.43 E+03$ \\
\hline $7774-413$ & $3 / 25 / 82$ & 0.419 & 136 & $1.00 E+00$ & $3.97 \mathrm{E}+02$ & $1.66 E+04$ & $3.00 E+03$ \\
\hline $7774-414$ & $3 / 25 / 82$ & 0.419 & 136 & $1.00 E+00$ & $4.56 E+02$ & $1.43 E+04$ & $3.00 E+03$ \\
\hline $7774-415$ & $3 / 25 / 82$ & 0.419 & 136 & $1.00 E+00$ & $4.36 E+02$ & $1.43 \mathrm{E}+04$ & $4.43 E+03$ \\
\hline $7774-416$ & $3 / 25 / 82$ & 0.419 & 136 & $1.00 \mathrm{E}+00$ & 3.17 E+02 & $1.43 E+04$ & $4.43 E+03$ \\
\hline $7774-417$ & $3 / 25 / 82$ & 0.419 & 136 & $1.00 E+00$ & $3.97 E+02$ & $1.66 \mathrm{E}+04$ & $4.43 E+03$ \\
\hline $7774-418$ & $3 / 25 / 82$ & 0.419 & 136 & $1.00 E+00$ & $3.57 E+02$ & $1.66 \mathrm{E}+04$ & $3.00 \mathrm{E}+03$ \\
\hline $7774-419$ & $3 / 25 / 82$ & 0.419 & 136 & $1.00 E+00$ & $3.97 E+02$ & $1.43 E+04$ & $3.00 E+03$ \\
\hline $7774-421$ & $3 / 25 / 82$ & 0.419 & 136 & $1.00 \mathrm{E}+00$ & $4.16 E+02$ & $1.66 \mathrm{E}+04$ & $4.43 E+03$ \\
\hline $7774-422$ & $3 / 25 / 82$ & 0.419 & 136 & $1.00 E+00$ & $4.16 E+02$ & $1.66 \mathrm{E}+04$ & $3.00 \mathrm{E}+03$ \\
\hline $7774-423$ & $3 / 25 / 82$ & 0.419 & 136 & $1.00 E+00$ & $3.96 E+02$ & $1.43 E+04$ & $3.00 E+03$ \\
\hline
\end{tabular}


Table 5-25. PFP Waste in Other Containers Containing More Than 300 Grams of TRU. (2 sheets)

\begin{tabular}{|l|c|c|c|c|c|c|c|}
\hline Container ID & $\begin{array}{c}\text { Disposal } \\
\text { date }\end{array}$ & $\begin{array}{c}\text { Volume } \\
\left(\mathbf{w}^{3}\right)\end{array}$ & $\begin{array}{c}\text { Weight } \\
(\mathrm{kg})\end{array}$ & $\begin{array}{c}\text { Total dose } \\
\text { rate } \\
(\text { mrem })\end{array}$ & $\begin{array}{c}\text { Plutonium } \\
(\mathrm{g})\end{array}$ & $\begin{array}{c}\text { Uranium } \\
\text { (natural) }\end{array}$ & $\begin{array}{c}\text { Uranium } \\
(\mathrm{deplet}) \mathrm{f})\end{array}$ \\
\hline $7774-424$ & $3 / 25 / 82$ & 0.419 & 136 & $1.00 \mathrm{E}+00$ & $4.29 \mathrm{E}+02$ & $1.43 \mathrm{E}+04$ & $4.43 \mathrm{E}+03$ \\
\hline $7774-425$ & $3 / 25 / 82$ & 0.419 & 136 & $1.00 \mathrm{E}+00$ & $6.70 \mathrm{E}+02$ & $1.66 \mathrm{E}+04$ & $3.00 \mathrm{E}+03$ \\
\hline $7774-426$ & $3 / 25 / 82$ & 0.419 & 136 & $1.00 \mathrm{E}+00$ & $4.29 \mathrm{E}+02$ & $1.43 \mathrm{E}+04$ & $4.43 \mathrm{E}+03$ \\
\hline
\end{tabular}

MOTE: Containers are stored in Burial Ground 218-W04C, Trench T01. All contain metal/iron/galvanized/sheet, and no listed hazardous constituents. 
WHC-EP-0621

Table 5-26. PFP (Z Plant) Plutonium Conversion to Oxide (200 West Area) (1973-76).

\begin{tabular}{|l|c|l|}
\hline \multicolumn{1}{|c|}{ Compound name } & Formula & \multicolumn{1}{|c|}{ Toxicity category } \\
\hline Hydrogen peroxide & $\mathrm{H}_{2} \mathrm{O}_{2}$ & $\begin{array}{l}\text { DOT oxidizer above } 8 \% \\
\text { TOX D- 8-20\% C-90\% }\end{array}$ \\
\hline Nitric acid & $\mathrm{HNO}_{3}$ & Oxidizer/corrosive \\
\hline Oxalic acid & $\mathrm{HO}_{2} \mathrm{CCO}_{2} \cdot 2 \mathrm{H}_{2} \mathrm{O}$ & Not regulated \\
\hline Potassium permanganate & $\mathrm{KMnO}_{4}$ & D/oxidizer \\
\hline
\end{tabular}

DOT - U.S. Department of Transportation.

TOX = Toxicity category. 
Table 5-27. PFP (Z Plant): Plutonium Reclamation and Waste Treatment (200 West Area) (1973-76). (2 sheets)

\begin{tabular}{|c|c|c|c|}
\hline Compound name & Formula & $\begin{array}{c}\text { EPA } \\
\text { category }\end{array}$ & $\begin{array}{l}\text { Comments and } \\
\text { DOT classification }\end{array}$ \\
\hline Aluminum & A1 & & $\begin{array}{l}\text { Feed constituent no } \\
\text { TOX data/flammable } \\
\text { solid }\end{array}$ \\
\hline $\begin{array}{l}\text { Aluminum nitrate } \\
\text { nonahydrate }\end{array}$ & $\mathrm{Al}\left(\mathrm{NO}_{3}\right)_{3} \cdot 9 \mathrm{H}_{2} \mathrm{O}$ & D & No DOT data \\
\hline $\begin{array}{l}\text { Aluminum nitrate } \\
\text { (monobasic) }\end{array}$ & $\mathrm{A} 1(\mathrm{OH})\left(\mathrm{NO}_{3}\right)_{2}$ & D & Oxidizer \\
\hline Beryllium & $\mathrm{Be}$ & & Feed constituent \\
\hline Calcium & $\mathrm{Ca}$ & & $\begin{array}{l}\text { Feed constituent } \\
\text { flammable sol id also } \\
\text { requires "Dangerous } \\
\text { When Wet" label }\end{array}$ \\
\hline $\begin{array}{l}\text { Carbon } \\
\text { tetrachloride }\end{array}$ & $\mathrm{CCl}_{4}$ & D & ORM-A \\
\hline $\begin{array}{l}\text { Dibutyl butyl } \\
\text { phosphonate }\end{array}$ & $\mathrm{CH}_{3}\left(\mathrm{CH}_{2}\right)_{3} \mathrm{PO}_{3}\left[\mathrm{CH}_{3}\left(\mathrm{CH}_{2}\right)_{33}\right]_{2}$ & & $\begin{array}{l}\text { Insufficient for data/ } \\
\text { no DOT information }\end{array}$ \\
\hline Dodecane & $\mathrm{CH}_{3}\left(\mathrm{CH}_{2}\right)_{10} \mathrm{CH}_{3}$ & & $\begin{array}{l}\text { Feed constituent } \\
\text { insufficient TOX: data } \\
\text { no DOT information }\end{array}$ \\
\hline Anti-Foam B & Silicon emulsion & & Product name \\
\hline Gallium & Ga & & $\begin{array}{l}\text { Feed constituent no } \\
\text { TOX data ORM-B }\end{array}$ \\
\hline Gallium oxide & $\mathrm{Ga}_{2} \mathrm{O}_{3}$ & & $\begin{array}{l}\text { Feed constituent } \\
\text { insufficient TOX data } \\
\text { no DOT information }\end{array}$ \\
\hline Hydrazine & $\mathrm{H}_{2} \mathrm{NNH}_{2} \cdot \mathrm{H}_{2} \mathrm{O}$ & B & $\begin{array}{l}\text { Flammable liquid and } \\
\text { poison/corrosive }\end{array}$ \\
\hline Hydrochloric acid & $\mathrm{HCl}$ & D & $\begin{array}{l}\text { Nonfl ammable } \\
\text { gas/corrosive }\end{array}$ \\
\hline Hydrofluoric acid & HF & & $\begin{array}{l}\mathrm{LC}_{50} 1,276 \mathrm{ppm} / \text { inhala- } \\
\text { tion hazard / corrosive }\end{array}$ \\
\hline $\begin{array}{l}\text { Hydroxyl amine } \\
\text { hydrochloride }\end{array}$ & $\mathrm{NH}_{2} \mathrm{OH} \cdot \mathrm{HCI}$ & & $\begin{array}{l}\text { Insufficient TOX data } \\
\text { no DOT information }\end{array}$ \\
\hline $\begin{array}{l}\text { Hydroxylamine } \\
\text { nitrate }\end{array}$ & $\mathrm{NH}_{2} \mathrm{OH} \cdot \mathrm{HCO}_{3}$ & & No entry \\
\hline Iodine & $I_{2}$ & & $\begin{array}{l}\text { Feed constituent } \\
14 \mathrm{~g} / \mathrm{kg}-\text { LD50 no DOT } \\
\text { information }\end{array}$ \\
\hline Iron & $\mathrm{Fe}$ & & $\begin{array}{l}\text { Scrap container } \\
\text { LD50-30 g/kg no DOT } \\
\text { information }\end{array}$ \\
\hline
\end{tabular}


Table 5-27. PFP (Z Plant): Plutonium Reclamation and Waste Treatment (200 West Area) (1973-76). (2 sheets)

\begin{tabular}{|c|c|c|c|}
\hline Compound name & Formula & $\begin{array}{c}\text { EPA } \\
\text { category }\end{array}$ & $\begin{array}{l}\text { Comments and } \\
\text { DOT classification }\end{array}$ \\
\hline Magnesium oxide & MgO & & $\begin{array}{l}\text { Feed constituent } \\
\text { insufficient TOX data } \\
\text { no DOT information }\end{array}$ \\
\hline Mercuric nitrate & $\mathrm{Hg}\left(\mathrm{NO}_{3}\right)_{2} \cdot \mathrm{H}_{2} \mathrm{O}$ & $B$ & Oxidizer/poison \\
\hline Mistron ${ }^{2}$ & $\mathrm{MgSiO}_{3}$ & & $\begin{array}{l}\text { Product name talc - } \\
\text { asbestos no TOX } \\
\text { data/no DOT } \\
\text { information/ } \\
\text { insufficient TOX data }\end{array}$ \\
\hline Molybdenum & Mo & & $\begin{array}{l}\text { Feed constituent no } \\
\text { DOT information }\end{array}$ \\
\hline Nitric acid & $\mathrm{HNO}_{3}$ & C & Oxidizer/corrosive \\
\hline Oxalic acid & $\mathrm{HO}_{2} \mathrm{CCO}_{2} \mathrm{H} \cdot 2 \mathrm{H}_{2} \mathrm{O}$ & & \\
\hline $\begin{array}{l}\begin{array}{l}\text { Potassium } \\
\text { carbonate }\end{array} \\
\end{array}$ & $\mathrm{K}_{2} \mathrm{CO}_{3}$ & D & \\
\hline Sodium carbonate & $\mathrm{Na}_{2} \mathrm{CO}_{3}$ & & $\begin{array}{l}\text { Insufficient TOX } \\
\text { data/DOT-IM: oxidizer }\end{array}$ \\
\hline Sodium fluoride & $\mathrm{NaF}$ & & No TOX data/corrosive \\
\hline Sodium hydroxide & $\mathrm{NaOH}$ & & \begin{tabular}{|l|} 
Insufficient TOX \\
data/corrosive
\end{tabular} \\
\hline Sodium nitrate & $\mathrm{NaNO}_{3}$ & D & Oxidizer \\
\hline Sulfamic acid & $\mathrm{NH}_{2} \mathrm{SO}_{3} \mathrm{H}$ & D & $\begin{array}{l}\text { Feed const ituent } \\
\text { DOT-IM: corrosive } \\
\end{array}$ \\
\hline Tributyl phosphate & $\left(\mathrm{C}_{4} \mathrm{H}_{9}\right)_{3} \mathrm{PO}_{4}$ & & $\begin{array}{l}\text { LD50 } 3 \mathrm{~g} / \mathrm{kg} / \mathrm{no} \text { DOT } \\
\text { information }\end{array}$ \\
\hline
\end{tabular}

Anti-Foam B is a trademark of Dow Corning Corporation.

${ }^{2}$ Mistron is a trademark of Cyprus Mines Corporation.

DOT = U.S. Department of Transportation.

$E P A=$ U.S. Environmental Protection Agency.

IM = Irritating material.

ORM = Other regulated materials.

TOX = Toxicity catagory. 
Table 5-28. PFP (Z Plant): Analytical Laboratory (200 West Area) (-1973-79). (3 sheets)

\begin{tabular}{|c|c|c|c|}
\hline Compound name & Formula & $\begin{array}{c}\text { EPA } \\
\text { category }\end{array}$ & $\begin{array}{l}\text { Comments and } \\
\text { DOT classification }\end{array}$ \\
\hline Acetric acid & $\mathrm{CH}_{3} \mathrm{CO}_{2} \mathrm{H}$ & C & Corrosive \\
\hline Acetone & $\mathrm{CH}_{3} \mathrm{C}_{2} \mathrm{H}_{3} \mathrm{O}$ & & $\begin{array}{l}\text { LD50 } 20 \mathrm{~g} / \mathrm{kg} / \mathrm{fl} \text { ammable } \\
\text { liquid }\end{array}$ \\
\hline Alizarin yellow & $\mathrm{C}_{44} \mathrm{H}_{8} \mathrm{O}_{4}$ & & No TOX data/no DOT \\
\hline $\begin{array}{l}\text { Aluminum nitrate } \\
\text { nonahydrate }\end{array}$ & $\mathrm{Al}\left(\mathrm{NO}_{3}\right)_{3} \cdot 9 \mathrm{H}_{2} \mathrm{O}$ & & \\
\hline $\begin{array}{l}\text { Aluminum nitrate (mono } \\
\text { basic) }\end{array}$ & $\mathrm{Al}(\mathrm{OH})\left(\mathrm{NO}_{3}\right)_{2}$ & & \\
\hline Aluminum sulfate & $\mathrm{Al}\left(\mathrm{SO}_{4}\right)_{3}$ & & $\begin{array}{l}\text { Insufficient TOX } \\
\text { data/ORM-B }\end{array}$ \\
\hline Ammonium chloride & $\mathrm{NH}_{4} \mathrm{Cl}$ & D & No DOT \\
\hline Ammonium hydroxide & $\mathrm{NH}_{4} \mathrm{OH}$ & C & Corrosive \\
\hline Ammonium oxalate & $\left(\mathrm{NH}_{4}\right)_{2} \mathrm{C}_{2} \mathrm{O}_{4}$ & & No TOX data/ORM-A \\
\hline Ammonium sulfate. & $\left(\mathrm{NH}_{4}\right)_{2} \mathrm{SO}_{4}$ & D & No DOT \\
\hline Arsenazo III & Arsenic compounds & D & Product name \\
\hline Boric acid & $\mathrm{H}_{3} \mathrm{BO}_{3}$ & D. & No DOT \\
\hline Bromocresol purple & $\mathrm{C}_{7} \mathrm{H}_{6} \mathrm{OHBr}$ & & $\begin{array}{l}\text { No entry (blue and } \\
\text { green only) }\end{array}$ \\
\hline Carbon tetrachloride & $\mathrm{CCl}_{4}$ & & \\
\hline Ceric ammonium nitrate & $\mathrm{Ce}\left(\mathrm{NH}_{4}\right)_{2}\left(\mathrm{NO}_{3}\right)_{6}$ & & \\
\hline Dibutyl phosphate & $\left(\mathrm{n}-\mathrm{C}_{4} \mathrm{H}_{9}\right)_{2} \mathrm{HPO}_{4}$ & & $\begin{array}{l}\text { No TOX data no DOT } \\
\text { information }\end{array}$ \\
\hline $\begin{array}{l}\text { Ferric ammonium } \\
\text { sulfate }\end{array}$ & $\mathrm{FeNH}_{4} \mathrm{SO}_{4}$ & & No entry \\
\hline Ferric nitrate & $\mathrm{Fe}\left(\mathrm{NO}_{3}\right)_{3} \cdot 6 \mathrm{H}_{2} \mathrm{O}$ & & No TOX data/oxidizer \\
\hline $\begin{array}{l}\text { Ferrous ammonium } \\
\text { sulfate }\end{array}$ & $\left(\mathrm{NH}_{4}\right)_{2} \mathrm{SO}_{4} \mathrm{FeSO}_{4} \cdot 6 \mathrm{H}_{2} \mathrm{O}$ & & No TOX data/no DOT \\
\hline Ferrous sulfamate & $\mathrm{Fe}\left(\mathrm{SO}_{3} \mathrm{NH}_{2}\right)_{2}$ & & No entry \\
\hline Hydrazime & $\mathrm{N}_{2} \mathrm{H}_{4} \cdot \mathrm{H}_{2} \mathrm{O}$ & & \\
\hline Hydrobromic acid & $\mathrm{HBr}$ & & $\begin{array}{l}\text { LC50 } 2858 \mathrm{ppm} / \\
\text { inhal ation hazard/ } \\
\text { nonfl amable gas/ } \\
\text { corrosive }\end{array}$ \\
\hline
\end{tabular}


Table 5-28. PFP (Z P1ant): Analytical Laboratory (200 West Area)(1973-79). (3 sheets)

\begin{tabular}{|c|c|c|c|}
\hline Compound name & Formula & $\begin{array}{c}\text { EPA } \\
\text { category }\end{array}$ & $\begin{array}{l}\text { Comments and } \\
\text { DOT classification }\end{array}$ \\
\hline Hydrochloric acid & $\mathrm{HCl}$ & & \\
\hline Hydrofluoric acid & HF & & \\
\hline Hydrogen peroxide & $\mathrm{H}_{2} \mathrm{O}_{2}$ & & \\
\hline Hydroiodic acid & $\mathrm{HI}$ & & Corrosive/no TOX data \\
\hline $\begin{array}{l}\text { Hydroxyl amine } \\
\text { hydrochloride }\end{array}$ & $\mathrm{HN}_{2} \mathrm{OH} \cdot \mathrm{HCl}$ & & \\
\hline Hydroxylamine nitrate & $\mathrm{NH}_{2} \mathrm{OH} \cdot \mathrm{HNO}_{3}$ & & \\
\hline Methanol & $\mathrm{CH}_{3} \mathrm{OH}$ & D & Flammable liquid \\
\hline Napthyl amine & $\mathrm{C}_{10} \mathrm{H}_{9} \mathrm{~N}$ & D & DOT IM: poison \\
\hline Nitric acid & $\mathrm{HNO}_{3}$ & C & Oxidizer, corrosive \\
\hline Oxalic acid & $\mathrm{HO}_{2} \mathrm{CCO}_{2} \mathrm{H} \cdot 2 \mathrm{H}_{2} \mathrm{O}$ & & \\
\hline Phosphoric acid & $\mathrm{H}_{3} \mathrm{PO}_{4}$ & D & Corrosive \\
\hline Potassium acetate & $\mathrm{KC}_{2} \mathrm{H}_{3} \mathrm{O}_{2}$ & D & No DOT \\
\hline Potassium dichromate & $\mathrm{K}_{2} \mathrm{CrO}_{7}$ & & $\begin{array}{l}\text { Insufficient TOX } \\
\text { data/ORM-A }\end{array}$ \\
\hline Potassium iodate & $\mathrm{KIO}_{3}$ & & $\begin{array}{l}\text { Insuffictent TOX } \\
\text { data/no DOT }\end{array}$ \\
\hline Potassium permanganate & $\mathrm{KMnO}_{4}$ & & \\
\hline Silver oxide & AgO & D & No DOT \\
\hline Sodium bisulfate & $\mathrm{NaHSO}_{4}$ & & No TOX data/ORM-B \\
\hline Sodium carbonate & $\mathrm{Na}_{2} \mathrm{CO}_{3}$ & & \\
\hline Sodium fluoride & $\mathrm{NaF}$ & & \\
\hline Sodium hydroxide & $\mathrm{NaOH}$ & & \\
\hline Sodium nitrate & $\mathrm{NaNO}_{3}$ & & \\
\hline Sodium nitrite & $\mathrm{NaNO}_{2}$ & & No entry \\
\hline Sodium oxalate & $\mathrm{Na}_{2} \mathrm{C}_{2} \mathrm{O}_{4}$ & & $\begin{array}{l}\text { Insufficient TOX } \\
\text { data/no DOT }\end{array}$ \\
\hline Sodium tartrate & $\mathrm{Na}_{2} \mathrm{C}_{2} \mathrm{H}_{4} \mathrm{O}_{6} \cdot 2 \mathrm{H}_{2} \mathrm{O}$ & & $\begin{array}{l}\text { Insufficient TOX } \\
\text { data/no DOT }\end{array}$ \\
\hline Sulfamic acid & $\mathrm{NH}_{2} \mathrm{SO}_{3} \mathrm{H}$ & & \\
\hline Sulfonic acid (chloro) & $\mathrm{ClHSO}_{3}$ & & No TOX data/corrosive \\
\hline
\end{tabular}


Table 5-28. PFP (Z Plant): Analytical Laboratory (200 West Area) (1973-79). (3 sheets)

\begin{tabular}{|l|l|c|l|}
\hline \multicolumn{1}{|c|}{ Compound name } & \multicolumn{1}{|c|}{ Formula } & $\begin{array}{c}\text { EPA } \\
\text { category }\end{array}$ & \multicolumn{1}{|c|}{$\begin{array}{c}\text { Comments and } \\
\text { DOT classification }\end{array}$} \\
\hline Sulfuric acid & $\mathrm{H}_{2} \mathrm{SO}_{4}$ & D & Corrosive \\
\hline Thenoyltrifluoracetone & $\left(\mathrm{CH}_{3} \mathrm{SCOCH}_{2} \mathrm{COCF}_{3}\right.$ & & No entry \\
\hline Thymophthalein & $\mathrm{C}_{29} \mathrm{H}_{30} \mathrm{O}_{4}$ & & No entry \\
\hline Toluene & $\mathrm{C}_{6} \mathrm{H}_{5} \mathrm{CH}_{3}$ & D & Fl ammable liquid \\
\hline Tributyl phosphate & $\left(\mathrm{C}_{4} \mathrm{H}_{9}\right)_{3} \mathrm{PO}_{4}$ & & \\
\hline Tritsooctylamine & $\mathrm{C}_{26} \mathrm{H}_{31} \mathrm{~N}$ & D & No DOT \\
\hline $\begin{array}{l}\text { Tris(hydroxymethyl) } \\
\text { amino methane }\end{array}$ & $\left(\mathrm{CH}_{2} \mathrm{OH}\right)_{3} \mathrm{CNH}_{2}$ & & Not regulated/no DOT \\
\hline Xylene & $\mathrm{C}_{6} \mathrm{H}_{4}\left(\mathrm{CH}_{3}\right)_{2}$ & D & Flammable liquid \\
\hline
\end{tabular}

DOT = U.S. Department of Transportation.

$E P A=U . S$. Environmental Protection Agency.

ORM = Other regulated material.

TOX = Toxicity category. 
Table 5-29. TRU Mixed Waste Stored in 55-Gallon Drums by Storage Facility.

\begin{tabular}{|c|c|c|c|c|c|c|c|c|c|c|c|c|c|c|c|c|c|c|c|c|c|c|}
\hline 234-52 TRU T & $\begin{array}{l}\text { Wast } \\
1970\end{array}$ & $\begin{array}{l}\text { (inc } \\
1971\end{array}$ & $\begin{array}{l}\text { Eludin } \\
1972\end{array}$ & $\begin{array}{l}8 \text { PCB) } \\
1973\end{array}$ & $\begin{array}{l}559 a \\
1974\end{array}$ & $\begin{array}{l}110 n c \\
1975\end{array}$ & $\begin{array}{l}\text { druns } \\
1976\end{array}$ & $\begin{array}{l}-\operatorname{coun} \\
1977\end{array}$ & $\begin{array}{l}\text { by S } \\
1978\end{array}$ & $\begin{array}{c}\text { torage } \\
1979\end{array}$ & $\begin{array}{c}\text { Area } \\
1980\end{array}$ & 1981 & 1982 & 1983 & 1984 & 1985 & 1986 & 1987 & 1988 & 1989 & 1990 & 1991 \\
\hline \multicolumn{23}{|l|}{$\begin{array}{l}2183 \sqrt{3 A} \\
218448\end{array}$} \\
\hline 218 anc & & & & & & & & & & & & & 1 & 16 & 3 & 13 & & & & & & \\
\hline \multicolumn{23}{|l|}{21845} \\
\hline $224-T$ & & & & & & & & & & & & & & & & & & & & 34 & 20 & 18 \\
\hline $2401-4$ & & & & & & & & & & & & & 6 & 4 & & 14 & & & 10 & 8 & 1 & \\
\hline \multicolumn{23}{|l|}{24024} \\
\hline $\begin{array}{l}240248 \\
\text { FS8 }\end{array}$ & & & & & & & & & · & & & & & & & & & & & 1 & & \\
\hline Total Drums & 0 & 0 & 0 & 0 & 0 & 0 & 0 & 0 & 0 & 0 & 0 & 0 & 7 & 20 & 3 & 27 & 0 & 0 & 10 & 43 & 21 & 18 \\
\hline
\end{tabular}


Table 5-30. TRU Mixed Waste Containers Other Than 55-Gallon Drums by Storage Facility.

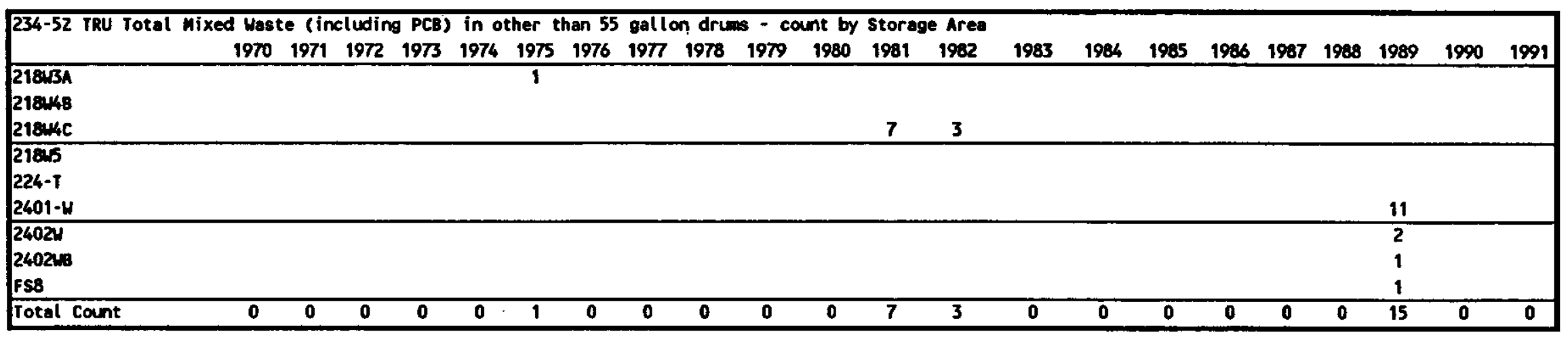

$\frac{1}{N}$ 
Table 5-31. Number and Percent of TRU Mixed Waste Drums Stored at 218W4C Containing Hazardous Constituents.

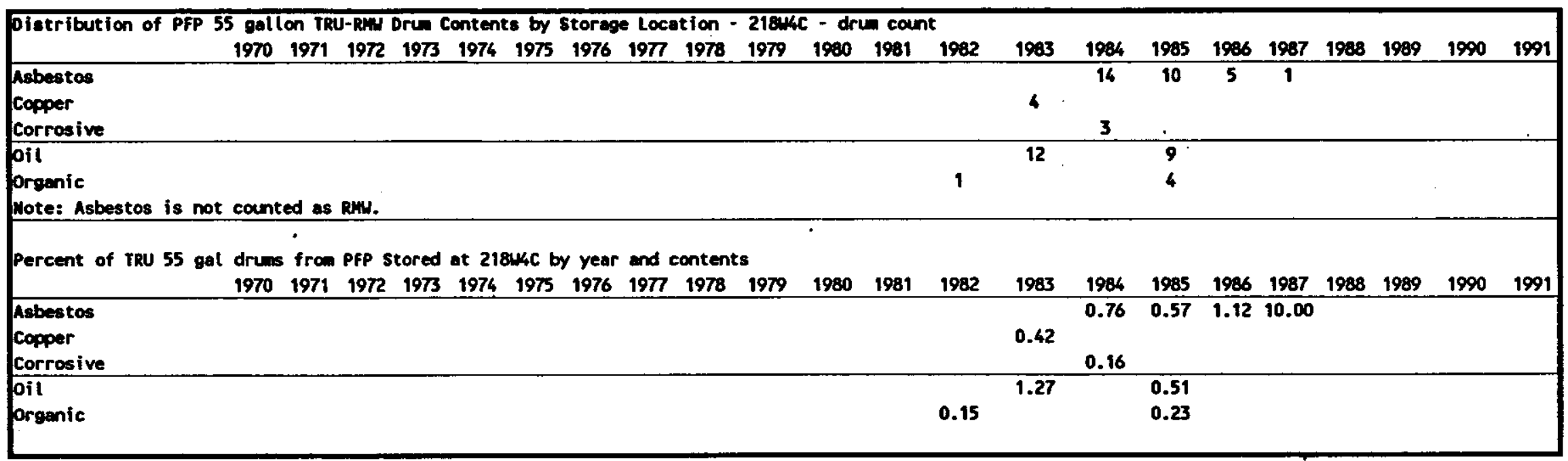


Table 5-32. Number and Percent of TRU Mixed Waste Drums Stored at 224-T Containing Hazardous Constituents.

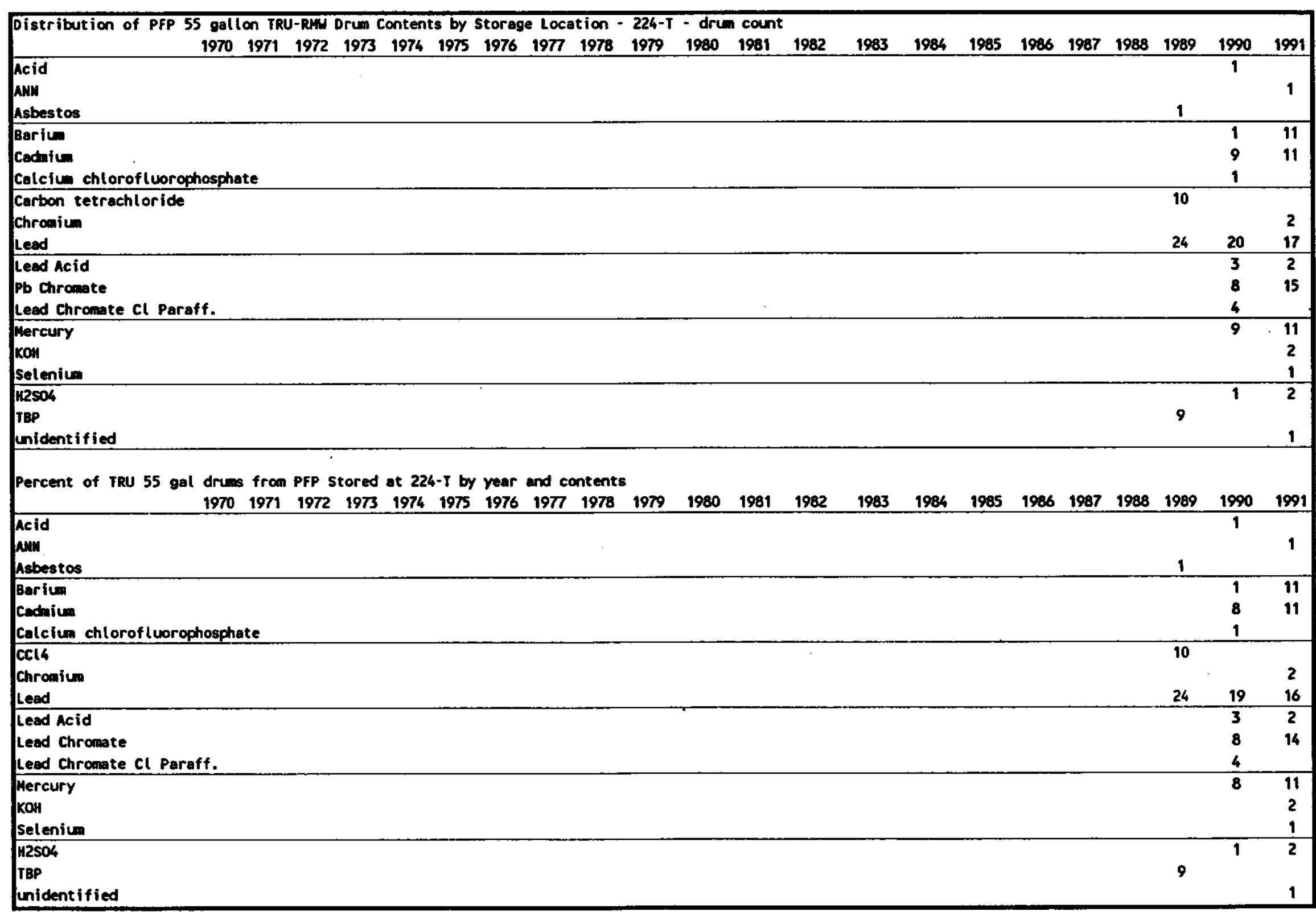


Table 5-33. Number and Percent of TRU Mixed Waste Drums Stored at 2401W Containing Hazardous Constituents.

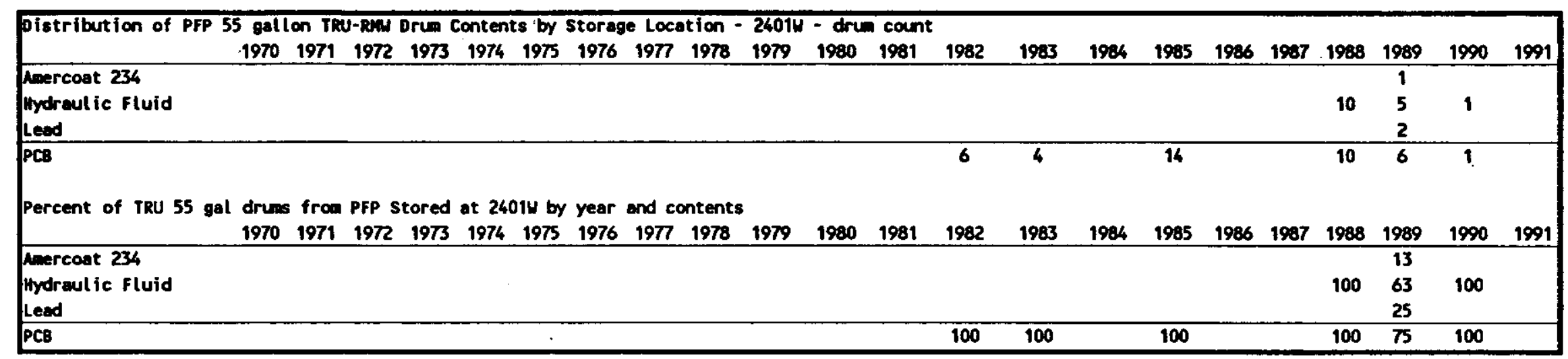

$\stackrel{\text { In }}{\text { G }}$ 
Table 5-34. Number and Percent of TRU Mixed Waste Drums Stored at 2402 WB Containing Hazardous Constituents.

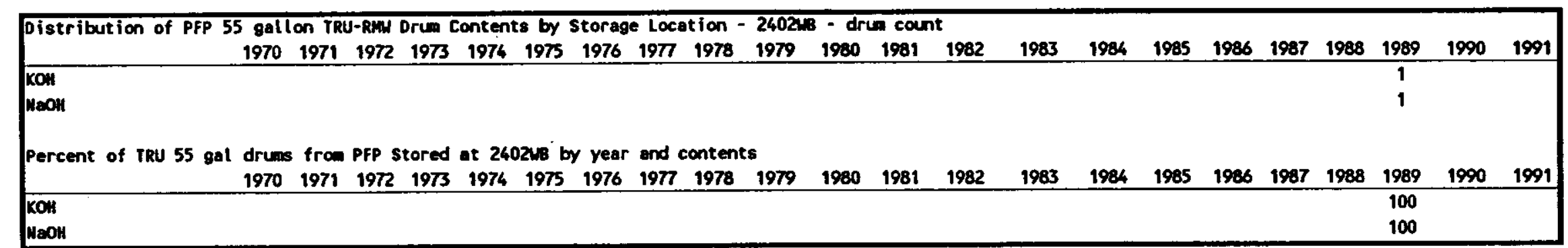

$\frac{1}{\text { ò }}$ 
Table 5-35. Number and Percentage of TRU Mixed Waste Containers Other Than 55-Gallon Drums Stored at 218W3A Containing Hazardous Constituents.

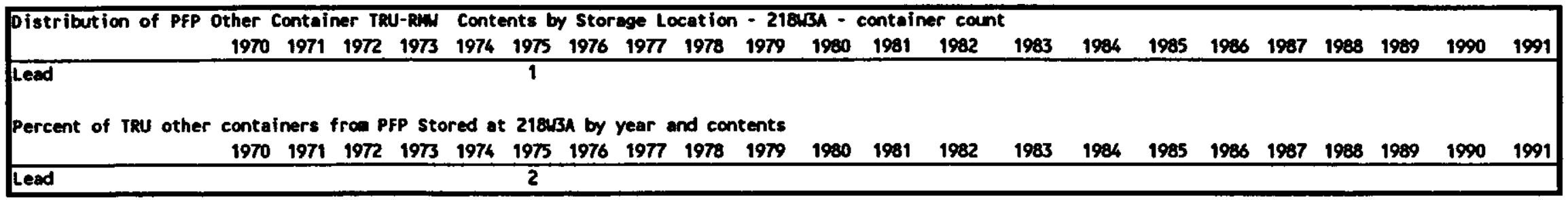


Table 5-36. Number and Percentage of TRU Mixed Waste Containers Other Than 55-Gallon Drums Stored at 218W4C Containing Hazardous Constituents.

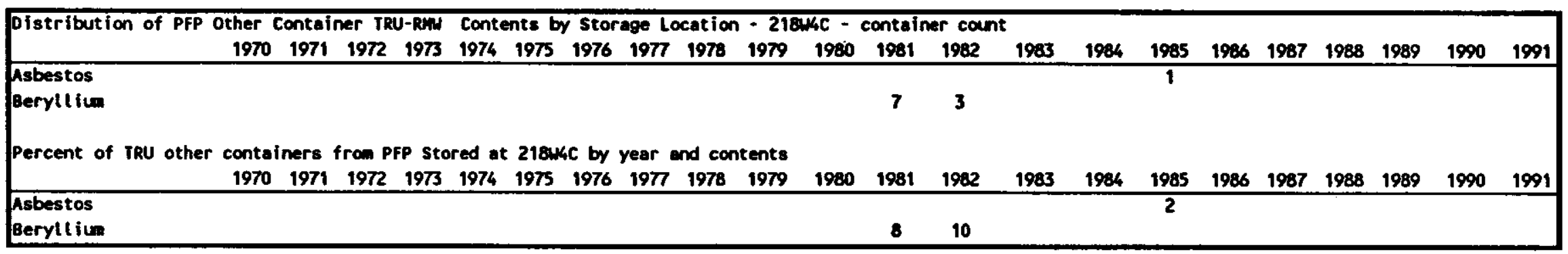


Table 5-37. Number and Percentage of TRU Mixed Waste Containers Other Than 55-Gallon Drums Stored at 2401W Containing Hazardous Constituents.

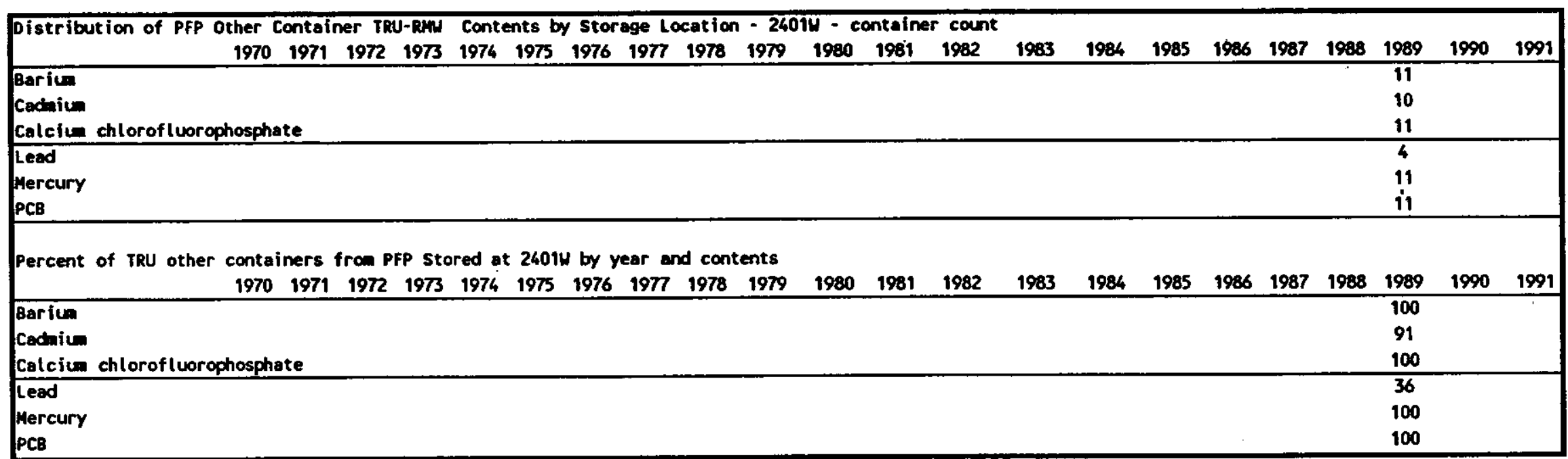


Table 5-38. Number and Percentage of TRU Mixed Maste Containers Other Than 55-Gallon Drums Stored at 2402W Containing Hazardous Constituents.

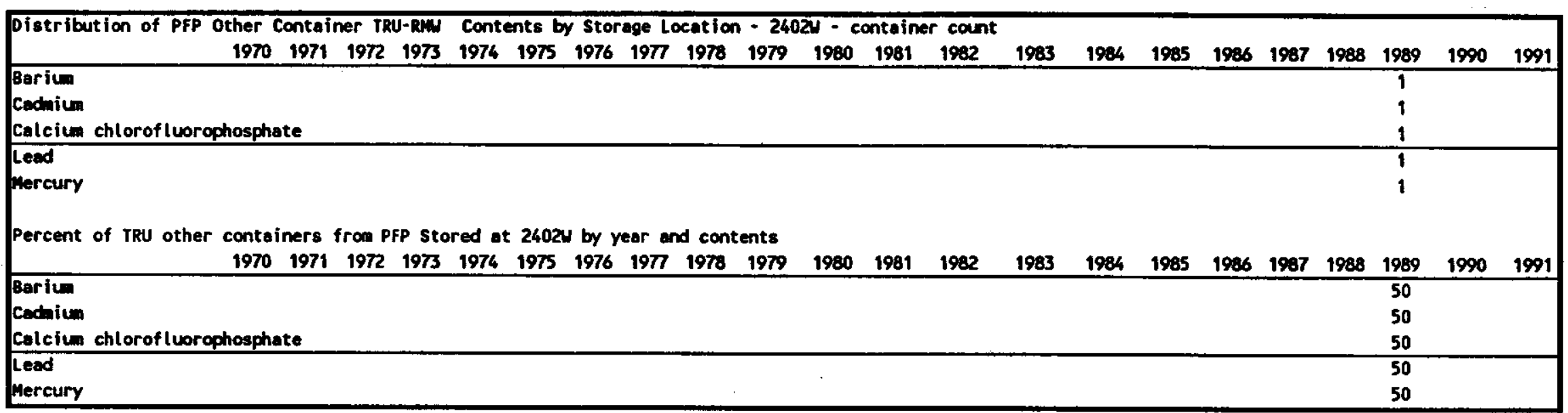


Table 5-39. Number and Percentage of TRU Mixed Waste Containers Other Than 55-Gallon Drums Stored at 2402WB Containing Hazardous Constituents.

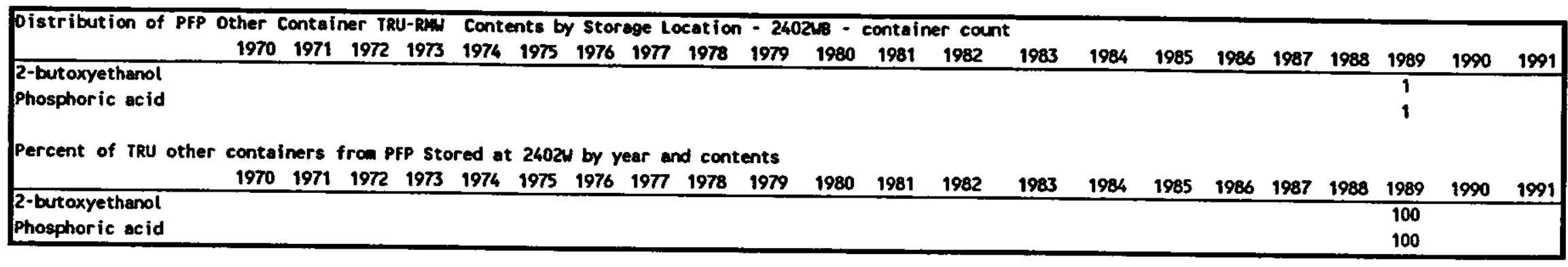


Table 5-40. Number and Percentage of TRU Mixed Waste Containers Other Than 55-Gallon Drums Stored at FS8 Containing Hazardous Constituents.

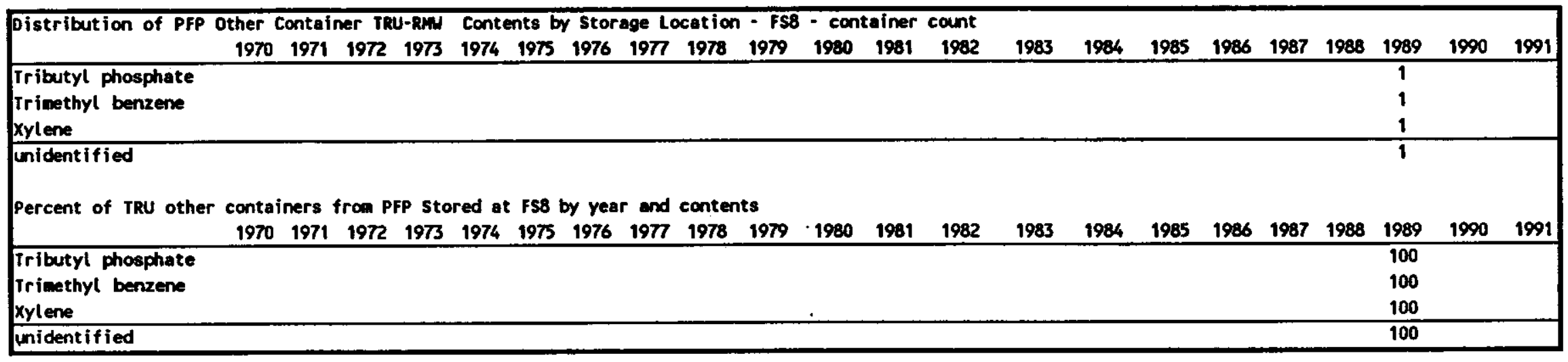


Table 5-41. Summary of Information from Solid Waste Storage Disposal Records for Containers of TRU Mixed Waste. (10 sheets)

\begin{tabular}{|c|c|c|c|c|c|c|}
\hline $\begin{array}{c}\text { Date } \\
\text { accepted }\end{array}$ & $\begin{array}{c}\text { Pin } \\
\text { number }\end{array}$ & $\begin{array}{l}\text { Manifest } \\
\text { number }\end{array}$ & $\begin{array}{c}\text { SWSDR } \\
\text { number }\end{array}$ & $\begin{array}{c}\text { Hazardous } \\
\text { constituents }\end{array}$ & $\begin{array}{l}\text { Waste } \\
\text { codes }\end{array}$ & $\begin{array}{l}\text { Weight } \\
(\mathrm{kg}) / \%\end{array}$ \\
\hline $05 / 07 / 81$ & $390 . A$ & $N / A$ & 810089 & $\mathrm{Pu} / \mathrm{Be}$ sources & N/A & $N / A$ \\
\hline $05 / 07 / 81$ & $240 . A$ & N/A & 810188 & $\mathrm{Pu} / \mathrm{Be}$ sources & $N / A$ & $N / A$ \\
\hline 05/07/81 & $381 . A$ & $N / A$ & 810186 & $\mathrm{Pu} / \mathrm{Be}$ sources & $N / A$ & $N / A$ \\
\hline $05 / 07 / 81$ & 441 & $N / A$ & 810187 & Pu/Be sources & $N / A$ & $N / A$ \\
\hline $02 / 14 / 83$ & Z0RGA9173 & $N / A$ & 830009 & Organic absorbed & N/A & N/A \\
\hline $02 / 14 / 83$ & ZORGA9172 & N/A & 830010 & Organic absorbed & $N / A$ & N/A \\
\hline $02 / 14 / 83$ & Z0RGA9183 & $N / A^{*}$ & 830011 & Organic absorbed & $N / A$ & N/A \\
\hline $02 / 14 / 83$ & Z0RGA9189 & $N / A$ & 830012 & Organic absorbed & $N / A$ & N/A \\
\hline $02 / 14 / 83$ & Z0RGA9181 & $N / A$ & 830008 & Organic absorbed & $N / A$ & $N / A$ \\
\hline $02 / 14 / 83$ & ZORGA9188 & $N / A$ & 830013 & Organic absorbed & N/A & $N / A$ \\
\hline $01 / 20 / 83$ & Z0RGA9126 & $N / A$ & 830003 & Fab oil absorbed & $N / A$ & $N / A$ \\
\hline $01 / 20 / 83$ & ZORGA9143 & $\mathbf{N} / \mathbf{A}$ & 830006 & Fab oil absorbed & $N / A$ & N/A \\
\hline $01 / 20 / 83$ & Z0RGA9139 & $N / A$ & 830004 & Fab oil absorbed & $N / A$ & $N / A$ \\
\hline $01 / 20 / 83$ & ZORGA9140 & N/A & 830005 & Fab oil absorbed & $N / A$ & $N / A$ \\
\hline $01 / 20 / 83$ & Z0RGA9144 & N/A & 830007 & Fab oil absorbed & $N / A$ & $N / A$ \\
\hline $01 / 20 / 83$ & ZORGA9125 & $N / A$ & 830002 & Fab oil absorbed & $N / A$ & $N / A$ \\
\hline $11 / 29 / 83$ & 8303 A9232 & $N / A$ & 890042 & PCB 56.3 ppm & $N / A$ & $N / A$ \\
\hline $11 / 29 / 83$ & $8303 A 9230$ & $N / A$ & 890044 & PCB $56.3 \mathrm{ppm}$ & $N / A$ & N/A \\
\hline $11 / 29 / 83$ & $8303 A 9632$ & $N / A$ & 890045 & PCB 56.3 ppm & $N / A$ & $N / A$ \\
\hline
\end{tabular}


Table 5-41. Summary of Information from Solid Waste Storage Disposal Records for Containers of TRU Mixed Waste. (10 sheets)

\begin{tabular}{|c|c|c|c|c|c|c|}
\hline $\begin{array}{c}\text { Date } \\
\text { accepted }\end{array}$ & $\begin{array}{c}\text { Pin } \\
\text { number }\end{array}$ & $\begin{array}{c}\text { Manifest } \\
\text { number }\end{array}$ & $\begin{array}{l}\text { SHSDR } \\
\text { number }\end{array}$ & $\begin{array}{l}\text { Hazardous } \\
\text { constituents }\end{array}$ & $\begin{array}{l}\text { Waste } \\
\text { codes* }\end{array}$ & $\begin{array}{l}\text { Weight } \\
(\mathrm{kg}) / \%\end{array}$ \\
\hline $11 / 29 / 83$ & 830319231 & N/A & 890043 & PCB 56.3 ppm & $N / A$ & N/A \\
\hline $06 / 30 / 82$ & ZORGA8996 & $N / A$ & 890041 & Contaminated oil & N/A & $N / A$ \\
\hline $06 / 30 / 82$ & ZORGA8815 & $N / A$ & 830001 & Organics aḅsorbed & $N / A$ & $N / A$ \\
\hline $06 / 30 / 82$ & ZORGA8993 & N/A & 890038 & Contaminated oil & $N / A$ & $N / A$ \\
\hline $06 / 30 / 82$ & ZORGA8994 & N/A & 890039 & Contaminated oil & $N / A$ & $N / A$ \\
\hline $06 / 30 / 82$ & ZORGA8995 & $N / A$ & 890040 & Contaminated oil & $N / A$ & N/A \\
\hline $06 / 09 / 89$ & RHZ-212-A19191 & PFP08904 & 890431 & Lead gloves & WT01, D008 & 27.84 \\
\hline $06 / 09 / 89$ & RHZ-212-A19567 & PFP08904 & 890435 & Lead gloves & WT01, D008 & 24.55 \\
\hline $06 / 09 / 89$ & RHZ-212-A18446 & PFP08907 & 890429 & $\mathrm{CCl}_{4}, \mathrm{TBP}$ & WCOl, WPOI, WTO1 & $\begin{array}{l}\mathrm{CCl}_{4} \\
\mathrm{TBP}^{4} \\
5.9\end{array}$ \\
\hline $06 / 09 / 89$ & RHZ-212-A19296 & PFP08904 & 890432 & Lead gloves & WT01, D008 & $37.35 / 52$ \\
\hline $06 / 09 / 89$ & RHZ-213-A19574 & PFP08904 & 890434 & Lead: brick/glass & WT01, D008 & $83.45 / 90$ \\
\hline $06 / 09 / 89$ & RHZ-212-A18445 & PFP08907 & 890428 & $\mathrm{CCl}_{4}, \mathrm{TBP}$ & WCO1, WPO1, WTO1 & $\begin{array}{l}\mathrm{CCl}_{4} 9.5 \\
\mathrm{TBP}^{5} 5.9\end{array}$ \\
\hline $06 / 09 / 89$ & RHZ-212-A19446 & PFP08904 & 890433 & Lead gloves & Wrol, D008 & $39.4 / 43$ \\
\hline $06 / 09 / 89$ & RHZ-212-A18447 & PFP08907 & 890430 & $\mathrm{CCl}_{4}, \mathrm{TBP}$ & WC01, WP01, WT01 & $\begin{array}{l}\mathrm{CCl}_{4} \\
\mathrm{TBP}^{4} \mathbf{5 . 9}\end{array}$ \\
\hline $06 / 09 / 89$ & RHZ-212-A18444 & PFP08907 & 890427 & $\mathrm{CCl}_{4}, \mathrm{TBP}$ & WC01, WP01, WT01 & $\begin{array}{ll}\mathrm{CCl}_{4} & 9.5 \\
\mathrm{TBP}^{5} & 5.9\end{array}$ \\
\hline $06 / 13 / 89$ & RHZ-213-A19387 & PFP08906 & 890001 & $\begin{array}{l}\text { Hydrol ic fluid/PCB } \\
(760 \mathrm{ppm})\end{array}$ & WT02 & $\begin{array}{l}\text { Fluid } 2.6 \\
\text { PCB } 0.002\end{array}$ \\
\hline
\end{tabular}


Table 5-41. Summary of Information from Solid Waste Storage Disposal Records for Containers of TRU Mixed Waste. (10 sheets)

\begin{tabular}{|c|c|c|c|c|c|c|}
\hline $\begin{array}{l}\text { Date } \\
\text { accepted }\end{array}$ & $\begin{array}{l}\text { Pin } \\
\text { number }\end{array}$ & $\begin{array}{l}\text { Manifest } \\
\text { number }\end{array}$ & $\begin{array}{c}\text { SWSDR } \\
\text { number }\end{array}$ & $\begin{array}{c}\text { Hazardous } \\
\text { constituents }\end{array}$ & $\begin{array}{l}\text { Waste } \\
\text { codes* }\end{array}$ & $\begin{array}{l}\text { Weight } \\
(\mathrm{kg}) / \%\end{array}$ \\
\hline $06 / 13 / 89$ & RHZ-213-A19394 & PFP08906 & 890002 & $\begin{array}{l}\text { hydrol ic fluid/PCB } \\
\text { (760 ppm) }\end{array}$ & WT02 & $\begin{array}{l}\text { Fluid } 5.9 \\
\text { PCB } 0.005\end{array}$ \\
\hline $06 / 13 / 89$ & RHZ-213-A19396 & PFP08906 & 890004 & $\begin{array}{l}\text { Hydrol ic fluid/PCB } \\
\text { (760 ppm) }\end{array}$ & WT02 & $\begin{array}{l}\text { Fluid } 24.18 \\
\text { PCB } 0.19\end{array}$ \\
\hline $06 / 13 / 89$ & RHZ-213-A19395 & PFP08906 & 890003 & $\begin{array}{l}\text { Hydrol ic fluid/PCB } \\
(760 \mathrm{ppm})\end{array}$ & WTO2 & $\begin{array}{l}\text { Fluid } 18.13 \\
\text { PCB } 0.03\end{array}$ \\
\hline $07 / 14 / 89$ & RHZ-212-A19826 & PFP08913 & 890467 & Lead & D008, WTO1 & $4.0 / 13$ \\
\hline $07 / 14 / 89$ & RHZ-220-A19847 & PFP08914 & 890473 & Lead gloves & D008, WT01 & 5.0 \\
\hline $07 / 14 / 89$ & RHZ-212-A19862 & PFP08913 & 890470 & Lead gloves & D008, WT01 & 2.0 \\
\hline $07 / 14 / 89$ & RHZ-212-A19860 & PFP08913 & 890468 & Lead metal & D008, WT01 & $2.5 / 4$ \\
\hline $07 / 14 / 89$ & RHZ-220-A19846 & PFP08914 & 890472 & Lead: gloves, shield & D008, WT01 & $18.0 / 42$ \\
\hline $07 / 14 / 89$ & RHZ-212-A19135 & PFP08913 & 890463 & Lead (metal tool) & D008, WTOl & $64.5 / 73$ \\
\hline $07 / 14 / 89$ & RHZ-212-A19808 & PFP08913 & 890465 & Lead & D008, WT01 & $34.98 / 71$ \\
\hline $07 / 14 / 89$ & RHZ-212-A19518 & PFP08913 & 890466 & Lead & D008, WT01 & $15.67 / 49$ \\
\hline $07 / 14 / 89$ & RHZ-212-A19715 & PFP08913 & 890464 & Lead (metal tool) & D008, WTOl & $25.5 / 37$ \\
\hline $07 / 14 / 89$ & RHZ-213-A19790 & PFP08914 & 890469 & Lead & D008, WT01 & $43.1 / 52$ \\
\hline $07 / 14 / 89$ & RHZ-212-A19812 & PFP08913 & 890471 & Lead: metal, glass & D008, WTO1 & $22.1 / 37$ \\
\hline $07 / 20 / 89$ & RHZ-213-A19411 & PFP08920 & 890494 & Lead gloves & WT01, D008 & $24.5 / 45$ \\
\hline $07 / 20 / 89$ & RHZ-213-A19867 & PFP08920 & 890493 & Lead gloves & WT01, D008 & $37.5 / 39$ \\
\hline $07 / 20 / 89$ & RHZ-212-A19866 & PFP08920 & 890495 & Lead: gloves, apron & WT01, D008 & $37.5 / 38$ \\
\hline $07 / 25 / 89$ & RHZ-212-A19813 & PFP08921 & 890502 & Lead gloves & WT01, D008 & $1.0 / 2$ \\
\hline
\end{tabular}


Table 5-41. Summary of Information from Solid Waste Storage Disposal Records for Containers of TRU Mixed Waste. (10 sheets)

\begin{tabular}{|c|c|c|c|c|c|c|}
\hline $\begin{array}{l}\text { Date } \\
\text { accepted }\end{array}$ & $\underset{\text { number }}{\text { Pin }}$ & $\begin{array}{c}\text { Manifest } \\
\text { number }\end{array}$ & $\begin{array}{c}\text { SWSDR } \\
\text { number }\end{array}$ & $\begin{array}{l}\text { Hazardous } \\
\text { constituents }\end{array}$ & $\begin{array}{l}\text { Haste } \\
\text { codes* }\end{array}$ & $\begin{array}{l}\text { Weight } \\
(\mathrm{kg}) / \%\end{array}$ \\
\hline $07 / 25 / 89$ & RHZ-212-A18517 & PFP08902 & 890501 & $\mathrm{CCl}_{4}:$ TB̈̈ absorbed & WCO1, WP01, WTO1 & $\begin{array}{l}\mathrm{CCl}_{4} \\
\mathrm{TBP}_{5} \\
\mathbf{5 . 9}\end{array}$ \\
\hline $07 / 25 / 89$ & RHZ-212-A18497 & PFP08920 & 890500 & $\mathrm{CCl}_{4}:$ TBP absorbed & WT01, WCO1, WPO1 & 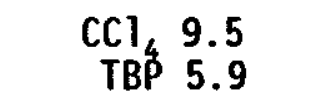 \\
\hline $07 / 25 / 89$ & $\begin{array}{c}\text { RHZ-212-A19729 } \\
* W I S\end{array}$ & PFP08903 & 900001 & $\mathrm{CCl}_{4}:$ TBP absorbed & WT01, WC01, WP01 & $\begin{array}{l}\mathrm{CCl}_{4} 6.5 \\
\mathrm{TBP} \\
4.0\end{array}$ \\
\hline $07 / 25 / 89$ & RHZ-212-A18517 & PFP08920 & 890501 & $\mathrm{CCl}_{4}:$ TBP absorbed & WT01, WC01, WP01 & $\begin{array}{l}\mathrm{CCl}_{4} \\
\mathrm{TBP} \\
5.9\end{array}$ \\
\hline $07 / 25 / 89$ & $\mathrm{RHZ}-212-\mathrm{Al} 8496$ & PFP08920 & 890498 & $\mathrm{CCl}_{4}:$ TBP absorbed & WT01, WC01, WP01 & $15.4 / 35$ \\
\hline $07 / 25 / 89$ & $\mathrm{RHZ}-212-\mathrm{A} 18497$ & PFP08902 & 890500 & $\mathrm{CCl}_{4}, \mathrm{TBP}$ & WCO1, WP01, WTO1 & $\begin{array}{l}\mathrm{CCL}_{4} 9.5 \\
\mathrm{TBP}^{5.9}\end{array}$ \\
\hline $07 / 25 / 89$ & $\begin{array}{c}\text { RHZ-212-A19862 } \\
\text { *WIS }\end{array}$ & PFP08913 & 900002 & Lead gloves & WT01, D008 & 2.0 \\
\hline $07 / 25 / 89$ & RHZ-212-A18496 & PFP08902 & 890499 & $\mathrm{CCl}_{4}, \mathrm{TBP}$ & WC01, WP01, WT01 & $15.4 / 35$ \\
\hline $07 / 26 / 89$ & RHZ-213-A19318 & PFP08926 & 890028 & $\begin{array}{l}\text { PCB (760 ppm) } \\
\text { hydraul ic fluid }\end{array}$ & WT02 & $\begin{array}{c}0.011 / 72 \\
33.989\end{array}$ \\
\hline $07 / 26 / 89$ & RHZ-213-A19875 & PFP08925 & 890029 & PCB (760 ppm) & WT02 & 0.002 \\
\hline $07 / 31 / 89$ & RHZ-87-025 & PFP08930 & 890030 & Above (no Pb) & ABOVE -D008 & 0.03139 \\
\hline $07 / 31 / 89$ & RHZ-87-029 & PFP08931 & 890031 & Above (no $\mathrm{Pb}$ ) & ABOVE -D008 & 0.28041 \\
\hline $08 / 02 / 89$ & RHZ-87-037 & PFP08933 & 890032 & Lead + above list & $\mathrm{D} 008+\mathrm{ABOVE}$ & $\begin{array}{l}\text { Pb207.0/3.5 } \\
\text { Trace } 0.06378\end{array}$ \\
\hline $08 / 04 / 89$ & RHZ-86-14 & PFP08936 & 890001 & $\mathrm{~N}_{2} \mathrm{PO}_{4}$ 2-butoxyethanol & D002 & $0.322 /$ \\
\hline
\end{tabular}


Table 5-41. Summary of Information from Solid Waste Storage Disposal Records for Containers of TRU Mixed Waste. (10 sheets)

\begin{tabular}{|c|c|c|c|c|c|c|}
\hline $\begin{array}{c}\text { Date } \\
\text { accepted }\end{array}$ & $\begin{array}{c}\text { Pin } \\
\text { number }\end{array}$ & $\begin{array}{c}\text { Manifest } \\
\text { number }\end{array}$ & $\begin{array}{c}\text { SWSDR } \\
\text { number }\end{array}$ & $\begin{array}{c}\text { Hazardous } \\
\text { constituents }\end{array}$ & $\begin{array}{l}\text { Waste } \\
\text { codes* }\end{array}$ & $\begin{array}{l}\text { Weight } \\
(\mathrm{kg}) / \%\end{array}$ \\
\hline $08 / 07 / 89$ & RHZ-87-041 & PFP08943 & 890033 & Lead + above list & $\begin{array}{l}\text { D006, D009, WT01, } \\
\text { WCO1, WCO2 }\end{array}$ & $\begin{array}{l}\text { Pbl137.5/15 } \\
\text { Trace } 0.03139\end{array}$ \\
\hline $08 / 07 / 89$ & RHZ-87-044 & PFP08937 & 900034 & Above list (no Pb) & ABOVE - D008 & 0.03139 \\
\hline 08/08/89 & RHZ-87-039 & PFP08941 & 890037 & $\begin{array}{l}\text { Trace PCB (500 ppm) } \\
\mathrm{Hg}, \mathrm{Ba}, \mathrm{Cd}, \mathrm{CaClFIPO}\end{array}$ & $\begin{array}{l}\text { D009, WT001, } \\
\text { D006, WC01, WC02 }\end{array}$ & 0.03139 \\
\hline $08 / 08 / 89$ & RHZ-85-001 & PFP08939 & 890002 & $\begin{array}{l}\text { Trace } \mathrm{Hg}, \mathrm{Cd}, \mathrm{Ba}, \\
\mathrm{CaClPO}_{4}\end{array}$ & $\begin{array}{l}\text { D009, D006, WT01, } \\
\text { WCO2 }\end{array}$ & 0.03136 \\
\hline $08 / 08 / 89$ & RHZ-87-028 & PFP08946 & 890036 & $\begin{array}{l}\text { Trace PCB }(500 \mathrm{ppm}) \\
\mathrm{Hg}, \mathrm{Ba}, \mathrm{Cd}, \mathrm{CaClFlPO}\end{array}$ & $\begin{array}{l}\text { D009, HT001, } \\
\text { D006, WCO1, WCO2 }\end{array}$ & 0.09417 \\
\hline $08 / 08 / 89$ & RHZ-86-002 & PFP08938 & 890035 & Lead + above list & D008 + ABOVE & $\begin{array}{c}\mathrm{Pb} 724.0 / 17 \\
\text { Trace } 0.102225\end{array}$ \\
\hline $08 / 08 / 89$ & RHZ-87-023 & PFP08945 & 890001 & Lead & DW008, WR01 & $103.4 / 20$ \\
\hline $08 / 09 / 89$ & $\mathrm{RHZ}-86-010$ & PFP08944 & 890015 & $\begin{array}{l}\text { Trace PCB, } \mathrm{Hg}, \mathrm{Cd} \text {, } \\
\mathrm{Ba}, \mathrm{CaClFiPO} \mathrm{C}_{4}+\text { Lead }\end{array}$ & $\begin{array}{l}\text { D008, WT01, D009, } \\
\text { D006, WCO1, WC02 }\end{array}$ & $\begin{array}{c}\text { Pbl03.4/10 } \\
\text { Trace } 0.03139\end{array}$ \\
\hline $08 / 10 / 89$ & RHZ-87-031 & PFP08932 & 890017 & Above (no $\mathrm{Pb}$ ) & ABOVE -D008 & 0.113357 \\
\hline $08 / 10 / 89$ & RHZ-85-002 & PFP08940 & 890016 & Above (no Pb) & ABOVE -DO08 & 0.06278 \\
\hline $08 / 22 / 89$ & RHZ-212-A18597 & PFP08929 & 890001 & $\begin{array}{l}\text { Scintillation fluid } \\
(60 \% \text { xylene, } 15 \% \\
\text { Tri/meth, benzone, } \\
\left.1 \% \text { TBP, } 4 \% \mathrm{CCl}_{4}\right)\end{array}$ & F003, D001, WTOl & $6.7 / 12$ absorbed \\
\hline $08 / 29 / 89$ & RHZ-212-A19843 & PFP08948 & NA & Lead gloves & WT01, D008 & $7.21 / 11$ \\
\hline $08 / 29 / 89$ & RHZ-212-A19931 & PFP08948 & 890592 & Lead: glove/shield & WT01, D008 & $59.0 / 86$ \\
\hline $08 / 29 / 89$ & RHZ-212-A19843 & PFP08948 & 890591 & Lead gloves & WT01, D008 & $7.21 / 11$ \\
\hline
\end{tabular}


Table 5-41. Summary of Information from Solid Waste Storage Disposal Records for Containers of TRU Mixed Waste. (10 sheéts)

\begin{tabular}{|c|c|c|c|c|c|c|}
\hline $\begin{array}{l}\text { Date } \\
\text { accepted }\end{array}$ & $\begin{array}{c}\text { Pin } \\
\text { number }\end{array}$ & $\begin{array}{l}\text { Manifest } \\
\text { number }\end{array}$ & $\begin{array}{l}\text { SWSDR } \\
\text { number }\end{array}$ & $\begin{array}{l}\text { Hazardous } \\
\text { const ituents }\end{array}$ & $\begin{array}{l}\text { Waste } \\
\text { codes* }\end{array}$ & $\begin{array}{l}\text { Weight } \\
(\mathrm{kg}) / \%\end{array}$ \\
\hline $11 / 07 / 89$ & RHZ-212-A19998 & PFP08958 & 890459 & Lead (shield, glove) & D008, WT01 & $24.5 / 54$ \\
\hline $12 / 14 / 89$ & $\mathrm{RHZ}-212-\mathrm{A} 20060$ & PFP08960 & 890002 & $\mathrm{NaOH}, \mathrm{KOH}$ & D002 & $6.0 / 25$ \\
\hline $12 / 14 / 89$ & RHZ-213-A20072 & PFP08963 & 890462 & $\begin{array}{l}\text { Lead (glove, brick), } \\
\text { Amercoat } 232 \quad\left(\mathrm{PbCrO}_{4}\right)\end{array}$ & D008, WTO1, SCO2, & $68.5 / 62 \quad 0.05 /$ \\
\hline $12 / 19 / 89$ & RHZ-212-A20061 & PFP08964 & 890610 & Lead gloves & WT01, D008 & $41.6 / 54$ \\
\hline $01 / 17 / 90$ & RHZ-220-A20220 & & 900001 & Lead, lead acid & D008, HTO1, DOO2 & $\begin{array}{l}\text { Pb } 123.36 \\
\text { Acid } 0.14\end{array}$ \\
\hline $02 / 02 / 90$ & RHZ-220-A20356 & 09003 & 900003 & $\begin{array}{l}\mathrm{Pb}, \mathrm{Hg} \text {, Trace: } \mathrm{Ba} \text {, } \\
\mathrm{Cd}, \mathrm{CaClFIPO}{ }_{4}\end{array}$ & $\begin{array}{l}\text { D008, WT01, , D005, } \\
\text { D006, D009, WC02 }\end{array}$ & $\begin{array}{c}\mathrm{Pb} \mathrm{26.32} \\
\mathrm{Hg} 0.01, \text { rest < }\end{array}$ \\
\hline $02 / 02 / 90$ & RHZ-212-A20312 & 09003 & 900002 & Lead, lead acid & D008, WT01, 0002, & $\begin{array}{l}\mathrm{Pb} 44.42 \\
\text { Acid } 0.2\end{array}$ \\
\hline $03 / 01 / 90$ & WHZ-220-A20361 & 09004 & 900004 & $\mathrm{~Pb}, \mathrm{Ba}, \mathrm{Cd}, \mathrm{Hg}$ & $\begin{array}{l}\text { D008, WTO1, D005, } \\
\text { D006, D007, D009, } \\
\text { WCO2 }\end{array}$ & $\begin{array}{ll}\mathrm{Pb} & 70.0 \\
\mathrm{Ba} & 0.05 \\
\mathrm{Cd} & 0.05 \\
\mathrm{Hg} & 0.1\end{array}$ \\
\hline $04 / 23 / 90$ & RHZ-212-A20499 & 09007 & 900039 & $\mathrm{~Pb}, \mathrm{Ba}, \mathrm{Cd}, \mathrm{Hg}$ & $\begin{array}{c}\text { D008, WTO1, D005, } \\
\text { D006, D007 }\end{array}$ & $\begin{array}{ll}\mathrm{Ba} & 0.08 \\
\mathrm{Cd} & 0.08 \\
\mathrm{Hg} & 0.08 \\
\mathrm{~Pb} & 19.0\end{array}$ \\
\hline $04 / 23 / 90$ & RHZ-213-A20536 & 09007 & 900041 & $\begin{array}{l}\mathrm{Pb}, \mathrm{Ba}, \mathrm{Cd}, \mathrm{Hg}, \\
\mathrm{PbCrO}_{2}, \text { (Cl. parafin) }\end{array}$ & $\begin{array}{l}\text { D008, WT01, D007, } \\
\text { WC02, D005, D006, } \\
\text { D009 }\end{array}$ & $\begin{array}{l}\mathrm{Pb} 36.9 \\
\mathrm{CrO}_{2} 1.0 \\
\mathrm{Ba} 0.001 \\
\mathrm{Hg} 0.001 \\
\mathrm{Cd} 0.001\end{array}$ \\
\hline
\end{tabular}


Table 5-41. Summary of Information from Solid Waste Storage Disposal Records for Containers of TRU Mixed Waste. (10 sheets)

\begin{tabular}{|c|c|c|c|c|c|c|}
\hline $\begin{array}{l}\text { Date } \\
\text { accepted }\end{array}$ & $\underset{\text { number }}{\text { Pin }}$ & $\begin{array}{l}\text { Manifest } \\
\text { number }\end{array}$ & $\begin{array}{c}\text { SWSDR } \\
\text { number }\end{array}$ & $\begin{array}{l}\text { Hazardous } \\
\text { constituents }\end{array}$ & $\begin{array}{l}\text { Waste } \\
\text { codes* }\end{array}$ & $\begin{array}{l}\text { Weight } \\
(\mathrm{kg}) / \%\end{array}$ \\
\hline $04 / 23 / 90$ & RHZ-212-A20576 & 09007 & 900040 & $\begin{array}{l}\mathrm{Pb}, \mathrm{Ba}, \mathrm{Cd}, \mathrm{Hg}, \\
\mathrm{PbCrO}_{2},(\mathrm{Cl}, \text { parafin) }\end{array}$ & $\begin{array}{l}\text { D008, WT01, D007, } \\
\text { WC02, D005, D006, } \\
\text { D009 }\end{array}$ & $\begin{array}{cc}\mathrm{Pb} & 43.5 \\
\mathrm{CrO}_{2} & 0.02 \\
\mathrm{Ba}^{2} & 0.03 \\
\mathrm{Cd} & 0.03 \\
\mathrm{Hg} & 0.04\end{array}$ \\
\hline $05 / 22 / 90$ & RHZ-212-A20759 & 09008 & 900095 & $\begin{array}{l}\mathrm{Ba}, \mathrm{Cd}, \mathrm{Pb}, \mathrm{Hg} \text {, } \\
\mathrm{PbCrO}_{2}, \text { (Cl. parafin) }\end{array}$ & $\begin{array}{l}\text { D008, D007, WC02, } \\
\text { WT01, D005, D006, } \\
\text { D009 }\end{array}$ & $\begin{array}{cc}\mathrm{Hg} & 0.1 \\
\mathrm{Cd} & 0.1 \\
\mathrm{Ba} & 0.8 \\
\mathrm{~Pb}^{2} & 40.95 \\
\mathrm{CrO}_{2} & 0.2 \\
\end{array}$ \\
\hline $05 / 22 / 90$ & RHZ-220-A20646 & 09008 & 900094 & $\begin{array}{l}\mathrm{Pb}, \mathrm{PbCrO}_{2}, \quad(\mathrm{Cl} . \\
\text { parafin) }\end{array}$ & D007, D008, WT01, & $\begin{array}{l}\mathrm{Pb} 27.0 \\
\mathrm{CrO}_{2} 1.0\end{array}$ \\
\hline $06 / 01 / 90$ & RHZ-211-A20917 & 09013 & 900001 & $\begin{array}{l}\text { Hydrolic oil } 190 \text { ppm } \\
\text { PCB }\end{array}$ & WTOl & $1.0 / 5$ \\
\hline $07 / 20 / 90$ & RHZ-220-A20790 & PFP09014 & 900114 & $\mathrm{Ba}, \mathrm{Cd}, \mathrm{Pb}, \mathrm{Hg}, \mathrm{H}_{2} \mathrm{SO}_{4}$ & $\begin{array}{l}\text { D002, D005, D006, } \\
\text { D008, D009, WT01, } \\
\text { WC02 }\end{array}$ & $\begin{array}{c}\text { Ba } 0.02, \text { Cd } 0.02 \\
\text { Pb 43.06, } \mathrm{Hg} \\
0.02, \text { SUL. } 0.02\end{array}$ \\
\hline $07 / 20 / 90$ & RHZ-213-A20916 & PFP09014 & 900113 & $\begin{array}{l}\mathrm{Pb}, \mathrm{PbCrO}_{2}, \quad(\mathrm{Cl} . \\
\text { parafin) }\end{array}$ & D007, D008, WTO1, & $\begin{array}{c}\mathrm{Pb} 53.8865 \\
\mathrm{CrO}_{2} 0.1\end{array}$ \\
\hline $07 / 20 / 90$ & RHZ-220-A20834 & PFP09014 & 900115 & Lead & D008, WT01 & $51.0 / 26$ \\
\hline $09 / 13 / 90$ & RHZ-211-A21030 & 09020 & 900259 & $\mathrm{~Pb} ; \mathrm{PbCrO}_{2}$ & D008, D007, WTO1, & $\begin{array}{l}\mathrm{Pb} 21.74 \\
\mathrm{CrO}_{2} 0.2\end{array}$ \\
\hline $10 / 31 / 90$ & $\mathrm{RHZ}-213-\mathrm{A} 21275$ & 09025 & 900371 & $\mathrm{~Pb}, \mathrm{PbCrO}_{2}$ & D008, D007, WTO1, & $\begin{array}{l}\mathrm{Pb} 32.14 \\
\mathrm{CrO}_{2} 0.08\end{array}$ \\
\hline
\end{tabular}


Table 5-41. Summary of Information from Solid Waste Storage Disposal Records for Containers of TRU Mixed Waste. (10 sheets)

\begin{tabular}{|c|c|c|c|c|c|c|}
\hline $\begin{array}{c}\text { Date } \\
\text { accepted }\end{array}$ & $\underset{\text { Pin }}{\text { number }}$ & $\begin{array}{l}\text { Manifest } \\
\text { number }\end{array}$ & $\begin{array}{c}\text { SWSDR } \\
\text { number }\end{array}$ & $\begin{array}{l}\text { Hazardous } \\
\text { constituents }\end{array}$ & $\begin{array}{l}\text { Waste } \\
\text { codes* }\end{array}$ & $\begin{array}{l}\text { Weight } \\
(\mathrm{kg}) / \%\end{array}$ \\
\hline $10 / 31 / 90$ & RHZ-220-A21302 & 09025 & 300372 & $\begin{array}{l}\mathrm{Ba}, \mathrm{Cd}, \mathrm{Pb}, \mathrm{PbCrO}_{2} \text {, } \\
\mathrm{Hg}\end{array}$ & $\begin{array}{l}\text { D008, WT01, D007, } \\
\text { WC02, D005, D006, } \\
\text { D009 }\end{array}$ & $\begin{array}{c}\mathrm{Ba} 0.01, \mathrm{Cd} 0.01 \\
\mathrm{~Pb} 47.85, \\
\mathrm{CrO}_{2} 0.1, \mathrm{Hg} 0.01\end{array}$ \\
\hline $10 / 31 / 90$ & RHZ-212-A21207 & 09025 & 900370 & $\mathrm{~Pb}, \mathrm{PbCrO}_{2}$ & D008, D007, WTO1, & $\begin{array}{l}\mathrm{Pb} 68.17 \\
\mathrm{CrO}_{2} 0.69\end{array}$ \\
\hline $12 / 10 / 90$ & $\mathrm{RHZ}-212-\mathrm{A} 21303$ & PFP09026 & 900445 & Lead & D008, WT01 & $24.38 / 40$ \\
\hline $12 / 10 / 90$ & RHZ-220-A21295 & PFP09026 & 900444 & $\begin{array}{l}\mathrm{Ba}, \mathrm{Cd}, \mathrm{Pb}, \mathrm{Hg} \text {, } \\
\mathrm{PbCrO}_{2} \text {, Lead acid }\end{array}$ & $\begin{array}{l}\text { D002, D005, D006, } \\
\text { D007, D008, WT01, } \\
\text { WC02 }\end{array}$ & $\begin{array}{cc}\mathrm{Ba} & 0.01 \\
\mathrm{Cd} & 0.01 \\
\mathrm{Hg} & 0.01 \\
\mathrm{~Pb} & 66.94 \\
\mathrm{Acid} & 0.01 \\
\mathrm{CrO}_{2} & 0.06\end{array}$ \\
\hline $12 / 10 / 90$ & RHZ-220-A21343 & PFP09026 & 900447 & $\mathrm{~Pb}, \mathrm{PbCrO}_{2}$ & $\begin{array}{c}\text { D007, D008, WT01, } \\
\text { WC02 }\end{array}$ & $\begin{array}{l}\mathrm{Pb} \mathrm{38.17} \\
\mathrm{CrO}_{2} 0.01 \\
\end{array}$ \\
\hline $12 / 10 / 90$ & RHZ-212-A21410 & PFP09026 & 900446 & $\begin{array}{l}\text { Lead, lead acid, } \\
\mathrm{PbCrO}_{2}\end{array}$ & $\begin{array}{l}\text { D002, D007, D008, } \\
\text { WTO1, WCO1 }\end{array}$ & $\begin{array}{l}\mathrm{Pb} \mathrm{33.57} \\
\text { Acid } 0.03 \\
\mathrm{CrO}_{2} 0.05\end{array}$ \\
\hline $02 / 04 / 91$ & RHZ-213-A21545 & 09101 & 910003 & $\mathrm{~Pb}, \mathrm{PbCrO}_{2}$, lead acid & $\begin{array}{l}\text { D008, WT01, D002, } \\
\text { D009, D007, } W C 02\end{array}$ & $\begin{array}{c}\text { Pb } 50.5, \mathrm{CrO}_{2} 1.5, \\
\text { Acid } 1.5\end{array}$ \\
\hline $02 / 04 / 91$ & RHZ-212-A21462 & 09101 & $910001^{\circ}$ & $\begin{array}{l}\mathrm{Pb}, \mathrm{PbCrO}_{2}, \mathrm{Hg}, \mathrm{Ba} \text {, } \\
\mathrm{Cd}\end{array}$ & $\begin{array}{l}\text { D008, WT01, D006, } \\
\text { D009, WC02, D007 }\end{array}$ & $\begin{array}{c}\mathrm{Pb} 7.5, \mathrm{CrO}_{2} 0.04, \\
\mathrm{Ba} 0.01, \mathrm{Cd}^{0.01}, \\
\mathrm{Hg} 0.01\end{array}$ \\
\hline $02 / 04 / 91$ & RHZ-212-A21603 & 09101 & 910002 & $\mathrm{~Pb}, \mathrm{PbCrO}_{2}$ & D008, WT01, D007, & 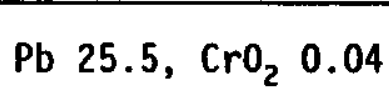 \\
\hline
\end{tabular}


Table 5-41. Summary of Information from Solid Waste Storage Disposal Records for Containers of TRU Mixed Waste. (10 sheets)

\begin{tabular}{|c|c|c|c|c|c|c|}
\hline $\begin{array}{c}\text { Date } \\
\text { accepted }\end{array}$ & $\begin{array}{c}\text { Pin } \\
\text { number }\end{array}$ & $\begin{array}{c}\text { Manifest } \\
\text { number }\end{array}$ & $\begin{array}{l}\text { SWSDR } \\
\text { number }\end{array}$ & $\begin{array}{c}\text { Hazardous } \\
\text { constituents }\end{array}$ & $\begin{array}{l}\text { Waste } \\
\text { codes* }\end{array}$ & $\begin{array}{l}\text { Weight } \\
(\mathrm{kg}) / \%\end{array}$ \\
\hline $\begin{array}{c}28 \\
03 / 21 / 91\end{array}$ & RHZ-212-A21606 & 91037 & 910040 & $\begin{array}{l}\mathrm{Ba}, \mathrm{Cd}, \mathrm{Pb}, \mathrm{PbCrO}_{2}, \\
\mathrm{Hg}\end{array}$ & $\begin{array}{l}\text { D006, D007, D008, } \\
\text { D009, WC02, WTO1 }\end{array}$ & $\begin{array}{c}\text { Ba } 0.01, \text { Cd } 0.01, \\
\text { Pb } 6.49, \mathrm{CrO}_{2} \\
0.08, \mathrm{Hg} 0.01\end{array}$ \\
\hline $05 / 20 / 91$ & RHZ-212-A21763 & 10487 & 910042 & $\begin{array}{l}\mathrm{Pb}, \mathrm{PbCrO}_{2}, \mathrm{Hg}, \mathrm{Ba} \text {, } \\
\mathrm{Cd}\end{array}$ & $\begin{array}{l}\text { D008, WT01, D006, } \\
\text { D009, WC02, D007 }\end{array}$ & $\begin{array}{l}\mathrm{Pb} 7.84, \mathrm{CrO}_{2} \\
0.13, \mathrm{Hg} 0.01, \mathrm{Ba} \\
0.01, \mathrm{Cd} 0.01\end{array}$ \\
\hline $05 / 20 / 91$ & RHZ-212-A21799 & 10487 & 910044 & Lead gloves & D008, WT01 & $34.32 / 25$ \\
\hline $05 / 20 / 91$ & RHZ-213-A21803 & 10487 & 910045 & $\begin{array}{l}\text { Lead, } \mathrm{PbCrO}, \mathrm{Pb} \\
\text { acid, } \mathrm{Hg}, \mathrm{Cd}, \mathrm{Ba}\end{array}$ & $\begin{array}{l}\text { D008, D007, WC02, } \\
\text { WT01, D009, } 0006\end{array}$ & $\begin{array}{c}\mathrm{Pb} 32.31, \\
\mathrm{CrO}_{2} 2.7, \text { Acid } \\
0.01, \mathrm{Ba} 0.01, \\
\mathrm{Hg} 0.01, \mathrm{Cd} 0.01\end{array}$ \\
\hline $05 / 20 / 91$ & RHZ-213-A21768 & 10487 & 910043 & $\begin{array}{l}\text { Lead, } \mathrm{PbCrO}_{2} \text {, lead } \\
\text { acid }\end{array}$ & $\begin{array}{l}\text { D007, D008, WCO2, } \\
\text { WT01, D009 }\end{array}$ & $\begin{array}{l}\mathrm{Pb} 8.43, \mathrm{CrO}_{2} \\
0.025 \text {, Acid } 8.0\end{array}$ \\
\hline $05 / 20 / 91$ & RHZ-213-A21306 & 10487 & 910041 & $\begin{array}{l}\mathrm{Pb}, \mathrm{Hg}, \mathrm{Ba}, \mathrm{Cd}, \\
\mathrm{PbCrO}_{2},\end{array}$ & $\begin{array}{l}\text { D008, WTO1, D006, } \\
\text { D009, WC02, D007 }\end{array}$ & 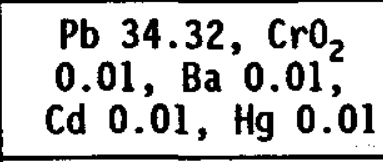 \\
\hline $07 / 25 / 91$ & RHZ-213-A21899 & 10760 & 910234 & $\begin{array}{l}\mathrm{Pb}, \mathrm{PbCrO}_{2}, \mathrm{Hg}, \mathrm{PbOH}, \\
\mathrm{Ba}, \mathrm{Cd}\end{array}$ & $\begin{array}{l}\text { D008, WT01, D006, } \\
\text { D009, WC02, D002 }\end{array}$ & $\begin{array}{c}\mathrm{Pb}, 44.47, \mathrm{CrO}_{2} \\
0.2, \mathrm{PbOH} 0.07 \\
\mathrm{Hg} 0.02, \mathrm{Ba} 0.02, \\
\mathrm{Cd} 0.02\end{array}$ \\
\hline $07 / 25 / 91$ & RHZ-212-A21860 & 10760 & 910231 & Lead (glove, paint) & ABOVE -D008 & $37.1 / 35$ \\
\hline $07 / 25 / 91$ & RHZ-212 A21910 & 10760 & 910232 & $\begin{array}{l}\text { Lead chromate, lead } \\
\mathrm{Hg}, \mathrm{Ba}, \mathrm{Cd} \text {. }\end{array}$ & $\begin{array}{l}\text { D008, WT01, D007, } \\
\text { WC01, WC02, D009, } \\
\text { D006 }\end{array}$ & $\begin{array}{c}\mathrm{Pb} 21.25 \\
\mathrm{CrO}_{2}, \mathrm{Hg} 0.01, \\
\mathrm{Ba} 0.01, \mathrm{Cd} 0.01\end{array}$ \\
\hline
\end{tabular}


Table 5-41. Summary of Information from Solid Waste Storage Disposal Records for Containers of TRU Mixed Waste. (10 sheets)

\begin{tabular}{|c|c|c|c|c|c|c|}
\hline $\begin{array}{l}\text { Date } \\
\text { accepted }\end{array}$ & $\begin{array}{l}\text { Pin } \\
\text { number }\end{array}$ & $\begin{array}{c}\text { Manifest } \\
\text { number }\end{array}$ & $\begin{array}{c}\text { SWSDR } \\
\text { number }\end{array}$ & $\begin{array}{l}\text { Hazardous } \\
\text { constituents }\end{array}$ & $\begin{array}{l}\text { Waste } \\
\text { codes* }\end{array}$ & $\begin{array}{l}\text { Weight } \\
(\mathrm{kg}) / \%\end{array}$ \\
\hline $07 / 25 / 91$ & RHZ-213-A21869 & 10760 & 910233 & $\begin{array}{l}\mathrm{KOH}, \mathrm{Hg}, \mathrm{Ba}, \mathrm{Cd}, \mathrm{Pb} \text {, } \\
\mathrm{PbCrO}_{2}\end{array}$ & $\begin{array}{c}\text { D002, D009, WT01, } \\
\text { D006, WCO1, D008, } \\
\text { WCO2, D007 }\end{array}$ & $\begin{array}{c}\mathrm{KOH} 0.11, \\
\mathrm{Hg} 0.01, \mathrm{Ba} .01, \\
\mathrm{Cd} 0.01, \mathrm{~Pb} 37, \\
\\
\mathrm{CrO}_{2} .1\end{array}$ \\
\hline $07 / 25 / 92$ & RHZ-212-A19730 & PFP08903 & 890497 & $\mathrm{CCl}_{4}:$ TBP absorbed & WTO1, WCO1, WP01 & $10.5 / 28$ \\
\hline $07 / 25 / 92$ & RHZ-212-A19729 & PFP08903 & 890496 & $\mathrm{CCl}_{4}:$ TBP absorbed & WTO1, WCO1, WP01 & $\mathrm{CCl}_{4}: 6.5$ TBP: 4.0 \\
\hline $07 / 25 / 92$ & RHZ-212-A19731 & PFP08903 & 890498 & $\mathrm{CCl}_{4}:$ TBP absorbed & WTO1, WCOl, WPOI & $\mathrm{CCl}_{4}: 6.5 \mathrm{TBP}: 4.0$ \\
\hline
\end{tabular}

${ }^{1}$ Amercoat is a trademark of the American Paint Association.

${ }^{2}$ Waste Codes are from the Washington Administrative Code 173-303.

$\mathrm{PCB}=$ Polychlorinated byphenyl .

$T B P=$ Tributyl Phospate. 


\subsection{REFERENCES}

AEC, 1970, Immediate Action Directive 0511-21, U.S. Atomic Energy Commission, Washington, D.C.

AEC, 1973, Atomic Energy Commission Manual, Chapter 0511, "Radioactive Waste Management," U.S. Atomic Energy Commission, Washington, D.C.

Anderson, B. C., J. D. Anderson, J. A. Demiter, D. R. Duncan, L. A. Fort, D. C. McCann, and S. J. Stone, 1991, Contact-Handled Transuranic Waste Characterization Based on Existing Records, WHC-EP-0225, Revision 1, Westinghouse Hanford Company, Richland, Washington.

Bruns, L. E., 1965, Geometrically Favorable Plutonium Scrap Recovery Plant, CONF-65-0559-10, Atlantic Richfield Hanford Company, Richl and, Washington.

DOE, 1982, Management of Transuranic Material, DOE Order 5820.1, U.S. Department of Energy, Washington, D.C.

DOE, 1987, "Radioactive Waste: By-Products Material Final Rule," Title 10, Code of Federal Regulations, Part 962, 52 Federal Register, 159397-1592, U.S. Department of Energy, Washington, D.C.

DOE, 1988, Radioactive Waste Management, DOE Order 5820.2A, U.S. Department of Energy, Washington, D.C.

DOT, 1989, "Transportation," Title 49, Code of Federal Regulations, Parts 100 to 199, U.S. Department of Transportation, Washington, D.C.

Ecology, 1989, "Dangerous Waste Regulations," Chapter 173-303, Washington Administrative Code, Washington State Department of Ecology, 01 ympia, Washington.

EPA, 1989a, "Interim Status Standards for Owners and Operators of Hazardous Waste Treatment, Storage, and Disposal Facilities," Title 40, Code of Federal Regulations, Part 265, U.S. Environmental Protection Agency, Washington, D.C.

EPA, 1989b, "Spill Table," Title 40, Code of Federal Regulations, Part 302.4, U.S. Environmental Protection Agency, Washington, D.C.

EPA, 1990, "Licensing Requirements for Land Disposal of Radioactive Waste," Title 10, Code of Federal Regulations, Part 61, U.S. Environmental Protection Agency, Washington, D.C.

ERDA, 1975, Final Environmental Statement, Waste Management Operations, Hanford Reservation, ERDA-1538, U.S. Energy Research and Development Administration, Washington, D.C.

$\mathrm{Klem}$, M. J., 1990, Inventory of Chemicals Used at Hanford Site Production Plants and Support Operations (1944-80), WHC-EP-0172, Rev. 1, Westinghouse Hanford Company, Richland, Washington. 
Louk, W. L., 1991, Solid Waste Repackaging and Disposal - PFP Analytical Laboratory, L0-100-323, Rev/Mod C-0, Westinghouse Hanford Company, Richl and, Washington.

Reddinger, R. W., 1992, Reactive and Unstable Chemicals in TRU Retrievable Waste at the Hanford Site -A Review of Available Data, WHC-EP-0603, Westinghouse Hanford Company, Richland, Washington.

Resource Conservation and Recovery Act of 1976, 42 USC 6901 et seq.

RHO, 1985, 200 Areas Fact Book, Rockwell Hanford Operations, Richl and, Washington.

WEC, 1989, TRU Waste Acceptance Criteria for the Waste Isolation Pilot Plant, WIPP-DOE-069, Revision 3, Westinghouse Electric Corporation, Carlsbad, New Mexico.

WHC, 1991, Plutonium Finishing Plant Safety Analysis Report, WHC-SD-CP-SAR-021, Revision 0, Westinghouse Hanford Company, Richland, Washington.

Willis, N. P., and Triner, 1991, Hanford Site Solid Waste Acceptance Criteria, WHC-EP-0063-3, Westinghouse Hanford Company, Richland, Washington. 
WHC-EP-0621

(from WHC 1991)

APPENDIX A

PFP FLOWSHEETS

A-1 
WHC-EP-0621

(from WHC 1991)

This page intentionally left blank. 
WHC-EP-0621

(from WHC 1991)

\section{PLUTONIUN FINISHING PLANT FLONSHEETS}

\subsubsection{Flowsheets}

This section provides flow diagrams showing flow of materials and material balances for the plutonium handling and storage, plutonium recovery, and plutonium conversion operations. Most of the processes are "batch" operations; consequently, material balances are based on normal "batch" size.

No heat balances are provided since the amount of heat varies depending on the amount and type of materials being processed. The number of processes where heat is input is relatively small. The heat input is controlled by automatic temperature controllers. Most of the heat generated is dissipated to the ventilation system, primarily to the E-4 (offgas) system and thence discharged to the atmosphere via the $200-\mathrm{ft}$ stack. Cooling water is provided to the following pieces of process equipment:

1. Reflux condensers in MT-5 in the PRF. The amount of heat generated by the heating plates varies over a wide range depending on the time a particular batch of recoverable material is heated. These operations are temperature controlled $\left(\sim 105^{\circ} \mathrm{C}\right)$ and sufficient cooling water is supplied to condense all the water vapor and other condensibles.

2. The concentrator condensers (two) in the PRF are supplied with cooling water sufficient to condense the water vapors from the solutions being concentrated. The concentrators are steam heated and controlled to a temperature $\left(\sim 110^{\circ} \mathrm{C}\right)$; the amount of heat input vartes over a wide range depending on plutonium concentration in the feed and the plutonium concentration desired in the product.

3. The MT vacuum pump has a closed-loop cooling system. A fixed flow (20\% of full scale) is set and then is not changed during operation. The heat is dissipated to the protected process water (PPW) system which discharges to the Z-20 crib.

4. Most of the process cell organic tanks' recirculating lines are equipped with tube-in-tube cooling water jackets. These jackets are used to remove heat added by recirculating pumps and radiolysis. Recirculation is used to prevent precipitation and minimize concentration gradient (piutonium polymer can form at low acid concentration or high temperatures). The amount of heat generated varies depending on circulation rates, time of operation, and concentration of plutonium. Water flow is set at a constant rate, sufficiently high to remove the generated heat.

5. The copper induction colls for the reduction furnaces in glovebox HC-15 are provided with cooling water flowing at a fixed rate. The total amount of heat input to this stream also varies between batches depending on batch size and how much heat is dissipated by the offgas ventilation system. 
6. The FLUR KOH scrubber solution is cooled by a closed-loop cooling systen. A secondary cooling loop dissipates the heat to the plant cooling water which discharges to the $\mathrm{z}-20 \mathrm{crib}$. The amount of heat generated varies depending on the amount of HF reacting with the $\mathrm{KOH}$. This also varies depending on the feedrate of $\mathrm{PuO}_{2}$ to the FLUR and the amount of downtime of the FLUR while HF is flowing. Adequate cooling is provided to assure that $\mathrm{KOH}$ scrubber solution is maintained below $79^{\circ} \mathrm{C}$.

Flowsheets are seldom required in the PPSL. Most of the projects are small chemistry denonstrations done in batch steps. Many of the processing steps cannot be decided until the results of the previous step are determined. Larger continuous experiments frequently demonstrate process flowsheets planned for use in production facilities. These planned flowsheets are demonstrated either full sized or scaled down to pilot plant size, where the principles of the operations can be demonstrated at a smaller scale. Flowsheets are incorporated into test plan documents unless the flowsheet is so complex that a separate document is required.

6.1.2.1 Fisstle Matorial Receiving, Handling, Shipping, and Storage. Fissile material is received at the PFP at Bullding 2736-ZC, the loading dock at the 2736-Z Support Facility. The single exception is the PUREX plutonium nitrate received in PR cans at 234-ZC, the loading dock at the west side of Building 234-5Z.

The overall schematic flow diagram for dry ftssile material handling at PFP is shown in Figure 6.1-6.

The schematic flow diagram for receipt and $L I / L O$ of plutonium nitrate solution from PUREX and PRF ts shown in Figure 6.1-7.

6.1.2.2 Plutoniun Recovery Flowsheet. The PRF uses a continuous SX process to convert plutonium-bearing materials to a concentrated plutonium nitrate product suitable for converstion to plutonium metal. Feed to the SX process is obtained from the dissolution plutonium-recoverable materials, filtrate from the RMC Line, and other aqueous solutions. This section describes the process flowsheets and material balances for each process. ${ }^{(6.9,6.8)}$

Figures 6.1-8 and 6.1-9 show the overall flow of plutonfum-recoverable material to provide feed for SX. Precise material bal ances vary depending on the feed material. Figures $6.1-10,6.1-11$, and $6.1-12$ show typical flowsheets for hydrolysis (glovebox HC-60), scrap dissolution (MT-5), and solution preparation clarification (MT-6), respectively. A flowsheet for ash calcining (glovebox HA-40F) is not shown since it is normally not required and both it and glovebox HA-46F are not operational. Glovebox HC-60 al so is currently not operational due to a not plate temperature controller problem but a fix may be relatively simple. Both HC-60 and MT-5 rely on hot plate control systems to limit solution temperature. The solution temperature is measured and the power to the hot plate is shut off on high temperature or low deviation on heat-up rate. 
WHC-EP-0621

from WHC 1991)

Figure 6.1-13 shows the flowsheet for dissolution of a typical lot of S\&C material in the S\&C dissolvers. Note there are two reaction rates, a fast dissolution rate of the calcium and iron components followed by a slow dissolution rate of the materials in the crucible and sand.

Each SaC dissolver has two interlock circuits. One is on the differential pressure (DP) between the protected process cooling water (PPCW) and the dissolver process. On low DP, the PPCW pump is shut off and vaives shut to isolate the cooling jacket. The other is on the charging ball valves. Both valves cannot be opened simultaneously without bypassing this interlock circuit. In that case, the S\&C bypass interlock drains the dissolver to assure the S\&C system, including the S\&C glovebox, cannot be pressurized by inadvertently charging a can with both vaives open and acid in the dissolver.

Figure 6.1-14 shows a schematic diagram of the feed preparation tankage for plutonium reclamation. The crude feed composition will vary over a wide range but generally will not exceed those shown in Figure 6.1-15. The composition is adjusted by adding appropriate amounts of $\mathrm{HNO}_{3}, \mathrm{ANN}$, and an anti-emulsifying agent to the feed to optimize CA column operation. Safety instrumentation of importance include the $\mathrm{CCl}_{4}$ detector in the chemtcal makeup room 40 and tankage liquid volume detectors (WF).

A typical SX flowsheet (currently in use) for the entire process system is shown in Figure 6.1-15. Included are the subsystems for chemical makeup, feed preparation, plutonium extraction and stripping, plutonium product concentration solvent cleanup, and waste handing (inciuding filtrate and miscellaneous recycle stream concentration). The actual composition and flowrates of the various streams will vary from those shown, depending on the feed composition and the actual operation of the various columns. Part of the CCP (product) strean from the top of the CC column is recycled as CAIS to the CA column and part of the stream is bled off as feed to the product concentrator. This recycling operation increases the concentration of plutonium in the product stream up to about $60 \mathrm{~g} / \mathrm{L}$, thereby reducing the load on the product concentrator. 
Figure 6.1-6. 2736-Z Support Facility Solid Fissile Material Handing Flow Diagram.

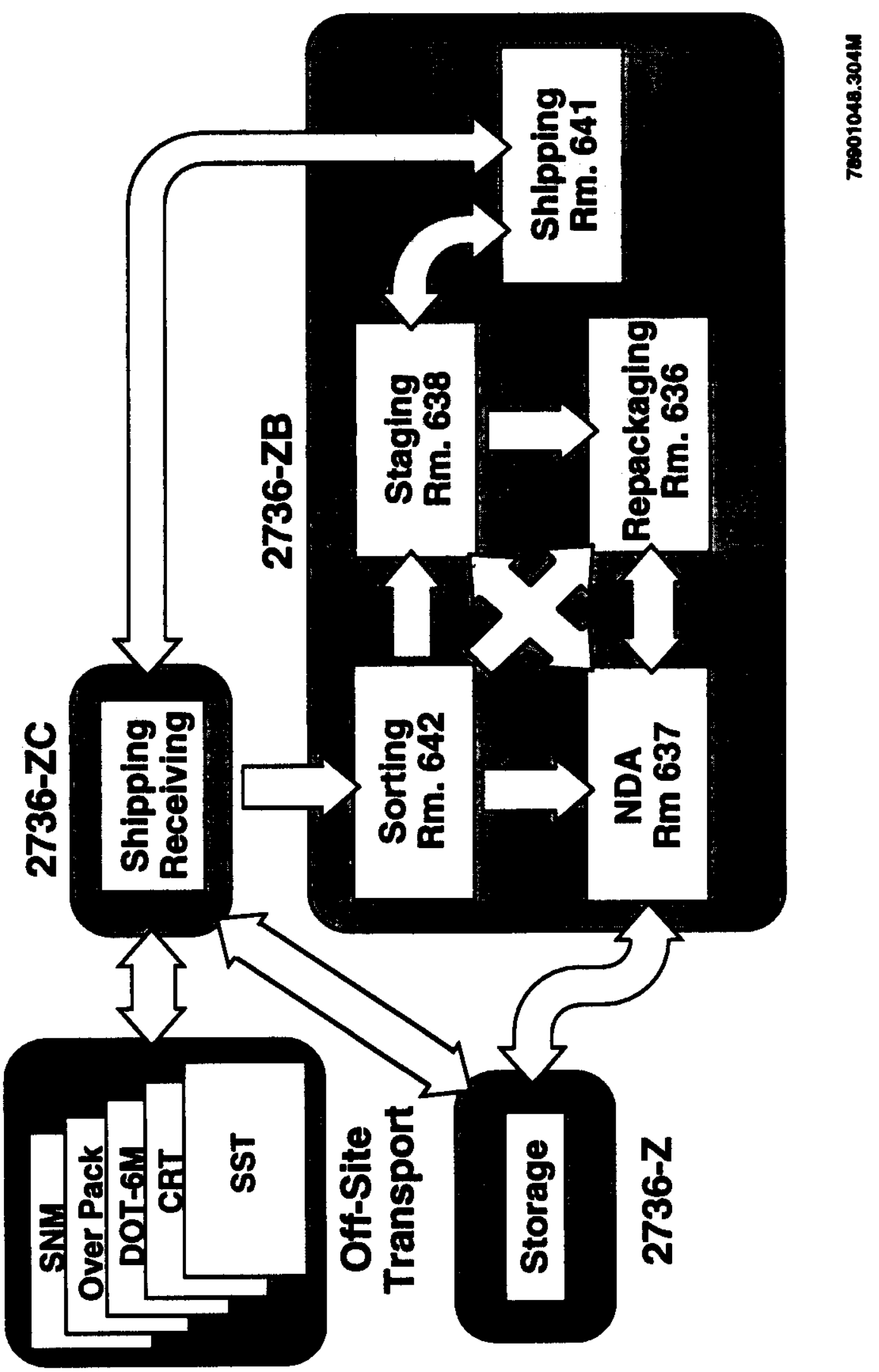


WHC-EP-0621

(from WHC 1991)

Figure 6.1-7. Load-In/Load-Out Operations Flow Diagram.

RMC

Load In Concentrated Plutonium Nitrate Solutions

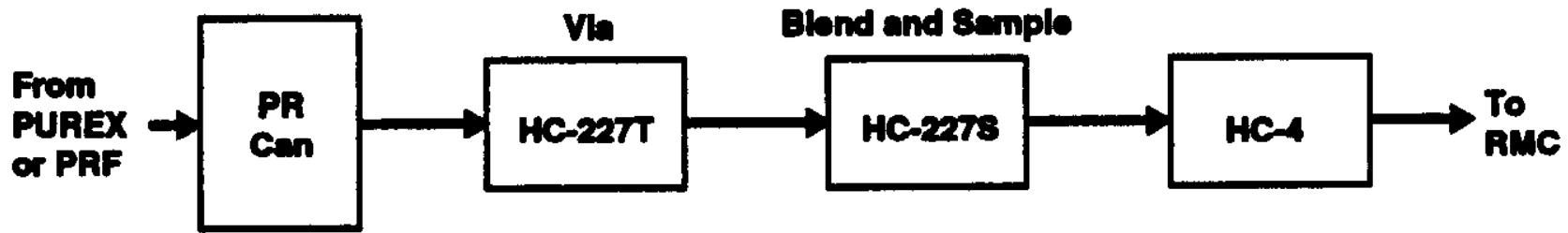

Load Out Dilute Solutions

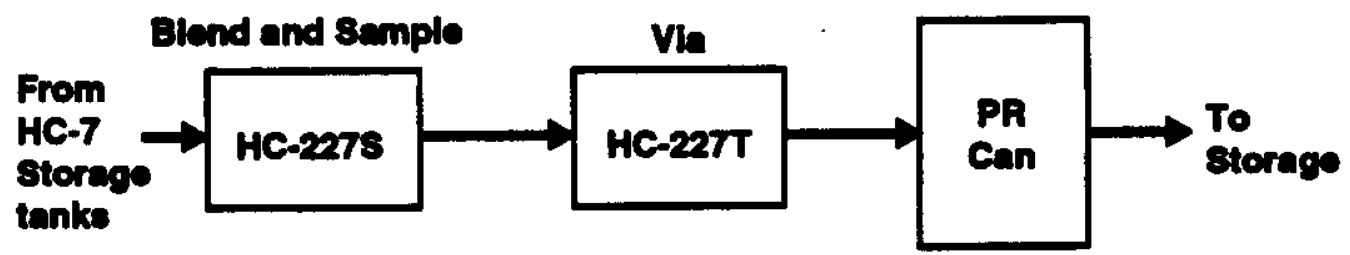

\section{PRF}

Load In Concentrated Filtrate or Other Dilute Plutonium Nitrate Solutions

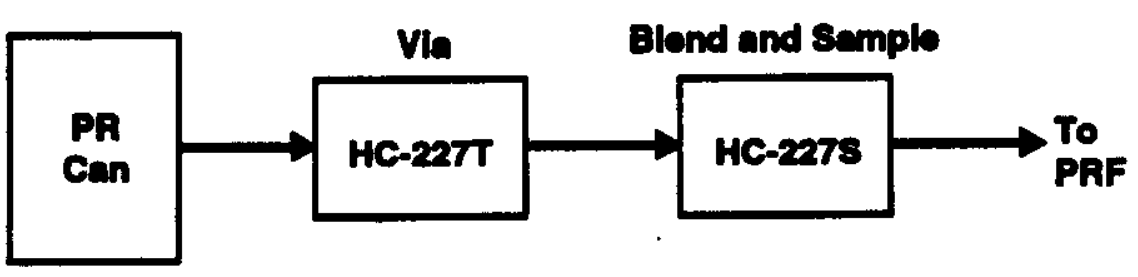

Load Out Concentrated Plutonlum Nitrate or Filtrate Solutions

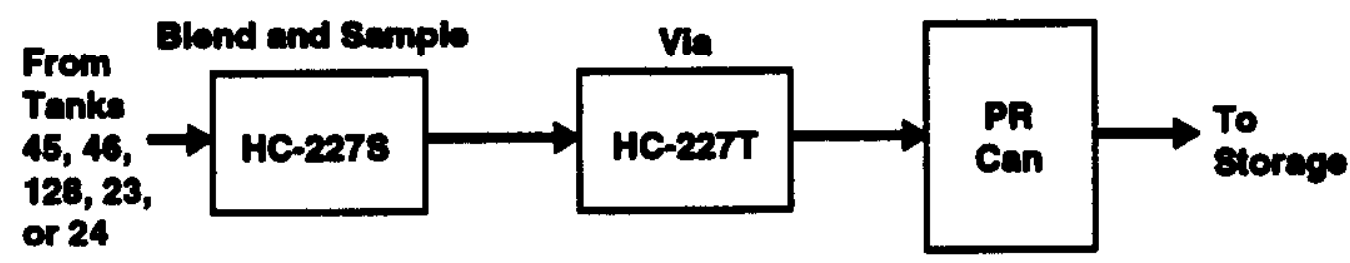

$79901040.302 M$ 
WHC-EP-0621

(from WHC 1991)

Figure 6.1-8. Recoverable Material Dissolution Flowsheet.

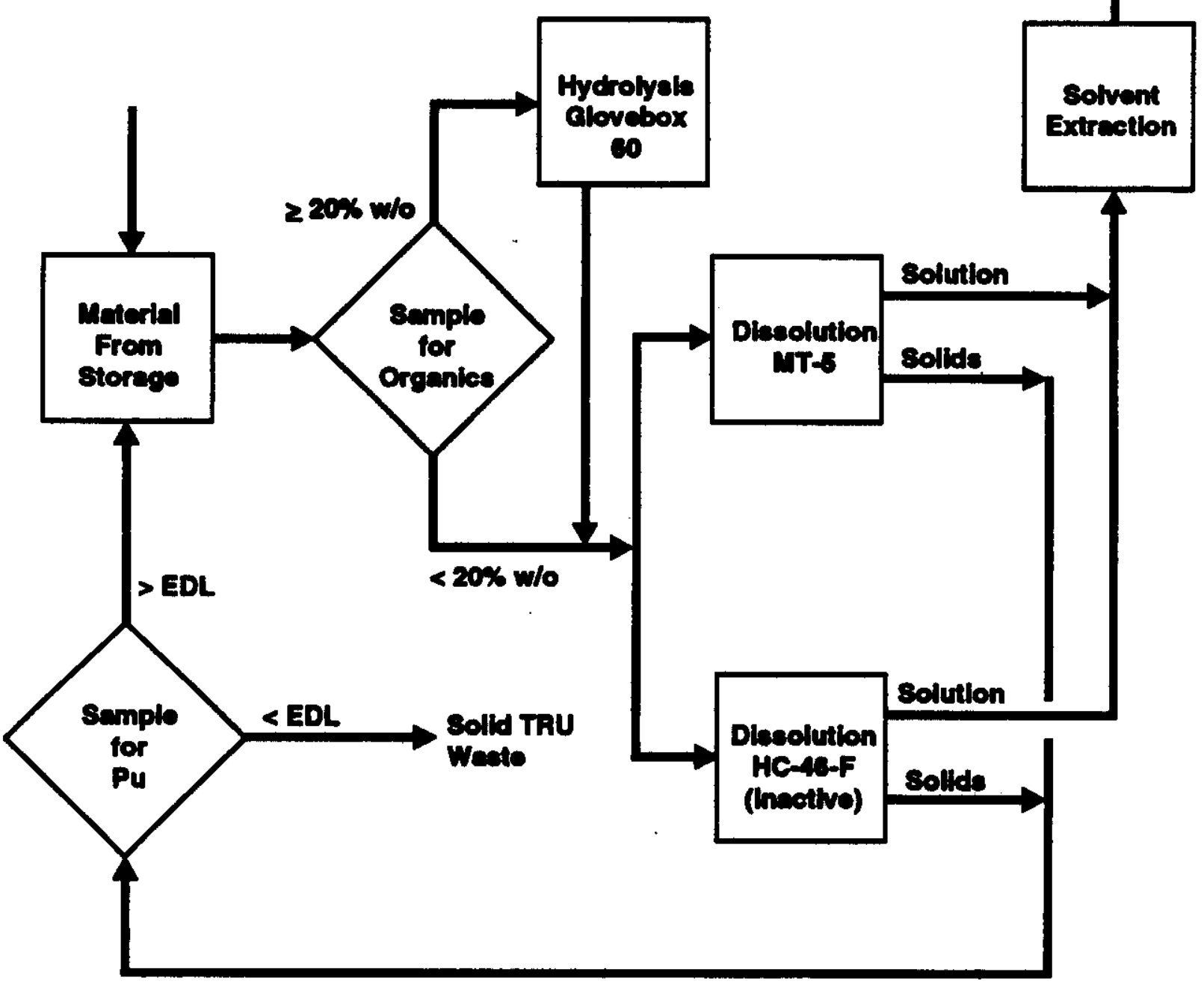

7ocotonsesou 


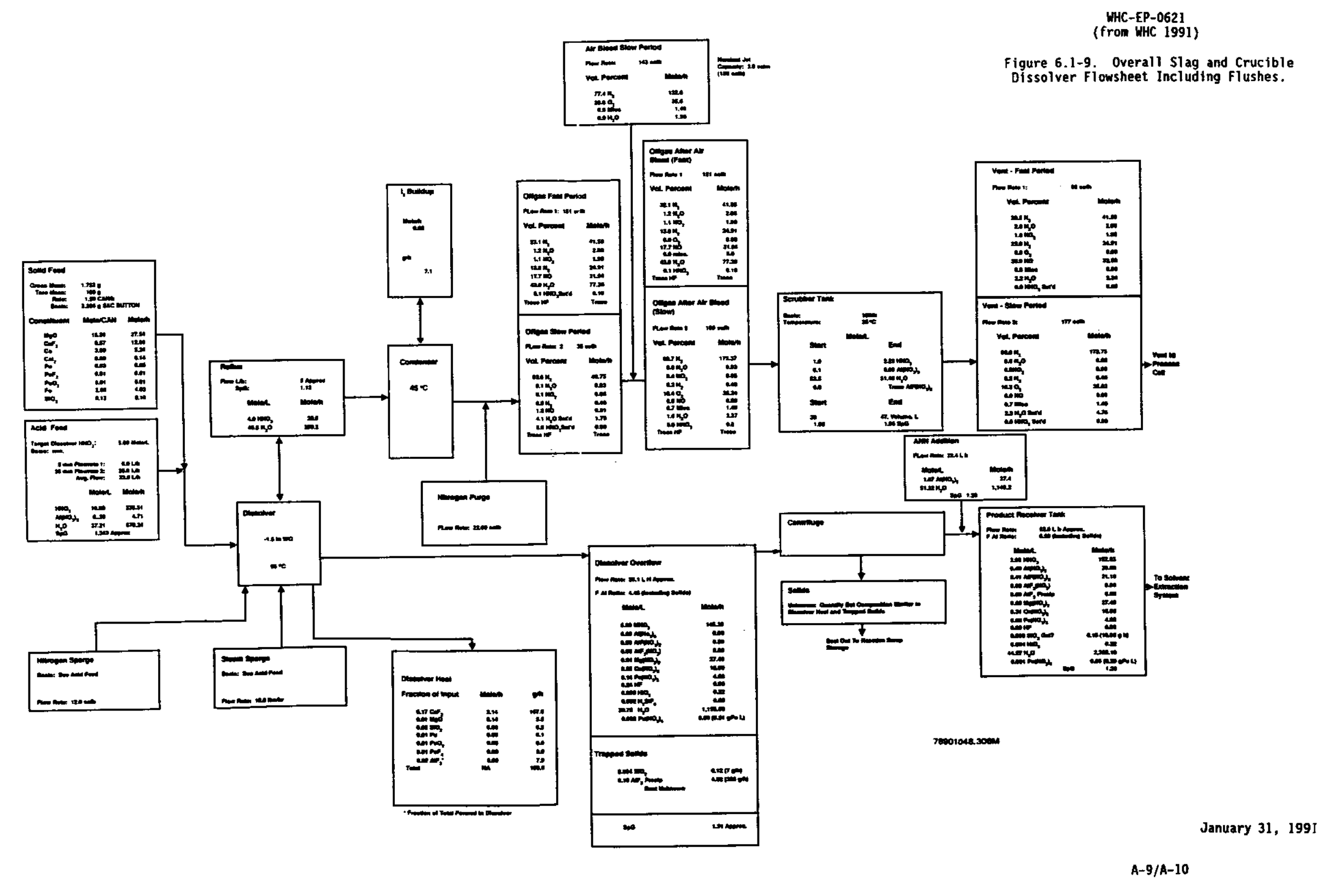


Figure 6.1-10. Glovebox 60 Flowsheet.

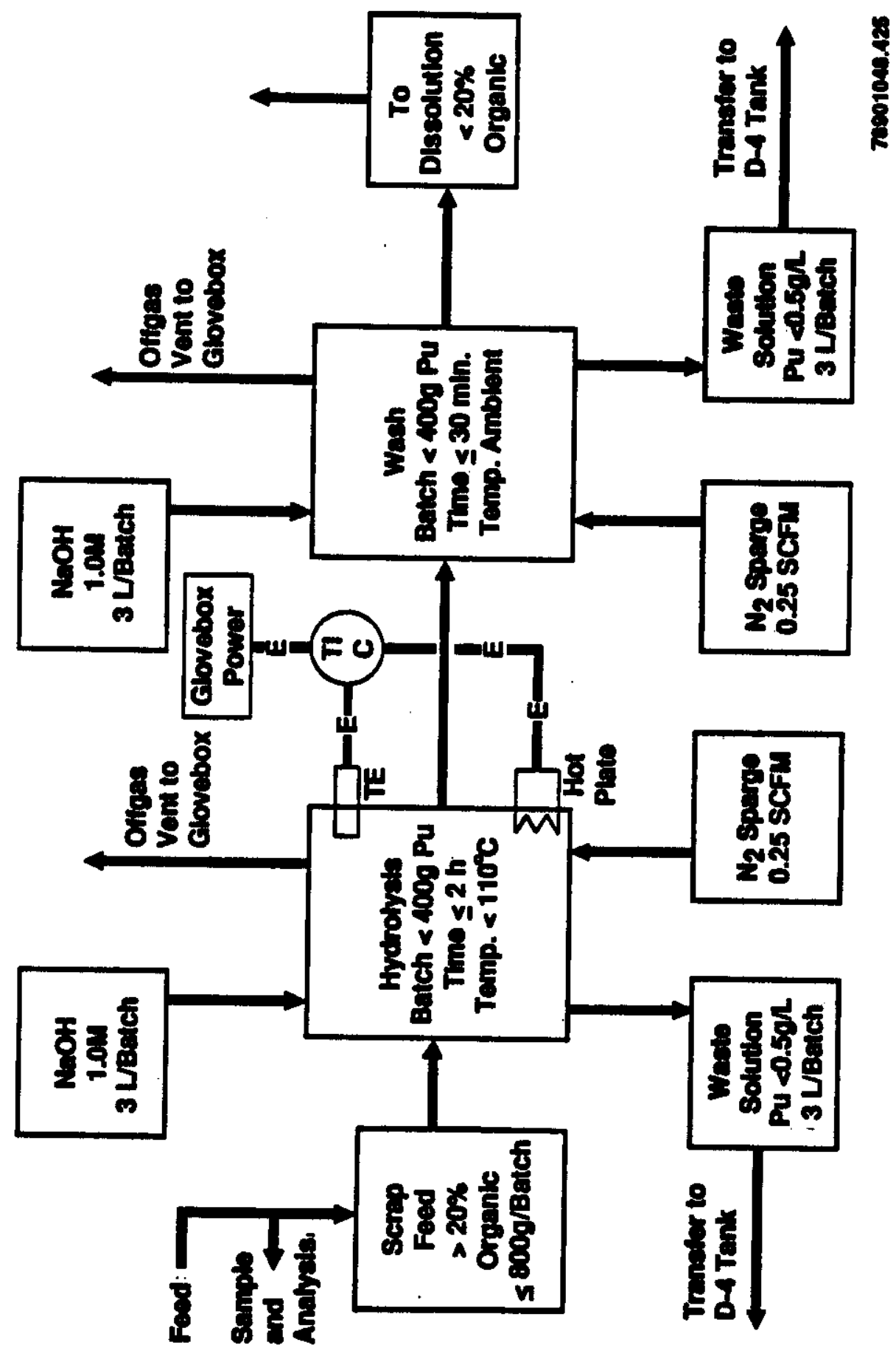


WHC-EP-0621

(from WHC 1991)

Figure 6.1-11. Miscellaneous Treatment Glovebox 5 Flowsheet.

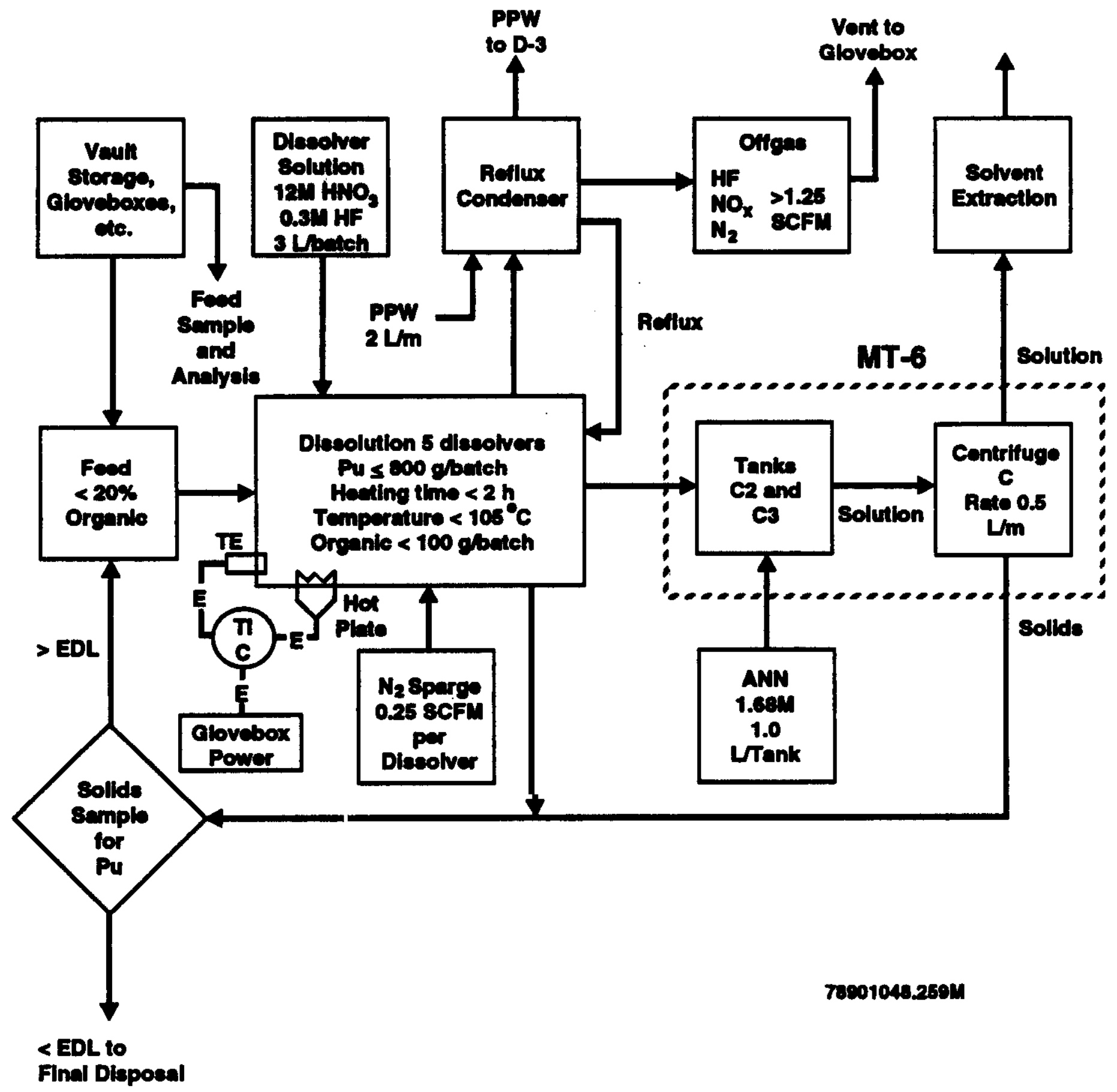


Figure 6.1-12. Miscellaneous Treatment Glovebox 6.

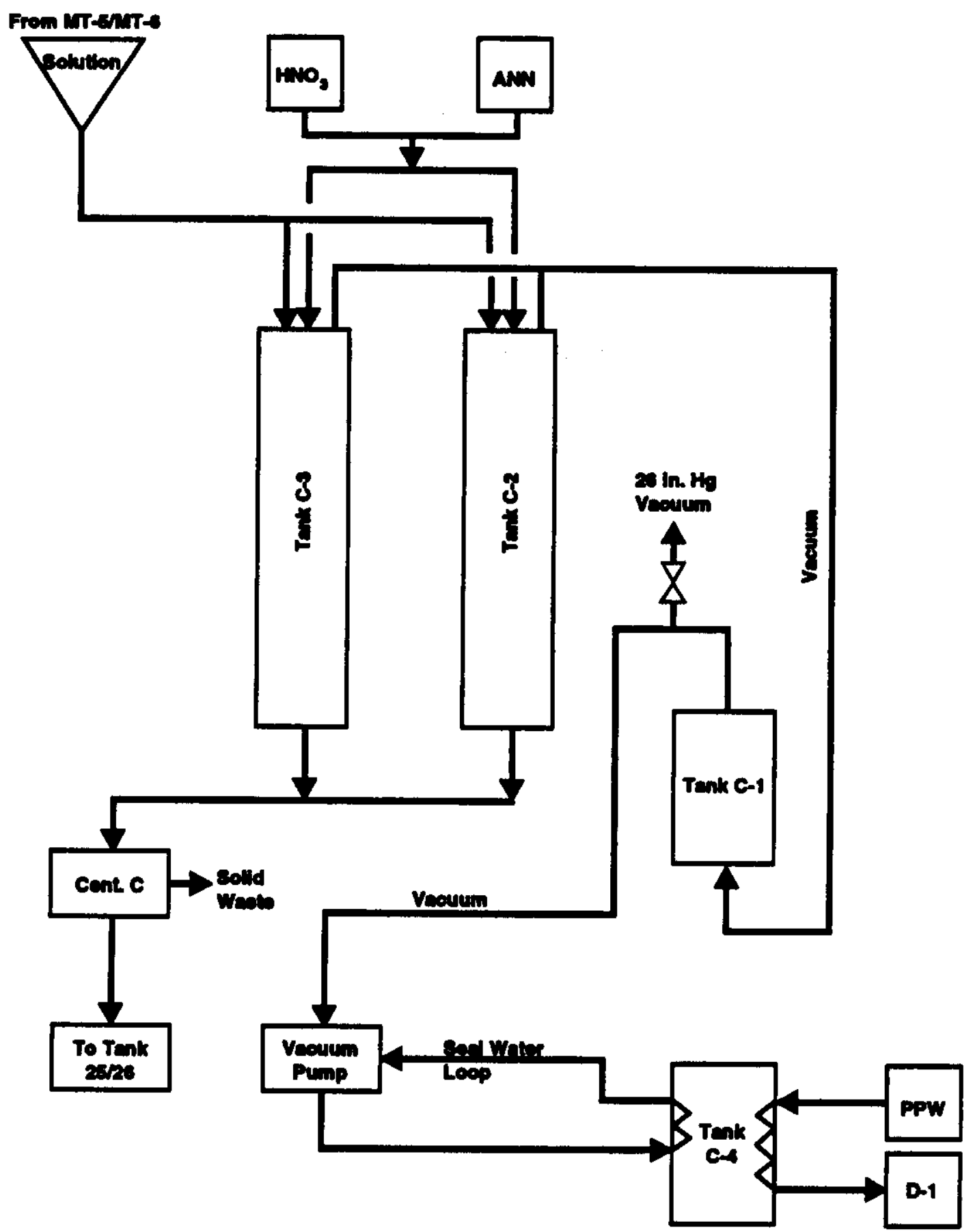

70010492074 
WHC-EP-0621

(from WHC 1991)

This page intentionally left blank. 


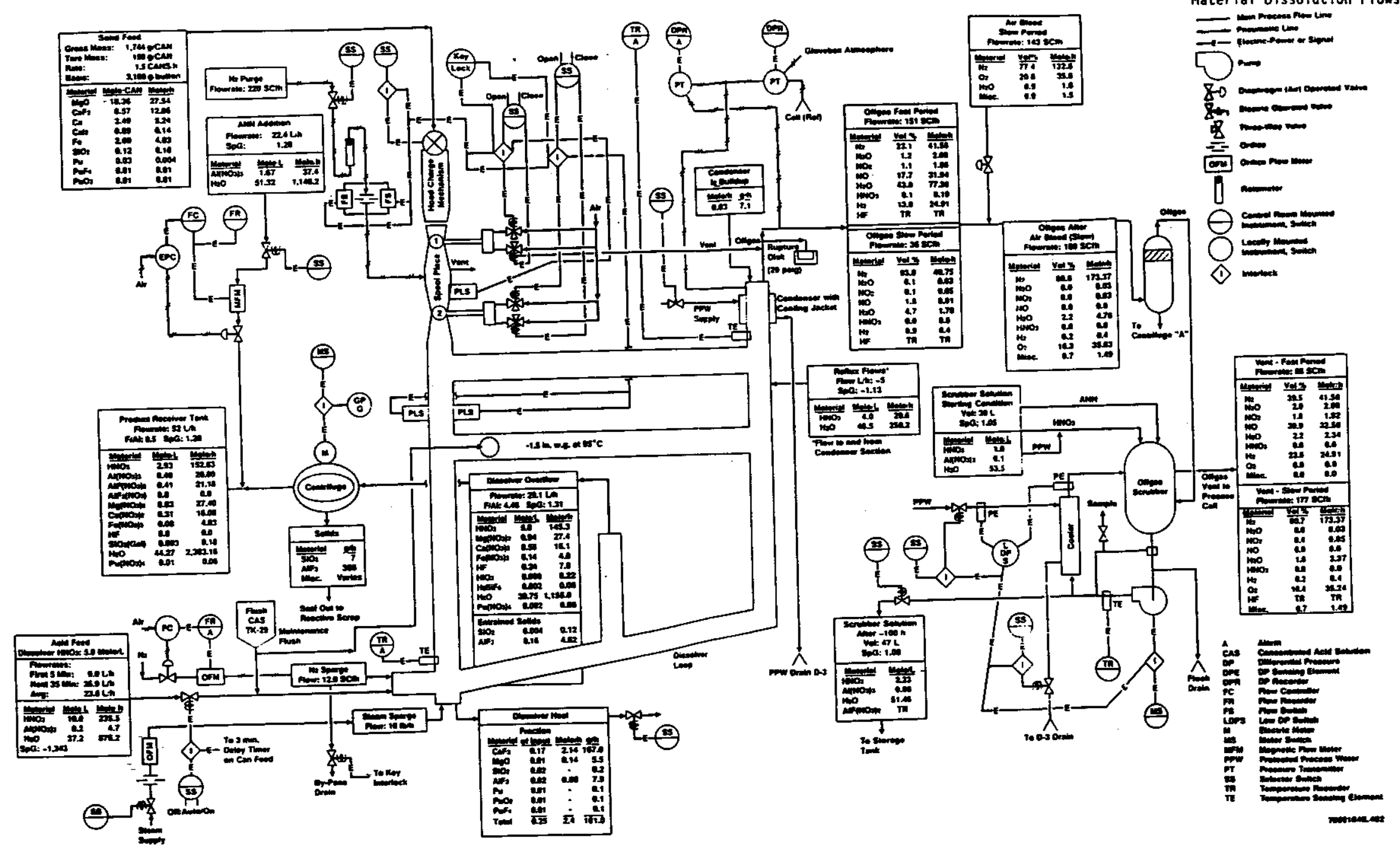


WHC-EP-0621

(from WHC 1991)

Figure 6.1-14. Flow Sketch Feed Preparation Process.

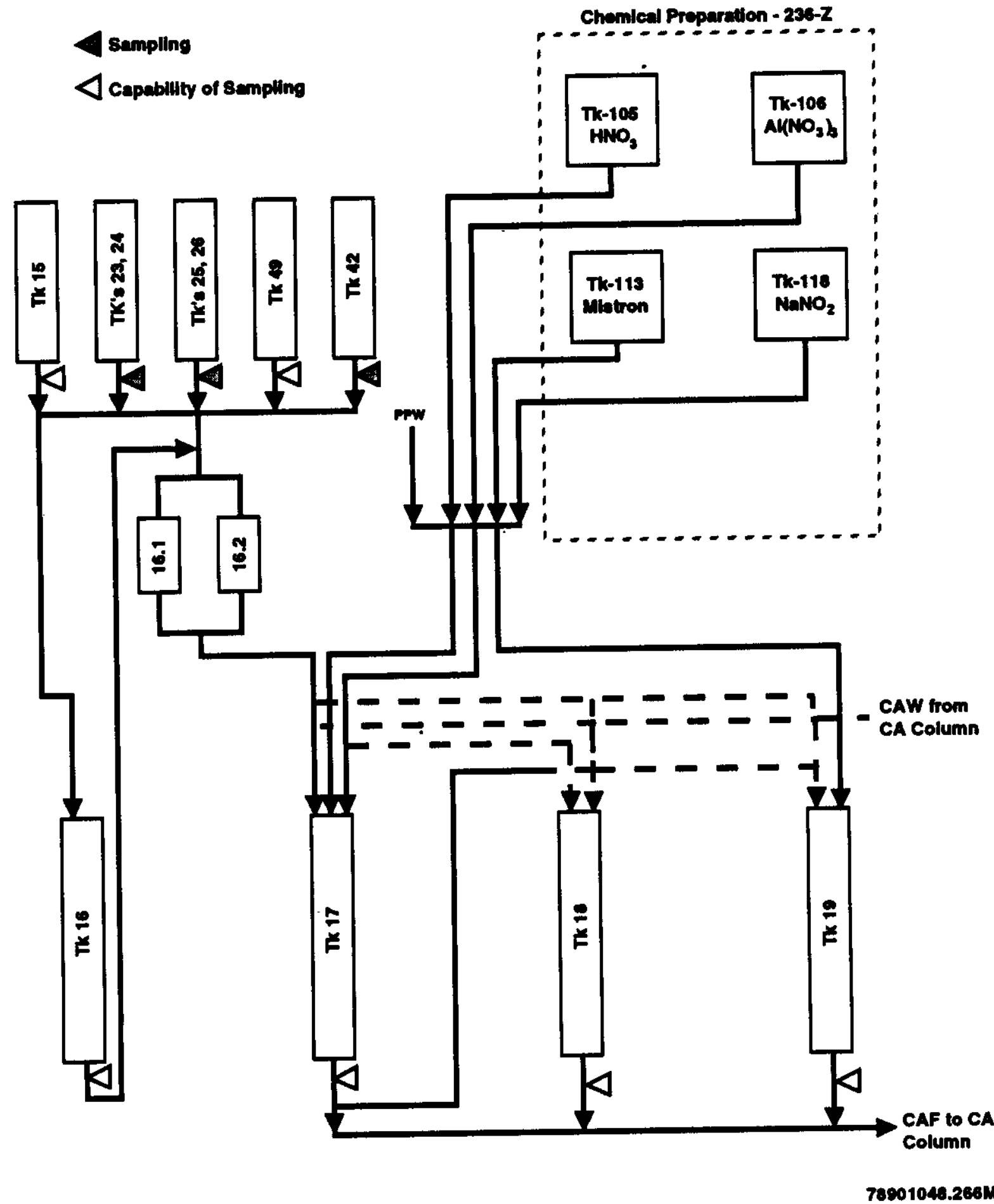


WHC-EP-0621

(from WHC 1991)

This page intentionally left blank. 


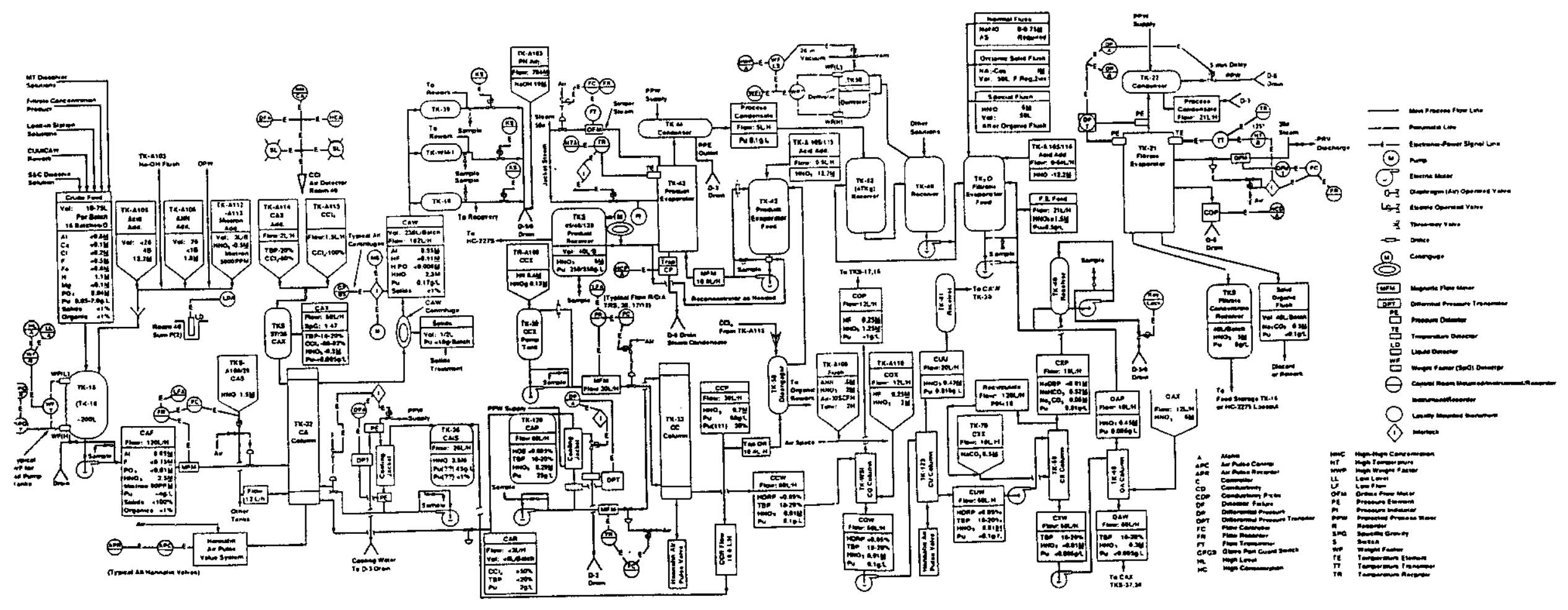


Important safety systems are shown in Table 6.1-1 and are 11lustrated in Figure 6.1-15. Table 6.5-2 provides a listing of safety class instrumentation for PRF. Table 6.1-2 lists primary PRF systems important to product quality.

An al ternate flowsheet, shown in Figure 6.1-16 and in PFD-2-180-00002, was used in the recent past for coextraction of uranium and plutonium in the CA column. ${ }^{\text {(6.2) }}$ The plutonium and uranium were separated by preferentially stripping the plutonium from the TBP in the CC column, with the uranium remaining in the organic phase to be stripped later and discarded. The separation is accomplished by reducing the plutonium from its normal tetravalent state to the trivalent state by the use of HN. The trivalent plutonium is easily stripped into a dilute $\mathrm{HNO}_{3}-\mathrm{NH}_{2} \mathrm{NH}_{2}$ solution.

At present, tankage is not available to operate with the alternate flowsheet. However, if needed, tankage can be realigned or replaced as needed for this alternate flowsheet or other possible flowsheets.

The plutonium product from the $\mathrm{CC}$ column is concentrated by evaporation. The flowsheet for product concentration is included in Figure 6.1-17. To reduce the possibility of nitrated organic-plutonium compounds from being formed during product concentration, the CCP stream is first contacted with clean $\mathrm{CCl}_{4}$.

Table 6.1-3 shows a listing of the drawing numbers of the piping and instrumentation diagrams (P\&ID) for the in-cell tanks. Table 6.1-4 shows the list for the associated out-of-cell tanks, and Table 6.1-5 shows the list for the chemical preparation tanks.

The P\&IDs for MT tanks in the PRF are shown in Drawings H-2-28013 and $\mathrm{H}-2-27984$.

Typical plutonium inventories at various locations are in Table 6.1-6. 
Table 6.1-1. Primary Plutonium Reclamation Factlity Safety Systems.

\begin{tabular}{|c|c|c|}
\hline System & Sample point & Control point \\
\hline TK-50 Hi WF interlock & $\begin{array}{l}\text { Level instrument - } \\
50 \text { WFT A }\end{array}$ & $\begin{array}{l}\text { Vacuum/vent valve-EMV } 50 \\
\text { D }\end{array}$ \\
\hline $\begin{array}{l}\text { TK-36 Cooling water } \\
\text { jacket DP interlock }\end{array}$ & $\begin{array}{l}\text { DP Cell on cooling } \\
\text { jacket - } 36 \text { DPT }\end{array}$ & $\begin{array}{l}\text { Relay to shut off } \\
36 \text { pump }\end{array}$ \\
\hline $\begin{array}{l}\text { TK-120 Cooling water } \\
\text { jacket DP interlock }\end{array}$ & $\begin{array}{l}\text { DP Cell on cooling } \\
\text { jacket - } 120 \text { DPT }\end{array}$ & $\begin{array}{l}\text { Relay to shut off } \\
120 \text { pump }\end{array}$ \\
\hline $\begin{array}{l}\text { TK-43 Hi temperature } \\
\text { interlock }\end{array}$ & $\begin{array}{l}\text { Temperature probe in TK- } \\
43-43 \mathrm{~T}\end{array}$ & $\begin{array}{l}\text { Steam supply valve } \\
\text { DOV } 43 \mathrm{C} \text {. }\end{array}$ \\
\hline $\begin{array}{l}\text { TK-43 Steam jacket } \\
\text { conductivity alarm }\end{array}$ & $\begin{array}{l}\text { Conductivity probe in } \\
\text { condensate line-C } 43 \mathrm{E}\end{array}$ & Alarm in control room \\
\hline $\begin{array}{l}\text { TK-21 Hi temperature } \\
\text { interlock }\end{array}$ & $\begin{array}{l}\text { Temperature probe in TK- } \\
21-\mathrm{TE} 21 \mathrm{C}\end{array}$ & $\begin{array}{l}\text { Steam supply valve } \\
\text { DoV } 21 \mathrm{E}\end{array}$ \\
\hline $\begin{array}{l}\text { TK-21 Steam jacket } \\
\text { conductivity alarm }\end{array}$ & $\begin{array}{l}\text { Conductivity probe in } \\
\text { condensate } 1 \text { ine-C } 21 \text { E }\end{array}$ & Alarm in control room \\
\hline $\begin{array}{l}\text { TK-21 Process condensate } \\
\text { diversion }\end{array}$ & $\begin{array}{l}\text { Demister DP cel1-21 } \\
\text { DPT D }\end{array}$ & $\begin{array}{l}\text { EMV on Process } \\
\text { condensate 1ine - } \\
\text { EMV } 21 \text { D }\end{array}$ \\
\hline $\begin{array}{l}\text { Chemical preparation } \\
\mathrm{CCl}_{4} \text { monitor }\end{array}$ & By TK-A115 & $\begin{array}{l}\text { Alarm/light in chemical } \\
\text { preparation and control } \\
\text { room }\end{array}$ \\
\hline CAN Discharge to TK-D5 & $\begin{array}{l}\text { CAW Tank sample valves } \\
-251,-275 \text {, and }-291\end{array}$ & $\begin{array}{l}\text { Key operated discharge } \\
\text { valves-EMVs MH-1 A, } \\
-19 \mathrm{~A} \text {, and }-39 \mathrm{G} \text {, }\end{array}$ \\
\hline
\end{tabular}

Table 6.1-2. Plutonium Reclamation Facility Product Quality Systems.

\begin{tabular}{|l|l|l|}
\hline \multicolumn{1}{|c|}{ System } & \multicolumn{1}{|c|}{ Sample point } & \multicolumn{1}{c|}{ Control point } \\
\hline CCX Makeup & CCX Tank sample valve & $\begin{array}{l}\text { Makeup Plant Operating } \\
\text { Procedure and D butting } \\
\text { instructions }\end{array}$ \\
\hline $\begin{array}{l}\text { Feed tank salting } \\
\text { strength }\end{array}$ & Tank 17 SpG monitor & Feed DOV \\
\hline Feed tank acid & Tank conductivity probe & Acid addition EMV \\
\hline $\begin{array}{l}\text { Recycle tank acid } \\
\text { concentration }\end{array}$ & Flowrate charts & $\begin{array}{l}\text { Tank 36 acid addition } \\
\text { controller }\end{array}$ \\
\hline $\begin{array}{l}\text { All process stream flow } \\
\text { controller }\end{array}$ & Flow element & Flow control valve \\
\hline
\end{tabular}


Figure 6.1-16. Plutonium-Uranium Coextraction Partitioning.

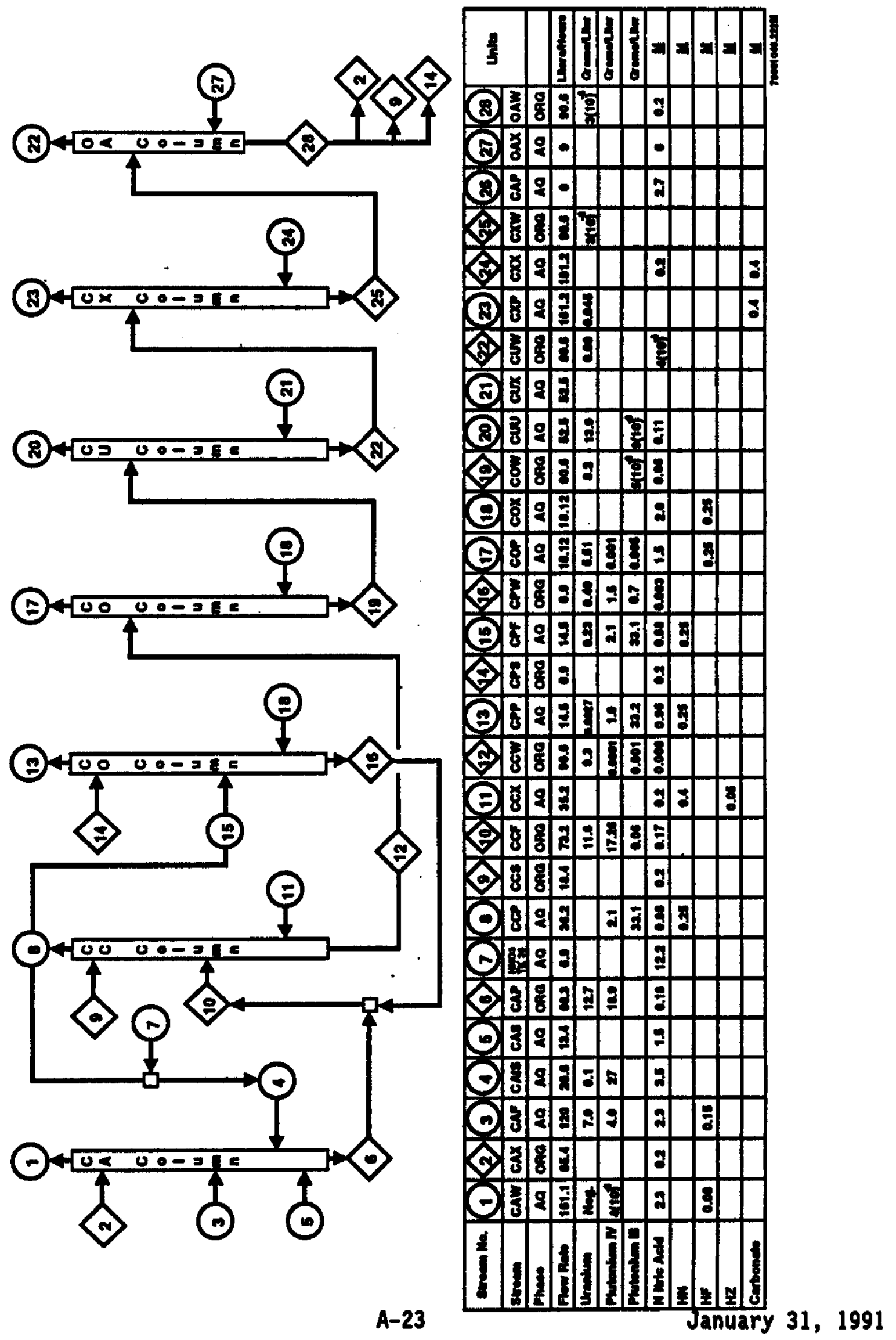


WHC-EP-0621
(from WHC 1991)

Figure 6.1-17. Product Concentration Flowsheet:

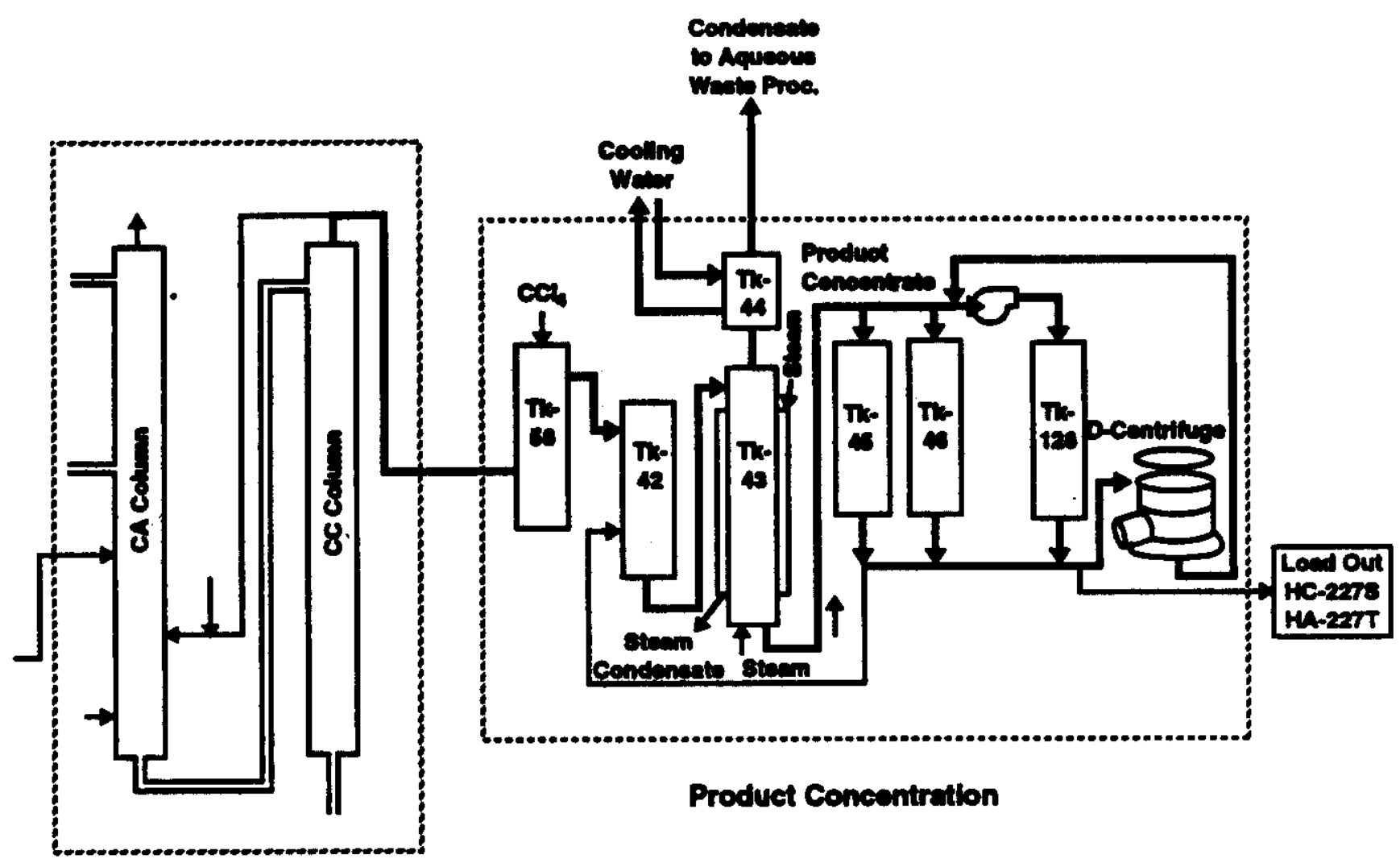

Plutonium Extraction

and stripping

70001945.191M 
WHC-EP-0621

(from WHC 1991)

Table 6.1-3. Drawing List of Plutonium Reclamation Facility In-Cell Tanks Piping and Instrumentation Diagrams. (2 sheets)

\begin{tabular}{|c|c|c|c|}
\hline Tank & Position & Designation & System PEFS \\
\hline TK-08 & EC & Dissolver & $\mathrm{H}-2-93533$ \\
\hline TK-09 & EC & Condenser & $\mathrm{H}-2-93533$ \\
\hline TK-10 & EG & Dissolver offgas scrubber & $\mathrm{H}-2-93496$ \\
\hline TK-12 & EH & Dissolver offgas scrubber & $\mathrm{H}-2-93496$ \\
\hline TK-15 & EJ & Dissolver receiver, filtrate receiver & $\mathrm{H}-2-28022$ \\
\hline TK-16 & EK & Storage vessel & $\mathrm{H}-2-28022$ \\
\hline TK-17 & WG & CAF Make-up & $\mathrm{H}-2-28021$ \\
\hline TK-18 & WH & CAF & $\mathrm{H}-2-28021$ \\
\hline TK-19 & WJ & Waste receiver & $\mathrm{H}-2-28041$ \\
\hline TK-20 & ES & Filtrate evaporator feed & $\mathrm{H}-2-28026$ \\
\hline TK-21 & ET & Filtrate evaporator & $\mathrm{H}-2-28026$ \\
\hline TK-22 & ET & Condenser & $\mathrm{H}-2-28026$ \\
\hline TK-23 & ER & Filtrate concentrator receiver & $\mathrm{H}-2-28026$ \\
\hline TK-24 & EP & Filtrate concentrator receiver & $\mathrm{H}-2-28026$ \\
\hline TK-25 & EN & Miscellaneous storage & $\mathrm{H}-2-28023$ \\
\hline TK-26 & EM & Miscellaneous storage & $\mathrm{H}-2-28023$ \\
\hline TK-27 & EL. & Organic recycle & $H-2-28018$ \\
\hline TK-29 & WD & CAS Pump & $\mathrm{H}-2-28015$ \\
\hline TK-30 & WC & CCX Pump & $\mathrm{H}-2-28019$ \\
\hline TK-32 & WD & CA Column & $\mathrm{H}-2-28019$ \\
\hline TK-33 & WC & CC Column & H-2-28019 \\
\hline TK-36 & WT & CAIS Pump & $\mathrm{H}-2-28019$ \\
\hline TK-37 & WM & Organic pump & $\mathrm{H}-2-20817$ \\
\hline TK-38 & WN & Organic pump & H-2-20817 \\
\hline TK-39 & WL & Wash receiver & $\mathrm{H}-2-28040$ \\
\hline TK-40 & WP & Wash receiver & H-2-28036 \\
\hline TK-41 & WK & Wash receiver & $\mathrm{H}-2-28036$ \\
\hline TK-42 & WS & CCP Evaporator feed & $\mathrm{H}-2-28027$ \\
\hline TK-43 & WR & CCP Stripper evaporator & H-2-28027 \\
\hline
\end{tabular}


WHC-EP-0621

(from WHC 1991)

Table 6.1-3. Drawing List of Plutontum Reclamation Facility In-Cell Tanks Piping and Instrumentation Diagrams. (2 sheets)

\begin{tabular}{|c|c|l|c|}
\hline Tank & Position & \multicolumn{1}{|c|}{ Designation } & System PEFS \\
\hline TK-44 & WR & Condenser & $\mathrm{H}-2-28027$ \\
\hline TK-45 & WS & CCP PR & $\mathrm{H}-2-28027$ \\
\hline TK-46 & WT & CCP PR & $\mathrm{H}-2-28027$ \\
\hline TK-49 & WV & Miscell aneous solution receiver & $\mathrm{H}-2-28016$ \\
\hline TK-50 & WZ & Vacuum receiver & $\mathrm{H}-2-28016$ \\
\hline TK-69 & WU & CX Colunn & $\mathrm{H}-2-28020$ \\
\hline TK-70 & WU & Wash receiver & $\mathrm{H}-2-28036$ \\
\hline TK-120 & WF & CAP Pump tank & $\mathrm{H}-2-28019$ \\
\hline TK-123 & WF & CU Column & $\mathrm{H}-2-28020$ \\
\hline TK-124 & ED & Dtssolver (spare) & $\mathrm{H}-2-93533$ \\
\hline TK-125 & ED & Condenser (spare) & $\mathrm{H}-2-93533$ \\
\hline WH-1 & WE & Recelver & $\mathrm{H}-2-28041$ \\
\hline TK-126 & EF & Storage vessel & $\mathrm{H}-2-28022$ \\
\hline TK-127 & EE & Storage vessel & $\mathrm{H}-2-28022$ \\
\hline TK-128 & WV & CCP PR & $\mathrm{H}-2-28027$ \\
\hline
\end{tabular}


Table 6.1-4. Drawing List of Plutonium Reclamation Facility Out-of-Cell Tanks Piping and Instrumentation Diagrams.

\begin{tabular}{|l|c|c|l|c|}
\hline \multicolumn{1}{|c|}{ Tank } & Position & Floor & \multicolumn{1}{|c|}{ Designation } & System PEFS \\
\hline TK-48 & WJ & 2 & OA Column tank & $\mathrm{H}-2-28020$ \\
\hline TK-52.1 & EW & 1 & Overflow tank & $\mathrm{H}-2-28032$ \\
\hline TK-52.2 & EW & 1 & Overflow tank & $\mathrm{H}-2-28032$ \\
\hline TK-52.3 & WW & 1 & Overflow tank & $\mathrm{H}-2-28032$ \\
\hline TK-52.4 & WW & 1 & Overflow tank & $\mathrm{H}-2-28032$ \\
\hline TK-54 & EW & 1 & Spare & $\mathrm{H}-2-28028$ \\
\hline TK-57 & W Gallery & 3 & Chemical addition tank & \\
\hline TK-58 & & 5 & Disengager tank & $\mathrm{H}-2-28027$ \\
\hline TK-82 & WX & 2 & Organic LO tank & $\mathrm{H}-2-28017$ \\
\hline TK-DWE-1 & EU & 2 & Disengager tank & $\mathrm{H}-2-28024$ \\
\hline TK-WM-2 & & 2 & Static mixer & $\mathrm{H}-2-28025$ \\
\hline 84 & EE & 1 & Organic removal tank & $\mathrm{H}-2-28037$ \\
\hline 85 & EC & 1 & Organic removal tank & $\mathrm{H}-2-28037$ \\
\hline $85 A$ & EE & 2 & $\begin{array}{l}\text { Organic removal tank and } \\
\text { "A centrifuge }\end{array}$ & $\mathrm{H}-2-28027$ \\
\hline
\end{tabular}


WHC-EP-0621

(from WHC 1991)

Table 6.1-5. Drawing List of Plutonium Reclamation Facility Chemical Preparation Tanks Piping and Instrumentation Diagrams.

\begin{tabular}{|c|c|c|c|}
\hline Tank & $\begin{array}{c}\text { Stream } \\
\text { designation }\end{array}$ & Function & System PEFS \\
\hline$A-101$ & -- & Dissolver feed & $H-2-28014$ \\
\hline$A-102$ & -- & Dissolver feed & H-2-28014 \\
\hline A-103 & -- & I4 $\mathrm{NaOH}$ & H-2-28014 \\
\hline$A-104$ & -- & Miscellaneous & H-2-28014 \\
\hline A-105 & -- & $57 \% \mathrm{HNO}_{3}$ & $\mathrm{H}-2-28014$ \\
\hline$A-106$ & $-\infty$ & 50\% ANN & $\mathrm{H}-2-28014$ \\
\hline A-107 & $\mathrm{cxX}$ & CX Column extractant & $\mathrm{H}-2-28014$ \\
\hline A-108 & CAS & $1.5 \mathrm{M} \mathrm{HNO}_{3}$ & $\mathrm{H}-2-28014$ \\
\hline$A-109$ & $\operatorname{ccx}$ & CC Column extractant & $\mathrm{H}-2-28014$ \\
\hline$A-110$ & $\operatorname{cox}$ & CO Column extractant & $\mathrm{H}-2-28014$ \\
\hline$A-112$ & $\cdots$ & S\&C Dissolver flush & H-2-28014 \\
\hline$A-113$ & ELS & Mistron" & $\mathrm{H}-2-28014$ \\
\hline$A-114$ & CAX & $20 \mathrm{v} / 0 \mathrm{TBP}$ and $80 \mathrm{v} / 0 \mathrm{CCl}_{4}$ & $\mathrm{H}-2-28014$ \\
\hline$A-115$ & $\mathrm{CCl}_{4}$ & $\mathrm{CCl}_{4}$ & $\mathrm{H}-2-28014$ \\
\hline$A-116$ & -- & Antifoam & $\mathrm{H}-2-28014$ \\
\hline$A-117$ & -- & B-acid & $\mathrm{H}-2-28014$ \\
\hline$A-118$ & -- & $\mathrm{NaNO}_{2}$ & $\mathrm{H}-2-28014$ \\
\hline$A-119$ & --- & $72 \% \mathrm{HNO}_{3}$ & $\mathrm{H}-2-28014$ \\
\hline
\end{tabular}


Table 6.1-6. Plutonium Inventories--Plutoniun Recovery System. (4 sheets)

\begin{tabular}{|c|c|c|c|c|c|c|c|c|}
\hline \multirow{2}{*}{ Equipant } & \multirow{2}{*}{ Location } & \multirow{2}{*}{ Function } & \multirow{2}{*}{ Description } & \multirow{2}{*}{$\begin{array}{l}\text { Working } \\
\text { volume } \\
\text { (L) }\end{array}$} & \multicolumn{2}{|c|}{$\begin{array}{l}\text { Noninal inventory } \\
\text { (s PU) }\end{array}$} & \multirow{2}{*}{$\begin{array}{l}\text { Chemical } \\
\text { form }\end{array}$} & \multirow{2}{*}{$\begin{array}{l}\text { Criticality } \\
\text { specification } \\
\text { limit (g/L) }\end{array}$} \\
\hline & & & & & Mormal & Ranose & & \\
\hline $\begin{array}{l}\text { TK-32 } \\
\text { (ca colum) }\end{array}$ & Proceses cell & $\begin{array}{l}\text { Extracts plutonin from } \\
\text { equeors feed }\end{array}$ & $\begin{array}{l}\text { 4-in. } x \text { 45-ft steinleas } \\
\text { steeil ind Pyrex }\end{array}$ & 146 & 1,400 & $900 \cdot 1,800$ & $\operatorname{Pux}_{\sin }(3)_{4}$ & 450 \\
\hline $\begin{array}{l}\text { TK-33 } \\
\text { (cc colum) }\end{array}$ & Procens cell & $\begin{array}{l}\text { Stripe plutoniun from } \\
\text { orgenic feed }\end{array}$ & $\begin{array}{l}4-\text { in. } x \text { 45-ft stainless } \\
\text { steel and Purex }\end{array}$ & 144 & 5,100 & $3,400-6,000$ & $\left.\operatorname{Pux} \operatorname{mop}_{3}\right)_{4}$ & 450 \\
\hline $\begin{array}{l}\text { TK-us-1 } \\
\text { (co column) }\end{array}$ & Process cell & Plutenilu serubbing & $\begin{array}{l}\text { 6-in. } \times 19-\mathrm{ft} \text { 10-in. } \\
\text { Staint cess stcel, Toflon". } \\
\text { tined }\end{array}$ & 90 & 9 & $5 \cdot 1,200$ & $\left.\operatorname{Pux}_{\text {ond }}\right)_{4}$ & 450 \\
\hline $\begin{array}{l}7 K-123 \\
\text { (cul colum) }\end{array}$ & Process cell & Urantue stripping & $\begin{array}{l}4-i n_{i} \times 23-f t \text { Stainleas } \\
\text { stcel }\end{array}$ & 65 & 1 & $0 \cdot 7$ & $\operatorname{Puxh}_{\text {and }}$ & 450 \\
\hline $\begin{array}{l}\text { TK-69 } \\
\text { (CX Colum) }\end{array}$ & Proceses cell & Dep scrubbing & $\begin{array}{l}\text { 6-in. } \times 12-f t \text { 7-in. } \\
\text { Stainl cess stcel, Teflon". } \\
\text { lined }\end{array}$ & $\pi$ & 1 & $0-35$ & $\begin{array}{l}\text { Trece } \\
\text { plutenilum } \\
\text { ond }\end{array}$ & 450 \\
\hline $\begin{array}{l}\mathrm{TK}-48 \\
(0 \mathrm{an} \text { colum }\end{array}$ & $\begin{array}{l}\text { 2nd Floor } \\
\text { meat section }\end{array}$ & Organic reacidification & $\begin{array}{l}\text { 5-in. } \times 4-f t \text { 6-in. stainleas } \\
\text { steel, Tefton-lined } \\
\end{array}$ & 18 & 0 & $0-2$ & $\begin{array}{c}\text { Trece } \\
\text { plutenium }\end{array}$ & 450 \\
\hline $7 x-15$ & Process cell & sec Product receiver & $\begin{array}{l}5-\text { in }_{\text {in }} \times 3-\text { ft } 9-\text { in. Stoinless } \\
\text { stel }\end{array}$ & 45 & 10 & $0 \cdot 1,700$ & $\operatorname{Pu}\left(\mathrm{NO}_{3}\right)_{4}$ & 450 \\
\hline$T K-16$ & Process cell & Miscellemens storese & $\begin{array}{l}5-\text { in. } \times 15-f t b^{-i n} . \\
\text { Stainleass stcel } b^{\text {in }}\end{array}$ & 185 & 2,700 & $1-20,000$ & 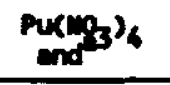 & 450 \\
\hline TK-17 & Proceses cell & feed makeep tenk & $\begin{array}{l}5-\text { in. } \times 15-f t \\
\text { Stainleas steel }\end{array}$ & 185 & 500 & $5 \cdot 5,000$ & $\operatorname{Pu}\left(\mathrm{N}_{3}\right)_{4}$ & 450 \\
\hline TK- 18 & Procees cell & $\begin{array}{l}\text { Feed trenefer to } \\
\text { Ca colum }\end{array}$ & $\begin{array}{l}5-\text { in. } \times 15-f t \\
\text { Stainleas steet }\end{array}$ & 185 & 500 & $5 \cdot 5,000$ & $\operatorname{Pu}\left(\mathrm{mon}_{3}\right)_{4}$ & 450 \\
\hline TK-19 & Process cell & cur receiver cell & $\begin{array}{l}5-\text { in. } \times 15-f t 4_{-i n} \\
\text { stainteas steel }\end{array}$ & 185 & 3 & $0 \cdot 140$ & $P_{x}\left(\log _{3}\right)_{4}$ & 450 \\
\hline $7 K-127$ & Process cell & Hiscelleneous storege & $\begin{array}{l}5-\text { in. } \times 15-f t \\
\text { stainteas steel } b^{-i n} .\end{array}$ & 185 & 2,700 & $0-20,000$ & Pu( $\left(\mathrm{NO}_{3}\right)_{4}$ & 450 \\
\hline$T K-126$ & Proceses cell & Miscel teneous storege & 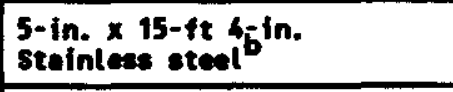 & 185 & 2,700 & $0-20,000$ & $\operatorname{Pxx}_{\operatorname{mat}}(3) 4$ & 450 \\
\hline$T K-36$ & Process cell & $\begin{array}{l}\text { Cais pup tank to } \\
\text { Ca colven }\end{array}$ & $\begin{array}{l}\text { 5-in } \\
\text { steel } \\
\end{array}$ & 45 & 1,700 & $100-4,000$ & Pu(mon $)_{3}$ & 450 \\
\hline$T K-37$ & Process cell & CAX Pup tank & $\begin{array}{l}\text { 5-in. } \times 15-f t \\
\text { Stainless steel }\end{array}$ & 185 & 0 & $0 \cdot 30$ & Tecte end & 450 \\
\hline TK-38 & Process cell & CAX Pupp tank & $\begin{array}{l}\text { 5-in } \\
\text { steel } \times 48-\text { in. Stainless }\end{array}$ & 185 & 0 & $0 \cdot 30$ & $\mathrm{CCl}_{4}^{\mathrm{TeP}}$ & 450 \\
\hline
\end{tabular}


Table 6.1-6. Plutonium Inventories--Plutoniun Recovery System. (4 sheets)

\begin{tabular}{|c|c|c|c|c|c|c|c|c|}
\hline \multirow{2}{*}{ Equipwent } & \multirow{2}{*}{ Location } & \multirow{2}{*}{ Function } & \multirow{2}{*}{ Description } & \multirow{2}{*}{$\begin{array}{l}\text { Working } \\
\text { volume } \\
\text { (L) }\end{array}$} & \multicolumn{2}{|c|}{$\begin{array}{c}\text { Mominal imventory } \\
\text { (Q Pu) }\end{array}$} & \multirow{2}{*}{$\begin{array}{l}\text { Chemical } \\
\text { form }\end{array}$} & \multirow{2}{*}{$\begin{array}{l}\text { Criticality } \\
\text { specification } \\
\text { linit (g/L) }\end{array}$} \\
\hline & & & & & Normal & Rempe & & \\
\hline TK-39 & Process cell & $\begin{array}{l}\text { Can Receiver tenk } \\
\text { (weste) }\end{array}$ & 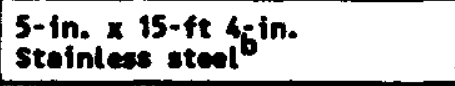 & 185 & 3 & $0-140$ & $\operatorname{Pu}\left(\mathrm{m}_{3}\right)_{4}$ & 450 \\
\hline$T K-40$ & Process coll & $\begin{array}{l}\text { Cup receiver tenk } \\
\text { (weste) }\end{array}$ & 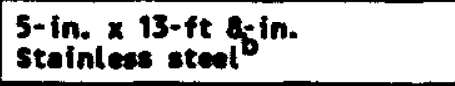 & 185 & 7 & $0-500$ & $\operatorname{Pu}\left(\mathrm{mog}_{3}\right)_{4}$ & 450 \\
\hline TK-41 & Proceess cell & $\begin{array}{l}\text { all receiver tenk } \\
\text { (meste) }\end{array}$ & $\sin _{\text {stcel }_{i}} \times 45-$ in. Steinless & 45 & 0 & $0-30$ & $\operatorname{Pu}\left(\mathrm{IO}_{3}\right)_{4}$ & $\cdot 450$ \\
\hline$T K-42$ & Process cell & Concentrator feed tank & 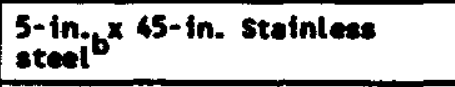 & 45 & 1,700 & $100-14,000$ & $\operatorname{Pu}\left(\mathrm{moO}_{3}\right)_{4}$ & 450 \\
\hline$T K-43$ & Process cell & Product concentrator & $\begin{array}{l}4-\text { in. } \times 10-4 t \text { 9-in. } \\
\text { stainless steel with on } \\
\text { comular stee jecket }\end{array}$ & 5 & 1,000 & $1,000-3,500$ & $\left.\mathrm{PuXNO}_{3}\right)_{4}$ & 450 \\
\hline$T K-44$ & Process cell & Condenser & $\begin{array}{l}\text { 6-in. } \times 3-f t \text { 8-in. Staintess } \\
\text { steel }\end{array}$ & $\mathbf{n} / \mathbf{a}$ & 100 & $100-200$ & $\mathbf{m} / \mathbf{A}$ & 450 \\
\hline$T K-45$ & Process cell & PR Tenk & s-in ${ }_{\text {steel }} \times 45-i n$. Stainless & 45 & 9,000 & $200-20,000$ & $\operatorname{Pu}\left(\mathrm{moO}_{3}\right)_{4}$ & 450 \\
\hline$T K-66$ & Proceses cell & PR Tmik & $\begin{array}{l}\text { 5-in. } \\
\text { steel } b^{x}\end{array}$ & 45 & 9,000 & $200 \cdot 20,000$ & Pur(mols $)_{4}$ & 450 \\
\hline$T K-20$ & Process call & Eveporator feed & $\begin{array}{l}5-\ln _{0} \times \\
\text { steel }\end{array}$ & 45 & 3 & $0 \cdot 15$ & $\left.\operatorname{Pu}\left(\mathrm{m}_{3}\right)_{3}\right)_{4}$ & 450 \\
\hline TK-21 & Process celt & Eveporator & 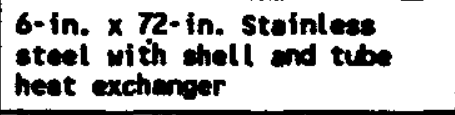 & 25 & 200 & $1-2,000$ & $\operatorname{Pu}\left(\mathrm{NO}_{3}\right)_{4}$ & 450 \\
\hline TK-22 & Process coll & $\begin{array}{l}\text { Condeneer for } \\
\text { eveporntor } \mathrm{TK}-21\end{array}$ & $\begin{array}{l}6-\ln _{\text {. }} \times 46-\text { in. Stainless } \\
\text { steel }\end{array}$ & $m / n$ & 5 & $0-10$ & $\omega / n$ & 450 \\
\hline TK-23 & Process cell & $\begin{array}{l}\text { Evaporator receiver for } \\
\text { IK-21 }\end{array}$ & 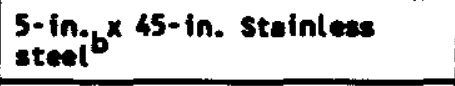 & 45 & 300 & $0 \cdot 4,000$ & $\operatorname{Pu}\left(\mathrm{NO}_{3}\right)_{4}$ & 450 \\
\hline TK-24 & Procees cell & $\begin{array}{l}\text { Evaporstor receiver for } \\
\text { TK-21 }\end{array}$ & 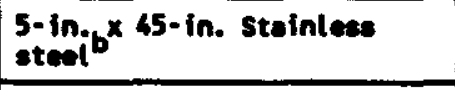 & 45 & 300 & $0 \cdot 4,000$ & $\operatorname{mu}\left(\mathrm{m}_{3}\right)_{4}$ & 450 \\
\hline TK-25 & Process cell & WT Receiver tenk & 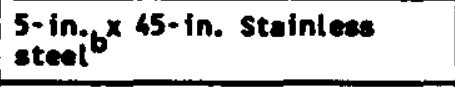 & 45 & 2,800 & $0-16,000$ & Pus(ung $)_{4}$ & 450 \\
\hline $\mathrm{TK}-26$ & Process cell & MT Receiver tenk & $\begin{array}{l}5-\text { in }_{\text {. }}{ } \times 45-i n . \text { Steintess } \\
\text { steel }\end{array}$ & 45 & 2,600 & $0-16,000$ & Pu( $\left(\mathrm{NO}_{3}\right)_{4}$ & 450 \\
\hline TK-27 & Process coll & CAR supply to Ca colum & $\operatorname{stcel}_{\text {stin }} b^{\times}$45-in. stainless & 45 & $\pi$ & $0-700$ & $\operatorname{Pu}\left(\mathrm{NO}_{3}\right)_{4}$ & 450 \\
\hline
\end{tabular}


Table 6.1-6. Plutonium Inventories--Plutonium Recovery System. (4 sheets)

\begin{tabular}{|c|c|c|c|c|c|c|c|c|}
\hline \multirow{2}{*}{$\begin{array}{l}\text { Equipment } \\
\text { number }\end{array}$} & \multirow{2}{*}{ Location } & \multirow{2}{*}{ Function } & \multirow{2}{*}{ Description } & \multirow{2}{*}{$\begin{array}{l}\text { Working } \\
\text { volune } \\
\text { (L) }\end{array}$} & \multicolumn{2}{|c|}{$\begin{array}{l}\text { Mominal imventory } \\
\text { (g Pu) }\end{array}$} & \multirow{2}{*}{$\begin{array}{l}\text { Chemical } \\
\text { form }\end{array}$} & \multirow{2}{*}{$\begin{array}{l}\text { Criticality } \\
\text { specification } \\
\text { limit (g/L) }\end{array}$} \\
\hline & & & & & Normal & Remere & & \\
\hline TK-29 & Process cell & $\begin{array}{l}\text { Cas pup tenk to } \\
\text { CA collum }\end{array}$ & $\operatorname{steel}_{\text {stein }} 6^{\times} 45-i n$. stuinless & 45 & 0 & 0 & $\boldsymbol{M} / \boldsymbol{A}$ & 450 \\
\hline TK-30 & $\begin{array}{l}\text { Process } \\
\text { cell }\end{array}$ & $\begin{array}{l}\text { CCX Pup tank to } \\
\text { CC collun }\end{array}$ & $\begin{array}{l}5-\text { in }_{\text {steel }} \\
b^{\times}\end{array}$ & 45 & 0 & o & $n / n$ & 450 \\
\hline$T K-128$ & Process cell & PR Tenk & ${ }_{\text {steal }}^{5-\text { in }_{i}} \times$ 45-in. Stainleas & 45 & 9,000 & $20-20,000$ & $\operatorname{Pu}\left(\mathrm{NO}_{3}\right)_{4}$ & 450 \\
\hline TK-49 & Process cell & $\begin{array}{l}\text { Miscel tenous transfor } \\
\text { tent }\end{array}$ & $\begin{array}{l}\text { 5-inn } \\
\text { stcel }\end{array}$ & 45 & 100 & $1 \cdot 20,000$ & $P\left(\mathrm{NO}_{3}\right)_{4}$ & 450 \\
\hline $\begin{array}{l}7 K-52.1 \\
7 K-52.2\end{array}$ & East glovebox & $\begin{array}{l}\text { East drain hoeder } \\
\text { receiver terks }\end{array}$ & $\begin{array}{l}\text { 6-in. } \times 2-f t \text { 6-in. Gless } \\
\text { pipe }\end{array}$ & 20 & 50 & $10 \cdot 7,000$ & $\operatorname{Pu}\left(\mathrm{mog}_{3}\right)_{4}$ & 450 \\
\hline $\begin{array}{l}T K-52.3 \\
T K-52.4\end{array}$ & Nest slovebox & $\begin{array}{l}\text { West droin header } \\
\text { receiver tenks }\end{array}$ & $\begin{array}{l}\text { 6-in. } \times 2-f t \text { 6-in. Glass } \\
\text { pipe }\end{array}$ & 20 & 50 & $10 \cdot 7,000$ & Pu(nopis) & 450 \\
\hline rk-70 & Process cell & CXX Pup tank & $\begin{array}{l}\text { 5-in. } \\
\text { steel }\end{array}$ & 45 & 0 & $0 \cdot 1$ & Pur(nOa $)_{4}$ & 450 \\
\hline TK-58 & $\begin{array}{l}\text { Room } 50 \\
\text { glovebox }\end{array}$ & Orgenic deentrainment & $\begin{array}{l}\text { 4-in. } \times 2 \text {-ft 6-in. Gless } \\
\text { pipe }\end{array}$ & 10 & 240 & $100-300$ & $\operatorname{Pu}\left(\mathrm{CH}_{3}\right)_{4}$ & 450 \\
\hline$T K-120$ & Process cell & CAP Pup tank & ${ }_{\text {steet }}^{5-\text { in }_{j}} \times$ 7-ft 5-in. Stainlese & 45 & 800 & $100 \cdot 2,000$ & - & 450 \\
\hline rK-MK-1 & Process cell & CAN Receliver & $\begin{array}{l}5-6-\text { in. } \times 14-f t \text { 6-in. } \\
\text { stainl ess steel, } 3 \text { tibes }\end{array}$ & 185 & 3 & $0-140$ & $\operatorname{Pux}\left(\mathrm{IO}_{3}\right)_{4}$ & 450 \\
\hline D Centrifupe & Weet glovebax & $\begin{array}{l}\text { Sol ids removal fron } \\
\text { product }\end{array}$ & Stainleas stcel & 2.7 & 500 & $100-1,000$ & $\left.P_{\left(n N_{3}\right.}\right)_{4}$ & $3,000 g^{c}$ \\
\hline $\begin{array}{l}\text { B1 and } 182 \\
\text { Centrifuges }\end{array}$ & MT-6 gtowbax & $\begin{array}{l}\text { Sollids removat from } \\
\text { meste steem (caiw) }\end{array}$ & Stainless steel & 2.7 & 15 & $1-50$ & $\left.\operatorname{Pux}_{\text {mat }} \ln _{3}\right)_{4}$ & $3,000 g^{c}$ \\
\hline Filter 16.1 & East slovebox & $\begin{array}{l}\text { Sollide removal from } \\
\text { feed solution }\end{array}$ & $n / a$ & $w / A$ & 10. & $5-50$ & $\operatorname{Pu}\left(\mathrm{mO}_{3}\right)_{4}$ & 450 \\
\hline filter 16.2 & East glovebox & $\begin{array}{l}\text { Sollide removal from } \\
\text { feed solution }\end{array}$ & $\mathbf{m} / \boldsymbol{A}$ & $M / A$ & 10 & $5-50$ & $\mathrm{Pu}\left(\mathrm{NO}_{3}\right)_{4}$ & 450 \\
\hline Filter 5.1 & Weat glovebox & $\begin{array}{l}\text { Fitter for sol ids } \\
\text { removal }\end{array}$ & $\begin{array}{c}4-i n_{\text {. }} \times 1-f t \text { 6-in. Stainteas } \\
\text { stcel }\end{array}$ & & 1 & 1 & $\operatorname{Pu}\left(\mathrm{mO}_{3}\right)_{4}$ & 450 \\
\hline TK-CI & MT-6 glovebox & $\begin{array}{l}\text { Vacule catch tank for } \\
\text { TK-C2 and TK-C3 }\end{array}$ & Glass & 5.6 & 5 & $5-20$ & $\operatorname{Pu}\left(\mathrm{NO}_{3}\right)_{4}$ & 450 \\
\hline
\end{tabular}


Table 6.1-6. Plutonium Inventories--Plutonium Recovery System. (4 sheets)

\begin{tabular}{|c|c|c|c|c|c|c|c|c|}
\hline \multirow{2}{*}{$\begin{array}{c}\text { Equipmant } \\
\text { nuber }\end{array}$} & \multirow{2}{*}{ Location } & \multirow{2}{*}{ Function } & \multirow{2}{*}{ Deecription } & \multirow{2}{*}{$\begin{array}{l}\text { Horking } \\
\text { volum } \\
\text { (L) }\end{array}$} & \multicolumn{2}{|c|}{$\begin{array}{l}\text { Mominal inventory } \\
\text { (o PU) }\end{array}$} & \multirow{2}{*}{$\begin{array}{l}\text { Chemical } \\
\text { form }\end{array}$} & \multirow{2}{*}{$\begin{array}{l}\text { Criticality } \\
\text { specification } \\
\text { linit }(g / L)\end{array}$} \\
\hline & & & & & Normal & Renese & & \\
\hline TK-C2 & MT-6 gloveorx & $\begin{array}{l}\text { Recoiver tank for MT } \\
\text { solutions }\end{array}$ & Glese & 13.9 & 200 & $5 \cdot 400$ & $\operatorname{Pu}\left(\mathrm{NOO}_{3}\right)_{4}$ & 450 \\
\hline TK-CB & m-6 glovebox & $\begin{array}{l}\text { Receliver tenk for MT } \\
\text { solutions }\end{array}$ & Glase & 14.4 & 200 & $5 \cdot 400$ & $\operatorname{Pu}\left(m_{3}\right)_{4}$ & 450 \\
\hline C Centrifupe & mT-6 glovebox & $\begin{array}{l}\text { Solids ravovel from } \\
\text { liquid feed for solvent } \\
\text { extrection }\end{array}$ & Staintess steet & 2.7 & 100 & $5-200$ & $\operatorname{Pu}\left(\mathrm{NO}_{3}\right)_{4}$ & $3,000 g^{c}$ \\
\hline
\end{tabular}

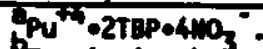

Equels 4 tubes per tenk.

solid.

$\stackrel{⿱ 乛 龰}{\mathbf{w}}$ 
6.1.2.3 Plutonium Conversion Flowsheets. The plutonium conversion to metal process is semi-continuous. ${ }^{(6.9)}$ A lot of plutonium nitrate solution is prepared in the HC-227S bank tanks and staged in the HC-4 tanks. Batches of solution are transferred to the PRT in glovebox $\mathrm{HC}-7$ for acid and plutonium valence adjustment. The solution is drained to the THT as needed for cont inuous pumping to the RCTR vessel in glovebox HC-9B. The operations in HC-9B where the plutonium nitrate solution is converted to PuF 4 powder are continuous. Thereafter, the operation is conducted on a batch basis, with lag storage of powder pans and metal buttons between unit operations.

Figure 6.1-18 provides a flow diagram of the process through PuF production. Table 6.1-7 provides the material balances for this part of the operation.

Figure 6.1-19 shows a flow diagram of the remainder of the process through producing a plutonium metal button. Table $6.1-8$ shows the corresponding material balances. A small sample of the button is taken after the last step shown, but it amounts to about a 10-g quantity which is well within the variance of net metal production among individual buttons.

The flow diagrams also show all Process Safety Class 2 and most of Safety Class 3 instrumentation and controls (I\&C). Not shown are the two HF detectors (Safety Class 2) at the 9AB filterbox. The exhaust from the middle of glovebox $H C-9 B$ leads to the 98 and $9 A B$ filters (in series) and bypasses the E-4 offgas system, thus providing added assurance of E-4 filter integrity. Table 6.1-9 1ist the significant RMC Line safety systems. 6.10$)$ Table 6.1-10 lists the Safety Class 2 and 3 RMC Line process instruments. Table $6.1-11$ lists the safety-related interlocks. Table 6.1-12 1ists the piping and instrumentation (P\&I) drawing numbers which show schematics of the process and instrumentation. A detalled discuission of the safety class instruments is provided in Section 6.5. Table 6.1-13 provides the maximum expected inventories of plutonium (and its form) at various points in the process.

6.1.2.4 PPSL Flowsheets. Flowsheets are seldom required in the PPSL. Most of the projects are small chemistry demonstrations done in batch steps. Many of the processing steps cannot be decided until the resuits of the previous step are determined. Larger continuous experiments (i.e., pilot plants) frequently demonstrate process flowsheets planned for use in production facilities. These planned flowsheets are either used full-scale for the PPSL demonstration or scaled down to fit the sometimes smaller nature of the demonstration. Flowsheets are incorporated into test plan documents except where the flowsheet is so large or complex that a separate flowsheet document is required. Two flow diagrams used for the TRUEX process demonstration are shown for illustrative purposes (Figures 6.1-20 and 6.1-21). The associated flowsheet document for TRUEX is SD-WM-ER-040, Rev. 0, Conceptual PFP TRUEX Flowsheet. (6.11) Procedures, where needed, are also usually incorporated into the flowsheet document. Separate procedures may be provided if they are lengthy. 
Figure 6.1-18. Remote Mechanical C Line Flow Diagram Nitrate Solution Through Puf, Production.

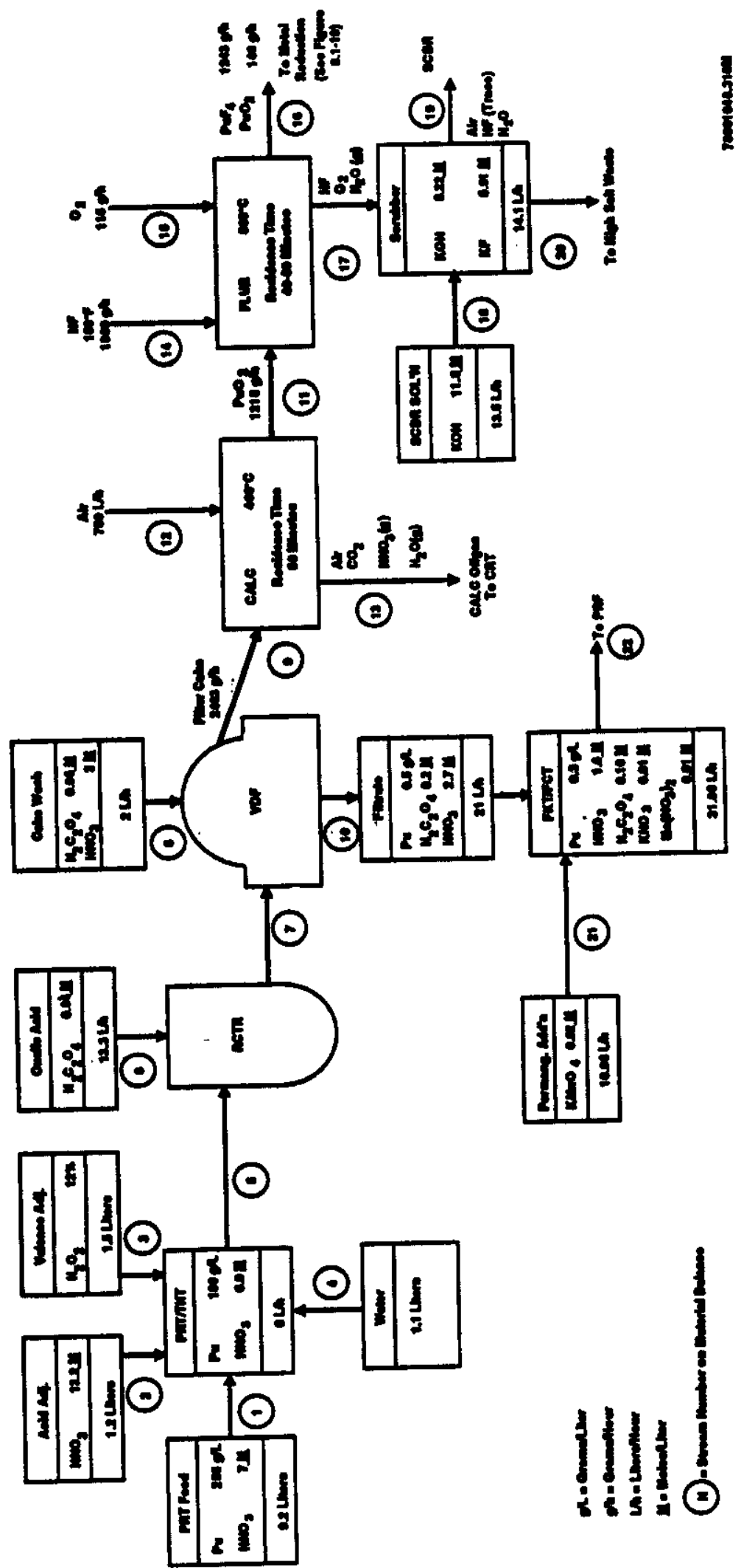


Table 6.1-7. Remote Mechanical C Line Material Balance Nitrate Solution Through PuF Production. (2 sheets)

\begin{tabular}{|c|c|c|c|c|c|c|c|c|c|c|c|c|}
\hline & strean & 1 & 2 & 3 & 4 & 5 & 6 & 7 & 8 & 9 & 10 & 11 \\
\hline & Descripeion & PRT fead & $\begin{array}{l}\text { Acid } \\
\text { ajuet }\end{array}$ & $\begin{array}{l}\text { Valence } \\
\text { djust }\end{array}$ & $\begin{array}{l}\text { Nater } \\
\text { edjust }\end{array}$ & netr fend & $c_{2} H_{2} O_{4}$ & $\begin{array}{c}\text { RCTR } \\
\text { Proctuct }\end{array}$ & Coke weh & calc fend & filtrate & flur feed \\
\hline & Phese & tiquid & Liquid & Liquid & Liquid & tiquid & Llquid & slurry & tiquid & solld & tiquid & solid \\
\hline & $\begin{array}{l}L_{\text {L }} \\
g_{\text {spo }}\end{array}$ & $\begin{array}{c}9.20 \\
0.0 \\
255.00 \\
1.66\end{array}$ & $\begin{array}{c}1.20 \\
\cdots \\
\cdots \\
1.35\end{array}$ & $\begin{array}{c}1.50 \\
\ldots . . \\
1.04\end{array}$ & $\begin{array}{l}1.10 \\
\cdots \ldots \\
1.00\end{array}$ & $\begin{array}{c}6.00 \\
180.46\end{array}$ & \begin{tabular}{c}
13.54 \\
\hdashline .03
\end{tabular} & $\begin{array}{c}19.34 \\
55.99 \\
\cdots\end{array}$ & $\begin{array}{c}\cdots 0 \\
2.00 \\
\cdots \\
1.06\end{array}$ & $\begin{array}{l}\ldots \\
\cdots \\
\cdots \\
\cdots\end{array}$ & $\begin{array}{l}20.87 \\
0.52 \\
1.09\end{array}$ & $\begin{array}{l}\cdots \\
\cdots \\
\cdots\end{array}$ \\
\hline & \multicolumn{12}{|c|}{ Components <-a. } \\
\hline & 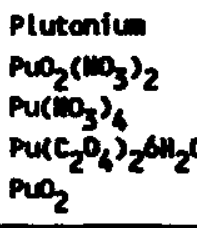 & $\begin{array}{c}(2,346) \\
581 \\
4.063 \\
\ldots \\
\ldots\end{array}$ & $\begin{array}{l}\ldots \\
\ldots \\
\ldots \\
\ldots\end{array}$ & $\begin{array}{l}\cdots \\
\cdots \\
\cdots \\
\cdots\end{array}$ & $\begin{array}{l}\cdots \\
\cdots \\
\cdots \\
\cdots\end{array}$ & $\begin{array}{c}(1,0.03) \\
\ldots \\
2.206 \\
\ldots \\
\ldots\end{array}$ & $\begin{array}{l}\ldots \\
\ldots \\
\ldots \\
\cdots\end{array}$ & $\begin{array}{c}(1,0.3) \\
\ldots \\
22 \\
2.345 \\
\ldots\end{array}$ & $\begin{array}{l}\ldots \\
\ldots \\
\ldots \\
\ldots \\
\ldots\end{array}$ & $\begin{array}{c}(1,072) \\
\ldots \\
\ldots \\
2,345 \\
\ldots\end{array}$ & $\begin{array}{l}(11) \\
\ldots \\
22 \\
\cdots \\
\ldots\end{array}$ & $\begin{array}{c}(1,072) \\
\ldots \\
\ldots \\
\ldots \\
1,215\end{array}$ \\
\hline w & $\begin{array}{l}\mathrm{PuF}_{4} \\
\mathrm{MnO}_{3} \\
\mathrm{H}_{2} \mathrm{O}_{2} \\
\mathrm{H}_{2} \mathrm{C}_{2} \mathrm{O}_{4} \\
\mathrm{NinnO}_{4}\end{array}$ & $\begin{array}{c}\cdots \\
4.151 \\
\ldots \\
\ldots \\
\ldots\end{array}$ & $\begin{array}{l}\ldots 23 \\
923 \\
\ldots- \\
\ldots \\
\ldots-\end{array}$ & $\begin{array}{l}\ldots- \\
\ldots \\
187 \\
\ldots \\
\ldots\end{array}$ & $\begin{array}{l}\ldots \\
\ldots \\
\ldots \\
\ldots\end{array}$ & $\begin{array}{c}\ldots \\
2,256 \\
\ldots \\
\ldots \\
\ldots\end{array}$ & $\begin{array}{c}\ldots \\
\ldots \\
\ldots \\
1.129 \\
\ldots\end{array}$ & $\begin{array}{c}\ldots \\
3,386 \\
\ldots 22 \\
\ldots\end{array}$ & $\begin{array}{c}\cdots \\
239 \\
\ldots \\
9 \\
\ldots\end{array}$ & $\begin{array}{c}\cdots \\
23 \\
\cdots \\
1 \\
\cdots\end{array}$ & $\begin{array}{c}\cdots \\
3,603 \\
\ldots \\
330 \\
\cdots\end{array}$ & $\begin{array}{l}\ldots \\
\ldots \\
\ldots \\
\ldots\end{array}$ \\
\hline & $\begin{array}{l}\mathrm{KOH} \\
\mathrm{KF} \\
\mathrm{MF} \\
\mathrm{O}_{2} \\
\mathrm{n}_{2}\end{array}$ & $\begin{array}{l}\cdots \\
\ldots \\
\ldots \\
\ldots\end{array}$ & $\begin{array}{l}\ldots \\
\ldots \\
\ldots \\
\ldots\end{array}$ & $\begin{array}{l}\cdots \\
\ldots \\
--. \\
-.-\end{array}$ & $\begin{array}{l}\ldots \\
\ldots \\
\ldots \\
\ldots\end{array}$ & $\begin{array}{l}\ldots- \\
\ldots \\
\ldots \\
\ldots \\
\ldots-\end{array}$ & $\begin{array}{l}\ldots \\
\ldots \\
\ldots \\
\ldots \\
\ldots\end{array}$ & $\begin{array}{l}\ldots \\
\ldots \\
\ldots \\
\ldots\end{array}$ & $\begin{array}{l}\ldots \\
\ldots \\
\ldots \\
\ldots \\
\ldots\end{array}$ & $\begin{array}{l}\cdots \\
\ldots \\
\ldots \\
\ldots \\
\ldots\end{array}$ & $\begin{array}{l}\ldots \\
\ldots \\
\ldots \\
\ldots \\
\ldots\end{array}$ & $\begin{array}{l}\ldots \\
\ldots \\
\ldots \\
\ldots \\
\cdots\end{array}$ \\
\hline$\stackrel{c}{3}$ & $\begin{array}{l}\mathrm{co}_{2} \\
\mathrm{x}_{1} \mathrm{n}_{3} \\
\mathrm{nn}\left(\mathrm{m}_{3}\right)_{2} \\
\mathrm{n}_{2} \mathrm{O}\end{array}$ & $\begin{array}{l}-.- \\
\cdots \\
-.- \\
6,293\end{array}$ & $\begin{array}{l}\ldots \\
\ldots \\
\cdots\end{array}$ & $\begin{array}{l}\ldots \\
\ldots \\
1,373\end{array}$ & $\begin{array}{c}\ldots \\
\ldots \\
\ldots \\
1,100\end{array}$ & $\begin{array}{l}\ldots \\
\ldots \\
\ldots . \\
4,392\end{array}$ & $\begin{array}{c}\ldots \\
\ldots \\
\ldots \\
12,609\end{array}$ & $\begin{array}{c}\ldots \\
\ldots \\
\ldots \\
16,517\end{array}$ & $\begin{array}{l}\ldots \\
\ldots \\
1,872\end{array}$ & $\cdots$ & $\begin{array}{c}\ldots \\
\cdots \\
\ldots \\
18.295\end{array}$ & $\cdots$ \\
\hline & Total & 15,088 & 1,620 & 1,560 & 1,100 & 8.854 & 13,738 & 22,592 & 2,120 & 2,463 & 22,250 & 1,215 \\
\hline
\end{tabular}


Table 6.1-7. Remote Mechanical C Line Material Balance Mitrate Solution Through Puf, Production. (2 sheets)

\begin{tabular}{|c|c|c|c|c|c|c|c|c|c|c|c|}
\hline strem & 12 & 13 & 14 & 15 & 16 & 17 & 18. & 19 & 20 & 21 & $2 ?$ \\
\hline Deseription & $\begin{array}{c}\text { Air to } \\
\text { calc }\end{array}$ & $\begin{array}{c}\text { cauc } \\
\text { offrese }\end{array}$ & $\begin{array}{l}\text { Wf to } \\
\text { plue }\end{array}$ & $\begin{array}{l}\text { o. to } \\
\text { fluge }\end{array}$ & $\begin{array}{c}\text { Flui } \\
\text { Product }\end{array}$ & $\begin{array}{l}\text { Fun } \\
\text { offryes }\end{array}$ & $\begin{array}{l}\text { Scrubter } \\
\text { eolution }\end{array}$ & $\begin{array}{l}\text { serviber } \\
\text { effluent }\end{array}$ & $\begin{array}{l}\text { Scrutber } \\
\text { exhenet }\end{array}$ & $\begin{array}{l}\text { Perempa } \\
\text { eddition }\end{array}$ & $\begin{array}{l}\text { Fil trate } \\
\text { to PRf } \\
\end{array}$ \\
\hline Phese & ass & $\mathrm{Ces}$ & $\mathrm{ses}$ & Gas & solid & Gas & Liquild & $\cos$ & Lieuid & Licuid & Liquid \\
\hline $\begin{array}{l}\text { L/n } \\
\text { g PuLL } \\
\text { spes }\end{array}$ & $\begin{array}{c}\ldots-.00 \\
\cdots \\
\cdots\end{array}$ & $\begin{array}{l}\ldots . \\
\cdots . \\
\cdots\end{array}$ & $\begin{array}{l}\ldots \\
\cdots \\
\cdots\end{array}$ & $\begin{array}{l}-. . \\
\cdots .- \\
-. . \\
\end{array}$ & $\begin{array}{l}\ldots . \\
\cdots . \\
\cdots\end{array}$ & 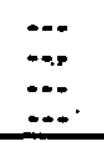 & $\begin{array}{c}13 . \\
13.50 \\
-.42\end{array}$ & $\begin{array}{l}\cdots \\
\cdots \\
\cdots\end{array}$ & \begin{tabular}{c}
14.07 \\
\hdashline-62 \\
1.42
\end{tabular} & $\begin{array}{c}\ldots . \\
10.96 \\
\ldots . . \\
\end{array}$ & $\begin{array}{l}31.85 \\
0.03 \\
1.06 \\
\end{array}$ \\
\hline
\end{tabular}

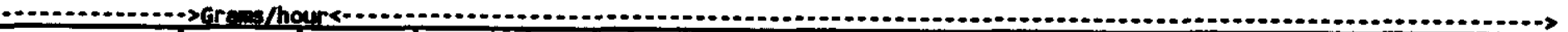

\begin{tabular}{|c|c|c|c|c|c|c|c|c|c|c|c|}
\hline 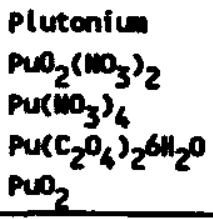 & $\begin{array}{l}\cdots \\
\cdots \\
\cdots \\
\cdots\end{array}$ & $\begin{array}{l}\cdots \\
\cdots \\
\cdots \\
\cdots\end{array}$ & $\begin{array}{l}\cdots \\
\cdots \\
\cdots \\
\cdots\end{array}$ & $\begin{array}{l}\cdots \\
\cdots \\
\cdots \\
\cdots\end{array}$ & $\begin{array}{c}(1,072) \\
\ldots \\
\ldots \\
\ldots \\
146\end{array}$ & $\begin{array}{l}\cdots \\
\ldots \\
\ldots \\
\ldots\end{array}$ & $\begin{array}{l}\cdots \\
\cdots \\
\cdots \\
\cdots\end{array}$ & $\begin{array}{l}\cdots \\
\cdots \\
\cdots \\
\ldots\end{array}$ & $\begin{array}{l}\cdots \\
\ldots \\
\cdots \\
\cdots\end{array}$ & $\begin{array}{l}\cdots \\
\ldots \\
\ldots \\
\ldots\end{array}$ & $\begin{array}{c}(11) \\
\ldots \\
22 \\
\ldots \\
\ldots\end{array}$ \\
\hline $\begin{array}{l}P_{4} f_{4} \\
\mathrm{mma}_{3} \\
\mathrm{H}_{2} \mathrm{O}_{2} \\
\mathrm{n}_{2} \mathrm{C}_{2} \mathrm{O}_{4} \\
\mathrm{xim}_{4} \mathrm{O}_{4}\end{array}$ & $\begin{array}{l}\cdots \\
\cdots \\
\cdots \\
\cdots\end{array}$ & $\begin{array}{l}\cdots \\
23 \\
\cdots \\
\cdots\end{array}$ & $\begin{array}{l}\cdots \\
\cdots \\
\cdots \\
\cdots\end{array}$ & $\begin{array}{l}\cdots \\
\cdots \\
\cdots \\
\cdots \\
\cdots\end{array}$ & $\begin{array}{c}1,263 \\
\ldots \\
\ldots \\
\ldots\end{array}$ & $\ldots$ & $\begin{array}{l}\cdots \\
\cdots \\
\cdots \\
\cdots \\
\cdots\end{array}$ & $\begin{array}{l}\ldots \\
\ldots \\
\ldots \\
\ldots \\
\cdots\end{array}$ & $\begin{array}{l}\cdots \\
\cdots \\
\cdots \\
\cdots\end{array}$ & $\begin{array}{l}\ldots \\
\ldots \\
\ldots \\
\ldots \\
35\end{array}$ & $\begin{array}{l}\ldots . \\
3,561 \\
\ldots- \\
280 \\
\ldots\end{array}$ \\
\hline $\begin{array}{l}\mathrm{KOH} \\
\mathrm{kf} \\
\mathrm{HF} \\
\mathrm{O}_{2} \\
\mathrm{H}_{2}\end{array}$ & $\begin{array}{l}\ldots \\
\ldots \\
\cdots \\
185 \\
716\end{array}$ & $\begin{array}{l}\cdots \\
\cdots \\
41 \\
716\end{array}$ & $\begin{array}{c}\ldots \\
\ldots \\
\ldots \\
\ldots\end{array}$ & $\begin{array}{l}\cdots- \\
\cdots \\
\cdots- \\
115 \\
\cdots\end{array}$ & $\begin{array}{l}\cdots \\
\ldots \\
\ldots \\
\ldots\end{array}$ & $\begin{array}{c}\ldots \\
\ldots \\
1,664 \\
115 \\
\ldots\end{array}$ & $\begin{array}{c}8,706 \\
\ldots \\
\ldots \\
\ldots \\
\ldots\end{array}$ & $\begin{array}{l}\ldots . \\
\ldots . \\
\ldots- \\
115 \\
\ldots-\end{array}$ & $\begin{array}{c}4,119 \\
4,853 \\
\ldots \\
\ldots \\
\ldots\end{array}$ & $\begin{array}{l}\ldots \\
\ldots \\
\ldots \\
\ldots\end{array}$ & $\begin{array}{l}\cdots \\
\cdots \\
\cdots \\
\cdots \\
\cdots\end{array}$ \\
\hline Total & 901 & 2,147 & 1,980 & 115 & 1,309 & 1,921 & 19,170 & 1,115 & 19,977 & 10,901 & 33,229 \\
\hline
\end{tabular}




\section{WHC-EP-0621 \\ (from WHC 1991)}

Figure 6.1-19. Remote Mechanical C Line Flow Diagran Puf, Through Plutonium Button Production.

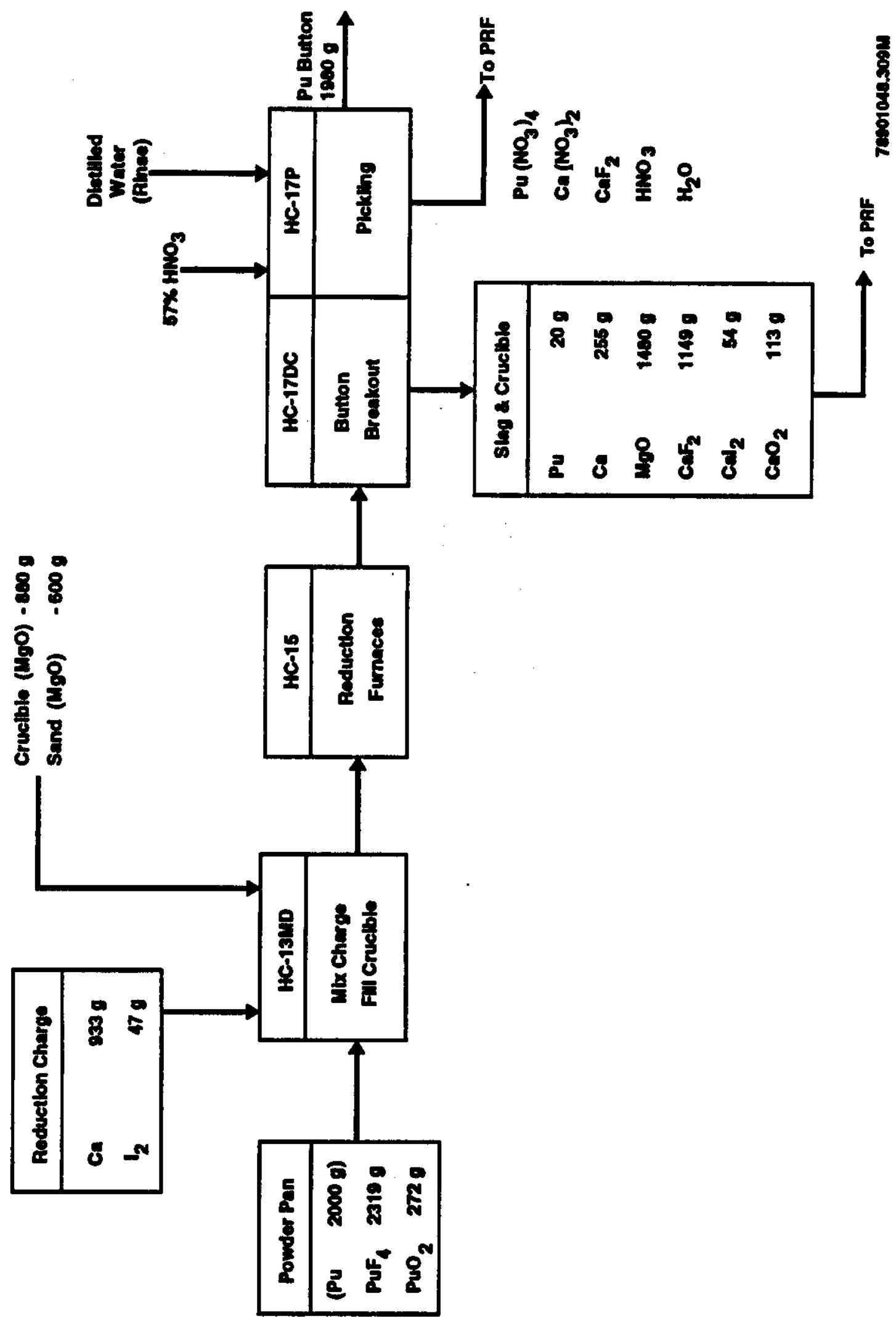


WHC-EP-0621

(from WHC 1991)

Table 6.1-8. Remote Mechanical C Line Material Balance PuF Through Metal Button Production.

\begin{tabular}{|c|c|c|c|c|c|c|}
\hline Stream & 1 & 2 & 3 & 4 & 5 & 6 \\
\hline Description & $\begin{array}{l}\text { Powder } \\
\text { pan }\end{array}$ & $\begin{array}{l}\text { Reduction } \\
\text { charge }\end{array}$ & Crucible & Sand & $\begin{array}{c}\text { Metal } \\
\text { button }\end{array}$ & S\&C \\
\hline \multicolumn{7}{|c|}{ 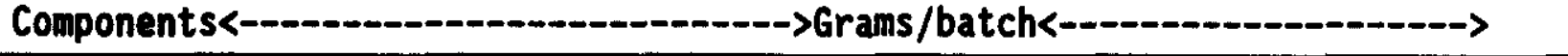 } \\
\hline 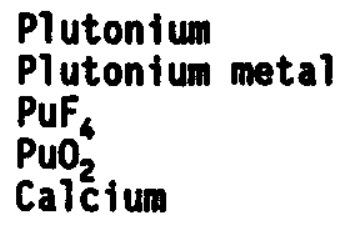 & $\begin{array}{c}(2,000) \\
-\overline{319} \\
272 \\
-\end{array}$ & $\begin{array}{l}--- \\
--- \\
--- \\
933\end{array}$ & $\begin{array}{l}--- \\
--- \\
--- \\
--\end{array}$ & --- & $\begin{array}{c}(1,980) \\
1,980 \\
--- \\
--\end{array}$ & $\begin{array}{c}(20) \\
20 \\
--- \\
-\overline{225}\end{array}$ \\
\hline $\begin{array}{l}\mathrm{I}_{2} \\
\mathrm{MgO}_{9} \\
\mathrm{CaF}_{2} \\
\mathrm{CaI}_{2} \\
\mathrm{CaO}^{2}\end{array}$ & $\begin{array}{l}--- \\
--- \\
--\end{array}$ & $\begin{array}{c}47 \\
-- \\
--- \\
---\end{array}$ & $\begin{array}{l}--- \\
880 \\
--- \\
---\end{array}$ & $\begin{array}{l}--- \\
600 \\
-- \\
--\end{array}$ & $\begin{array}{l}--- \\
--- \\
--- \\
---\end{array}$ & $\begin{array}{r}-\overline{480} \\
1,489 \\
1,149 \\
54 \\
113\end{array}$ \\
\hline Total & 2,591 & 980 & 880 & 600 & 1,980 & 3,071 \\
\hline
\end{tabular}


Table 6.1-9. Remote Mechanical C Line Safety Systems. (2 sheets)

I. FLUR Temperature

Detection and Control: Three independently measured and controlled temperature zones exist within the FLUR; feedback temperature control is provided by the PM-550 programmable controller. Low temperature al arms are set at $450^{\circ} \mathrm{C}$ on the manual controllers and annunciator board, LTAL-9B-2. Record of temperatures is on $R-9 B-1$, channels $D, E$, and $F$.

\section{FLUR Vaculim}

Detection and Control: An automatic control system maintains the desired vacuum in the FLUR. A set point deviation alarm, LVAL-9B-10, alerts operations personnel if the desired vacuum is deviated from. Record of vacuum is on $R-9-2$, channel $A$.

\section{HF Line High Temperature}

Detection and Control: The HF facility room temperature is monitored on a readout at the HF facility at least once every $6 \mathrm{~h}$ and recorded on the HF Facility Survelllance Data Sheet, TI-HF.

\section{HF Facility AHU High Temperature Shutdown}

Detection and Control: The AHU high temperature shutdown setting is set every 6 mo per a Preventive Maintenance Procedure. Instruments are labeled SCR-HF-A and SCR-HF-B.

VI. Seismic Vibration Shutoff of the HF Line

Detection and Control: Seismic vibration detection on VE-HF-1, VE-HF-3, and VE-HF-4 will close AOBV-HF-1, AOBV-HF-2. The shutoff alarms in the RMC Line control room HVAL-HF-I.

VII. HF Gas Detection, HF Facility

Detection and Control: The detector is in the Facility; it has a local alarm; and it alarms in the RMC Line control room.

\section{HF Gas Detection, RIC Line Process Room 228-A}

Detection and Control: Two detectors are located next to the HF flow control valve and the HF rotameter and they both alarm in the RMC Line control room, HFA-9B-1.

IX. HF Gas Detection in 6lovebox HC-9B Midsection Exhaust

Detection and Control: The HF concentration in the exhaust from the midlevel of glovebox $\mathrm{HC}-9 \mathrm{~B}$ is continuously monitored by two separate HF detectors which read out in the control room and alarm at HF levels of $5 \mathrm{ppm}$ and $10 \mathrm{ppm}$. The HF concentration reads out on instruments C-HF-9B-1 and C-HF-9B-2. 
WHC-EP-0621

(from WHC 1991)

Table 6.1-9. Remote Mechanical C Line Safety Systems. (2 sheets)

$X$. HF Gas Detection, HF Scrubber offgas to E-4 Ventilation

Detection and Control: The detector is after the HA-46, F-HF6 demister and it alarms in room 232, HFA-HA46-1.

$X I$. Huaidity. Exposure in Dry Air Gloveboxes

Detection and Control: The frost point monitor (hygrometer) samples four separate gloveboxes (not simultaneously) and has a high frost point alarm in the RMC Line control room. Record of humidity is on $R-9 B 2$, channel $E$. 
Table 6.1-10. Remote Mechanical C Line Safety Instruments. (3 sheets)

\begin{tabular}{|l|c|c|l|}
\hline \multicolumn{1}{|c|}{ Location } & $\begin{array}{c}\text { PISCES } \\
\text { Loop }\end{array}$ & $\begin{array}{c}\text { Safety } \\
\text { Class }\end{array}$ & \multicolumn{1}{|c|}{ Description } \\
\hline HA-46 & $B 0057$ & 3 & $\begin{array}{l}\text { Low secondary-process-cool ing-water } \\
\text { pressure to stop pump P-HF4-1 }\end{array}$ \\
\hline HA-46 Canyon & $B 0199$ & 3 & $\begin{array}{l}\text { Moisture separator (F-HF6) high liquid } \\
\text { level }\end{array}$ \\
\hline HC-1 & $B 0336$ & 3 & HC-1 Conveyor Key interlock switches \\
\hline HC-1 & $B 0392$ & 3 & $\begin{array}{l}\text { HC-1 Low vacuum, dry air supply, shutoff } \\
\text { valve }\end{array}$ \\
\hline HC-1 & $B 0393$ & 3 & HC-1 Emergency E-4 vacuum regulator valve \\
\hline HC-1 & $B 0394$ & 3 & HC-1 Emergency E-4 vacuum regulator valve \\
\hline HC-10 & 80060 & 3 & HC-10 Balance, SNM weigh scale \\
\hline HC-10 HVAC & & 3 & Low hood DP, shut off dry air to hood \\
\hline HC-12S Sand & $B 0094$ & 3 & Sand heater temperature control \\
\hline HC-15 & $B 0043$ & 3 & HC-15A, -B, -C Argon pressure indicators \\
\hline HC-15A & $B 0032$ & 3 & HC-15A Cooling water pressure \\
\hline HC-15A & $B 0035$ & 3 & HC-15A Hydraulic booster pressure \\
\hline HC-15B & $B 0033$ & 3 & HC-15B Cool ing water pressure \\
\hline HC-15B & $B 0036$ & 3 & HC-15B Hydraulic booster pressure \\
\hline HC-15C & $B 0034$ & 3 & HC-15C Cooling water pressure \\
\hline HC-15C & $B 0037$ & 3 & HC-15C Hydraulic booster pressure \\
\hline HC-17P & $B 0346$ & 3 & Metering tank water addition controls \\
\hline HC-17P & $B 0347$ & 3 & Metering tank nitric addition controls \\
\hline HC-17P & $B 0348$ & 3 & Metering tank solution drop control \\
\hline HC-17SBB HVAC & & 3 & Low hood DP, shut off dry air to hood \\
\hline HC-2 & $B 0030$ & 3 & HC-2 Conveyor high pressure alarm \\
\hline HC-2 & $B 0349$ & 3 & HC-2 Conveyor power desk control \\
\hline HC-2 & $B 0350$ & 3 & HC-2 Conveyor key interlock switches \\
\hline HC-227S & & 3 & HC-227S Sump probe \\
\hline HC-227S & $B 0091$ & 3 & HC-227S Vacuum trap 1 qquid detector \\
\hline HC-4 & & 3 & HC-4 Sump probe \\
\hline HC-4 & 30089 & 3 & HC-4 Vacuum trap liquid detector \\
\hline
\end{tabular}


Table 6.1-10. Remote Mechanical C Line Safety Instruments. (3 sheets)

\begin{tabular}{|l|c|c|l|}
\hline \multicolumn{1}{|c|}{ LoCation } & $\begin{array}{c}\text { PISCES } \\
\text { LO0p }\end{array}$ & $\begin{array}{c}\text { Safety } \\
\text { Class }\end{array}$ & \multicolumn{1}{|c|}{ Description } \\
\hline HC-6 & & 3 & HC-6 Sump probe \\
\hline HC-7 & $B 0090$ & 3 & HC-7 Vacuum trap liquid detector \\
\hline HC-7 & $B 0059$ & 3 & HC-7 Sump al arm \\
\hline HC-7 PRT & $B 0369$ & 3 & PRT Agitator/peroxide interlock \\
\hline HC-7 PRT & $B 0022$ & 3 & Peroxide to PRT flow control \\
\hline HC-9B & $B 0020$ & 3 & Overflow tank high level al arm \\
\hline HC-9B & $B 0058$ & 3 & HC-9B Sump alarm \\
\hline HC-9B CALC & $B 0026$ & 3 & CALC Purge low flow alarm \\
\hline HC-9B CALC & $B 0188$ & 3 & CALC High temperature heater shutdown \\
\hline HC-9B CALC & & 3 & CALC Inlet CCTV \\
\hline HC-9B FLUR & & 3 & FLUR Tube CCTV \\
\hline HC-9B FLUR & $B 0001$ & 3 & FLUR Inlet heater control \\
\hline HC-9B FLUR & $B 0002$ & 3 & FLUR Center heater control \\
\hline HC-9B FLUR & $B 0003$ & 3 & FLUR Outlet heater control \\
\hline HC-9B FLUR & $B 0007$ & 2 & $\begin{array}{l}\text { FLUR Vacuum control, low vacuum/HF flow } \\
\text { interlock }\end{array}$ \\
\hline HF & $B 0023$ & 3 & HF Supply line, low temperature alarm \\
\hline HF & & 3 & $\begin{array}{l}\text { HF Supply line, heat trace, and control } \\
\text { system }\end{array}$ \\
\hline HF & $B 0013$ & 2 & $\begin{array}{l}\text { HF Supply line pressure, high and low } \\
\text { pressure/HF flow interlock }\end{array}$ \\
\hline HF & $B 0017$ & 3 & HF Supply line temperature, high alarm \\
\hline HF & $B 0087$ & 3 & HF Detector, room 228-A \\
\hline HF & $B 0088$ & 3 & HF Detector, HF faciltty \\
\hline HF & $B 0162$ & 3 & HF Detector, glovebox HA-46 \\
\hline HF & $B 0163$ & 2 & HF Detector l, 9B filterbox \\
\hline HF & $B 0009$ & 2 & HF Detector 2, 9B filterbox \\
\hline HF-Facility & 2 & HF Bank A heater control \\
\hline HF-Facility & $B 0093$ & 2 & HF Bank B heater control \\
\hline HF-Facility & $B 0096$ & 3 & HF Room temperature indicator \\
\hline
\end{tabular}


Table 6.1-10. Remote Mechanical C Line Safety Instruments. (3 sheets)

\begin{tabular}{|l|c|c|l|}
\hline \multicolumn{1}{|c|}{ Location } & $\begin{array}{c}\text { PISCES } \\
\text { LOOD }\end{array}$ & $\begin{array}{c}\text { Safety } \\
\text { class }\end{array}$ & \multicolumn{1}{|c|}{ Description } \\
\hline HF-Facility & B0198 & 2 & Earthquake vibration, HF flow interlock \\
\hline RMC EMER & B0168 & 3 & RMC EMER Shutdown of KMnO 4 in HC-7 \\
\hline RMC EMER & B0338 & 3 & RMC EMER Shutdown controls \\
\hline RMC Calcium & B0196 & 3 & Calcium overpack PRV \\
\hline RMC Dry Air & $B 0042$ & 3 & RMC Line dry air monitor-hygrometer \\
\hline RMC Dry Air & B0095 & 3 & Dry air header pressure control \\
\hline RMC Ory Air & B0395 & 3 & Hygrometer for HC-21C, HA-2OMB, and HA-211 \\
\hline RMC HVAC & $\begin{array}{c}\text { B0370 } \\
\text { through } \\
\text { B0388 }\end{array}$ & 3 & RMC Glovebox DPs \\
\hline
\end{tabular}


Table 6.1-11. Remote Mechanical C Line Emergency Shutdown Button Interlocks.

\begin{tabular}{|c|c|c|c|}
\hline Location & $\begin{array}{c}\text { PISCES } \\
\text { Loop }\end{array}$ & $\begin{array}{l}\text { Safety } \\
\text { class } \\
\end{array}$ & Description \\
\hline $\mathrm{HC}-7$ & B0338 & 3 & $\begin{array}{l}\text { RMC Emergency shutdown pumps } M P-7 C-2 A \text { and } \\
\text { MP-7C-2B, EV-7C-26 (DOV-7C-26), from } \\
\text { relay } K-E S-3 \text {. }\end{array}$ \\
\hline$H C-9 B$ & B0338 & 3 & $\begin{array}{l}\text { RMC Emergency shutdown CALC heaters, FLUR } \\
\text { heaters, EV-9B-21 for VDF oxal ic wash from } \\
\text { relay K-ES-5. }\end{array}$ \\
\hline $\begin{array}{l}\text { Electrical } \\
\text { Pane1 120-A }\end{array}$ & B0338 & 3 & 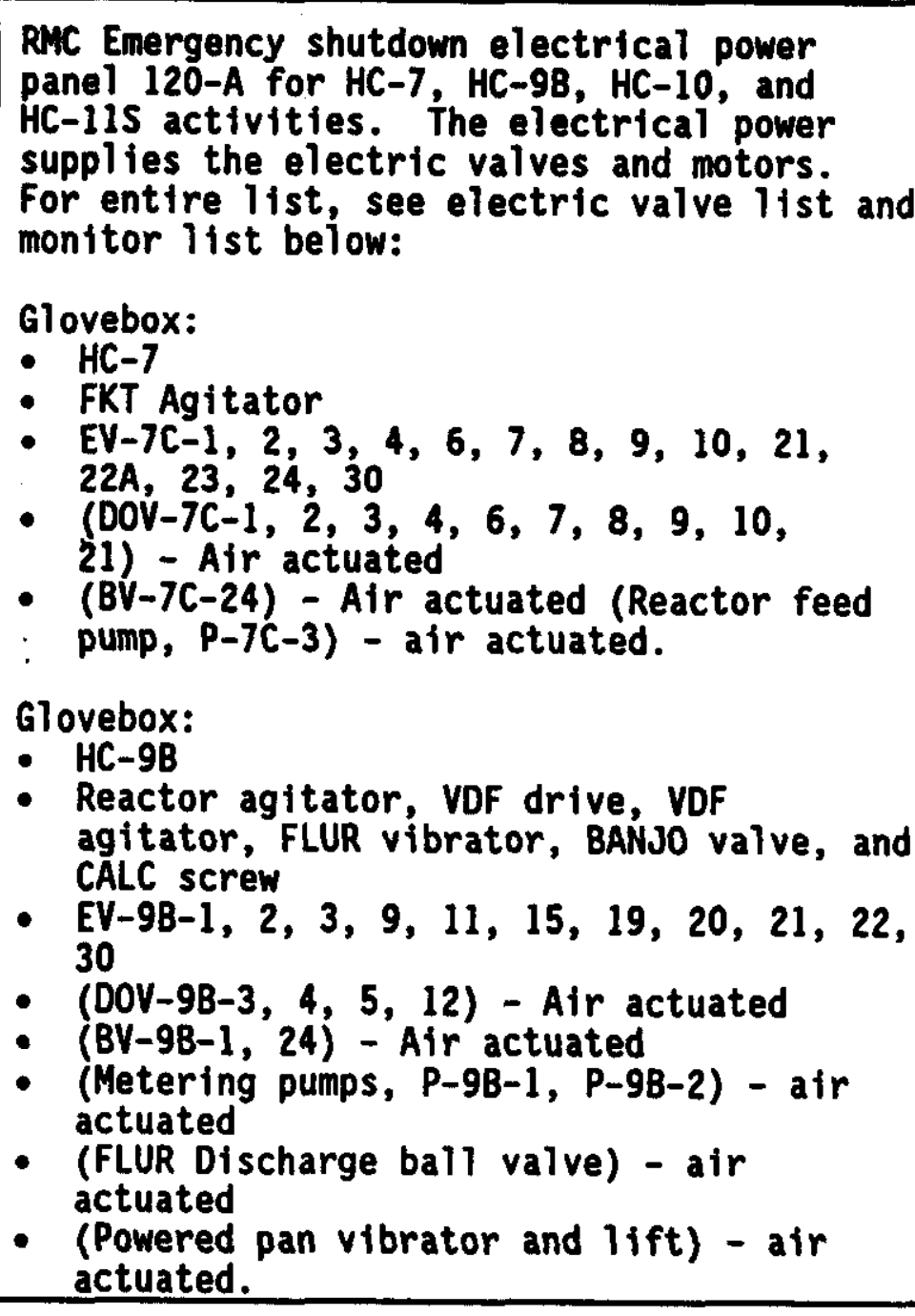 \\
\hline $\begin{array}{l}\text { Electrical } \\
\text { panel } 120-B\end{array}$ & B0338 & 3 & $\begin{array}{l}\text { RMC Emergency shutdown electrical power } \\
\text { pane1 } 120-B \text { for } H C-13 M D \text { through } H C-18 \\
\text { activities. }\end{array}$ \\
\hline
\end{tabular}


WHC-EP-0621

(from WHC 1991)

Table 6.1-12. Piping and Instrumentation Diagram Numbers Remote Mechanical C Line.

\begin{tabular}{|c|c|c|}
\hline Operation & Glovebox & P\&I Drawing number \\
\hline Solution staging & $\mathrm{HC}-4$ & H-2-93504 Sheet 1 \\
\hline $\begin{array}{l}\text { Valence adjustment } \\
\mathrm{C}_{2} \mathrm{H}_{2} \mathrm{O}_{4} \mathrm{Kill}\end{array}$ & $\begin{array}{l}\mathrm{HC}-7 \\
\mathrm{HC}-7\end{array}$ & $\begin{array}{l}\mathrm{H}-2-93504 \text { Sheet } 3 \\
\mathrm{H}-2-93504 \text { Sheet } 3\end{array}$ \\
\hline $\begin{array}{l}\text { Precipitation } \\
\text { Calcination } \\
\text { Fluorination }\end{array}$ & $\begin{array}{r}H C-9 B \\
. H C-9 B \\
H C-9 B\end{array}$ & $\begin{array}{l}\text { H-2-93504 Sheet } 4 \\
\text { H-2-93504 Sheet } 4 \\
\text { H-2-93504 Sheet } 4\end{array}$ \\
\hline $\begin{array}{l}\text { Powder weighing } \\
\text { Powder storage } \\
\text { Vessel sanding } \\
\text { Mixing and dumping } \\
\text { Reduction furnace }\end{array}$ & $\begin{array}{c}H C-10 \\
H C-11 \\
H C-12 S \\
H C-13 M D \\
H C-15 A B C\end{array}$ & $\begin{array}{l}\mathrm{H}-2-93504 \text { Sheet } 5 \\
\mathrm{H}-2-93504 \text { Sheet } 5 \\
\mathrm{H}-2-93504 \text { Sheet } 5 \\
\mathrm{H}-2-93504 \text { Sheet } 5 \\
\mathrm{H}-2-93504 \text { Sheet } 5\end{array}$ \\
\hline $\begin{array}{l}\text { Crucible cutter } \\
\text { Dump and can } \\
\text { Pickling } \\
\text { Sampling, weighing } \\
\text { Button storage }\end{array}$ & $\begin{array}{l}H C-16 C C \\
H C-17 D C \\
H C-17 P \\
H C-27 S B B \\
H C-18 B S\end{array}$ & $\begin{array}{l}\mathrm{H}-2-93504 \text { Sheet } 6 \\
\mathrm{H}-2-93504 \text { Sheet } 6 \\
\mathrm{H}-2-93504 \text { Sheet } 6 \\
\mathrm{H}-2-93504 \text { Sheet } 6 \\
\mathrm{H}-2-93504 \text { Sheet } 6\end{array}$ \\
\hline Cold chemical pump & --- & H-2-93504 Sheet 7 \\
\hline
\end{tabular}


Table 6.1-13. Remote Mechanical C Line Maximum Plutonium Inventories. (2 sheets)

\begin{tabular}{|c|c|c|c|c|c|c|c|c|}
\hline \multirow{2}{*}{$\begin{array}{l}\text { Equipment } \\
\text { (vessel) }\end{array}$} & \multirow{2}{*}{ Location } & \multirow{2}{*}{ Function } & \multirow{2}{*}{ Deseription } & \multirow{2}{*}{$\begin{array}{l}\text { working } \\
\text { volume (L) }\end{array}$} & \multicolumn{2}{|c|}{ Mominal imventory } & \multirow{2}{*}{$\begin{array}{l}\text { Chemical } \\
\text { form }\end{array}$} & \multirow{2}{*}{$\begin{array}{l}\text { Criticality } \\
\text { specification } \\
\text { limit }\end{array}$} \\
\hline & & & & & $\begin{array}{l}\text { Liquid } \\
(g / L)\end{array}$ & solfid (kg) & & \\
\hline FST & $M c-7$ & $\begin{array}{l}\text { seppline and } \\
\text { tronsfor of filtrate } \\
\text { solution to paf }\end{array}$ & 6-in. $\times 6-f t$ class & $33 \cdot 36$ & 0.3 & $w / A$ & $\operatorname{Pu}\left(\mathrm{MO}_{3}\right)_{4}$ & $450 \mathrm{~g} / \mathrm{L}$ \\
\hline VDF and Pan & $M C-9 B$ & $\begin{array}{l}\text { Plutoniun axalate } \\
\text { sol lide are sepprated } \\
\text { frow the filtrete }\end{array}$ & $\begin{array}{l}5-\text { in. } x 11-\text { in.-die. } \\
8-\text { in. } x 7-i{ }_{0} 8 \\
8.25 \text { in. } x 10 \text { in. } x \\
0.5 \text { in. }\end{array}$ & $\begin{array}{c}2 \\
2 \\
2.2\end{array}$ & $\underset{w / a}{w / A}$ & $\begin{array}{c}0.24=0.27 \\
0.24=0.27 \\
0.76\end{array}$ & $\operatorname{Pu}\left(\mathrm{HO}_{3}\right)_{4}$ & $13 \mathrm{~kg}$ \\
\hline Calcíner & MC- 98 & $\begin{array}{l}\text { Discomposes } \\
\text { plutonitu oxalate } \\
\text { solids to } \mathrm{Plg}_{2}\end{array}$ & $\begin{array}{l}\text { 6-ft 9-in. Stainless } \\
\text { steil trowh } x \\
\text { 4-in.-dia. screw } \\
\end{array}$ & $\mathbf{m} / \mathbf{A}$ & $m / n$ & 2.27 & $\mathrm{PuO}_{2}$ & $17 \mathrm{~kg}$ \\
\hline Fluorinator & MC-9B & $\begin{array}{l}\text { Conversion of } \mathrm{PLO}_{2} \\
\text { to Puff }\end{array}$ & $\begin{array}{l}\text { 5-ft 6-in. Lone } x \\
4-\text { in. hish } \\
\text { Incond yith of } \\
\text { platimum-iridium liner }\end{array}$ & $\omega / A$ & $m / A$ & 0.66 & Puf $_{4}$ & $17 \mathrm{ks}$ \\
\hline Pounder pens & $\begin{array}{l}M C-1 \\
M C-11\end{array}$ & $\begin{array}{l}\text { Interin storage and } \\
\text { transport of Puf } 4 \\
\text { pouder }\end{array}$ & pein. $\times 6$-in. Wigh & 1.7 & $w / n$ & $0 \cdot 2.2$ & $\mathrm{Puf}_{4}$ & $\begin{array}{l}5.1 \mathrm{~kg}^{*} \\
5.1 \mathrm{~kg}^{*}\end{array}$ \\
\hline Mixer/dupor & HC-13:D & $\begin{array}{l}\text { Mixine calcium } \\
\text { metel, iodine } \\
\text { cryetals with Puf, } \\
\text { pouder }\end{array}$ & $\begin{array}{l}\text { 10-in.-diam } \times 3-\text { in. } \\
\text { High iexen mixer that } \\
\text { tepers conically to } \\
7 \text { in. }\end{array}$ & $\begin{array}{l}6.5 \\
\cdots\end{array}$ & $M / n$ & $0-2.2$ & PuF $_{4}$ & $5.1 \mathrm{~kg}$ \\
\hline $\begin{array}{l}\text { Induction } \\
\text { furnoces (3) }\end{array}$ & $M C-15$ & $\begin{array}{l}\text { Firing of rectuction } \\
\text { charge }\end{array}$ & 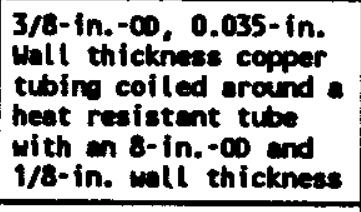 & $w / n$ & $M / n$ & $0 \cdot 2.2$ & Puf $_{4}$ & $5.1 \mathrm{kg*}$ \\
\hline PV & $\begin{array}{l}M C-1 \\
C H-125 \\
C H-13 \% 0 \\
M C-15 \\
M C-16 C C \\
M C-17 D C\end{array}$ & $\begin{array}{l}\text { Contoine Puf, charge } \\
\text { along with chemicals } \\
\text { for reduction, } \\
\text { crucible and inos } \\
\text { send }\end{array}$ & $\begin{array}{l}\text { 7-in. } x 12.7-\text { in. } \\
\text { Hestelloy }-x\end{array}$ & 4.3 . & $\mathbf{M / A}$ & $0 \cdot 2.2$ & $\begin{array}{l}\text { Puf } \\
\text { Plutonín } \\
\text { metal } \\
\text { Plutonic } \\
\text { sec }\end{array}$ & $5.6 \mathrm{~kg}^{*}$ \\
\hline $\begin{array}{l}\text { Desper hamer } \\
\text { will }\end{array}$ & HC-17DC & $\begin{array}{l}\text { Separate the } \\
\text { plutonic metal and } \\
\text { grind up the sand, } \\
\text { sec }\end{array}$ & m/a & $w / A$ & $M / n$ & $2.5 \pm 10 x$ & $\begin{array}{l}\text { Plutonium } \\
\text { untal } \\
\text { Plutonium } \\
\text { sec }\end{array}$ & $4 \mathrm{~kg}$ \\
\hline B valve & MC-9s & $\begin{array}{l}\text { Transfer } \mathrm{PuO}_{2} \text { from } \\
\text { calciner to } \\
\text { fluorinator }\end{array}$ & $m / n$ & $m / a$ & $\omega / \boldsymbol{A}$ & 0.1 & $\mathrm{PLO}_{2}$ & $M / A$ \\
\hline
\end{tabular}


Table 6.1-13. Remote Mechanical C Line Maximum Plutonium Inventories. (2 sheets)

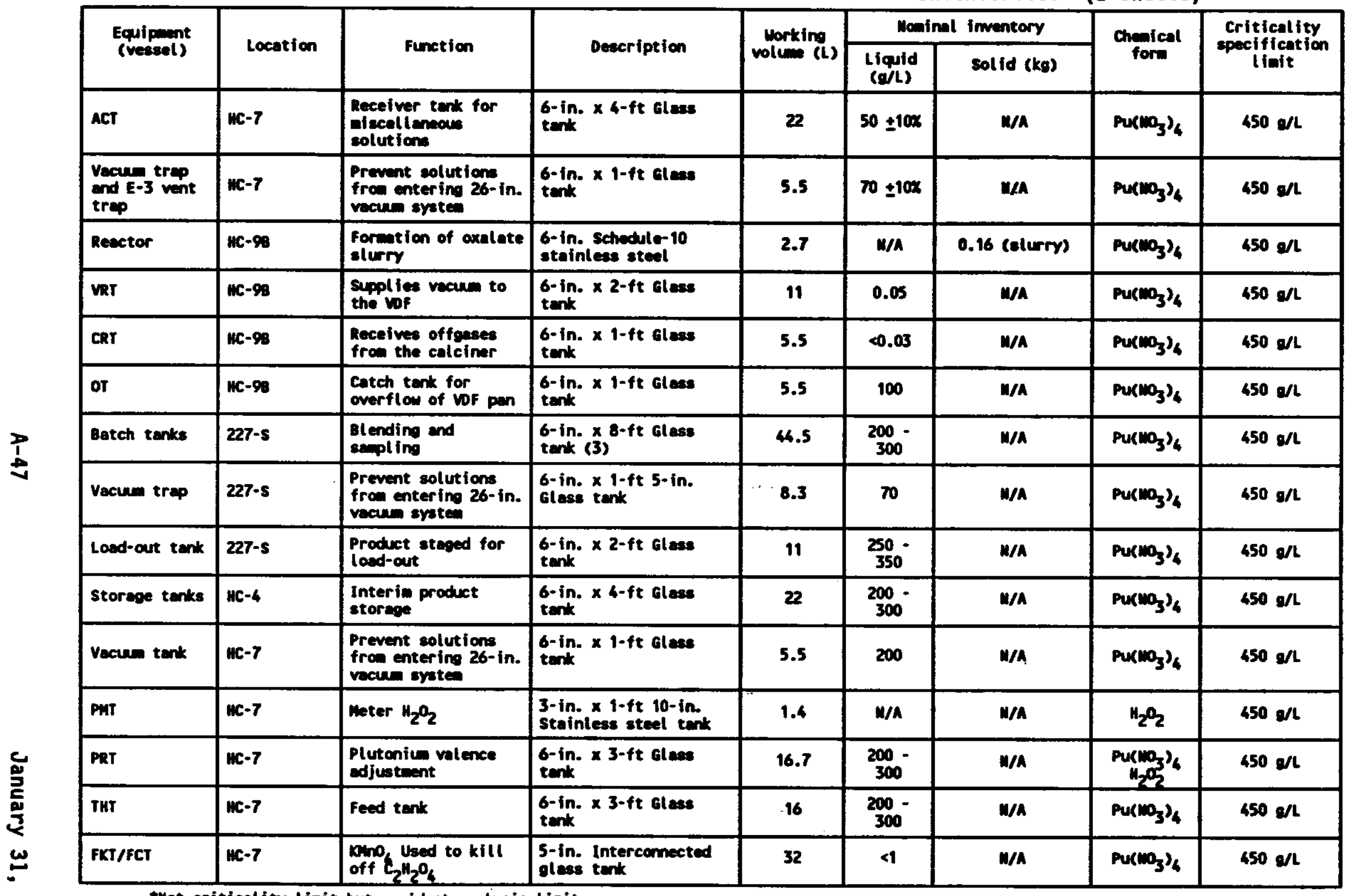


Figure 6.1-20. Transuranic Extraction Solvent Extraction Flow Diagram.

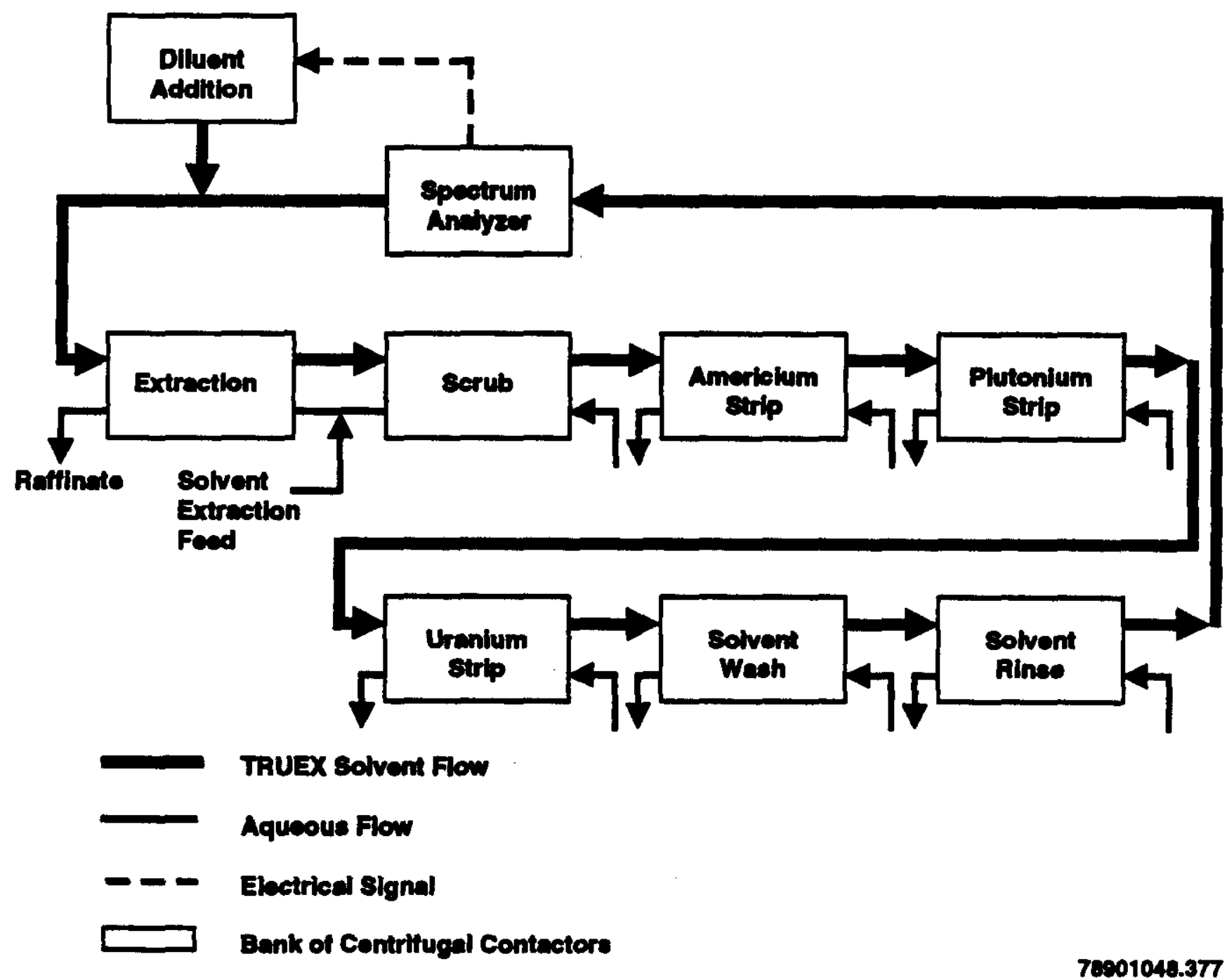


WHC-EP-0621

(from WHC 1991)

Figure 6.1-21. Overall Transuranic Extraction Process Flow Diagram.

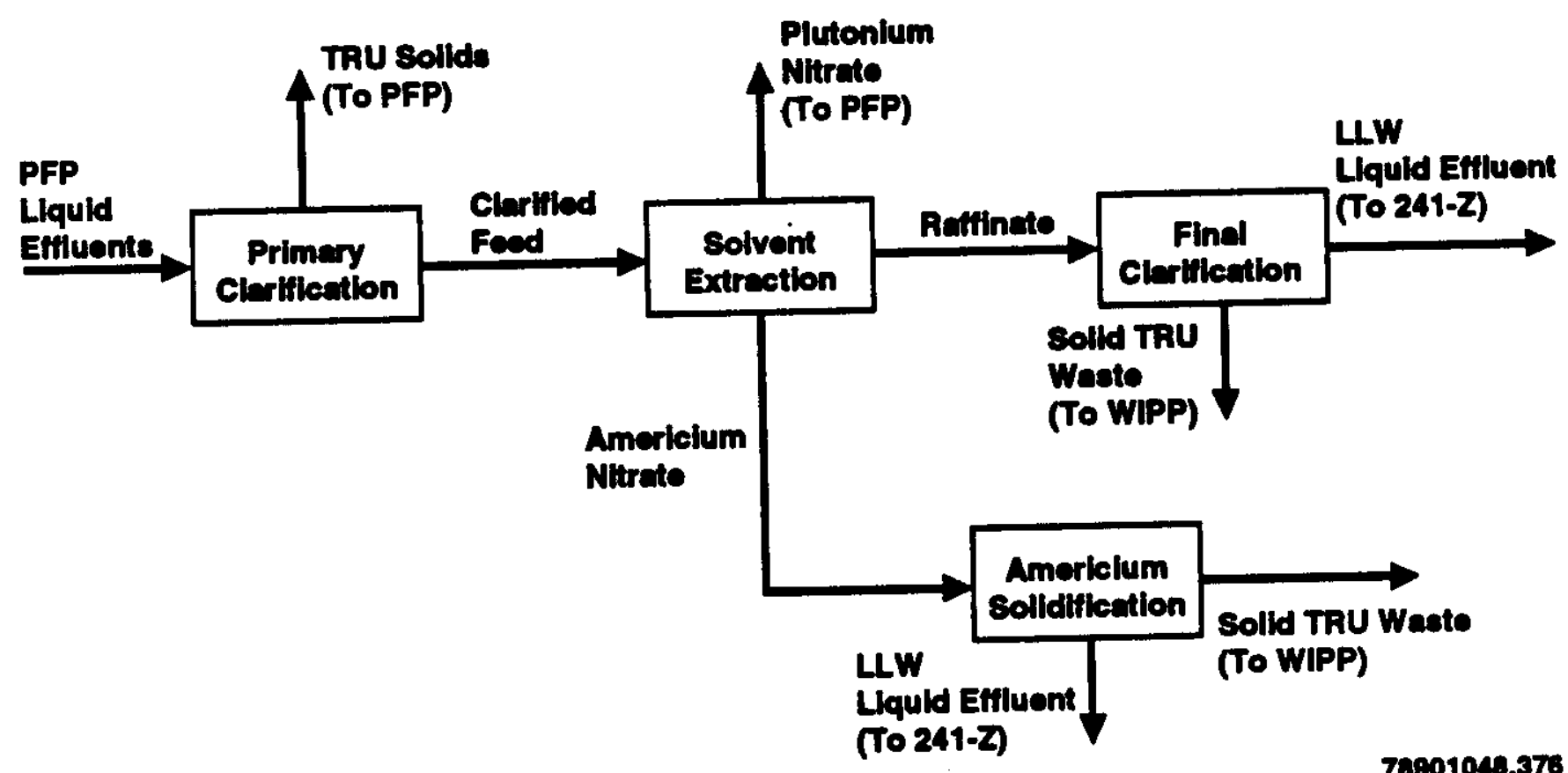


WHC-EP-0621

(from WHC 1991)

\section{REFERENCES}

WHC, 1988, Conceptual Plutonium Finishing Plant TRUEX Flowsheet, WHC-SD-WM-ER-040, Westinghouse Hanford Company, Richland, Washington.

WHC, 1991, Plutonium Finishing Plant Final Safety Analysis Report, Vol. 3, WHC-SD-CP-SAR-021, Westinghouse Hanford Company, Richland, Washington. 
WHC-EP-0621

APPENDIX B

DATA ON 234-5Z WASTE GENERATION FRON THE SOLID WASTE INFORMATION TRACKING SYSTEM

B-1 
WHC-EP-0621

This page intentionaliy left blank.

B-2 


\section{DATA ON 234-5Z WASTE GENERATION FRON THE SOLID WASTE INFORMATION TRACKING SYSTEM}

The information found in this appendix is from the Solid Waste Information and Tracking System (SWITS) database. The older Richland Solid Waste Information Management System (R-SWIMS) database was incorporated into SWITS. SWITS is used to track information on radioactive and other wastes stored or disposed of at the Hanford Site.

Each SWITS data run in this appendix is preceded by the query used to generate the data. A brief explanation of the run and any additional information needed to understand the data presented is also included.

The bulk of the data provided is limited to information about transuranic (TRU) waste generated at the Plutonium Finishing Plant (PFP); however, some general information on the non-TRU waste is included for completeness. The term non-TRU is used in lieu of low-level waste (LLW) because a small percentage of the waste has been designated only as "not TRU." It is believed that most, if not all, of the non-TRU waste is LLW.

The data runs in this appendix are further segregated by waste container type. Because initial retrieval efforts and Waste Receiving and Processing Facility (WRAP) 1 will focus on 55-gal drums, these container types are considered separately.

Some general information about the SWITS database and the codes used follows. A disclaimer also follows. 


\section{Disclaimer for Solid Waste Information and Tracking System Data}

Requests for information from the Solid Waste Information and Tracking System (SWITS) are normally relatively limited in scope. The requests are for specific data fields or summary data. The responses to these requests undergo review during data collection, summary, and response preparation.

The responses to these requests represent a simple reproduction of the SWITS database. Transmittal of information is made with the following disclaimers.

- The information contained in this transmittal is raw data, and represents information provided to Solid Waste Engineering (SWE) on burtal records or other documents. These data have not been validated.

- The information contained in this transmittal is subject to change without notice. Continual updates of SWITS information and improvement of the software system make it impossible to ensure these data correspond with the database after transmittal.

- This information is current as of May 15, 1992.

- The U.S. Department of Energy (DOE) and Westinghouse Hanford Company (WHC) are not responsible for the accuracy, completeness, or any other representation of this information. 
WHC-EP-0621

SWIR401

Solid Waste Information and Tracking System

$05 / 01 / 92$

$13: 25$

Pàge: 1

Primary Waste Type code Table

Primary Waste Type code

Ar

CM

$\mathrm{H}$

HP

$M$

MP

N

$\mathbf{P}$

$\mathbf{R}$

RP

$\mathrm{U}$
Primary Waste Type Description HAz, ADWIN HoLd MORÁTORIUUA CONTROLID

HAZARDOUS

HAZARDOUS WASTE PCB

MIXED

MIXED PCB

NON-REGUIATED

PCB

RADIOACTIVE

RADIOACTIVE PCB

UNKNOWN

\begin{tabular}{ll} 
Ship & \\
Time & Rad \\
\hline 89 & $-N$ \\
89 & $N$ \\
89 & $N$ \\
89 & $N$ \\
29 & $N$ \\
89 & $Y$ \\
29 & $Y$ \\
& N \\
29 & $N$ \\
29 & $Y$ \\
29 & $Y$ \\
& N
\end{tabular}


Secondary Waste Type Code Table

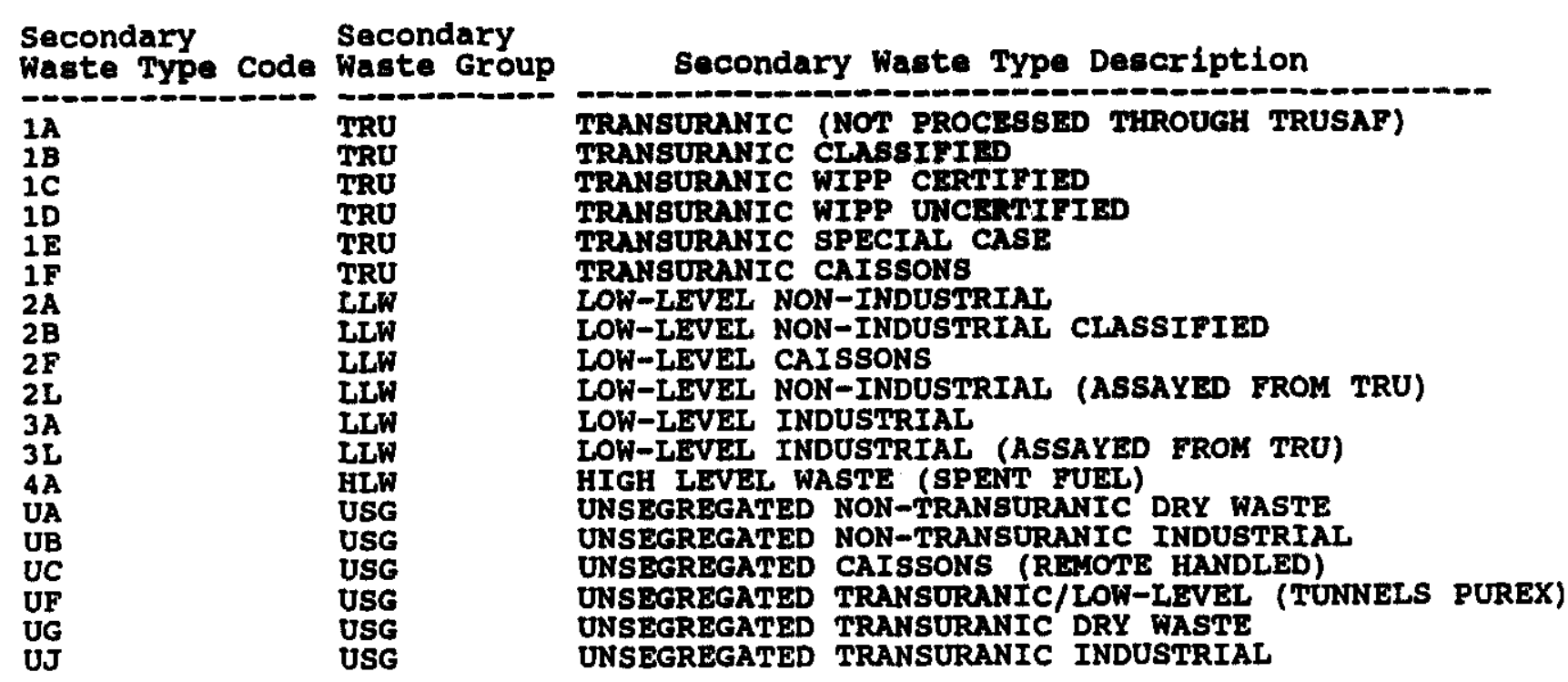


WHC-EP-0621

APPENDIX B.1

CONTAINER NUMBER AND WEIGHT OF TRU WASTE IN 55-GALLON DRUNS - SORTED BY WASTE TYPE 


\section{WHC-EP-0621}

This page intentionally left blank. 


\section{CONTAINER NUMBER AND WEIGHT OF TRU WASTE IN 55-GALLON DRUMS - SORTED BY WASTE TYPE}

This data run provides an overall look at the waste types, container numbers and total weight (in kilograms) of the radioactive wastes generated annually at the Plutonium Finishing Plant (PFP). These data are for wastes stored in 55-gal drums only. The meanings of primary and secondary waste codes can be found in the introductory materials of this appendix.

The data in the average weight column should be studied cautiously, especially for low-level waste (LLW) codes. Concern is warranted because LLW records are still done on a batch basis. This means that one weight datum may be the composite weight for a group of drums. The computer program that was used simply divided the sum of all weight values by the number of values used to compute the total weight not the actual number of containers that value represents.

From 1970 until 1978 individual container weights were not required to be recorded for drums of transuranic (TRU) waste. During the data re-entry program in the mid-1980's standard weights were assigned for all container types; 55-gal drums were given a standard weight of $68 \mathrm{~kg}$. This is why the average weight per drum of TRU waste is so consistent during this period. 


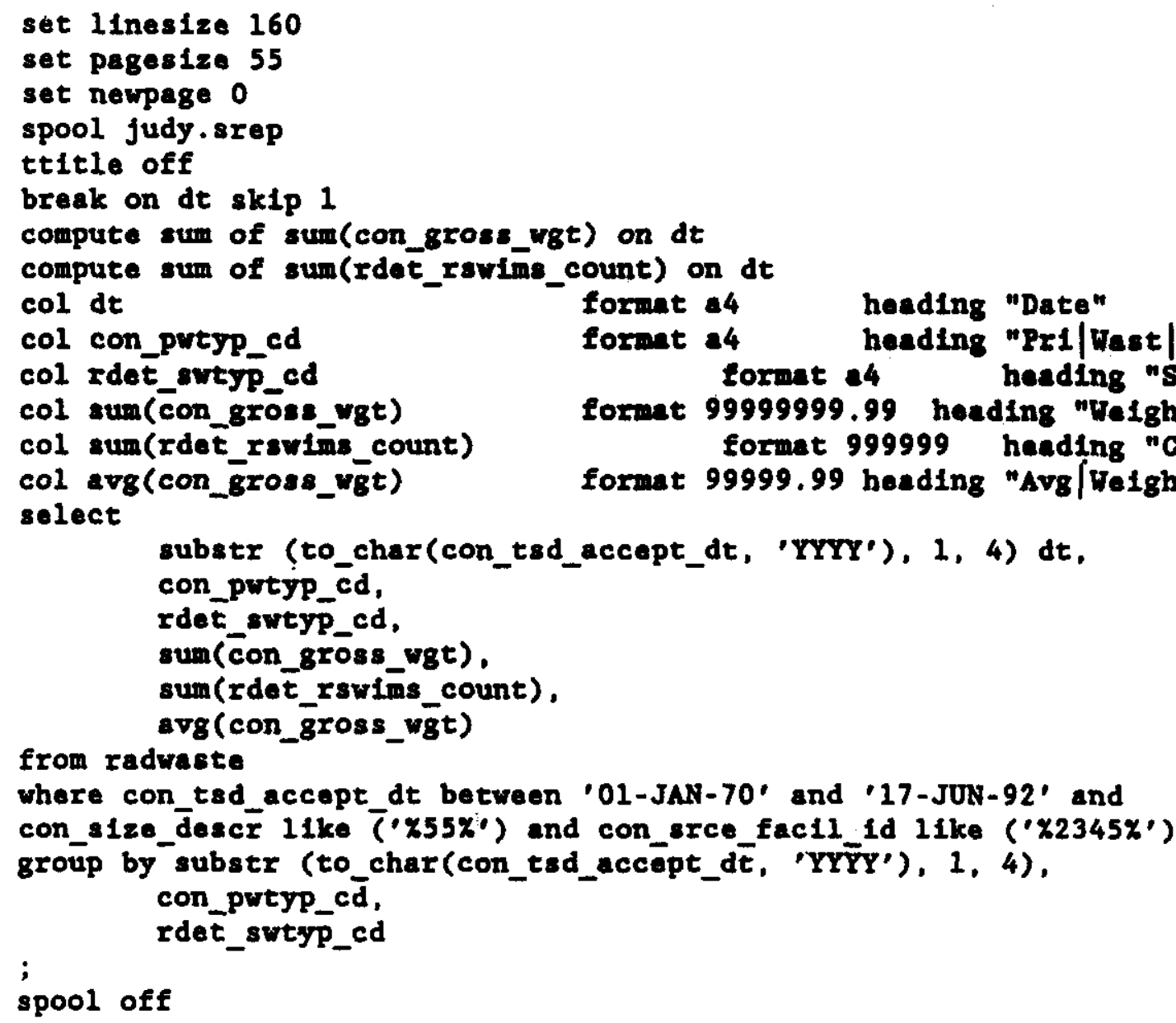


WHC-EP-0621

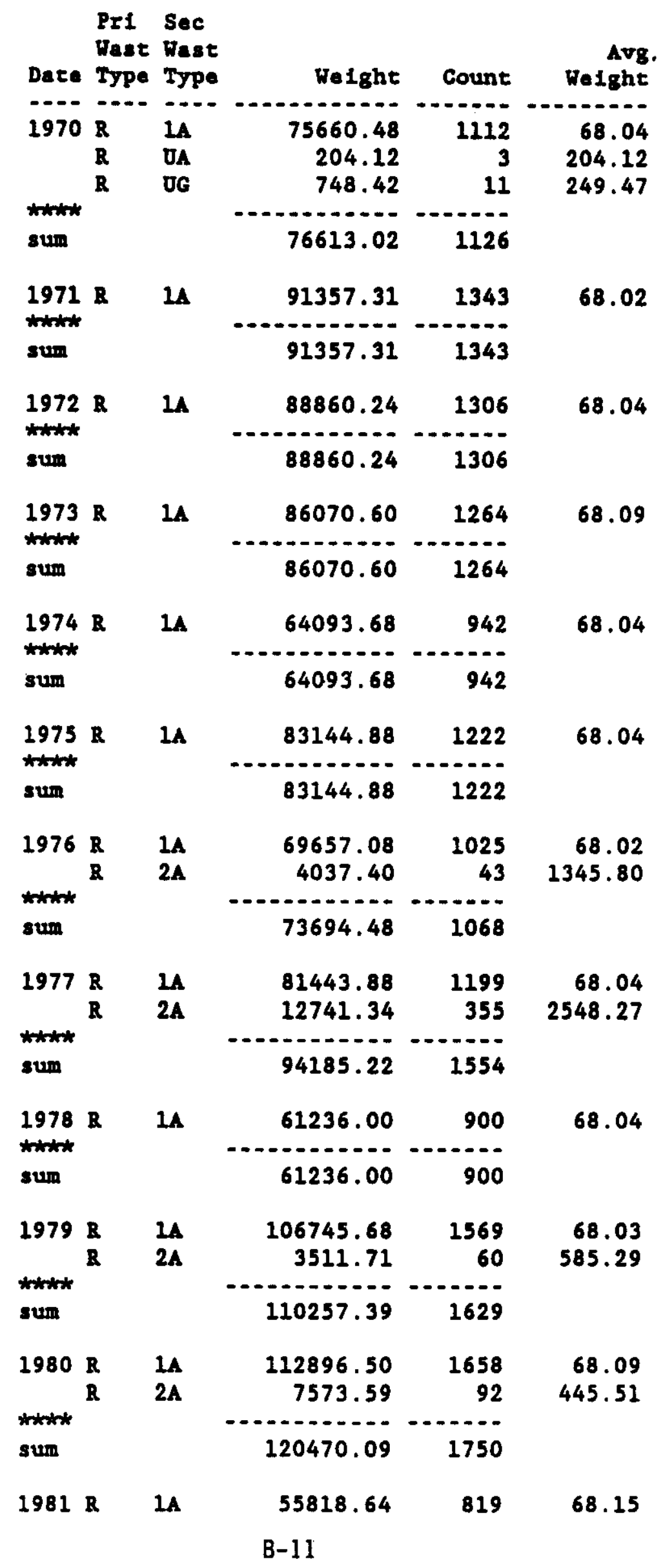




\begin{tabular}{|c|c|c|c|c|c|}
\hline Date & $\begin{array}{l}\text { Pr1 } \\
\text { Wast } \\
\text { Type }\end{array}$ & $\begin{array}{l}\text { Sec } \\
\text { Wast } \\
\text { Type }\end{array}$ & Neight & Count & $\begin{array}{r}\text { Avg } \\
\text { Weight }\end{array}$ \\
\hline 1981 & $\mathbb{R}_{\mathbf{R}}^{-\cdots}$ & $2 A$ & $\begin{array}{l}16437.66 \\
10899.77\end{array}$ & $\begin{array}{r}182 \\
73\end{array}$ & $\begin{array}{l}1174.12 \\
2724.94\end{array}$ \\
\hline sum & & & 83156.07 & 1074 & \\
\hline 1982 & $\begin{array}{l}\mathbf{Y} \\
\mathbf{L P} \\
\mathbf{R} \\
\mathbf{R}\end{array}$ & $\begin{array}{l}14 \\
14 \\
14 \\
24\end{array}$ & $\begin{array}{r}68.04 \\
482.62 \\
45099.39 \\
1172.53\end{array}$ & $\begin{array}{r}1 \\
6 \\
665 \\
25\end{array}$ & $\begin{array}{r}68.04 \\
80.44 \\
67.82 \\
390.84\end{array}$ \\
\hline thints & & & 46822.58 & 697 & \\
\hline 1983 & $\begin{array}{l}\mathrm{H} \\
\mathbf{U} \\
\mathbf{M P} \\
\mathrm{R} \\
\mathrm{R} \\
\mathrm{R}\end{array}$ & $\begin{array}{l}1 A \\
1 D \\
1 A \\
1 A \\
1 D \\
2 A\end{array}$ & $\begin{array}{r}909.00 \\
297.56 \\
319.32 \\
45858.69 \\
15389.84 \\
7437.52\end{array}$ & $\begin{array}{r}12 \\
4 \\
4 \\
713 \\
216 \\
51\end{array}$ & $\begin{array}{r}75.75 \\
74.39 \\
79.83 \\
64.23 \\
71.25 \\
1239.59\end{array}$ \\
\hline $\begin{array}{ll}t+6 x-\pi \\
\sin \end{array}$ & & & 70211.93 & 1000 & \\
\hline 1984 & $\begin{array}{l}\mathbf{M} \\
\mathbf{R} \\
\mathbf{R} \\
\mathbf{R}\end{array}$ & $\begin{array}{l}1 \mathrm{~A} \\
1 \mathrm{~A} \\
1 \mathrm{~B} \\
1 \mathrm{D}\end{array}$ & $\begin{array}{r}197.31 \\
15023.40 \\
56.70 \\
106113.78\end{array}$ & $\begin{array}{r}3 \\
172 \\
1 \\
1679\end{array}$ & $\begin{array}{l}65.77 \\
87.35 \\
56.70 \\
63.24\end{array}$ \\
\hline twith & & & 121391.19 & 1855 & \\
\hline 1985 & $\begin{array}{l}\boldsymbol{Y} \\
\mathbf{Z P} \\
\mathbf{R} \\
\mathbf{R} \\
\mathbf{R} \\
\mathbf{R} \\
\mathbf{R} \\
\mathbf{R}\end{array}$ & $\begin{array}{l}1 D \\
1 A \\
1 A \\
1 D \\
2 A \\
2 L \\
3 A \\
3 L\end{array}$ & $\begin{array}{r}1023.07 \\
1319.74 \\
438.18 \\
113112.98 \\
892.21 \\
266.98 \\
3612.39 \\
3513.15\end{array}$ & $\begin{array}{r}13 \\
14 \\
6 \\
1749 \\
8 \\
5 \\
27 \\
66\end{array}$ & $\begin{array}{r}78.70 \\
94.27 \\
73.03 \\
64.67 \\
892.21 \\
53.40 \\
1204.13 \\
53.23\end{array}$ \\
\hline twith & & & 124178.70 & 1888 & \\
\hline 1986 & $\begin{array}{l}\mathbf{R} \\
\mathbf{R} \\
\mathbf{R} \\
\mathbf{R} \\
\mathbf{R} \\
\mathbf{R}\end{array}$ & $\begin{array}{l}1 C \\
1 D \\
2 A \\
2 L \\
3 A \\
3 L\end{array}$ & $\begin{array}{r}3076.94 \\
30424.55 \\
7597.63 \\
6420.95 \\
1627.93 \\
43551.91\end{array}$ & $\begin{array}{r}59 \\
505 \\
128 \\
117 \\
20 \\
813\end{array}$ & $\begin{array}{r}52.15 \\
60.25 \\
1085.38 \\
54.88 \\
1627.93 \\
53.57\end{array}$ \\
\hline $\begin{array}{l}\text { **t*k } \\
\text { sum }\end{array}$ & & & 92699.91 & 1642 & \\
\hline 1987 & $\begin{array}{l}\mathbf{Y} \\
\mathbf{M} \\
\mathbf{R}\end{array}$ & $\begin{array}{l}2 A \\
3 A \\
1 C\end{array}$ & $\begin{array}{r}40.82 \\
86.00 \\
21820.00\end{array}$ & $\begin{array}{r}1 \\
2 \\
339\end{array}$ & $\begin{array}{l}40.82 \\
86.00 \\
64.37\end{array}$ \\
\hline
\end{tabular}


WHC-EP-0621

\begin{tabular}{|c|c|c|c|c|c|}
\hline Dete & $\begin{array}{l}\text { Pr1 } \\
\text { Dast } \\
\text { Type }\end{array}$ & $\begin{array}{l}\text { Sec } \\
\text { Wast } \\
\text { Type }\end{array}$ & Neight & Count & $\begin{array}{r}\text { Avg } \\
\text { WeIght }\end{array}$ \\
\hline 1987 & $\begin{array}{l}\vec{R} \\
\mathbf{R} \\
\mathbf{R}\end{array}$ & $\begin{array}{l}0- \\
1 D \\
32\end{array}$ & $\begin{array}{r}6829.98 \\
7323.65 \\
71215.62\end{array}$ & $\begin{array}{r}83 \\
193 \\
1263\end{array}$ & $\begin{array}{r}82.29 \\
665.79 \\
56.39\end{array}$ \\
\hline $\sin$ & & & 107316.07 & 1881 & \\
\hline 1988 & $\begin{array}{l}\mathbf{Z} \\
\mathbf{X P} \\
\mathbf{Z P} \\
\mathrm{R} \\
\mathrm{R} \\
\mathrm{R} \\
\mathrm{R} \\
\mathrm{R} \\
\mathrm{R}\end{array}$ & $\begin{array}{l}2 A \\
1 D \\
2 A \\
1 C \\
1 D \\
2 A \\
21 \\
3 A \\
3 L\end{array}$ & $\begin{array}{r}1214.98 \\
699.99 \\
12437.25 \\
8279.32 \\
3369.61 \\
7567.24 \\
994.03 \\
9778.96 \\
41260.60\end{array}$ & $\begin{array}{r}10 \\
10 \\
177 \\
133 \\
47 \\
110 \\
17 \\
146 \\
705\end{array}$ & $\begin{array}{r}121.50 \\
70.00 \\
70.27 \\
62.25 \\
71.69 \\
1261.21 \\
58.47 \\
1222.37 \\
58.53\end{array}$ \\
\hline sum & & & 85601.98 & 1355 & \\
\hline 1989 & $\begin{array}{l}M \\
M \\
M \\
M P \\
M P \\
R \\
R \\
R \\
R \\
R\end{array}$ & $\begin{array}{l}1 C \\
1 D \\
2 A \\
1 D \\
2 A \\
1 C \\
1 D \\
2 L \\
3 A \\
3 L\end{array}$ & $\begin{array}{r}67.00 \\
3186.87 \\
2911.17 \\
438.63 \\
1621.62 \\
3393.84 \\
998.62 \\
63.50 \\
1220.16 \\
28386.15\end{array}$ & $\begin{array}{r}1 \\
36 \\
38 \\
6 \\
27 \\
51 \\
15 \\
1 \\
21 \\
488\end{array}$ & $\begin{array}{l}67.00 \\
88.52 \\
76.61 \\
73.11 \\
60.06 \\
66.55 \\
66.57 \\
63.50 \\
58.10 \\
58.17\end{array}$ \\
\hline $\sin$ & & & 42287.56 & 684 & \\
\hline 1990 & $\begin{array}{l}\mathbf{H} \\
\mathbf{M} \\
\mathbf{M P} \\
\mathbf{M P} \\
\mathbf{R} \\
\mathbf{R} \\
\mathbf{R} \\
\mathbf{R} \\
\mathbf{R} \\
\mathbf{R}\end{array}$ & $\begin{array}{l}1 D \\
2 A \\
1 D \\
2 A \\
1 C \\
1 D \\
2 A \\
2 L \\
3 A \\
3 L\end{array}$ & $\begin{array}{r}2469.03 \\
3051.02 \\
45.99 \\
530.07 \\
1643.08 \\
7193.07 \\
2658.57 \\
2175.01 \\
8804.85 \\
28428.27\end{array}$ & $\begin{array}{r}20 \\
21 \\
1 \\
8 \\
24 \\
116 \\
43 \\
35 \\
166 \\
498\end{array}$ & $\begin{array}{r}123.45 \\
145.29 \\
45.99 \\
66.26 \\
68.46 \\
63.10 \\
61.83 \\
62.14 \\
53.04 \\
58.98\end{array}$ \\
\hline thin & & & 56998.96 & 932 & \\
\hline 1991 & $\begin{array}{l}\mathbf{H} \\
\mathbf{M} \\
\mathbf{M} \\
\mathbf{M P} \\
\mathbf{R} \\
\mathbf{R} \\
\mathbf{R}\end{array}$ & $\begin{array}{l}1 A \\
1 D \\
2 A \\
2 A \\
1 A \\
1 C \\
1 D\end{array}$ & $\begin{array}{r}386.00 \\
1325.99 \\
1815.03 \\
662.01 \\
1386.00 \\
905.97 \\
4676.05\end{array}$ & $\begin{array}{r}4 \\
14 \\
28 \\
10 \\
21 \\
14 \\
69\end{array}$ & $\begin{array}{l}96.50 \\
94.71 \\
64.82 \\
66.20 \\
66.00 \\
64.71 \\
67.77\end{array}$ \\
\hline
\end{tabular}




\section{WHC-EP-0621}

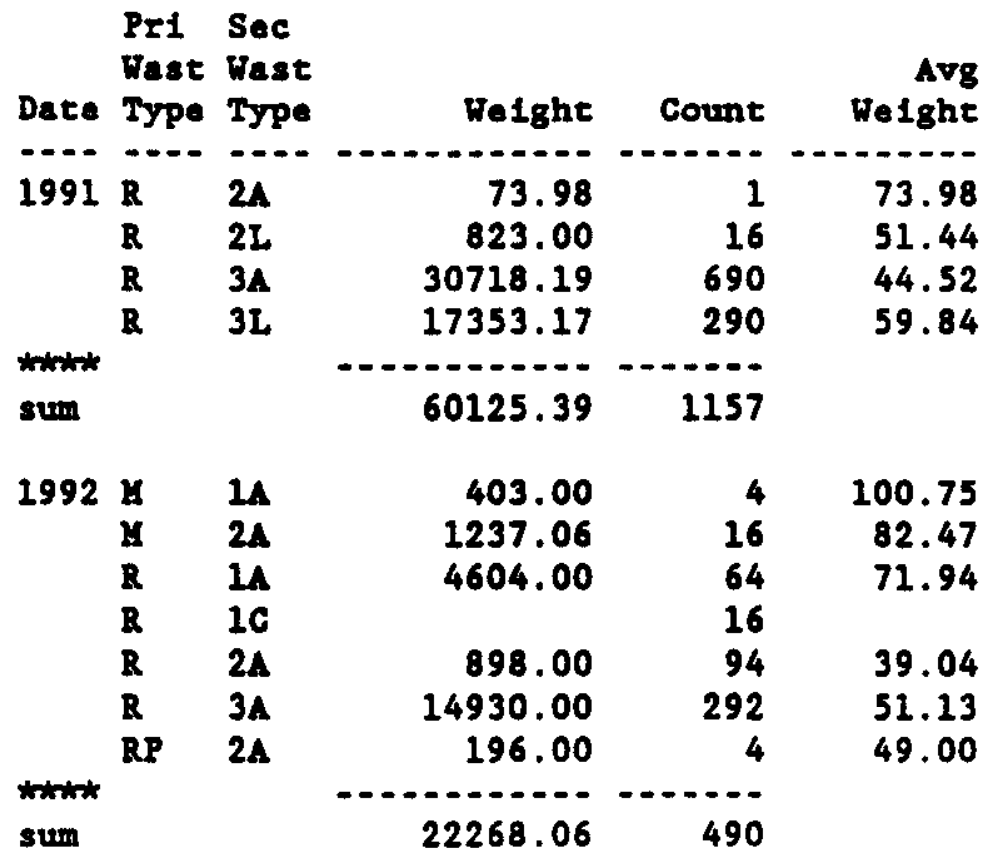

101 rows selected. 
WHC-EP-0621

APPENDIX B.2

TRU WASTE IN CONTAINERS OTHER THAN 55-GALLON DRUMS - SORTED BY WASTE TYPE 
WHC-EP-0621

This page intentionally left blank. 


\section{TRU WASTE IN CONTAINERS OTHER THAN 55-GALLON} DRUMS - SORTED BY WASTE TYPE

The data included in this subappendix are waste type container numbers and total weight (in kilograms) of radioactive wastes packaged in containers other than 55-gal drums. The primary and secondary waste type codes are explained in the introduction to Appendix B.

The data in the average weight column should be studied cautiously, especially for low-level waste (LLW). Records for LLW are still done for batches. This means that one recorded weight on a single Solid Waste Information and Tracking System (SWITS) record may be the composite weight for a group of containers. The computer program used simply divided the sum of all weights by the number of values used to compute the sum, not by the actual number of containers those values represent.

Also, it should be remembered that individual container weights for transuranic (TRU) waste packages were not required before 1978. The weight values for containers generated between 1970 and 1978 represent standard values assigned during the Richland Solid Waste Information Management System (R-SWIMS) data re-entry program in the mid-1980's. 


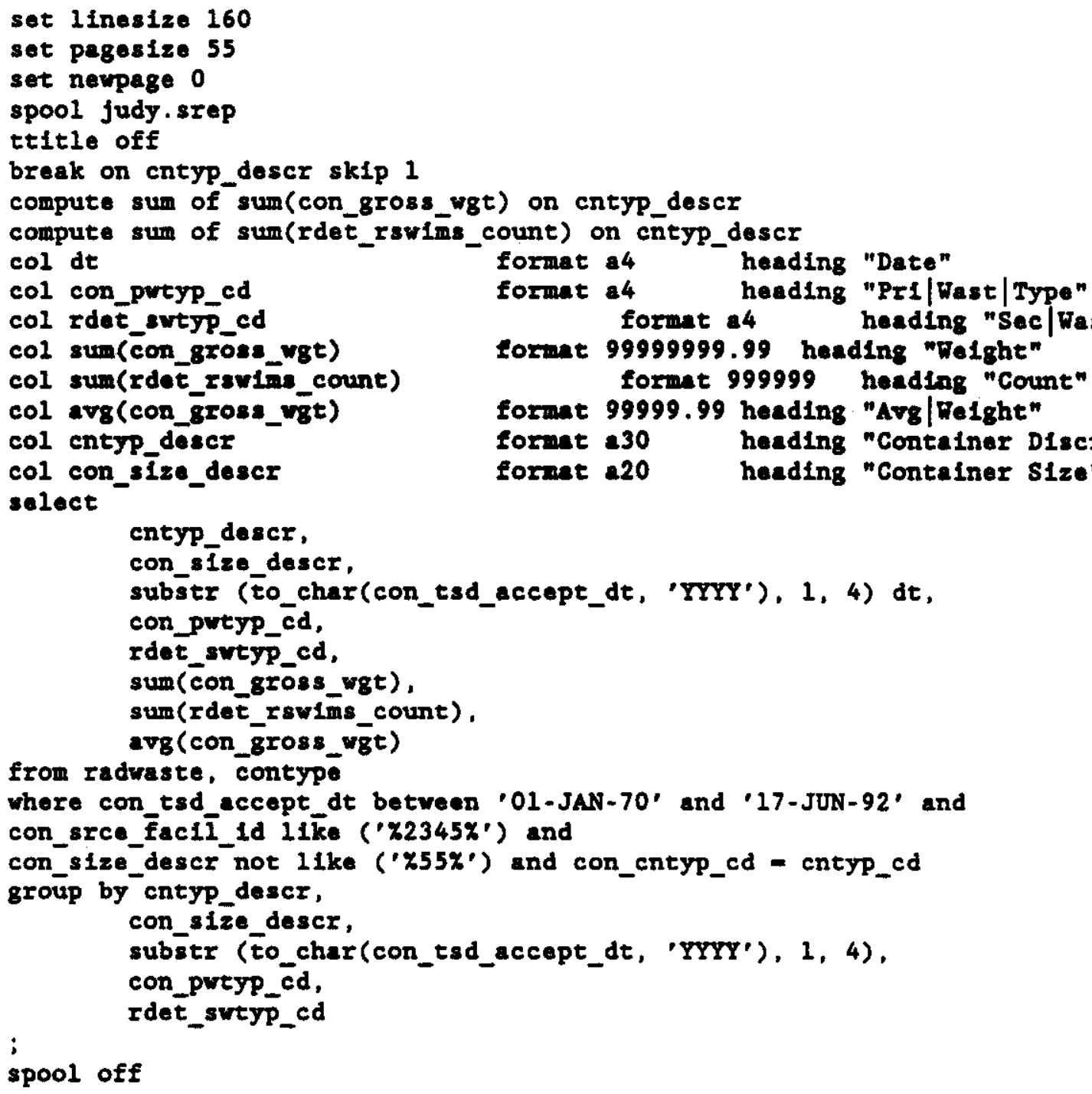




\begin{tabular}{|c|c|c|c|c|c|c|c|}
\hline Container Discription & Contalner Size & Date & $\begin{array}{l}\text { Pri } \\
\text { Wast } \\
\text { Type }\end{array}$ & $\begin{array}{l}\text { Sec } \\
\text { Wast } \\
\text { Type }\end{array}$ & We1ght & Count & $\begin{array}{r}\text { Av } \\
\text { We1gh }\end{array}$ \\
\hline BURLAP, CLOTH, PAPER OR PLASTI & Udranons & 1970 & R & Ua & 1638.36 & 13 & 327.6 \\
\hline & 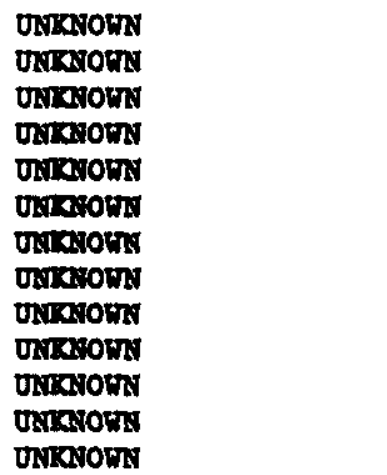 & $\begin{array}{l}1970 \\
1976 \\
1979 \\
1980 \\
1981 \\
1982 \\
1983 \\
1985 \\
1986 \\
1987 \\
1988 \\
1989 \\
1990\end{array}$ & $\begin{array}{l}\mathbf{R} \\
\mathbf{R} \\
\mathbf{R} \\
\mathbf{R} \\
\mathbf{R} \\
\mathbf{R} \\
\mathbf{R} \\
\mathbf{R} \\
\mathbf{R} \\
\mathbf{R} \\
\mathbf{R} \\
\mathbf{R} \\
\mathbf{R}\end{array}$ & $\begin{array}{l}0 G \\
2 A \\
2 A \\
2 A \\
2 A \\
2 A \\
2 A \\
2 A \\
2 A \\
2 A \\
2 A \\
2 A \\
2 A\end{array}$ & $\begin{array}{r}129.72 \\
37.15 \\
254.01 \\
18168.55 \\
226.80 \\
181.44 \\
791.06 \\
907.18 \\
2721.54 \\
4082.31 \\
1905.08 \\
2721.54 \\
810.00\end{array}$ & $\begin{array}{r}6 \\
1 \\
12 \\
706 \\
2 \\
4 \\
2 \\
2 \\
2 \\
3 \\
5 \\
2 \\
18\end{array}$ & $\begin{array}{r}32.4 \\
57.1 \\
254.0 \\
6056.1 \\
226.8 \\
181.4 \\
395.5 \\
907.1 \\
1360.7 \\
1360.7 \\
952.5 \\
1360.7 \\
45.0\end{array}$ \\
\hline 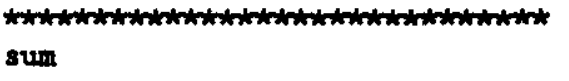 & & & & & 34594.74 & 778 & \\
\hline CONCBETE BOXES & $\begin{array}{l}\text { onrasons } \\
\text { ontratown }\end{array}$ & $\begin{array}{l}1970 \\
1970\end{array}$ & $\begin{array}{l}\mathbf{R} \\
\mathbf{R}\end{array}$ & $\begin{array}{l}2 \mathbf{A} \\
\mathbf{0 A}\end{array}$ & $\begin{array}{r}20638.35 \\
331.12\end{array}$ & 10 & $\begin{array}{r}20638.3 \\
331.1\end{array}$ \\
\hline 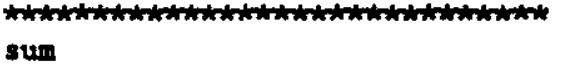 & & & & & 20969.47 & 11 & \\
\hline DURAP TRUCK & 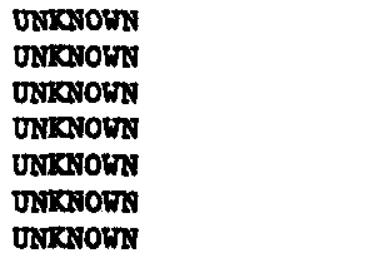 & $\begin{array}{l}1981 \\
1985 \\
1986 \\
1987 \\
1988 \\
1989 \\
1990\end{array}$ & $\begin{array}{l}\mathbf{R} \\
\mathbf{R} \\
\mathbf{R} \\
\mathbf{R} \\
\mathbf{R} \\
\mathbf{R} \\
\mathbf{R}\end{array}$ & $\begin{array}{l}2 A \\
2 A \\
2 A \\
2 A \\
2 A \\
2 A \\
2 A\end{array}$ & $\begin{array}{r}2721.54 \\
3235.91 \\
4082.31 \\
3628.72 \\
14968.47 \\
2177.23 \\
680.84\end{array}$ & $\begin{array}{l}2 \\
3 \\
3 \\
3 \\
7 \\
5 \\
1\end{array}$ & $\begin{array}{r}1360.7 \\
1078.6 \\
1360.7 \\
1209.5 \\
2138.3 \\
435.4 \\
680.8\end{array}$ \\
\hline 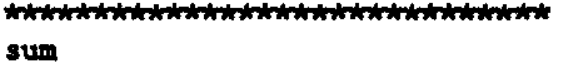 & & & & & 31495.02 & 24 & \\
\hline $\begin{array}{l}\text { BIBERBOARD/PLASTIC BOXES, GART } \\
\text { ONS, CASES }\end{array}$ & $2 \mathrm{CD} \mathrm{ET}$ & 1970 & $\mathbf{R}$ & OA & 76.20 & 14 & 25.4 \\
\hline & 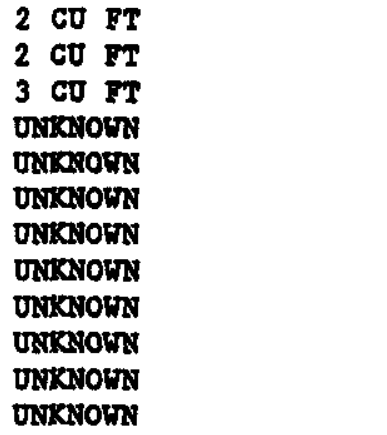 & $\begin{array}{l}1970 \\
1980 \\
1981 \\
1970 \\
1970 \\
2973 \\
1979 \\
1980 \\
1981 \\
1981 \\
1982 \\
1983\end{array}$ & $\begin{array}{l}\mathbf{R} \\
\mathbf{R} \\
\mathbf{R} \\
\mathbf{R} \\
\mathbf{R} \\
\mathbf{R} \\
\mathbf{R} \\
\mathbf{R} \\
\mathbf{R} \\
\mathbf{R} \\
\mathbf{R} \\
\mathbf{R}\end{array}$ & $\begin{array}{l}0 G \\
2 A \\
3 A \\
0 A \\
0 G \\
2 A \\
2 A \\
2 A \\
2 A \\
3 A \\
2 A \\
2 A\end{array}$ & $\begin{array}{r}21.77 \\
10.89 \\
1387.99 \\
9449.19 \\
6641.47 \\
38.10 \\
546.12 \\
3195.10 \\
615.97 \\
283.49 \\
152.41 \\
101.60\end{array}$ & $\begin{array}{r}4 \\
2 \\
153 \\
744 \\
523 \\
3 \\
11 \\
218 \\
45 \\
25 \\
12 \\
8\end{array}$ & $\begin{array}{r}7.2 \\
10.8 \\
694.0 \\
497.3 \\
415.0 \\
38.1 \\
546.1 \\
290.4 \\
123.1 \\
283.4 \\
152.4 \\
101.6\end{array}$ \\
\hline $\sin 1$ & & & & & 22520.30 & 1762 & \\
\hline
\end{tabular}




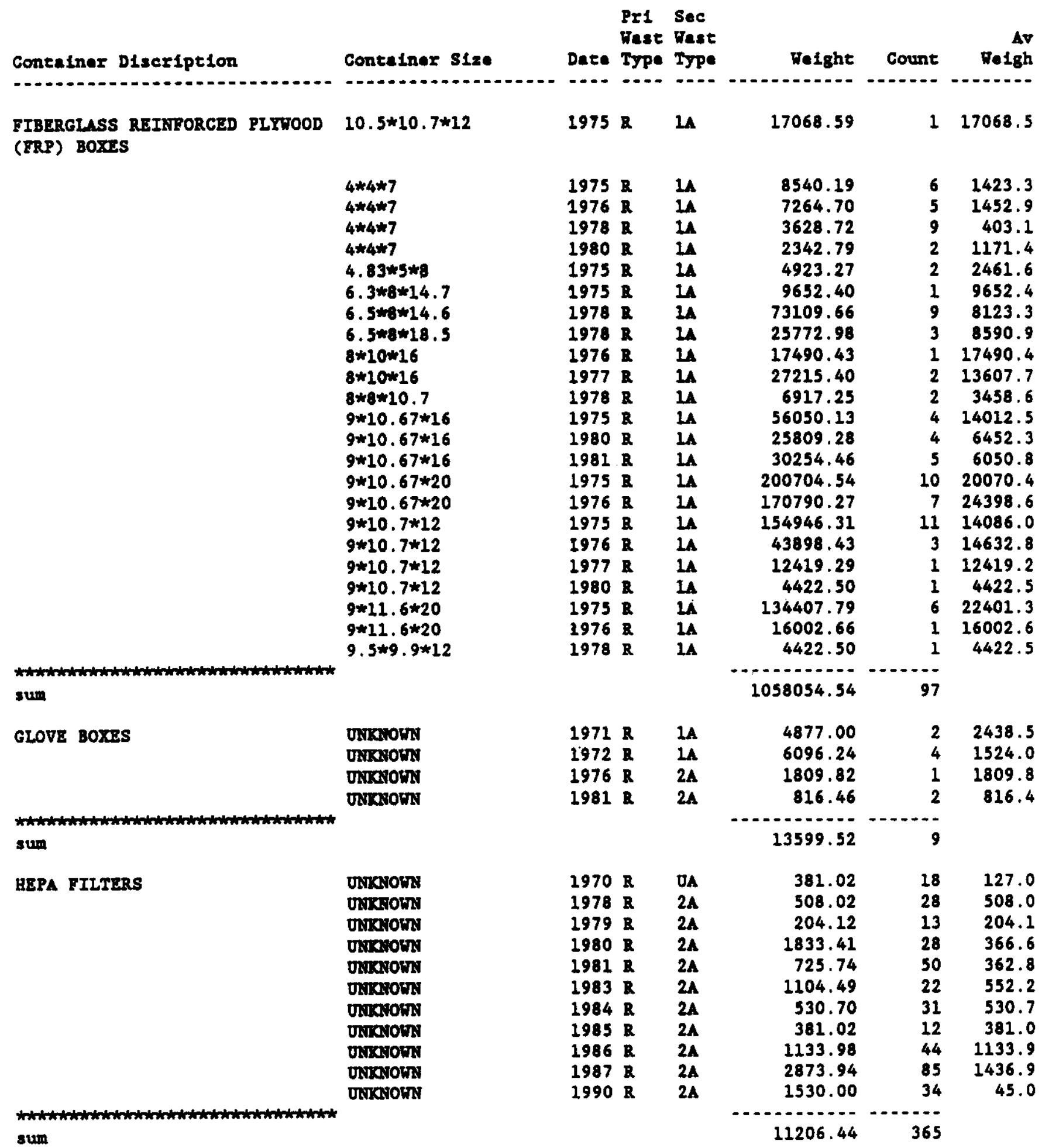




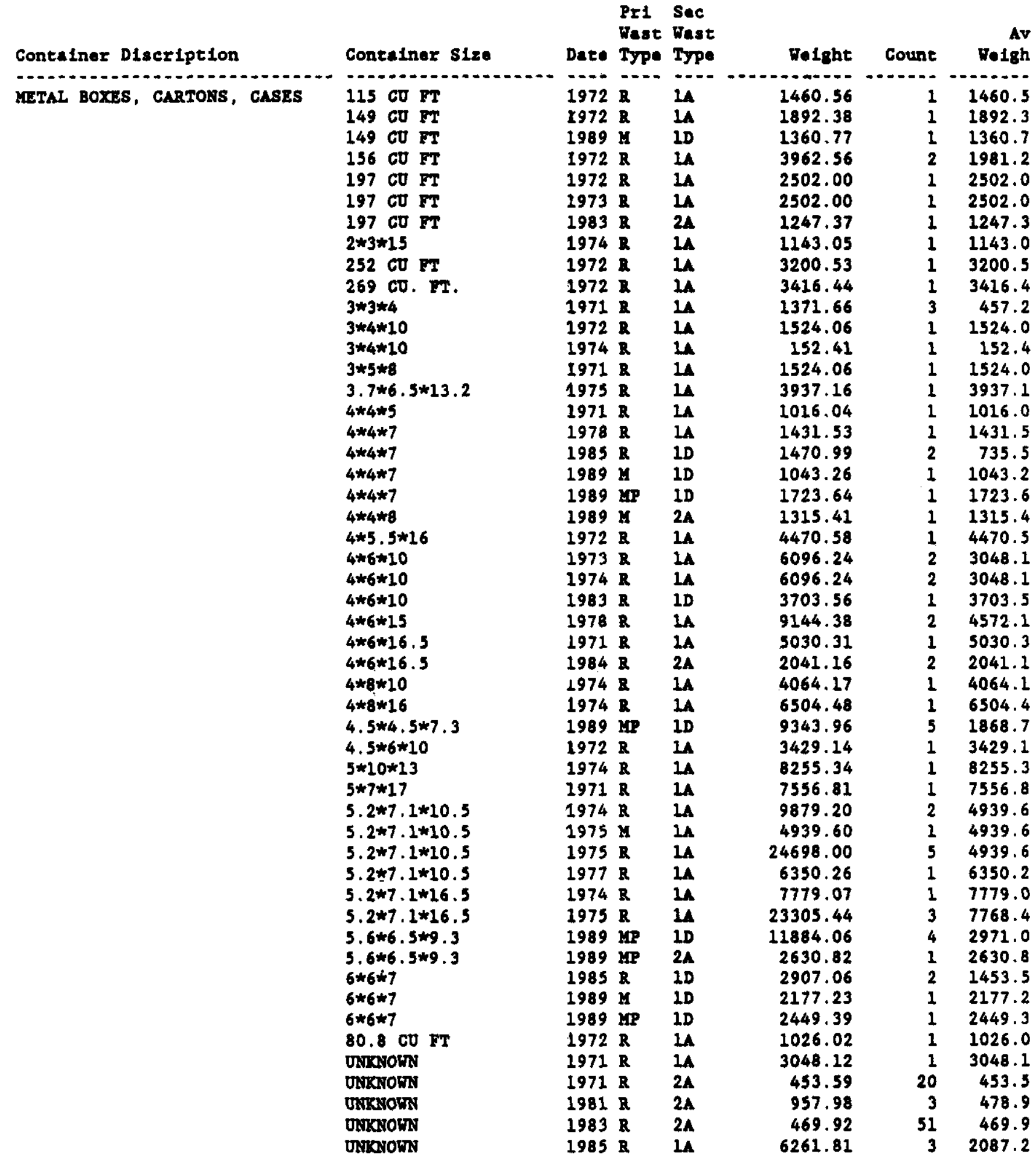




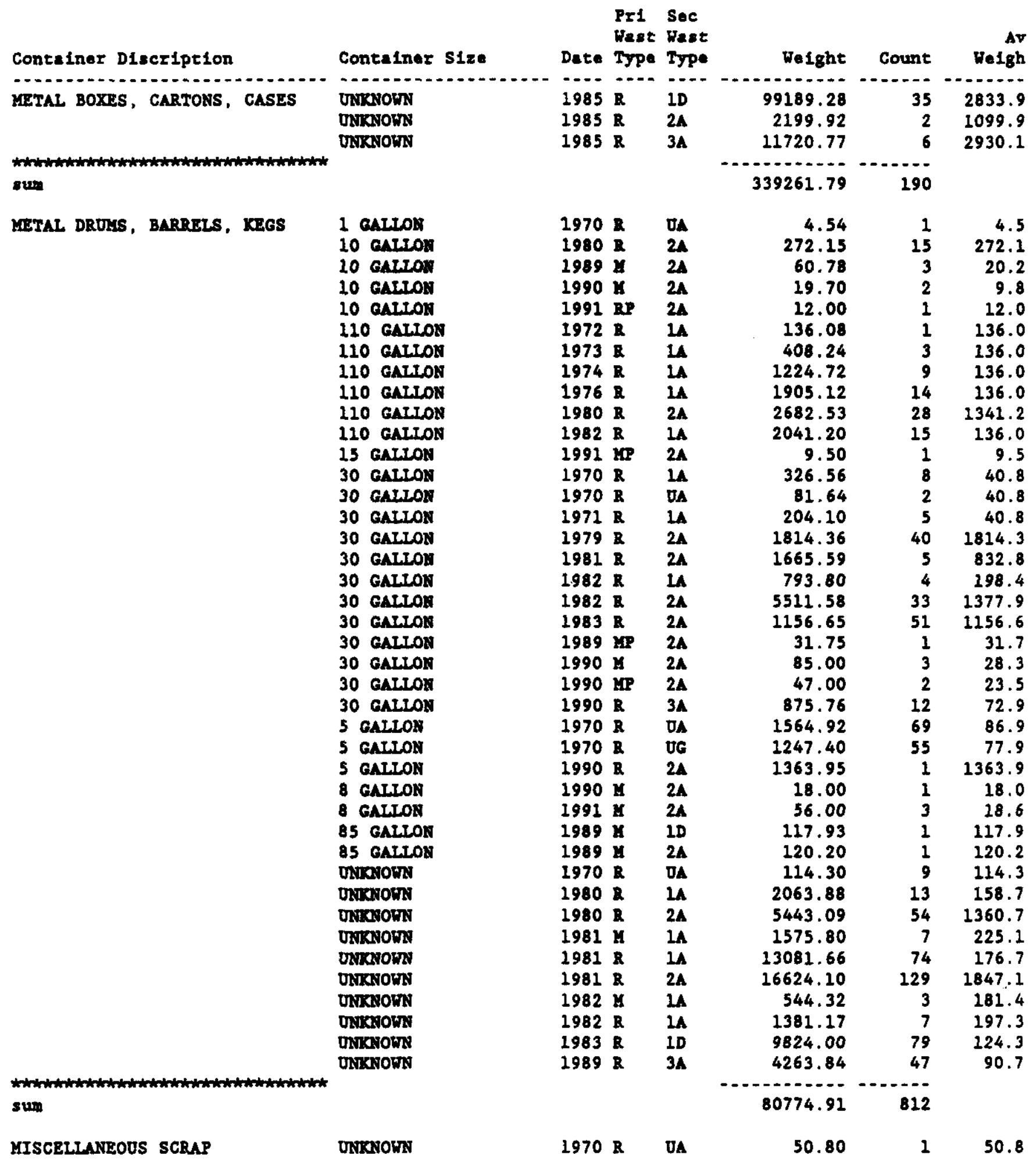




\begin{tabular}{|c|c|c|c|c|c|c|c|}
\hline Container Discription & Container Size & Date & $\begin{array}{l}\text { Prt } \\
\text { Wast } \\
\text { Type }\end{array}$ & $\begin{array}{l}\text { Sec } \\
\text { Wast } \\
\text { Type }\end{array}$ & We1ght & Count & Av \\
\hline 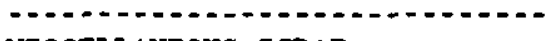 & 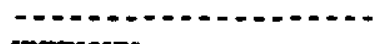 & $\ddot{-n}$ & --- & --- & $n-n-n-n$ & 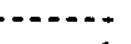 & 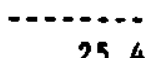 \\
\hline MISCELLANEOUS SCRAP & $\begin{array}{l}\text { UnNewOHN } \\
\text { UNKNYOWN }\end{array}$ & $\begin{array}{l}1970 \\
1971\end{array}$ & $\begin{array}{l}\mathbf{R} \\
\mathbf{R}\end{array}$ & $\begin{array}{l}\text { UG } \\
2 A\end{array}$ & $\begin{array}{r}25.40 \\
381.02\end{array}$ & $\begin{array}{l}1 \\
3\end{array}$ & $\begin{array}{r}25.4 \\
381.0\end{array}$ \\
\hline & DLTENOWN & 1975 & $\mathbf{R}$ & $2 \mathbf{A}$ & 2540.10 & 1 & 2540.1 \\
\hline & Unarown & 1977 & $\mathbf{R}$ & 24 & 5250.30 & 2 & 2625.1 \\
\hline & DRANOAN & 1978 & $\mathbf{R}$ & $2 \mathbf{A}$ & 7783.61 & 6 & 1297.2 \\
\hline & DSTWONA & 1979 & $\mathbf{R}$ & $2 A$ & 333.39 & 21 & 333.3 \\
\hline & Dorasom & 1980 & $\mathbf{R}$ & $2 \mathbf{A}$ & 4272.82 & 26 & 854.5 \\
\hline & DaRom & 1981 & $\mathbf{R}$ & $2 \boldsymbol{A}$ & 1461.46 & 26 & 730.7 \\
\hline & oncrown & 1981 & $\mathbf{R}$ & $3 \mathbf{A}$ & 435.45 & 20 & 435.4 \\
\hline & Dixarots & 1982 & $\mathbf{R}$ & 24 & 113.40 & 14 & 113.4 \\
\hline & Unaromy & 1983 & $\mathbf{R}$ & $\mathbf{2 4}$ & 1977.66 & 2 & 988.8 \\
\hline & UNALOWN & 1984 & $\mathbf{R}$ & $\mathbf{L A}$ & 86.18 & 1 & 86.1 \\
\hline 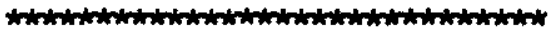 & & & & & $n-\cdots+-n$ & 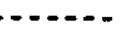 & \\
\hline sum & & & & & 24711.59 & 124 & \\
\hline SELF CONTAINED, EQUIPLENT & UNRTOWN & 1970 & $\mathbf{R}$ & $2 \mathbf{A}$ & 254.01 & 1 & 254.0 \\
\hline & onxarown & 1970 & $\mathbf{R}$ & UA & 190.51 & 3 & 95.2 \\
\hline & Unxwown & 1970 & $\mathbf{R}$ & UG & 920.34 & 26 & 102.2 \\
\hline & UARAOHN & 1971 & $\mathbf{R}$ & 14 & 3657.74 & 2 & 1828.8 \\
\hline & DNRwowns & 1976 & $\mathbf{R}$ & $2 \mathbf{A}$ & 4368.98 & 6 & 2184.4 \\
\hline & Unaroin & 1978 & $\mathbf{R}$ & $2 A$ & 748.43 & 9 & 374.2 \\
\hline & Unavors & 1979 & $\mathbf{R}$ & $2 A$ & 1519.53 & 69 & 506.5 \\
\hline & URTRYOWN & 1980 & $\mathbf{R}$ & $2 \mathbf{A}$ & 1655.61 & 56 & 551.8 \\
\hline & Dixarown & 1981 & $\mathbf{R}$ & $2 A$ & 20028.74 & 423 & 2503.5 \\
\hline & OnKwown & 1981 & $\mathbf{R}$ & 34 & 5007.63 & 2 & 5007.6 \\
\hline & UNKNOWN & 1982 & $\mathbf{R}$ & $2 A$ & 1315.42 & 21 & 438.4 \\
\hline & URTCWOWN & 1983 & $\mathbf{R}$ & $2 A$ & 2755.56 & 16 & 1377.7 \\
\hline & ONXNOWN & 1984 & $\mathbf{R}$ & $2 A$ & 1292.73 & 1 & 1292.7 \\
\hline & UNRNORN & 1985 & $\mathbf{R}$ & $2 \mathbf{A}$ & 898.12 & 34 & 299.3 \\
\hline & ONREOWN & 1987 & $\mathbf{R}$ & $2 A$ & 907.18 & 1 & 907.1 \\
\hline & unvarown & 1988 & $\mathbf{R}$ & $2 A$ & 646.37 & 57 & 646.3 \\
\hline & UnKarown & 1988 & $\mathbf{R}$ & $3 A$ & 7112.30 & 98 & 1185.3 \\
\hline & UnKNOWN & 1989 & $\mathbf{R}$ & $2 \mathbf{A}$ & 5964.71 & 12 & 497.0 \\
\hline & UNKNOWN & 1990 & $\mathbf{R}$ & $2 A$ & 4353.93 & 8 & 544.2 \\
\hline 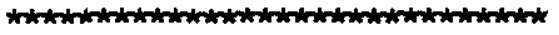 & & & & & 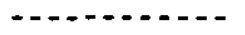 & $\cdots$ & \\
\hline sum & & & & & 63597.84 & 845 & \\
\hline TANKS, PORTABLE & ONRWOWN & 1985 & $\mathbf{R}$ & 2A & 734.82 & 4 & 734.8 \\
\hline 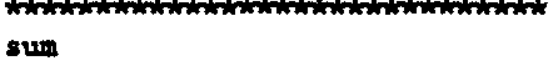 & & & & & 734.82 & 4 & \\
\hline \multirow{8}{*}{$\begin{array}{l}\text { TROCKS, FLATBEDS, COMPACTOR, I } \\
\text { OADLUGGER }\end{array}$} & ONRANOWN & 1970 & $\mathbf{R}$ & DG & 45.36 & 1 & 45.3 \\
\hline & UNKWOWN & 1972 & $\mathbf{R}$ & $2 A$ & 6782.08 & 3 & 3391.0 \\
\hline & UNKANOWN & 1973 & $\mathbf{R}$ & $2 A$ & 13067.92 & 4 & 4355.9 \\
\hline & UNKWOWN & 1974 & $\mathbf{R}$ & $2 A$ & 4343.58 & 2 & 4343.5 \\
\hline & UNKNOWN & 1975 & $\mathbf{R}$ & $2 A$ & 8686.25 & 2 & 4343.1 \\
\hline & UNKNOWN & 1976 & $\mathbf{R}$ & $2 A$ & 24820.44 & 5 & 8273.4 \\
\hline & UNKNOWN & 1977 & $\mathbf{R}$ & $2 A$ & 17336.22 & 6 & 2889.3 \\
\hline & UNKNOWN & 1978 & $\mathbf{R}$ & $2 A$ & 12383.02 & 6 & 2063.8 \\
\hline
\end{tabular}




\begin{tabular}{|c|c|c|c|c|c|c|c|}
\hline Container Discription & Container SLze & Date & $\begin{array}{l}\text { Pri } \\
\text { Weat } \\
\text { Type }\end{array}$ & $\begin{array}{l}\text { Sec } \\
\text { Wate } \\
\text { Type }\end{array}$ & Weight & Count & $\begin{aligned} \text { Av } \\
\text { We1gh }\end{aligned}$ \\
\hline TRUCKS, PLATBEDS, COMPACTOR, I & Dinworn & 1979 & $\mathbf{R}$ & $\ddot{2 A}$ & 6191.51 & 8 & 773.9 \\
\hline & 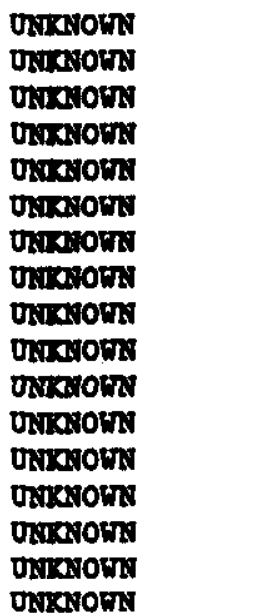 & $\begin{array}{l}1980 \\
1981 \\
1981 \\
1982 \\
1983 \\
1984 \\
1984 \\
1984 \\
1985 \\
1986 \\
1986 \\
1987 \\
1988 \\
1989 \\
1990 \\
1990 \\
1991\end{array}$ & $\begin{array}{l}\mathbf{R} \\
\mathbf{H} \\
\mathbf{R} \\
\mathbf{R} \\
\mathbf{R} \\
\mathbf{X} \\
\mathbf{R} \\
\mathbf{R} \\
\mathbf{R} \\
\mathbf{H} \\
\mathbf{R} \\
\mathbf{R} \\
\mathbf{R} \\
\mathbf{R} \\
\mathbf{R} \\
\mathbf{R} \\
\mathbf{R}\end{array}$ & $\begin{array}{l}2 A \\
2 A \\
2 A \\
2 A \\
2 A \\
2 A \\
2 A \\
3 A \\
2 A \\
2 A \\
2 A \\
2 A \\
2 A \\
2 A \\
2 A \\
3 A \\
2 A\end{array}$ & $\begin{array}{r}37826.70 \\
4535.90 \\
48557.72 \\
2478.87 \\
2494.75 \\
1587.57 \\
20320.83 \\
4535.90 \\
37359.97 \\
8391.43 \\
18143.61 \\
17690.01 \\
12246.93 \\
1534.04 \\
8847.78 \\
1362.12 \\
4999.93\end{array}$ & $\begin{array}{r}18 \\
1 \\
16 \\
38 \\
3 \\
1 \\
8 \\
1 \\
28 \\
4 \\
11 \\
10 \\
6 \\
6 \\
8 \\
3 \\
3\end{array}$ & $\begin{array}{r}2101.4 \\
4535.9 \\
3468.4 \\
619.7 \\
831.5 \\
1587.5 \\
2540.1 \\
4535.9 \\
1334.2 \\
2097.8 \\
1649.4 \\
1965.5 \\
2041.1 \\
255.6 \\
1105.9 \\
454.0 \\
1666.6\end{array}$ \\
\hline $\begin{array}{l}\text { hum } \\
\text { sum }\end{array}$ & & & & & 326570.43 & 202 & \\
\hline TOODEA BOXES, CARTONS, CASES & 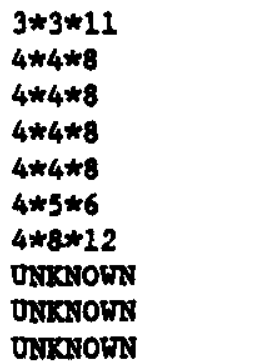 & $\begin{array}{l}1970 \\
1990 \\
1991 \\
1992 \\
1992 \\
1971 \\
1987 \\
1970 \\
1971 \\
1985\end{array}$ & $\begin{array}{l}\mathbf{R} \\
\mathbf{R} \\
\mathbf{R} \\
\mathbf{R} \\
\mathbf{R} \\
\mathbf{R} \\
\mathbf{R} \\
\mathbf{R} \\
\mathbf{R} \\
\mathbf{X}\end{array}$ & $\begin{array}{l}0 A \\
3 A \\
3 A \\
2 A \\
3 A \\
1 A \\
2 A \\
0 A \\
2 A \\
3 A\end{array}$ & $\begin{array}{r}1257.35 \\
4871.56 \\
39288.01 \\
909.00 \\
20083.00 \\
10160.42 \\
1360.77 \\
203.21 \\
1033.73 \\
150.00\end{array}$ & $\begin{array}{r}1 \\
8 \\
35 \\
1 \\
17 \\
1 \\
1 \\
4 \\
2 \\
2\end{array}$ & $\begin{array}{r}1257.3 \\
608.9 \\
1122.5 \\
909.0 \\
1181.3 \\
10160.4 \\
1360.7 \\
203.2 \\
1033.7 \\
150.0\end{array}$ \\
\hline 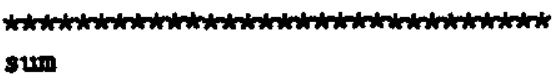 & & & & & 79317.05 & 72 & \\
\hline
\end{tabular}

239 rows selected. 
WHC-EP-0621

APPENDIX B.3

ANNUAL VOLUME OF TRU AND NON-TRU WASTE IN 55-GALLON DRUMS - SORTED BY WASTE TYPE 
WHC-EP-0621

This page intentionally left blank. 


\section{ANNUAL VOLUME OF TRU AND NON-TRU WASTE IN 55-GALLON DRUMS - SORTED BY WASTE TYPE}

The data included in this subappendix are the waste types, total volume (in cubic meters), and total number of 55-gal drums generated annually at the Plutonium Finishing Plant (PFP). The primary and secondary waste type codes are explained in the introduction to Appendix $B$.

The data in the average volume column should be studied cautiously, especially for low-level waste (LLW). Records for LLW containers are still batched. This means that the volume recorded in a single Solid Waste Information Tracking System (SWITS) record may be a composite volume for a group of drums. The computer program used simply divided the sum of all volumes recorded by the number of values present, not by the actual number of containers those values represent. 


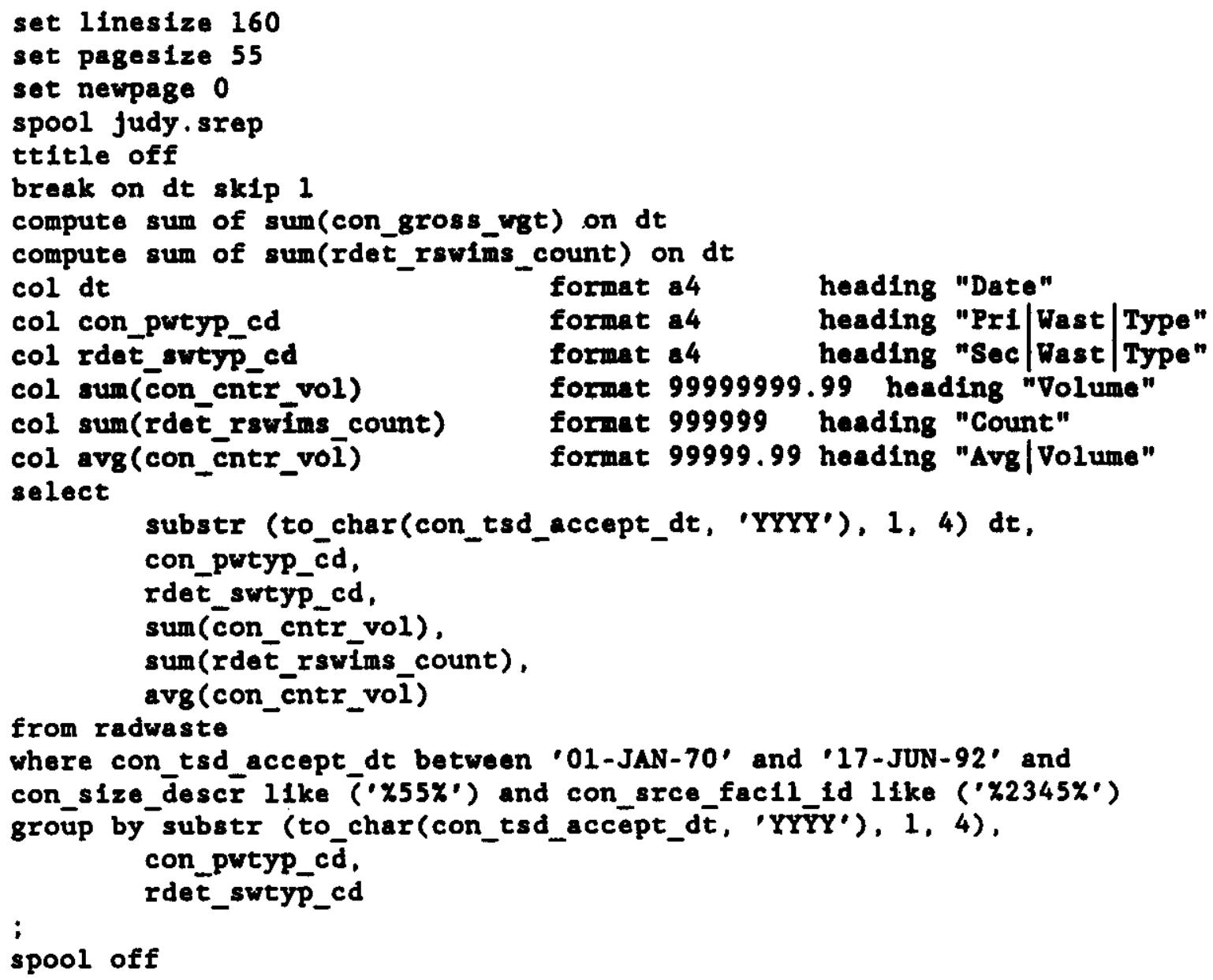




\section{WHC-EP-0621}

\begin{tabular}{|c|c|c|c|c|c|}
\hline Dete & $\begin{array}{l}\text { Pri } \\
\text { Wast } \\
\text { Type }\end{array}$ & $\begin{array}{l}\text { Sec } \\
\text { Wast } \\
\text { Type }\end{array}$ & Volume & Count & $\begin{array}{l}\text { Avg } \\
\text { Volume }\end{array}$ \\
\hline$\cdots$ & $\ldots$ & $\ldots$ & ....... & - & $\ldots \ldots$ \\
\hline 1970 & $R$ & $1 A$ & 233.52 & 1112 & .21 \\
\hline & $R$ & UA & .63 & 3 & .63 \\
\hline & $R$ & Uo & 2.31 & 11 & .77 \\
\hline twt & & & & $\cdots$ & \\
\hline sum & & & & 1126 & \\
\hline 1971 & R & $1 A$ & 282.03 & 1343 & .21 \\
\hline sum & & & & 1343 & \\
\hline 1972 & $R$ & $1 A$ & 274.26 & 1306 & .21 \\
\hline $\sin$ & & & & 1306 & \\
\hline 1973 & R & $1 A$ & 265.65 & $\begin{array}{r}1264 \\
\ldots \ldots\end{array}$ & .21 \\
\hline sum & & & & 1264 & \\
\hline 1974 & $\mathbf{R}$ & $1 A$ & 197.82 & 942 & .21 \\
\hline $\sin$ & & & & 942 & \\
\hline 1975 & $R$ & $1 A$ & 256.62 & 1222 & .21 \\
\hline$\omega \omega$ & & & & . & \\
\hline sum & & & & 1222 & \\
\hline 1976 & $R$ & $1 A$ & 215.25 & 1025 & .21 \\
\hline & $\mathbf{R}$ & $2 A$ & 9.09 & 43 & 3.03 \\
\hline t+men & & & & ........ & \\
\hline sun & & & & 1068 & \\
\hline 1977 & $R$ & $1 A$ & 252.08 & 1199 & .21 \\
\hline & $R$ & $2 A$ & 74.40 & 355 & 14.88 \\
\hline H*\#* & & & & $\ldots \ldots$ & \\
\hline $\sin$ & & & & 1554 & \\
\hline 1978 & R & 14 & 189.00 & 900 & .21 \\
\hline sum & & & & 900 & \\
\hline 1979 & $\mathbf{R}$ & $1 A$ & 329.49 & 1569 & .21 \\
\hline & R & $2 A$ & 12.59 & 60 & 2.10 \\
\hline$m$ mat & & & & $\cdots$ & \\
\hline מטs & & & & 1629 & \\
\hline 1980 & $\mathbf{R}$ & $1 A$ & 368.39 & 1658 & .21 \\
\hline & $R$ & $2 A$ & 20.08 & 92 & 1.18 \\
\hline 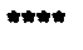 & & & & $\cdots$ & \\
\hline sum & & & & 1750 & \\
\hline 1981 & $R$ & $1 A$ & 172.05 & 819 & .21 \\
\hline & & & $B-29$ & & \\
\hline
\end{tabular}


WHC-EP-0621

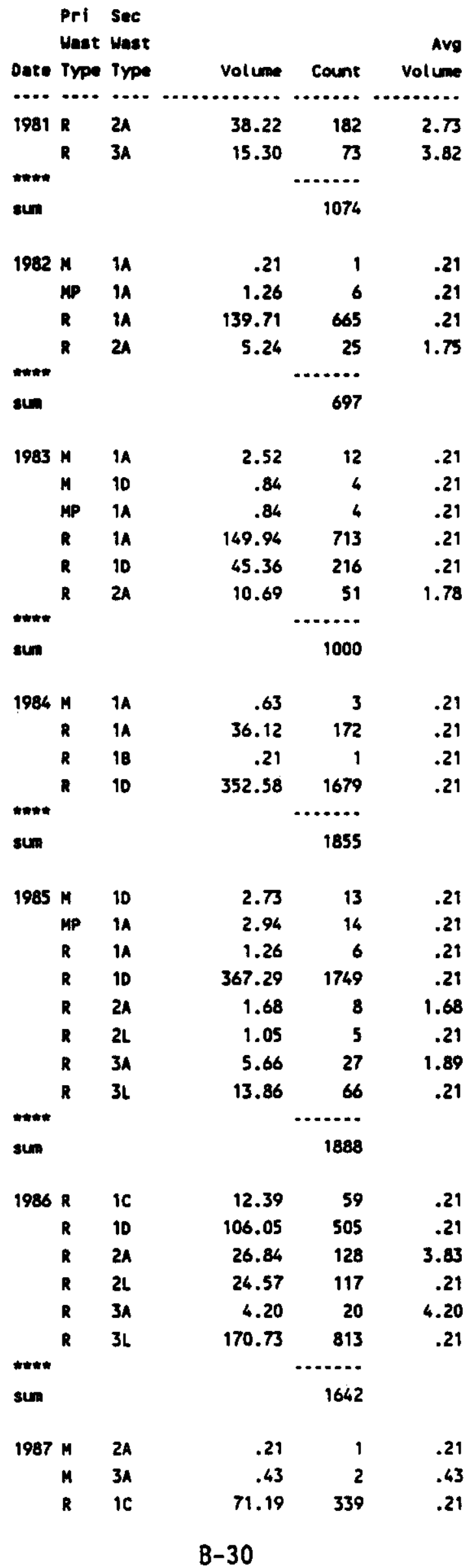




\section{WHC-EP-0621}

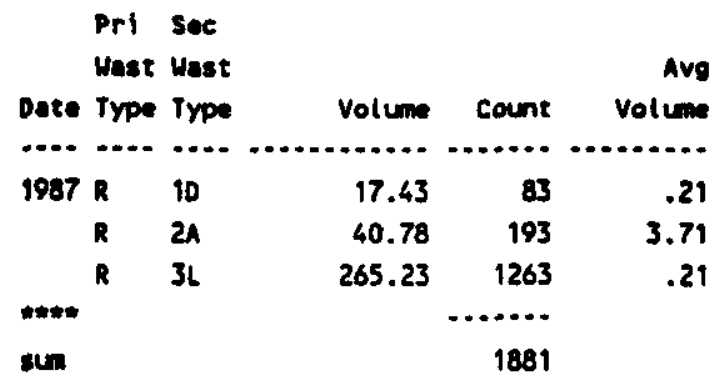

1988 M 2A

$\begin{array}{lll}2.12 & 10 & .21\end{array}$

MP 10

MP $2 A$

$2.10 \quad 10$

.21

R IC

37.17

177

.21

R 10

$27.93 \quad 133$

.21

R 2A

9.87

$24.17 \quad 110 \quad 4.03$

R $2 \mathrm{~L}$

$3.57 \quad 17$

$\begin{array}{lll}30.80 & 146 \quad 3.85\end{array}$

$\begin{array}{ll}R & 3 A \\ R & 3 i\end{array}$

148.05

705

sut

1355

$1989 n$ IC

H 10

M 2A

MP 10

MP 2A

R $1 C$

R 10

R 2 L

R $3 A$

R $3 L$

sum

$\begin{array}{lll}.21 & 1\end{array}$

$\begin{array}{lll}7.56 & 36 & .21\end{array}$

$\begin{array}{lll}7.98 & 38 & .21\end{array}$

$\begin{array}{lll}1.26 & 6 & .21\end{array}$

$\begin{array}{lll}5.67 & 27 & .21\end{array}$

$10.71 \quad 51$

.21

$3.15 \quad 15$

.21

$\begin{array}{lll}.21 & 1\end{array}$

$\begin{array}{lll}4.61 & 21 & .21\end{array}$

$102.48 \quad 488 \quad .21$

1990 M 10

1990 M
MP

MP 10

MP $2 A$

$R$ IC

........

684

R 10

R $2 A$

$4.20 \quad 20$

.21

$4.61 \quad 21$

.21

$.21 \quad 1 \quad .21$

$\begin{array}{lll}1.68 & 8 & .21\end{array}$

$\begin{array}{lll}5.04 & 24 & .21\end{array}$

$18.48 \quad 88 \quad .21$

$13.93 \quad 43 \quad .32$

$13.23 \quad 63 \quad .21$

$34.86 \quad 166 \quad .21$

$104.58 \quad 498 \quad .21$

$\sin$

932

1991 M 1A

M 10

M $2 A$

MP $2 A$

R IA

R IC

R 10

ia

$\begin{array}{rrr}.83 & 4 & .21 \\ 2.94 & 14 & .21 \\ 5.82 & 28 & .21 \\ 2.10 & 10 & .21 \\ 1.25 & 6 & .21 \\ 2.92 & 14 & .21 \\ 14.28 & 68 & .21\end{array}$


WHC-EP-0621

\begin{tabular}{|c|c|c|c|c|c|}
\hline Date & $\begin{array}{l}\text { Pri } \\
\text { West } \\
\text { Typo }\end{array}$ & $\begin{array}{l}\text { Sec } \\
\text { Wast } \\
\text { iype }\end{array}$ & Volume & Count & $\begin{array}{l}\text { Avg } \\
\text { volume }\end{array}$ \\
\hline$\cdots$ & $\cdots$ & $\cdots$ & 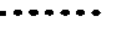 & 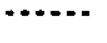 & $\cdots$ \\
\hline 1991 & R & $2 A$ & .21 & 1 & .21 \\
\hline & $R$ & $2 L$ & 6.69 & 32 & .21 \\
\hline & R & $3 \AA$ & 144.82 & 690 & .21 \\
\hline & $\mathbf{R}$ & $3 L$ & 60.86 & 290 & .21 \\
\hline twit: & & & & $\ldots$ & \\
\hline $\sin$ & & & & 1157 & \\
\hline 19921 & M & $1 A$ & .82 & 4 & .21 \\
\hline & M & $2 A$ & 3.64 & 18 & .20 \\
\hline & 8 & 14 & 13.31 & 64 & .21 \\
\hline & $R$ & $1 c$ & 3.71 & 18 & .21 \\
\hline & $R$ & $2 \lambda$ & 13.52 & 65 & .21 \\
\hline & $R$ & $3 A$ & 66.59 & 321 & .21 \\
\hline & $R P$ & $2 A$ & .81 & 4 & .20 \\
\hline 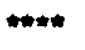 & & & & $\ldots$ & \\
\hline sum & & & & 494 & \\
\hline
\end{tabular}

101 rows selocted. 
WHC-EP-0621

APPENDIX 8.4

ANNUAL VOLUME OF TRU WASTE IN CONTAINERS OTHER

THAN 55-GALLON DRUMS - SORTED BY WASTE TYPE 
WHC-EP-0621

This page intentionally left blank. 


\section{ANNUAL VOLUME OF TRU WASTE IN CONTAINERS OTHER THAN 55-GALLON DRUMS - SORTED BY WASTE TYPE}

The data contained in this subappendix are the waste types, total volume (in cubic meters) and total number of transuranic (TRU) waste containers other than 55-gal drums generated annually at the Plutonium Finishing Plant (PFP). The primary and secondary waste type codes are explained in the introduction to Appendix B.

The secondary waste codes used to generate the data in this subappendix included only those codes beginning with a "1." This excluded a small number of TRU waste containers with secondary waste codes that did not begin with the number 1. These containers were picked up on the data run which is in Appendix B.6. 


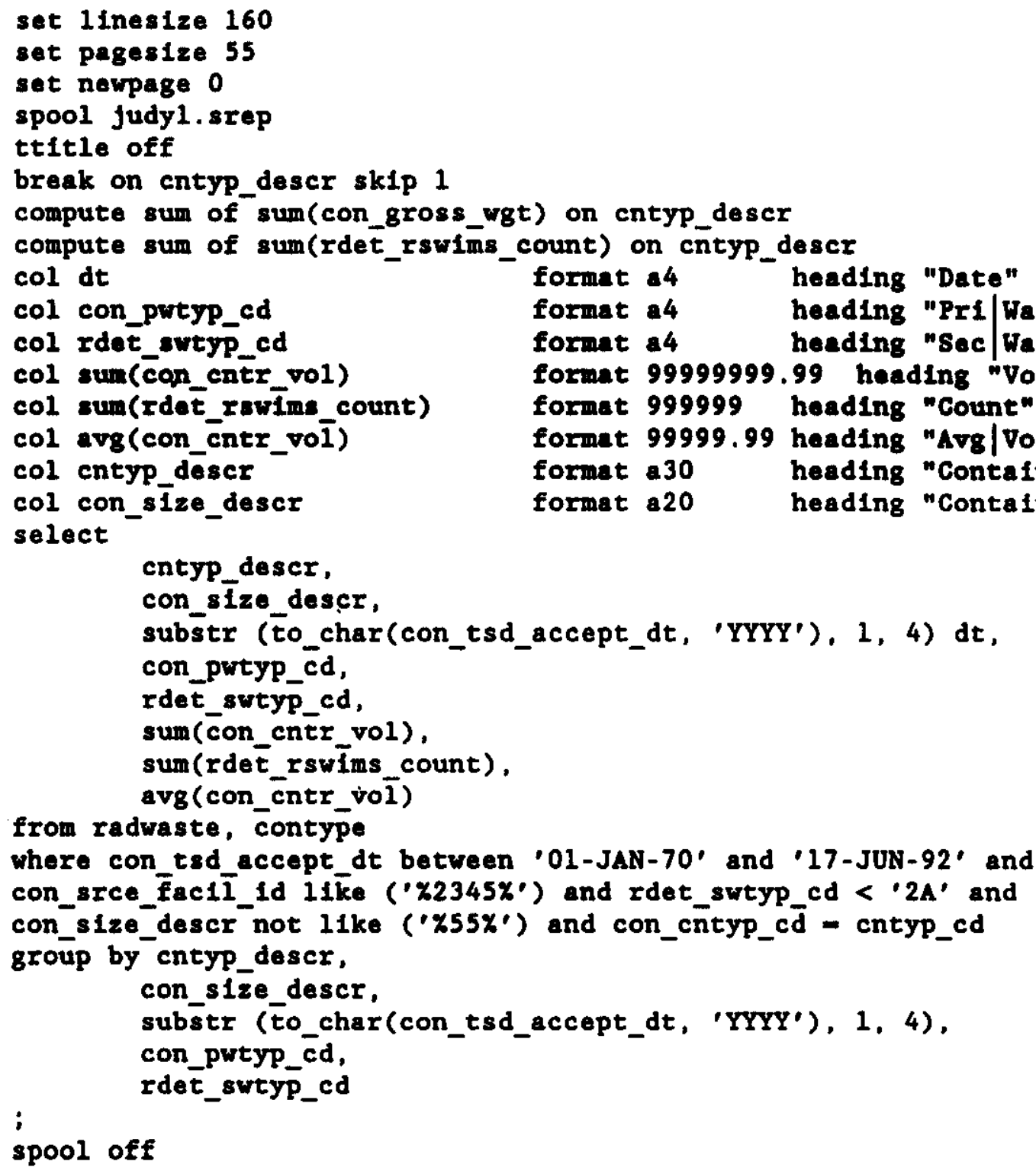


WHC-EP-0621

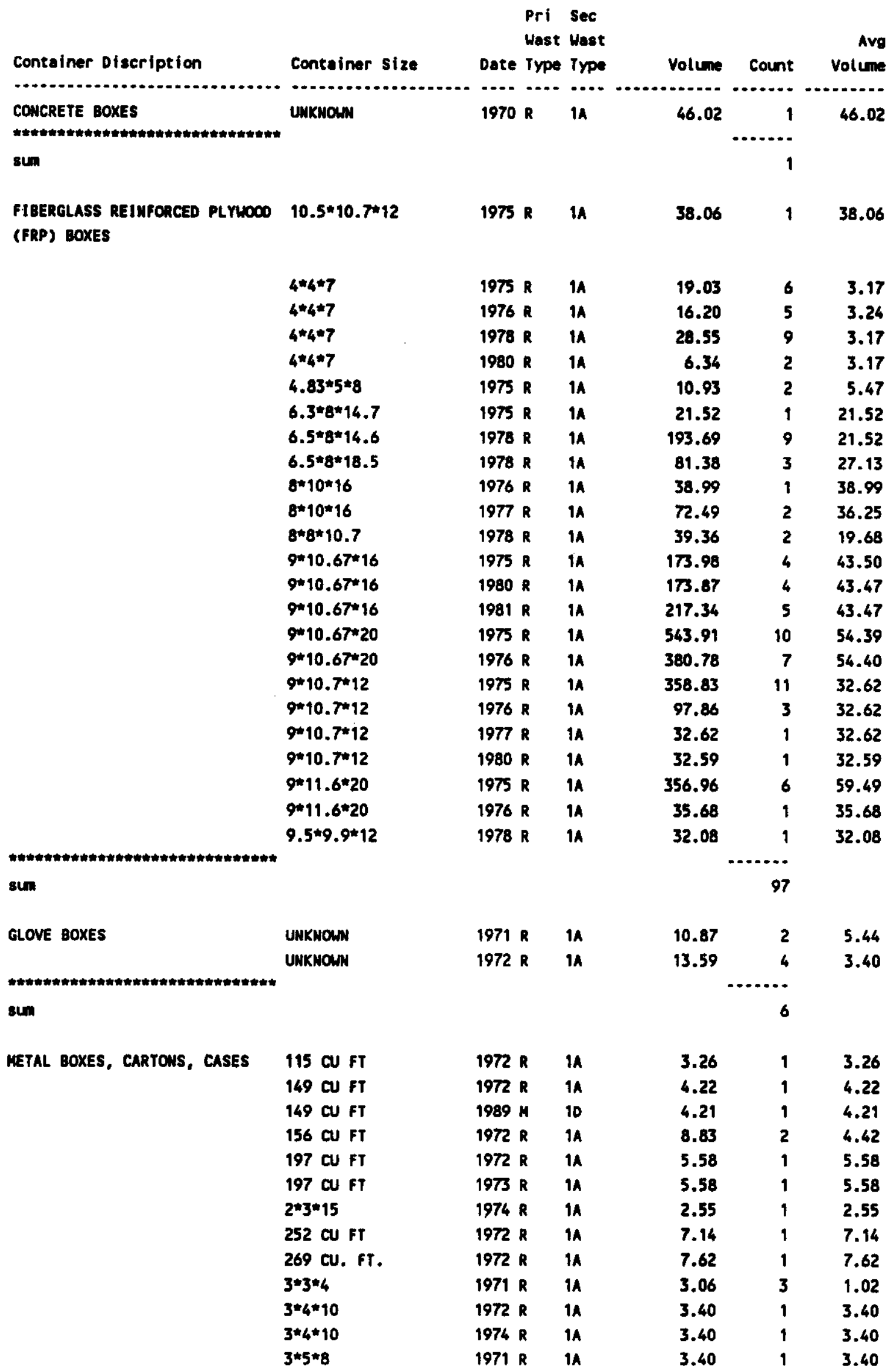


WHC-EP-0621

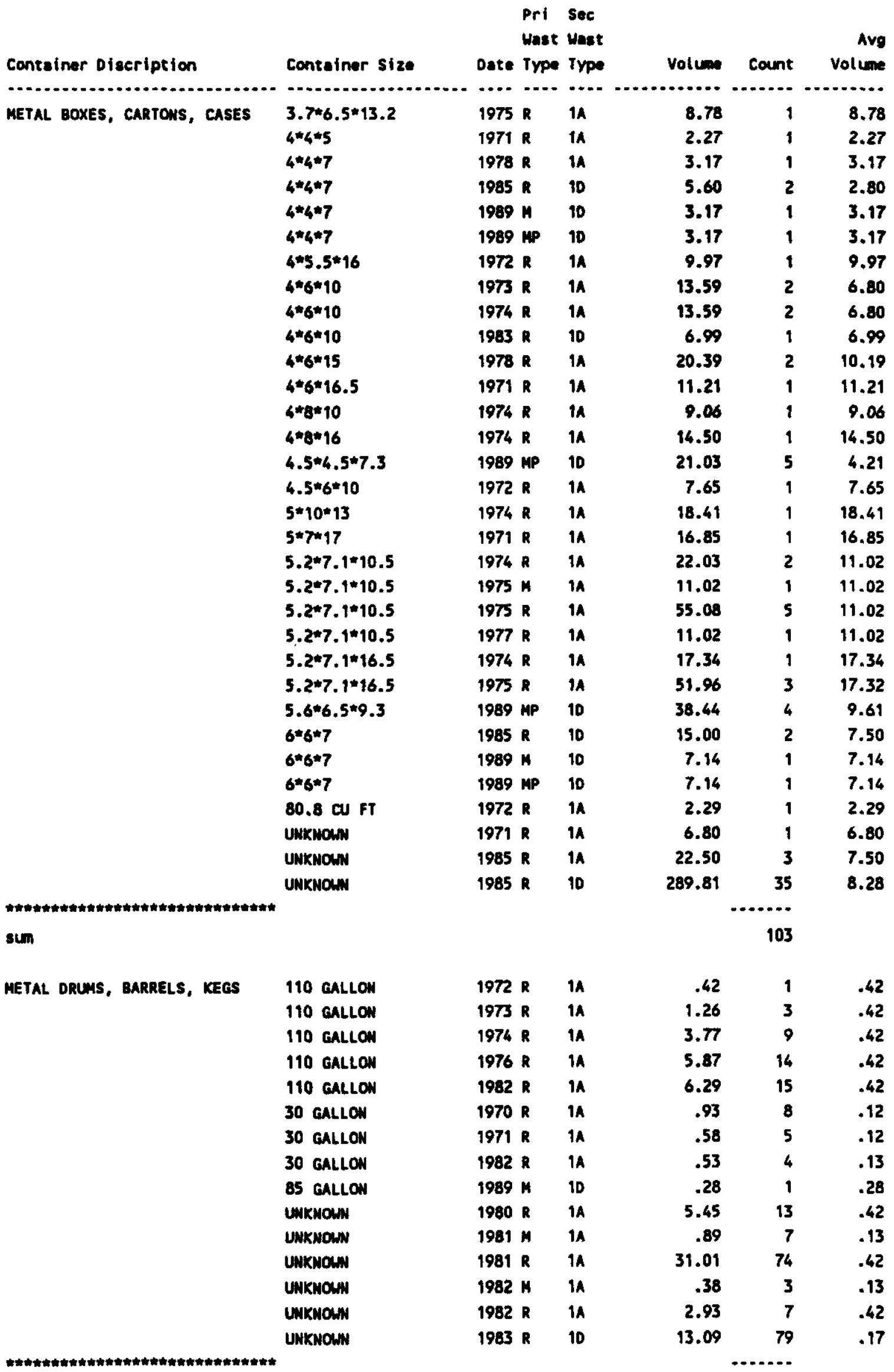


WHC-EP-0621

\begin{tabular}{|c|c|c|c|c|c|c|c|}
\hline Container Discription & Container size & Date & $\begin{array}{l}\text { Pri } \\
\text { Wast } \\
\text { Type }\end{array}$ & $\begin{array}{l}\text { Sec } \\
\text { Wast } \\
\text { Type }\end{array}$ & Volume & Count & $\begin{array}{r}\text { Avg } \\
\text { volume }\end{array}$ \\
\hline sum & (n) & $\cdots$ & $\cdots$ & $\cdots$ & (n) & 243 & (n) \\
\hline $\begin{array}{l}\text { HISCELLANEOUS SCRAP } \\
\text { sum }\end{array}$ & UNKNONH & 1984 & $\mathbf{R}$ & $1 A$ & .21 & $\begin{array}{r}1 \\
1\end{array}$ & .21 \\
\hline $\begin{array}{l}\text { SELF CONTAINED, EOUIPNENT } \\
\text { sum }\end{array}$ & UNKNOUH & 1971 & $\mathbf{R}$ & $1 A$ & 8.16 & $\begin{array}{r}2 \\
2\end{array}$ & 4.08 \\
\hline $\begin{array}{l}\text { WOOOEN BOXES, CARTONS, CASES } \\
\text { sum }\end{array}$ & $4 * 5 * 6$ & 1971 & $\mathbf{R}$ & $1 A$ & 22.65 & $\begin{array}{r}1 \\
1 \\
\cdots \cdots+\cdots\end{array}$ & 22.65 \\
\hline
\end{tabular}




\section{WHC-EP-0621}

This page intentionally left blank. 
WHC-EP-0621

APPENDIX B.5

ANNUAL VOLUMES OF NON-TRU WASTE IN CONTAINERS

OTHER THAN 55-GALLON DRUMS 
WHC-EP-0621

This page intentionally left blank. 
ANNUAL VOLUMES OF NON-TRU WASTE IN CONTAINERS OTHER THAN 55-GALLON DRUMS

The data contained in this subappendix include the total volume (in cubic meters) and total number of low-level waste (LLW) (secondary waste code $=2 A$ ) containers other than 55-gal drums generated at the Plutonium Finishing Plant (PFP) between 1970 and 1991 .

Please note that not all waste that has been categorized as LLW has the " $2 A$ " secondary waste code. Data for packages with the "UA" and " $3 A$ " codes, which also contain LLW, are given in Appendix B.6. 


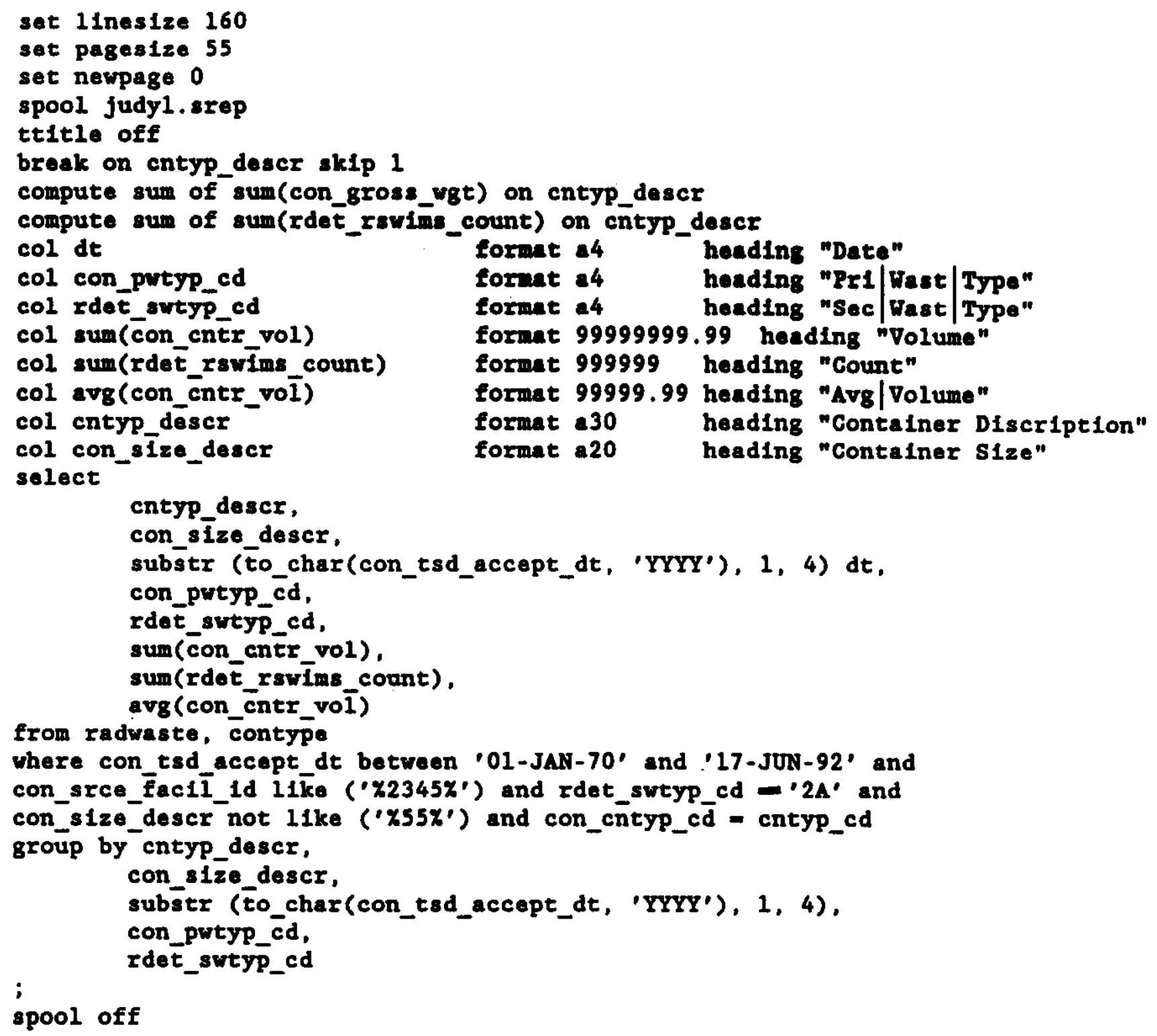




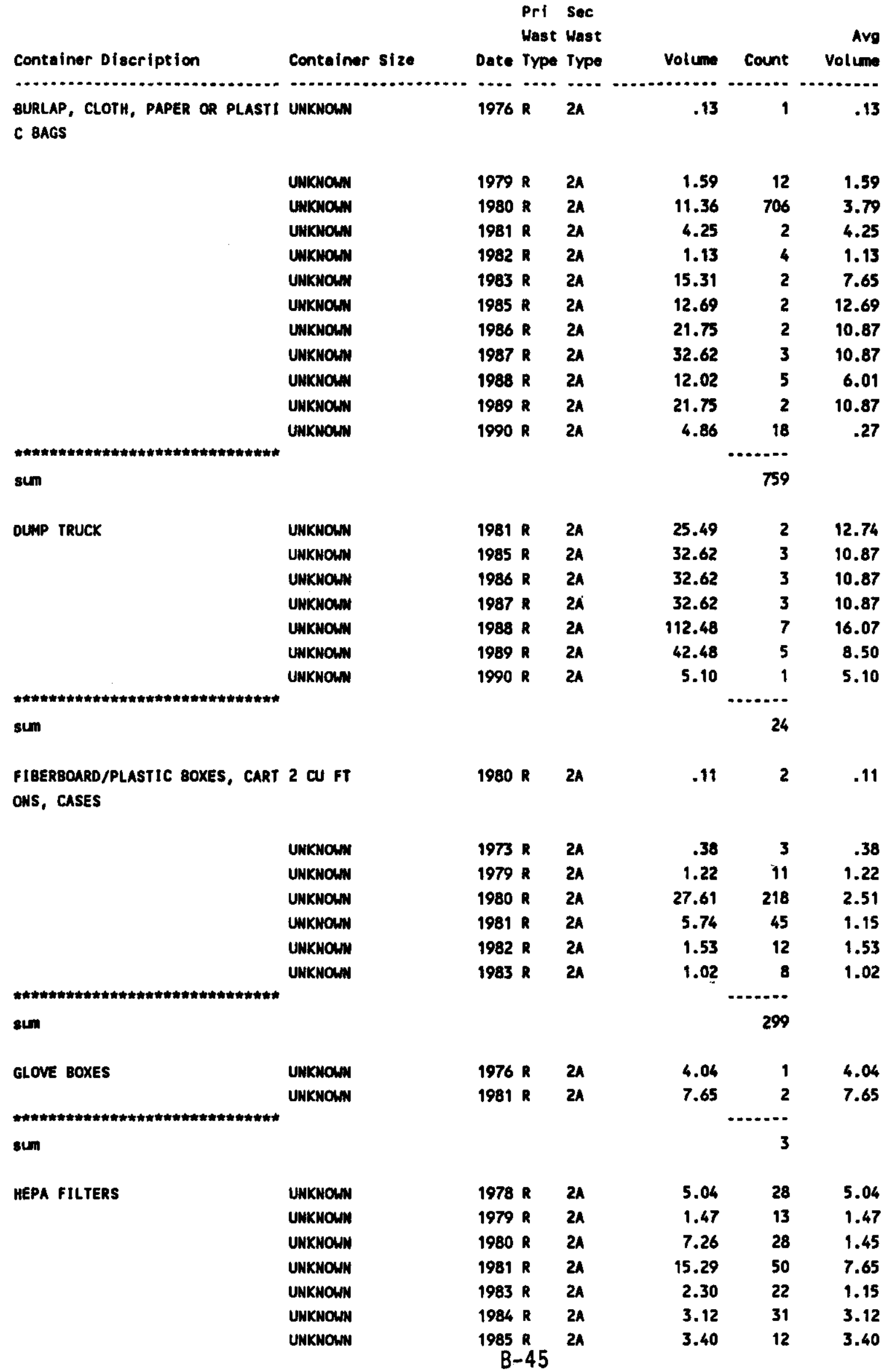




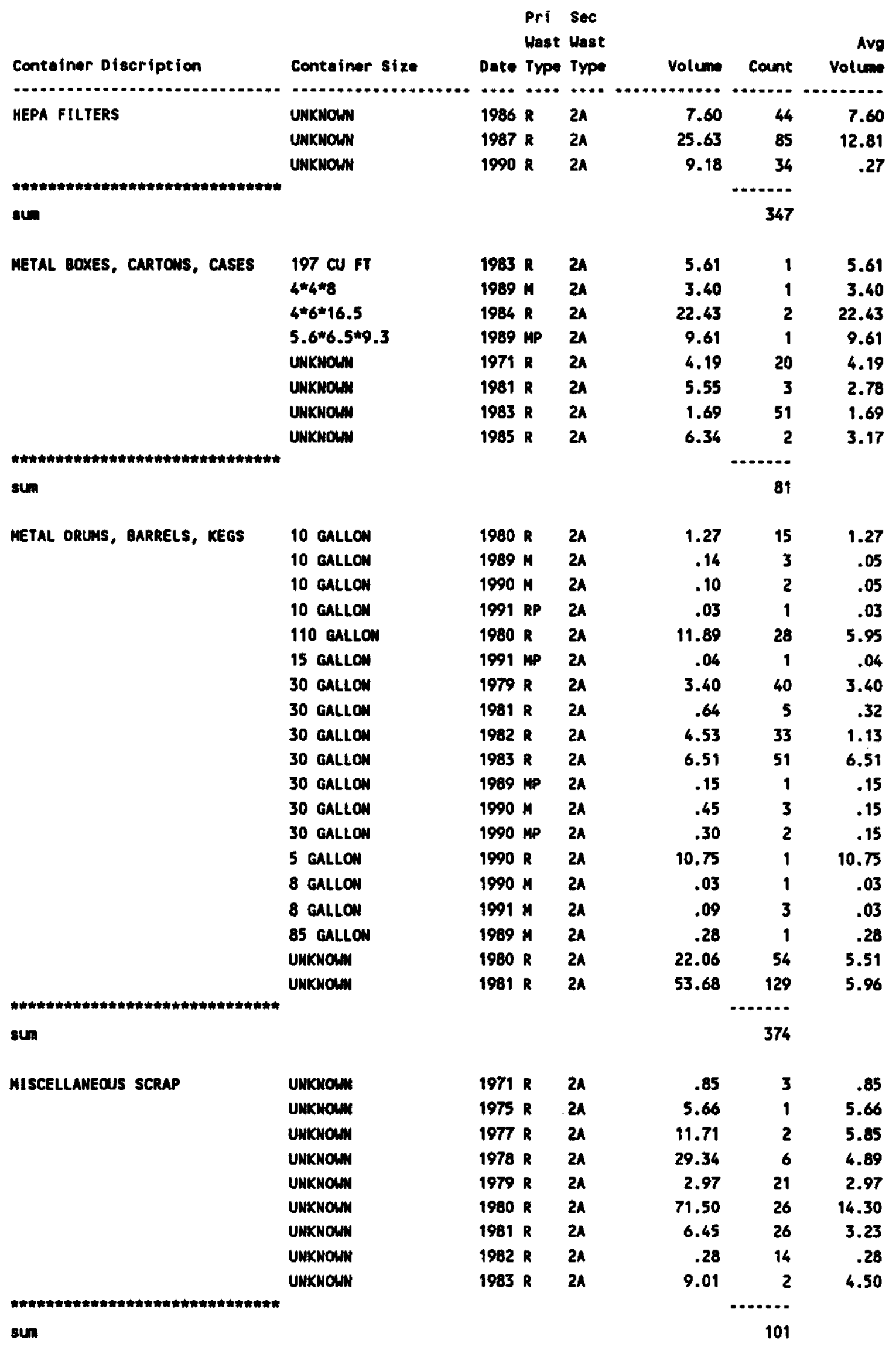




\begin{tabular}{|c|c|c|c|c|c|c|c|}
\hline Container Diseription & Container size & oate & $\begin{array}{l}\text { Pri } \\
\text { Hest } \\
\text { Type }\end{array}$ & $\begin{array}{l}\text { Sec } \\
\text { Wast } \\
\text { Type }\end{array}$ & Volume & Count & $\begin{array}{l}\text { Avg } \\
\text { Volume }\end{array}$ \\
\hline - & nas & $\cdots$ & $\cdots$ & $\cdots$ & ......... & ...... & ....... \\
\hline \multirow[t]{14}{*}{ SELF CONTAINED, EQUIPMENT } & UNKNOWN & 1970 & $\mathbf{R}$ & $2 \AA$ & .57 & 1 & .57 \\
\hline & UAKNOWN & 1976 & $\mathbf{R}$ & $2 \AA$ & 9.74 & 6 & 4.87 \\
\hline & UNKNOWN & 1978 & R & $2 A$ & 1.70 & 9 & .85 \\
\hline & UNKNOM & 1979 & $\mathbf{R}$ & $2 A$ & 7.40 & 69 & 2.47 \\
\hline & UMKNOWN & 19801 & $\mathbf{R}$ & 2A & 42.90 & 56 & 14.30 \\
\hline & UNKNOAN & 19811 & R & $2 \mathbf{a}$ & 32.88 & 423 & 4.11 \\
\hline & UNKNOM & 1982 & R & $2 \mathrm{a}$ & 15.97 & 21 & 5.32 \\
\hline & UNKNOWN & 1983 & R & $2 \mathrm{~A}$ & 9.95 & 16 & 4.97 \\
\hline & UNKHOWM & $1984 \mathrm{I}$ & $\mathbf{R}$ & $2 a$ & 5.69 & 1 & 5.69 \\
\hline & UMKNOWN & 1985 & R & 2A & 7.51 & 34 & 2.50 \\
\hline & UNKNOWN & 1987 & $\mathbf{R}$ & 2A & 10.87 & 1 & 10.87 \\
\hline & UNKNOWN & 1988 & $\mathbf{R}$ & $2 \mathrm{~A}$ & 5.04 & 57 & 5.04 \\
\hline & UNKNOWN & $1989 \mathrm{r}-\mathrm{r}-\mathrm{r}-\mathrm{r}$ & $\mathbf{R}$ & $2 \AA$ & 86.08 & 12 & 7.17 \\
\hline & UAKMOWN & 1990 & R & $2 \AA$ & 50.89 & 8 & 6.36 \\
\hline 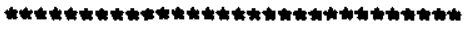 & & & & & & n...... & \\
\hline $\sin$ & & & & & & 714 & \\
\hline $\begin{array}{l}\text { TANKS, PORTABLE } \\
\text { IA }\end{array}$ & UNKHOWM & 1985 & $\mathbf{R}$ & $2 A$ & 2.29 & $\begin{array}{l}4 \\
. .\end{array}$ & 2.29 \\
\hline sum & & & & & & 4 & \\
\hline \multirow{23}{*}{$\begin{array}{l}\text { TRUCKS, FLATBEDS, COMPACTOR, L } \\
\text { OADLUGGER }\end{array}$} & UMKNOWN & 1972 & $\mathbf{R}$ & $2 A$ & 15.12 & 3 & 7.56 \\
\hline & UNKNONM & 1973 & $\mathbf{R}$ & $2 A$ & 29.14 & 4 & 9.71 \\
\hline & UNKNOWN & 1974 & $\mathbf{R}$ & $2 \mathrm{~A}$ & 9.68 & 2 & 9.68 \\
\hline & UNKNOWN & 1975 & $\mathbf{R}$ & $2 \AA$ & 19.37 & 2 & 9.68 \\
\hline & UNKNOWN & 1976 & $\mathbf{R}$ & $2 A$ & 55.33 & 5 & 18.44 \\
\hline & UNKNOWN & 1977 & $R$ & $2 A$ & 38.65 & 6 & 6.44 \\
\hline & UNKNONH & 1978 & $\mathbf{R}$ & $2 A$ & 25.03 & 6 & 4.17 \\
\hline & UNKNONM & 1979 & $\mathbf{R}$ & $2 \mathbf{a}$ & 36.62 & 8 & 4.58 \\
\hline & UNKNOWN & 1980 & $\mathbf{R}$ & $2 A$ & 141.66 & 18 & 7.87 \\
\hline & UNKNOWN & 19811 & M & $2 A$ & 2.83 & 1 & 2.83 \\
\hline & UNKNOWN & 1981 & R & $2 A$ & 164.84 & 16 & 11.77 \\
\hline & UNKNOWN & 1982 & $\mathbf{R}$ & $2 A$ & 72.07 & 38 & 18.02 \\
\hline & UNKNOWN & 1983 & $\mathbf{R}$ & $2 A$ & 32.45 & 3 & 10.82 \\
\hline & UNKNOWN & 1984 & M & $2 A$ & 24.47 & 1 & 24.47 \\
\hline & UNKNOWN & 1984 & $\mathbf{R}$ & $2 A$ & 125.84 & 8 & 15.73 \\
\hline & UNKNOWM & 1985 & $\mathbf{R}$ & $2 \AA$ & 281.47 & 28 & 10.05 \\
\hline & UNKNOWM & 1986 & M & $2 \mathbf{A}$ & 21.64 & 4 & 5.41 \\
\hline & UHKMON & 1986 & & $2 \mathrm{~A}$ & 116.10 & 11 & 10.55 \\
\hline & UHKNOWN & 1987 & & $2 \mathbf{A}$ & 83.99 & 10 & 9.33 \\
\hline & UNKNOWN & 1988 & & $2 \mathrm{~A}$ & 58.11 & 6 & 9.68 \\
\hline & UNKNOWN & 1989 & & $2 \lambda$ & 31.43 & 6 & 5.24 \\
\hline & UNKNOWN & 1990 & & $2 A$ & 82.95 & 8 & 10.37 \\
\hline & UNKNOWN & 1991 & $\mathbf{R}$ & $2 A$ & 42.90 & 3 & 14.30 \\
\hline 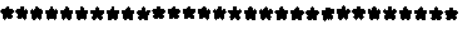 & & & & & & (n...... & \\
\hline sum & & & & & & 197 & \\
\hline \multirow[t]{2}{*}{ WOOOEN BOXES, CARTONS, CASES } & $4 * 4 * 8$ & 1992 & $\mathbf{R}$ & $2 \mathrm{~A}$ & 4.11 & 1 & 4.11 \\
\hline & $4 * 8 * 12$ & $\begin{array}{r}1987 \\
B-C\end{array}$ & $\begin{array}{l}R \\
47\end{array}$ & $2 A$ & 10.87 & 1 & 10.87 \\
\hline
\end{tabular}


WHC-EP-0621

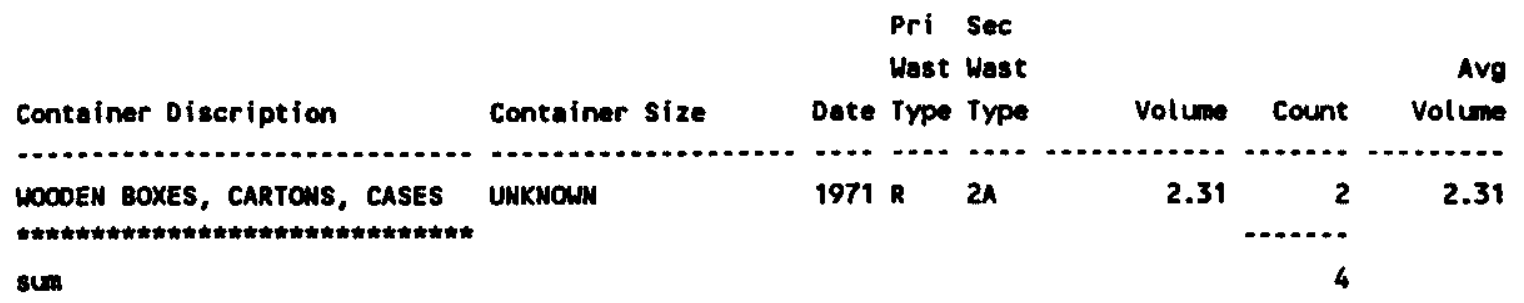

115 rows selected. 
WHC-EP-0621

APPENDIX B.6

ANNUAL VOLUMES OF WASTE WITH SECONDARY WASTE CODES GREATER THAN 2A STORED IN WASTE CONTAINERS OTHER THAN 55-GALLON DRUMS 
WHC-EP-0621

This page intentionally left blank. 


\section{ANNUAL VOLUMES OF WASTE WITH SECONDARY WASTE CODES \\ GREATER THAN 2A STORED IN WASTE CONTAINERS \\ OTHER THAN 55-GALLON DRUMS}

The data contained in this subappendix include the total volume (in cubic meters) and total number of containers other than 55-gal drums with secondary waste codes greater than $2 A$. This group includes the following waste categories:

- UA, unsegregated nontransuranic (non-TRU) dry waste

- UG, unsegregated transuranic (TRU) dry waste

- $3 A$, low-level industrial waste.

This computer run was made to address oversights in the queries in subappendixes B.4 and B.5. 


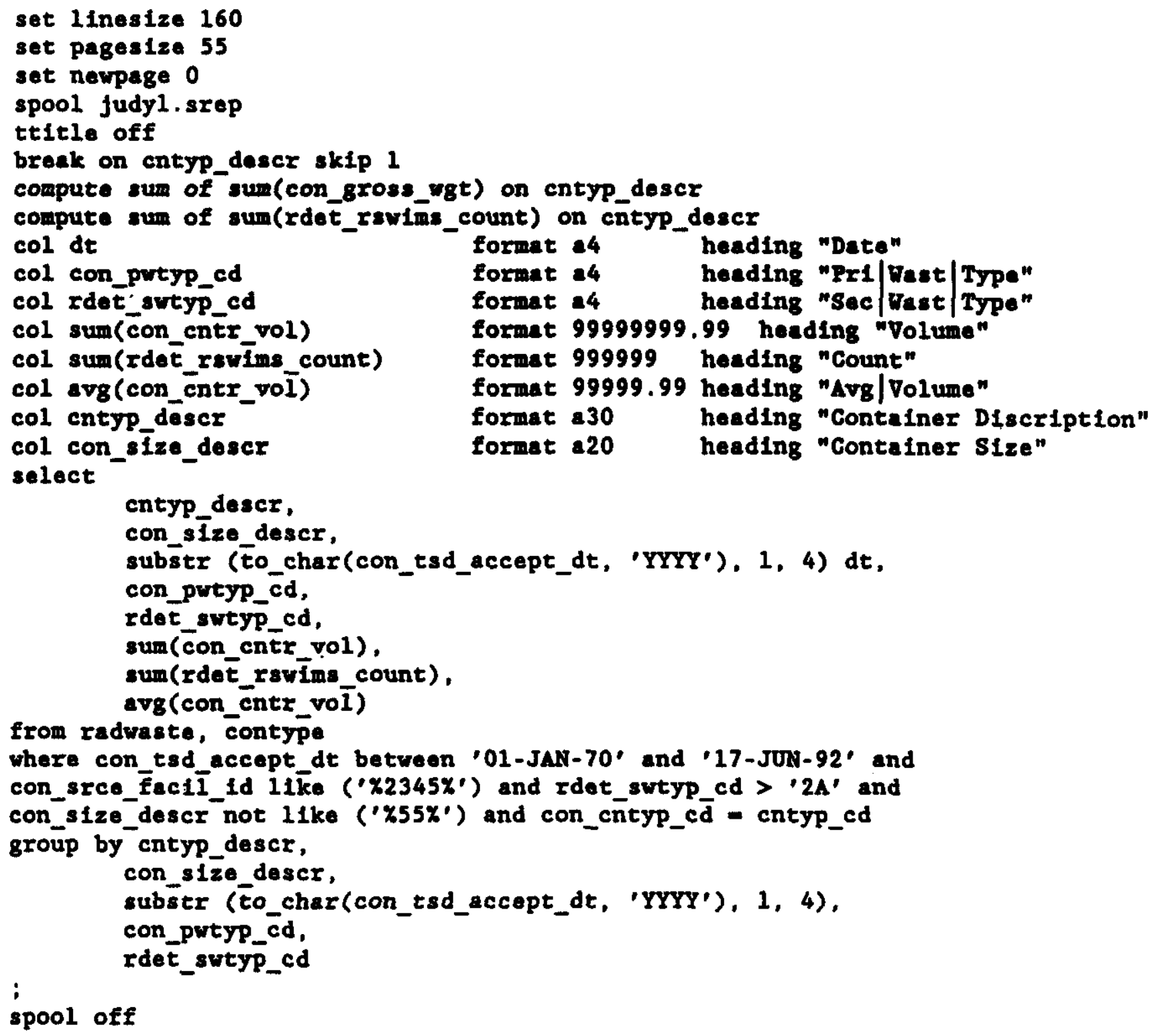




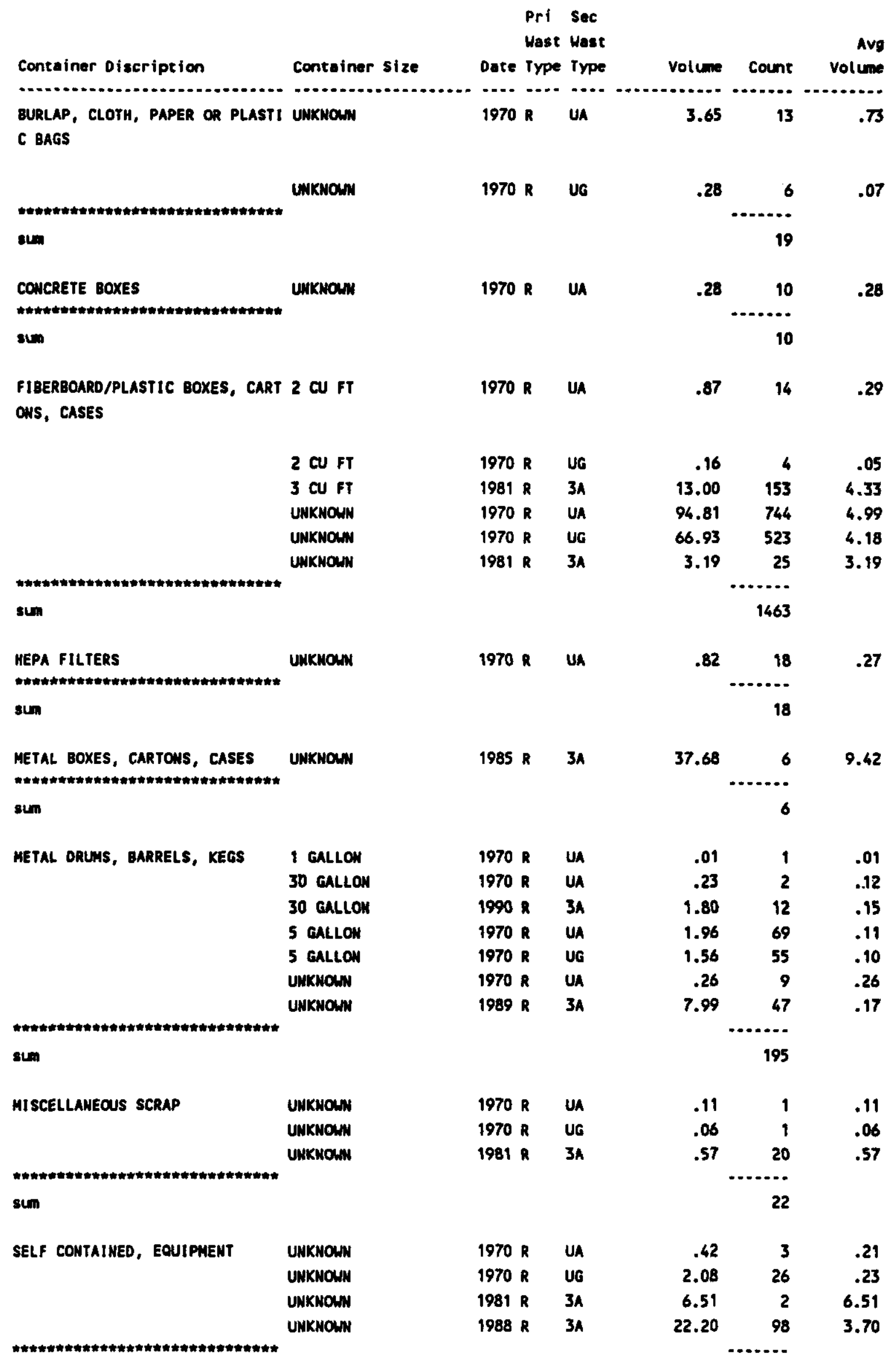




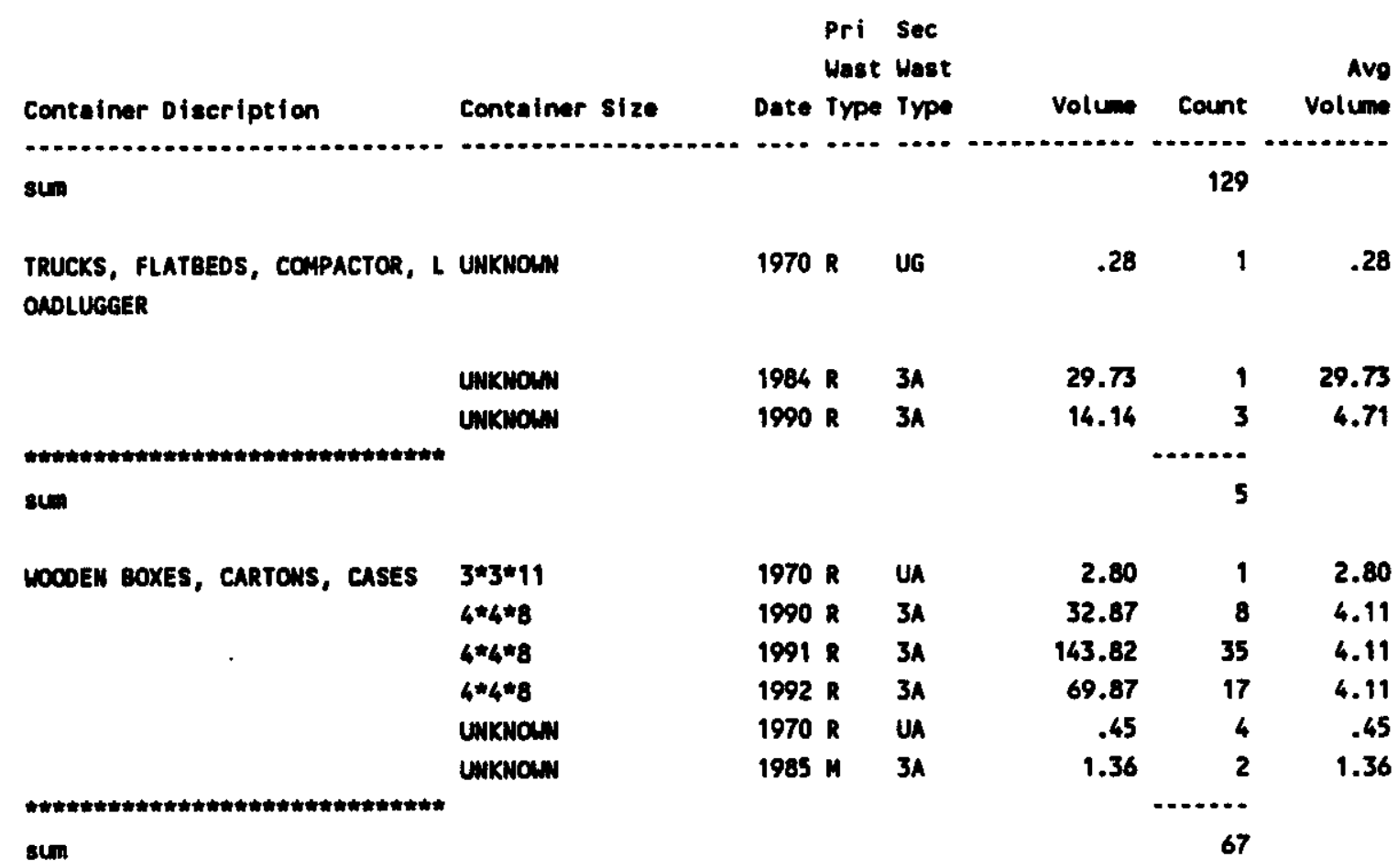

34 rows selected. 
WHC-EP-0621

APPENDIX B.7

TRU WASTE STORAGE FACILITIES - SORTED BY PRIMARY WASTE TYPE, CONTAINER TYPE, AND YEAR 
WHC-EP-0621

This page intentionally left blank. 


\section{TRU WASTE STORAGE FACILITIES - SORTED BY PRIMARY}

WASTE TYPE, CONTAINER TYPE AND YEAR

This subappendix provides information on the type, weight, and primary waste type of transuranic (TRU) waste containers stored at the various TRU waste storage facilities on the Hanford Site. The data are sorted by the primary waste type (mixed waste [M] is listed first followed by PCB contaminated mixed waste [MP] and radioactive [R] waste). 


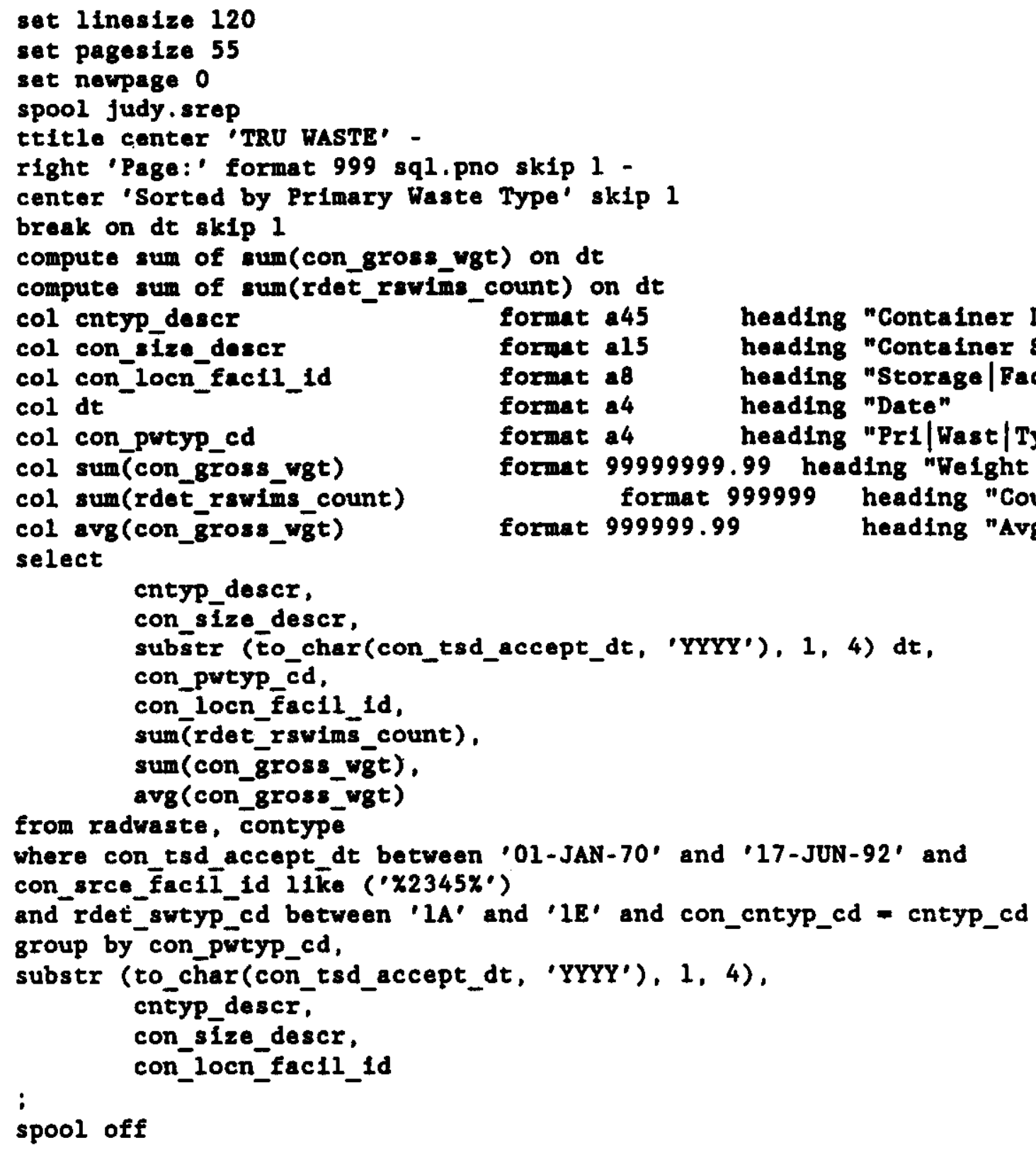


TRU WASTE

Page: 1

Sorted by Primary Waste Type

Pri

Wast storage Avg

Contoiner Diseription

Container size oute Typa facility count Woight (kg) Woight

metal boxes, cartows, CAsEs

METAL DRUNS, BARRELS, KEGS
METAL DRUNS, BARRELS, KEGS
METAL DRLAS, BARRELS, KEGS

1975 (1)

..........

c........ -

4939.60

4939.60

ntre $\quad$.....................

th 14939.60

Encrom

1981 n $21844 C$
sum

$7 \quad 1575.80$

225.11

1575.00

35 enllow

1982 n 218 auc

unkwoum

METAL DRLAS, BARRELS, KECS

METAL DRUNS, BARRELS, KEGS

N $21844 C$

108.04

68.04

sta

$\begin{array}{rr}3 & 546.32 \\ 4 \ldots \ldots & 612.36\end{array}$

181.44

MeTAL Dauns, BaraeLs, Keos

metal dRLAS, BarRels, KEgS

METAL DRUnS, GARRELS, KEGS

METAL BOXEs, cartons, cases METAL BOXES, CARTOWS, CASES MTAL boxes, cartows, cases METAL dRLAs, Barrels, kEes metal dauns, andrels, KEos metal oRuns, BarRets, KEGS METAL DRLMS, BARRELS, KEES

METAL DRUAS, BARRELS, KEgS

METAL DRLAS, BARRELS, KEGS

METAL ORLMS, BARRELS, KEGS

METAL DRUWS, BARRELS, KEGS
55 GALLON

1983 n 2184hC
ond

$\begin{array}{rr}16 & 1206.56 \\ 16 & 1206.56\end{array}$

55 GALLON

1924 M

218146

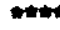

sup

$\begin{array}{rr}3 & 197.31 \\ 3 & 197.31\end{array}$

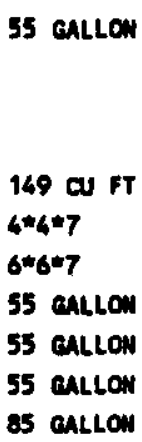

1985 in 218446
$\sin$

$\begin{array}{rr}13 & 1023.07 \\ 13 & 1023.07\end{array}$

1989 N 2602W

M 24024B

M 24024

M 2267

H 24014

H 240219

H4⿻十

sta

55 GALLON

1990 M 2245

trite

sum

55 axlon

$\$ 991$ M 2265

minte

su

55 GALLON

$1992 \mathrm{~N}$

$226 T$

काต

(1)

\begin{tabular}{rrr}
1 & 1360.77 & 1360.77 \\
1 & 1043.26 & 1043.26 \\
1 & 2177.23 & 2177.23 \\
34 & 2955.86 & 86.94 \\
2 & 218.63 & 109.32 \\
1 & 79.38 & 79.38 \\
\hline 1 & 117.93 & 117.93 \\
\hline 41 & 7953.06 & \\
\hline
\end{tabular}

$20 \quad 2669.03$

123.45

$20 \quad 2669.05$

$\begin{array}{rrr}18 & 1711.99 \\ 18 & 1711.99\end{array}$

$\begin{array}{rr}4 & 403.00 \\ 4 & 403.00\end{array}$

55 GALLON

$6 \quad 482.62$

80.44

$\begin{array}{lrrrr}1982 \text { MP } 2401 \mathrm{~W} & 6 & 482.62 & 80.44\end{array}$


sorted by Primary waste Type

Pri

Wast storege Avg

Container Diseription

Container size Date Type Facility count Weloht (kg) Weight

retal Daum, saracts, Kegs

metal Dauns, BankELs, Kegs

MeTAL ofuns, eARRELs, KeES

METAL BOXES, CARTONS, CASES metal Boxes, cantons, cases metal eOKes, cartens, cases metal BoXES, cantows, cases mETAL oRLnS, BARRELS, KegS

METAL DRLMS, BarRELS, KEGS

cowcete boxes

metal detus, Barrels, Kegs metal DRuns, Barrels, KEgS

G.ove Boxss

metal nouss, cartows, cases METAL soxes, cantous, cases metal souss, cantous, cases METAL Boxes, cartous, cases METAL Bouks, cartous, cases METAL DoKes, cantows, cases MEtal oxuns, aARnEls, keEs metal dRuns, aARrels, KeES metat oRuns, BARRELS, KEgS SELF CONTAIMED, ECUIPIENT WOOEM BOXES, CARTONS, CASES

GLOVE Boxes

MeTAL BOKES, cartows, cases

$$
\text { su }
$$

55 andon

$$
\begin{aligned}
& 1983 \\
& \text { sum }
\end{aligned}
$$

55 callow

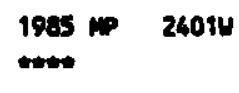

$\sin$

55 gallow

1988 mp 24014

$\sin$

4"4*7

$4.5 * 4.5 * 7.3$

$5.6 * 6.5 * 9.3$

$6 * 6 \div 7$

55 alloM

ss ant

55 GALLON

UnKnow
30 eALLon
55 cAlLON

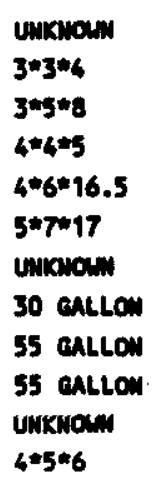

UnKMow

$115 \mathrm{CU} F \mathrm{FT}$

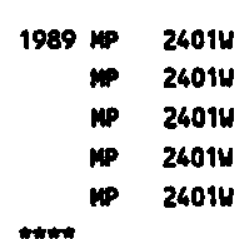

wtw

su

1990 ip $2401 \mathrm{~W}$

sus

$1970: \quad 218448$
$? \quad 21043 A$
$R \quad 21843 A$

su
$1974 R \quad 218448$$$
\text { R 218:48 }
$$
R 218 468
R 218448
R 218468
R 218448
R 21813a
R 21 sive
R 21arba
R 21846
R 210448
R 218468
tatio:

sin

1972 R 218448

R 218468
6

482.62

$\begin{array}{rr}4 & 319.32 \\ 4 & 319.32\end{array}$

79.83

\begin{tabular}{rrr}
14 & 1319.74 \\
\hdashline$\ldots \ldots$ & 1319.74
\end{tabular}

96.27

\begin{tabular}{rrr}
10 & 699.99 & 70.00 \\
10 & 699.99 & \\
1 & 1723.64 & 1723.64 \\
5 & 9343.96 & 1868.79 \\
4 & 11884.06 & 2971.02 \\
1 & 2469.39 & 2469.39 \\
6 & 438.63 & 73.11 \\
\hline 17 & 25839.68 &
\end{tabular}

45.99

$\begin{array}{rr}1 & 45.99 \\ 1 & 45.99\end{array}$

\begin{tabular}{rrr}
1 & 20638.35 & 20638.35 \\
8 & 326.56 & 40.82 \\
1112 & 75660.48 & 68.04 \\
\hline 1121 & 96625.39 &
\end{tabular}

2438.50

457.22

1524.06

1016.06

5030.31

7556.81

$\begin{array}{rr}3048.12 & 3048.12 \\ 204.10 & 40.82\end{array}$

$\begin{array}{rr}204.10 & 40.02 \\ 56537.48 & 68.06\end{array}$

$54819.03 \quad 68.01$

$3657.74 \quad 1828.87$

10160.42

$1361 \quad 129803.57$

$4 \quad 6096.24 \quad 1524.06$ 
TRU WASTE

Page: 3

Sorted by Prienry waste Type

Pri

Wast storage
Conteiner size Date Type Fecility count Waight (kg) Meight

Wast Storage
Conteiner size Date Type Fecillity count Woight (kg) Weight

Container Diseription

metal boxes, cartons, casts

metAl boxes, cantons, cases

METAL BoxEs, cARTous, cases

Metal bones, cantows, cases

METAL eocus, cantous, cases

netal cours, chrtows, cases

netal coxes, cantons, cares

metal soxes, cantoms, casts

mital sockes, cantous, cases

metal dRLws, Barrels, Kecs

METAL dRUAS, BarRELS, KEGS

METAL BOXES, CARTOAS, CAsES metal roxes, cartous, cases METAL DRums, BARRELS, KEES METAL DRUMS, BARRELS, KEGS

METAL BOXES, cARTOAS, CASES metal boxes, cantows, cases retal boxes, cartons, cases netal noxes, cartons, cases metal coxes, cantons, cases METAL coxes, cartons, cases METAL soxes, CAHTONs, CASEs metal DOKES, cartons, cases metal DRLMS, BarreLL, KECS METAL DRLWS, BARRELS, KEES

piberglass Reimforced PLYNOCO (FRP) boxes

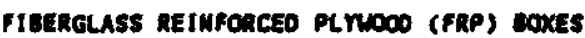

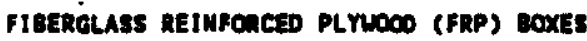
FIBERELASS REIMFCnCED PLYWCO (FRP) DOXES

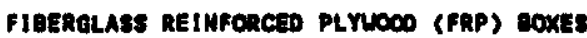

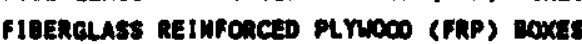
Ftaerglass REIMFORCED PLYNoD (FRP) BOXES

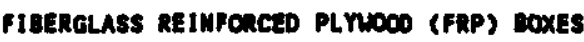
METAL Boxes, caktons, cases METAL 8OXEs, cantows, cases METAL BoXes, cartons, cases metal boxes, cantons, cases metal boXes, cartows, cases METAL dRUMS, BARRELS, KECS

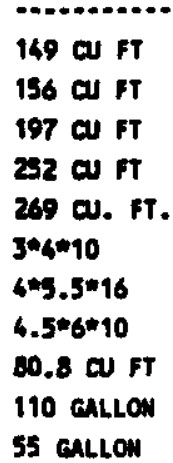

197 CU FT
$4 " 6 * 10$
110 GALLON
55 GALLON

$2 * 3 * 15$
$3 * 4 * 10$
$4 * 6 * 10$
$4 * g * 10$
$4 * 8 * 16$
$5 * 10 * 13$
$5.2 * 7.1 * 10.5$
$5.2 * 7.1 * 16.5$
110 EALLON
55 EALLON

4047

$4.83 * 5 * 8$

$6.3+8 * 14.7$

9*10.67*16

$9 * 10.67 * 20$

$9 * 10.7 \div 12$

$9+11.6 * 20$

$3.7 * 6.5 * 13.2$

5.2*7.1*10.5

$5.2 * 7.1 * 10.5$

$5.2 * 7.1 * 16.5$

$5.2 * 7.1 * 16.5$

55 GALLON

\begin{tabular}{|c|c|}
\hline 1972 & 218448 \\
\hline$R$ & 218uk8 \\
\hline$R$ & 218440 \\
\hline $\mathbf{R}$ & $218 \mathrm{se}$ \\
\hline n & 21ane \\
\hline$R$ & $2180 \times 5$ \\
\hline R & $218 \mathrm{mat}$ \\
\hline R & 21844 \\
\hline त & 218Wh \\
\hline R & 21844B \\
\hline R & $218 \times 40$ \\
\hline
\end{tabular}

$\begin{array}{rr}1973 R & 218448 \\ R & 218468 \\ R & 218468 \\ R & 218448 \\ & \end{array}$

sum

$\begin{array}{rr}1974 R & 218448 \\ R & 218446 \\ R & 218446 \\ R & 218146 \\ R & 218464 \\ R & 218458 \\ R & 218448 \\ R & 218048 \\ R & 218448 \\ R & 218448\end{array}$

sum

$\begin{array}{rr}1973 R & 21843 A \\ R & 21843 A \\ R & 21843 A \\ R & 21843 A \\ R & 21813 A \\ R & 21843 A \\ R & 21843 A \\ R & 21843 A \\ R & 218448 \\ R & 21843 A \\ R & 218468 \\ R & 21843 A \\ R & 218448 \\ R & 218448\end{array}$

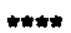

$\begin{array}{rr}1 & 1892.38 \\ 2 & 3962.56 \\ 1 & 2502.00 \\ 1 & 3200.53 \\ 1 & 3616.64 \\ 1 & 1524.06 \\ 1 & 4670.58 \\ 1 & 3429.16 \\ 1 & 1026.02 \\ 1 & 136.08 \\ 1306 & 88260.24 \\ \cdots 1322 & 121976.83\end{array}$

\begin{tabular}{rrr}
1 & 2502.00 & 2502.00 \\
2 & 6096.26 & 3048.12 \\
3 & 408.24 & 136.08 \\
1264 & 86070.60 & 68.09 \\
\hline 1270 & 95077.08 & \\
& & \\
1 & 1943.05 & 1143.05 \\
1 & 152.41 & 152.41 \\
2 & 6096.24 & 3048.12 \\
1 & 4064.17 & 4064.17 \\
1 & 6504.48 & 6504.48 \\
1 & 8255.34 & 8255.34 \\
2 & 9879.20 & 4939.60 \\
1 & 7779.07 & 779.07 \\
9 & 1224.72 & 136.08 \\
942 & 64093.68 & 68.04 \\
\hline.. & $\ldots \ldots+\ldots$ & \\
961 & 109192.36 &
\end{tabular}

17068.59

$8540.19 \quad 1423.37$

$4923.27 \quad 2461.64$

$9652.40 \quad 9652.40$

56050.1314012 .53

200704.5420070 .45

$154946.31 \quad 14086.03$

$134407.79 \quad 22401.30$

$3937.16 \quad 3937.16$

$14818.80 \quad 4939.60$

$9879.20 \quad 4939.60$

$7760.92 \quad 7760.92$

$15544.52 \quad \pi 72.26$

$1222 \quad 83144.88 \quad 68.04$ 


\section{WHC-EP-0621}

TRU UASTE

Page: 4

Sorted by Prinary Waste Type

Pri

Haat Storage Avg

Contoiner Diseription

Conteiner size Date Iype facility count Woight (kg) Waight

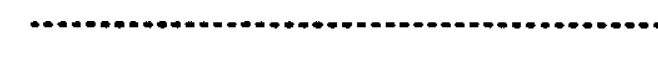

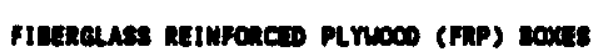

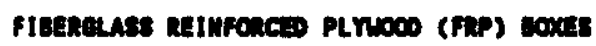
fibenelase Returonced plnoce (fup) coves

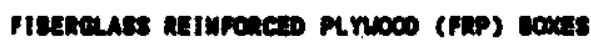

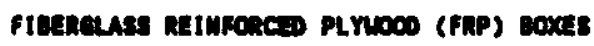

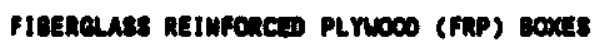
metal deuns, sanidels, KEes

metal oduns, Barrels, KegS

FJBerglass REIMFORCED PLYNOO (FRP) DOXES

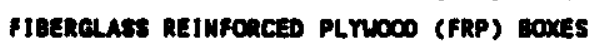
metal bones, cantous, cases METAL DEMS, BanRELS, KEgS

FlBengLASS REIMFOnCED PLYNOCO (FRP) BOXES

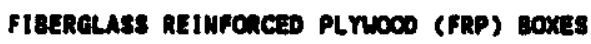
fiberclass retmfonced plynoo (FRP) boxes FJBERGLASS REIMFORCED PLYNDOD (FRP) BOXES FIBERCLASS REIMFCACED PL nDOD (FRP) GoXKES METAL BoxES, CARTOUS, CASES metal coxes, cartous, cases METAL ORLMS, BARRELS, KEGS

METAL DRUAS, BarReLS, KEGS

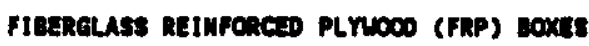
PILERGLaes ReIMfonced PL noco (fRP) notes

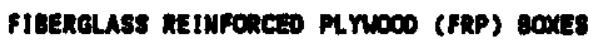
metal onuns, candels, KECS meTAL dRuis, BarReLs, xets

FIBERGLASS REIWFORCED PLYWOCO (FRP) BOXES metal deruHs, Baraels, Kecs metal dRuns, Barrels, Kegs sum

$4 * 4 \div 7$

4iㅏㄴㅏ

$8+10+16$

$9 \times 10.67 \div 20$

$9 * 10.7 \div 12$

$9 * 11.6 * 20$

110 GALLOI

55 CALLON

$8 * 10 * 16$
$9 * 10.7 * 12$
$5.2 * 7.1 * 10.5$
55 GALLOW

$4 * 4 * 7$
$6.5 * 8 * 14.6$
$6.5 * g * 18.5$
$8 * 8 * 10.7$
$9.5 * 9.9 * 12$
$4 * 4 * 7$
$4 * 6 * 15$
55 GALLOW

55 GALLON

$4 * 4 * 7$

$9+10.67 \times 16$

$9 \times 10.7 \div 12$

55 callon

unirnoun

$9+10.67 \div 16$

55 GALLON

unkavery

\begin{tabular}{|c|c|c|}
\hline 1976 & & $21813 \mathrm{~A}$ \\
\hline & R & 218 ins \\
\hline & $\mathbf{k}$ & 218ma \\
\hline & $R$ & $218 \mathrm{an}$ \\
\hline & $\mathbf{R}$ & 218.uBA \\
\hline & R & $218 \mathrm{~B}$ \\
\hline & $\mathbf{R}$ & $218: 48$ \\
\hline & $\mathbf{R}$ & 218468 \\
\hline
\end{tabular}

1977 R 21013A

$$
\begin{aligned}
& \text { R 218MBA } \\
& \text { R 21843A } \\
& \text { R 218460 } \\
& \text { twot }
\end{aligned}
$$$$
\text { sum }
$$

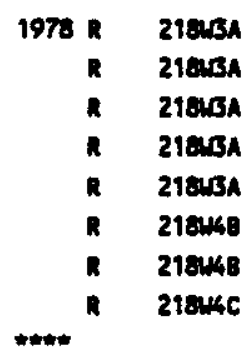$$
\sin
$$

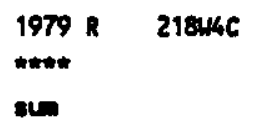

$1980 R \quad 21844 C$ R 210u4C R $2104 \mathrm{ke}$

a 21ausc

R 218ukc

$\rightarrow \infty$

sin
$1981 R \quad 21804 C$ R 218 ac
R $21844 \mathrm{C}$
min:

sum
1272721378.70

\begin{tabular}{rrr}
2 & 2844.92 & 1422.46 \\
3 & 4419.76 & 1475.26 \\
1 & 17490.43 & 17490.43 \\
7 & 170790.27 & 26398.61 \\
3 & 43890.43 & 14632.81 \\
1 & 16002.66 & 16002.66 \\
14 & 1905.12 & 136.08 \\
1025 & 69657.08 & 60.02 \\
\hline 1056 & 327008.69 & \\
\hline
\end{tabular}

13607.70

12619.29

6350.26

68.04

$1199 \quad 81443.88$

$1203 \quad 127428.83$

\begin{tabular}{rrr}
9 & 3628.72 & 403.19 \\
9 & 73109.66 & 8123.30 \\
3 & 25772.90 & 8590.99 \\
2 & 6917.25 & 3458.63 \\
1 & 4422.50 & 4622.50 \\
1 & 1431.53 & 1431.53 \\
2 & 9144.38 & 4572.19 \\
900 & 61236.00 & 68.04 \\
\hline 927 & 185663.02 & \\
1569 & 106745.68 & 68.03 \\
\hline 1569 & 106745.68 & \\
\hline
\end{tabular}

$1569 \quad 106745.68$

1171.40

6452.32

4422.50

60.09

138.76

\begin{tabular}{rrr}
13 & 2063.88 & 158.76 \\
\hline 1678 & 147534.95
\end{tabular}

\begin{tabular}{rrr}
5 & 30254.46 & 6050.89 \\
819 & 55818.64 & 68.15 \\
74 & 13081.66 & 176.78 \\
\hline$\ldots .$. &.$\ldots .6 .0$ & \\
898 & 99154.76 &
\end{tabular}




\section{WHC-EP-0621}

TRU MASTE

Page: 5

Sorted by Prianry Waste Type

Pri

Wast Storage

Avg

Container Discription Conteiner size Date Type facility count Weight (kg) Woight

metal DRUAS, BarRels, KEgS METAL ORUNS, BARRELS, KEGS METAL DRuNS, BARRELS, KEeS metal owurs, eaknets, Kees

METAL EOXES, CARTOHS, CAEES METAL DALSS, BarezLS, KEFS METAL DRUAS, BARRELS, KECS

METAL DRLAS, BARRELS, KEgS metal dELAS, BARRELS, KEgS miscellaneous SCRAP

metal coxes, carrows, cases METAL BOXES, CARTONS, cases METAL Boxes, cartows, cases METAL ORUNS, BARRELS, KEGS

METAL DRUNS, BARRELS, KEGS METAL DRLAS, BARRELS, KEGS

METAL DRUNS, BARRELS, KEGS metAl DRLAS, Barrels, KEgS

METAL DRLNS, BARRELS, KEGS METAL DRUMS, BARRELS, KEGS

METAL DRUMS, BARRELS, KEGS

METAL DRLMS, GARRELS, KEGS METAL DRUMS, BARRELS, KEGS METAL DRLAMS, BARRELS, KEGS
110 GALLOH

30 GALLON

55 galLON

unkeon

55 eAllon

unxinow

55 GALLON

55 GALLOH

UnKMOW

$4 * 4 * 7$

6"6"7

UnKinown

55 GALLON

55 GALLON

55 GALLON

55 GALLON

55 calto

55 GALLON

55 GALLON

5 Gallon

55 GALLON
1982 R $21844 C$

R 218 ac

R 218 anc

l 218 unc

$\rightarrow+\infty$

$\sin$

1983 a 21 aukc

R 218 anc

R 218ukC

แแ⿰幺幺

sum

$\begin{array}{rr}1984 R & 21843 A \\ R & 21844 C \\ R & 21844 C\end{array}$

twet

sin

$1985 R \quad 21844 C$

R $21844 C$

R 218 4he

R $218 \mathrm{WC}$

mant

sun

$\begin{array}{rl}1986 & R \\ R & 21844 C \\ R & 224 T\end{array}$

$m$

sum

$\begin{array}{rl}1987 \times & 21844 C \\ R & 226 T\end{array}$

เum

$\begin{array}{ll}1988 \text { R } & 21844 C \\ \text { R } & 226 T \\ \sin & \end{array}$

1989 R $224 T$

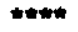

sum

$1990 R \quad 21844 C$

$R \quad 21845$

R $224 \pi$
$793.80 \quad 198.45$

$45099.39 \quad 67.82$

$1381.17 \quad 197.31$

$699 \quad 49315.56$

\begin{tabular}{rrr}
1 & 3703.56 & 3703.56 \\
929 & 61248.53 & 65.86 \\
79 & 9824.00 & 124.35 \\
\hline$\ldots .$. &. .2. & \\
1009 & 74776.09 &
\end{tabular}

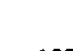

56.70

$1851 \quad 121137.18$

86.18

65.48

86.18

$1853 \quad 121280.06$

\begin{tabular}{rrr}
2 & 1470.99 & 735.50 \\
2 & 2907.06 & 1453.53 \\
38 & 105451.09 & 2775.03 \\
1755 & 113551.16 & 64.70 \\
\hline 1797 & 223380.30 &
\end{tabular}

$\begin{array}{rrr}445 & 25680.72 & 57.71 \\ 119 & 7820.77 & 65.72 \\ \ldots . . . & . .7 . . . & \\ 564 & 33501.49 & \end{array}$

\begin{tabular}{rrr}
10 & 582.03 & 58.20 \\
412 & 28067.95 & 68.13 \\
\hline 422 & 28649.98 &
\end{tabular}

\begin{tabular}{rrr}
11 & 681.94 & 61.99 \\
169 & 10966.99 & 64.89 \\
\hline 180 & 11648.93 &
\end{tabular}

$\begin{array}{crr}66 & 4392.46 & 66.55\end{array}$

4392.46

$\begin{array}{rrr}53 & 3094.25 & 58.38 \\ 1 & 54.02 & 54.02\end{array}$


TRU MASTE

sorted by Primary waste Type

Pri

Vast stortge

Container Discription

.................................................

METAL oRUMS, MarRELS, Keos

meTal ondis, annele, kess

metal dRuns, BarReLs, xeos
Container slze Date Type Facility Count Weight (kg) Avg

sin

55 axlon

55 alcon

1991

R 218446

-

su

2247

$1992 R$

mone

sum

$140 \quad 8836.15$

\begin{tabular}{|c|c|c|}
\hline $\begin{array}{l}18 \\
8\end{array}$ & $\begin{array}{l}1181.01 \\
5727.01\end{array}$ & $\begin{array}{l}65.61 \\
67.20\end{array}$ \\
\hline 106 & 6968.02 & \\
\hline
\end{tabular}

$82 \quad 4604.00$

71.94

4604.00

Page: 0

139 rous solected. 
WHC-EP-0621

APPENDIX B.8

RADIOLOGICAL DATA ON TRU WASTE CONTAINERS SORTED BY DATE, PRIMARY WASTE TYPE, AND STORAGE FACILITY 
WHC-EP-0621

This page intentionally left blank. 


\section{RADIOLOGICAL DATA ON TRU WASTE CONTAINERS - SORTED BY DATE, PRIMARY WASTE TYPE, AND STORAGE FACILITY}

Subappendix B.8 summarizes the radiological data for all transuranic (TRU) waste containers generated at the Plutonium Finishing Plant (PFP) between 1970 and 1991. Specifically, this computer run provides the total grams of TRU elements in a given container type in a given year. In addition, the average gram loading for a given container type in a given year has been calculated.

In 1991 and 1992 the number of curies of alpha radiation was included on the solid waste burial records (SWBRs). These data are included in this run.

The "Max Dose" column contains the highest value recorded for a given container in a given year.

This computer run is sorted according to primary waste type (mixed [M], mixed with PCB contamination [MP] and radioactive [R]), year, and the facility where the given containers are stored pending retrieval.

Additional information about the isotopes present in containers can be found in subappendixes B.13 and B.14. 


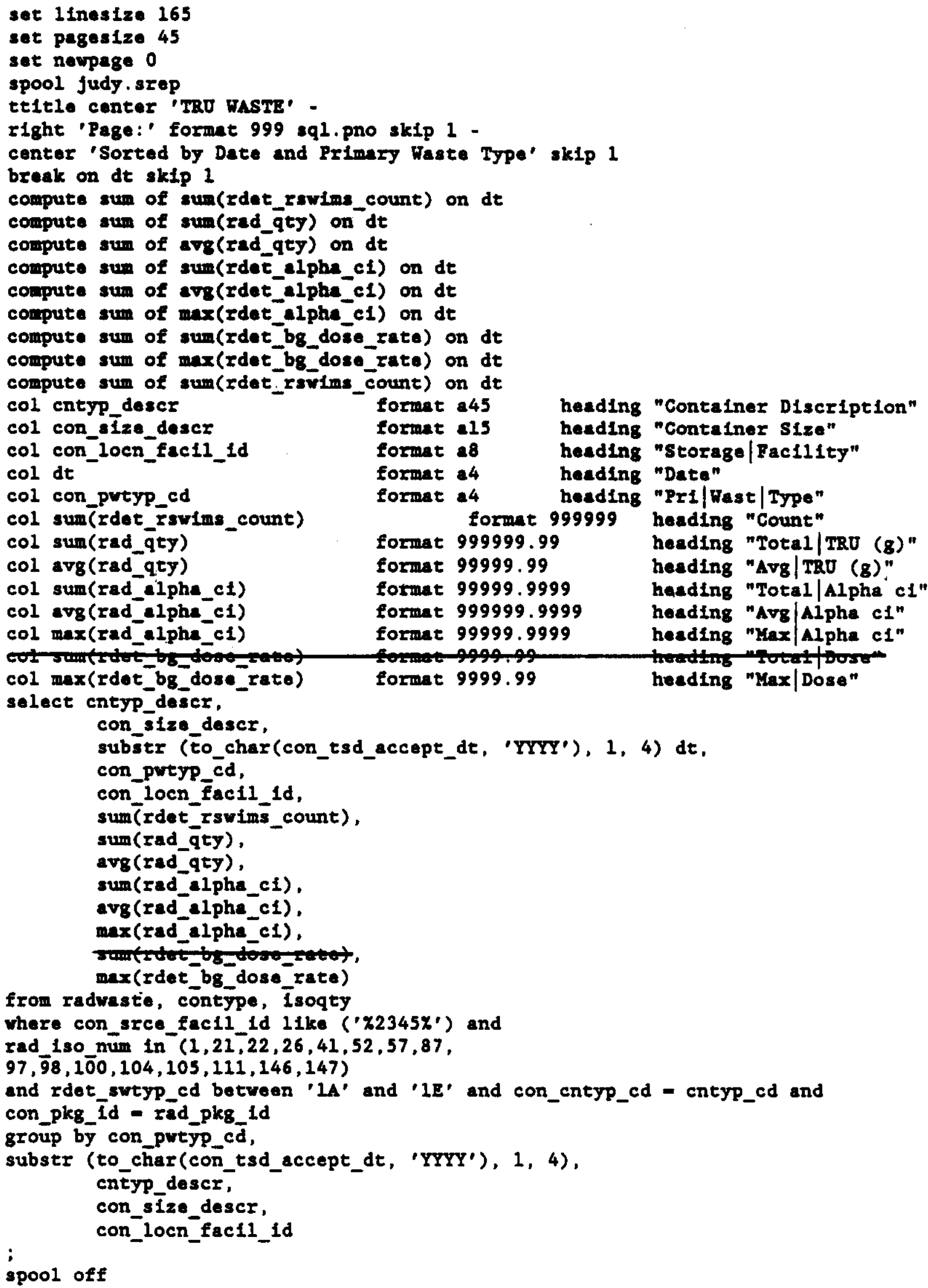


Wast Storage Total Avg Total Avg Max

Container Discription

Container Size Date Type Facility count

TRU (g) TRU (g) Alpho ci Alpha ci Alpha ci

wetal boxes, cartous, cases

11.00

1.00

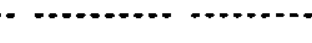

$.0000 \quad .0000 \quad .0000$

sin

$1.00 \quad 1.00$

1.00

METAL DRLNS, aARRELS, KEGS

unknow

1981 in 218 atc

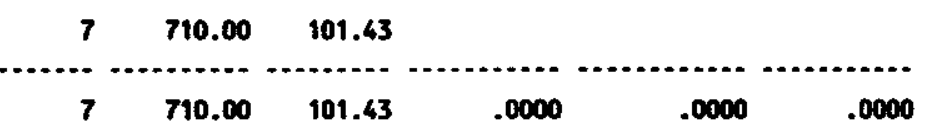

70.00

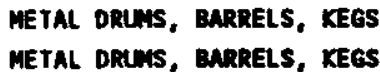

55 GALLOM

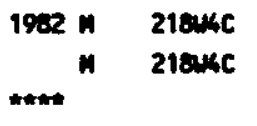

100

unkwoun

METAL DRLANS, BARRELS, KEGS

55 GALLOO

sum

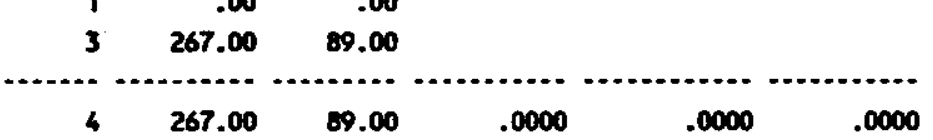

METAL DRUMAS, BARRELL, KEGS

METAL DRUNS, BARKELS, KEGS

metal boxes, cartons, cases metal boXEs, cartows, cases METAL BOXEs, CaRTows, CASES METAL DRLNTS, BaRRELS, KEGS METAL DRUES, BARRELS, KEGS METAL DRUNS, BarRELS, KEGS METAL DRUMS, BARRELS, KEgS

METAL DRLAS, BaRRELS, KEgS

55 GALLON
55 GALLON
149 Cl FT
$4 * 4 * 7$
$6 * 6 * 7$
55 GALLON
55 GALLON
55 GALLO
85 GALLON

55 GALLON

\section{9}

sin

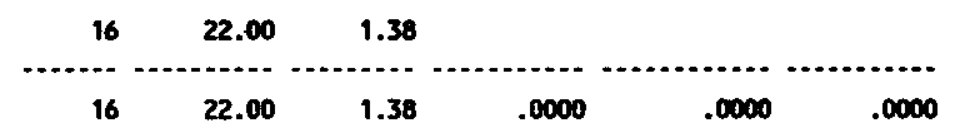

1904 n $21844 \mathrm{C}$

trit

sin

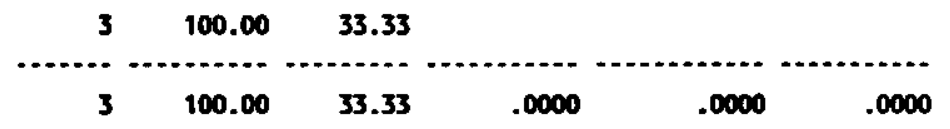

1905 in 21844c

-...

$13 \quad 168.00 \quad 12.92$

sum

\begin{tabular}{|c|c|c|}
\hline $13 \quad 168.00$ & 12.92 & .0000 \\
\hline
\end{tabular}

10.00

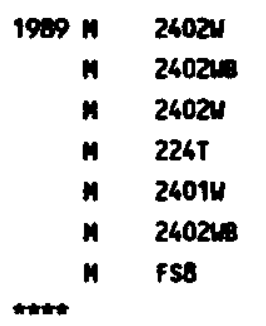

\begin{tabular}{|c|c|c|}
\hline 1 & 7.00 & 77.00 \\
\hline 1 & 11.00 & 11.00 \\
\hline 1 & 76.00 & 76.00 \\
\hline 34 & 1038.13 & 30.53 \\
\hline 2 & 10.00 & 5.00 \\
\hline 1 & 1.00 & 1.00 \\
\hline 1 & .31 & .31 \\
\hline
\end{tabular}

$\begin{array}{r}.50 \\ .50 \\ .50 \\ 5.00 \\ 1.00 \\ .50 \\ 1.00 \\ \hline 9.00\end{array}$

1990 n 224

su

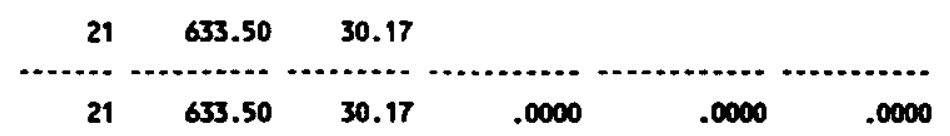


Container Diseription

metal dRuns, barReLs, Kegs

METAL DRUAS, BARRELS, KECS

METAL ORLiHS, BARRELS, KEGS

METAL DRUMS, BARRELS, KEgS

metAL BOXES, CARTONS, CASES metal boxes, cartons, cases METAL BOXES, CARTONS, CASES METAL BOXES, CARTONS, CASES METAL DRLMS, BARRELS, KEGS

metal oruhs, barrels, kegs

CONCRETE BOXES

METAL DRLAHS, BARRELS, KEGS

mETAL DRLAS, BARRELS, KEGS

GLOVE BOXES

\begin{tabular}{|c|c|c|c|c|c|c|c|c|c|}
\hline Container size & Date & $\begin{array}{l}\text { Hast } \\
\text { Type }\end{array}$ & $\begin{array}{l}\text { Storage } \\
\text { fecitity }\end{array}$ & count & $\begin{array}{r}\text { Total } \\
\text { TRU (g) }\end{array}$ & $\begin{array}{r}\text { Avg } \\
\text { TRU (g) }\end{array}$ & $\begin{array}{r}\text { Total } \\
\text { Alphe ci }\end{array}$ & $\begin{array}{r}\text { Avg } \\
\text { Alphe ci }\end{array}$ & $\begin{array}{r}\text { Max } \\
\text { Alphe ci }\end{array}$ \\
\hline ; & $\cdots$ & $\cdots$ & a.......... & ........ & .............. & (........... & a........... & - & (n........ \\
\hline \multirow[t]{2}{*}{55 aAllow } & 1991 & M & $224 \mathrm{~T}$ & 18 & 166.44 & 9.25 & 5.7417 & 1.4354 & 3.0666 \\
\hline & sum & & & 18 & 166.44 & 9.25 & 5.7417 & 1.4354 & 3.0666 \\
\hline \multirow[t]{2}{*}{55 exlom } & 1992 & n & 2261 & 4 & 194.00 & 48.50 & 15.2828 & 3.8207 & 7.3982 \\
\hline & $\sin$ & & & 4 & 194.00 & 48.50 & 15.2828 & 3.8207 & 7.3982 \\
\hline \multirow[t]{2}{*}{55 callow } & $19 \% 2$ & m & 24014 & 6 & 8.00 & 1.33 & & & \\
\hline & $\operatorname{sen}$ & & & 6 & 8.00 & 1.33 & .0000 & .0000 & .0000 \\
\hline \multirow[t]{2}{*}{55 CALLON } & 1985 I & P & 24014 & 14 & 1.55 & .11 & & & \\
\hline & sin & & & 14 & 1.55 & .11 & .0000 & .0000 & .0000 \\
\hline \multirow[t]{2}{*}{55 callon } & 1938 & $\mathbf{m}$ & $2401 \mathrm{~N}$ & 10 & 2.81 & .28 & & & \\
\hline & sin & & & 10 & 2.81 & .28 & .0000 & .0000 & .0000 \\
\hline $4 * 4 * 7$ & 1909 & $\operatorname{mip}$ & 24014 & 1 & 72.00 & 72.00 & & & \\
\hline $4.5 * 4.5 * 7.3$ & & $\mathbb{M P}$ & $2401 \mathrm{~W}$ & 5 & 128.00 & 25.60 & & & \\
\hline $5.6 * 6.5 * 9.3$ & & $\operatorname{mp}$ & $2401 \mathrm{~N}$ & 4 & 448.00 & 112.00 & & & \\
\hline $6 * 6 * 7$ & & $\mathbf{M P}$ & 26014 & 1 & 316.30 & 316.30 & & & \\
\hline 55 cAlloN & & $\mathbf{m p}$ & 24014 & 6 & .55 & .09 & & & \\
\hline
\end{tabular}

55 callow

\section{MP 2401K}

sum

Lnxuorin

30 Gallow

55 callow

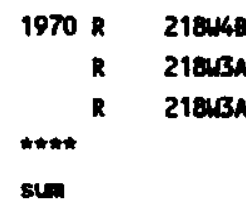

Unknow

1971 R 218448

$\begin{array}{rrrrrr}1 & .05 & .05 & & & \\ \ldots \ldots & \ldots & .05 & .00 & \end{array}$

Max
Dose
$\ldots .00$
$\ldots \ldots$
7.00
2.70
$\ldots .70$
1.00
$\ldots \ldots$
1.00
1.00
1.00
1.00

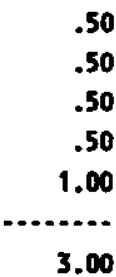

.50

.50

1.00

1.00

2.00

1.00 
TRU MASTE

Page: 3

Sorted by Date and Primary Waste Type

Pri

Wast Storage Totel Avg Total Avg Max

Container Diseription

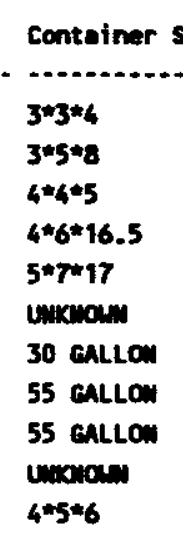

GLOVE BOXES

METAL BOXES, CARTOUS, CASES

P Metal bookes, cartous, cases

W Metal boXes, cartons, cases

METAL BOXES, CARTOUS, CASES

metal boxes, cartous, cases

METAL goxes, cartous, cases

netal boxes, cartoms, cases

METAL boKes, cartous, cases

METAL BOXES, CARTONS, CASES

metal boXes, cartons, cases

METAL DRUMS, BARRELS, KEGS

METAL dRUnS, Bannels, KEGS

metal BoXEs, cartous, cases

METAL BOXES, CARTOUS, CASES

METAL DRLALS, BARRELS, KECS

metal dRtus, BarRels, KEes

metal boXes, cartows, cases

metal boxes, cartons, cases

\begin{tabular}{|c|c|c|c|c|c|c|c|c|c|}
\hline $3 * 3 * 4$ & 1971 & $R$ & 218448 & 3 & 5.02 & 1.67 & & & \\
\hline $3 * 5 * 8$ & & R & 218448 & 1 & 1.67 & 1.67 & & & \\
\hline $4 * 4 * 5$ & & $R$ & 21844B & 1 & 2.05 & 2.05 & & & \\
\hline $4 * 6 * 16.5$ & & $R$ & 218468 & 1 & .00 & .00 & & & \\
\hline $5^{\star 7 * 17}$ & & R & $210 \mathrm{k} 6 \mathrm{~B}$ & 1 & 1.67 & 1.67 & & & \\
\hline Luxnoun & & 8 & 2184BA & 1 & .01 & .01 & & & \\
\hline 30 GALLON & & R & 218448 & 5 & 10.24 & 2.05 & & & \\
\hline 55 CALLON & & $\mathbf{R}$ & 218ur3a & 537 & 1067.27 & 1.99 & & & \\
\hline 55 EALLON & & $\mathbf{R}$ & 218448 & 806 & 1581.33 & 1.96 & & & \\
\hline unnown & & R & 218468 & 2 & .00 & .00 & & & \\
\hline \multirow[t]{2}{*}{$4=5 * 6$} & & R & 218448 & 1 & 2.05 & 2.05 & & & \\
\hline & sin & & & 1361 & 3191.32 & 275.13 & .0000 & .0000 & .0000 \\
\hline UnKwonn & 1972 & $\mathrm{R}$ & 218468 & 4 & 199.90 & 49.98 & & & \\
\hline $115 \propto \mathrm{FT}$ & & R & 218048 & 1 & 25.00 & 25.00 & & & \\
\hline $149 \mathrm{cU} F$ & & $R$ & 218046 & 1 & 8.75 & 8.75 & & & \\
\hline $156 \mathrm{CU} \mathrm{FT}$ & & R & $2180 \times 5$ & 2 & 300.00 & 150.00 & & & \\
\hline $197 \mathrm{CU}$ fT & & $R$ & 218448 & 1 & 8.75 & 8.75 & & & \\
\hline 252 ad fT & & $\mathbf{R}$ & 218468 & 1 & 8.75 & 8.75 & & & \\
\hline 269 cu. $\mathrm{FT}$. & & $R$ & $218 \times 48$ & 1 & 8.75 & 8.75 & & & \\
\hline $3 * 4 * 10$ & & $\mathbf{R}$ & $218 \times 68$ & 1 & .00 & .00 & & & \\
\hline $4 * 5.5 * 16$ & & R & 218448 & 1 & .00 & .00 & & & \\
\hline $6.5 * 6 * 10$ & & R & 218468 & 2 & 3.49 & 1.74 & & & \\
\hline $80.8 \mathrm{cu} \mathrm{ft}$ & & $R$ & 218468 & 1 & 20.00 & 20.00 & & & \\
\hline 110 GALLON & & R & 218468 & 1 & 2.70 & 2.70 & & & \\
\hline \multirow[t]{3}{*}{55 EALLOW } & & $\mathbf{R}$ & 210468 & 1527 & 3208.91 & 2.10 & & & \\
\hline & 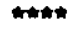 & & & $\cdots$ & 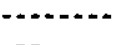 & $-\cdots$ & ........ & $\ldots .$. & ......... \\
\hline & sum & & & 1544 & 3794.99 & 286.52 & .0000 & .0000 & .0000 \\
\hline 197 Cl FT & 1973 & R & 218uks & 1 & 200.00 & 200.00 & & & \\
\hline $4 * 6 * 10$ & & $\mathbf{R}$ & 218046 & 2 & 54.00 & 27.00 & & & \\
\hline 110 GALLO & & R & 218148 & 3 & 19.50 & 6.50 & & & \\
\hline \multirow[t]{3}{*}{55 CALLOM } & & R & 218:14B & 1264 & 3070.10 & 2.43 & & & \\
\hline & "tw" & & & …... & -1.2. & . & & 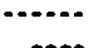 & $\cdots$ \\
\hline & sun & & & 1270 & 3343.60 & 235.93 & .0000 & .0000 & .0000 \\
\hline 15 & 1974 & R & 218ankB & 1 & 10.00 & 10.00 & & & \\
\hline $4 * 10$ & & $\mathbf{R}$ & 218448 & 1 & 10.00 & 10.00 & & & \\
\hline
\end{tabular}


Sorted by Date and Primary Waste Type

Pri

West Storage Total Avg Totol Avg Max Max

Container Diseription

Dose

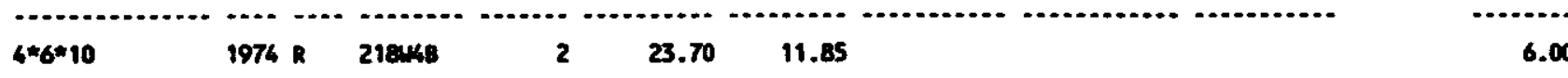

Metal goxes, cartons, cAses

METAL bockes, cartous, cases

METAL BOXES, CARTOUS, CASES

METAL BOXES, CARTOWS, CASES

METAL BOXEs, CaRToms, CASES

METAL BOXES, CARTOWS, CASES

METAL DRUNS, BARRELS, KECS

METAL DRAWH, BARRELS, KEGS

FIBERGLASS REIMFORCED PLVUDCO (FRP) GOXES FIBERGLASS REIWFORCED PLYNOCD (FRP) BOXES Fiberglass REIWFORCED PLYNDOD (FRP) BOXES FIBERGLASS REIUFORCED PLYNOD (FRP) BOXES F IBERGLASS REINFORCED PL NDOD (FRP) BOXES

PO FIBERGLASS REINFGRCED PLYNOD (FRP) BOXES FIBERGLASS REINFCRCED PLNDCD (FRP) BOXES FIBERGLASS REIMFCRCED PLTWODO (FRP) BOXES metal BOXEs, CARTONS, CASES METAL BOXES, CARTOUS, CASES METAL BOXES, CARTOMS, CASES METAL BOXES, CaRTONS, CASES METAL BOXEs, Cartows, CASES METAL ORLMAS, BarRels, KECS

FIBERGLASS REIMFORCED PLYWDOD (FRP) BOXES F1BERGLASS REINFCRCED PInNOD (FRP) BOXES fIBERgLASS REIMFORCED PLNNOO (FRP) BOXES FIBERGLASS REIMFORCED PL NMOD (FRP) BOKES fibercLass REIWFORCEd PLnDOO (FRP) BOXES FIBERGLASS REINFCRCED PLTMDOD (FRP) BOXES METAL DRUMS, BARRELS, KEES metal dRUAS, BarRets, KegS

$4 * 6 * 10$
$4 * 8 * 10$
$4 * 8 * 16$
$5 * 10 * 13$
$5.2 * 7.1 * 10.5$
$5.2 * 7.1 * 16.5$
110 GALLON
55 GALLON

$10.5 * 10.7 * 12$
$4 * 4 * 7$
$4.83 * 5 * 8$
$6.3 * 8 * 14.7$
$9 * 10.67 * 16$
$9 * 10.67 * 20$
$9 * 10.7 * 12$
$9 * 11.6 * 20$
$3.7 * 6.5 * 13.2$
$5.2 * 7.1 * 10.5$
$5.2 * 7.1 * 10.5$
$5.2 * 7.1 * 16.5$
$5.2 * 7.1 * 16.5$
$55 * 410 *$

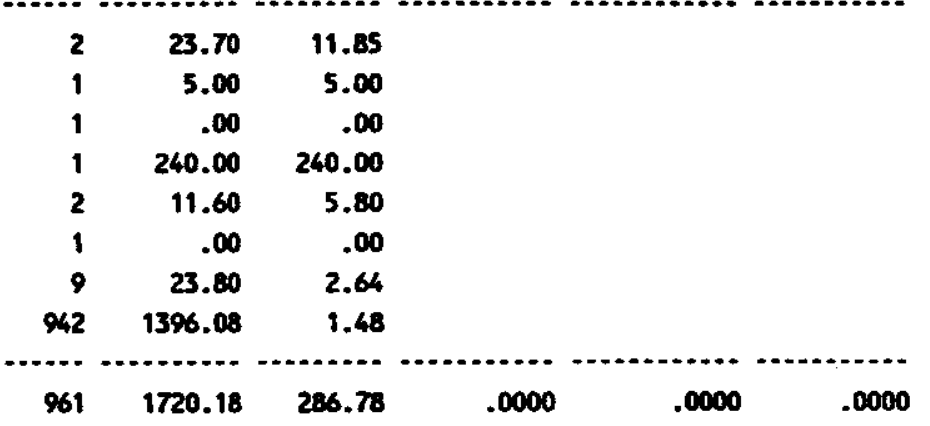

$\sin$

30.00

1975 R 218uBA

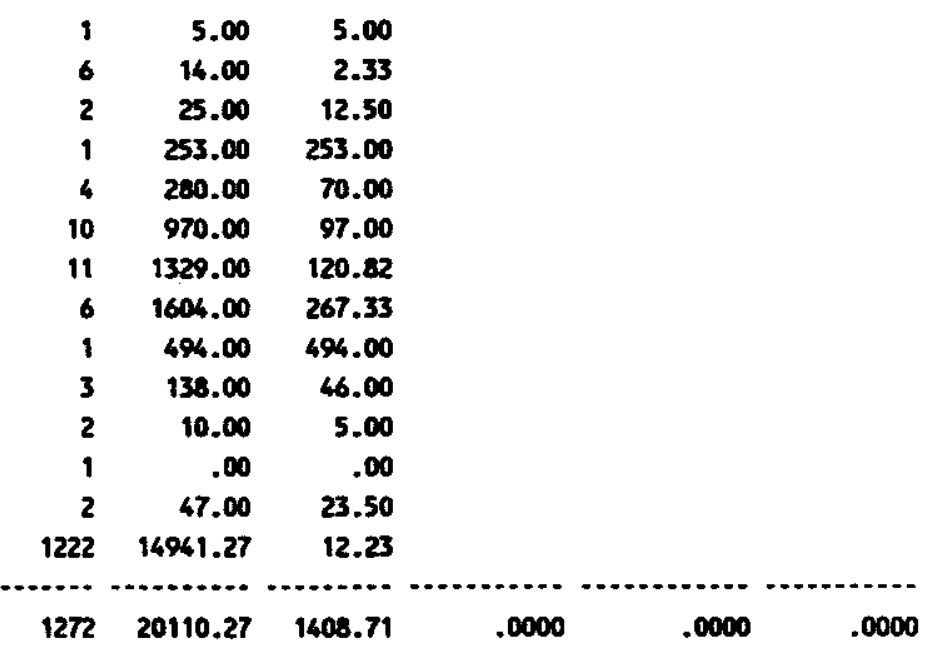

55 callom

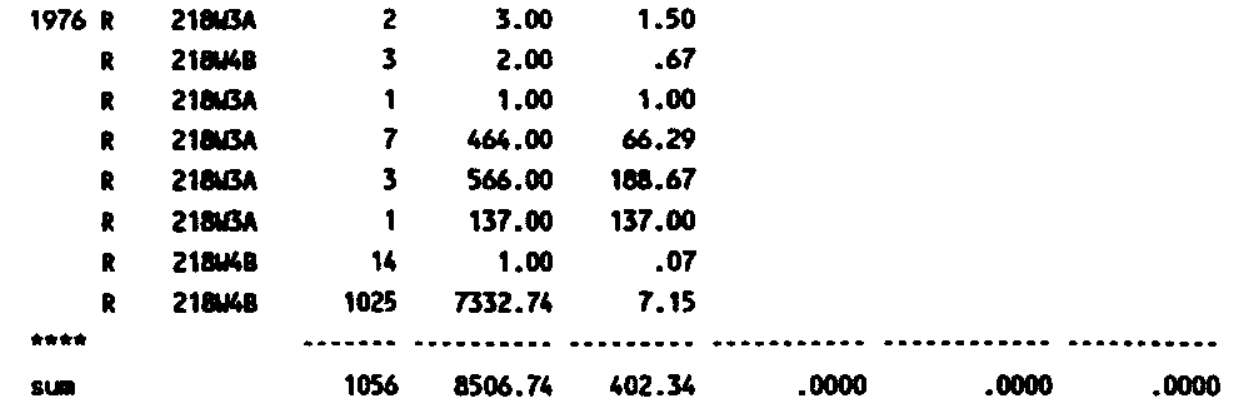

5.00
1.00
$\ldots . .0$
22.00

5.00
1.00
5.00
1.00
5.00
5.00
1.00
5.00
$\ldots . .$.
28.00


Sorted by Date and Primery Waste Type Pri

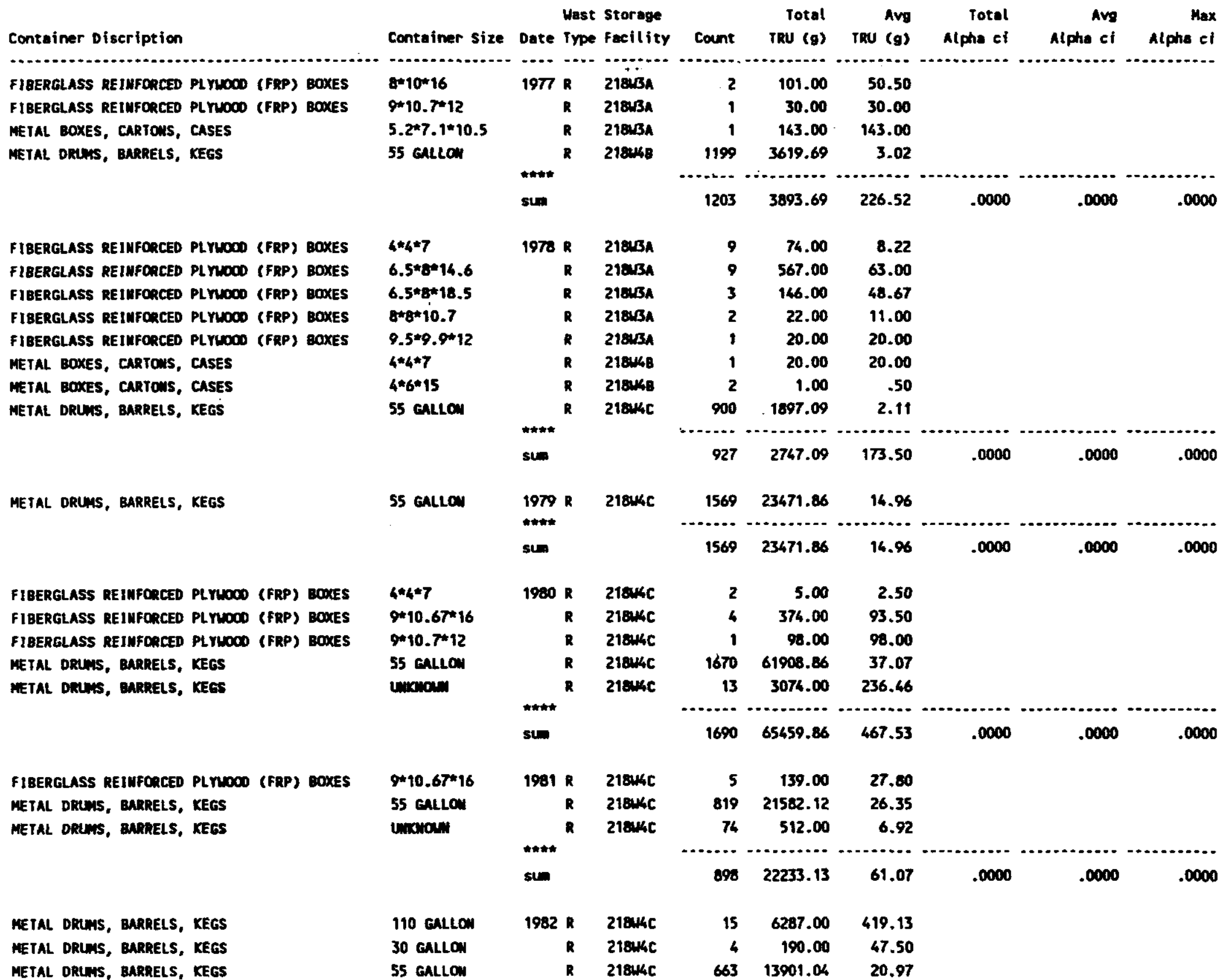

METAL DRLONS, Burgess,

30 GALLON

$21844 \mathrm{C}$ 
Sorted by Date and Primery Waste Type

Pri

Mast Storoge

Container Discription

Conteiner size Date Type facility Count TRU (g) TRU (g) Alpha ci Alphe ci Alphe ci

metal druns, barrets, ketos

unkwow

1982 R 218 MC
sum

$7 \quad 4588.00 \quad 655.43$

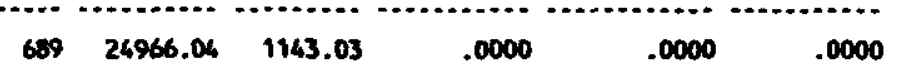

METAL BOXES, CARTOUS, CASES

$4 * 6 * 10$

METAL DRUAS, BARRELS, KEES

METAL DRUNS, BARRELS, KEGS

55 GALLON

unknow

METAL DRUNS, BARRELS, KEES METAL DRUMS, BaRRELS, KEGS miscellameOUS SCRAP

METAL BOKES, CARTOUS, cases METAL bOXES, CaRTOHS, CASES METAL BOXES, CARTONS, CASES METAL Douns, BARRELS, KEGS

METAL dRLAS, BARRELS, KEGS mETAL DRUNS, BARRELS, KETS

metAl dRLAS, BARRELS, KEGS metal dRLMS, BarRELS, KEGS

METAL dRLAS, BarRELS, KEgS METAL DRLAS, BarRels, Kegs

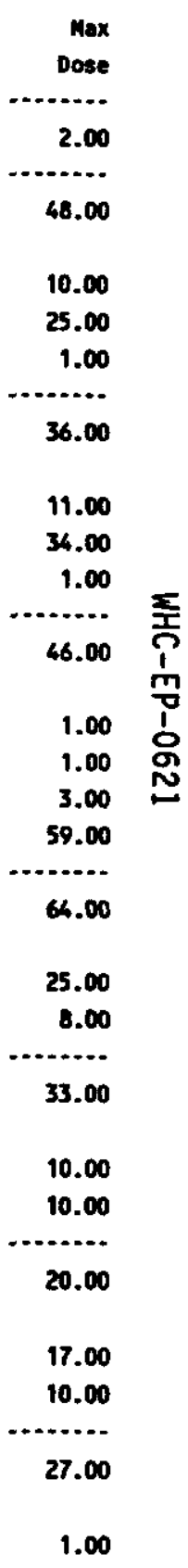


TRU MASTE

Page: 7

Sorted by Date and Primary Waste Type

$$
\text { Pri }
$$

Wast storage

Avg

Container Discription

Container size Date Type Facility

count

tht

-...........

suin

Metal dRLAS, Barrels, Kegs

55 GALLOM

$1990 R \quad 2241$

-

Avg Total Avg Max

Max Dose (n) 1.00

METAL DRUMS, BARRELS, KEGS METAL DRUNS, BARRELS, KEGS

METAL DRUNS, BarReLS, KEGS

sin

$\begin{array}{llllll}14 & 343.00 & 24.50 & .0000 & .0000 & .0000\end{array}$

1.00

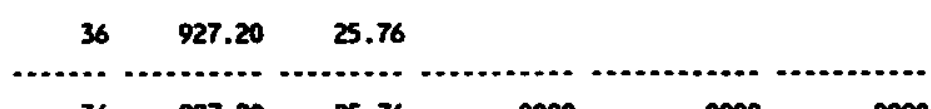

1.00

$\begin{array}{lllllllll}55 \text { GALLON } & 1991 \mathrm{R} & 21844 \mathrm{C} & 18 & 16.00 & .89 & 7.9311 & .4957 & .5230\end{array}$

55 GaLloN

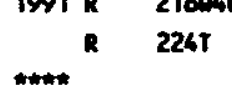

4057

sin

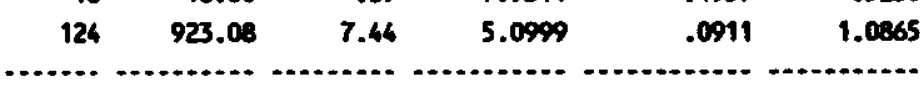

142939.08

8.33

13.0310

$.5868 \quad 1.6095$

\begin{tabular}{|c|c|c|c|c|c|c|c|}
\hline $1992 R$ & $224 \mathrm{~T}$ & 64 & 64.00 & 1.00 & 33.4720 & .5230 & .5230 \\
\hline$* * * * *$ & & & & & & & \\
\hline $\sin$ & & 64 & 64.00 & 1.00 & 33.4720 & .5230 & .5230 \\
\hline
\end{tabular}


WHC-EP-0621

This page intentionally left blank. 
WHC-EP-0621

APPENDIX B.9

PHYSICAL CONTENTS DESCRIPTION FOR 55-GALLON DRUMS CONTAINING TRU WASTE - SORTED BY DATE, PRIMARY WASTE TYPE, AND STORAGE FACILITY 
WHC-EP-0621

This page intentionally left blank. 


\section{PHYSICAL CONTENTS DESCRIPTION FOR 55-GALLON DRUMS \\ CONTAINING TRU WASTE - SORTED BY DATE, PRIMARY WASTE TYPE, AND STORAGE FACILITY}

This subappendix describes the physical contents of the 55-gal drums of transuranic (TRU) waste generated at the Plutonium Finishing Plant (PFP). Before 1978 physical contents were not required to be listed on the burial records, so a great many of the early records list the contents of the drum as "Miscellianeous."

The printout for this computer run sorts the contents data by storage facility, date, and primary waste type. The introduction to Appendix B contains a table of waste codes and their meanings. 


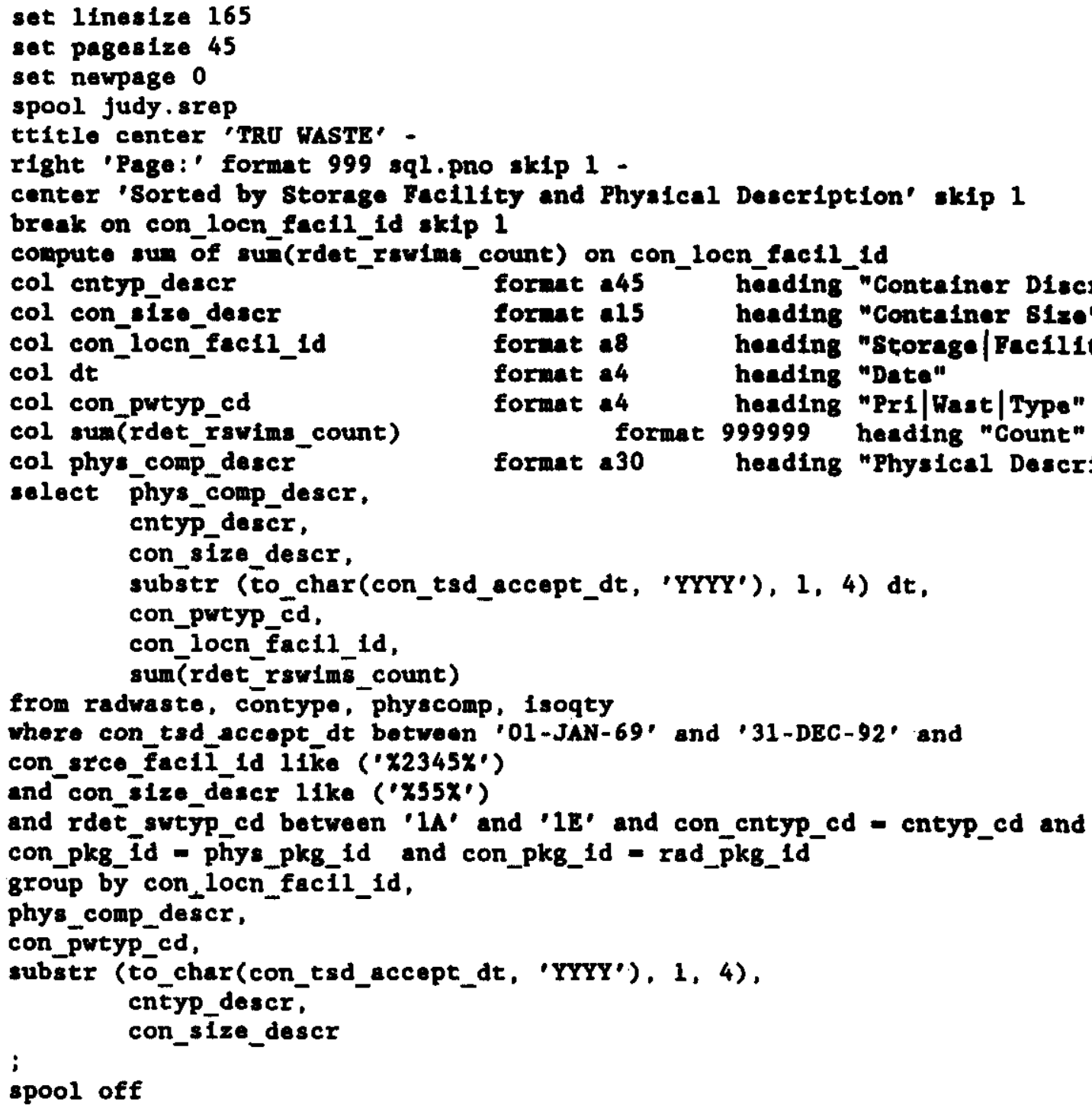


Wast Storage Container size Date Type Facility count

Physical Description

Conteiner Discription

MeTAL/IROW/GULVANIZED/SHEET METAL DENS, BNIRELS, KEGS

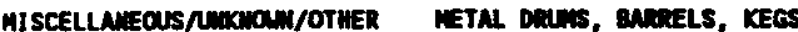

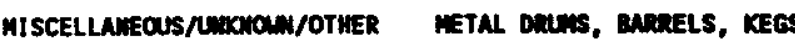

55 EALLOH

55 calta

55 callow

2180

3
1074

1.

3301

55 exlow

55 callow

55 callo:

55 callon

55 exlom

55 callo

55 GALLOM

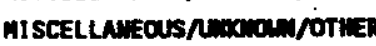

MISCELLAMEOUS/NWOMOUN/OTHER

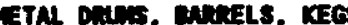

METAL Dedns, saraels, KECS

ABSORBANT/KITY LTR/VERAICULITE METAL DAMS, EAMALLS, KEGS ABSORBANT/KITY LTR/VERMIOULITE METAL DetRS, ANRELLS, KEGS ABSORBANT/KITY LTR/VERenICULITE METRL DEUN, MadeLS, KEES ABSORBANT/KITY LTR/VERHICULITE METAL Dans, BaneLL, KEGS ABSORBEUT/KITY LTR/NERHICOLITE METAL DeUAS, MWRELS, KEGS AIR

ALLMIIIIM

ASBESTOS

ASBESTOS

ASEESTOS

ASHES

ASHES

ASHES

CENEMT

CERAMICS

CLOTH/RAGS/WILO

CLOTH/RACS/AYLOH

CLOTH/RACS/ATLOO

CLOTM/RAGS/WYLON

CLOTH/RaGs/MrLon

CLOTH/RAGS/WYLON

CLOTH/RaCS/MYLO

CLOTH/RACS/MYLO
METAL DAMs, anirets, KECS

neTAL Dours, murets, KEGS

METAL Duns, sancels, KECS

METAL Dans, matels, KEeS

METAL Dans, maciss, KeES

retal Dans, moctis, KeEs

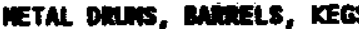

METAL Dans, eunects, KECS

METAL Dans, Manels, KECS

METAL Dauns, BunRELS, KECS

METAL oums, Bamets, KECS

reTAL Deans, Bapatels, KECS

METAL onurs, bunecLs, KEGS

METAL DPans, Bundels, KECS

METAL Desuss, Bnerels, KEES

METAL DEUUS, BarRELS, KEES

METAL Dalrs, whereLs, KEES

METAL Deluss, andeELS, XEES
55 callon

55 Gatlom

55 callom

55 exlon

55 eNlon

55 enllom

55 calla

55 calla

55 callo.

55 GALON

55 callon

55 callom

55 callom

55 callon

55 cNLLO

55 eallon

55 EALLO

55 exlon

55 enLon

55 CALLON

55 Gallo

55 cullon

55 callow

$\begin{array}{llr}1971 \text { R } & 218448 & 1612 \\ 1972 \text { R } & & 1668 \\ 1973 \text { R } & & 28 \\ 1974 \text { R } & 176 \\ 1975 \text { R } & 3 \\ 1976 \text { R } & 5 \\ 1977 \text { R } & & 5 \\ & & 16 \\ & \text { sin } & 3508\end{array}$

1901 R $21844 C$

$1902 \mathrm{R}$

$1906 R$

$1991 \mathrm{R}$

$1902 R$

$1905 \mathrm{R}$

$1905 \mathrm{R}$

$1906 R$

1937 R 2

$1931 \mathrm{R}$

$1962 R$

$1905 \mathrm{R}$

$1905 \mathrm{R}$

$1905 \mathrm{R}$

$1978 R$

$1979 \mathrm{R}$

$1981 R$

1982 R 197

$1983 R$

1994 R 1943

1985 R 2449

$1900 \mathrm{R} \quad 2230$ 
TRU UASTE

Sorted by storage facility and Physical Description

Pr

Wast Storege

Physicat Description

Conteiner Discription Container size Date Type Facility count

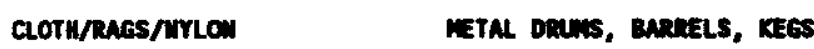

CLOTH/RMES/WYLOW

CLOTH/RMES/MrTow

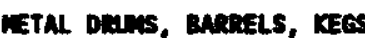

metal Dans, Bathels, KEes

METAL DaUns, BABRels, KeES

METAL Dians, Menels, KEes

CLOTH/RACS/WYLON

METAL Daus, BAmels, KECS

METAL bans, Butats, KES

metal Deuns, Backels, KEes

METal Dans, Banders, kEes

methe Dans, anders, KeEs

metal Dans, anceats, keos

meTal Douns, meicels, reas

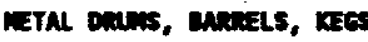

retal bans, wricels, kess

metal Dauns, wovels, Lees

metal Dauns, Baneets, KeEs

metal DRLNS, Bathels, KeES

metal Danns, ankels, kecs

retal deans, Baraels, KECS

meTAL DRLMS, BaRRELS, KECS

metal deins, Baneles, kEgs

weTAL Dauns, BuneELS, KECS

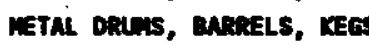

METAL Denus, cankels, KegS

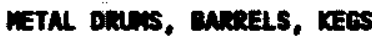

meTal dauns, Barrets, KEes

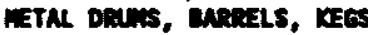

metal Denus, Barpels, kEas

vetal Dadrs, aurrels, kEes

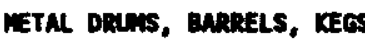

MeTAL DRuns, Barkets, KEeS

metal Deans, menels, keCS

metal Delms, Barketels, KeEs

METAL Dadus, Buratels, KECS

metal dRuns, barrels, KEes

METAL DRLNS, BARRELS, KECS

MetAl DRUnS, barRELS, KEGS

PetAl DRLUS, BarRELS, KECS

55 callow

55 ealion

55 GALLOW

55 ealom

55 callow

55 callon

55 exlow

55 EALLON

55 callam

55 eallom

55 encom

55 enlom

55 eallom

55 cultom

55 ENLLOM

55 enllan

55 callon

55 EALLOW

55 eAllow

55 CALloN

55 callor

55 exllow

55 culion

55 exllow

55 callow

55 cullon

55 cullow

55 callow

55 callon

55 callow

55 callon

55 callon

55 eallon

55 GALLOW

55 callan

55 callow

GLASS

55 EAllon

55 callow

GLASS

metaL dRLNAS, BarReLLS, KEGS

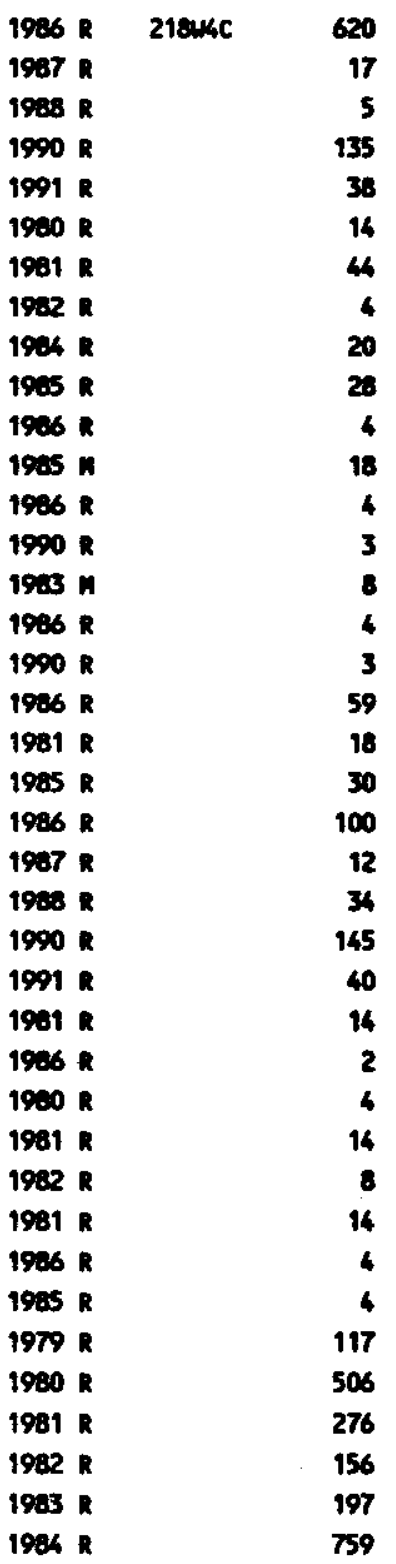


TRU MASTE

sorted by Storage Facility and Physical Description

Pri

Physical Description

Containar Discription

Wast Storage

Glass

GLASS

IETAL DRLALS, Batrels, KEOS

metal Delnis, Barrels, KEes

METAL Dauns, BARRELS, KEES

METAL Divns, Banrels, KEGS

METAL DeLUAS, BarreLs, KECS

METAL Dauns, BARRELS, KECS

retal dounts, marRels, xÉes

mitAl Datus, Mardels, KETS

METAL banes, BariRELS, KEES

meTAL Douns, BARRELS, KEES

META DQUnS, BARRELS, KEGS.

meTAL Dans, anRels, KECS

METAL Deurs, anRrELS, KEeS

meTAl Danes, Burrels, KEeS

METAL DeAns, earrelels, KECS

META Dants, BARRELS, KECS

METAL Detus, BarRELS, KEOS

meTAl Dauns, Barrels, KEeS

metal Dans, Barafels, KEES

meTAL DMins, sarkels, KEOS

METAL DeAns, BabreLs, KEES

meTAL Dauns, Barrels, KEes

METAL bauns, meaELS, XEos

meTAL Dauns, Barpels, KEES

vetAl Deuns, BARnels, KEES

mETAL Douns, Barrels, KECS

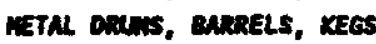

METAL Danus, MnReLS, KEgS

METAL Danes, carrets, KEeS

netal baurs, menels, kEtos

METAL DEAns, sarrels, KEGS

reTAL Daus, BARRels, KEeS

neTAL Douns, marRELS, KECS

METAL Deums, BareELS, KEeS

RETAL DRARS, BARRELS, KEÇS

metal dents, Barrels, KEOS

iETAL DRUMS, BARRELS, KEES

METAL denuis, Barrels, KEGS

Container size Date Iype Facility Count

55 callom

55 Gulom

55 eallow

55 Gallow

55 culcon

55 exllom

55 callom

55 callow

55 exilam

55 Ealloi

55 eallo:

55 callom

55 exlon

55 callom

55 EAlLOW

55 enleon

55 exlon

55 enlom

55 enllow

55 enlom

55 callow

55 callo:

55 enllon

55 entow

55 extury

55 exuo

55 callow

55 cula

55 enlion

55 exlou

55 callou

55 exlow

55 exlow

55 culloy

55 callow

55 Eallow

55 callow

55 enlow

55 cullom

\begin{tabular}{|c|c|c|}
\hline $1945 \mathrm{R}$ & $21844 c$ & 590 \\
\hline $\begin{array}{l}1906 \mathrm{R} \\
1908 \mathrm{R}\end{array}$ & & $\begin{array}{r}324 \\
5\end{array}$ \\
\hline $\begin{array}{l}1908 \mathrm{R} \\
1990 \mathrm{R}\end{array}$ & & $\begin{array}{l}3 \\
3\end{array}$ \\
\hline $1991 \mathrm{R}$ & & 8 \\
\hline $1985 \mathrm{R}$ & & 8 \\
\hline $1986 \mathrm{R}$ & & 2 \\
\hline 1982 n & & 2 \\
\hline 1963 N & & 32 \\
\hline $1979 \mathrm{R}$ & & 133 \\
\hline 1900 R & & 923 \\
\hline $1981 \mathrm{R}$ & & 706 \\
\hline 1962 R & & 504 \\
\hline $1983 \mathrm{R}$ & & 354 \\
\hline $1984 R$ & & 1118 \\
\hline 1985 R & & 748 \\
\hline $1986 \mathrm{R}$ & & 489 \\
\hline 1987 R & & 34 \\
\hline 1908 R & & 22 \\
\hline $1990 \mathrm{R}$ & & 47 \\
\hline $1991 \mathrm{R}$ & & 28 \\
\hline $1978 \mathrm{R}$ & & 62 \\
\hline 1979 R & & 64 \\
\hline $1900 \mathrm{R}$ & & 16 \\
\hline $19.5 \mathrm{H}$ & & 18 \\
\hline 1902 in & & 2 \\
\hline 1933 и & & 24 \\
\hline $1905 n$ & & 8 \\
\hline 1989 R & & 2 \\
\hline $1905 \mathrm{R}$ & & 2 \\
\hline 1986 n & & 6 \\
\hline 1905 n & & 8 \\
\hline $1976 \mathrm{R}$ & & 6 \\
\hline $1979 R$ & & 24 \\
\hline $1900 \mathrm{R}$ & & 2321 \\
\hline $1981 \mathrm{R}$ & & 822 \\
\hline $1992 R$ & & 830 \\
\hline 1983 & & 1482 \\
\hline 1984 & & 3217 \\
\hline
\end{tabular}

PAPER/CARDPOARD 
TRa UASTE

Sorted by Storage Facility and Physical Description

Prí

Uast Storage

Physical Deseription

PAPER/CADopaso

PAPER/Cariosonso

PAPER/CARDecando

PAPER/CAODSOAND

PAPER/CARDeOAND

PAPER/CARDEOARD

PLASTIC/POLYvatune

PLASTIC/POL YURATMNE

PLASTIC/Pochratuane

PLastic/pocrumatmaie

PLasticfporizatmae

PLASTIC/Pol Vuatuave

PLASTIC/PoL TuRathuE

PLASTIC/POL VuRATHNE

PLASTIC/POL VURATMUEF

PLAstic/polneatmane

$\underset{\infty}{\infty}$
Conteiner Discription

metal Douns, menels, reas

METAL boums, Babeats, kes

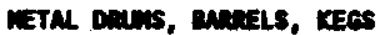

metal douns, meecls, ress

METAL Dodus, mareELS, wees

metal douns, manels, kECS

METAL Deuns, Rutrefels, KECS

METal opuns, meneLs, KEOS

METAL Dans, Bnidels, KECS

netal Deuns, muness, KEes

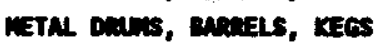

METal Dans, mabasas, rees

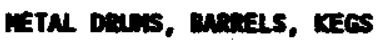

METAL Deanis, Bunets, KEes

METAL Demus, BabeELS, KECS

METAL Daurs, Banetels, KECS

METAL Douns, ameels, KECS

METAL Dans, munels, KECS

METAL Deans, encelis, KEES

metal Deurs, enceres, KEes

metal deuns, manels, kEeS

metal douns, minels, Kess

meTal Deuss, merels, KEes

metal DAuns, anucels, KEES

meTAL Dears, mateis, kecs

METAL Dans, morsts, reses

metal deuns, maness, kess

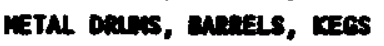

metal deans, Buncels, crees

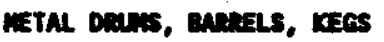

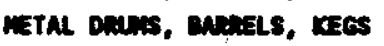

metal oedns, monels, reos

METAL Defus, Bamels, KEES

METAL Deuns, Barels, KEES

metal Daus, buvels, KEes

MeTAL DAMns, encuels, keEs

METAL DRLurs, BaneELL, KECOS

meTAL Deluns, Bumats, KEeS

meTAL DRUAS, maneELS, KECS
Conteinar size Date Type Facility count

55 culom 1905 R 21844

55 enLLON $1986 R \quad 866$

55 Gullon $1987 R$

55 cullow $1988 R$

55 GALLON $1990 \%$

55 callom $1991 \%$

55 Gallow 1984 N 6

55 CALLON 1985 M 26

55 CALLOW 1978 R 6

55 enlan 1979 R

55 EALLOW 1990 R 2315

55 GNLOW $1981 R$

55 cullol $1992 R$

55 ClLLON $1983 R$

55 CAllOW 1984 R 3634

55 callow $1905 R \quad 3463$

55 exlow $1986 R$

55 GLLLOW 1987 R

55 cillon $1928 R$

55 CAlLOW $1990 \mathrm{R}$

55 Exllow $1991 R \quad 40$

55 Callow 1966 ? 2

55 callon $19 \% 4 \mathrm{n}$

55 callow 1978 R 6

55 eqLiO $1979 R$

55 culan $1900 R$

55 EULLON $1981 R \quad 940$

55 GAllon 1962 R

55 exlow $1983 R \quad 1444$

55 CAlLON $1904 R$

55 CALLON $1905 R$ $R$

55 cAllOW $1996 \mathrm{R}$

55 callow $1987 R$

55 GALLON 1988 R 14

55 anlon $1990 R \quad 141$

55 EALLON $1991 \mathrm{R}$

55 callow $1986 R \quad 10$

55 callow 1982 R 39

55 GALLON 1981 R 
TRU MASTE

Sorted by Storage facility and Physical Description

Pri

Wast Storage

Physical Description

Container Discription

STAIMLESS STEEL

STAIMLESS STEEL

STAIRLESS STEEL

UOCO/LUMER/PL YNOOD

UDCo/LUNER/PL YNDOO

UOCD/LUEAER/PL NDOOD

1000/LUABER/PL YNOOO

WOCO/LLIMER/PLYNOCO

HOOD/LLEER/PL NOCOO

YOCO/LLIBER/PL YNDOD

UOOO/LURBER/PLYVOOD

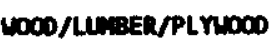

LOOD/LUWBER/PLYMCOO

$\underset{1}{1}$

CLOTH/RAES/MYLOU

DIRT/SOIL/DIATOMACEUS EARTM

PAPER/CARDBOARD

PLASTIC/POL YURATHAME

RUBBER

TETAL Dauns, BABRELS, KECS

veTal Dauns, marels, KeÉS

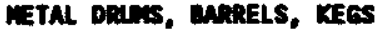

METAL Dalns, Bumels, KeES

metal douns, marReLs, KECS

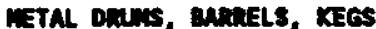

METAL Deuns, BabeELL, KEGS

METML DRUAS, BABRELS, KEES

METAL DRLMS, BARRELS, KEES

metal dRUns, anrrels, 'KEes

METAL DeUnS, BarRELS, KEES

metal druns, barkels, KeES

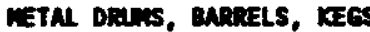

metal deans, Babrels, Kecs

METAL DAUHS, GARELLS, KEES

metal deuns, anderels, Kees

MEtAL Dauns, BarReLS, KECS

METAL DEurs, BuAELL, KEGS

metal dRLns, baknets, KEes

ABSORBANT/KITY LTR/VERHICULITE METAL DRUMS, BARRELS, KEGS

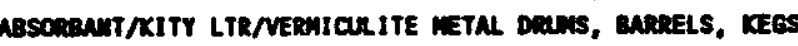
ABSORRANT/KITY LTR/VERHICULITE METAL DEAES, BARELL, KEGS ALinitivin

ASBESTOS

BATTERIES

BRICK/FIREBRICK

CARBORUMUU

CERENT

CEMENT

CERAMICS

CERAMICS

CLOTH/RAES/ATLOW

CLOTH/RAES/NYLOW
METAL Deans, BankeLS, KEGS

METAL DEAIS, BARRELS, KEES

metal DRuss, Barrels, KEeS

metal douns, Bureets, KEes

METAL DEAMS, Bancels, KEOS

metal Dans, marets, KECS

MeTAL Douns, Baceels, KEES

METAL DRuns, ankRELS, KEGS

METAL DRUMAS, MARRELS, KEES

METML Deans, BARRELS, KEGS

METAL DRLAS, BARRELS, KECS
Conteiner Size Date Type Fecility Count

55 enlan 1984 a $21844 \mathrm{C} \quad 2$

55 callon $1905 R$

55 callon $1996 ?$

55 callom $1905 \mathrm{n}$

55 callow $1980 R$

55 callon $1991 R$

55 EALLON $1982 R$

55 CAllon $1984 R$

55 GAlOH $1905 R$

55 Ellow 1986 R 36

55 callom

55 callow $1980 R$

55 callow $1990 R$

55 callow $1981 R$

$\sin \quad 58303$

55 Gallon

55 eallon

55 ealiom

55 exlLow

55 exlow

$\begin{array}{llr}1990 \text { R } & 21845 & 3 \\ 1990 \text { R } & & 3 \\ 1990 \text { R } & & 3 \\ 1990 \text { R } & & 3 \\ 1990 \text { R } & & 3 \\ & & \text { SU }\end{array}$

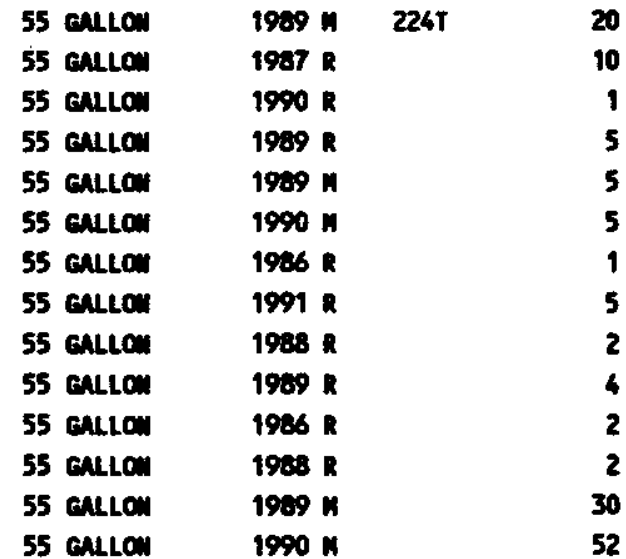


TRU MASTE

Sorted by Storage Facillty and Physical Deacription

Prí

Wast Storage

hysical Description

Container Discription

Conteiner size Date Type Facility count

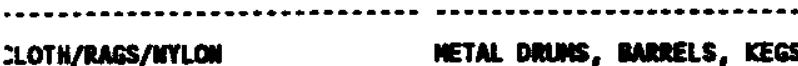

METAL DAurs, Baniels, KEeS

MeTAL Dauns, Babaels, KeES

reTal douns, manels, KEes

METAL Dauns, Bumets, KEES

veTal Daus, Bunets, KECS

MeTAl douns, Buncels, KeES

metal Deves, Bunets, KECS

METAL Denus, maets, KEGS

reTAL Deuns, maneLS, KECS

nETML Denus, maxels, KEOS

MeTM Daurs, manels, KEGS

wetal Danns, watels, ketes

veTAl deuns, manels, KECS

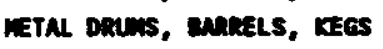

METAL Denuns, marels, KEGS

METAL DRLAHS, MarkELS, KEES

METAL DRLUS, BAREELS, KECS

METAL DQuns, mareELS, KECS

$\cos$

METAL Denns, BaneELS, KECS

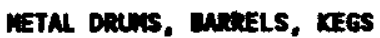

JRT/SOIL/OIATOMCEOUS EARTH

JIRT/SOIL/DIATOACEOUS EARTH

JIRT/SOIL/DIATOACEOUS EARTH

OIRT/SOIL/DIATOUCEOUS EARTH

OIRT/SOIL/DIATOMCEOUS EARTH

JIRT/SOIL/DIATOWCEOUS EARTH

OIRT/SOIL/OIATOACEOUS EARTH

DIRT/SOIL/OIATOACEOUS EARTH

DIRT/SOIL/OIATOACEOUS EARTH

DIRT/SOIL/DIATOMCEOUS EARTH FIBERCLASS

GLass

GLASS

METAL DRUNS, Baretels, KeES

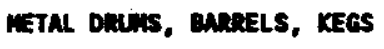

METAL DRUNS, CanRELS, KECS

retal Denus, Bakels, xeES

METAL Deuns, Barrels, KEGS

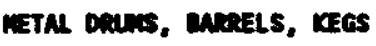

METAl Delurs, marels, KEES

retal deans, menels, KEeS

METAL Deuns, BuneLS, KECS

METAL Dauns, BAreELS, KECS

mETM Dans, Bunels, KECS

veTAL deans, Bninels, kECS

veTAL owns, andels, KEES

METAL Douns, ankELS, KEES

metal dRAOHS, BunReLs, KEES

metal Daus, whaELS, KECS

metal Dauns, Banels, KECS

GLASS

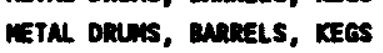

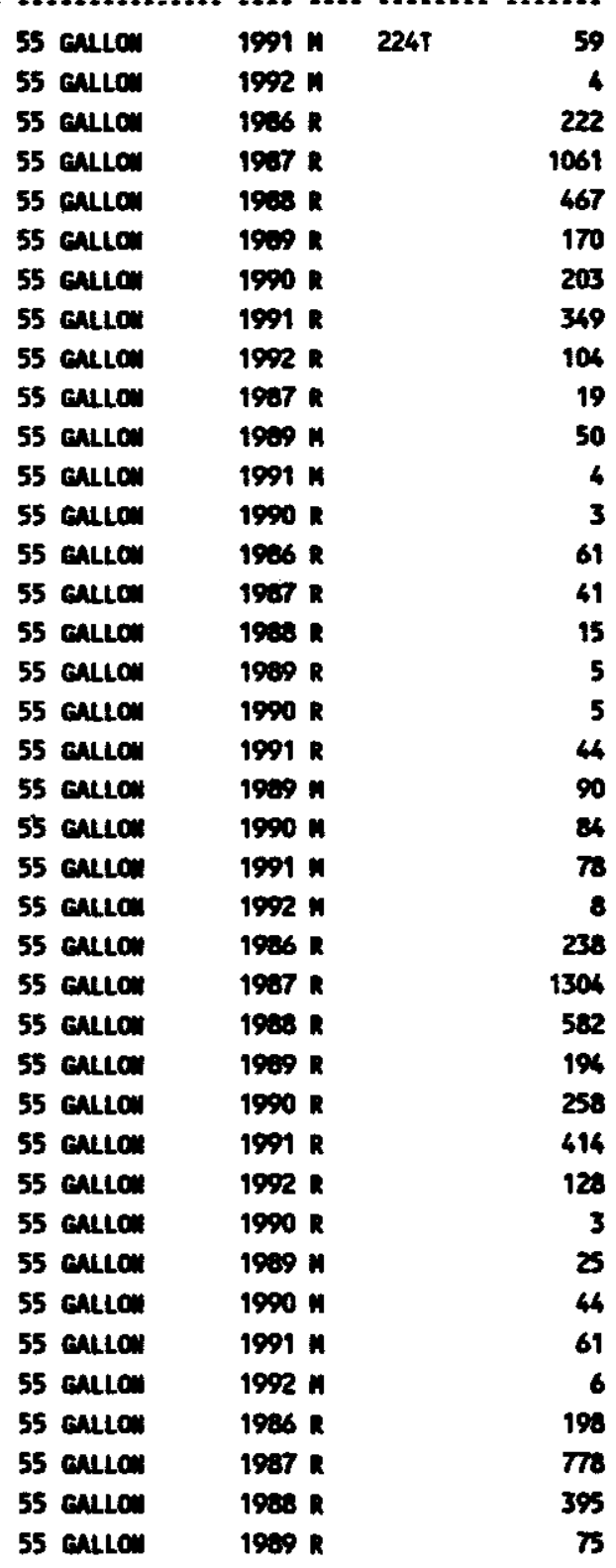


Physical Description

Conteiner Discription

GLASS
GLASS
GRAPHITE
GROUI

EROUT

mizreous constituents

mazaroous constituents

thazARDous ConstituENTS

Insehation mon-AseEstos

tustration MON-ASEESTOS

LEAD

LEAD

LEAD

LEAD

LEAD SHIELDING

LEATHER

LEATHER

D 1 LEATHER

LEATHER

MERCURY

METAL/IROW/ELLVAMIZSD/SHEET METAL/IROW/ERLVAUIZED/SHEET

METAL/IROW/GULVAWIZED/SHEET

NETAL/IROW/GALVAMIZSD/SHEET

METAL/IROW/GALVAMIZED/SHEET

METAL/IROW/ENLVMIZED/SHEET

METAL/IROW/EULVANIZEO/SHEET

IETAL/IROU/GULVANIZED/SHEET

METAL/IROW/EALVAMIZED/SHEET

NETAL/IROW/GALVANIZED/SHEET

METAL/IROW/CALVAMIZED/SHEET

HI SCELLAMEOUS/LARMOUM/OTHER

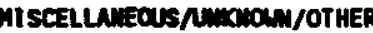

organics

PAIUTS/LUCITE

PAIMTS/LUCITE

PAPER/CARDBOAND

PAPER/CAREBCATE

PAPER/CAROBOARD
METal bauss, ankeELS, KEES

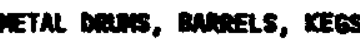

METAL mans, aukets, KEos

METRL DEISs, macels, KECS

METAL Deves, maceses, KEDS

Metul beavs, aderels, KEes

MeTal bems, marels, kEes

MeTal deans, maxels, KEes

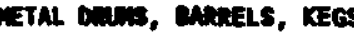

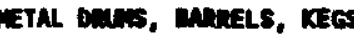

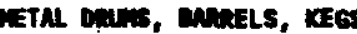

MeTal bauss, manels, KEes

METAL Danns, merets, KEeS

VETML Danss, chatels, KEes

METAL Dens, ineels, KEES

METAL Dalss, mavels, KECS

METRL bouss, maneLs, KEeS

KETAL Dams, matiels, KEOS

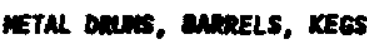

METAL Dans, EMoELLS, KECS

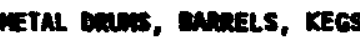

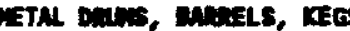

METAL Daurs, MBeetS, KEES

MeTal Denss, masels, KECS

METAL ONers, muets, KECS

metal bears, matefls, KEes

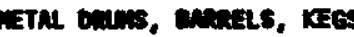

METAL Demns, amacels, KEES

Metal banss, anvets, KEgS

Metal bans, menels, kEes

METAL Dams, wasers, KEes

METAL Desns, macuzls, KEes

MeTML Banss, Bubets, KEGS

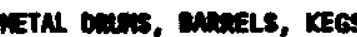

Metal Deans, Bnoels, KEgS

METAL Dhans, mexets, KEES

MeTAL Deans, waets, KEGS

METAL Deans, marezLs, KEES

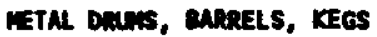

Conteiner size Date trpe Facility count

\begin{tabular}{|c|c|c|}
\hline \multirow{2}{*}{\multicolumn{3}{|c|}{35 CALLOW }} \\
\hline & & \\
\hline 55 CALLOM & $1967 \mathrm{R}$ & \\
\hline 55 CALLOW & $1991 \mathrm{~K}$ & \\
\hline 55 Gellow & $1990 \mathrm{n}$ & \\
\hline 55 GNLLOW & $1991 \mathrm{n}$ & \\
\hline 55 GALLOW & $1992 \mathrm{n}$ & \\
\hline 55 GACLOW & $1907 \mathrm{R}$ & \\
\hline 55 Gallow & 1909 R & \\
\hline 55 callow & $1900 \mathrm{~N}$ & \\
\hline 55 EULLOW & 1990 N & \\
\hline 55 GALLOW & $1991 \mathrm{n}$ & \\
\hline 55 EALLOW & $1992 \mathrm{~N}$ & \\
\hline 55 ERLLOW & $1990 \mathrm{n}$ & \\
\hline 55 CALLOO & $1909 \mathrm{n}$ & \\
\hline S5 anllow & $1990 \mathrm{n}$ & \\
\hline 55 GLLOW & 1986 R & \\
\hline 55 GULLOW & $1967 \mathrm{R}$ & \\
\hline 55 GALLOW & $1909 \mathrm{R}$ & \\
\hline $55 \mathrm{cAcLOW}$ & $1990 \mathrm{n}$ & \\
\hline 55 ENLLO & $1909 \mathrm{n}$ & \\
\hline 55 GALLOW & $1990 \mathrm{n}$ & \\
\hline 55 callow & $1991 \mathrm{~N}$ & \\
\hline 55 GuLlOW & $1992 \mathrm{n}$ & \\
\hline 55 GALLOM & $1986 \mathrm{R}$ & \\
\hline 55 EALLOW & 1987 R & \\
\hline 55 anclom & $1968 \mathrm{R}$ & \\
\hline 55 Gellom & $1909 \mathrm{R}$ & \\
\hline 55 GALLOW & 1990 R & \\
\hline 55 CALLOW & $1991 \mathrm{R}$ & \\
\hline 55 EALLOW & $1992 \mathrm{R}$ & \\
\hline 55 GULLOW & 1907 : & \\
\hline 55 CALLOW & 1968 R & \\
\hline 55 GULOW & 1909 n & \\
\hline 55 EnLlow & 1991 \& & \\
\hline 35 callow & $1992 \mathrm{R}$ & \\
\hline 55 EACLOW & $1909 \mathrm{n}$ & \\
\hline 55 CALlon & 1990 in & \\
\hline $55 \mathrm{~cm}$ & $1991 \mathrm{NH}$ & \\
\hline
\end{tabular}


TRU MASTE

Sorted by Storage facility and Physical Deacription

Pri

West Storage

Physical Description

Contoiner Discription

Contoiner size Date Type Facility count

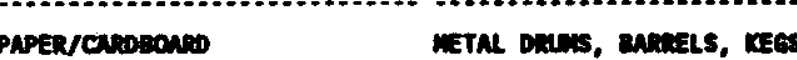

metal bens, encedels, Kees

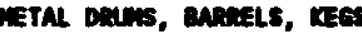

ETAL Daus, anciets, teEs

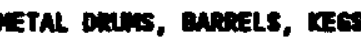

metal bours, subachs, tees

metal dents, enchels, wes

metal douns, mokts, kes

METAL Diuns, maxicLs, KEOS

METAL Dans, makels, KECs

ETAL Daurs, Baratels, REc

metal deuns, cantiels, Kecs

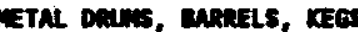

METAL Denes, manels, KeES

MeTAL Dauns, BabaELS, KEes

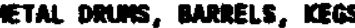

METAL OAUMS, BARRELS, KECS

izTAL Denus, Barkels, KEes

METAL DAUns, Barkets, KEes

cetal onuns, Bahiets, KEes

meTAL Douss, Bakiels, kEes

metal Deins, mankets, wees

meTAL Danis, Banagers, KEES

EeTAL Dembs, Bunatels, KEes

metal Donss, Babnels, kees

ietal Dauns, Burkels, kege

metal Deuns, Bunkels, rees

metal doens, monets, kegs

metal deniss, merets, Kees

ietal Douns, enmels, kees

metal doum, Bakels, KeEs

retal douns, butazis, Kees

IETAL DAuns, ankels, KEOS

METAL Deuns, BunkELS, reEs

METAL Dans, anRELS, KECS

nETAL Deans, Barrels, KECS

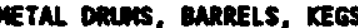

MeTAL DEuns, BarRels, Kecs

METAL DRINS, BARRELS, KEGS

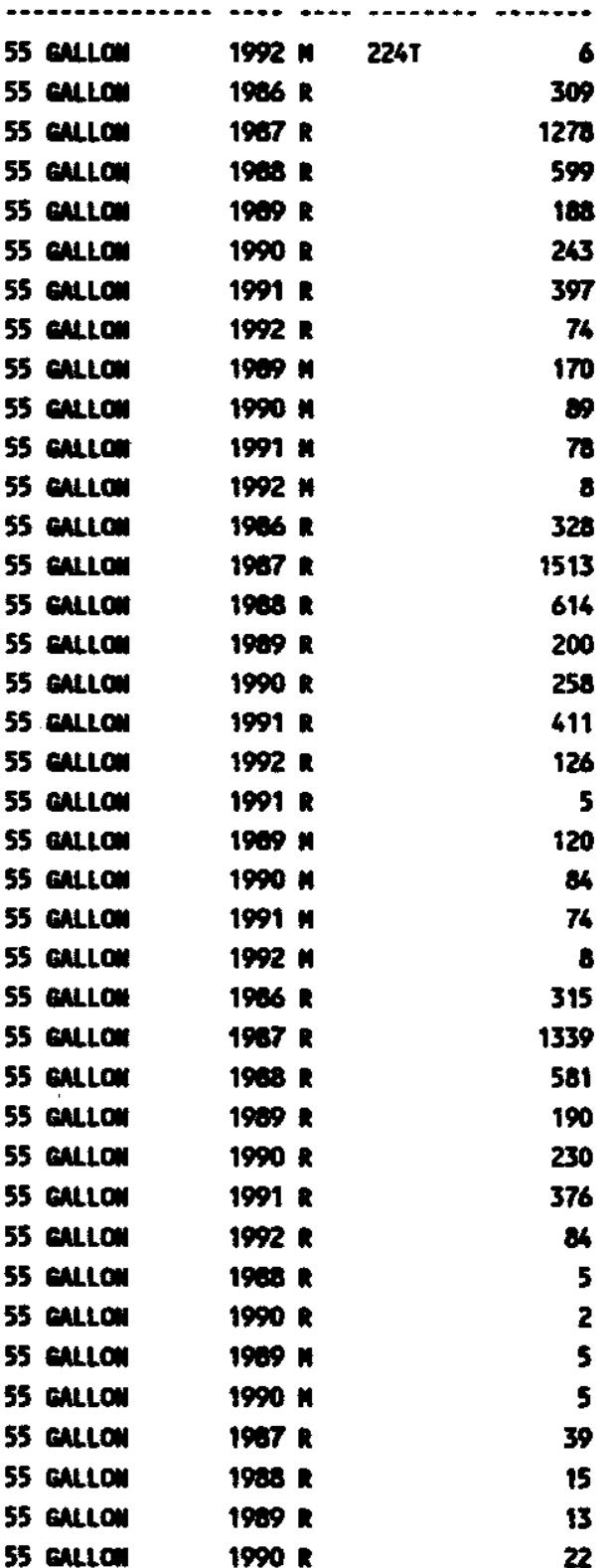


TRU WASTE

Sorted by storage Facility and Physical Description

Physical Deacription

WOCO/LUMEER/PLINOOD

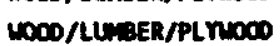

UDCo/LUMBER/PL YNOOD

LOCO/LUABER/PL YNOCD

WOCO/LGPER/PLYMOD

nOOO/LUnBER/PL MUOD

WOOD/LUMEER/PL NONO

UDCO/LUMEER/PL YNOD

YOCO/LUMER/PLYNODO

LOCD/LUMER/PL NOCO

UOCO/LUEBER/PL YNOOD
MaSORBANT/KITY LTR/VERUICULITE METAL DEUMS, BARRELS, KEGS

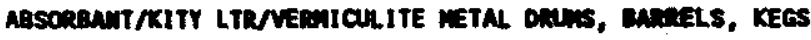
CLOTH/RAGS/MTLOW CLOTH/RAGS/WTLOM CLOTH/RAGS/MrLOM COMUEB PADS COMNEB PRDS COMEB PAOS

DIRT/SOIL/DIATOMACEOUS EARTH DIRT/SOIL/OIATOUCEOUS EARTH

LEND

METAL/IROW/EALVAWIZED/SHEET METAL/IRCW/COLYMIZED/SWEET MTAL/IROW/GALVAIIZED/SHEET

oILs

Oits

otLs

OILS

concanics

PAPER/CAROPOANO

PAPER/CARoBonio

PAPER/CARDEQREO

PCD

PLAST IC/POL YRATMaNE
Container Discription

MetAl opens, Marels, Keos

METAL DeAus, Baraels, KEgS

metal deanus, barkels, Kegs

METAL DRUUS, BARRELS, KECS

metal donus, andels, KEgS

METAL Drans, Barhels, KECS

wETAL DRUNS, BARRELS, KECS

METAL Denus, Banels, KEES

metal DRLUS, RaRrels, KegS

METAL DAMUS, BAMRELS, KEES

MEJAL DRLUMS, BNRRELS, KEGS

metal dauns, Barrels, Kecs

NeTAL Denus, anders, KEGS

METAL Daurs, eniaels, KEGS

metal Danis, mutials, Ketes

METAL DEUNS, MARELS, KEGS

metal dauis, carrels, KEgS

METAL Daus, BatRELS, KEGS

retal beans, andacls, KEOS

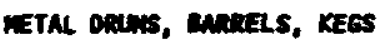

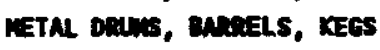

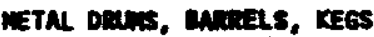

METAL Dalus, raninels, KECS

METAL DaAns, BanezLs, KeES

NETAL Dous, ennazs, KECS

METAL Dauns, merels, KESS

reTAL Daws, Bunels, ReES

netal baurs, chocets, kees

METAL Dalus, marels, KegS

METAL Dauns, andels, KEES

metal Dauns, Bneazls, KeES

METAL DRUNS, BARRELS, KECS

METAL DAUns, BARRELS, KECS

METAL Daurs, Baraels, KeES
Pri

West storage

Container Size Date Trpe Facility Count

55 culcom

55 callow

55 cillon

55 cillow

55 callow

55 callom

55 calla

55 allo

55 eulion

55 callom

55 callom

55 callow

55 callom

55 exllom

55 callow

55 enlion

55 callow

55 eallo

55 exllom

55 eAllo

55 callom

55 Gallow

55 exlom

55 aller

55 cullom

55 callo:

55 callom

55 cula

55 extcon

55 cullom

55 exlod.

55 callow

55 entam

55 enlion

55 callo

55 cultom

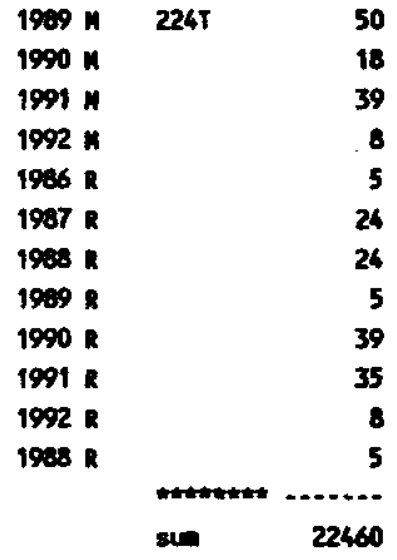

1909 in

$194 \mathrm{n}$

1908 in

$1998 \mathrm{mp}$

1903 in

$1989 \mathrm{mp}$

$1990 \mathrm{mp}$

$1909 \mathrm{n}$

$1909 \mathrm{~m}$

$1909 \mathrm{n}$

$1989 n$

1948 in

1989 is

$1902 \mathrm{mp}$

1905 mp

Pי 1909

$1990 \mathrm{mos}$

$1908 \mathrm{~m}$

1909 n

$1902 \mathrm{mp}$

1905 in

$1990 \mathrm{mo}$

1909 \% 
TRU UASTE

Sorted by Storage facility and Physical Description

Pri

Uast Storage

Phyalcal Description

PLASTIC/PoL notathate

PLASTIC/POL MURATMANE

PLASTIC/POL YuRATMAME

PLASTIC/POL MRATHANE

PLASTICPPOLnaATMUE

Aubaer

Asaes

Ruper

Aras?

WOO/LUEER/PLYWOO

CHEAICALS

Cloth/RAGS/WrLOO

\& OIRT/SOIL/DIATOMACEOUS EARTH

O METAL/IROM/GALVAMIZED/SHEET

PLASTIC/POL YURATMANE

RUBBER

METAL DRUNS, BARRELS, KECS

METAL DRLTS, MARRELL, KEES

retal DRLis, Barrels, Kegs

metal Deuns, Barrels, KeES

mETAL DELis, Barrels, KECS

metal deuns, barrels, Kegs
Container size Date Type Facility Count

55 eallon 1962 up 24014

55 ENLLO 1985 In

55 CALLON 1908 ip 50

55 callom $\quad 1999$ ip 30

55 EAlom $1990 \mathrm{MP}$

55 callow $1909 n$

55 cullom $1902 \mathrm{mp}$

55 ealion $1908 \mathrm{ip}$

55 GAlloN 1989 IP 10

55 callon $1989 \mathrm{n}$

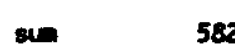

55 GALLON 1989 M 240218

55 CALLOM 1989 M

55 calcon $1909 \mathrm{~N}$

55 cullow $1909 \mathrm{M}$

55 axton $1989 n$

55 callon 1989 N 5

$\sin 30$

352 rows selected. 
WHC-EP-0621

APPENDIX B. 10

PHYSICAL CONTENTS DESCRIPTION FOR TRU WASTE CONTAINERS OTHER THAN 55-GALLON DRUMS - SORTED BY DATE, PRIMARY WASTE TYPE, AND STORAGE FACILITY 
WHC-EP-0621

This page intentionally left blank. 
PHYSICAL CONTENTS DESCRIPTION FOR TRU WASTE CONTAINERS

OTHER THAN 55-GALLON DRUMS - SORTED BY DATE, PRIMARY WASTE TYPE, AND STORAGE FACILITY

This subappendix describes the physical contents of transuranic (TRU) waste containers other than 55-gal drums. Note that, before 1978, physical contents were not required to be on the burial records, so a great many of the early records list the physical contents for a container as "Miscellaneous."

This computer run is sorted by the waste container's storage facility, type of container, and year. 


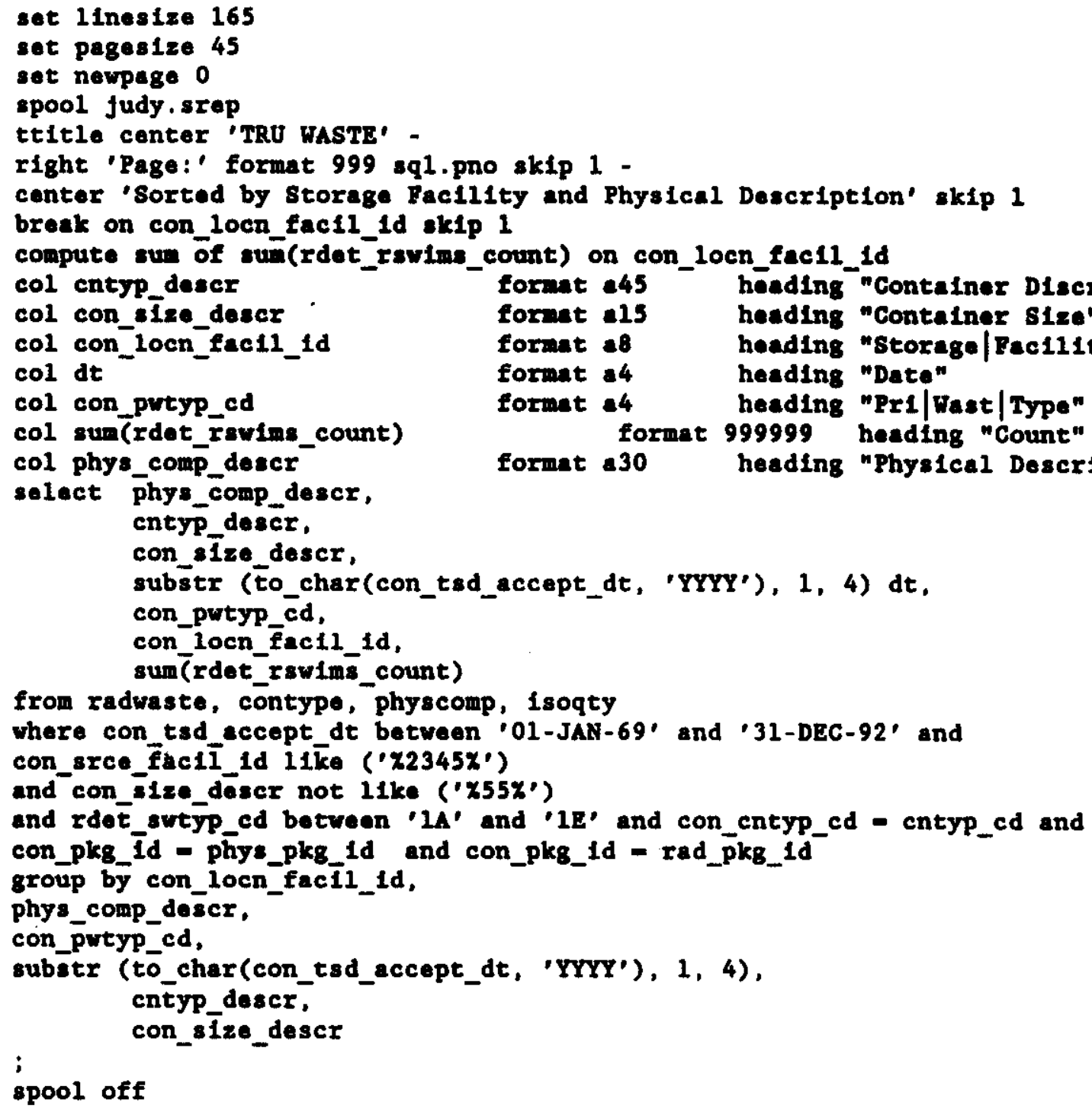


Thu ASTE

Sorted by Storage facility and Physical Description

Pri

Nast Storage

Physical Description

Container Discription

MISCELLAREOUS/NWMONWW/OTHER

MI SCELLAMEOUS/NMOMOWH/OTHER

METAL Dauns, maneLs, KEES

metal boxes, cartons, cases

MISCELLANEOUS/NWWWOMYOTKER MISCELLANEOUS/UWKWOM/OTHER MISCELLANEOUS/NMWMOMW/OTHER MI SCELLANEOUS/NMXMOMH/OTHER MI SCELLWNEOUS/NUXWOMIOTHER

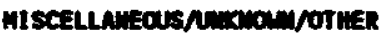
MISCELLUEOUS/NWMOMV/OTHER MI SCELLLAMEOS/M mWOOM/OTHER

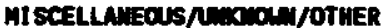
MI SCELLAMEOUS/NMKMOUM/OTHER MI SCELLANEOUS/NMXINOW/OTHER MISCELLAMECUS/MWHOWNOTHER MI SCELLAMEQUS/Mumaciw/OTHER MISCELLAMEOUS/NMKMOUM/OTMER

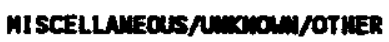

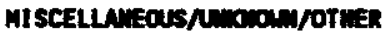
MISCELLANEOUS/NWMOMT/OTEER MISCELLANEOS/OMMOMOM/OTHER

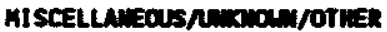

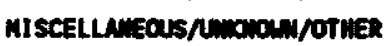

\section{GOVE Boxes}

meTal boues, cartoms, cases metal eoves, cartous, cases METAL cones, cantous, cases netal bones, cartons, cases metal vexes, cartous, cases METAL Diuns, enterens, KEGS SELF COWTAINED, EWIPATEWT WOCOEW BOXES, CARTONS, CASES GLOVE Boxes

meTAL Boucs, cartows, CAsES Metal bouses, cartous, cases metal bones, cartons, cases wetal boces, cartoms, cases metal bexes, cartoms, cases metal oontes, cartous, cases METAL Danss, BabaELS, KECS netal bocks, cantoms, cases METAL DOXES, CANTOHS, CASES metal boces, cartous, cases

ABSORBANT/KITY LTR/NERIICHITE METAL BOEES, CARTOMS, CASES Alontion cestem

CLoth/RACS/mrLom cloth/rags/mrion CONCRETE

FILTERS

FILTERS

FILTERS

GLAsS

GLASS

METAL DOEES, CARTOUS, CASES
metAL EOKES, CARTOWS, CAsES

METAL boxes, cantous, cases

METAL Dowes, cantoms, cases

metal Dans, marels, KEes

MeTAl DouEs, cantows, cases

METAL BOXES, CARTOUS, CASES

metal boutes, cartous, cases

FIBERELASS REIMFORCED PL nDOD (FRP) BOXES

Metal soxes, cantons, cases
Container Size Date Type Facility Count

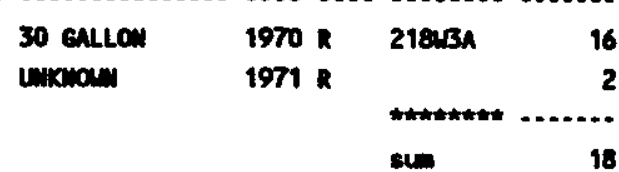

\begin{tabular}{|c|c|c|}
\hline 3erman & $\begin{array}{l}1971 R \\
1971 R\end{array}$ & 2184aB \\
\hline $3 \leftarrow 5 * 8$ & $1971 R$ & \\
\hline $4 \pi 4+5$ & $1971 \mathrm{R}$ & \\
\hline $4 * 6 * 16.5$ & $1971 \mathrm{R}$ & \\
\hline $5 * 7 * 17$ & $1971 k$ & \\
\hline 30 cAllOM & 1971 R & \\
\hline umaran & $1971 \mathrm{R}$ & \\
\hline $4-5 * 6$ & $1971 \mathrm{R}$ & \\
\hline Lumour & $1972 \times$ & \\
\hline $169 \mathrm{Cr} F$ & $1972 R$ & \\
\hline $197 \mathrm{dr}$ & $1972 R$ & \\
\hline $252 \mathrm{CJ}$ & 1972 R & \\
\hline 269 al. FT. & $1972 R$ & \\
\hline $4-5.5 * 16$ & $1972 R$ & \\
\hline $4.5 * 6 * 10$ & $1972 R$ & \\
\hline 110 enLon & $1972 R$ & \\
\hline $3.7-6.5=13.2$ & $1975 R$ & \\
\hline $4 \div 4 \div 7$ & 1978 R & \\
\hline \multirow[t]{3}{*}{$4 * 6 * 15$} & 1970 R & \\
\hline & & 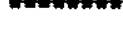 \\
\hline & & $\sin$ \\
\hline Unown & $1985 \&$ & 21Banc \\
\hline $4 * 4 * 7$ & $1985 \mathrm{R}$ & \\
\hline Lnicion & $1985 \mathrm{R}$ & \\
\hline 4"4*7 & $1985 \mathrm{R}$ & \\
\hline Langen & $1985 R$ & \\
\hline 30 CALLON & $1982 \times$ & \\
\hline $4 * 47$ & $1995 R$ & \\
\hline $6 * 6 * 7$ & $1985 R$ & \\
\hline 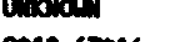 & 1985 R & \\
\hline 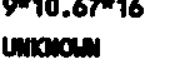 & $\begin{array}{l}1900 R \\
1905 R\end{array}$ & \\
\hline
\end{tabular}


TRU MAste

Sorted by Storage facility and Physical Description

Physical Description

METAL/IROW/GALVANIZED/SHEET

METAL/IROW/GALVANIZED/SWEET

METAL/IRON/GALVAHIZED/SWEET

METAL/IROM/GALVAMIZED/SHEET

METAL/IROW/GALVAMIZED/SHEET

METAL/IROM/GALVAMIZED/SHEET

METAL/IROW/GALVANIZED/SHEET

METAL/IROW/CALVANIZED/SUEET

METAL/JROW/GALVALIZED/SUEE

METML/1ROW/GULVNIZED/SWEET

METAL/TROW/CULVAMIZED/EWEET

METAL/IRON/CALVAMIZED/SWEET

METAL/IROU/GULVAIZED/S:FET

METAL/IROW/CMVVUIZED/SWEET

METAL/IROW/CALVAWIZED/SWEET

DP METAL/IROM/EALVAMIZED/SHEET

\& PAPER/CABDeOAvD

PAPER/CADDEOAMO

PAPER/Carobeando

PAPER/CALOEOARO

PLASTIC/PCR VuRATMU:

PLASTIC/POL VuRATMa:

PLAstic/pocromatume

PLASTIC/PoL YuRatuaue

PLASTIC/POL YUDATHANE

PLAstic/por rotarme

PLAstic/polnuatmaie

PLATIC/PoLnuatmave

PLASTIC/PoLVuRatma:

PLASTIC/POL VuRATHane

PLASTIC/POL VuraTunE

PLASTIC/POL VuRatMaNE

PLEXICLASS

PLEXIGLASS

RLBBER

RUBBER

sum

SILICA SEL

SILICA SEL
Conteiner Discription

Wast Storage

Container Size Date Irpe Facility count

Metal Dauns, eabaels, KeES

Metal deanes, enceets, KEES

fIeERGLASS REIMFORCED PLnDOD (FRP) BOXES

FIBERGLASS REIWFOCEED PLnW00 (FRP) BOXES

FIDERGLASS REIMFORCED PLYNOD (FRP) BOXES

metal Danis, manets, xEos

FJbergLASS REIUFORCED PLYNDOD (FEP) SOXES

meTAL dauns, chaneLs, KECS

METAL Daus, makets, KeES

METAL Daves, chareas, KeEs

metal cones, chrtows, cheses

METAL Buns, EAnazLs, KEOS

MISCELLLMEous sone

metal cones, chrtous, cases

metal Doxes, cantons, cases

METAL bones, cantous, cases

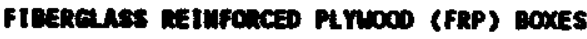

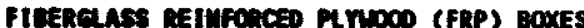

metal nowes, cantons, cases

metal Doures, cantons, cases

METML Danos, anacels, KEeS

Metal Dans, anceets, KEES

fIDERGLSS REIHFonced PLN000 (FRP) BOXES

FIBERGLASS REIMFOnCED PL nDOO (FRP) DOXES

FIEERelass REIMFonced PLnDod (FRP) BOXEs

FIBerelass REIUFOACED PLnNoD (FRP) bOXES

metal dans, mutels, keEs

metal Dalus, Bunels, kecs

MISCELLANEOUs SCRAP

METAL BonkEs, cakrows, cases

METAL Dowes, cartons, cases

METAL bones, cartons, cases

metal soxes, cantons, cases

METAL Bowes, Cantous, cAsEs

METAL Boutes, CARTOUS, CASES

metal bouces, cartons, cases

metal boxes, caktous, cases

METAL DhLEs, BarReLs, KEES

metal douns, barrels, Kegs

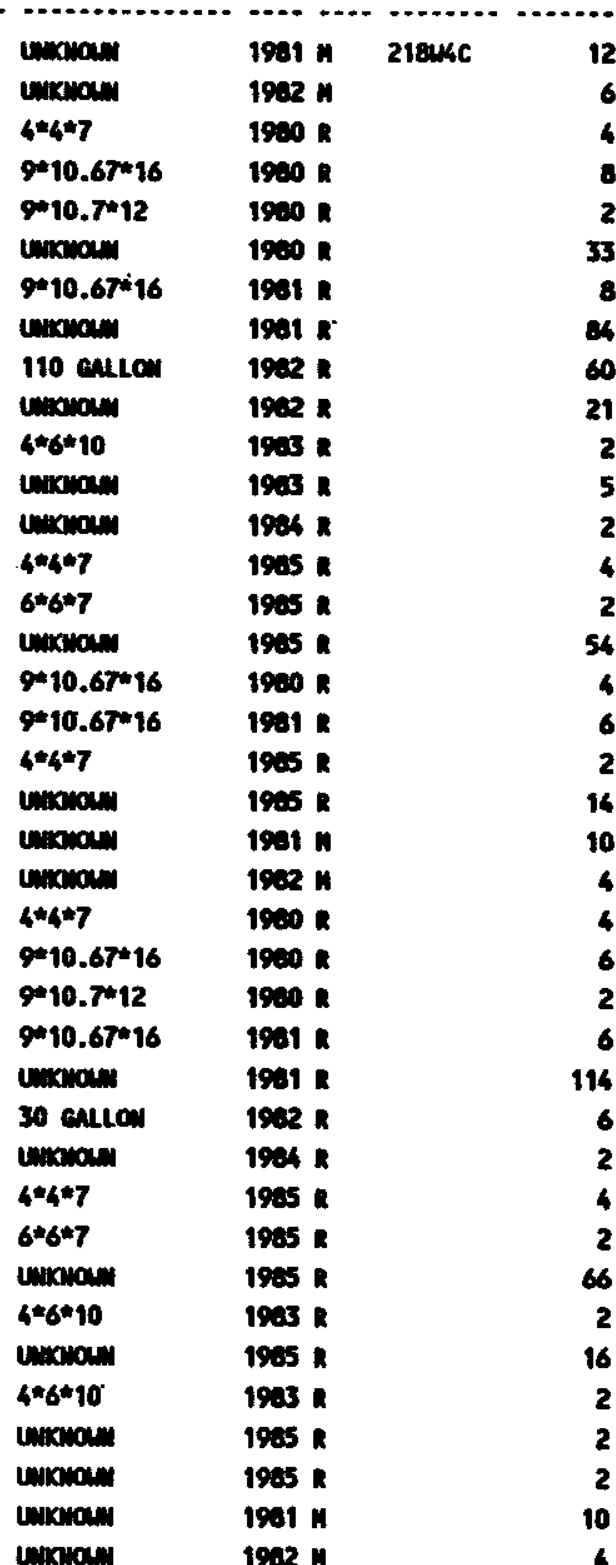


Pri

Physical Description

Conteiner Discription

Hast Storage

STAIMLESS STEEL

STAIMLESS STEEL

TEFLOW

DOOD/LURER/PLYMCOO

YOCD/LINEER/PLY YNOO

1000/LUrEeR/PLYNCOD

1000/LLIEER/PL YWOOD

CLOTH/RAGS/WYLON

CLOTH/RAGS/UMLLON

CONCRETE

GLASS

GLASS

GLASS

gLASS

L

LEAD

LEAD

METAL/IROW/GALVAMIZED/SHEET

METAL/IROW/GRYVNIZED/SHEET

METAL/IROM/GULVAMIZED/SHEET

ETAL/IROU/EALVAMIZED/SHEET

OILS

PAPER/CARDEOAPO

PLASTIC/POC TERATHANE

PLASTIC/POC NRATHANE

PLASTIC/POL TRaTmave

PLASTIC/POC VURATHAEE

1000/LURBER/PLYNOOD

$1000 /$ LUVEeR/PL nDoD

WOOD/LUEER/PLYNDOD

CLOTH/RAGS/MTLO

OIRT/SOIL/DIATOUAEOUS EARTH

LEAD
METAL BOCES, cartous, CASES

METAL BOXES, CARTOHS, CASES

metal DanNS, Barrets, KEgS

FIBERGLASS REINFCRCED PLYNOCD (FRP) BOXES

FIBERGLASS REIWFORCED PLYNOCD (FRP) BOXES

FIBERGLASS REINFCRCED PL YLDCD (FRP) BOXES

METAL BOKES, CARTOWS, CASES

metal boXes, cartons, cases

vetal bones, cartons, cases

metal boxes, cartons, cases

METAL BOWES, CARTOUS, CASES

METAL Eowes, cartous, cases

metal boutes, cantous, cases

MeTAL cones, CARTONS, CASES

meTAL EOEES, CARTOUS, CASES

METAL menes, CARTOUS, CASES

METAL DONES, CARTONS, CASES

METAL woutes, cartons, cases

METAL COUES, CARTOWS, CASES

metal benes, cartons, cases

MetAl cones, cartons, cases

METAL Bones, cartous, cases

metal Bours, cartous, cases

wetal Eones, cartows, cases

mia mones, cantous, cases

METAL EOUES, cartous, cases

netal boues, cartoms, cases

MeTAL coukes, cartons, cases

METAL Dowes, cartons, cases

METAL BoXEs, CARTOUS, CASES

METAL BOWES, cartons, CASES

metal bones, cartons, cases
Conteiner Size Date Iype Facility count

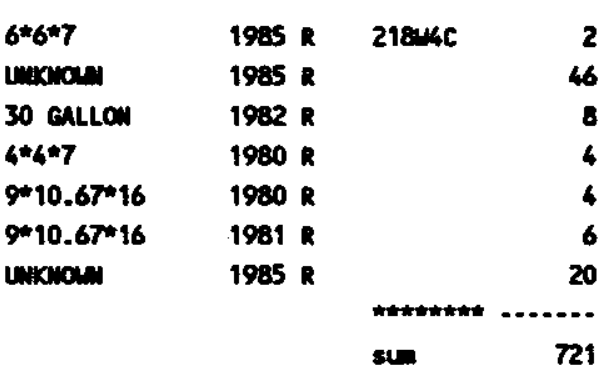

$4.5 * 4.5 * 7.3 \quad 1909$ ip $24014 \quad 10$

$6 * 6 * 7$

$5.6 * 6.5 * 9.3 \quad 1909 \mathrm{ip} \quad 5$

4*4*7 1989 m

$4.5 \star 4.5 * 7.3 \quad 1909 \approx 0$

$5.6 * 6.5 * 9.3 \quad 1909 \mathrm{mp} \quad 5$

$6 \$ 6 \div 7$

$4 * 4 * 7$

$4.5 * 4.5 * 7.3 \quad 1989$

$5.6 * 6.5 * 9.3 \quad 1989 \div$

4*4*7 1909

$4.5 * 4.5 * 7.3 \quad 1909$

$5.6 * 6.5 * 9.3 \quad 1909$ m

6*6"7 1909

$4.5 * 4.5 * 7.3 \quad 1989$ \%

$4.5 * 4.5 * 7.3 \quad 1989 \mathrm{mp}$

4*4*7 1909 ip

4.5*4.5*7.3 1909 ip

$5.6 \times 6.5 \times 9.3 \quad 1909$ mp

$6 * 6 * 7 \quad 1909$ im

$4.5 \star 4.5 * 7.3 \quad 1909$

$5.6 * 6.5 \times 9.3 \quad 1909$ in

$6 * 6 \star 7 \quad 1909$

sin

245

6*6*7

1909 n 2402

1909 n

1989 n

$\begin{array}{lll}149 \text { C FT } & 1909 \text { H } & 5 \\ 149 \text { C FT } & 1909 N & 5\end{array}$


TRE WASTE

Sorted by Storage Facility and Physical Deacription

prí

Wast storege

Physical Description

Container Diseription

Container size Date Type Facility count

METAL/IRON/GALVNIZED/SHEET HETAL BOXES, CARTOMS, CASES

metal BOUES, CARTOUS, cases

METAL BOXES, CARTOUS, CASES

METAL/IFOO/GALYAIIZED/SHEET

mETAL BOXES, CARTOMS, CASES

PLASTIC/POLYRRATHAME

METAL BOXES, CARTOUS, CASES

METAL BCOXES, CARTOWS, CASES

$1000 / L$ UBBER/PL YNOOD

METAL BdXEs, cartows, cases

Coneainer size Date Type Facklity count

149 al FT 1989 M $2402 \mathrm{~W} \quad 5$

$6 * 6 * 7$

149 ad FT

149 CU FT

1999

$1909 \mathrm{n}$

149 ad FT

$1989 \mathrm{n}$

1909 *

OOO/LUMER/PL YIDCO

METAL BOXES, CARTOUS, CASES

CLOTH/RAGS/RTLO

METAL BOXKE, CARTOUS, CASES

METAL/IROM/GALVANIZED/SMEET

metal bOXEs, CARTOLS, CASES

eER/CADOPONDO

PLASTIC/POL MURATHAME METAL BOXES, CARTOMS, CASES
RUBBER
METAL BOXES, cartons, cases

COMNEB PADS

METAL DRUNS, BarRELS, KEGS

DIRT/SOIL/OIATOUACEOUS EARTH METAL DRUSS, BARRELS, KEGS

ORGANICS

meTAL Denuns, gurpels, KECS

PLASTIC/POLYRATHAME

METAL DRUnes, Barretels, KECS

WOOD/LUABER/PLYNDO

METAL DRUNS, BARRELS, KECS

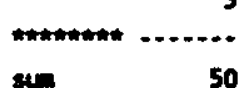

$4 * 4 * 7$

$4 * 4 * 7$

$4 * 4 * 7$

$4 * 4 * 7$

$4 * 4 * 7$

$\begin{array}{llr}1989 \text { n } & 240218 & 5 \\ 1909 \text { n } & & 5 \\ 1909 \text { n } & & 5 \\ 1909 \text { n } & & 5 \\ 1989 \text { n } & & 5 \\ & \\ & \text { sin } & 25\end{array}$

85 GAlLON

1989 N FS8

85 callon

85 Gallon

85 callow

1989 n

$1909 n$

1909 n

85 EALLON

1989 n

FS8

5

5

5

su 25

122 rows selected. 
WHC-EP-0621

APPENDIX B.11

\section{HAZARDOUS CONSTITUENTS LISTED FOR 55-GALLON} DRUMS CONTAINING TRU WASTE 
WHC-EP-0621

This page intentionally left blank. 


\section{HAZARDOUS CONSTITUENTS LISTED FOR 55-GALLON DRUMS CONTAINING TRU WASTE}

The presence of hazardous constituents in a radioactive waste drum designates the waste as "Mixed" (primary waste code " $M$ "). In this subappendix the hazardous contents of 55-gal drums generated at the Plutonium Finishing Plant (PFP) are sorted by storage facility.

Information concerning the hazardous constituents of waste containers was not required before 1986. During the Richl and Solid Waste Information Management System (R-SWIMS) data re-entry program in the mid-1980's an attempt was made to add any avallable information on the hazardous materials present, however, this informatton was limited. 


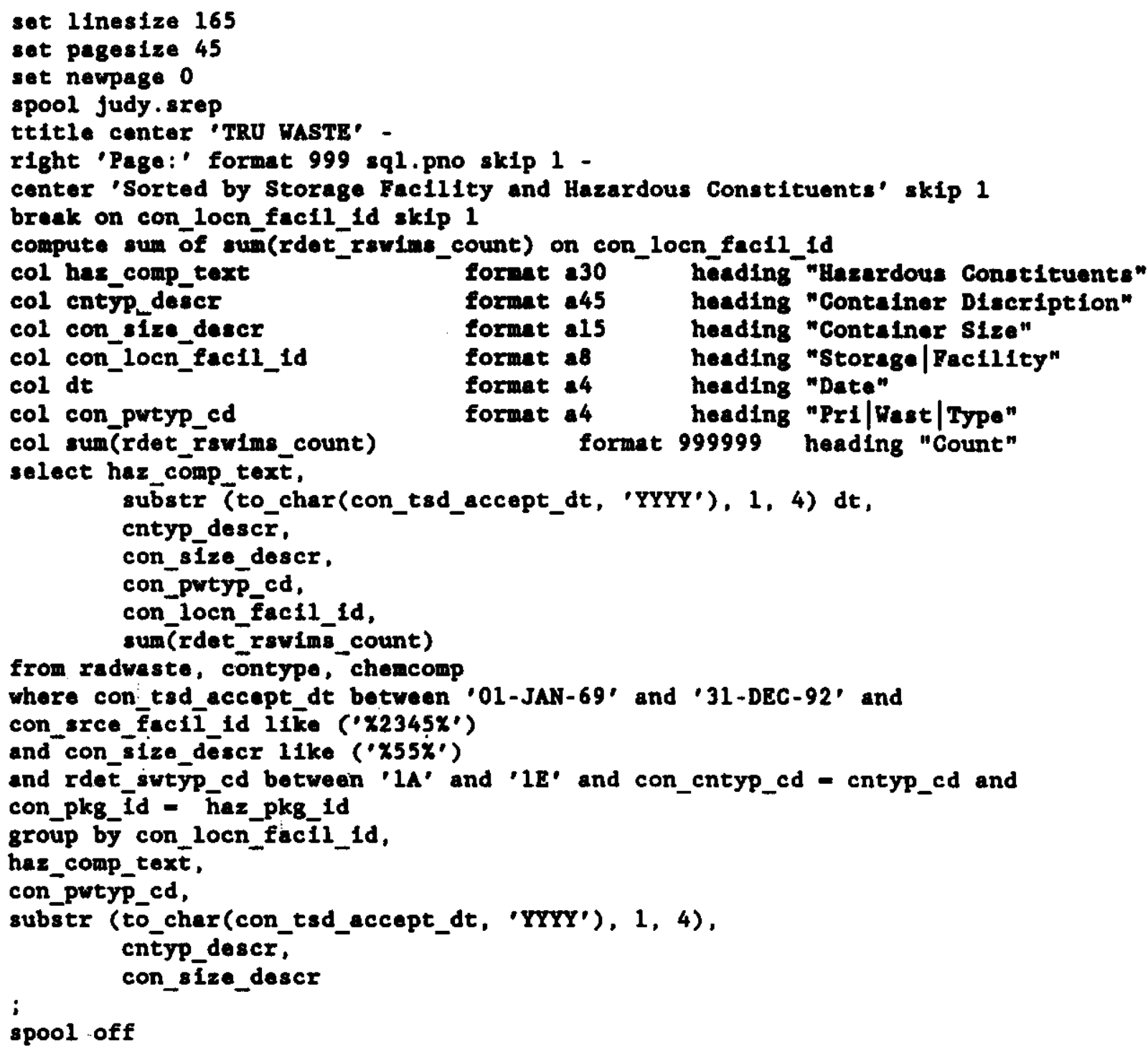


Sorted by Storege Facility and Hezardous Constituents

Pri

Wast Storage

tazardous Constituents

Date Container Discription

Container size Type Facility count

ISBESToS 1984 METAL DRUNS, BarReLS, KEGS

1985 metal Douns, marRels, KECS

1986 METAL DRUMS, BarRELS, KEES

1987 mETAL DRUnS, BARRELS, KEGS

1983 METAL ORAHS, BARRELS, KEGS

19 Q

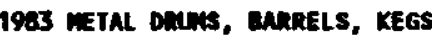

1985 METAL DEUMS, MARRELS, KEGS

1942 meTAL Dolns, BarreLs, KEGS

1985 metal ontris, mRRELS, XEGS

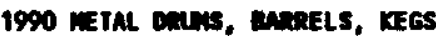

55 culon $21846 \mathrm{C}$

55 cullow

55 GALLOW

55 callow

55 callow

55 Gatton

55 callow

55 GALLOW

55 GALLOW

ReAnIC

55 culom

$\begin{array}{rlr}R & \text { 218W4C } & 16 \\ R & & 10 \\ R & & 5 \\ R & & 1 \\ M & & 4 \\ M & & 3 \\ M & & 12 \\ M & & 9 \\ M & & 1 \\ M & & 4 \\ & & 63\end{array}$

ICID

55 catlon

55 callow

55 Gillom

ISBestos

1909 METAL DOMEs, maneLL, KEGS

1990 mETh owns, munets, KEGS

1991 wETR omne, BambeLS, KEES

1992 METAL omms, manels, KEeS

1990 metal buins, manats, KeES

1991 IETAL Dams, mutels, KEGS

1992 vetAL Deums, ancets, KEGS

55 entom

55 enlom

55 callom

55 callom

55 cullow

zanilts

1990 METAL Deums, MAREELS, KECS

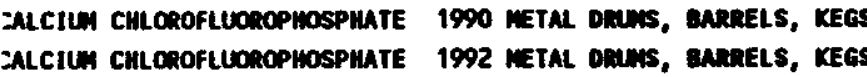

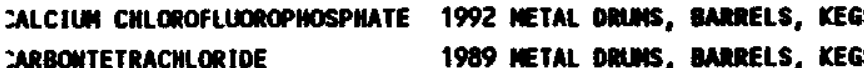

1991 METAL. Dans, whaets, KECS

1909 retal beuns; anaels, KEgS

1990 retal dans, anects, KEES

1991 MeTAL ouns, manels, KECS

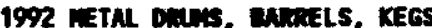

1990 METAL omns, Bhenels, KEGS

1991 meTht Dalns, wherels, KEGS

1990 meTAL dunns, minkELS, KEGs

1991 meTAL daums, warkeLS, KEGS

1992 METAL Douns, BarRELS, KECS

1992 METAl oxus, BanREtS, KEgS

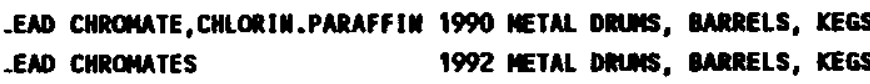

ERCURY

1990 metal deUMS, BarreLs, KEES

55 callom

55 Gallom

55 Gallow

55 GALLO

55 callow

55 GALLO

55 Gallow

55 Gallon

55 culton

55 Gallon

55 Gallow

55 Gution

55 callon

55 callow

55 callow

55 callow

55 exlon

55 callom 
TRU UASTE

Sorted by Storage Facility and Hazardous Corstituents

Pri

Uast Storage

Hazardous Constituents

MERCRY

nercury

POTASSIUN IMDROXIDE

POTASSIUN MTDROXIDE

SELENIUN

SULFURIC ACID

SULFURIC ACID

TRI GUTYL PHOSPHATE

Hropalitic FUID

HYDRALLIC FLUID

DP HORAMLIC FLUID

○ LEAD

PCB

PCB

PCB

PCS

PCE

PCB

POTASSILA HYROOXIDE

SCOIUN moroxide
Date Container Discription

1991 MTTAL DAUS, BABRELS, KEGS

1992 WETAL Duns, minels, keEs

1991 METAL DAuns, ButeELS, KEES

1992 METAl Dans, ankels, KEes

1991 metal dewns, Barrels, KEES

1990 metal Dants, BarreLs, KEES

1991 metAL Dauns, BarReLs, KECS

1909 METAL Dauns, wakels, KEES

1991 retal DAuns, BurReLs, KEES

1909 METAL DAUNS, BarkELS, KEGS 1908 weTAL Deans, Barkels, KEES 1909 metal beuns, BanRELs, KEES 1990 mETML DRUns, BakReLs, KECS 1989 metMl Dalns, anReELS, KECS 1998 METAL DRUMS, CaRkeLS, KECS 1982 metA DAus, Barkets, KECS 1983 METAL DAMS, BAnELLS, KEES 1905 METM Dans, Bariels, KEes 1980 METAL DRLHS, Barkels, KEES 1909 metAL DRurs, Barrels, KECS 1990 METAL DRUNS, Barkels, KEGS

1989 METAL DRLMS, BARRELS, KECS 1909 METAL DRUIS, Barkels, KEES container size Type Facility count

5e

55 callow

55 EALIO

55 exllow

55 GULLO

55 enlon

55 cullow

55 cullow

55 culcon

55 callow

55 calloy

55 cullon.

55 callom

55 callom

55 CALLO

55 callon

55 callom

55 callow

55 culcon

55 cullon

55 callow

$\begin{array}{llr}M & 2241 & 11 \\ M & & 4 \\ M & & 2 \\ M & & 4 \\ M & & 1 \\ M & & 1 \\ M & & 9 \\ & & 1 \\ & & 9\end{array}$

H $24014 \quad 1$

10

$\operatorname{mop}$

M

$\operatorname{mos}$

m

m

$m$

ip

sum

55 EALLO

55 cullon

$\begin{array}{lr}240218 & 1 \\ & 1 \\ \text { sum } & 2\end{array}$

59 rows selected. 
WHC-EP-0621

APPENDIX B.12

HAZARDOUS CONSTITUENTS LISTED FOR TRU WASTE CONTAINERS OTHER THAN 55-GALLON DRUMS 
WHC-EP-0621

This page intentionally left blank. 


\section{HAZARDOUS CONSTITUENTS LISTED FOR TRU WASTE CONTAINERS OTHER THAN 55-GALLON DRUMS}

In this subappendix, the hazardous constituents of transuranic (TRU) waste containers other than 55-gal drums are relisted by storage facility. Because the presence of hazardous materials in a radioactive waste drums designates the waste as "Mixed," most of the containers have a primary wake code of "M" for mixed waste or "MP" for mixed waste containing polychlorinated biphenyls (PCBs).

Information concerning the hazardous constituents of waste containers was not required before 1986. During the Richland Solid Waste Information Management System (R-SWIMS) data re-entry program in the mid-1980's, an attempt was made to add all available information on the hazardous materials present in TRU waste containers. This information is limited. 


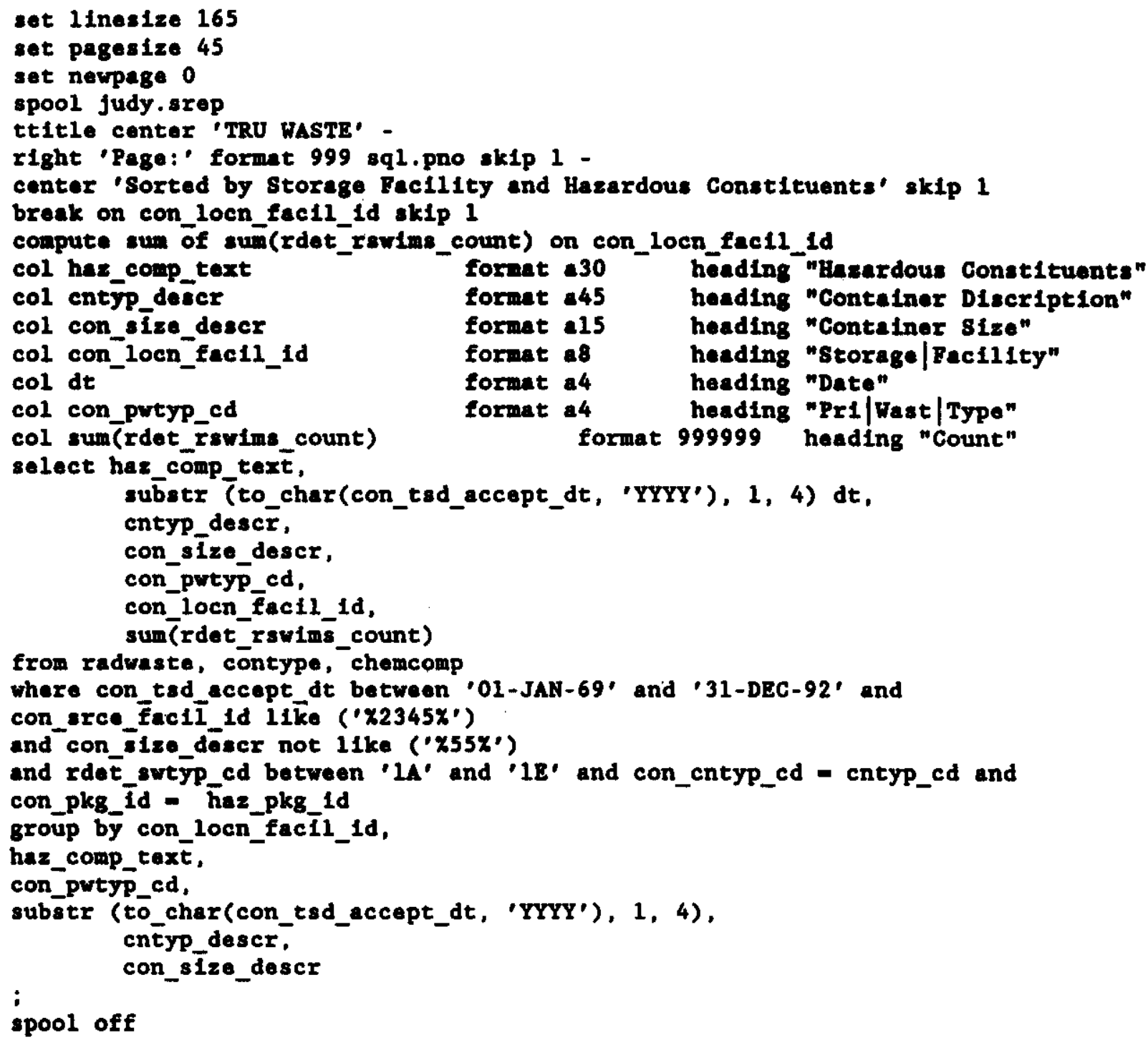


fazardous Constituents

Date Container Discription

Container size Type facility count

....

1975 metAL boxes, caRtons, cases

ISBESTOS

3ERYLLIU:

3ERYLLIUN

1985 METAL BOXES, CARTONS, CASES

1981 metAL DRLNS, BARRELS, KEES

1982 metAL DRLAS, BARRELS, KEGS

1909 RETAL BOXKE, CARTOUS, CASES 1969 METAL BOXES, CARTOUS, CASES 1909 metal boxes, cartous, cases 1909 METAL BOXES, CARTOUS, CASES 1909 mETA BOXES, CARTOWS, CASES 1909 METAL BOXES, CARTOWS, CASES 1989 METAL BOXES, CARTOUS, CASES 1989 METAL BOXES, CARTOUS, CASES

apmiu

OLCIUN CHLOROFLUCROPHOSPHATE 1909 METAL BOOES, CNRTOHS, CASES zaLCIUN CHLOROFLUOROPHOSPHATE 1909 RETAL EOKES, CARTOUS, CASES - ALCIU, CHLCROFLUOROPHOSPHATE 19\%9 METAL DOXES, CANTOUS, CASES ZALCIUN CHLCBOFLUOROPHOSPHATE 1909 METAL BOKES, CARTOUS, CASES

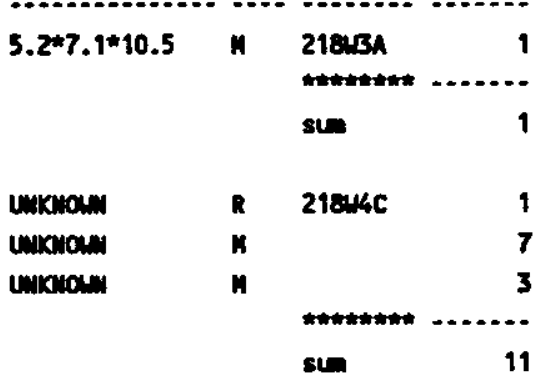

$4 * 4 * 7$

$4.5 * 4.5 * 7.3$

$5.6 * 6.5 * 9.3$

6*6*7

$4 \times 4 \div 7$

$4.5 * 4.5 * 7.3$

$5.6 * 6.5 * 9.3$

$6 \star 6 * 7$

$4 * 4 * 7$

$6.5 * 4.5 * 7.3$

$5.6 * 6.5 * 9.3$

$6 * 6 * 7$

$4 * 4 * 7$

$4.5 * 4.5 * 7.3$

$5.6 * 6.5 * 9.3$

4*4*7

$4.5 * 4.5 * 7.3$

$5.6 * 6.5 * 9.3$

$6 * 6 * 7$

$4 * 4 * 7$

$4.5 * 4.5 * 7.3$

$5.6 * 6.5 * 9.3$

$6 * 6 * 7$

$2601 \mathrm{~W} \quad 1$

in 5

im

im

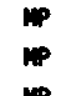

ip

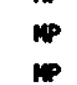

in

is

in 4

m

$\boldsymbol{m p}$

in

in

in

in

im

in

in

$m$

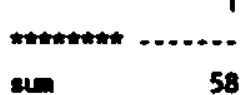

58

$6 * 6 * 7$

24024

$6 * 6 * 7$

$6 * 6 * 7$ EAB

EAD

EAD

GERCURY

ERCARY

IERCURY

IERCuRY

'CB

$\cos$

$\mathrm{CB}$

ca:

1909 METAL BOXES, CARTOWS, CASES

1989 METAL BOKES, CARTOUS, CASES 1909 netal boxes, cartous, cases 1909 METAL Boxes, cartous, CASES 1909 metAl bouces, cartons, cases 1989 RETAL BOKES, CARTOUS, CASES 1989 METAL BOXES, CARTOUS, CASES 1989 METAL BOXES, CARTONS, CASES 1909 METAL BOAES, CARTONs, CASES 1909 IETAL Boxes, cartons, cases 1909 mETAL boxes, cartows, cases 
THU UASTE

Sorted by Storage Facility and Hazardous Constituents

Pr

Mast Storage

Hozardous Const ituents

Date Container Discription Container size Type facility count

LEAD 1909 math BOKES, CARTOWS, CASES

MERCURY

1909 METAL BOXES, CARTOUS, CASES

1999 metAl bouces, cantous, cases

2-BUTOXYETHANOH

PHospinoestc ACID

TRIBUTYLLPMOSPHATE

TRIMETHYLBEMZEWE

XYLENE

0
1
0
0
1909 RETAL DANHS, BARRELS, KEES

1909 IETAL Danns, BARRELS, KEGS

1909 metrl Douns, ankels, KEGS

1909 meTAL DAMHS, aARkELS, KEOS
149 ad FT

$6 * 6 * 7$

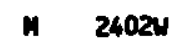

sin

$4 * 4 * 7$

4थ4

H 24021:

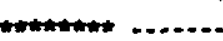

$\sin$

85 callon

85 cullo

85 callon

o5 callo

$\begin{array}{llr}n & \text { Fs8 } & 1 \\ n & & 1 \\ n & & 1 \\ & & 1 \\ & \sin & 4\end{array}$

38 rows selected. 
WHC-EP-0621

APPENDIX B.13

ISOTOPES IN 55-GALLON DRUMS THAT CONTAIN TRU WASTES 
WHC-EP-0621

This page intentionally left blank. 


\section{ISOTOPES IN 55-GALLON DRUMS \\ CONTAIN TRU WASTES}

This subappendix contains a listing of the Solid Waste Information Management System's (SWITS's) isotope information for transuranic (TRU) waste from the Plutonium Finishing Plant (PFP) stored in 55-gal drums. The isotope field in SWITS contains the following types of information:

- Specific isotope (e.g., ${ }^{241} \mathrm{Am},{ }^{237} \mathrm{~Np},{ }^{233} \mathrm{U}$, or ${ }^{238} \mathrm{Pu}$ )

- Generic isotope listings (e.g., plutonium, uranium-enriched, or uranium-depleted)

- Plutonium-239 fissile gram equivalents (Pu239 FSL GR equiv)

- Total alpha

- Total beta/gamma.

A SWITS record for a single container may list one or all of the above in the isotope field.

This computer run contains the isotope field listing along with the number of TRU waste drums with that listing. The run is sorted by storage facility and year. 


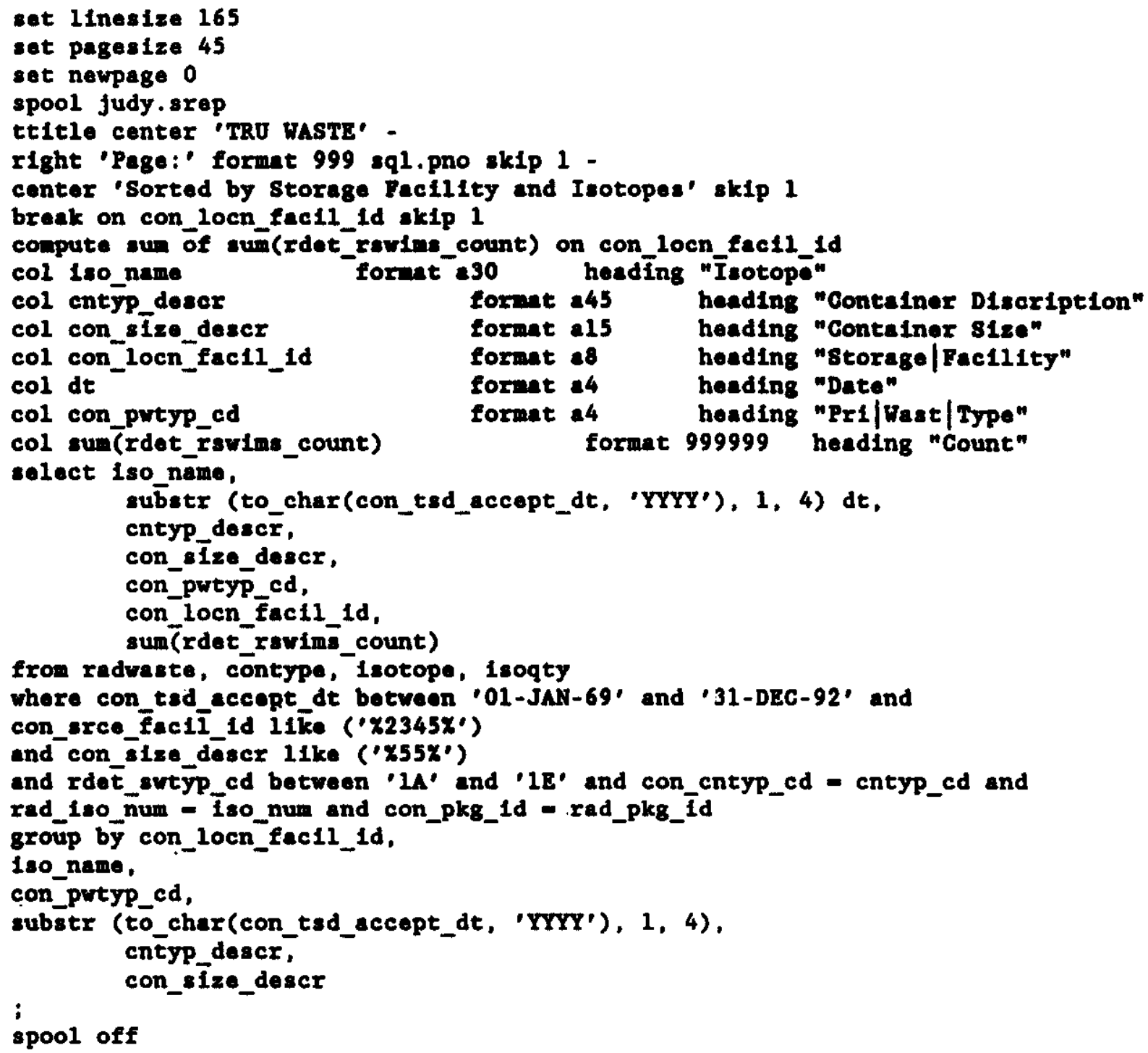


Sorted by storese Fecility and isotopes

Pri

Vest Storage

Isotepe

Date Contaliner Discription

Containar size Type facility count

1970 mital Dauns, arerets, KEES

1971 viTh oums, wherELs, KECS

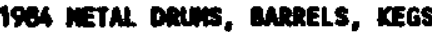

1970 METAL Dans, BurRets, KEGS

1971 METML bans, manels, KEeS

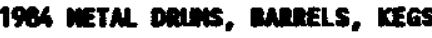

TOTAL BETAVANM

TOTAL BeTNGMma

VRANIUN-ELERICWEO

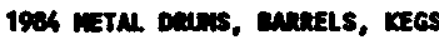

1972 metal Dadis, Banels, KEgS

1972 METAL onus, monels, KEGS

1971 MeTAL deums, manels, KEes

1972 mithl buns, mbacts, KeES

1973 METML Dans, munels, mes

1974 METM Dans, andiels, KECS

1975 KETML Dans, watels, KEGS

1976 MTTM bans, munezls, kECS

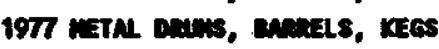

1971 METML Danis, maxts, Keos

1972 reTM druns, macels, KEGS

1973 METML ouns, manels, KEGS

1974 METM owns, Mnwets, KEGS

1975 METAL ounus, ankels, XEES

1976 METAL odAns, wirets, KEGS

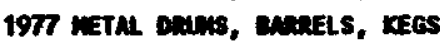

1972 metal ouns, chuets, kees

Containar size Type Facility Count

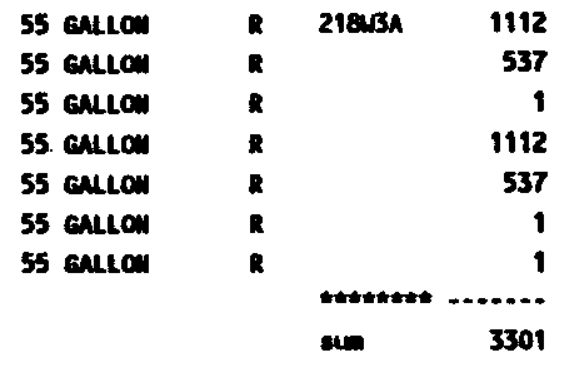

TOTAL BETA/GAYU

TOTAL BETMGGMU

TOTAL BETA/GNMA

J-233

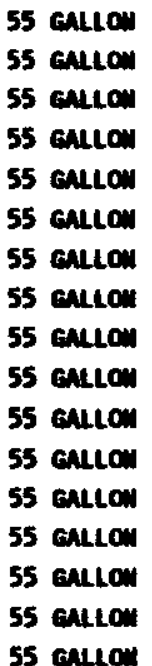

218ukB

7

69
806

306

1301

962

1222

1025

1199

806

1285

1264

909

708
1009

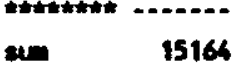

\section{MH-241 \\ MP- 237 \\ PE-CI \\ PE-CI \\ PE-CI \\ PE-CI \\ PE-CI \\ PU}

19as wetal Deuns, caneets, KEGS 19e4 METAL Dans, mapaets, KEGS 1926 METML Deuns, aneetets, KEGS 1907 metal deuns, canacts, Kegs

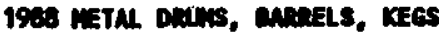
1990 metal Dans, Bunetel, KECS 1991 METML DEDrs, MankELS, KEGS 1962 metal dRens, manels, KECS 1903 mETAL DRtMS, Barrets, KEGS

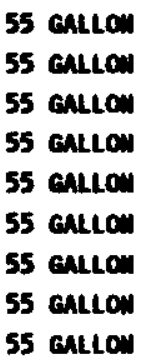

55 eallon

Gallow

35 GAlLO

5 culan

55 Gallon

$\begin{array}{rr}21844 c & 1 \\ 2 \\ 11 \\ 7 \\ 8 \\ 46 \\ 2 \\ 1 \\ 16\end{array}$


sorted by storage fecillity and lsotope:

Pri

Wast storage

Isotope

Date Container Diseription

Contoiner size Iype fecility count

PU 1994 merMl Dans, makls, KEes

Conteiner size Iype fecility count

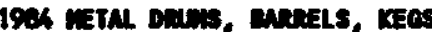

3

1905 metal beuns, aunets, Kecs

55 callow

55 callow

55 callow

1979 EETAL owns, andeELS, KECS

55 callow

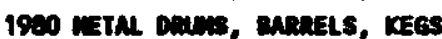

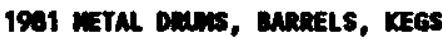

55 callon

55 callon

1902 metal dauns, menets, Kees

1903 METAL Danns, mareELis, KEGS

55 cullam

55 cAllom

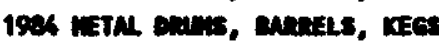

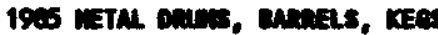

55 callom

55 callom

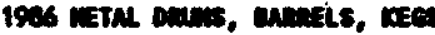

1907 isTAL Dums, whacts, Mess

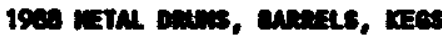

55 encen

55 callam

55 culcom

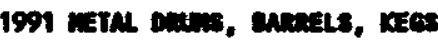

1900 ratul omens, enrats, ress

55 envia

55 enclow

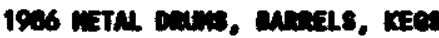

PU239 FSL ER EOUIY

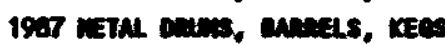

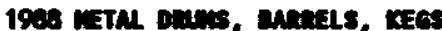

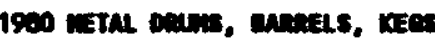

1902 METM Deuns, subects, KEes

1903 KETAL sums, shatels, KEes

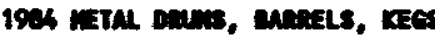

1905 metal oums, aneces, KEG

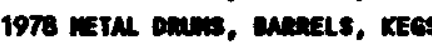

1979 rata onus, Mants, KECS

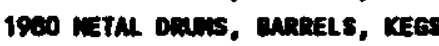

1901 matM Dann, makets, KEES

1902 mith Dans, marels, Kees

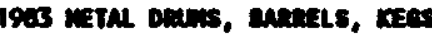

19at metal bans, waels, reas

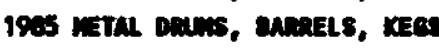

1906 mital dans, warists, rees

1997 metal dans, suncts, kegs

1908 meTM Dalms, sakikets, KEes

1990 metal Douns, anoels, KEos

1991 metAL DRuns, marRets, KEGS

1996 meral omms, manels, KEeS

1967 metal Dequs, anaels, KEes

1986 metal dranis, barrels, KEGS

55 cullow

55 culcon

55 culloy

55 caruen

55 callow

55 cullom

55 cellom

55 cullow

55 callom

55 exlow

55 cullow

55 callow

55 callom

55 culcom

55 callo:

55 callo:

55 exllow

55 callo

55 callow

55 eAllow

55 ealion

55 exlow

MPA

55 callow

55 callow

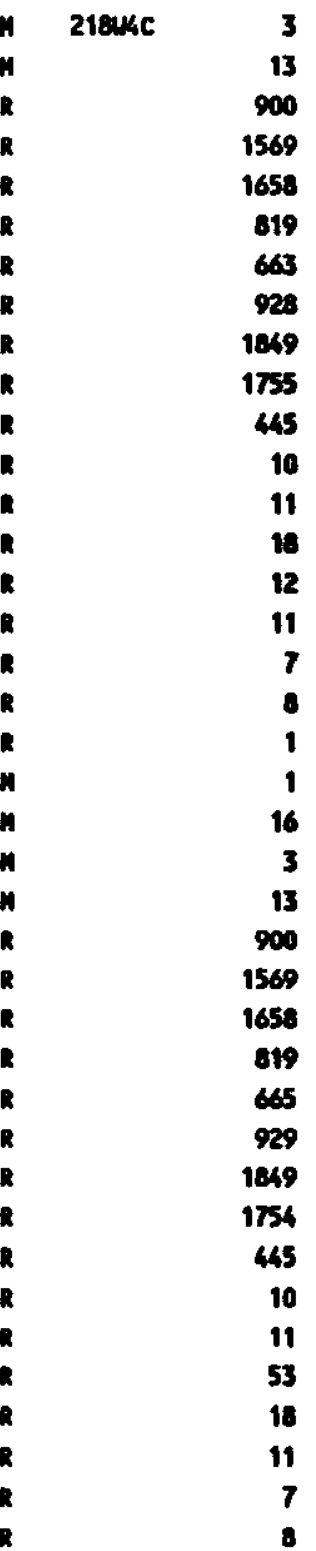


Sorted by Storage facility and Isotopes

Pri

Wast Storage

I sotope

TOTAL-ALPHA

TOTAL-ALPMA

LEANILN-DEPLETED

LANIUN-DEPLETED

URANILA-DEPLETED

UANIUN-EMRICHED

VRAMJUN-EURICMED

Lanilu-EMRicuso

URAIIU-EMRICHED

crantun-matuall

LeANIUN-MATUaAl

PE-CI

rotal esta/esmu

TOTAL-ALPMA

A4-261

서-241

PE-CI

PE-CI

PE-CI

PE-CI

PE-CI

PE-CI

गE-CI

PE-CI

2E-CI

x

w

xu

iv

iv

y

y
Date Conteiner Discription

Container size Type facility count

1990 matal dedns, anRRELS, KEES 1991 istal Douns, eanrels, KEGS 1979 mital daus, eanrels, regs 1900 ristal Dans, Bacrels, KEGS 1982 istal dauns, Burrels, KEgS 1900 ristal ouns, barrels, KEes 1922 matal Dodus, anroels, KEGS

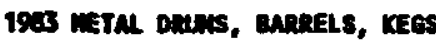
1904 MTTAL DAuns, BanRELS, KECS 1900 mital Danss, maprels, KEes 1922 mital, Danes, Bardels, KECS

1990 MSTAL Danis, anReELS, KECS 1990 inTML onus, BARRELs, KECS 1990 IFTM DRUAS, BARRELS, KEES

1990 metMl Dadns, BarRels, KEgS 1991 meTh Dans, BnRels, KEes 1909 MSTAL dedns, enreels, KEGS 1990 istal Dans, anRels, kECS $19 \%$ mital baus, Bandels, KECS

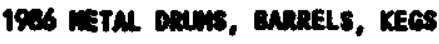
1907 mital duns, cannels, kees 1908 intal bunts, Bareets, rees 1909 rstal Daus, cararels, LEeS

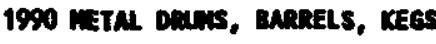
1991 mith odums, candels, yees

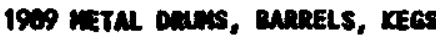
1990 mital nans, andrels, KEes 1991 metal nouss, manels, KECS 1992 mital onurs, andarels, KECS 1996 minal bouns, anRels, KEGS 1987 mital Dans, anrRELs, KEOS 1988 mstal Dauns, Barrets, KEGS 1989 ustal dans, BarRELS, KECS

\begin{tabular}{|c|c|c|c|}
\hline 55 CALLOM & R & $218 u 4 c$ & 46 \\
\hline 55 cullou & R & & 2 \\
\hline 55 culan & $R$ & & 10 \\
\hline 55 Gallon & R & & 9 \\
\hline 55 GALLO & R & & 13 \\
\hline 55 calloy & R & & 2 \\
\hline 55 Galloy & $R$ & & 2 \\
\hline 55 entzon & $\mathbf{R}$ & & 1 \\
\hline 55 eAllon & R & & 1 \\
\hline 55 GALLON & $R$ & & 26 \\
\hline 55 culon & $\mathbf{R}$ & & 1 \\
\hline
\end{tabular}

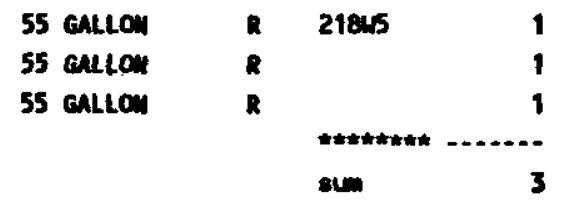

55 allon

55 aulon

55 callon

55 cullon

55 cultom

55 cullom

55 ealloy

55 callou

55 cullon

55 cullom

55 cultom

55 anlom

55 calloy

55 callon

SS enLloy

55 callow

55 callon

55 GAllow

55 callon
1

10

16

50

260

98

42

57

70

34

20

18 
TRU MASTE

Sorted by storage Facillity and Isotopes

Pri

Wast Storage

Isotope

Date Conteiner Discription

Contoiner size type facility count

PU

1990 METAL DOME, BAREELS, KFES

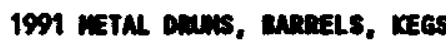

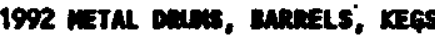

1991 istal Dans, Mrenets, KEes

1991 meTal ouns, manets, Kees

991 reTal dans, monels, KEos

1991 METM Dans, Muatels, KEos

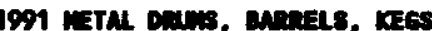

1909 METM deuns, minets, Keas

1990 Methl deins, mandets. KEes

1991 MeTAL ouns, Bublets, KECS

1906 metal Days, manels, KECS

1987 metal Dans, Mratels, KEES

1988 METAL Dedus, Bariels, KEGS

1989 metal Deans, BabneLs, KEGS

1990 meTAL Deuns, BABaELS, KEGS

1991 METAL DAums, BARRELS, KEGS

1989 METML DEMS, MARELS, KEES

1990 metal oums, Bunels, KEES

1991 meTAL Danes, BAPeELS, KEES

1992 METAL Danes, ManELS, KEES

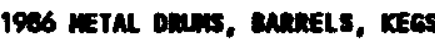

1907 METML Dans, chenels, KEOS

1908 METAL Deuns, Barrels, KEGS

1909 veTAL Dums, maneLS, KECS

1990 METAL DEm, manels, KEGS

1991 veTaL deums, masels, KEGS

1992 METML muns, Manels, KECS

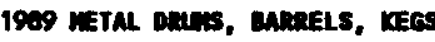

1990 METAL Danis, matrels, KEES

1991 METAL Daus, MarRELS, KEgS

1906 METAL Danss, MRRELL, KEGS

1907 METM Dants, BARRELS, KEGS

1908 META Dauns, Barrels, KEES

1909 METAL DRus, anRRELS, KECS

1990 METAL Dauns, Barrels, KECS

55 enlom 2261

55 eulow

55 Gulaw

55 culcu

55 eallon

55 culam

55 culam

55 anlow

55 callom

55 callom

55 callo"

55 allom

55 Gallow

55 callow

55 Gallon

55 callow

55 callow

55 Gallow

55 callon

55. callon

55 callom

55 callom

55 allom

55 exllom

55 cullom

55 callom

55 exlom

55 callow

55 Gallon

55 calior

55 cAllon

55 anllow

55 GALLOM

55 callow

55 callon

55 Gallom

TOTAL-ALPHA

1991 meTAL ORUAS, anReELS, KEGS

55 GALLOM

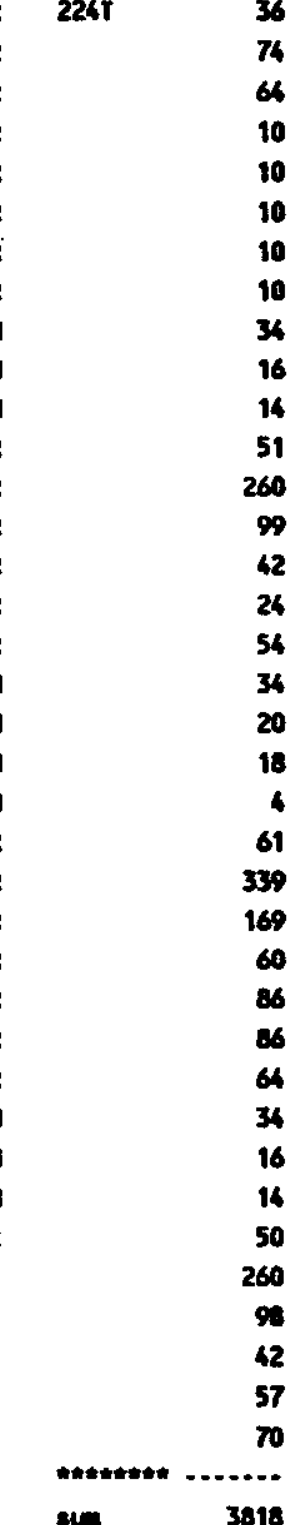




\section{Isotope}

Date Conteiner Discription

PE-CI

PE-CI

PE-CI

PE-CI

PE-CI

PU

PU

PU

PU

Pu

PU

PU239 fSt GR EOUIV PU239 FSL GR ECUIV PU239 FSL GR EQUIV PU239 FSL GR EOUIV 39 FSL GR ECUIV

TOTAL BETA/GNANA

TOTAL BETA/GNWA

TOTAL BETA/GANA

TOTAL BETA/GANU

TOTAL BETA/CAMA

TOTAL BETA/GNWA

TOTAL BETA/GUmU

TOTAL-ALPMA

TOTAL-ALPMA

TOTAL-ALPHA

TOTAL-ALPHA

TOTAL-ALPHA

\section{PE-CI}

PU

PU239 FSL GR EOUIV TOTAL BETA/GNWA TOTAL-ALPIA
1909 Metal doums, anerels, Kegs 1955 Metal deans, Baperels, KEes $19 a 8$ Metal deans, Barrets, KEtS 1909 METAL Dauts, ankeleL, KEES 1990 METAL DEMAs, anRRELS, KEES 1909 metal Deans, ankrels, KEeS 1902 metal obuns, sarrels, KEES 1905 VETML DAUns, BARRELS, KECS 1900 WETML DRUMS, BuRRELS, KEGS 1909 METAL DRUAs, Barreis, KEGS 1990 meTAl DRLAS, BarreLS, KEGS 1909 METAL DRUMS, BarRELS, XEGS 1905 metal DRuns, Barkels, KEGS 1908 METAL DRAMS, BARRELS, KEGS 1999 METAL DRUnS, aARRELS, KEGS 1990 METAL DRUNS, aARRELS, KECS 1989 METAL DRAMS, BarRELS, KECS 1902 metal Dwnus, Banrels, KEgS 1903 METAL Dams, EarRets, KECS 1905 METAL DAMns, BarRels, KECS 1908 Metal Dauns, BanRELS, KECS 1909 metal deuns, carrets, KEGS 1990 METAL Deuns, earaels, KECS 1909 METAL Douns, BarRELS, KEOS $19 e 5$ mETAL DAuns, BarRels, KEes 1903 METAL DRAns, ankRELS, KEES 1909 Metal DRANS, marrels, KEGS 1990 metal deans, Barnels, KEgS

1989 metal DRAns, BARRELS, KEGS 1989 METAL Douns, carreLs, KEGS 1909 METML DRAnS, arRrels, KEGS 1909 mETML DAuns, BARRELS, KEGS 1909 meTM dauns, BARrELS, KEgS
Wast Storage Container size Type facility count

55 Gulou.

55 cullom

55 cullom

55 GALOW

55 cultom

55 callon

55 cillow

55 callo

55 callom

55 callon

55 callow

55 Gailow

55 callow

55 Gallow

55 callon

55 callow

55 GALLOM

55 callow

55 Gallow

55 culton

55 cullon

55 Gallon

55 culcon

55 Eulom

55 callom

55 GAllon

55 callo

55 calton

.......................

1 $24014 \quad 2$

iip 14

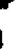

14

$\operatorname{mp}$

PP

P

2

TP

14

10

10

in 1

M

16

im 10

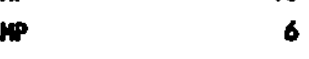

ט191

55 anlom

55 eullon

55 cullom

55 cullon

55 mellom

$\begin{array}{llr}n & 2402 M B & 1 \\ n & & 1 \\ n & & 1 \\ n & & 1 \\ n & & 1 \\ & & \\ & & \end{array}$


WHC-EP-0621

This page intentionally left blank. 
WHC-EP-0621

APPENDIX B.14

ISOTOPES IN TRU WASTE CONTAINERS

OTHER THAN 55-GALLON DRUMS 
WHC-EP-0621

This page intentionally left blank. 


\section{ISOTOPES IN TRU WASTE CONTAINERS \\ OTHER THAN 55-GALLON DRUMS}

This subappendix contains a listing of the Solid Waste Information Tracking System's (SWITS's) isotope information for transuranic (TRU) waste from the Plutonium Finishing Plant (PFP) which is stored in containers other than 55-gal drums. The isotope field in SWITS contains the following types of information:

- Specific isotope (e.g., ${ }^{249} \mathrm{Am},{ }^{237} \mathrm{~Np}$, or ${ }^{233} \mathrm{U}$ )

- Generic isotope (e.g., plutonium, uranium-enriched, or uraniumdepleted)

- Plutonfum-239 equivalent curies (PE-CI)

- Total alpha

- Total beta/gamma.

A SWITS record for a single container may list one or all of the above in the isotope field.

This computer run contains the isotope field listing along with the number of TRU waste containers with that listing. The run is sorted by storage facility and year. 


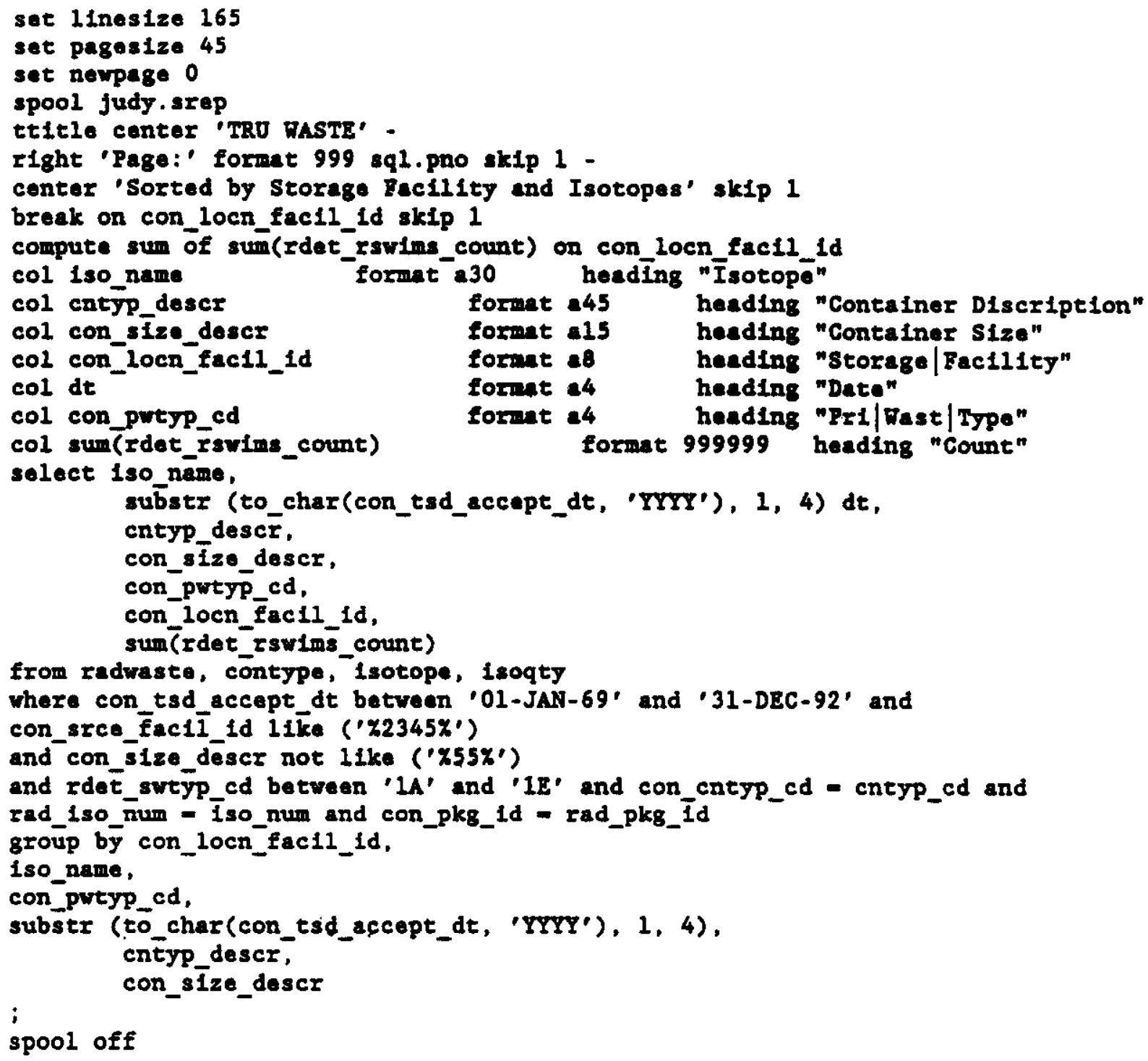


Wast Storage

Isotope

Date Container Discription

1975 METAL BonEs, cartous, CASES

1970 METML Deans, anRRELS, KEGS

1971 EETAL Dowes, cartons, CASES

1974 IETAL BOXES, CARTOWS, CASES

1975 flaEacLASS REIHFORCED PLnNOD (FAP) BOXES

1975 fIBERCLSS REIMFORCED PL YNDOO (FRP) BOXES 1975 fIBeneLASS BEIUFORCED PL YUDOD (FRP) gOXES 1975 fIREaGLASS REIMFORCED PLYUDCD (FRP) BOXES 1975 fIBEngLASS REIMFORCED PLYUDCD (FRP) BOXES 1975 fIBERGLASS REIMFORCED PL nDOD (FRP) BOXES 1975 FIBERGLASS REINFDRCED PLYWDOD (FRP) BOXES 1975 fIDERELASS REIMFORCED PLYNDOD (FRP) BOXES 1975 METAL woXes, CARTOUS, CASES

1975 METAL Eowes, cartons, CASES

1976 FIDEneuns REIWFCRCED PLVWOD (FRP) BOXES 1976 ftoERGLASS REIMFCRCED PL YNOD (FRP) BOXES 1976 FIDEAGASS REIUFORCED PLYNOCD (FRP) BOXES 1976 FIBERGLASS REIMFORCED PLYNDOD (FRP) BOXES 1976 ftDERGASS REIMFORCED PLYNOOD (FRP) BOXES 1977 FIREREASS REIMFORCED PLYNOCD (FRP) BOXES 1977 FIRERGLASS REIMFORCED PL YNOCD (FRP) BOXES 1977 mETAL Boves, CARTOUS, CASES

1973 fIBERGLASS REIMFORCED PLYUDCD (FRP) BOXES 1970 FIBERGLASS REINFORCED PL YUDCO (FRP) BOXES 1978 fIBERGLASS REIMFaRCED PLYUDCO (FRP) BOXES 1978 fiberelass REIUFORCED PLYWOO (FRP) gOXES 1978 fIBERGLASS MEINFORCED PLYWOD (FRP) BOXES 1975 weTAl boxes, cartous, cases 1970 METhl outrs, BhaELS, KEGS 1971 methe eowess, chetous, cases 1975 FJBERGLASS REIMFORCED PL YNOCD (FRP) BOXES

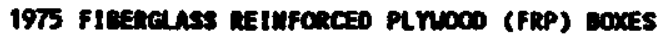
1975 fIBERCLASS REINFCRCED PLINOCD (FRP) BOXES 1975 FIDERGLASS REIUFCRCED PLYUDOD (FRP) BOXES 1975 fIRERCLASS REINFORCED PLYUDCD (FRP) BOXES 1975 FIBERCLASS REIMFORCED PL YUDOO (FRP) BOXES 1975 METAL BonES, CARTOWS, CAsES

1976 FIBERELASS REIMFOCCED PLYNOCO (FRP) BOXES 1976 FIBERGLASS REIMFORCED PLYWOCO (FRP) BOXES
Contalner size Type facility count

\begin{tabular}{|c|c|}
\hline $\begin{array}{l}5.2 * 7.1 * 10.5 \\
30 \text { GaLLON }\end{array}$ & $\begin{array}{l}\text { n } \\
\text { R }\end{array}$ \\
\hline unurion & $\mathbf{R}$ \\
\hline $5 * 10 * 13$ & $\boldsymbol{R}$ \\
\hline $10.5 * 10.7 * 12$ & R \\
\hline $4 * 4 * 7$ & $R$ \\
\hline $4.83 * 5 * 8$ & $\mathbf{R}$ \\
\hline $6.3 * 8 * 14.7$ & $\mathbf{R}$ \\
\hline $9 * 10.67 * 16$ & $R$ \\
\hline $9 * 10.67 * 20$ & $\mathbf{R}$ \\
\hline $9 * 10.7 * 12$ & $R$ \\
\hline $9 * 11.6 * 20$ & $\mathbf{R}$ \\
\hline $5.2 * 7.1 * 10.5$ & $\mathbf{R}$ \\
\hline $5.2 * 7.1 * 16.5$ & $R$ \\
\hline $4 * 4 * 7$ & R \\
\hline $8 * 10=16$ & R \\
\hline $9=10.67 m 20$ & $\mathbf{R}$ \\
\hline $9 * 10.7 * 12$ & $\mathbf{R}$ \\
\hline $9+11.6 * 20$ & $\mathbf{R}$ \\
\hline $8 * 10 * 16$ & $R$ \\
\hline $9+10.7 * 12$ & R \\
\hline $5.2 * 7.1 * 10.5$ & $R$ \\
\hline $4 * 4 * 7$ & R \\
\hline $6.5+8 * 14.6$ & $R$ \\
\hline $6.5 * 8 * 18.5$ & $R$ \\
\hline $8 * 8 * 10.7$ & $R$ \\
\hline $9.5 * 9.9 * 12$ & R \\
\hline $5.2 * 7.1 * 10.5$ & H \\
\hline 30 callow & R \\
\hline LuKacew & $\mathbf{R}$ \\
\hline $10.5 * 10.7 * 12$ & $R$ \\
\hline $4 * 4 * 7$ & $\mathbf{R}$ \\
\hline $9 * 10.67 * 16$ & R \\
\hline $9 * 10.67 * 20$ & $R$ \\
\hline $9 * 10.7 * 12$ & $R$ \\
\hline $9 * 11.6=20$ & $\mathbf{R}$ \\
\hline $5.2 * 7.1 * 10.5$ & $\mathbf{R}$ \\
\hline $4 * 4 * 7$ & $\mathbf{R}$ \\
\hline $8 * 10 * 16$ & $R$ \\
\hline
\end{tabular}


Pri

Wast Storage

Isotope

Date Container Discription

Container size Type Facility count

TOTAL Betaverima

TOTAL BETAVEMAM

TOTAL BETAVGNA

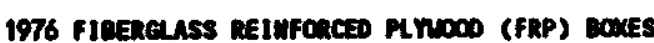

TOTAL BETA/GNWA

TOTAL BETAVAVIA

TOTAL BETA/GAm

TOTAL BETAVANM

TOTAL BETA/GNWM

TOTAL BETA/Cuma

TOTAL EETA/GAYUA

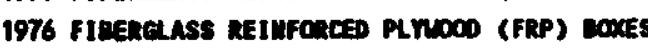

1976 FIPERGLASS REIMFCRCED PLYDOOD (FRP) BOXES

1977 FIBERELASS REIMFORCED PLYNDO (FRP) BOXES

1977 fIBerelass REINFOACED PLNOOD (FRP) BOXES

197 vetal boxes, cantous, chses

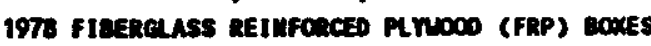

1976 FIDERGLASS REIMFORCED PLYNDOD (FRP) DOXES

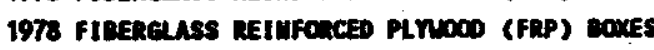

1976 fIBERGLASS REIWFOnCED PLYNOCD (FRP) COKES

$9 * 10.67 \div 20$

$9+10.7 \div 12$

$9 * 11,6 * 20$

$9 * 10 * 16$

$9 * 10.7 * 12$

$5.2 * 7.1 * 10.5$

6.500114 .6

$6.5 * 9 * 18.5$

$8+8 * 10.7$

$9.5 * 9.9 * 12$

$\begin{array}{llr}R & 21843 A & 7 \\ R & & 3 \\ R & & 1 \\ R & & 2 \\ R & & 1 \\ R & & 1 \\ R & & 9 \\ R & & 3 \\ R & & 2 \\ R & & 1 \\ & & 156\end{array}$

1970 COUCRete bOXKS

1971 GLWe soxes

1971 metal boxes, cartous, cases

1971 METAL BonES, CARTOUS, CASES

1971 METML coxes, cartons, cases

1971 meTAL boxes, cuntons, cases

1971 metal DOXES, CARTOUS, CASES

1971 wetMl Demus, BareELS, KEES

1971 SELF COMTAINED, EWUIPWENT

1971 uDOOEN cones, cantous, cases

1972 GLONE woxes

1972 METAL BOKES, CARTONS, CASES

1972 metal cones, cantons, cuses

1972 MEtAl moxes, cAntows, cAsEs

1972 vETAL DouEs, cantous, CASES

1972 vetal Dowes, cantous, cAsEs

1972 METAl bowes, CARTous, CASES

1972 METAL boves, chrtous, cases

1972 METM EONES, CARTOUS, CASES

1972 metal eockes, cartows, cases

1972 metAl boues, cintons, cases

1972 METAL DHASS, BAMRELS, KECS

1973 IETAL DOXES, CARTOUS, CASES

1973 meTAL Boxes, caktous, cases

1973 METAL Danns, BNRRELS, KEGS

1974 metal goxes, cartows, cases

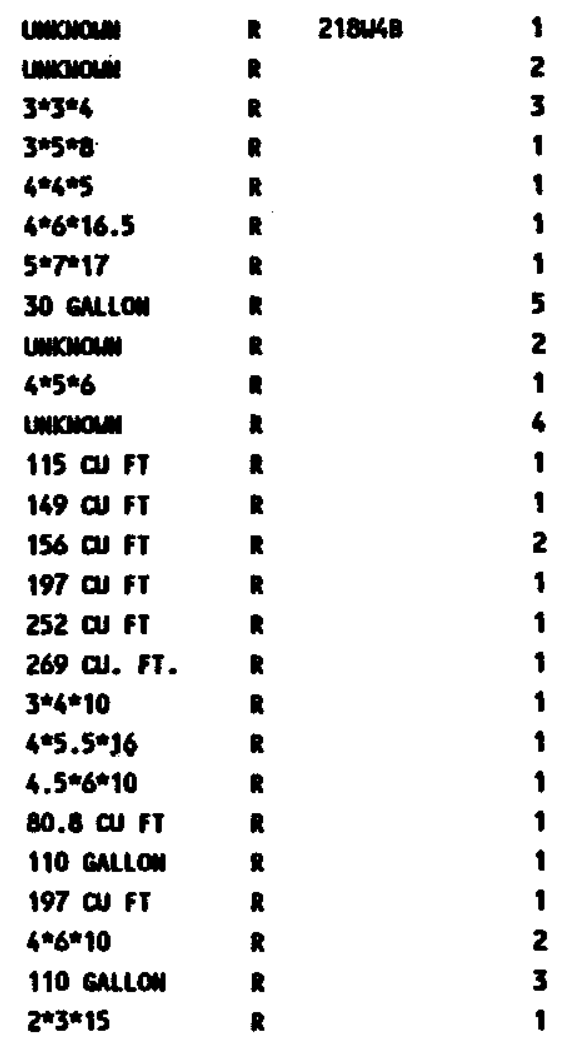


Sorted by storege facility and Isotopes

Pri

Mast Storage

Isotope

Date Contoiner Discription

container size Trpe facility count

PU 1974 METM voues, chatous, cases

1974 METML EonEs, CARTOUS, CASES

1974 metal cones, cantous, cases

1974 METAL contes, cantows, cises

1976 METAL cones, caktoms, cases

1976 metal cowes, cantous, cases

1974 METAL omums, monets, KEES

1975 wetal senes, cantows, cases

1975 wetMl couss, Cantows, cases

1975 mithl wouss, caktows, cases

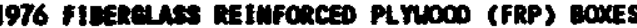

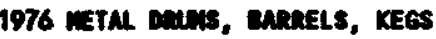

1978 metM cowes, cantous, cases

1978 metal noues, curtous, cases

1971 GLove cowes

1971 MeTMl cones, cARTons, CASES

1971 META conces, CARTOUS, CASES

1971 methl cones, cantous, cases

1971 METAL CowEs, cantous, CASES

1971 METAL coxes, cantows, cAses

1971 vetal omis, enanels, KEgS

1971 SELF COMTAINED, ECUJPUEWT

1971 wOCOEN bouks, cartous, cases

1972 GLOVE DOXES

1972 METM DOXES, CARTOWS, CASES

1972 METM. noutes, cantows, cases

1972 METAL nouEs, cantons, cases

1972 meth cowes, chrtous, cases

1972 METAL Eoucs, cutous, cAsEs

1972 metal coures, cantous, cases

1972 METAL monces, cantons, CASES

1972 METAL CONES, CARTOUS, CASES

1972 metAL ECOES, CARTOWS, CASES

1972 METAL bouKes, cartous, CASES

1972 METAL Douns, Barrels, KEGS

1973 METAL EOOEES, CARTONS, CASES

1973 metal oRtms, Barrets, KEGS

1974 metal boxes, cartous, cases

1974 metal gOXES, Cartons, CASES

\begin{tabular}{|c|c|}
\hline $3 * 4 * 10$ & R 21844B \\
\hline $4 * 6 * 10$ & R \\
\hline $4 * 8 * 10$ & $R$ \\
\hline $4 * 8+16$ & $R$ \\
\hline $5.2 * 7.1 * 10.5$ & $R$ \\
\hline $5.2 * 7.1 * 16.5$ & $\mathbf{R}$ \\
\hline 110 GALLOM & R \\
\hline $3.7 * 6.5=13.2$ & $\mathbf{R}$ \\
\hline $5.2 * 7.1 * 10.5$ & $\mathbf{R}$ \\
\hline $5.2 * 7.1 * 16.5$ & $\mathbf{n}$ \\
\hline $4 * 4 * 7$ & R \\
\hline 110 GALLON & $\mathbf{R}$ \\
\hline $4 * 4 * 7$ & $\mathbf{R}$ \\
\hline $4 * 6 * 15$ & $R$ \\
\hline unkuoun & $\mathbf{R}$ \\
\hline $3 * 3 * 4$ & $\mathbf{R}$ \\
\hline $3 * 5 * 8$ & $R$ \\
\hline $4 * 4 * 5$ & $R$ \\
\hline $4 * 6 * 16.5$ & $\mathbf{R}$ \\
\hline $5 * 7 * 17$ & $R$ \\
\hline 30 GAlLON & $R$ \\
\hline unkrioun & $\mathbf{R}$ \\
\hline $4 * 5 * 6$ & $R$ \\
\hline wruoum & $R$ \\
\hline $115 \mathrm{CU} F$ & R \\
\hline $149 \mathrm{COFT}$ & $R$ \\
\hline $156 \mathrm{CU} \mathrm{FT}$ & a \\
\hline $197 \propto \mathrm{FT}$ & R \\
\hline $252 \mathrm{Cl} \mathrm{FT}$ & $R$ \\
\hline 269 cl. FT. & $R$ \\
\hline $3 * 4 * 10$ & R \\
\hline $4 * 5.5 * 16$ & R \\
\hline $4.5 * 6 * 10$ & $R$ \\
\hline $80.8 \mathrm{CJ}$ FT & R \\
\hline 110 GALLOH & $R$ \\
\hline $4 * 6 * 10$ & R \\
\hline 110 Gallom & $R$ \\
\hline $2 * 3 * 15$ & $\mathbf{R}$ \\
\hline $4 * 6 * 10$ & $R$ \\
\hline
\end{tabular}


Pri

Wast Storage

1sotope

TOTAL BETNGAma

TOTAL BETAVGAMA

TOTAL BETA/CANM

TOTAL BetA/Gima

TOTAL BETANermu

TOTAL BETAVEAmA

total Beta/cesma

TOTAL BETA/Germa

TOTAL Beta/cumu

U-233

\section{CF-252}

PU

PU

DP

$\begin{array}{ll}\infty & P U \\ 1 & P U\end{array}$

PU

PU

PU

Pu

PU

PU

PU

PU

PU

PU

PU

TOTAL BETA/GNWU

TOTAL BETA/GAWM

TOTAL BETA/GNMAM

TOTAL BETA/GaWA

TOTAL BETA/GAWM

TOTAL BETA/GAWU

TOTAL BETA/GNMU

TOTAL BETA/GAMPA
Date Container Discription

Conteiner size Type Facility count

1976 METAL EOWES, currous, cAses

1976 METAL boxes, cantows, cases

1976 METAL COXES, cantous, cAsEs

1974 METAL Dams, sanets, KEes

1975 IETAL CowEs, cantons, cheses

1976 FIDEgelas REILFOCED PLnOD (FRP) DOXES

1976 ratal bans, manels, Kees

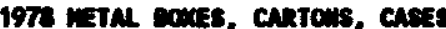

1978 wital Eares, cantous, cases

1972 METAL BOWES, CARTOUS, CASES

1982 META douns, Bankels, rees

1991 MFIA Dans, Barbets, KEes

1902 METAL Deuns, Babeets, KECS

1900 fIBERGLAS REIHFORCED PLVDDOD (FRP) BOXES

1980 FIRERGLASS REIMFOACED PLVUDOD (FRP) BOXES

1960 FIEERELASS REIMFCRCED PLINDCD (FRP) BOXES

1960 mith Dans, Barexts, KEes

1981 FICERCLASS REIUFOCEED PLMDOO (FRP) BOXES

1901 metal douns, manets, KEes

1902 METML Dams, Bapats, KECS

1992 vetal oums, Bundels, wess

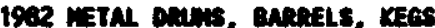

1903 metal couss, cartous, cases

1903 mETM oums, Baceats, KEos

1924 MISCELLAMEOUS SCPNP

1965 mital eokes, cantous, cases

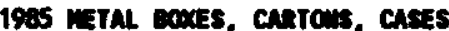

1985 mETAL boxes, cartous, cases

1901 meTAL Deuns, Baninets, KEGS

1992 meth ouns, Bankels, KECS

1930 fleEkCLAS REIMFCRCED PLNDOD (FRP) BOXES

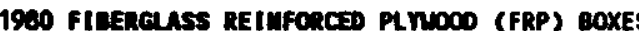

1900 FIBERGLASS REIMFORCED PLMNOD (FRP) BOXES

1900 METAL DRUNS, BakneLs, KEGS

1981 FIQERCLASS REIMFCRCED PLYWDOD (FRP) BOXES

1981 metal dRUAS, BARRELS, KEgS

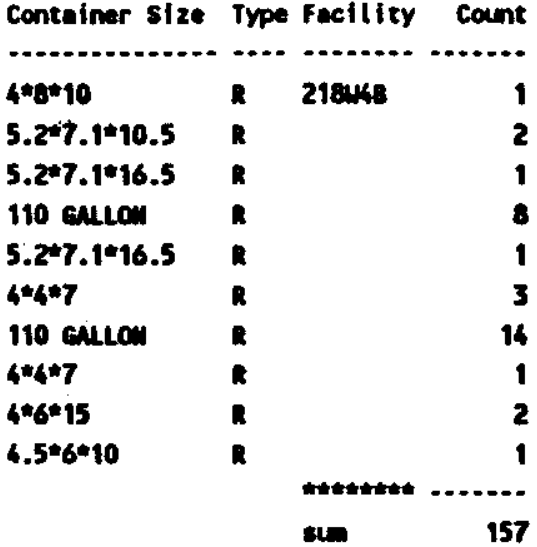

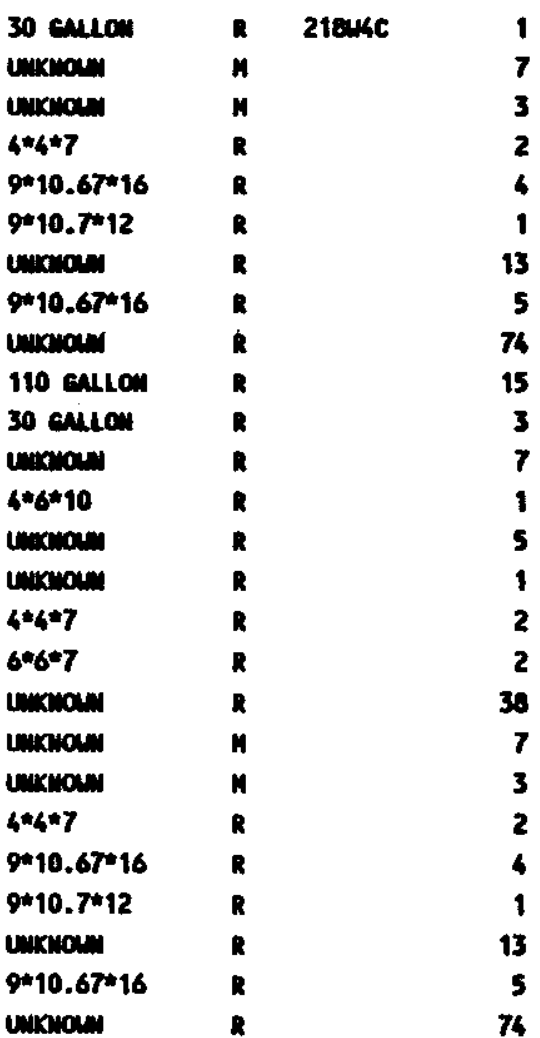




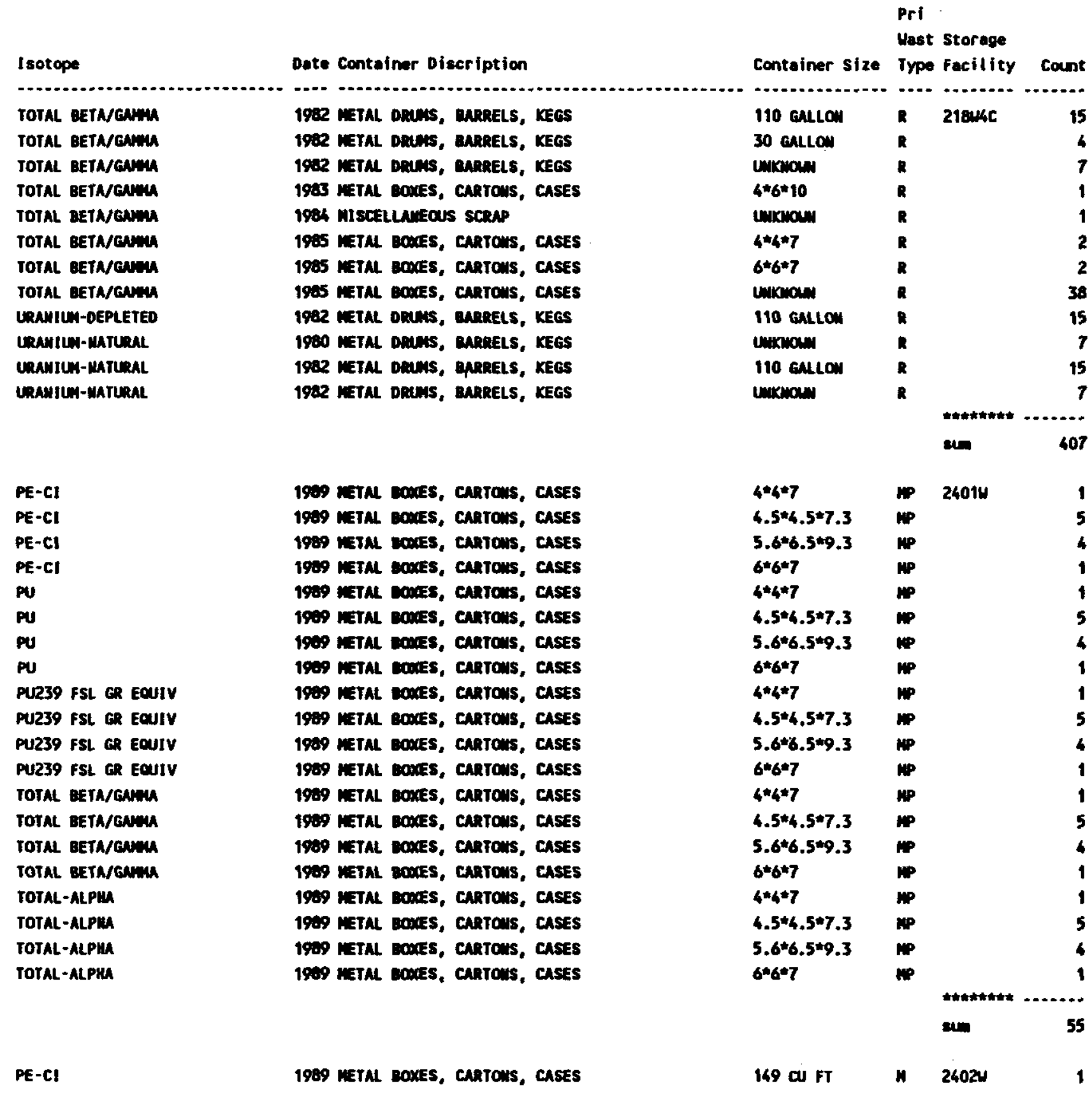


Sorted by Storage facility and Isotopes

Pri

West Storage

I sotope

PE-CI

PU

Pu

PU239 FSL ER EOUIV PU239 FSL ER ECUIV

TOTAL BETAVGAvA

TOTAL BETAJerma

TOTAL-ALPMA

TOTAL-ALPHA

PE-CI

PU

PU239 FSL GR EQUIV

TOTAL BETA/GAMA

TOTAL - ALPHA

'E-CI

v

Y239 FSL ER EOUIV

OTAL BETAJGAMA

OTAL-ALPHA
Date Contelmer biscription

1909 intal bores, cantous, casts

1909 mith sowst, chrous, casas

1909 mithl sones, cantous, cases

1908 motal vares, cantons, chess

1909 mith ratzs, cuntous, caecs

1909 mitht nouss, cantous, cases

1909 mathl wouts, cartous, cases

1909 IETAL EONES, CARTOUS, CASES

1909 metal boxes, cartons, cases

1909 METAL BOXES, CARTOUS, CASES

1909 mital cones, cantous, cases

1909 metal bouxs, cartons, cases

1909 meTA conts, cartous, cases

1909 metal cones, cantons, cases

1909 mTAL Dents, Bunels, KEes

1989 miTM Denns, BARRELS, KEgS

1909 mETAL Dans, BabaELS, KECS

1989 mTA dans, marels, kecs

1989 uETAL DQLUNS, BArRELS, KEeS
Container size Type facility count

$6 * 6 * 7$

169 ad $5 T$

6*6*7

149 CU FT

6*6*7

$149 \mathrm{~cd} h$

6*6*7

$149 \mathrm{CW} F$

$6 * 6 * 7$

$\begin{array}{lll}n & 24024 & 1 \\ n & & 1 \\ n & & 1 \\ n & & 1 \\ n & & 1 \\ n & & 1 \\ n & & 1 \\ n & & 1 \\ & & 1\end{array}$

sin

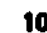

$4 * 4 * 7$

4*4*7

4*4*7

4"4-7

$4 * 4=7$

5 Gallo

85 aAllo

85 Gallon

85 cella

85 callar

8

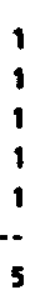

22 rows selected. 
WHC-EP-0621

\section{APPENDIX C}

ORIGINAL SOLID WASTE INFORMATION AND TRACKING SYSTEM RECORDS FOR ALL 55-GALLON WASTE DRUMS WITH WEIGHTS $\geq 150$ KILOGRAMS 
WHC-EP-0621

This page intentionally left blank. 


\section{ORIEIMAL SOLID WASTE INFORMATION AND TRACKING SYSTEN \\ RECORDS FOR ALL 55-GALLON WASTE DRUMS WITH \\ WEIGHTS $\geq 150$ KILOGRAMS}

This appendix contains complete Solid Waste Information and Tracking System (SWITS) records for the twenty six 55-gal drums weighing $\geq 150 \mathrm{~kg}$ each. Table 5-2 is based on the data in this appendix. 
WHC-EP-0621

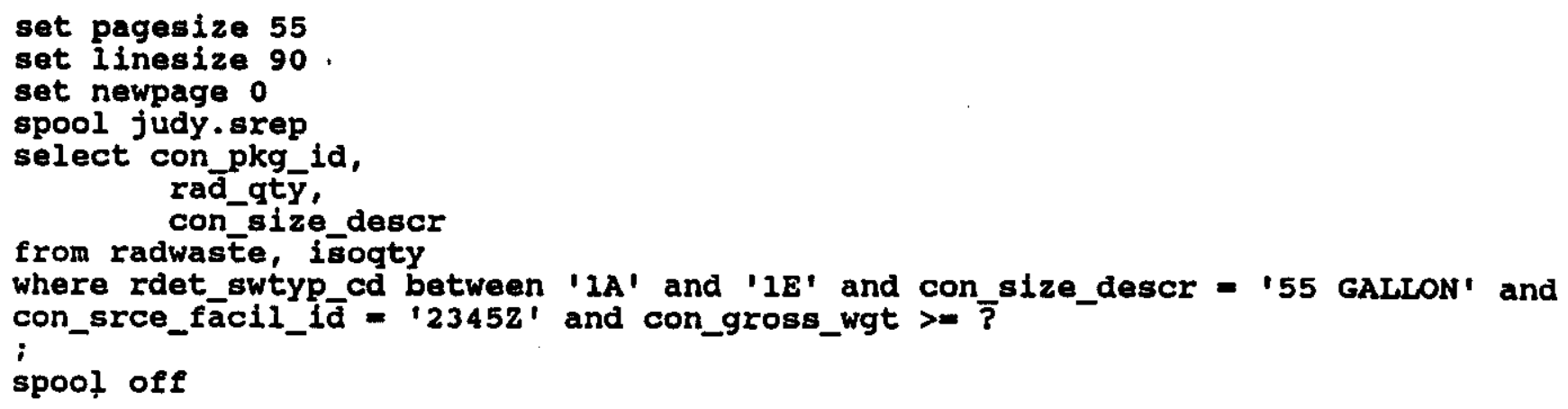

$C-4$ 
for Generating Company: $x$

for Facility ID: $\boldsymbol{x}$

for Primary Waste Type Code: $x$

for Secondary Waste Type Code: $x$

Container ID: A11392

Location Beginning Coordinates: N N40190 W w77708 Location Ending Coordinates: N N40190 W w77684 Content Analysis Return Date: Packaged Date:

TSD Accept Date: 11/08/84

Certification Date:

Container Type Code: DM

Container Count: 1

Dose Rate: 1.000E+00 Neutron Dose Rate: Container Volume:

0.210 Gross Wt:

Previous Container ID:

Current Container ID:

Organic Volume:

Organic Wt.:

139.00

Compaction Wt. (Prorated)

z Content Compaction:

Compactor PIN:

Comments/Description:

Designation Code: DW Waste

Generator Information:

Name:

ID: WHC

Facility ID: $2345 Z$

Charge Code: K6

Location Information:

Area Nane: 200W Facility ID: 218W4C

Tier Level: Tier Position:

Module: Surface Area:

Lab Pack Description:

Lab Pack Flag: N

Physical State Code: S Chemical Nature Code: Primary Waste Type Code: $R$ Secondary Waste Type Code: 1D

Container Size Description: 55 GALLON

Container Storage Category Code:

Container Empty Tare Wt.:

SWSDR \#: 850055

Content Wrap Category:

Radioactive Information:

Total Alpha (Ci):

Total Pu Equivalent (C1):

27.00 Container Thermal Power: 1.000E-01 Container Total Wt.:

SDAR Approval \#:

SWEA General Comments:

Total Beta-Gamma (Ci): 5.000E-02

Total Pu Fissile Gran Equivalent: 
Isotope Number

Isotope Name

1 PU

19 TOTAL BETA/GAMYA

\begin{abstract}
Alpha C1
PE-Ci
\end{abstract}

PU-FGE

-........ ..........
Isotope Activity Unit

1......................

1. $000 \mathrm{E}+00$

5. $000 \mathrm{E}-02$

Hazardous Chemical Components: Component Text

PPM

Weight (kg) \%

1.

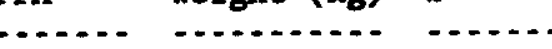

Physical Components:

Content Description

Volume $x$ Weight $(\mathrm{kg})$

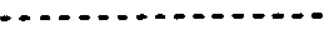

CLOTH/RAGS/NYLON 5

GLASS 6

METAL/IRON/GALVANIZED/SHEET 70

PAPER/CARDBOARD 10

$\begin{array}{lll}P & \text { PLASTIC/POLYURATHANE } & 4 \\ \text { o } & \text { RUBBER } & 5\end{array}$

Relocation History:

Shipment Information: 
for Generating Company: $x$

for Facility ID: $x$

for Primary Waste Type Code: $x$

for Secondary Waste Type Code: $x$

Container ID: A11478

Location Beginning Coordinates: N N39050 w w77500 Location Ending Coordinates: N N39050 W w77476 Content Analysis Return Date: Packaged Date:

TSD Accept Date: 10/25/84

Certification Date:

Container Type Code: DM Container Count:

Dose Rate: 1.000E+00 Neutron Dose Rate: Container Volume:

0.210 Gross Wt:

189.01

Previous Container ID:

Organic Volume:

Current Container ID:

Compaction Wt. (Prorated):

Comments/Description:

Designation Code:

DW Waste \#:

Generator Information:

Name:

Location Information:

Area Name: 200W Facility ID: 218W4C Module: Surface Area:

Tier Level: Tier Position: Unit: T29

Lab Pack Description:

Lab Pack Flag: N

Physical State Code: $S$ Chemical Nature Code:

Container Size Description: 55 GALLON

Container Storage Category Code: SWIMS Waste Description Code: DS

Container Empty Tare Wt.: 27.00 Container Thermal Power:

Content Wrap Category: SDAR Approval \#:

Radioactive Information:

Total Alpha (Ci):

Total Pu Equivalent ( $C 1)$ :

SWEA General Comments:

Total Beta-Gamma (Ci): 5.000E-02

Total Pu Fissile Gram Equivalent:
SWSDR \#: 840232

Container Total Wt.: 


$\begin{array}{rlrrrr}\text { Isotope Number } & \text { Isotope Name } & \text { Alpha Ci } & \text { PE-Ci } & \text { PU-FGE } & \text { Isotope Activity Unit } \\ 11 & \text { PU } & & & 6.000 E+00 & \text { GI } \\ 19 & \text { TOTAL BETA/GAMMA } & & & 5.000 E-02 & \text { CI }\end{array}$

Hazardous Chemical Components: Component Text

PPM Weight (kg) $x$ Coxt

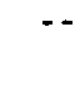

Physical Components: Content Description

Volume $₹$ Weight $(\mathrm{kg})$

METAL/IRON/GALVANIZED/SHEET

PLASTIC/POLYURATHANE

21

Shipment· Information: 
for Generating Company: $x$

for Facility ID: $x$

for Primary Waste Type Code: $z$

for Secondary Waste Type Code: $x$

Container ID: Al1608

Location Beginning Coordinates: N N39050 W w77548 Location Ending Coordinates: N N39050 W w77524

Content Analysis Return Date:

Certification Date:

Packaged Date:

TSD Accept Date: $01 / 22 / 85$

Container Type Code: DM Container Count: Container Volume: $\quad 0.210$ Gross Wt:

Previous Container ID:

Current Container ID:

Organic Volume:

Organic Wt.: $\quad \mathbf{7 8 . 0 0}$

Compaction Wt. (Prorated) :

* Content Compaction:

Compactor PIN:

Designation Code: DW Waste \#:

Generator Information:

Name :

Location Information: Area Nane: 200W Facility ID: 218W4C Tier Level: Tier Position: Unit: T29

Module: Surface Area:

Lab Pack Description:

Lab Pack Flag: N

Physical State Code: S Chemical Nature Code: Primary Waste Type Code: R Secondary Waste Type Code: 1D Container Size Description: 55 GaLLON

Container Storage Category Code:

Container Empty Tare Wt.: SWSDR $f: 850277$

Content Wrap Category:

Radioactive Information:

Total Alpha (Ci):

Total Pu Equivalent ( $\mathrm{Ci}$ ): SDAR Approval \#:

SWIMS Waste Description Code: DS

SWEA General Comments:

Total Beta-Gamma (Ci): 5.000E-02

Total Pu Fissile Gram Equivalent: 


\begin{tabular}{|c|c|c|c|c|c|}
\hline Isotope Number & Isotope Name & Alpha Ci & PE-CI & PU-FGE & Isotope Activity \\
\hline $\begin{array}{r}1 \\
19\end{array}$ & PU & 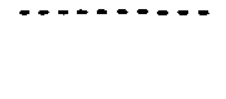 & (n) & $\cdots-$ & $\begin{array}{l}1.790 \mathrm{E}+02 \\
5.000 \mathrm{E}-02\end{array}$ \\
\hline
\end{tabular}

Hazardous Chemical Components:

Component Text

PPM

Weight (kg) $x$

(.................

Physical Components:

Content Description

Volume $x$ Weight $(\mathrm{kg})$

CONCRETE

12

METAL/IRON/GALVANIZED/SHEET 38

PLASTIC/POLYURATHANE 38

RUBBER

12.

$\stackrel{1}{0}$

Relocation History:

Shipment Information: 
for Primary Waste Type Code: $x$

for Secondary Waste Type Code: $x$

\section{Container ID: Al1778}

Location Beginning Coordinates: N N39050 w w77500 Location Ending Coordinates: N N39050 W w77476 Content Analysis Return Date: Certification Date:

Dose Rate: 1.000E+00 Neutron Dose Rate: Packaged Date:

TSD Accept Date: 12/26/84 Container Type Code: DM Container Count: 1 Container Volume:

0.210 Gross Wt:

Previous Container ID:

Current Container ID:

Compaction Wt. (Prorated):

Organic Volume:

x Content Compaction:
Organic Wt.:

Compactor PIN:

Designation Code: DW Waste \#:

Generator Information:

Name:

ID: WHC

Facility ID: $2345 Z$

Charge Code: K6

Location Information:

Area Name: 200W Facility ID: 218W4C Module: Surface Area:

Tier Level: Tier Position: Unit: T29

Lab Pack Description:

Lab Pack Flag: N

Physical State Code: S. Chemical Nature Code:

Gontainer Size Description: 55 GALLON

Container Storage Category Code:
Container Empty Tare Wt.:

Content Wrap Category:

SWIMS Waste Description Code: DS

Primary Waste Type Code: $R$ Secondary Waste Type Code: ID

Radioactive Information:

Total Alpha (CI):

Total Pu Equivalent (Ci):
SWEA General Comments:

27.00 Container Thermal Power: $1.000 \mathrm{E}-01$ Container Total Wt.:

SDAR Approval \#:

Total Beta-Gamma (CI): 5.000E-02

Total Pu Fissile Gran Equivalent: 


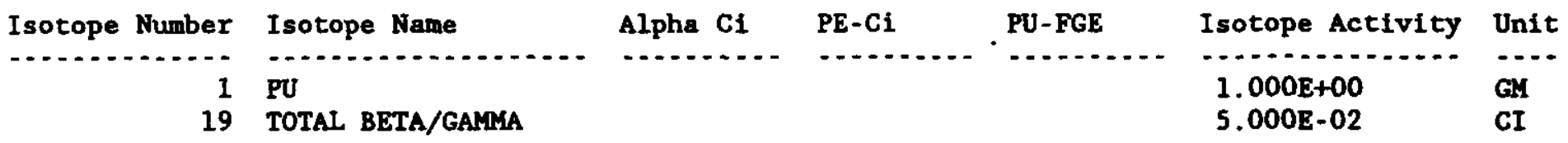

Hazardous Chemical Components:

Component Text

PPM

Weight (kg) $x$

....... ...........

Physical Components:

Content Description

METAL/IRON/GALVANIZED/SHEET

Volune $x$ Weight (kg)

-.......

80

PLASTIC/POLYURATHANE

Relocation History:

Shipment Information: 
for Generating Company: $x$

for Facility ID: $\mathbf{x}$

for Primary Waste Type Code: $z$

for Secondary Waste Type Code: $x$

Container ID: A11787

Location Beginning Coordinates: N N40190 W w77708 Location Ending Coordinates: N N40190 W W77684 Content Analysis Return Date: Packaged Date:

TSD Accept Date: $12 / 03 / 84$

Certification Date:

Container Type Code: DM Container Count: Container Volume: $\quad 0.210$ Gross Wt:

Previous Container ID:

Current Container ID:

Organic Volume:

Organic Wt.: 157.00

Compaction Wt. (Prorated) :

$x$ Content Compaction:-

Compactor PIN:

Designation Code: DW Waste :

Generator Information:

$$
\text { Name: }
$$

Location Information:

Area Name: 200W Facility ID: 218W4C

Module: Surface Area:

Tier Level: Tier Position:

Unit: T01

Lab Pack Description:

Lab Pack Flag: N

Physical State Code: S Chemical Nature Code: Primary Waste Type Code: R Secondary Waste Type Code: 1D

Container Size Description: 55 GALLON

Container Storage Category Code:

SWIMS Waste Description Code: DS

SWSDR \#: 850069

Container Empty Tare Wt.: 27.00 Container Thermal Power: 1.000E-01 Container Total Wt.:

Content Wrap Category:

Radioactive Information:

Total Alpha (Ci):

Total Pu Equivalent (CI): SDAR Approval \#:

SWEA General Comments

Total Beta-Gamma (Ci): 5.000E-02 Total Pu Fissile Gram Equivalent: 


\begin{tabular}{|c|c|c|c|c|c|}
\hline Isotope Number & Isotope Name & Alpha Ci & PE-CI & PU-FGE & Isotope Activity \\
\hline $\begin{array}{r}1 \\
19\end{array}$ & $\begin{array}{l}\text { PU } \\
\text { TOTAL BETA/GAMAA }\end{array}$ & $\cdots$ & $\cdots . .$. & & $\begin{array}{l}1.000 E+00 \\
5.000 E-02\end{array}$ \\
\hline
\end{tabular}

Hazardous Chemical Components:

Component Text

PPM

Weight $(\mathrm{kg}) \mathrm{x}$

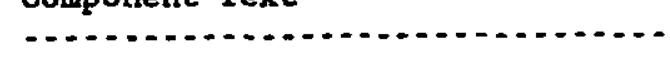

Physical Components: Content Description Con

METAL/IRON/GALVANIZED/SHEET

Volume $x$ Weight (kg)

PLASTIC/POLYURATHANE

20

$\stackrel{P}{A}$

Relocation History:

Shipment Information: 
for Generating Company: $x$

for Facility ID: $z$

for Primary Waste Type Code: $x$

for Secondary Waste Type Code: $x$

Container ID: A12236

Location Beginning Coordinates: N N39050 w w7524 Location Ending Coordinates: N N39050 w w77500 Content Analysis Return Date:

Certification Date:

Dose Rate: 6.000E+00 Neutron Dose Rate:

Packaged Date:

TSD Accept Date: 02/11/85

Container Type Code: DM Container Count:

Container Volume: $\quad 0.210$ Gross Wt:

Previous Container ID:

Current Container ID:

Organic Volume: $\quad$ Organic Wt.: 21.00

Compaction Wt. (Prorated) :

\% Content Compaction:

Comnents/Description:

Designation Code:

DW Waste \#:

Generator Information:

Nane:

ID: WHC

Location Information:

Area Nane: 200W Facillty ID: 218W4C

Tier Level: Tier Position:

Unit: T29

Facility ID: 23452

Charge Code: K6

Lab Pack Description:

Lab Pack Flag: N

Physical State Code: S Chemical Nature Code: Primary Waste Type Code: R Secondary Waste Type Code: 1D

Container Size Description: 55 GALLON

Container Storage Category Code:

SWIMS Waste Description Code: DS

SWSDR \#: 850154

Content Wrap Category:

27.00 Container Thermal Power: 1.000E-01 Container Total Wt.:

Radioactive Information:

Total Alpha (Ci): SDAR Approval \#:

Total Pu Equivalent (Ci):

Total Beta-Gamma (Ci): 5.000E-02

SWEA General Comments:

Total Pu Fissile Gram Equivalent: 


\begin{tabular}{|c|c|c|c|c|c|}
\hline isotope Number & Isotope Name & Alpha Ci & PE-Ci & PU-FGE & Isotope Activity \\
\hline $\begin{array}{r}1 \\
19\end{array}$ & $\begin{array}{l}\text { PU } \\
\text { TOTAL BETA/GAMMA }\end{array}$ & $\ldots \ldots$ & n. & .............. & $\begin{array}{l}1.250 \mathrm{E}+02 \\
5.000 \mathrm{E}-02\end{array}$ \\
\hline
\end{tabular}

Hazardous Chemical Components: Component Text:

PPM Weight $(\mathrm{kg}) \quad x$ -

\begin{tabular}{|c|c|c|}
\hline $\begin{array}{l}\text { Physical Components: } \\
\text { Content Description }\end{array}$ & Volume $x$ & Weight $(\mathrm{kg})$ \\
\hline (1) & $\cdots+\cdots$ & $\cdots$ \\
\hline CLOTH/RAGS/NYLON & 10 & \\
\hline METAL/IRON/GALVANIZED/SHEET & 21 & \\
\hline PLASTIC/POLYURATHANE & 42 & \\
\hline RUBBER & 27 & \\
\hline
\end{tabular}

Relocation History:

Shipment Information: 
Container ID: $A 12615$

Location Beginning Coordinates: N N40190 w w77780 Location Ending Coordinates: N N40190 W W77756 Content Analysis Return Date: Packaged Date: TSD Accept Date: 04/04/85 Certification Date: Container Type Code: DM Container Count: 1 Dose Rate: 1.000E+00 Neutron Dose Rate: Container Volume:

0.210 Gross wt:

Previous Container ID:

Current Container ID:

Compaction Wt. (Prorated):

Organic Volume:

Organic Wt. :

79.00

Comments/Description:

Designation Code:

$z$ Content Compaction:

Compactor PIN:

Name:

Location Information:
Area Name: 200w Facility ID: 218W4C Module: Surface Area:

DW Waste \#:

Tier Level: Tier Position: Unit: T01

Lab Pack Description:

Lab Pack Flag: N

Physical State Code: S Chemical Nature Code: Primary Waste Type Code: R Secondary Waste Type Code: 1D Container Size Description: 55 GALLON

Container Storage Category Code: SWIMS Waste Description Code: DS SWSDR \#: 850618

Container Empty Tare Wt.: 27.00 Container Thermal Power: 1.000E-01 Container Total Wt.:

Content Wrap Category: SDAR Approval \#:

Radioactive Information:

Total Alpha (Ci):

Total Pu Equivalent (Ci):

Total Beta-Gamma (Ci): 5.000E-02

SWEA General Comments:

Total Pu Fissile Gram Equivalent: 


\begin{tabular}{|c|c|c|c|c|c|}
\hline Isotope Number & Isotope Name & Alpha $\mathbf{C i}$ & $\mathrm{PE}-\mathrm{Ci}$ & PU-FGE & Isotope Activity \\
\hline $\begin{array}{r}1 \\
19\end{array}$ & $\begin{array}{l}\text { PU } \\
\text { TOTAL BETA/GAMMA }\end{array}$ & - n. & - & $\cdots$ & $\begin{array}{l}1.000 \mathrm{E}+00 \\
5.000 \mathrm{E}-02\end{array}$ \\
\hline
\end{tabular}

Hazardous Chemical Components:

Component Text

PPM Weight (kg) $x$

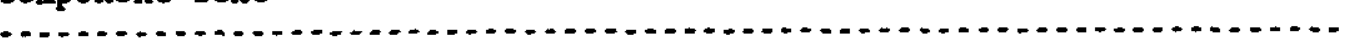

Per

Physical Components:

Content Description

Volume $x$ Weight $(\mathrm{kg})$

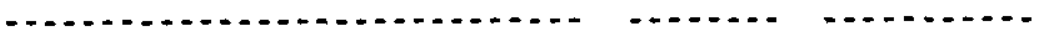

CLOTH/RAGS/NYLON

PAPER/CARDBOARD 40

PLASTIC/POLYURATHANE 30

RUBBER 15

Relocation History:

Shipment Information: 
for Generating Company: $z$

for Facility ID: $x$

for Primary Waste Type Code: $x$

for Secondary Waste Type Code: $x$

Container ID: Al2764

Location Beginning Coordinates: N N40190 w w77780 Location Ending Coordinates: N N40190 W w77756 Content Analysis Return Date:

Certification Date:

Dose Rate: 1.000E+00 Neutron Dose Rate:

Packrged Dațe:

TSD Accept Date: 04/30/85

Previous Container ID:

Conteiner Type Container Volume:

Current Container ID:

Compaction Wt. (Prorated):
Comments/Description:

Designation Code: DW Waste \#:

Organic Volume:

z Content Compaction:

Organic Wt.: Compactor PIN:

0.00 Generator Information:

$$
\text { Name: }
$$

Location Information:

Area Nane: 200W Facility ID: 218W4C

Tier Leve1: Tier Position:

ID: WHC

Facility ID: 23452

Charge Code: K6

ab Pack Description:

Lab Pack Flag: N

Physical State Code: S Chemical Nature Code: Primary Waste Type Code: R Secondary Waste Type Code: 1D

Container Size Description: 55 GalloN

Container Storage Category Code:

Container Empty Tare Wt.:

Content Wrap Category:

27.00 SWIMS Waste Description Code: DS

SWSDR \#: 850746

Radioactive Information:

Total Alpha (Ci):

Total Pu Equivalent (C1): SDAR Approval \#:

Module: Surface Area:

SWEA General Comments:
Total Beta-Gamma (Ci): 5.000E-02

Total Pu Fissile Gram Equivalent: 


\begin{tabular}{|c|c|c|c|c|c|}
\hline Isotope Number & Isotope Name & Alpha Ci & PE-Ci & PU-FGE & Isotope Activity \\
\hline $\begin{array}{r}1 \\
19\end{array}$ & PU & $\ldots$ & $\ldots \ldots$ & . & $1.000 \mathrm{E}+00$ \\
\hline
\end{tabular}

Hazardous Chemical Components:

Component Text

PPM

Weight (kg) $x$

Helght.......

Physical Components:

Content Description

Volume $x$ Weight $(\mathrm{kg})$

GLASS

METAL/IRON/GALVANIZED/SHEET

30

70

$I$
1

Relocation History:

Shipment Information: 
for Generating Company: $x$

for Facility ID: $\chi$

for Primary Waste Type Code: $x$

for Secondary Waste Type Code: $\chi$

\section{Container ID: Al3135}

Location Beginning Coordinates: N N39050 w w77548 Location Ending Coordinates: N N39050 w w77524 Content Analysis Return Date:

Certification Date:

Dose Rate: 4.000E+00 Neutron Dose Rate: Packaged Date:

Container Type Code: DM Container Count: 1

Previous Container ID:

Current Container ID:

Compaction Wt. (Prorated):

Container Volume:

TSD Accept Date: 08/08/85

165.02

Comments/Description:

Designation Code:

Organic Volume:

Organic Wt.:

97.00

Generator Information:

Name:

x Content Compaction:

Compactor PIN:

Location Information:

Area Name: 200W Facility ID: 218W4C

Tier Level: Tier Position:

ID: WHC

Facility ID: 23452

Charge Code: K6

Lab Pack Description:

Lab Pack Flag: N

Physical State Code: S Chemical Nature Code: Primary Waste Type Code: R Secondary Waste Type Code: 1D Container Size Description: 55 GALLON

Container Storage Category Code:

Container Empty Tare Wt.:

SWIMS Waste Description Code: DS

SWSDR \#: 850329

Content Wrap Category:

27.00 Container Thermal Power: 1.000E-01 Container Total Wt.:

Radioactive Information:

Total Alpha (Ci):

Total Pu Equivalent (Ci):

Total Beta-Gamma (Ci): 5.000E-02

SWEA General Comments:

Total Pu Fissile Gram Equivalent: 
Isotope Number Isotope Name

PU

19 TOTAL BETA/GAMMA
Alpha C1 PE-Ci PU-FGE

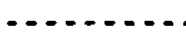

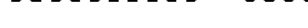

sotope Activity Unit

8. $200 \mathrm{E}+01$

5. O00E-02
CI

Hazardous Chemical Components: Component Text

Physical Components: Content Description

CLOTH/RAGS/NYLON
METAL/IRON/GALVANIZED/SHEET
PLASTIC/POLYURATHANE
RUBBER

N

\section{Volume $\chi$ Weight $(\mathrm{kg})$}

Volume $\mathrm{\gamma}$ Welght (kg)

1
32
3
47
18

Relocation History:

Shipment Information: 
for Generating Company: $z$

for Facility ID: $x$

for Primary Waste Type Code: $x$

for Secondary Waste Type Code: $x$

Container ID: A13200

Location Beginning Coordinates: N N39050 w w77548 Location Ending Coordinates: N N39050 W w77524

Content Analysis Return Date:

Certification Date:

Packaged Date:

TSD Accept Date: 07/31/85

Container-Type Code: D

Container Count: 1 Container Volume:

0.210 Gross Wt:

176.99

Previous Container ID:

Current Container ID:

Organic Volume:

Organic wt.:

5.00

Compaction Wt. (Prorated):

y Content Compaction:

Compactor PIN:

Comments/Description:

Designation Code:

DW Waste :

Generator Information:

Name:

ID: WHC

Facility ID: 23452

Charge Code: K6

Location Information:

Area Name: 200W Facility ID: 218W4C

Tier Level: Tier Position:

Module: Surface Area:

Lab Pack Description:

Lab Pack Flag: N

Physical State Code: S Chemical Nature Code: Primary Waste Type Code: $R$ Secondary Waste Type Code: 1D

Container Size Description: 55 GALLON

Container Storage Category Code:

Container Empty Tare Wt.

SWIMS Waste Description Code: DS

SWSDR \#: 850358

Content Wrap Category:

Radioactive Information:

Total Alpha (Ci):

27.00 Container Thermal Power: 1.000E-01 Container Total Wt.:

Total Pu Equivalent (Ci):

Total Beta-Gamma (Ci): 5.000E-02

SWEA General Coments:

Total Pu Fissile Gram Equivalent: 


\begin{tabular}{|c|c|c|c|c|c|}
\hline Isotope Number & Isotope Name & Alpha Ci & PE-Ci & PU-FGE & Isotope Activity \\
\hline 1 & PU & $\ldots$ & 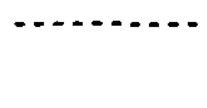 & $\cdots$ & $\begin{array}{l}1.000 \mathrm{E}+00 \\
5.000 \mathrm{E}-02\end{array}$ \\
\hline
\end{tabular}

Hazardous Chenical Components:

Component Text

PPM Welght (kg) $x$

Physical Components:

Content Description

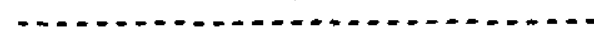

DIRT/SOIL/DIATOMACEOUS EARTH

PLASTIC/POLYURATHANE

Volume $x$ Weight (kg)

$97 . . . . . . . . .$.

97

$\stackrel{T}{\sim}$

Relocation History:

Shipment Information: 
for Secondary Waste Type Code: $x$

\section{Container ID: A13201}

Location Beginning Cooxdinates: N N39050 W w77548 Location Ending Coordinates: N N39050 W W77524

Content Analysis Return Date:

Certification Date:

Dose Rate: 1.000E+00 Neutron Dose Rate:

Packaged Date:

TSD Accept Date: $07 / 31 / 85$

Dose Rate: $1.000 E+00$
Previous Container ID

Container Type Code: DM Container Count:

Container Volume:

0.210 Gross Wt:

153.99

Current Container ID:

Compaction Wt. (Prorated):
Comments/Description:

Designation Code:

DW Waste \#:

Generator Information:

Name:

Organic Volume:

x Content Compaction: Compactor PIN:

ID: WHC

Facility ID: 23452

Charge Code: K6

Location Information:

Area Name: $200 \mathrm{~W}$ Facility ID: $218 \mathrm{WC}$ Module: Surface Area:
Tier Leve1: Tier Position: $\quad$ Unit: T29

Tier Level: Tier

Lab Pack Flag: N

Physical State Code: S Chemical Nature Code: Primary Waste Type Code: $R$ Secondary Waste Type Code: 1D

Container Size Description: 55 GALLON

Container Storage Category Code:

SWIMS Waste Description Code: DS

SWSDR \#: 850359

Container Empty Tare Wt.

Content Wrap Category:

27.00 Container Thermal Power: 1.000E-01 Container Total Wt.:

Radioactive Information:

Total Alpha (Ci):

Total Pu Equivalent (Ci):

Total Beta-Gamma (Ci): 5.000E-02

SWEA General Comments:
Total Pu Fissile Gran Equivalent: 


\begin{tabular}{|c|c|c|c|c|c|}
\hline Isotope Number & Isotope Name & Alpha CI & PE-Ci & PU-FGE & Isotope Activity \\
\hline $\begin{array}{r}1 \\
19\end{array}$ & $\begin{array}{l}\text { PU } \\
\text { TOTAL BETA/GAMAa }\end{array}$ & $\cdots$ & 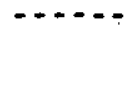 & ................ & $\begin{array}{l}2.000 \mathrm{E}+00 \\
5.000 \mathrm{E}-02\end{array}$ \\
\hline
\end{tabular}

\section{Hazardous Chemical Components:}

Component Text

PPM

Weight (kg) $x$

(............

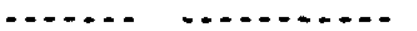
Physical Components: Content Description
Volume $x$ Weight $(\mathrm{kg})$
DIRT/SOIL/DIATOMACEOUS EARTH 97
PLASTIC/POLYURATHANE

Relocation History:

Shipment Information: 
for Generating Company: $z$

for Facility ID: $\chi$

for Primary Waste Type Code: $x$

for Secondary Waste Type Code: $x$

Container ID: Al3203

Location Beginning Coordinates: N N39050 w w77548 Location Ending Coordinates: N N39050 w w77524 Content Analysis Return Date:

Certification Date:

Packaged Date:

TSD Accept Date: 07/31/85

Dose Rate: $1.000 \mathrm{E}+00$

Container Count: 1

Previous Container ID:

$$
\text { Container Volume: } \quad 0.210 \text { Gross Wt: }
$$

Current Container ID:

Organic Volume:

Organic Wt.:

4.00

Compaction Wt. (Prorated) :

z Content Compaction:

Compactor PIN:

Comnents/Description:

Designation Code:

DW Waste \#:

Generator Information:

Name :

Location Information:

Area Nane: 200W Facility ID: 218W4C

Tier Level: Tier Position:

Module: Surface Area:

Lab Pack Description:

Lab Pack Flag: N

Physical State Code: S Chemical Nature Code: Primary Waste Type Code: $R$ Secondary Waste Type Code: 1D

Container Size Description: 55 GALLON

Container Storage Category Code:

SwIMS Waste Description Code: DS SWSDR \#: 850361

Container Empty Tare Wt.: 27.00 Container Thermal Power: 1.000E-01 Container Total Wt.:

Content Wrap Category: SDAR Approval \#:

Radioactive Information:

Total Alpha (Ci):

Total Pu Equivalent (Ci):

Total Beta-Gamma (Ci): 5.000E-02

SWEA General Comments:

Total Pu Fissile Gram Equivalent: 


\begin{tabular}{|c|c|c|c|c|c|c|}
\hline Isotope Number & Isotope Name & Alpha Ci & $\mathbf{P E}-\mathrm{Ci}$ & PU-FGE & Isotope Activity & Unit \\
\hline $\begin{array}{r}1 \\
19\end{array}$ & TOTAL BETA/GAMMA & 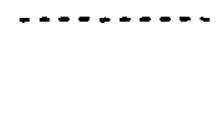 & 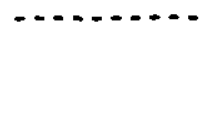 & 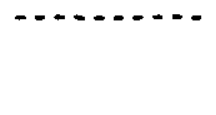 & $\begin{array}{l}1.000 \mathrm{E}+00 \\
5.000 \mathrm{E}-02\end{array}$ & GI \\
\hline
\end{tabular}

Hazardous Chemical Components: Component Text

PPM Welght (kg) $x$

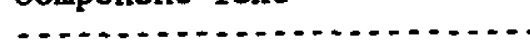

Physical Components:

Content Description

Volume $x$ Weight $(\mathrm{kg})$

DTRT/SOIL/DIATOYACEOUS EARTH

PLASTIC/POLYURATHANE

97

3

$\mathbb{N}_{\infty}^{1}$

Relocation History:

Shipment Information: 
for Generating Company: $x$

for Facility ID: $x$

for Primary Waste Type Code: $\chi$

for Secondary Waste Type Code: $x$

Container ID: A13205

Location Beginning Coordinates: N N39050 w w77548 Location Ending Coordinates: N N39050 W w77524 Content Analysis Return Date:

Certification Date:
Dose Rate: $1.000 \mathrm{E}+00$ Packaged Date:

TSD Accept Date: 07/31/85

Container Type Code: DM

Container Count: 1

Previous Container ID: Container Volune:

0.210 Gross Wt:

181.98

Current Container ID:

Compaction Wt. (Prorated):

Organic Volume:

Organic Wt.:

5.00

Corments/Description:

Designation Code: DW Waste \#:

Generator Information:

Name:

x Content Compaction:

Compactor PIN:

Location Information:

Area Name: 200 Facility ID: 218W4C Module: Surface Area:

Tier Level: Tier Position: Unit: T29

Lab Pack Description:

Lab Pack Flag: N

Physical State Code: S Chemical Nature Code: Primary Waste Type Code: R Secondary Waste Type Code: 1D

Container Size Description: 55 GALLON

Container Storage Category Code: SWIMS Waste Description Code: DS SWSDR \#: 850363

Container Empty Tare Wt.: $\quad 27.00$ Container Thermal Power: 1.000E-01 Container Total Wt.:

Content Wrap Category: SDAR Approval \#:

Radioactive Information

Total Alpha (Ci):

Total Pu Equivalent (Ci):

Total Beta-Gamma (Ci): 5.000E-02

SWEA General Cominents:

Total Pu Fissile Gran Equivalent: 


$\begin{array}{rlccccc}\text { Isotope Number } & \text { Isotope Name } & \text { Alpha CI } & \text { PE-Ci } & \text { PU-FGE } & \text { Isotope Activity Unit } \\ 1 & \text { PU } & & & & 1.000 E+00 & \text { GM } \\ 19 & \text { TOTAL BETA/GAMMA } & & & 5.000 E-02 & \text { CI }\end{array}$

Hazardous Chemical Components:

Component Text

PPM

Weight $(\mathrm{kg}) \quad x$

(2.

-....

..................

Physical Components:

Content Description

Volume $x$ Weight $(\mathrm{kg})$

DIRT/SOIL/DIATOMACEOUS EARTH

PLASTIC/POLYURATHANE

$\stackrel{T}{\omega}$

Relocation History:

Shipment Information: 
for Secondary Waste Type Code: $x$

\section{Container ID: Al3209}

Location Beginning Coordinates: N N39050 W W77548 Location Ending Coordinates: N N39050 W w77524 Content Analysis Return Date:

Certification Date:

Dose Rate: $.1 .000 E+00$ Neutron Dose Rate: Packaged Date:

TSD Accept Date: 07/31/85

Previous Container ID:

Container Type Code: DM Container Count: Container Volume:

0.210 Gross Wt:

Current Container ID:

Compaction Wt. (Prorated):

Comments/Description:

Designation Code: DW Waste \#:

Generator Information:

$$
\text { Name: }
$$

Organic Volume:

z Content Compaction:

Organic Wt.:

4.00 Compactor PIN:

ID: WHC

Facility ID: 23452

Charge Code: K6

Location Information:

Area Name: 200W Facility ID: 218W4C

Tier Level: Tier Position:

Lab Pack Description:

Lab Pack Flag: N

Physical State Code: S Chemical Nature Code: Primary Waste Type Code: $R$ Secondary Waste Type Code: 1D

Container Size Description: 55 GALLON

Container Storage Category Code:

SWIMS Waste Description Code: DS

SWSDR \#: 850328

Container Empty Tare Wt.: 27.00 Container Thermal Power: $1.000 E-01$ Container Total Wt.:

Content Wrap Category: SDAR Approval \#:

Radioactive Information:

Total Alpha (Ci):

Total Pu Equivalent (Ci):

Total Beta-Gamma (Ci): 5.0008-02

SWEA General Comments:
Total Pu Fissile Gram Equivalent: 


\begin{tabular}{|c|c|c|c|c|c|c|}
\hline Isotope Number & Isotope Name & Alpha Ci & $P E-C I$ & PU-FGE & Isotope Activity & Unit \\
\hline $\begin{array}{r}1 \\
19\end{array}$ & TOTAL BETA/GAMA & $\cdots \cdots+\cdots$ & 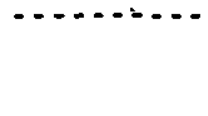 & $\ldots \ldots$ & $\begin{array}{l}3.000 \mathrm{E}+00 \\
5.000 \mathrm{E}-02\end{array}$ & $\begin{array}{l}\cdots \\
\text { GI }\end{array}$ \\
\hline
\end{tabular}

Hazardous Chemical Components:

Component Text

PPM Weight (kg) $x$

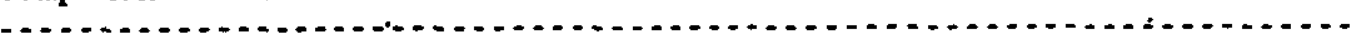

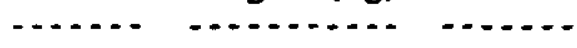

Physical Components:

Content Description

Volume $x$ Weight $(\mathrm{kg})$

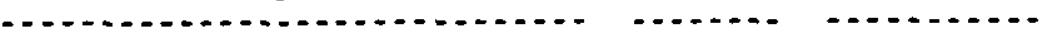

DIRT/SOIL/DIATOMACEOUS EARTH 97

PLASTIC/POLYURATHANE

Shipment Information: 
for Generating Company: $x$

for Facility ID: $x$

for Primary Waste Type Code: $x$

for Secondary Waste Type Code: $x$

Container ID: Al3220

Location Beginning Coordinates: N N40190 W w77804 Location Ending Coordinates: N N40190 w w77780

Content Analysis Return Date: Packaged Date: TSD Accept Date: 08/01/85

Certification Date:

Container Type Code: DM Container Count:

Dose Rate: 1.000E+00 Neutron Dose Rate: Container Volume:

0.210 Gross Wt:

176.99

Previous Container ID:

Current Container ID:

Organic Volume:

Organic Wt.: $\quad 77.00$

Compaction Wt. (Prorated):

x Content Compaction:

Compactor PIN:

Designation Code:

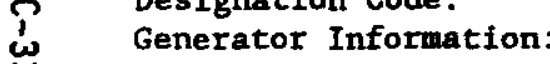

Name:

DW Waste *:

Location Information:

Area Nane: 200 Facility ID: 218W4C Module: Surface Area:

Tier Level: Tier Position:

Unit: T01

Lab Pack Description:

Lab Pack Flag: N

Physical State Code: S Chemical Nature Code: Primary Waste Type Code: $R$ Secondary Waste Type Code: 1D

Container Size Description: 55 GALLON

Container Storage Category Code:

SWIMS Waste Description Code: DS

SWSDR \#: 851087

Container Empty Tare Wt.: $\quad 27.00$ Container Theraal-Power: 1.000E-01 Container Total Wt.:

Content Wrap Category: SDAR Approval \#:

Radioactive Information:

Total Alpha (C1):

Total Pu Equivalent ( $\mathrm{Ci}$ ):

Total Beta-Gamma (Ci): 5.000E-02

SWEA General Comments:

Total Pu Fissile Gram Equivalent: 


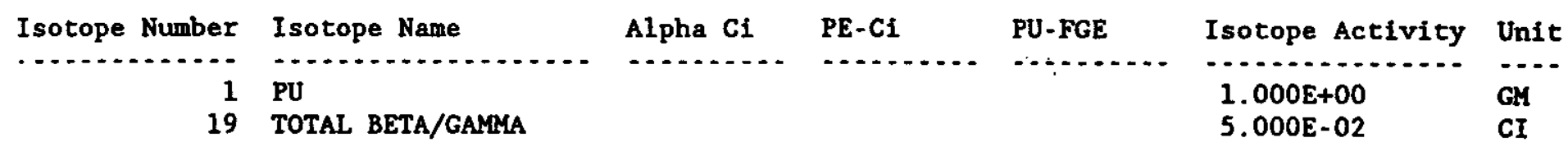

Hazardous Chemical Components:

Component Text

PPM

Weight (kg) $x$

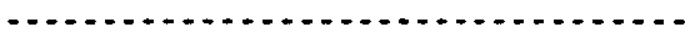

- ...... ...........

Physical Components:

Content Description

Volume $\chi$ Weight $(\mathbf{k g})$

CLOTH/RAGS/NYLON

DIRT/SOIL/DIATOMACEOUS EARTH 50

PAPER/CARDBOARD 15

PLASTIC/POLYURATHANE 30

$\stackrel{P}{1}$

Relocation History:

Shipment Information: 
for Generating Company:

for Facility ID: $x$

for Primary Waste Type Code: $x$

for Secondary Vaste Type Code: $x$

Container ID: A13237

Location Beginning Coordinates: N N40190 W w77804 Location Ending Coordinates: N N40190 W W77780

Content Analysis Return Date:

Certification Date:

Dose Rate: 1.000E+00 Neutron Dose Rate:

Packaged Date:

TSD Accept Date: 08/08/85

Container Type Code: DM Container Count: Container Volume: $\quad 0.210$ Gross Wt:

Previous Container ID:

Current Container ID:

Organic Volume: Organic Wt.: 10.00

Compaction Wt. (Prorated):

$x$ Content Compaction:

Comments/Description:

Designation Code: DW Waste :

Generator Information:

Name:

Location Information:

Area Name: 200W Facility ID: 218W4C Module: Surface Area:

Tier Level: Tier Position: Unit: T01

Lab Pack Description:

Lab Pack Flag: N

Physical State Code: S Chemical Nature Code: Primary Waste Type Code: $R$ Secondary Waste Type Code: 1D Container Size Description: 55 GALLON

Container Storage Category Code:

Container Empty Tare Wt.:

SWIMS Waste Description Code: DS

SWSDR : 851094

Content Wrap Category:

27.00 Container Thermal Power: 1.000E-01 Container Total Wt.

Radioactive Information:

Total Alpha (Ci):

Total Pu Equivalent (Ci):

Total Beta-Gamma (Ci): 5.000E-02

SWEA General Comments :

Total Pu Fissile Gram Equivalent: 


$\begin{array}{rlccccc}\text { Isotope Number } & \text { Isotope Name } & \text { Alpha } \mathrm{CI} & \text { PE-Ci } & \text { PU-FGE } & \text { Isotope Activity Unit } \\ 19 & \text { PU } & & & & 1.000 E+00 & \text { GH } \\ 19 & \text { TOTAL BETA/GAMRA } & & & 5.000 E-02 & \text { CI }\end{array}$

Hazardous Chemical Components:

Component Text

PPM Weight (kg) $x$

C...

\section{Physical Components:} Content Description

METAL/IRON/GALVANIZED/SHEET

PLASTIC/POLYURATHANE

Volume $x$ Weight $(\mathrm{kg})$

V.......-

95

5

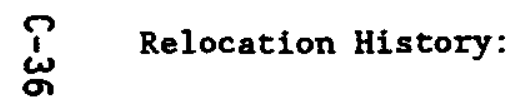

Shipment Information: 
for Generating Company: $x$

for Facility ID: $x$

for Primary Waste Type Code: $x$

for Secondary Waste Type Code: $\chi$

Container ID: A14053

Location Beginning Coordinates: N

Content Analysis Return Date:

Certification Date:

Dose Rate: 2.000E+00

Neutron Dose Rate:

\section{Location Ending Coordinates: N \\ W \\ TSD Accept Date: 12/15/86 \\ Container Type Code: D}

Previous Container ID:

Current Container ID:

1 Container Volure: 0.210 Gross Wt:

153.00

Compaction Wt. (Prorated):

Organic Volume: 45 Organic Wt.:

46.00

Comments/Description:

Designation Code:

DW Waste \#:

Generator Information:

$$
\text { Name: }
$$

ID: WHC

Facility ID: 23452

Charge Code:

Location Information:

Area Name: 200W Facility ID: $224 \mathrm{~T}$

Tier Level: Tier Position:

$x$ Content Compaction:

Compactor PIN:

Lab Pack Description:

Lab Pack Flag: N

Physical State Code: S Chemical Nature Code: Primary Waste Type Code: $R$ Secondary Waste Type Code: 1D

Container Size Description: 55 GALLON

Container Storage Category Code:

Container Empty Tare Wt.:

Content Wrap Category:

Radioactive Information:

Total Alpha (Ci):

Total Pu Equivalent (Ci):

Module: Surface Area:

WEA General Comments:

Total Pu Fissile Gran Equivalent: 
Isotope Number

-. -

1 PU

sotope Name -......
Alpha Ci PE-Ci

$\therefore \ldots . . .$.

.........
Isotope Activity Unit ..........................

1.230E+02

Hazardous Chemical Components:

Component Text

PPM

Weight (kg) $\%$

Physical Components:

Content Description

2..................

Volume $x$ Weight $(\mathrm{kg})$

CLOTH/RAGS/NYLON

-.....................

METAL/IRON/GALVANIZED/SHEET

PAPER/GARDBOARD

PLASTIC/POLYURATHANE

RUBBER

6
25

12

$\mathfrak{w}_{\infty}^{\infty}$

Relocation History:

Shipment Information: 
for Generating Company: $x$

for Facility ID: $x$

for Primary Waste Type Code: $z$

for Secondary Waste Type Code: $z$

Container ID: A15015

Location Beginning Coordinates: N

Content Analysis Return Date:

Certification Date:

Dose Rate: 3.000E+00 Neutron Dose Rate:

Previous Container ID:

Current Container ID:

Compaction Wt. (Prorated):

W

\section{Location Ending Coordinates: $\mathbf{N}$ \\ W \\ Packaged Date: \\ TSD Accept Date: 12/15/86 \\ Container Type Code: DM Container Count: 1}

1 Container Volume: 0.210 Gross Wt:

154.99

Organic Volume: $\quad 60$ Organic Wt.: $\quad 23.72$

* Content Compaction:- Compactor PIN :

Comments/Description:

Designation Code

Generator Information:

Name:

DW Waste \#:

Location Information:

Tier Level: Tier Position:

ID: WHC

Facility ID: 23452

Charge Code:

Tier Level: Tier
ab Pack Description:

Lab Pack Flag: N

Physical State Code: S Chemical Nature Code: Primary Waste Type Code: $R$ Secondary Waste Type Code: 1D

Container Size Description: 55 GALLON

Container Empty Tare Wt.:

SWIMS Waste Description Code: DS

SWSDR \# : 860139

Content Wrap Category:

Radioactive Information:

Total Alpha (Ci):

Total Pu Equivalent ( $\mathrm{Ci}$ ):

27.00 Container Thermal Power:.1.000E-01 Container Total Wt.: SDAR Approval \#:

WEA General Comments:
Total Beta-Gamma (Ci):

Total Pu Fissile Gram Equivalent: 
Isotope Number

Isotope Name

Alpha Ci

PE-Ci

1 PU
-.........
PU-FGE

..........
Isotope Activity Unit

-....................

1. $450 \mathrm{E}+02$

GM

Hazardous Chemical Components:

Component Text

PPM

Weight (kg) $x$

Component Text

Physical Components: Content Description

CLOTH/RAGS/NYLON

Volume $x$ Weight (kg)

METAL/IRON/GALVANI

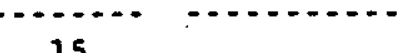

PAPER/CARDBOARD

40

PLASTIC/POLYURATHANE

$\stackrel{P}{0}$

RUBBER

20

\section{Relocation History:}

Shipment Information: 
for Generating Company: $x$

for Facility ID: $x$

for Primary Waste Type Code: $\%$

for Secondary Waste Type Code: $x$

Container ID: A15107

Location Beginning Coordinates: N

Content Analysis Return Date:

Certification Date:

Dose Rate: 2.000E+00 Neutron Dose Rate:

Previous Container ID:

Current Container ID:

Compaction Wt. (Prorated):

W

Location Ending Coordinates: N
Packaged Date:

TSD Accept Date: 12/08/86

Container Type Code: DM Container Count: 1

1 Container Volume: 0.210 Gross Wt:

153.99

Organic Volume: 66 Organic wt.: 13.95

* Content Compaction: Compactor PIN:

Designation Code: DW Waste \#:

Generator Information:

$$
\text { Name: }
$$

Location Information:

Area Name: 200 Facility ID: 224T

Tier Level: Tier Position:

ID: WHC

Facility ID: 23452

Charge Code:

Module: Surface Area:

Unit: L01

Lab Pack Description:

Lab Pack Flag: N

Physical State Code: S Chemical Nature Code: Primary Waste Type Code: $R$ Secondary Waste Type Code: 1D

Container Size Description: 55 GALLON

Container Storage Category Code: SWIMS Waste Description Code: DS SWSDR \#: 860114

Container Empty Tare Wt.: 27.00 Container Thermal Power: 1.000E-01 Container Total Wt.:

Content Wrap Category:

SDAR Approval \#:

Radioactive Information:

Total Alpha (Ci):

Total Pu Equivalent (Ci):

Total Beta-Gamma (Ci):

SWEA General Comments:

Total Pu Fissile Gram Equivalent: 


\begin{tabular}{clcccc} 
Isotope Number & Isotope Name & Alpha Ci & PE-Ci & PU-FGE & Isotope Activity Unit \\
\hline 1 & PU & & & $1.030 \mathrm{E}+02$ & GK
\end{tabular}

Hazardous Chemical Components:

Component Text

PPM

Weight $(\mathrm{kg}) \quad x$

C.........

..................

Physical Components:

Content Description

Volume $x$ Weight $(\mathrm{kg})$

CLOTH/RAGS/NYLON

- .'.................

METAL/IRON/GALVANIZED/SHEET 34

PAPER/CARDBOARD 13

PLASTIC/POLYURATHANE 30

RUBBER

20

$\stackrel{?}{\mathbb{N}}$

Relocation History:

Shipment Information: 
for Facility ID: $z$

for Primary Waste Type Code: $x$

for Secondary Waste Type Code: $x$

\section{Container ID: A17826}

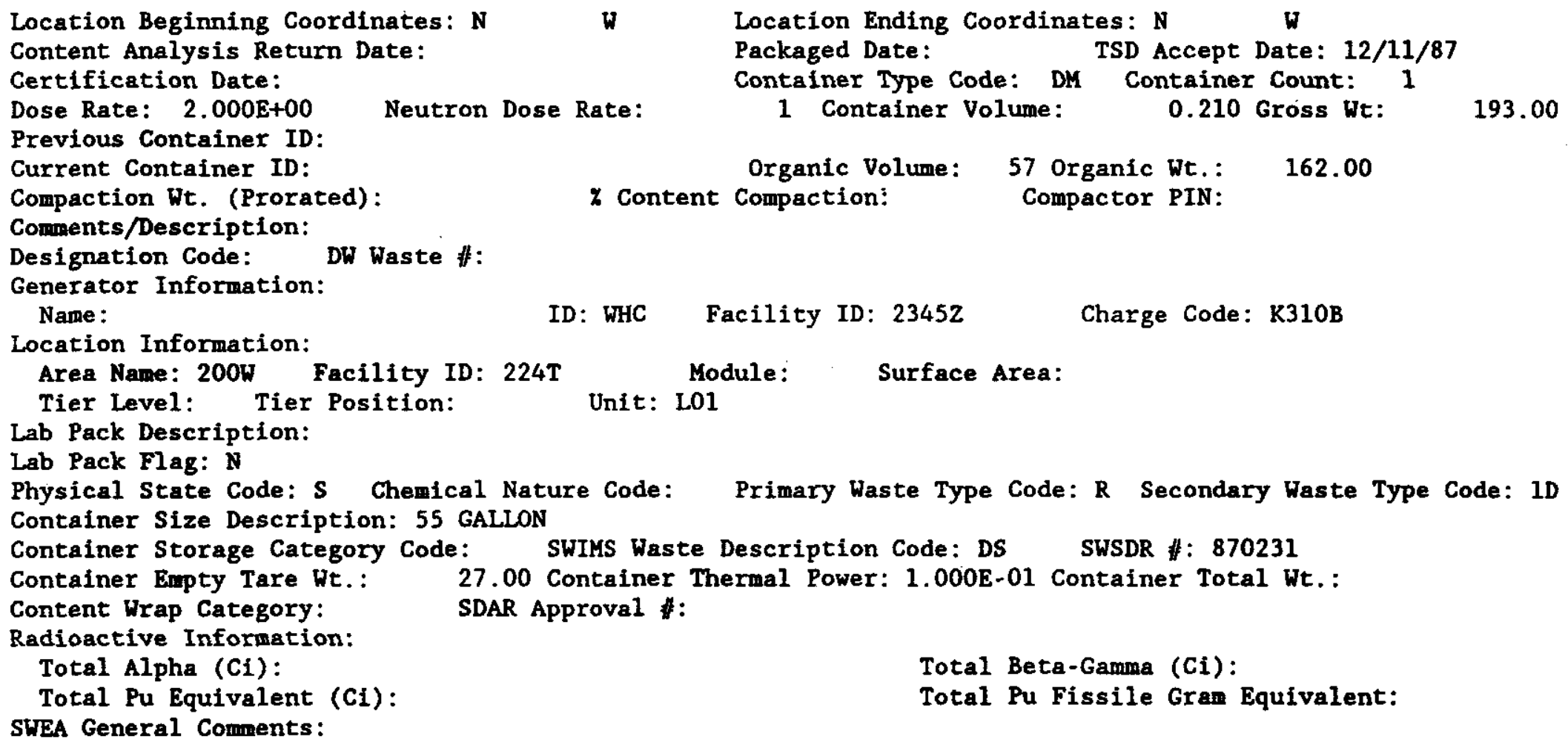

Location Information:
Area Name: 200w
Facility ID: 224T
Module: Surface Area:

Unit: L01

ab Pack Description:

Lab Pack Flag: N

Physical State Code: $S$ Chemical Nature Code: Primary Waste Type Code: R Secondary Waste Type Code: 1D Container Size Description: 55 GALLON

Container Storage Category Code:

Container Empty Tare Wt.:

SWIMS Waste Description Code: DS

SWSDR \#: 870231

Content Wrap Category:

27.00 Container Thermal Power: 1.000E-01 Container Total Wt.:

Radioactive Information:

Total Alpha (Ci):

SDAR Approval \#:

Total Pu Equivalent ( $C i)$ :

Total Beta-Gamma (Ci):

SWEA General Comments:

Total Pu Fissile Gram Equivalent: 
Isotope Number Isotope Name

Alpha Ci

PE-Ci

PU-FGE

PU
Alpha CI

-...

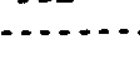

.......

\section{Isotope Activity Unit}

$2.400 \mathrm{E}+01$

(-.-

Hazardous Chemical Components:

Component Text

PPM

Weight (kg) $z$

-.................

Physical Components: Content Description

CLOTH/RAGS/NYLON

DIRT/SOIL/DIATOYACEOUS EARTH

METAL/IRON/GALVANIZED/SHEET

MISCELLANEOUS/UNKNOWN/OTHER

PAPER/CARDBOARD

PLASTIC/POLYURATHANE

RUBBER

Volume $x$ Weight $(\mathrm{kg})$

$\stackrel{1}{p}$

........

3

2

41

5

8

14

27

Relocation History:

Shipment Information: 
for Generating Company: $x$

for Facility ID: ' $x$

for Primary Waste Type Code: $x$

for Secondary Waste Type Code: $x$

Container ID: $220-\mathrm{A} 20220$

Location Beginning Coordinates: N Content Analysis Return Date:

Certification Date:

Dose Rate: 1.000E+00 Neutron Dose Rate:

W Location Endïng Coordinates: N

W

Packaged Date: TSD Accept Date: 01/17/90

Container Type Code: DM Container Count: Container Volume: $\quad 0.210$ Gross Wt:

Previous Container ID:

Current Container ID:

Compaction Wt. (Prorated):

Organic Volume: 22 Organic Wt.:

45.30

Comments/Description:

Designation Code: RE DW Waste \#: D002 D008 WT01

I Generator Information:
Name:
ID: WHC
Facility ID: 23452
Charge Code: K61AW

Location Information:

Area Nane: 200W Facility ID: 224T Module: Surface Area:

Tier Level: Tier Position:

Lab Pack Description:

Lab Pack Flag: N

Physical State Code: S Chemical Nature Code: Primary Waste Type Code: M Secondary Waste Type Code: 1D

Container Size Description: 55 GalloN

Container Storage Category Code:

Content Wrap Category: DM

SDAR Approval \#: 1-1B-1AM-0

Radioactive Information:

Total Alpha (Ci): $1.940 \mathrm{E}+00$

Total Pu Equivalent (Ci): 2.098E+00

Total Beta-Gamma (Ci): 1.000E-03

SWEA General Comments:

Total Pu Fissile Gram Equivalent: 2.366E+01 


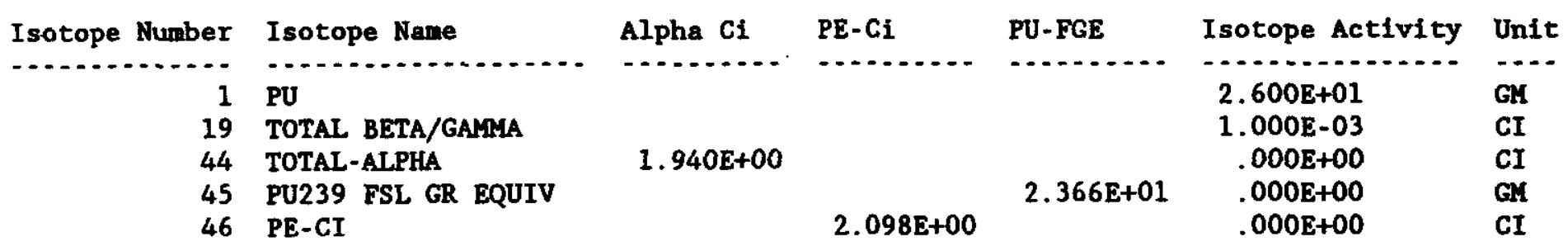

Hazardous Chemical Components:

Component Text

PPM Weight $(\mathrm{kg}) \quad z$

ACID

123.3765

Physical Components:

Content Description

Volume $z$ Weight $(\mathrm{kg})$

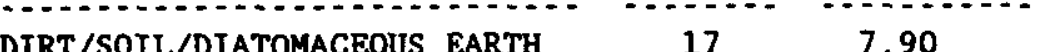

LEAD $60 \quad 123.40$

LEATHER

METAL/IRON/GALVANIZED/SHEET 1100.14

PAPER/CARDBOARD

3.95

18.70

PLASTIC/POLYURATHANE

RUBBER

22.40

STAINLESS STEEL

$\begin{array}{rr}10 & 22.40 \\ 1 & 0.30\end{array}$

Relocation History:

Shipnent Information:

DOE/NRC 741 \#: Profile \#: RSR \#:

Item \#: 1 Manifest \#: 09002 CWDR Number:

NMIT \#: TSD Process: SO1C 
for Generating Company: $x$

for Facility ID: $\boldsymbol{x}$

for Primary Waste Type Code: $x$

for Secondary Waste Type Code: $x$

Container ID: 212-A21207

Location Beginning Coordinates: N

Location Ending Coordinates: N

W

Content Analysis Return Date:

\section{Packaged Date:}

TSD Accept Date: 10/31/90

Certification Date:

Dose Rate: 2.000E+00 Neutron Dose Rate:

Container Type Code: DM Container Count: 1 Container Volume: $\quad 0.210$ Gross Wt:

Previous Container ID:

Organic Volume: 58 Organic Wt.: $\quad 46.41$

Compaction Wt. (Prorated):

\% Content Compaction: Compactor PIN

$\%$ Content Compaction: Compactor PIN

Designation Code: RE DW Waste \#: D007 D008 WT01 WC02

Generator Information:

Name :

ID: WHC

Facility ID: 23452

Charge Code: K6EWA

Location Information:

Area Name: 200W Facility ID: $224 \mathrm{~T}$

Tier Level: Tier Position:

Unit: 201

Module: Surface Area:

Lab Pack Description:

Lab Pack Flag: N

Physical State Code: S Chemical Nature Code: Primary Waste Type Code: M Secondary Waste Type Code: 1D

Container Size Description: 55 GALLON

Container Storage Category Code:

Container Empty Tare Wt.:

SWIMS Waste Description Códe: DS

SWSDR \#: 900370

Content Wrap Category: DM

27.00 Container Thermal Power: 1.000E-01 Container Total Wt.:

Radioactive Information:

Total Alpha (Ci): $3.034 \mathrm{E}+00$

Total Pu Equivalent (Ci): $3.426 \mathrm{E}+00$

Total Beta-Gamma (C1): 2.000E-03

SWEA General Comments: 


\begin{tabular}{|c|c|c|c|c|c|c|}
\hline Isotope Number & Isotope Name & Alpha $\mathrm{CI}$ & $\mathrm{PE}-\mathrm{CI}$ & PU-FGE & Isotope Activity & Unit \\
\hline , n & (1) & $\ldots$ & - . & $\cdots \ldots$ & (300r+o1 & GX \\
\hline $\begin{array}{r}1 \\
19\end{array}$ & $\begin{array}{l}\text { PU } \\
\text { TOTAL BETA/GAMMA }\end{array}$ & & & & $\begin{array}{l}3.900 \mathrm{E}+01 \\
2.000 \mathrm{E}-03\end{array}$ & $\begin{array}{l}\text { GII } \\
\text { CI }\end{array}$ \\
\hline 44 & TOTAL-ALPHA & $3.034 E+00$ & & & $.000 \mathrm{E}+00$ & CI \\
\hline 45 & PU239 FSL GR EQUIV & & & $3.707 E+01$ & $.000 \mathrm{E}+00$ & GI \\
\hline 46 & PE-CI & & $3.426 \mathrm{E}+00$ & & $.000 \mathrm{E}+00$ & CI \\
\hline
\end{tabular}

Hazardous Chemical Components:

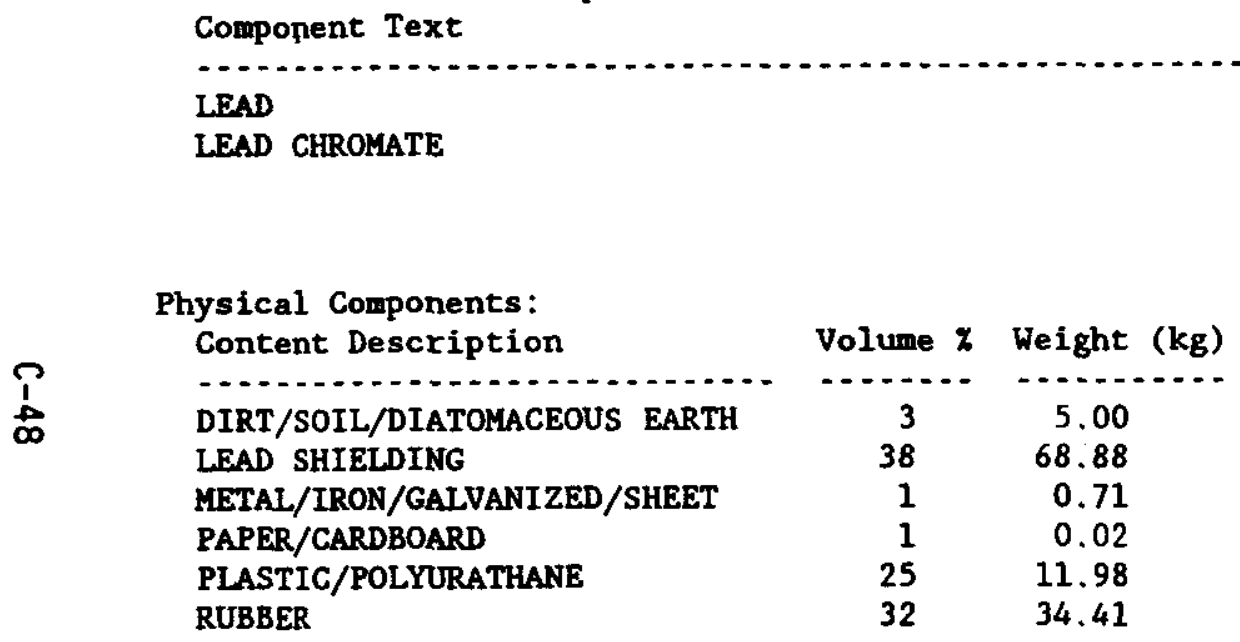

PPM Weight $(\mathrm{kg}) \quad x$

Component Text

LEAD $\quad 68.1746$

$\begin{array}{lr}\text { LEAD CHROMATE } & 0.6999\end{array}$

Relocation History:

Shipment Information:

DOE/NRC 741 \#: $\quad$ Profile \#: RSR \#:

Item \# 1 Manifest \#: 09025 CWDR Number:

NMIT \#: TSD Process: S01C 
for Generating Company: $*$

for Facility ID: $x$

for Primary Waste Type Code: $z$

for Secondary Waste Type Code: $x$

Container ID: $220-\mathrm{A} 21295$

Location Beginning Coordinates: $N$ Content Analysis Return Date:

Certification Date:

Dose Rate: 4.000E+00 Neutron Dose Rate:

Previous Container ID:

Current Container ID:

Compaction Wt. (Prorated):

Comments/Description:

Designation Code: RE DW Waste \#: D002 D005 D006 D007 D008 D009 WT01 WC02

Generator Information:

$$
\text { Name: }
$$

Location Information:

Area Name: 200W Facility ID: $224 \mathrm{~T}$

Tier Level: Tier Position:
W
Location Ending Coordinates:
Packaged Date:
Container Type Code: DM TSD

Container Type Code: DH
TSD Accept Date: 12/10/90

0.210 Gross wt:

150.00
Lab Pack Description:

Lab Pack Flag: N

Physical State Code: S Chemical Nature Code: Primary Waste Type Code: M Secondary Waste Type Code: 1D Container Size Description: 55 GALLON

Container Storage Category Code:

Container Empty Tare Wt.:

SWIMS Waste Description Code: DS

SWSDR \#: 900444

Content Wrap Category: DM

27.00 Container Thermal Power: 1.000E-01 Container Total wt.:

SDAR Approval \#: 1-1B-1AM-0

Radioactive Information:

Total Alpha (Ci):

Total Pu Equivalent (Ci):

Total Beta-Gamma (Ci): 4.000E-03

SWEA General Comments: 


$\begin{aligned} \text { Isotope Number } & \text { Isotope Name } & \text { Alpha Ci } & \text { PE-Ci } & \text { PU-FGE } & \text { Isotope Activity Unit } \\ 1 & \text { PU } & & & 4.400 E+01 & \text { GM } \\ 19 & \text { TOTAL BETA/GAMMA } & & & 4.000 E-03 & \text { CI }\end{aligned}$

Hazardous Chemical Components:
Component Text
BARIUM

Physical Components:

Content Description

Volume $\chi$ Weight $(\mathrm{kg})$

CLOTH/RAGS/NYLON

DIRT/SOIL/DIATOMACEOUS EARTH $\quad 16 \quad 16.62$

GLASS

LEAD

METAL/IRON/GALVANIZED/SHEET

PLASTIC/POLYURATHANE

1.20

67.06

0.53

4.70

RUBBER

28.29

Relocation History:

Shipment Information:

DOE/NRC 741 \#: Profile \#: RSR \#:

Item \#: 1 Manifest \#: (PFP)09026 CWDR Number:

NMIT \#: TSD Process: S01C 
Container ID: RHZ-213-A22311

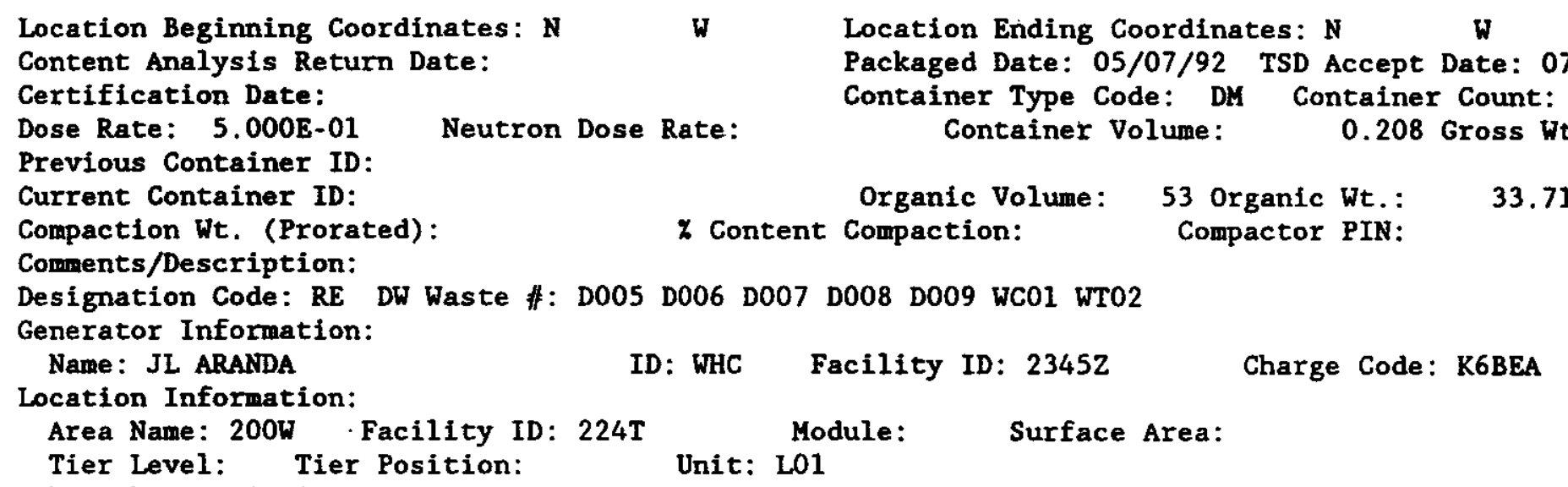

Packaged Date: 05/07/92 TSD Accept Date: 07/15/92

Content Analysis Return Date:

Cation :

Previous Container ID:

Generator Information:

Name: JL ARANDA

Tier Level: Tier Position:

Lab Pack Description:

Lab Pack Flag: N

Physical State Code: S Chemical Nature Code: I Primary Waste Type Code: M Secondary Waste Type Code: 1A Container Size Description: 55 GALLON

Container Storage Category Code: OMW SWIMS Waste Description Code: DS SWSDR \#:

Container Empty Tare Wt.: $\quad 31.00$ Container Thermal Power: 1.000E-01 Container Total Wt.: 142.00

Content Urap Category: DM SDAR Approval \#: 14-1D-1AM-0301

Radioactive Information:

Total Alpha (Ci): .000E+00

Total Pu Equivalent (Ci): $\quad .000 \mathrm{E}+00$

Total Beta-Gamma (Ci): .000E+00

SWEA General Comments: 


$\begin{array}{rlrrrrr}\text { Isstope Number } & \text { Isotope Name } & \text { Alpha } C i & \text { PE }-\mathrm{Ci} & \text { PU-FGE } & \text { Isotope Activity } & \text { Unit } \\ 1 & \text { PU } & 9.899 \mathrm{E}-01 & 1.111 \mathrm{E}+00 & 1.235 \mathrm{E}+01 & 1.291 \mathrm{E}+01 & \mathrm{GM} \\ 19 & \text { TOTAL BETA/GAMMA } & & & & & \\ 26 & \text { AM-241 } & 3.820 \mathrm{E}-02 & 3.820 \mathrm{E}-02 & 2.000 \mathrm{E}-04 & 8.585 \mathrm{E}-02 & \text { GI }\end{array}$

Hazardous Chemical Components:
Component Text
BARIUM

Physical Components:

Content Description

\begin{tabular}{rc} 
Volume $z$ & Weight $(\mathrm{kg})$ \\
\hline 1 & 0.01 \\
20 & 27.99 \\
1 & 0.60 \\
25 & 78.40 \\
1 & 1.30 \\
1 & 0.20 \\
8 & 5.50 \\
40 & 26.50 \\
3 & 1.50
\end{tabular}

Relocation History:

Shipment Information:

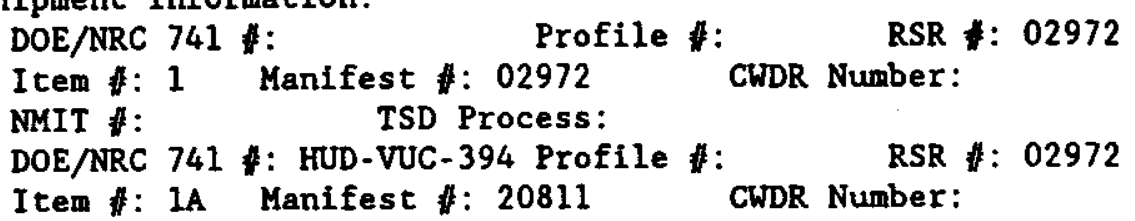


for Generating Company:

for Facility ID: $: T$

for Primary Waste Type Code: $z$

for Secondary Waste Type Code: $x$

\section{Container ID: RHZ-218-A22350}

Location Beginning Coordinates: $\mathbb{N}$ Content Analysis Return Date:

Certification Date:

Dose Rate: 2.700E-01 Neutron Dose Rate:

Previous Container ID:

Current Container ID:

Compaction Wt. (Prorated):

Comments/Description:

\section{Designation Code:}

Generator Information:

Name: JL ARANDA

Location Information:

Area Name: 200W Facility ID: 224T

Tier Leve1: Tier Position:

Lab Pack Description:

Lab Pack Flag: N

Physical State Code: S Chemical Nature Code: I Primary Waste Type Code: $R$ Secondary Waste Type Code: 1C Container Size Description: 55 GALLON

Container Storage Category Code:

Container Empty Tare Wt.:

Content Wrap Category: DM

Radioactive Information:

Total Alpha (Ci): $1.583 E+00$

Total Pu Equivalent (Ci): $1.768 \mathrm{E}+00$

SWIMS Waste Description Code: DS SWSDR \#:

31.00 Container Thermal Power: 1.000E-01 Container Total Wt.: 37.00 SDAR Approval \#: 14-1D-1B-0301

SWEA General Comments:
Total Beta-Gamma (Ci): .000E+00

Total Pu Fissile Gram Equivalent: $1.900 \mathrm{E}+01$ 


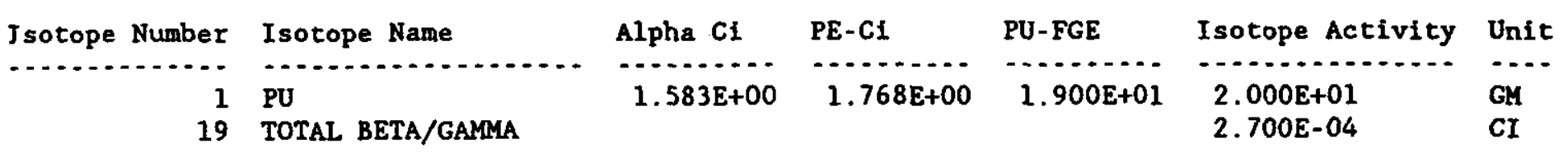

Hazardous Chemical Components:

$$
\text { Component Text }
$$

\begin{tabular}{|c|c|c|}
\hline $\begin{array}{l}\text { Physical Components: } \\
\text { Content Description }\end{array}$ & Volume $x$ & Weight (kg) \\
\hline (1) & ........ & 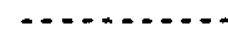 \\
\hline CLOTH/RAGS/NYLON & 15 & 5.00 \\
\hline DIRT/SOIL/DIATOMACEOUS EARTH & 10 & 4.00 \\
\hline METAL/IRON/GALVANIZED/SHEET & 20 & 8.00 \\
\hline PAPER/CARDBOARD & 20 & 7.00 \\
\hline PLASTIC/POLYURATHANE & 30 & 11.00 \\
\hline RUBBER & 5 & 2.00 \\
\hline
\end{tabular}

Relocation History:

Shipment Information: DOE/NRC 741 \#:

Item \#: 1 Manifest \#: 02935

Profile \#: $\quad$ RSR \#: 02935

NMIT \#:

TSD Process:

CWDR Number: 
Container ID: RHZ-213-A22515

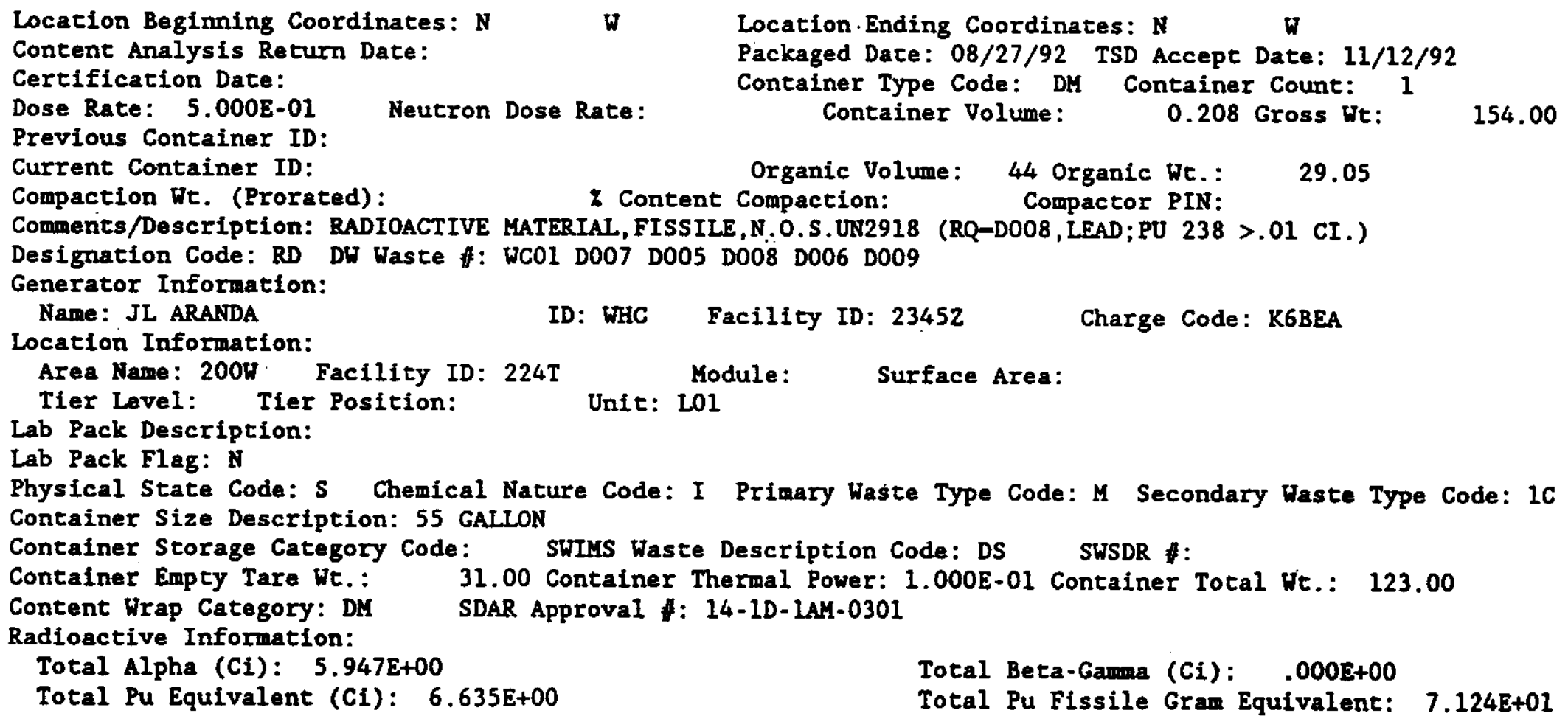




\begin{tabular}{|c|c|c|c|c|c|c|}
\hline pe Number & Isotope Nane & Alpha $\mathrm{Cl}$ & PE-CI & PU-FGE & Isotope Activity & nit \\
\hline & $\ldots \ldots$ & $\ldots \ldots$ & $\ldots \ldots$ & $\cdots$ & - & \\
\hline $\begin{array}{r}1 \\
19 \\
26\end{array}$ & $\begin{array}{l}\text { PU } \\
\text { TOTAL BETA/GANRA } \\
\text { AY-24I }\end{array}$ & $5.712 \mathrm{E}+00$ & $6.400 \mathrm{E}+00$ & $7.124 E+01$ & $\begin{array}{l}7.493 E+01 \\
5.000 E-04 \\
6.880 E-02\end{array}$ & $\begin{array}{l}\text { GI } \\
\text { CI } \\
\text { GM }\end{array}$ \\
\hline
\end{tabular}

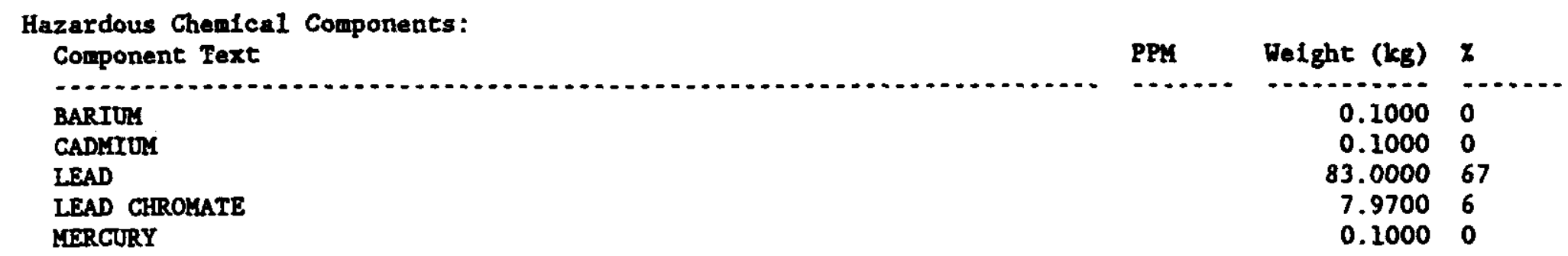

Physical Components: Content Description

\begin{tabular}{cc} 
Volume $x$ & Weight $(\mathbf{k g})$ \\
\hline 2 & 2.48 \\
2 & 0.20 \\
52 & 91.27 \\
3 & 0.50 \\
15 & 8.85 \\
23 & 19.50 \\
3 & 0.20
\end{tabular}

Relocation History:

Shipment Information: DOE/NRC 741 \#: Profile \#: RSR \#: 02942 Iten : 1 Manifest \#: 02942 CWDR Number:

NMIT : TSD Process:

DOE/NRC 741 \#: Profile \#: $\quad$ RSR \#: 02942

Item *: IA Manifest \#: 21429 CWDR Number:

NAIT \#: TSD Process: S01C 
WHC-EP-0621

APPENDIX D

ORIGINAL SOLIO WASTE INFORMATION TRACKING SYSTEM

RECORDS FOR ALL 55-GALLON WASTE DRUMS WITH GREATER THAN 300 GRAMS OF PLUTONIUM

D-1 
WHC-EP-0621

This page intentionally left blank.

D-2 


\section{ORIGINAL SOLID WASTE INFORMATION TRACKING SYSTEM \\ RECORDS FOR ALL 55-GALLON WASTE DRUMS WITH GREATER THAN 300 GRAMS OF PLUTONIUM}

This appendix contains complete Solid Waste Information Tracking System (SWITS) records for the ten 55-gal waste drums and 22 other containers that have over $300 \mathrm{~g}$ of plutonium stored in them.

Each SWITS data run in this appendix is preceded by the query used to generate the data. 
WHC-EP-0621

This page intentionally left blank. 
WHC-EP-0621

\section{APPENDIX 0.1}

\section{5-GALLON WASTE DRUNS WITH GREATER THAN} 300 GRAMS OF PLUTONIUM

D-5 
WHC-EP-0621

This page intentionally left blank.

D-6 


\section{5-GALLON WASTE DRUMS WITH GREATER THAN 300 GRAMS OF PLUTONIUM}

This subappendix contains the Solid Waste Information Tracking System (SWITS) records for the ten 55-gal drums from the Plutonium Finishing Plant (PFP) that contain greater than $300 \mathrm{~g}$ of plutonium. All 10 drums were filled to the standard weight of $68 \mathrm{~kg}$ and all were disposed of on October 28, 1980. 
set pagesize 55

set linesize 90

set newpage 0

spool judy.srep

select con_pkg_id, rad̄qty, con_size_descr

from radwaste, isoqty

where rdet swtyp cd between ' $1 A$ ' and ' $1 E$ ' and con size descr = '55 GALLON' and con_srce_facil_íd $=' 2345 \mathrm{~T} '$ and rad_iso_num in $(1,21,2 \overline{2}, 26,41,52,57,87,97,98$, $100,104, \overline{1} 11,14 \overline{6}, 147)$ and rad_qty $>=-130 \overline{0}$ and con_pkg_id = rad_pkg_id ; spool off 
for Secondary Waste Type Code: $x$

\section{Container ID: $\mathrm{T}-102$}

Location Beginning Coordinates: N N40190 w w77582 Location Ending Coordinates: N N40190 W w77557

Content Analysis Return Date:

Packaged Date:

TSD Accept Date: $10 / 28 / 80$

Certification Date:

Container Type Code: DM Container Count: 1

Dose Rate: 1.000E+00 Neutron Dase Rate:

Container Volume:

0.210 Gross Wt:

68.04

Previous Container ID:

Organic Volume:

Organic Wt.:

Compaction Wt. (Prorated):

$x$ Content Compaction:

Compactor PIN:

Comments/Description:

Designation Code:

DW Waste \#:

Generator Information:

Name:

ID: WHC

Facility ID: $2345 Z$

Charge Code:'

Location Information:

Area Name: 200W Facility ID: 218W4C Module: Surface Area:

Tier Level: Tier Position: Unit: T01

Lab Pack Description:

Lab Pack Flag: N

Physical State Code: S Chemical Nature Code: Primary Waste Type Code: $R$ Secondary Waste Type Code: 1A

Container Size Description: 55 GALLON

Container Storage Category Code: SWIMS Waste Description Code: DS

Container Empty Tare Wt.: 27.00 Container Thermal Power:

Content Wrap Category:

SDAR Approval \#:

SWSDR : 801541

Radioactive Information:

Total Alpha (Ci):

Total Pu Equivalent (Ci):

Total Beta-Gamma (Ci): 1.000E-03.

SWEA General Comments:

Total Pu Fissile Gram Equivalent: 


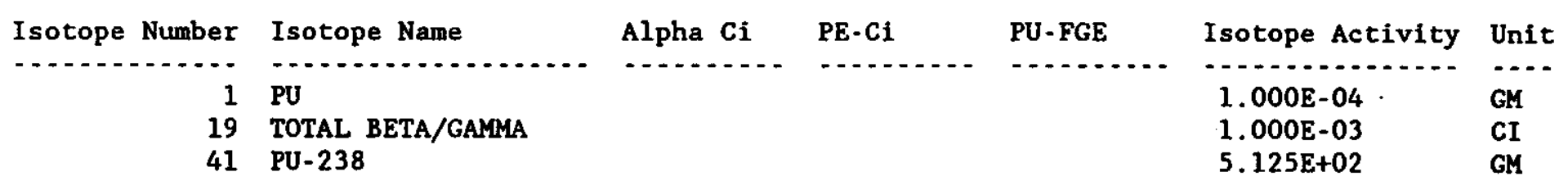

Hazardous Chemical Components: Component Text

-......

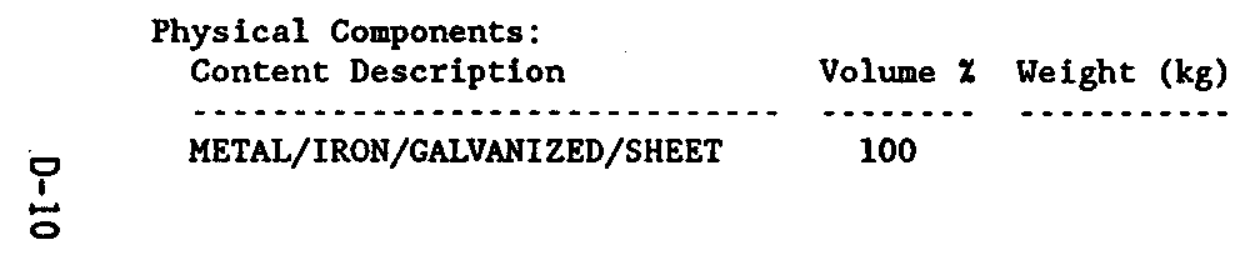

Relocation History:

Shipment Information: 
for Generating Company: $x$

for Facility ID: $x$

for Primary Waste Type Code: $\chi$

for Secondary Waste Type Code: $x$

Container ID: T-103

Location Beginning Coordinates: N N40190 W w77582 Location Ending Coordinates: N N40190 W w77557 Content Analysis Return Date:

Certification Date:

Dose Rate: 1.000E+00 Neutron Dose Rate:

Packaged Date:

TSD Accept Date: $10 / 28 / 80$

Container Type Code: DM Container Count:
Container Volume:
0.210 Gross Wt:
68.04

Previous Container ID:

Current Container ID:

Compaction Wt. (Prorated):

Organic Volume: Organic Wt.:

Comments/Description:

Designation Code: DW Waste \#:

Generator Information:

Name :

z Content Compaction:

Compactor PIN:

Location Information:

Area Name: 200W Facility ID: 218W4C Module: Surface Area:

Tier Level: Tier Position: Unit: T01

Lab Pack Description:

Lab Pack Flag: N

Physical State Code: S Chemical Nature Code: Primary Waste Type Code: R Secondary Waste Type Code: 1A

Container Size Description: 55 GALLON

Container Storage Category Code: SWIMS Waste Description Code: DS

Container Empty Tare Wt.: 27.00 Container Thermal Power:

Content Wrap Category:

SDAR Approval \#:

SWSDR : 801547

Radioactive Information:

Total Alpha (Ci):

Total Pu Equivalent (Ci):

Total Beta-Gamma (Ci): 1.000E-03

SWEA General Comments :

Total Pu Fissile Gram Equivalent: 


\begin{tabular}{|c|c|c|c|c|c|c|}
\hline Isotope Number & Isotope Name & Alpha $\mathrm{Ci}$ & $P E-C 1$ & PU-FGE & Isotope Activity & Unit \\
\hline $\begin{array}{llll}1 & \end{array}$ & PU & $\cdots$ & n. & $\ldots$ & $1.000 \mathrm{E}-04$ & \\
\hline & TOTAL BETA/GAMAMA & & & & $1.000 \mathrm{E}-03$ & I \\
\hline & $\mathrm{PU}-238$ & & & & $5.126 \mathrm{E}+02$ & MH \\
\hline
\end{tabular}

\section{Hazardous Chenicsl Components:}

Component Text

PPM Weight $(\mathrm{kg}) \quad x$

C.
Physical Components:
Content Description
Volume $z$ Weight $(\mathrm{kg})$

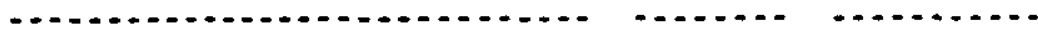
METAL/IRON/GALVANIZED/SHEET 100

Shipment Information: 
Container ID: $\mathrm{T}-104$

Location Beginning Coordinates: N N40190 w w77582 Location Ending Coordinates: N N40190 w w77557 Content Analysis Return Date: Packaged Date:

TSD Accept Date: $10 / 28 / 80$

Certification Date:

Container Type Code: DM Container Count:
Container Volume:
0.210 Gross wt:
68.04

Previous Container ID:

Current Container ID:

Compaction Wt. (Prorated):

Órganic Volume: Organic Wt.:

Comments/Description:

Designation Code:

DW Waste \#:

Generator Information:

Name :

ID: WHC

Facility ID: $2345 \mathrm{Z}$

Charge Code:

Location Information:

Area Name: 200W Facility ID: 218W4C

Tier Level: Tier Position:

Module: Surface Area:

Lab Pack Description:

Lab Pack Flag: N

Physical State Code: S Chemical Nature Code: Primary Waste Type Code: $R$ Secondary Waste Type Code: 1A

Container Size Description: 55 GALLON

Container Storage Category Code:

Container Empty Tare Wt.:

27.00 Container Thermal Power:

Content Wrap Category:

Compactor PIN:

Radioactive Information:

Total Alpha (Ci):

Total Pu Equivalent (Ci):

Total Pu Fissile Gram Equivalent:

SWEA General Comments: 


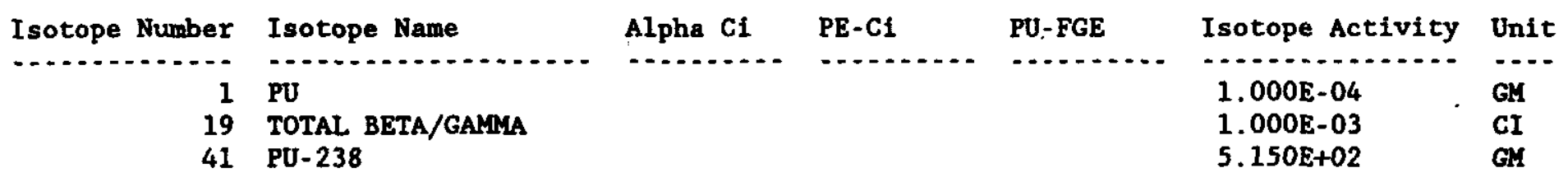

Hazardous Chemical Components:

Component Text

PPM

Weight $(\mathrm{kg}) \quad$

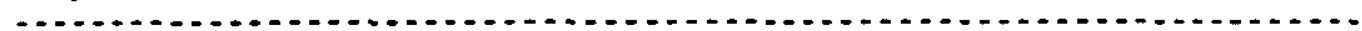

(.....................
Physical Components: Content Description
Volume $x$ Weight $(\mathrm{kg})$

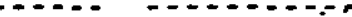
METAL/IRON/GALVANIZED/SHEET
100

$\stackrel{1}{\stackrel{1}{0}}$

Relocation History:

Shipment Information: 
for Generating Company: $\mathcal{X}$

for Facility ID: 7

for Primary Waste Type Code: $x$

for Secondary Waste Type Code: $x$

\section{Container ID: T-105}

Location Beginning Coordinates: N N40190 w w77582 Location Ending Coordinates: N N40190 W w77557

Content Analysis Return Date:

Certification Date:

Packaged Date: TSD Accept Date: 10/28/80

Dose Rate: $1.000 \mathrm{E}+00$

Neutron Dose Rate:

Container Type Code:

Container Count: 1

Previous Container ID:

Current Container ID:

- Container Volume:

0.210 Gross Wt:

68.04

Compaction Wt. (Prorated):

Organic Volume:

Organic Wt.:

Comments/Description:

Designation Code: DW Waste \#:

Generator Information:

Nane:

z Content Compaction:

Compactor PIN :

Location Information:

Area Nane: 200W Facility ID: 218W4C Module: Surface Area:

Tier Level: Tier Position:

Lab Pack Description:

Lab Pack Flag: N

Physical State Code: S Chemical Nature Code: Primary Waste Type Code: R Secondary Waste Type Code: 1A

Container Size Description: 55 GALLON

Container Storage Category Code: SWIMS Waste Description Code: DS

Container Empty Tare Wt.: 27.00 Container Thermal Power:

Content Wrap Category:

SDAR APproval \#:

SWSDR \#: 801548

Radioactive Information:

Total Alpha (Ci):

Total Pu Equivalent (Ci):

Total Beta-Gamma (Ci): 1.000E-03

SWEA General Comments: 


$\begin{array}{rlrrr}\text { Isotope Number Isotope Name } & \text { Alpha Ci } & \text { PE-Ci } & \text { PU-FGE } & \text { Isotope Activity Unit } \\ 1 & \text { PU } & & & 1.000 E-04 \\ 19 & \text { TOTAL BETA/GAMMA } & & & 1.000 E-03 \\ 41 & \text { PU-238 } & & & 5.143 E+02\end{array}$

Hazardous Chemical Components:

Component Text

PPM Weight (kg) $x$

...... -......... ......

Physical Components:

Content Description

-...-......

METAL/IRON/GALVANIZED/SHEET

Volume $x$ Weight $(\mathrm{kg})$

100....................

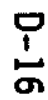

Relocation History:

Shipment Information: 
for Generating Company: $x$

for Facility ID: $x$

for Primary Waste Type Code: $x$

for Secondary Waste Type Code: $x$

Container ID: T-106

Location Beginning Coordinates: N N40190 w w77582 Location Ending Coordinates: N N40190 W w77557

Content Analysis Return Date:

Packaged Date:

TSD Accept Date: $10 / 28 / 80$

Certification Date:

Container Type Code: DM Container Count:
Container Volume:
0.210 Gross Wt:
68.04

Previous Container ID:

Organic Volume:

Organic Wt.:

Current Container ID:

Compaction Wt. (Prorated):

\% Content Compaction:

Compactor PIN:

Comments/Description:

Designation Code:

DW Waste \#:

Generator Information:

Name

ID: WHC

Facility ID: $2345 Z$

Charge Code:

Location Information:

Area Nane: 200W Facility ID: 218W4C Module: Surface Area:

Tier Leve1: Tier Position: Unit: T01

Lab Pack Description:

Lab Pack Flag: N

Physical State Code: S Chemical Nature Code: Primary Waste Type Code: R Secondary Waste Type Code: 1A

Container Size Description: 55 GALLON

Container Storage Category Code:

Container Empty Tare Wt.:

SWIMS Waste Description Code: DS

Content Urap Category:

27.00 Container Thermal Power

SWSDR \#: 801549

Radioactive Information

Total Alpha (Ci):

Total Pu Equivalent ( $\mathrm{Ci}$ ):

Total Beta-Gamma (Ci): 1.000E-03

SWEA General Comments:

Total Pu Fissile Gram Equivalent: 


\begin{tabular}{|c|c|c|c|c|c|c|}
\hline Isotope Number & Isotope Name & Alpha Ci & $\mathrm{PE}-\mathrm{CI}$ & PU-FGE & Isotope Activity & Unit \\
\hline 1 & PU & n. & (n. & , n & $1.000 \mathrm{E}-04$ & \\
\hline 19 & TOTAL BETA/GAMMA & & & & $1.000 E-03$ & CI \\
\hline
\end{tabular}

Hazardous Chemical Components:

Component Text

PPM

Weight $(\mathrm{kg}) \quad x$

C...

(.............................

Physical Components:

Content Description

METAL/IRON/GALVANIZED/SHEET

Volume $x$ Weight $(\mathrm{kg})$

100

Shipment Information: 
for Generating Company: $x$ THRU: $12 / 04 / 92$

for Facility ID: $x$

for Primary Waste Type Code: $x$

for Secondary Waste Type Code: $x$

Container ID: $\mathrm{T}-108$

Location Beginning Coordinates: N N40190 w w77582 Location Ending Coordinates: N N40190 w w77557

Content Analysis Return Date:

Packaged Date:

TSD Accept Date: $10 / 28 / 80$

Certification Date:

\section{Container Iype}

Dose Rate: 1.000E+00 Neutron Dose Rate: Container Volume:

0.210 Gross Wt:

68.04

Previous Container ID:

Current Container ID:

Compaction Wt. (Prorated):

Organic Volume:

Organic Wt.:

Comments/Description:

Designation Code:

z Content Compaction:

Compactor PIN:

Generator Information:

$$
\text { Name: }
$$

DW Waste \#:

Location Information:

Area Name: 200W Facility ID: 218W4C Module: Surface Area:

Tier Level: Tier Position: Unit: T01

Lab Pack Description:

Lab Pack Flag: N

Physical State Code: S Chemical Nature Code: Primary Waste Type Code: $R$ Secondary Waste Type Code: 1A Container Size Description: 55 GaLLON

Container Storage Category Code: SWIMS Waste Desoription Code: DS

Container Empty Tare Wt.: 27.00 Container Thermal Power:

Content Wrap Category: 27.00 Container
SDAR Approval \#:

SWSDR \# : 801550

Radioactive Information:

Total Alpha (Ci):

Total Pu Equivalent (Ci):

Total Beta-Gamma (Ci): 1.000E-03

SWEA General Comments:

Total Pu Fissile Gram Equivalent: 


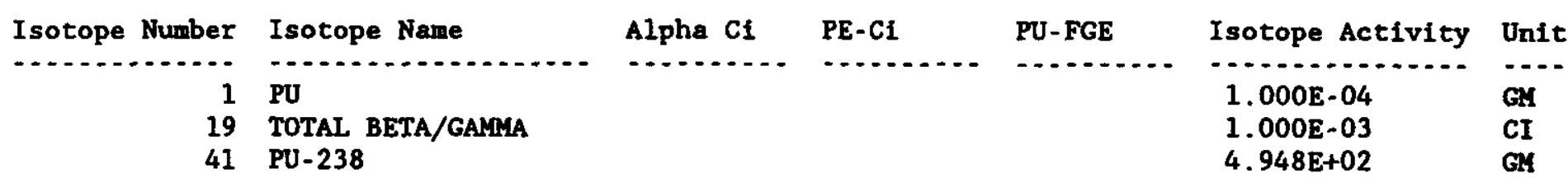

Hazardous Chemical Components:

$$
\text { Component Text }
$$

Physical Components: Content Description

METAL/IRON/GALVANIZED/SHEET

Volume $x$ Weight $(\mathrm{kg})$

-....... ..........

100

Shipment Information: 
for Facility ID: $\boldsymbol{Z}$

for Primary Waste Type Code: $x$

for Secondary Waste Type Code: $x$

Container ID: T-109

Location Beginning Coordinates: N N40190 W w77582 Location Ending Coordinates: N N40190 w w77557

Content Analysis Return Date:

Packaged Date:

TSD Accept Date: $10 / 28 / 80$

Certification Date:

Container Type Code: D

Dose Rate: 1.000E+00 Neutron Dose Rate:

Container Volume:

0.210 Gross Wt:

68.04

Previous Container ID:

Current Container ID:

Organic Volume: Organic Wt.:

Compaction Wt. (Prorated): $\quad$ Content Compaction: Compactor PIN:

Comments/Description:

Designation Code:

DW Waste :

I Generator Information:

Name:

ID: WHC

Facility ID: 23452

Charge Code:

Location Information:

Area Name: 200W Facility ID: 218W4C

Tier Level: Tier Position:

Module: $\quad$ Surface Area :

Lab Pack Description:

Lab Pack Flag: N

Physical State Code: S Chemical Nature Code: Primary Waste Type Code: $R$ Secondary Waste Type Code: IA Container Size Description: 55 GALLON

Container Empty Tare Wt.

SWIMS Waste Description Code: DS

Content Wrap Category:

27.00 Container Thermal Power: SDAR Approval \#:

Radioactive Information:

Total Alpha (Ci):

Total Pu Equivalent (Ci):

SWEA General Comments:

Container Total Wt.: 


\begin{tabular}{|c|c|c|c|c|c|}
\hline Isotope Number & Isotope Name & Alpha Ci & PE-Ci & PU-FGE & Isotope Activity \\
\hline $\begin{array}{r}1 \\
19\end{array}$ & $\begin{array}{l}\text { PU } \\
\text { TOTAL BETA/GAMMA } \\
\text { PU-238 }\end{array}$ & $\cdots$ & , & $\cdots$ & $\begin{array}{l}1.000 \mathrm{E}-04 \\
1.000 \mathrm{E}-03 \\
4.839 \mathrm{E}+02\end{array}$ \\
\hline
\end{tabular}

Hazardous Chemical Components: Component Text

PPM Weight $(\mathrm{kg}) \quad x$

Physical Components: Content Description (1) Volume $\chi$ Weight $(\mathrm{kg})$ METAL/IRON/GALVANIZED/SHEET

Relocation History:

Shipment Information: 
for Generating Company: $x$

for Facility ID: $x$

for Primary Waste Type Code: $z$

for Secondary Waste Type Code: $x$

Container ID: T-110

Location Beginning Coordinates: N N40190 W w77582 Location Ending Coordinates: N N40190 W W77557

Content Analysis Return Date:

Certification Date:

Packaged Date:

TSD Accept Date: $10 / 28 / 80$

Dose Rate: 1.000E+00 Neutron Dose Rate:

Container Type Code:

Container Volume:

Container Count:

68.04

Previous Container ID:

Current Container ID:

Organic Volume: Organic Wt.:

Compaction Wt. (Prorated): \% Content Compaction: Compactor PIN:

Comments/Description:

Designation Code: DW Waste \#:

Generator Infornation:

Name:

Location Information:

Area Nane: 200W Facility ID: 218W4C Module: Surface Area:

Tier Level: Tier Position: Unit: T01

Lab Pack Description:

Lab Pack Flag: N

Physical State Code: S Chemical Nature Code: Primary Waste Type Code: R Secondary Waste Type Code: 1A

Container Size Description: 55 GaLLON

Container Storage Category Code:

SwIMS Waste Description Code: DS

Container Expty Tare Wt.: 27.00 Container Thermal Power:

Content Wrap Category:

Radioactive Information:

Total Alpha (Ci):

Total Pu Equivalent (Ci):

SWEA General Coments: 


$\begin{array}{rlrrrr}\text { Isotope Number } & \text { Isotope Name } & \text { Alpha } \mathrm{Ci} & \text { PE-Ci } & \text { PU-FGE } & \text { Isotope Activity Unit } \\ 1 & \text { PU } & & & 1.000 E-04 & \text { GM } \\ 19 & \text { TOTAL BETA/GAMMA } & & & 1.000 E-03 & \text { CI } \\ 41 & \text { PU-238 } & & & 3.134 E+02 & \text { GH }\end{array}$

Hazardous Chemical Components:

Component Text

....

..................
Physical Components:
Content Description
Volume $\%$ Weight (kg)
100

METAL/IRON/GALVANIZED/SHEET

100

Shipment Information: 
for Generating Company:

for Facility ID: $x$

for Primary Waste Type Code: $z$

for Secondary Waste Type Code: $x$

Container ID: T-112

Location Beginning Coordinates: N 140190 w w77582 Location Ending Coordinates: N N40190 W w77557

Content Analysis Return Date:

Packaged Date:

TSD Accept Date: $10 / 28 / 80$

Certification Date:

Container Type Code: D

Container Count: 1

Dose Rate: ' 1.000E+00 Neutron Dose Rate:

Container Volume:

0.210 Gross Wt:

68.04

Previous Container ID:

Current Container ID:

Organic Volume:

Organic Wt.:

Compaction Wt. (Prorated) :

z Content Compaction:

Compactor PIN

Comments/Description:

Designation Code:

DW Waste \#:

Generator Information:

Name:

ID: WHC

Facility ID: 23452

Charge Code:

Location Information:

Area Name: 200W Facility ID: 218W4C

Module: Surface Area:

Tier Level: Tier Position:

Unit: T01

Lab Pack Description:

Lab Pack Flag: N

Physical State Code: S Chemical Nature Code: Primary Waste Type Code: $R$ Secondary Waste Type Code: 1A

Container Size Description: 55 GALLON

Container Storage Category Code:

Container Empty Tare Wt.:

27.00 Container Thermal Power:

SuIMS Waste Description Code: DS

Content Wrap Category: SDAR Approval \#:

Radioactive Information

Total Alpha (Ci):

Total Pu Equivalent (Ci):

SWEA General Comments:

Total Beta-Gamma (Ci): 1.000E-03

Total Pu Fissile Gram Equivalent:

SWSDR \#: 801545

Container Total Wt.: 


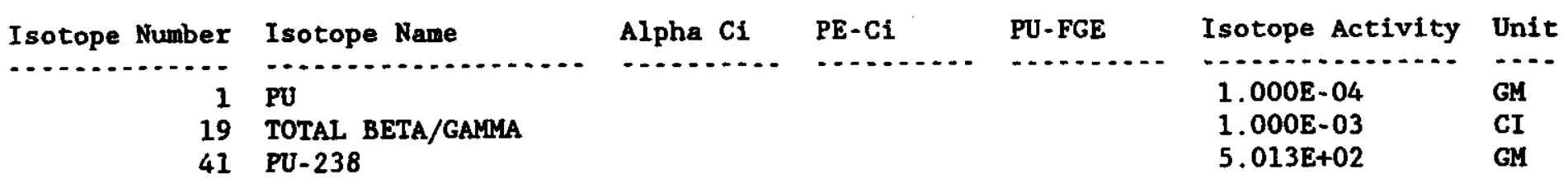

Hazardous Chemical Components:

Component Text

PPM

Weight (kg) $x$

-...... -..........

Physical Components:

Content Description

(1)

METAL/IRON/GALVANIZED/SHEET

Volume $x$ Weight $(\mathrm{kg})$

100

0
1
o

Relocation History:

Shipment Information: 
for Generating Company: $x$

for Facility ID: $z$

for Primary Waste Type Code: $\chi$

for Secondary Waste Type Code: $x$

\section{Container ID: $\mathrm{T}-113$}

Location Beginning Coordinates: N N40190 W w77582 Location Ending Coordinates: N N40190 W W77557

Content Analysis Return Date:

Certification Date:

Dose Rate: 1.000E+00 Neutron Dose Rate:

Packaged Date:

TSD Accept Date: $10 / 28 / 80$

Pose Rate: 1.000E+00

Current Container ID:

Compaction Wt. (Prorated) :
Comments/Description:

Container Type Code: DM Container Count:

$$
\text { Container. Volume: } \quad 0.210 \text { Gross Wt: }
$$

Designation Code:

Organic Volune: Organic Wt.:

Name:
Location Information:

DW Waste \#:

Area Name: 200w Facility ID: 218W4C

Tier Level: Tier Position:

$x$ Content Compaction:

Compactor PIN:

Lab Pack Description:

Lab Pack Flag: N

Physical State Code: S Chemical Nature Code: Primary Waste Type Code: R Secondary Waste Type Code: 1A Container Size Description: 55 GALLON

Container Storage Category Code:

Container Empty Tare Wt.

SWIMS Waste Description Code: DS

Content Wrap Category:

27.00 Container Thermal Power:

SDAR Approval \#:

Radioactive Information:

Total Alpha (Ci):

Total Pu Equivalent (Ci):

Total Beta-Gamma (Ci): 1.000E-03

SWEA General Comments:

Total Pu Fissile Gram Equivalent: 


\begin{tabular}{|c|c|c|c|c|c|c|}
\hline Isotope Number & Isotope Name & Alpha Ci & $P E-C I$ & PU-FGE & Isotope Activity & Unit \\
\hline $\begin{array}{r}1 \\
19 \\
41\end{array}$ & $\begin{array}{l}\text { PU } \\
\text { TOTAL BETA/GAMMA } \\
\text { PU-238 }\end{array}$ & $\ldots \ldots \ldots$ & $\ldots \ldots \ldots$ & $=\ldots \ldots \ldots$ & $\begin{array}{l}1.000 \mathrm{E}-04 \\
1.000 \mathrm{E}-03 \\
3.971 \mathrm{E}+02\end{array}$ & $\begin{array}{l}\text { GY } \\
\text { CI } \\
\text { GM }\end{array}$ \\
\hline
\end{tabular}

Hazardous Chemical Components: Component Text

\section{Physical Components:}

Content Description

Volume $x$ Weight $(\mathrm{kg})$

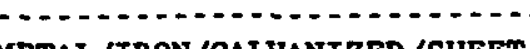

100

METAL/IRON/GALVANIZED/SHEET

Shipment Information: 
WHC-EP-0621

APPENDIX 0.2

CONTAINERS OTHER THAN 55-GALLON DRUMS WITH GREATER THAN 300 GRAMS OF PLUTONIUN 
WHC-EP-0621

This page intentionally left blank. 


\section{CONTAINERS OTHER THAN 55-GALLON DRUMS WITH GREATER THAN 300 GRAMS OF PLUTONIUM}

This subappendix contains Solid Waste Information Tracking System (SWITS) records for the 22 containers other then 55-gal drums that contain greater than $300 \mathrm{~g}$ of plutonium. These containers had weights ranging from 136 to $197 \mathrm{~kg}$ and were all disposed of between 1980 and 1982 . 
WHC-EP-0621

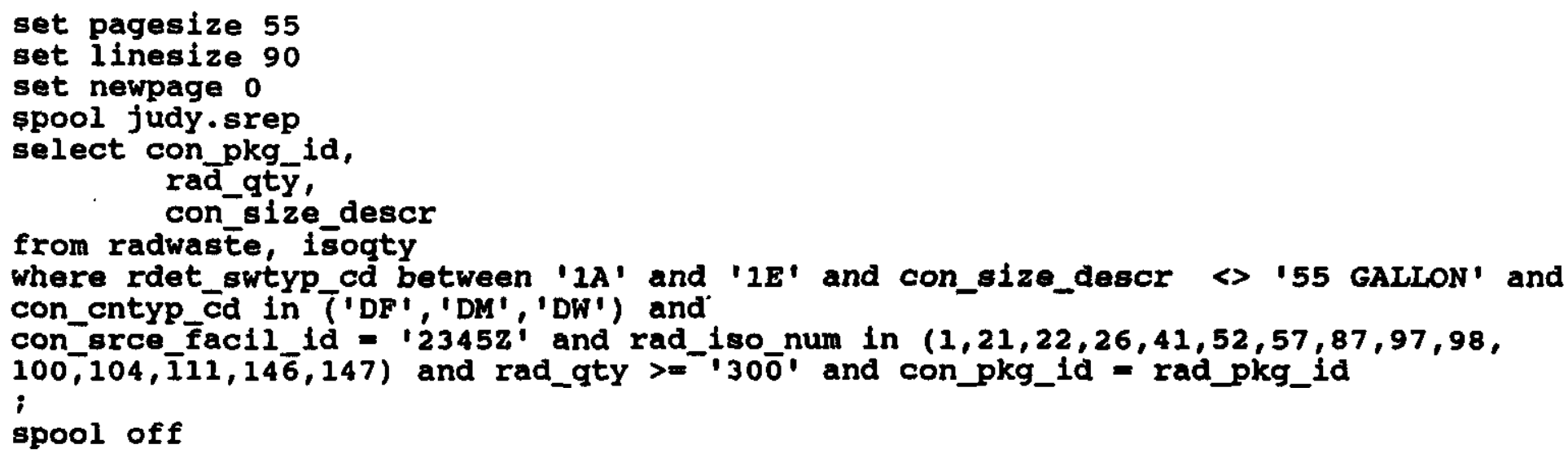


for Generating Company: $x$

for Facility ID: $\boldsymbol{x}$

for Primary Waste Type Code: $x$

for Secondary Waste Type Code: $x$

Container ID: $\mathbf{R 4 0 9}$

Location Beginning Coordinates: N N40190 W w77635 Location Ending Coordinates: N N40190 W W77609 Content Analysis Return Date:

Packaged Date:

TSD Accept Date: $10 / 16 / 80$

Certification Date:

Container Type Code: D

Dose Rate: 1.000E+00 Neutron Dose Rate: Container Volume:

0.419 Gross Wt:

158.76

Previous Container ID:

Organic Volume:

Organic Wt.:

Compaction Wt. (Prorated):

* Content Compaction:

Compactor PIN:

Comments/Description:

Designation Code: DW Waste

Generator Information:

Name:

ID: WHC

Facility ID: 23452

Charge Code: $\mathrm{K} 6$

Location Information:

Area Name: 200W Facility ID: 218W4C Module: Surface Area:

Tier Level: Tier Position: Unit: T01

Lab Pack Description:

Lab Pack Flag: N

Physical State Code: S Chemical Nature Code: Primary Waste Type Code: $R$ Secondary Waste Type Code: 1A

Container Size Description: UNKNOWN

Container Storage Category Code:

Container Empty Tare Wt.:

Content Wrap Category:

Container Thermal Power:

SWSDR \#: 830005

Radioactive Information:

Total Alpha (Ci):

Total Pu Equivalent (Ci): SDAR Approval \#:

Container Total Wt.:

Total Beta-Gamma (Ci): 1.000E-03

SWEA General Comments:

Total Pu Fissile Gram Equivalent: 


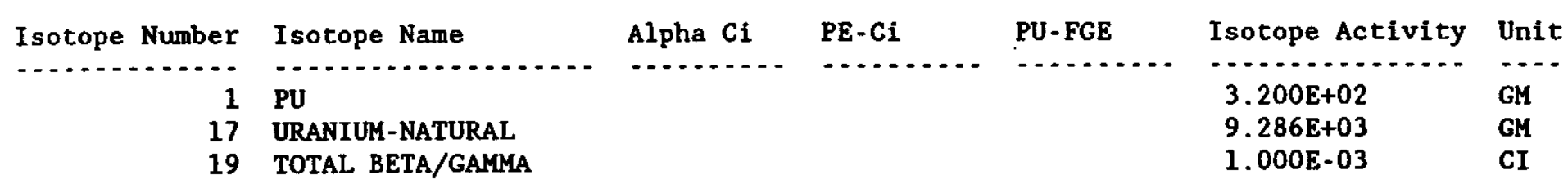

Hazardous Chemical Components:

Component Text

PPM Weight $(\mathrm{kg}) \quad \%$

C...

Weight (kg) $x$

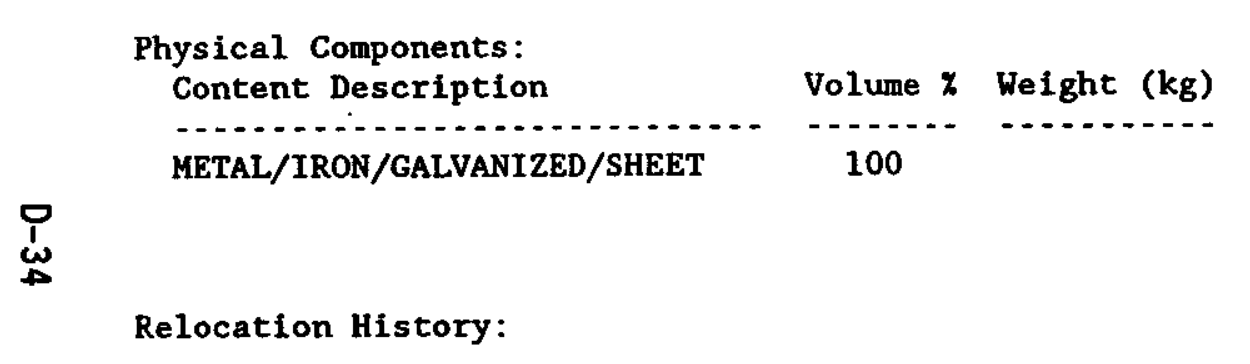

Shipment Information: 
for Generating Company: $x$

for Facility ID: $x$

for Primary Waste Type Code: $z$

for Secondary Waste Type Code: $x$

\section{Container ID: R488}

Location Beginning Coordinates: N N40190 W w77635 Location Ending Coordinates: N N40190 W w77609

Content Analysis Return Date:

Certification Date:

Dose Rate: 1.000E+00 Neutron Dose Rate:

Previous Container ID:

Current Container ID:

Compaction Wt. (Prorated):

Packaged Date:

Container Type Code: DM

TSD Accept Date: $10 / 16 / 80$

Container Volume:

0.419 Gross

158.76

Organic Volume: Organic We.:

y Content Compaction: Compactor PIN:

Comments/Description:

Designation Code:

DW Waste \#:

Generator Information:

$$
\text { Name : }
$$

ID: WHC

Facility ID: 23452

Charge Code: $\mathrm{K6}$

Location Information:

Area Name: 200W Facility ID: 218W4C Module: Surface Area:

Tier Level: Tier Position: Unit: T01

Lab Pack Description:

Lab Pack Flag: N

Physical State Code: S Chemical Nature Code: Primary Waste Type Code: R Secondary Waste Type Code: 1A Container Size Description: UNKNOWN

Container Storage Category Code:

Container Empty Tare Ut.:

Content Wrap Category:

SWSDR \#: 830003

SWIMS Waste Description
Container Thermal Power:

SDAR Approval \#:

Container Total Wt.

Radioactive Information:

Total Alpha (Ci):

Total Pu Equivalent (Ci):

Total Beta-Gamma (Ci): 1.000E-03

SWEA General Coments:
Total Pu Fissile Gram Equivalent: 


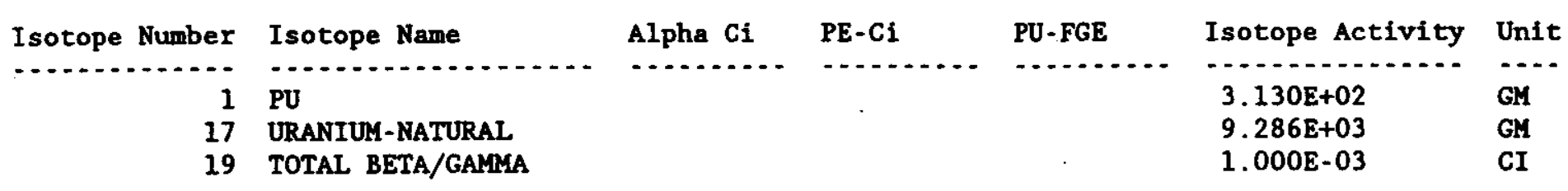

Hazardous Chemical Components: Component Text

PPM Weight (kg) $\%$

Physical Components: Content Description

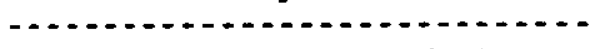

METAL/IRON/GALVANIZED/SHEET

Volume $\%$ Weight $(\mathrm{kg})$

.............................

100

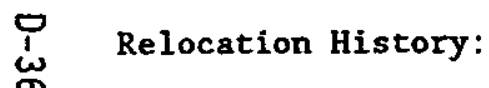

Shipment Information: 
for Generating Company:

for Facillty ID: $\dot{x}$

for Primary Waste Type Code: $x$

for Secondary Waste Type Code: $x$

Container ID: $\operatorname{ccs} 74-137$

Location Beginning Coordinates: N N40190 W W77635 Location Ending Coordinates: N N40190 W w77609

Content Analysis Return Date:

Packaged Date:

TSD Accept Date: 03/04/82

Certification Date:

Container Type Code: DM Container Count:

Container Volume: $\quad 0.419$ Gross Wt:

Previous Container ID:

Organic Volume: Organic Wt.:

Current Container ID:

Compaction Wt. (Prorated):

Neutron Dose Rate:

Comments/Description:

Designation Code:

DW Waste \#:

Generator Information:

Name:

z Content Compaction:

Compactor PIN :

Location Information:

Area Name: 200W Facility ID: 218W4C Module: Surface Area:

Tier Level: Tier Position: Unit: T01

Lab Pack Description:

Lab Pack Flag: N

Physical State Code: S Chemical Nature Code: Primary Waste Type Code: R Secondary Waste Type Code: 1A

Container Size Description: UNKNOWN

Container Storage Category Code:

Container Empty Tare Wt.:

Content Wrap Category:

SWIMS Waste Description Code: DS SWSDR \#: 830021

Radioactive Infornation:

Total Alpha $(C i)$ :

Total Pu Equivalent (Ci):

SDAR Approval \#:

Container Total Wt. :

SWEA General Comments: 


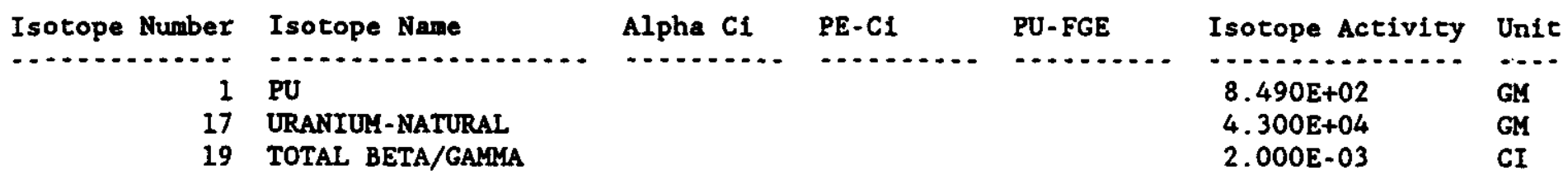

Hazardous Chemical Components:

Component Text

PPM

Weight $(\mathrm{kg}) \quad x$

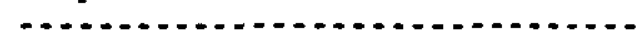

Physical Components:

Content Description

Volume $x$ Weight $(\mathrm{kg})$

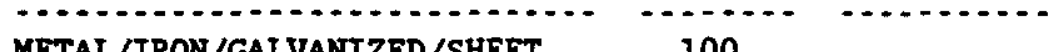

METAL/IRON/GALVANIZED/SHEET 100

Shipment Information: 
for Generating Company: $x$

for Facility ID: $x$

for Primary Waste Type Code: $z$

for Secondary Waste Type Code: $z$

Container ID: $\operatorname{ccs} 74-138$

Location Beginning Coordinates: N N40190 W w77635 Location Ending Coordinates: N N40190 W W77609

Content Analysis Return Date: Packaged Date: TSD Accept Date: 03/04/82

Certification Date:

Dose Rate: 2.000E+00 Neutron Dosé Rate:

Container Type Code: DM Container Count:

Previous Concainer ID:

Current Container ID:

Compaction Wt. (Prorated):

Organic Volume:

Organic We.:

Comments/Description:

Designation Code:

$x$ Content Compaction:

Compactor PIN:

Generator Information:

$$
\text { Name: }
$$

ID: WHC

Facilicy ID: $2345 \mathrm{Z}$

Charge Code: $\mathrm{k} 6$

Location Information:

Area Nane: 200W Facility ID: 218W4C Module: Surface Area:

Tier Level: Tier Position: Unit: T01

Lab Pack Description:

Lab Pack Flag: N

Physical State Code: $S$ Chemical Nature Code: Primary waste Type Code: R Secondary Waste Type Code: 1A

Container Size Description: UNKNOWN

Container Storage Category Code:

Container Enpty Tare Vt.:

Content Wrap Category:

SWSDR \#: 830019

Radioactive Information:

Total Alpha (Ci):

Total Pu Equivalent (Ci): SDAR Approval \#:

Container Total Wr.:

ToEA General Comments:

Total Beta-Ganma (Ci): 2.000E-03

Total Pu Fissile Gram Equivalent: 


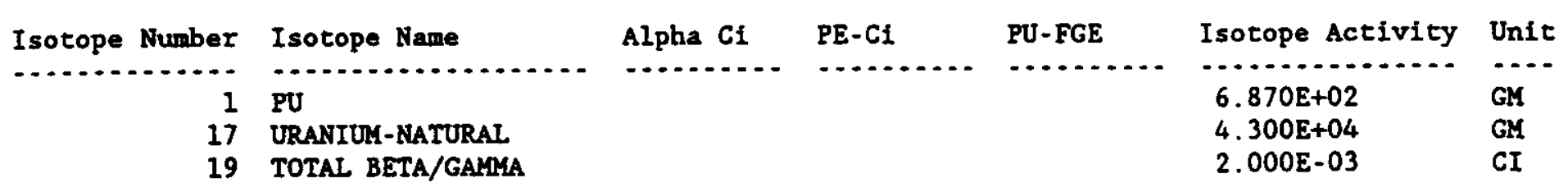

Hazardous Chenical Components:

Component Text

PPM Weight $(\mathrm{kg}) \cdot x$

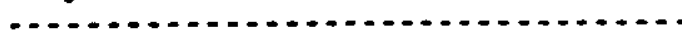

Physical Components:

Content Description

Volume $z$ Weight $(\mathrm{kg})$

.............

METAL/IRON/GALVANIZED/SHEET 100

Shipment Information: 
fot Secondary Waste Type Code: $x$

Container ID: $\operatorname{ccs} 74 \cdot 141$

Location Beginning Coordinates: N N40190 w w77635 Location Ending Coordinates: N N40190 W w77609 Content Aralysis Return Date:

Certification Dace:

Dose Rate: 2.000E+00 Neutron Dose Rate:

Packaged Date:

TSD Accept Date: 03/04/82

Dose Rate: $2.000 E+00$
Previous Container ID:

Container Type Code: DM Container Count: Container Volume: $\quad 0.419$ Gross Wt:

Current Container ID:

Organic Volume:

Organic Wt.:

Compaction Wt. (Prorated) :

* Content Compaction: Compactor PIN:

Comments/Description:

Designation Code:

DW Waste \#:

Generator Information:

Name:

ID: WHC

Facility ID: $2345 Z$

Charge Code: $\mathrm{K} 6$

Location Information:

Area Name: 200W Facility ID: 218W4C Module: Surface Area:

Tier Level: Tier Position: Unit: TOI

Lab Pack Description:

Lab Pack Flag: N

Physical State Code: S Chemical Nature Code: Primary Waste Type Code: R Secondary Waste Type Code: 1A

Container Size Description: UNKNOWN

Container Storage Category Code:

Container Empty Tare Wt.:

Content Wrap Category:

Radioactive Information:

Total Alpha (Ci):

Total Pu Equivalent (Ci): SDAR Approval \#:

SWIMS Waste Description Code: DS

Container Thermal Power:

SWSDR \#: 830018

Container Total Wt.:

SWEA General Comments:

Total Beta-Gamma (Ci): 2.000E-03

Total Pu Fissile Gram Equivalent: 


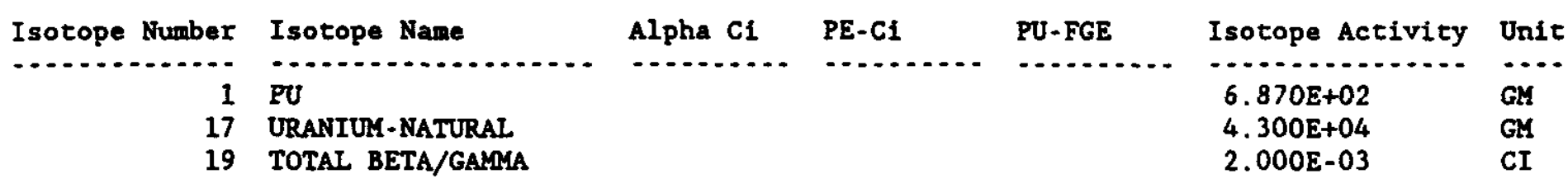

\section{Hazardous Chemical Components:}

Component Text

PPM

Weight (kg) $x$

....... -.........

Physical Components: Content Description

METAL/IRON/GALVANIZED/SHEET

Volume $x$ Weight $(\mathrm{kg})$

100

$\frac{p}{N}$

Relocation History:

Shipment Information: 
for Generating Company: $z$

for Facility ID: $x$

for Primary Waste Type Code: $\%$

for Secondary Waste Type Code: $\chi$

Container ID: $\operatorname{CCS} 74-142$

Location Beginning Coordinates: N N40190 w w77635 Location Ending Coordinates: N N40190 W w77609

Content Analysis Return Dace:

Certification Date:

Dose Rate: 2.000E+00 Neutron Dose Rate:

Packaged Date:

TSD Accept Date: 03/04/82

Container Type Code: DM Container Count: 1

$$
\text { Container Volume: } \quad 0.419 \text { Gross Wt: }
$$

Previous Container ID:

Current Container ID:

Compaction Wt. (Prorated):

Organic Volume: Organic Wt.:

Comments/Description:

Designation Code: DW Waste \#:

Generator Information:

Name :

$z$ Content Compaction:

Compactor PIN:

Location Information:

Area Name: 200w Facility ID:

$218 W 4 C$

ab Pack Description:

Lab Pack Flag: N

Physical State Code: S Chemical Nature Code: Primary Waste Type Code: $R$ Secondary Waste Type Code: 1A Container Size Description: UNKNOWN

Container Storage Category Code:

Container Empty Tare Wt.:

Content Wrap Category:

Container Thermal Power:

SWSDR \#: 830017

Radioactive Information:

Total Alpha (Ci):

Total Pu Equivalent (C1): SDAR Approval \#:

Container Total Wt.:

Total Pu Equivalent
SWEA General Comments:

Total Beta-Gamma ( $\mathrm{C}$ i): 2.000E-03

Total Pu Fissile Gram Equivalent: 


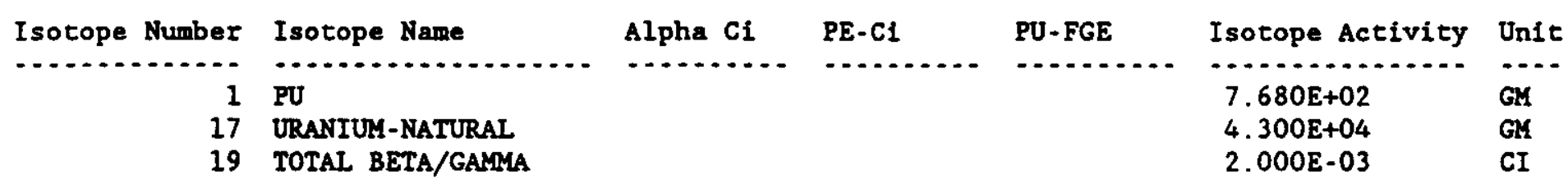

Hazardous Chemfcal Components:

Component Text

PPM

Weight $(\mathrm{kg}) \quad z$

(..................

Physical Components:

Content Description

Volume $\%$ Weight $(\mathrm{kg})$

................

100

Shipment Information: 
for Secondary Waste Type Code: $\%$

Container ID: $\operatorname{ccs} 74-143$

Location Beginning Coordinates: N N40190 W w77635 Location Ending Coordinates: N N40190 W77609 Content Analysis Return Date: Packaged Date:

TSD Accept Dare: 03/04/82

Certification Date:

Container Type Code: DM Container Count: 1

Dose Rate: 2.000E+00 Neutron Dose Rate: Container Volume:

0.419 Gross Wt:

Previous Container ID:

Organic Volume: Organic We.:

Compaction Wt. (Prorated): $\quad$ \& Content Compaction: Compactor PIN:

Comments/Description:

Designation Code:

Generator Information:

Nane:

DW Waste $\#$ :

Location Information:

Area Name: 200W Facility ID: 218W4C Module: Surfäce Area:

Charge Code: $\mathrm{K} 6$

Tier Level: Tier Position: Unit: Tol

Lab Pack Description:

Lab Pack Flag: N

Physical State Code: S Chemical Nature Code: Primary Wastë Type Code: R Secondary Waste Type Code: 1A

Container Size Description: UNKNOWN

Container Storage Category Code: SwIMS Waste Description Code: DS

Container Empty Tare Wt.:

Content Wrap Category:

Container Thermal Power:

SWSDR \#: 830015

Radioactive Information:

Total Alpha (Ci): SDAR Approval \#:

Container Total Wt.:

Total Pu Equivalent (C1):

SWEA General Comments:

Total Beta-Gamma (Ci): 2.000E-03

Total Pu Fissile Gram Equivalent: 


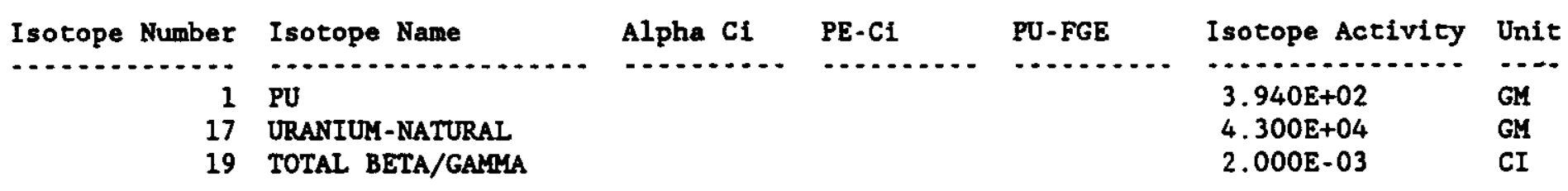

Hazardous Chenical Components:

Component Text

PPM Weight (kg) $x$

- .

Physical Components:

Content Description

Volume $x$ Weight $(\mathrm{kg})$

METAL/IRON/GALVANIZED/SHEET 100

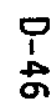

Relocation History:

Shipment Information: 
for Generating Company: $x$

for Facility ID: $x$

for Primary Waste Type Code: $x$

for Secondary Waste Type Code: $x$

Container ID: $\operatorname{ccs} 74-149$

Location Beginning Coordinates: N N40190 W w77635 Location Ending Coordinates: N N40190 W w77609

Content Analysis Return Date:

Certification Date:

Dose Rate: 2.000E+00 Neutron Dose Rate:

Packaged Date:

TSD Accept Date: 03/04/82

Contafner Type Code: DM Container Count: 1 Container Volume: $\quad 0.419$ Gross Wt:

Previous Container ID:

Current Container ID:

Compaction Wt. (Prorated) :

Organic Volume:

Organic Wt.:

Comments/Description:

Designation Code:

$z$ Content Compaction:

Compactor PIN:

Generator Information:

$$
\text { Name: }
$$

Location Information:

Area Name: 200W Facility ID: 218W4C Module: Sürface Area:

Tier Level: Tier Position: Unit: T01

Lab Pack Description:

Lab Pack Flag: N

Physical State Code: S Chemical Nature Code: Primary Waste Type Code: $R$ Secondary Waste Type Code: 1A

Container Size Description: UNKNOWN

Container Storage Caregory Code:

Container Empty Tare Wt.:

Content Wrap Category:

Container Thermal Power:

SWSDR \#: 830016

Radioactive Information:

Total Alpha (Ci):

SDAR Approval \#:

Container Total Wt.:

Total Pu Equivalent (Ci):

Total Beta-Gamma (Ci): 2.000E-03

SWEA General Comments: 


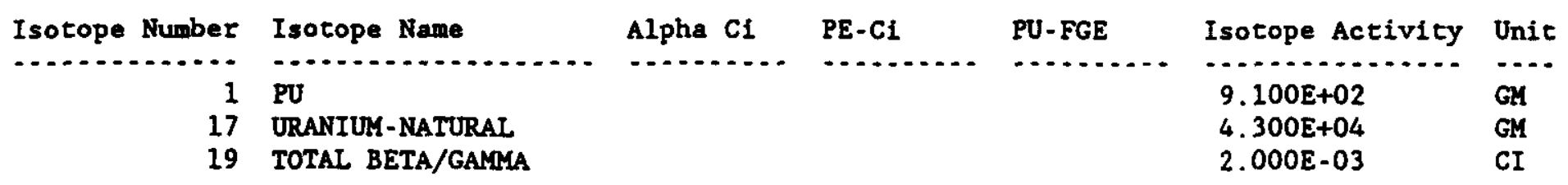

Hazardous Chemical Components:

Component Text

Component Text

Physical Components: Content Description

METAL/IRON/GALVANIZED/SHEET

Volume $x$ Weight $(\mathrm{kg})$

100

0
1
$\infty$

Relocation History:

Shipment Information: 
for Generating Company: $x$

for Facility ID: $\not$

for Primary Waste Type Code: $x$

for Secondary Waste Type Code: $x$

Container ID: $7774-412$

Location Beginning Coordinates: N N40190 W w77635 Location Ending Coordinates: N N40190 W w77609

Content Analysis Return Date:

Certification Date:

Dose Rate: 1.000E+00 Neutron Dose Rate:

Previous Container ID:

Current Container ID:

Compaction Wt. (Prorated):

Packaged Date:

Container Type Code: DM
Container Volume:

TSD Accept Date: 03/25/82

0.419 Gross Wt

136.08

Organic Volume: Organic Wt.:

* Content Compaction: Compactor PIN:

Comments/Description:

Designation Code: DW Waste \#:

Generator Information:

Name:

Location Information:

Area Name: 200W Facility ID: 218W4C Module: Surface Area:

Tier Level: Tier Position: Unit: TOI

Lab Pack Description:

Lab Pack Flag: N

Physical State Code: S Chemical Nature Code: Primary Waste Type Code: R Secondary Waste Type Code: 1A

Container Size Description: 110 GALION

Container Storage Category Code: SWIMS Waste Description Code: DS

Container Empty Tare Wt.:

Content Urap Category:

Container Thermal Power:

SWSDR \#: 820172

SDAR Approval \#:

Container Total Wt.:

Radioactive Information:

Total Alpha ( $\mathrm{Ci}$ ):

Total Pu Equivalent ( $\mathrm{Ci}$ ):

Total Beta-Gamma (Ci): 1.000E-02

SWEA General Comments:
Total Pu Fissile Gram Equivalent: 


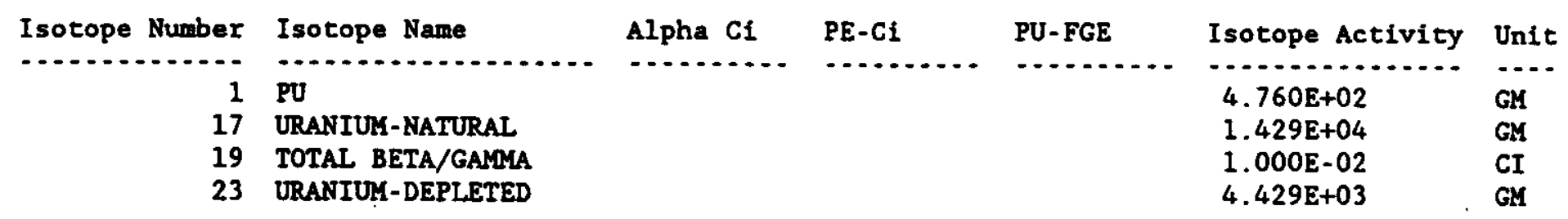

Hazardous Chemical Components:

Component Text

PPM

Weight $(\mathrm{kg})$ \%

(..................

Physical Components:

Content Description

Volume $z$ Weight $(\mathrm{kg})$

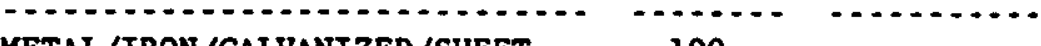

METAL/IRON/GALVANIZED/SHEET $\quad 100$

망

Relocation History:

Shipment Information: 


\section{Container ID: 7774.413}

Location Beginning Coordinates: N N40190 W w77635 Location Ending Coordinates: N N40190 W w77609

$\begin{array}{lll}\text { Content Analysis Return Date: } & \text { Packaged Date: } & \text { TSD Accept Date: 03/25/82 } \\ & \text { Container Type Code: DM Container Count: } 1\end{array}$

Certification Date:

Dose Rate: 1.000E+00 Neutron Dose Rate: Gontainer Volume:

0.419 Gross Wt:

Previous Container ID:

Current Container ID:

Organic Volume: Organic Wt.:

Compaction Wt. (Prorated): $\quad$ C Content Compaction: Compactor PIN:

Comments/Description:

Designation Code: DW Waste \#:

Generator Information:

Name :

ID: WHC

Facility ID: 23452

Charge Code: $\mathrm{K} 6$

Location Information:

Area Name: 200W Facility ID: 218W4C Module: Surface Area:

Tier Level: Tier Position: Unit: TOl

Lab Pack Description:

Lab Pack Flag: N

Physical State Code: S Chemical Nature Code: Primary Waste Type Code: R Secondary Waste Type Code: IA Container Size Description: 110 GaLLON

Container Storage Category Code:

Container Empty Tare Wt.:

Content Wrap Category:

Radioactive Information:

Total Alpha (Ci):

Total Pu Equivalent (Ci):

Container Thermal Power:

SWMS Waste Description Code: DS SWSDR \#: 820165

SDAR Approval \#:

Container Total Wt.:

SWEA General Comments: 


\begin{tabular}{|c|c|c|c|c|c|c|}
\hline Isotope Number & Isotope Name & Alpha CI & PE-Ci & PU-FGE & Isotope Activity & Unit \\
\hline 1 & PU & $\cdots$ & $\cdots \ldots$ & - . & 39705+0? & \\
\hline 17 & URANIUM-NATURAL & & & & $\begin{array}{l}3.970 \mathrm{E}+02 \\
1.663 \mathrm{E}+04\end{array}$ & $\begin{array}{l}\text { GM } \\
\text { GM }\end{array}$ \\
\hline 19 & TOTAL BETA/GAMMA & & & & $1.000 \mathrm{E}-02$ & CI \\
\hline 23 & URANIUM-DEPLETED & & & & $3.000 E+03$ & GM \\
\hline
\end{tabular}

Hazardous Chenical Components:

Component Text

PPM

Weight $(\mathrm{kg}) \quad x$

(n)

Physical Components:

Content Description

Volume $z$ Weight $(\mathrm{kg})$

METAL/IRON/GALVANIZED/SHEET 100

员

Relocation History:

Shipment Information: 
Container ID: $7774-414$

Location Beginning Coordinates: N N40190 W w77635 Location Ending Coordinates: N N40190 W w77609 Content Analysis Return Date:

Certification Date:

Dose Rate: $1.000 E+00$ Packaged Date:

TSD Accept Date: 03/25/82

Container Type Code: DM Container Count: 1

Current Container ID:

Container Volume:

0.419 Gross wt:

Compaction Wt. (Prorated):

Organic Volume:

Organic We.:

Comments/Description:

Designation Code:

\% Content Compaction: Compactor PIN:

Generator Information:

$$
\text { Name: }
$$

DW Waste \#:

Location Information:

Area Name: 200W Facility ID: 218W4C Module: Surface Area:

Tier Level: Tier Position: Unit: T01

Lab Pack Description:

Lab Pack Flag: N

Physical State Code: S Chemical Nature Code: Primary Waste Type Code: $R$ Secondary Waste Type Code: 1A

Container Size Description: 110 GALLON

Container Storage Category Code: SWIMS Waste Description Code: DS

Container Empty Tare Wr.:

Content Wrap Category:

Concainer Thermal Power:

SWSDR \#: 820158

Radioactive Information:

Total Alpha (Ci): SDAR Approval \#:

Container Total Wt.:

Total Pu Equivalent (Ci):

Total Beta-Gamma (Ci): 1.000E-02

SWEA General Coments: 


\begin{tabular}{|c|c|c|c|c|c|c|}
\hline Isotope Number & Isotope Name & Alpha Ci & $\mathrm{PE}-\mathrm{Ci}_{\mathbf{I}}$ & PU-FGE & Isotope Activity & Unit \\
\hline 1 & PU & 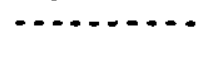 & $\cdots$ & (n.......... & $4,5608+02$ & 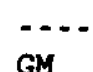 \\
\hline 17 & URANIUM-NATURAL & & & & $1.663 \mathrm{E}+04$ & GM \\
\hline 19 & TOTAL BETA/GALIMA & & & & $1.000 \mathrm{E}-02$ & CI \\
\hline 23 & URANIUM-DEPLETED & & & & $3.000 E+03$ & M \\
\hline
\end{tabular}

Hazardous Chemical Components:

Component Text

n.

-....... .............

Physical Components:

Content Description

Volume $\chi$ Weight $(\mathrm{kg})$

METAL/IRON/GALVANIZED/SHEET

100

虽

Relocation History:

Shipment Information: 
for Generating Company: $x$

for Facility ID: $x$

for Primary Waste Type Code: $x$

for Secondary Waste Type Code: $x$

Container ID: $7774-415$

Location Beginning Coordinates: N N40190 w w77635 Location Ending Coordinates: N N40190 W w77609 Content Analysis Return Date:

Certification Date:

Dose Rate: 1.000E+00 Neutron Dose Rate:

Packaged Date:

TSD Accept Date: 03/25/82

Container Type Code: DM Container Count: Container Volume: $\quad 0.419$ Gross Wt:

136.08

Previous Container ID:

Current Container ID:

Compaction Wt. (Prorated):

Organic Volume:

Organic Wt.:

Comments/Description:

Designation Code: DW Waste \#:

Generator Information:

Name :

z Content Compaction:

Compactor PIN:

Location Information:

Area Name: 200W Facility ID: 218W4C Module: Surface Area:

Tier Level: Tier Position: Unit: T01

Lab Pack Description:

Lab Pack Flag: N

Physical State Code: S Chemical Nature Code: Primary Waste Type Code: R Secondary Waste Type Code: 1A

Container Size Description: 110 GALLON

Container Storage Category Code: SWIMS Waste Description Code: DS

Container Empty Tare Wt.:

Content Wrap Category:

Radioactive Information:

Total Alpha (Ci): SDAR Approval \#:

Container Total Wt.:

Total Pu Equivalent ( $C i)$ :

Total Beta-Gamma (Ci): 1.000E-02

SWEA General Comments:

Total Pu Fissile Gram Equivalent: 


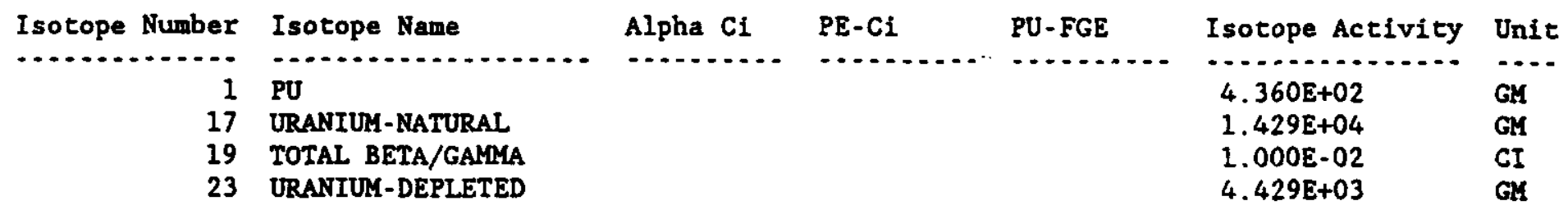

\section{Hazardous Chemical Components:}

Component Text
Physical Components: Content Description
Volume $\%$ Weight (kg)

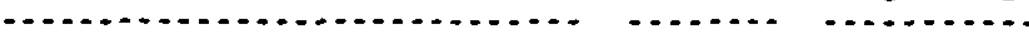
METAL/IRON/GALVANIZED/SHEET 100

Shipment Information: 
for Generating Company: $x$

for Facility ID: $x$

for Primary Waste Type Code: $x$

for Secondary Waste Type Code: $x$

Container ID: $7774-416$

Location Beginning Coordinates: N N40190 W w77635 Location Ending Coordinates: N N40190 W w77609 Content Analysis Return Date:

Packaged Dace:

Container Type Code: DH

TSD Accept Date: 03/25/82

Certification Date:

Dose Rate: 1.000E+00 Neutron Dose Rate:

Previous Container ID:

Current Container ID:

Compaction Wt. (Prorated): Container Volume:

Container Count:

0.419 Gross wt.

136.08

Organic Volume:

Organic Wt.:

\% Content Compaction: Compactor PIN :

Coments/Description:

Designation Code: DW Waste \#:

Generator Information:

$$
\text { Nane: }
$$

Location Information:

Area Name: 200W Facility ID: 218W4C Module: Surface Area:

Tier Level: Tier Position: Unit: T01

Lab Pack Description:

Lab Pack Flag: N

Physical State Code: S Chemical Nature Code: Primary Waste Type Code: R Secondary Waste Type Code: 1A Container Size Description: 110 GALLON

Container Storage Category Code:

Container Empty Tare Wt.:

Content Wrap Category:

SWIMS Waste Description Code: DS

Container Thermal Power:

SWSDR \#: 820167

Radioactive Information:

Total Alpha (Ci):

Total Pu Equivalent (Ci): SDAR Approval *:

Container Total Wt.

SWEA General Comments:
Total Beta-Gamma (Ci): 1.000E-02 Total Pu Fissile Gram Equivalent: 


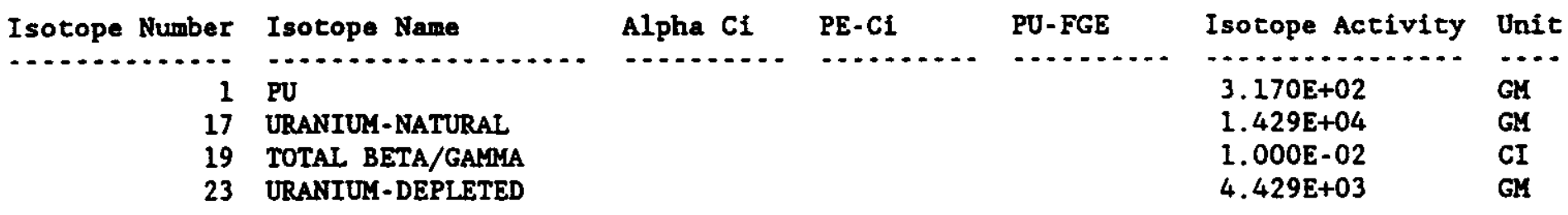

Hazardous Chemical Components:

Component Text

PPM Weight (kg) $z$

........ W - W.

Physical Components:

Content Description

Volume $x$ Weight. $(\mathrm{kg})$

METAL/IRON/GALVANIZED/SHEET 100

吕

Relocation History:

Shipment Information: 
Container ID: $7774-417$

Location Beginning Coordinates: N N40190 w w77635 Location Ending Coordinates: N N40190 W W77609 Content Analysis Return Date:

Certification Date:

Dose Rate: 1.000E+00 Neutron Dose Rate:

Packaged Date:

TSD Accept Date: 03/25/82

Container Type Code: DM Container Count:

Container Volume:

0.419 Gross Wt:

136.08

Previous Container ID:

Current Container ID:

Organic Volume: Organic Wt.:

Compaction Wt. (Prorated):

* Content Compaction:

Compactor PIN:

Designation Code: DW Waste \#:

Generator Information:

$$
\text { Name: }
$$

Location Information:

Area Nane: 200W Facility ID: 218W4C Module: Surface Area

Tier Level: Tier Position: Unit: T01

Lab Pack Description:

Lab Pack Flag: N

Physical State Code: S Chemical Nature Code: Primary Waste Type Code: $R$ Secondary Waste Type Code: 1A

Container Size Description: 110 GALLON

Container Storage Category Code: SWIMS Waste Description-Code: DS

Container Empty Tare Wt.:

Content Hrap Category:

Container Thermal Power:

SWSDR \# 820168

Radioactive Information:

Total Alpha (Ci): SDAR Approval \$:

Container Total Wt.:

Total Pu Equivalent (Ci):

SWEA General Coments:

Total Beta-Gamma (C1): 1.000E-02

Total Pu Fissile Gran Equivalent: 


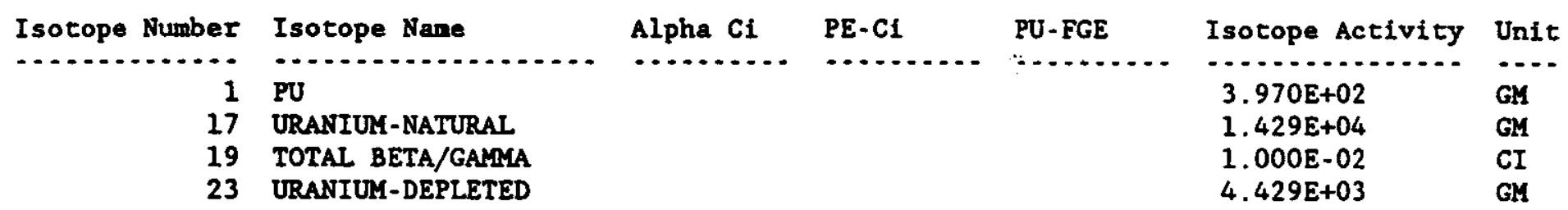

Hazardous Chemical Components:

Component Text

PPM Weight $(\mathrm{kg}) \quad \mathrm{z}$

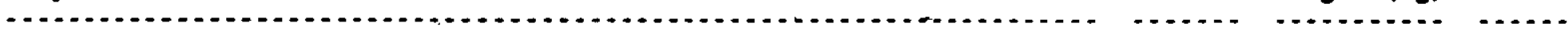

Physical Components:

Content Description

METAL/IRON/GALVANIZED/SHEET

Volume $\%$ Weight $(\mathrm{kg})$

(100.......................

100

Shipment Information: 
Location Beginning Coordinates: N N40190 W w77635 Location Ending Coordinates: N N40190 W w77609 Content Analysis Return Date: Certification Date:

Dose Rate: $1.000 \mathrm{E}+00$ Neutron Dose Rate: Packaged Date:

TSD Accept Date: 03/25/82

Container Type Code: Dir

Container Count:

Container Volume: $\quad 0.419$ Gross Wt:

136.08

Current Container ID:

Organic Volume:

Organic Wt.:

Compaction Wt. (Prorated):

* Content Compaction:

Compactor PIN:

Comments/Description:

Designation Code: DW Waste \#:

Generator Information:

Name:

Location Information:

Area Name: 200W Facility ID: 218w4C

Tier Level: Tier Position:

Lab Pack Description:

Lab Pack Flag: N

Physical State Code: S Chemical Nature Code: Primary Waste Type Code: R Secondary Waste Type Code: 1A Container Size Description: 110 GALLON

Container Storage Category Code: SWIMS Waste Description Code: DS

Container Empty Tare Wt.:

Content Wrap Category:

Container Thermal Power:

SWSDR \#: 820159

Radioactive Information:

Total Alpha (Ci):

Total Pu Equivalent (Ci): SDAR Approval \#:

Module: Surface Area:

nit: T01
Total Beta-Gamma (C1): 1.000E-02

Total Pu Fissile Gram Equivalent:

SWEA General Commenes: 


\begin{tabular}{|c|c|c|c|c|c|}
\hline Isotope Number & Isotope Name & Alpha Ci & PE-Ci & PU-FGE & Isotope Activity \\
\hline 1 & PU & 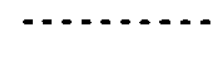 & $\cdots$ & . & $3.570 \mathrm{~s}+02$ \\
\hline 17 & URANIUM-NATURAL & & & & $1.663 \mathrm{E}+04$ \\
\hline 19 & TOTAL BETA/GAMYA & & & & $1.000 \mathrm{E}-02$ \\
\hline 23 & URANIUM-DEPLETED & & & & $3.000 E+03$ \\
\hline
\end{tabular}

Hazardous Chemical Components:

Component Text

PPM Weight $(\mathrm{kg}) \quad x$

Physical Components:

Content Description

Volume $\%$ Weight $(\mathrm{kg})$

METAL/IRON/GALVANIZED/SHEET 100

Shipment Information: 
Container ID: 7774.419

Location Beginning Coordinates: N N40190 W w77635 Location Ending Coordinates: N N40190 W w77609

Content Analysis Return Date:

Certification Date:

Dose Rate: 1.000E+00 Neutron Dose Rate:

Packaged Date:

TSD Accept Date: $03 / 25 / 82$

Container Type Code: DM Container Count:

Container Volume:

0.419 Gross Wt:

136.08

Previous Container ID

Current Container ID:

Organic Volume: Organic Wt.:

Compaction Wt. (Prorated): $\quad$ Content Compaction: Compactor PIN:

Comments/Description:

Designation Code: DW Waste \#:

Generator Information:

Name:

ID: WHC

Facility ID: 23452

Charge Code: K6

Location Information:

Area Name: 200W Facility ID: 218W4C Module: Surface Area:

Tier Level: Tier Position: Unit: T01

Lab Pack Description:

Lab Pack Flag: N

Physical State Code: S Chemical Nature Code: Primary Waste Type Code: $R$ Secondary Waste Type Code: $1 A$ Container Size Description: 110 GALLON

Container Storage Category Code: SWIMS Waste Description Code: DS

Container Empty Tare Wt.:

Content Wrap Category:

Container Thermal Power:

SWSDR \#: 820160

Radioactive Information: SDAR Approval \#

Total Alpha (Ci):

Total Pu Equivalent (CI):

SWEA General Comments:

Total Pu Fissile Gram Equivalent: 


\begin{tabular}{|c|c|c|c|c|c|c|}
\hline Isotope Number & Isotope Name & Alpha $\mathrm{Ci}$ & $\mathrm{PE}-\mathrm{CI}$ & PU-FGE & Isotope Activity & Unit \\
\hline . & 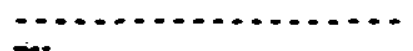 & - & (n.......... & $\cdots$ & $\cdots$ & \\
\hline 1 & & & & & $3.970 \mathrm{E}+02$ & GM \\
\hline 17 & URANIUM-NATURAL & & & & $1.663 \mathrm{E}+04$ & GM \\
\hline 19 & TOTAL BETA/GAMMA & & & & $1.000 \mathrm{E}-02$ & CI \\
\hline 23 & URANIUM-DEPLETED & & & & $3.000 E+03$ & GM \\
\hline
\end{tabular}

Hazardous Chemical Components:

Component Text

PPM

Weight $(\mathrm{kg}) \quad z$

Physical Components:

Content Description

Volume $\%$ Weight $(\mathrm{kg})$

METAL/IRON/GALVANIZED/SHEET 100

Shipment Information: 
for Generating Company: 2

for Facility ID: $z$

for Primary Waste Type Code: $z$

for Secondary Waste Type Code: $x$

Container ID: 7774.421

Location Beginning Coordinates: N N40190 w w77635 Location Ending Coordinates: N N40190 W w77609

Content Analysis Return Date:

Certification Date:

Dose Rate: 1.000E+00 Neutron Dose Rate:

Packaged Date:

TSD Accept Date: 03/25/82

Container Type Code: DM Container Count:

Containęr Volume: $\quad 0.419$ Gross Wt: 136.08

Previous Container ID:

Current Container ID:

Compaction Wt. (Prorated):

Organic Volume: Organic Wt.:

Comments/Description:

Designation Code: DW Waste \#:

Generator Information:

$$
\text { Name: }
$$

Location Information: Area Name: 200W Facility ID: 218W4C Module: Surface Area: Tier Level: Tier Position: Unit: T01

Lab Pack Description:

Lab Pack Flag: N

Physical State Code: S Chemical Nature Code: Primary Waste Type Code: $R$ Secondary Waste Type Code: 1A Container Size Description: 110 GALLON

Container Storage Category Code: SWIMS Waste Description Code: DS

Container Empty Tare Wt.:

Content Wrap Category:

Radioactive Information:

Total Alpha (Ci):

Total Pu Equivalent (Ci): SDAR Approval \#:

SWIMS Waste Description

SWEA General Comments:
Total Beta-Gamma (Ci): 1.000E-02

Total Pu Fissile Gram Equivalent: 


\begin{tabular}{|c|c|c|c|c|c|c|}
\hline Isotope Number & Isotope Name & Alpha $\mathrm{Ci}$ & $P E-C i$ & PU-FGE & Isotope Activity & Unit \\
\hline , & PU & $\ldots \ldots \ldots$ & $\cdots$ & $\ldots \ldots \ldots$ & $4.160 \mathrm{E}+02$ & \\
\hline 17 & URANIUM-NATURAL & & & & $1.429 E+04$ & $M$ \\
\hline 19 & TOTAL BETA/GAMMA & & & & $1.000 \mathrm{E}-02$ & CI \\
\hline 23 & URANIUM-DEPLETED & & & & $4.429 E+03$ & M \\
\hline
\end{tabular}

\section{Hazardous Chemical Components:}

Component Text

PPM Weight (kg) \%

Physical Components: Content Description

Volume $\approx$ Weight (kg)

METAL/IRON/GALVANIZED/SHEET

100

Shipment Information: 
for Generating Company: $x$

for Facility ID: $x$

for Primary Waste Type Code: $x$

for Secondary Waste Type Code: $z$

Container ID: $7774-422$

Location Beginning Coordinates: N N40190 W w77635 Location Ending Coordinates: N N40190 W77609 Content Analysis Return Date:

Certification Date:

Dose Rate: 1.000E+00 Neutron Dose Rate: Packaged Date:

TSD Accept Date: 03/25/82

Dose Rate: 1.000E+00

Container Type Code: DM Container Count: Container Volume:

0.419 Gross We:

Current Container ID:

Organic Volume: Organic Wt.:

Compaction Wt. (Prorated):

z Content Compaction:

Compactor PIN:

Comments/Description:

Designation Code:

DW Waste \#:

Generator Information:

Name:

ID: WHC

Facility ID: 23452

Charge Code: $\mathrm{K} 6$

Location Information:

Area Nane: 200W Facility ID: 218W4C Module: Surface Area:

Tier Level: Tier Position: Unit: T01

Lab Pack Description:

Lab Pack Flag: N

Physical State Code: S Chemical Nature Code: Primary Waste Type Code: R Secondary Waste Type Code: 1A

Container Size Description: 110 GALLON

Container Storage Category Code: SWIMS Waste Description Code: DS

Container Empty Tare Wt.:

Content Wrap Category:

Container Thermal Power:

SWSDR \#: 820162

Radioactive Information:

Total Alpha (Ci): SDAR Approval \#:

Container Total We.:

Total Pu Equivalent (Ci):

Total Beta-Gamma (CI): 1.000E-02

SWEA General Comments:

Total Pu Fissile Gram Equivalent: 


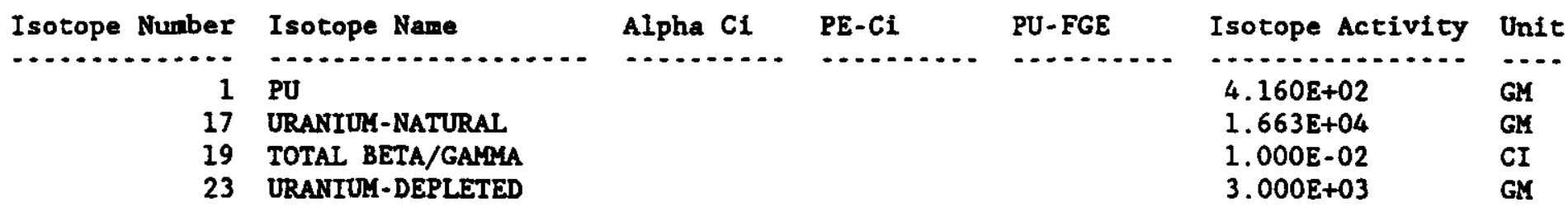

Hazardous Chenical Components:

Component Text

PPM

Weight (kg) $x$

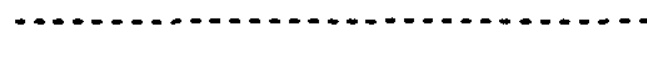

....... ...........

Physical Components:

Content Description

Volume $\%$ Weight $(\mathrm{kg})$

Desal

100

$\underset{\substack{1 \\ \hdashline}}{0}$

METAL/IRON/GALVANIZED/SHEET

Relocation History:

Shipment Information: 
for Secondary Waste Type Code: $x$

Container ID: $7774-423$

Location Beginning Coordinates: N N40190 w w77635 Location Ending Coordinates: N N40190 W w77609

Content Analysis Return Date:

Certification Date:

Dose Rate: 1.000E+00 Neutron Dose Rate:

Previous Container ID:

Current Container ID:

Compaction Wt. (Prorated):

Packaged Date:

TSD Accept Date: 03/25/82

Container Type Code: DM Container Count: Container Volume: $\quad 0.419$ Gross Wt:

136.08

Organic Volume: Organic Wt.:

* Content Compaction: Compactor PIN:

Designation Code: DW Waste \#:

Generator Information:

Name:

ID: WHC

Facility ID: $2345 Z$

Charge Code: $\mathrm{K} 6$

Location Information:

Area Name: 200W Facility ID: 218W4C Module: Surface Area:

Tier Level: Tier Position:

Unit: T01

Lab Pack Description:

Lab Pack Flag: N

Physical State Code: S Chemical Nature Code: Primary Waste Type Code: R Secondary Waste Type Code: lA

Container Size Description: 110 GALLON

Container Storage Category Code:

Container Empty Tare Wt.:

Content Wrap Category:

Radioactive Information:

Total Alpha (Ci):

Total Pu Equivalent (Ci):

Container Thermal Power: SDAR Approval \#:

SWEA General Comments:
Total Beta-Gamma (Ci): 1.000E-02

Total Pu Fissile Gram Equivalent:
SWSDR \#: 820163

Container Total Wt.: 


\begin{tabular}{|c|c|c|c|c|c|c|}
\hline Isotope Number & Isotope Name & Alpha $C_{i}$ & $\mathrm{PE}-\mathrm{Ci}$ & PU-FGE & Isotope Activity & Unit \\
\hline 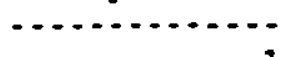 & (1) & $\cdots$ & $\cdots \cdots$ & $\ldots \ldots \ldots$ & and & $\cdots$ \\
\hline & & & & & $3.960 \mathrm{E}+02$ & GM \\
\hline 17 & URANIUM-NATURAL & & & & $1.663 E+04$ & GM \\
\hline 19 & TOTAL BETA/GAMMA & & & & $1.000 \mathrm{E}-02$ & CI \\
\hline 23 & URANIUIL-DEPLETED & & & & $3.000 E+03$ & GM \\
\hline
\end{tabular}

\section{Hazardous Chemical Components:}

Component Text

PPM
Component Text

Physical Components:

Content Description

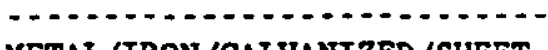

METAL/IRON/GALVANIZED/SHEET

Volume $\mathrm{x}$ Weight $(\mathrm{kg})$

......... -..........

100

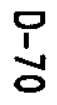

Relocation History:

Shipment Information: 
for Generating Company:

for Facility ID: $x$

for Primary Waste Type Code: $x$

for Secondary Waste Type Code: $x$

Container ID: $7774-424$

Location Beginning Coordinates: N N40190 W w77635 Location Ending Coordinates: N N40190 W w77609

Content Analysis Return Date:

Certification Date:

Packaged Date:

TSD Accept Date: $03 / 25 / 82$

Dose Race: 1.000E+00

Container Type Code: Dif

Container Volume:

0.419 Gross We:

136.08

Previous Container ID:

Current Container ID:

Organic Volume: Organic Wt.:

Compaction Wt. (Prorated): \% Content Compaction: Compactor PIN:

Coments/Description:

Designation Code: DW Waste \#:

P Generator Information:

$$
\text { Name: }
$$

Location Information:

Area Name: 200W Facility ID: 218W4C Module: Surface Area:

Tier Level: Tier Position:

ID: WHC

Facility ID: 23457

Charge Code: K6

Lab Pack Description:

Lab Pack Flag: N

Physical State Code: S Chemical Nature Code: Primary Waste Type Code: $R$ Secondary Waste Type Code: 1A

Container Size Description: 110 GALLON

Container Storage Category Code: SwIMS Waste Description Code: DS

Container Empty Tare Wt.:

Content Hrap Category:

Container Thermal Power:

SWSDR \#: 820170

SDAR Approval \#:

Radioactive Information:

Total Alpha (CI):

Total Pu Equivalent (Ci):

Total Beta-Gamma ( $\mathrm{C} i$ ): 1.000E-02

SWEA General Comments:

Total Pu Fissile Gram Equivalent: 


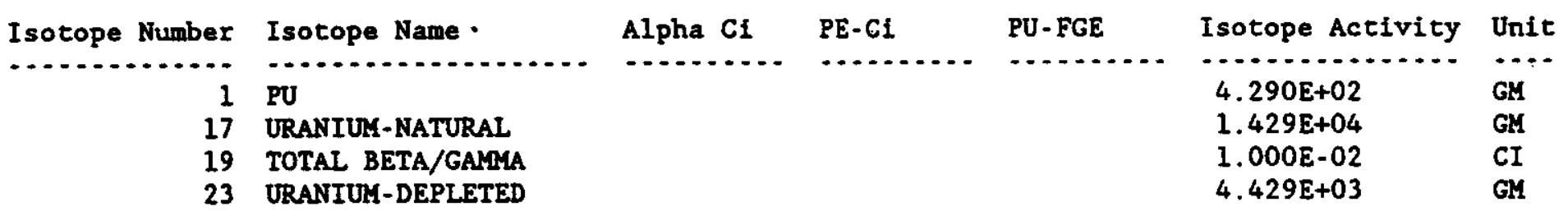

Hazardous Chemical Components:

$$
\text { Component Text }
$$

Physical Components:

Content Description

Volume $\%$ Weight (kg)

METAL/IRON/GALVANIZED/SHEET

Shipment Information: 
for Generating Company: $x$

for Facility ID: $\mathbf{z}$

for Primary Waste Type Code: $z$

for Secondary Waste Type Code: $x$

Container ID: $7774-425$

Location Beginning Coordinates: N N40190 W w77635 Location Ending Coordinates: N N40190 W

Content Analysis Return Date:

Certification Date:

Dose Rate: 1.000E+00 Neutron Dose Rate:

Previous Container ID:

Current Container ID:

Compaction Wt. (Prorated):

Packaged Date:

TSD Accept Date: 03/25/82

Container Type Code: DM Container Count:

Container Volume:

0.419 Gross Wt:

136.08

v. Organic Volume: Organic Wt.:

Comments/Description:

Designation Code: . DW Waste \#:

Generator Information:

Name:

z Content Compaction:

Compactor PIN:

ID : WHC

Facility ID: 23452

Charge Code: $\mathbf{6} 6$

Information:

Area Name: 200W Facility ID: 218W4C

Tier Level: Tier Position:

Module: Surface Area:

Lab Pack Description:

Lab Pack Flag: N

Physical State Code: S Chemical Nature Code: Primary Waste Type Code: R Secondary Waste Type Code: 1A

Container Size Description: 110 GALLON

Container Storage Category Code: SWIMS Waste Description Code: DS

Container Enpty Tare Wt.:

Content Wrap Category:

Container Thermal Power:

SWSDR \#: 820164

Radioactive Information:

Total Alpha (Ci):

Total Pu Equivalent (Ci): SDAR Approval \#:

Container Total Wt.

Total Beta-Gamma (Ci): 1.000E-02

SWEA General Comments:

Total Pu Fissile Gran Equivalent: 


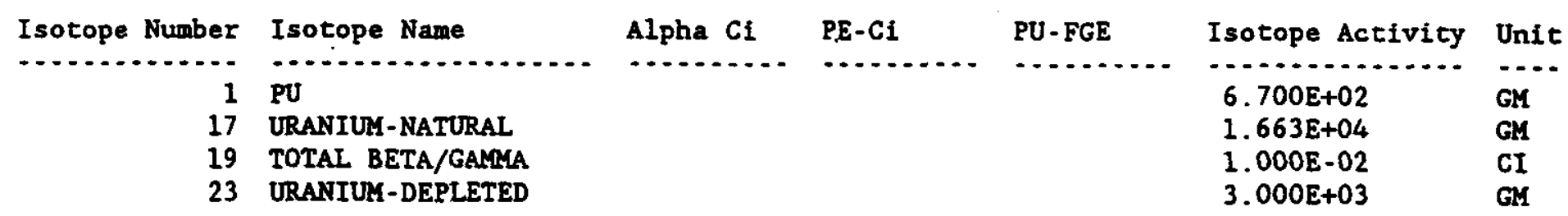

Hazardous Chemical Components:

Component Text

PPM Weight (kg) $\%$

- . .
Physical Components: Content Description

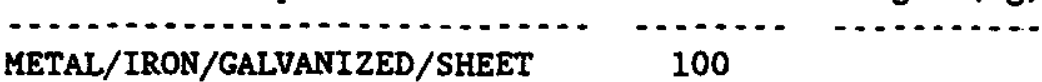

Shipment Information: 
for Secondary Waste Type Code: $z$

Container ID: $7774-426$

Location Beginning Coordinates: N N40190 w w77635 Location Ending Coordinates: N N40190 W w77609 Content Analysis Return Date:

Certification Date:

Container Type Code: DM

Container Count:

Dose Rate: 1.000E+00 Neutron Dose Rate: Container volume:

0.419 Gross Wt:

Previous Container ID:

Current Container ID:

Compaction Wt. (Prorated):

Organic Volume:

z Content Compaction:

Organic Wt.:

Compactor PIN:

Comments/Description:

Designation Code: DW Waste \#:

Generator Information:

Name:

Location Information:

Area Name: 200W Facility ID: 218W4C Module: Surface Area:

Tier Level: Tier Position:

Lab Pack Description:

Lab Pack Flag: N .

Physical State Code: S Chemical Nature Code: Primary Waste Type Code: R Secondary Waste Type Code: 1A

Container Size Description: 110 GALLON

Container Storage Category Code:

Container Eupty Tare Wt.:

Content Wrap Category:

Radioactive Information:

Total Alpha (Ci):

Total Pu Equivalent ( $\mathrm{Ci}$ ):

SWEA General Comments:
SWIMS Waste Description Code: DS Container Thermal Power: SDAR Approval \#:
SWSDR \#: 820171

Container Total wt.: 


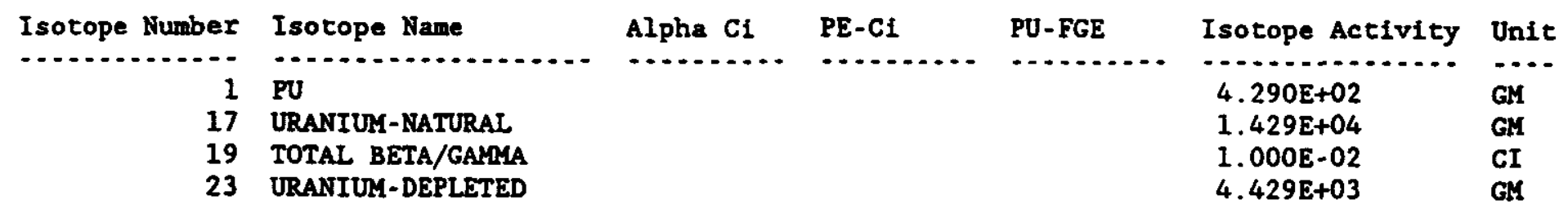

Hazardous Chemical Components: Component Text

PPM

Weight (kg) $\%$

-...........

(-...... - -...........

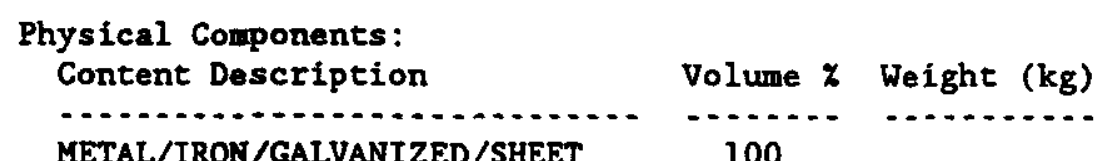

METAL/IRON/GALVANIZED/SHEET 100

Shipment Information: 
WHC-EP-0621

\section{APPENDIX E}

SOLID WASTE INFORMATION AND TRACKING SYSTEM RECORD FOR TRU CLASSIFIED DRUM RS-83-6-1 
WHC-EP-0621

This page intentionally left blank. 
SOLID WASTE INFORMATION AND TRACKING SYSTEM RECORD

FOR TRU CLASSIFIED DRUM RS-83-6-1

This appendix contains the complete Solid Waste Information and Tracking System (SWITS) record for the one classified transuranic (TRU) waste drum recorded for 234-SZ. 
for Secondary Waste Type Code: $x$

Container ID: RS-83-6-1

Location Beginning Coordinates: N N44540 w w77448 Location Enaing Coordinates: N N44540 W77756

Content Analysis Return Date: Packaged Date: TSD Accept Date: 07/03/84

Certification Date:

Container Type Code: DM Container Count: 1

Dose Rate: 1.100E+01 Neutron Dose Rate: Container Volume:

0.210 Gross

56.70

Previous Container ID:

Current Container ID:

Organic Volume: Organic Wt.:

Compaction Wt. (Prorated):

Comments/Description:

Designation Code:

I Generator Information:

Name:

Location Information:

$$
\begin{aligned}
& \text { Area Name: 200W Facility ID: 218\$3A } \text { Module: } \text { Surface Area: } \\
& \text { Tier Level: Tler Position: } \quad \text { Unit: T05 }
\end{aligned}
$$

Lab Pack Description:

Lab Pack Flag: N

Physical State Code: S Chemical Nature Code: Primary Vaste Type Code: R Secondary Waste Type Code: 1B

Container Size Description: 55 GALLON

Container Storage Category Code:

SuIMS Waste Description Code: DS

Container Empty Tare Wt.

27.00 Container Thermal Power:

SWSDR \#: 840139

Content Wrap Category: SDAR Approval \#:

Radioactive Information:

Total Alpha (CI):

Total Pu Equivalent (C1):

Tota1 Beta-Gamma (CI): 5.000E-02

SWEA General Comments: 


\begin{tabular}{|c|c|c|c|c|c|c|}
\hline Isotope Number & Isotope Name & Alpha $\mathrm{Ci}$ & PE-Ci & PU-FGE & Isotope Activity & Unit \\
\hline - & 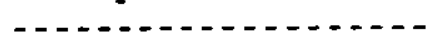 & 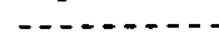 & $\cdots$ & $\ldots$ & - & \\
\hline 1 & & & & & $2.600 \mathrm{E}+01$ & GM \\
\hline 19 & TOTAL BETA/GAMMA & & & & $5.000 \mathrm{E}-02$ & CI \\
\hline 24 & URANIUM-ENRICHED & & & & $2.455 E+03$ & GM \\
\hline
\end{tabular}

Hazardous Chemical Components:

Component Text

PPM Weight (kg) \%

C...

-

Physical Components:

Content Description

Volume $\%$ Weight $(\mathrm{kg})$

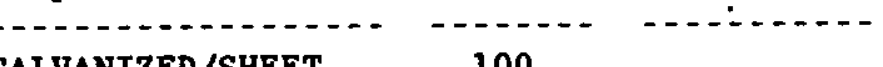

METAL/IRON/GALVANIZED/SHEET

100

i

Relocation History:

Shipment Information: 
WHC-EP-0621

This page intentionally left blank. 


\section{WHC-EP-0621}

\section{DISTRIBUTION}

Number of copies

OFFSITE

1

Los Alamos Technical Associates 8633 Gage Blvd.

Kennewick, Washington 99336

J. Pottmeyer

\section{ONSITE}

U.S. Department of EnerayRichland Field office

R. F. Guercia (2)

Westinghouse Hanford Company

D. R. Duncan (3)

H5-33

H5-33

B. A. Mayancsik

H5-33

M. M. McCarthy

G1 -19

E. P. Mertens

H5-33

J. G. Riddelle

R. J. Roberts

J. A. Swenson

Central Files

Document Processing and

$$
\begin{gathered}
\text { Distribution (2) } \\
\text { Information Release } \\
\text { Administration }
\end{gathered}
$$

H5-33

N3-13

G6- 45

L8-04

L8-04

L8-07

Pacific Northwest Laboratory

PNL Technical Files 